\title{
Physikalisch-technologische Untersuchungen zur Faserstofferzeugung für die Herstellung von Holzfaserdämmstoffen
}

\author{
Dissertation \\ zur Erlangung des Doktorgrades (Dr. rer. nat.) \\ der Fakultät für Forstwissenschaften und Waldökologie \\ der Georg-August-Universität Göttingen \\ vorgelegt von \\ Simon Valentin Barth \\ geboren am 18.11.1984 in Tübingen
}

Göttingen, September 2021 
1. Gutachter: Prof. Dr. Carsten Mai (Georg-August-Universität Göttingen)

2. Gutachter: Prof. Dr. Andreas Michanickl (Technische Hochschule Rosenheim)

3. Gutachter: Prof. Dr. Kai Zhang (Georg-August-Universität Göttingen)

Tag der mündlichen Prüfung: 12. November 2021 


\section{Inhaltsverzeichnis}

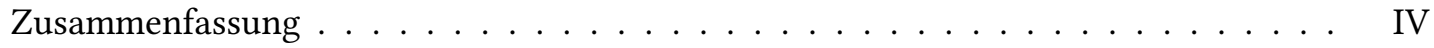

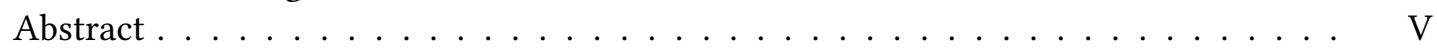

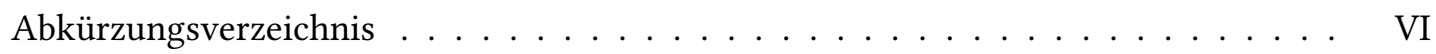

1 Einleitung 2

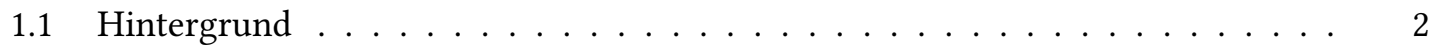

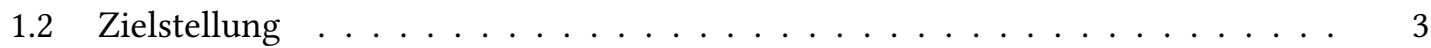

2 Charakterisierung von Holz als Faserrohstoff unter Berücksichtigung der Holzarten Fichte und Buche $\quad 5$

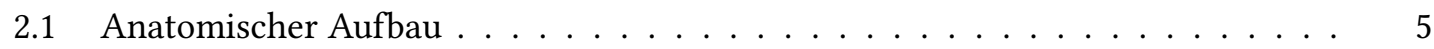

2.1.1 Strukturmerkmale . . . . . . . . . . . . . . . . . 5

2.1.2 Zusammensetzung und geometrische Variabilität des Holzgewebes . . . 6

2.2 Chemisch-struktureller Aufbau . . . . . . . . . . . . . . . . 8

2.2.1 Ultrastruktur und chemische Komponenten . . . . . . . . . . . . . 8

2.2.2 Verteilung der Komponenten im Zellverbund . . . . . . . . . . . . 10

2.3 Holzimmanente Eigenschaften . . . . . . . . . . . . . . . . . . . . . 11

2.3.1 Holzfeuchte . . . . . . . . . . . . . . . . . . . . . 11

2.3.2 Erweichungsverhalten . . . . . . . . . . . . . . . . . . 12

2.3.3 Wärmeleitung. . . . . . . . . . . . . . . . . . . . . . 15

3 Technologie der Faserstofferzeugung 17

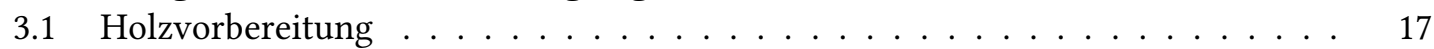

3.2 Chemischer Aufschluss (Zellstoffherstellung) . . . . . . . . . . . . . . . . 17

3.3 Mechanische Faserstofferzeugung . . . . . . . . . . . . . . . . . . . 17

3.3 .1 Holzschliff . . . . . . . . . . . . . . . . . . . . . . . . . . . 17

$3.3 .2 \quad$ RMP-Verfahren . . . . . . . . . . . . . . . . . . . 18

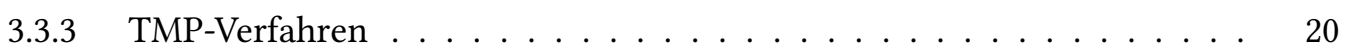

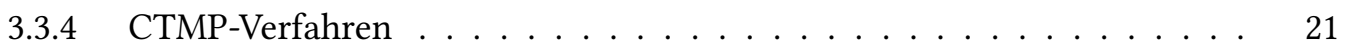

3.4 Mahlscheiben . . . . . . . . . . . . . . . . . . 22

3.4.1 Konstruktionsmerkmale . . . . . . . . . . . . . . . . 22

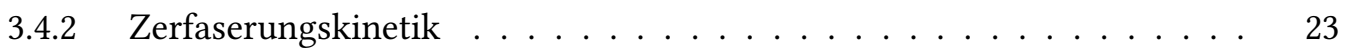

3.4 .3 Simulation . . . . . . . . . . . . . . . . . . 24

4 Charakterisierung der Beschaffenheit von Faserstoffen 26

4.1 Zusammensetzung und ausgewählte Eigenschaften . . . . . . . . . . . . . 26

4.2 Dispersitätseigenschaften und Partikelmesstechnik . . . . . . . . . . . . . . 27

4.3 Mikroskopische Untersuchungen . . . . . . . . . . . . . . . . . . . . 29

5 Herstellung und ausgewählte Eigenschaften von Holzfaserdämmstoffen $\quad 30$

5.1 Rohstoffe, Zusatzstoffe, Hilfsstoffe und Klebstoffe . . . . . . . . . . . . . . . . 30 
$5.2 \quad$ Spezielle Prozesstechnik . . . . . . . . . . . . . . . . . . . . . . . . . 31

5.3 Aspekte der Wärmeleitfähigkeit . . . . . . . . . . . . . . . . . . . . . 31

6 Material und Methoden $\quad 34$

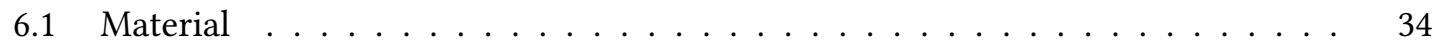

6.1.1 Beschreibung des Ausgangsmaterials . . . . . . . . . . . . . 34

6.1.2 Industrielle Zellstoffe, Holzstoffe und Holzfaserdämmstoffe . . . . . . 34

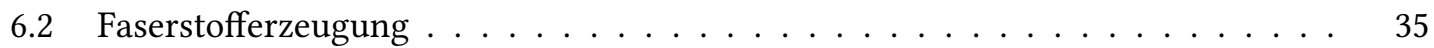

6.2.1 Spezifikationen der Mahlscheiben . . . . . . . . . . . . . . . . . 35

6.2.2 Konfiguration der Laborrefineranlage im TMP-Verfahren . . . . . . . . . 39

6.2.3 Konfiguration der Laborrefineranlage im RMP-Verfahren . . . . . . . . . . 41

6.2.4 Erfassung von Prozessparametern . . . . . . . . . . . . . . . . 43

6.3 Vliesbildung zur Bestimmung der Wärmeleitfähigkeit . . . . . . . . . . . . . . 43

6.4 Analytische Methoden . . . . . . . . . . . . . . . . . . . . . 44

6.4.1 Grundlegende Untersuchungen zur Prozessführung in der labortechnischen Faserstofferzeugung . . . . . . . . . . . . . . . . 44

6.4.2 Bestimmung der Holzfeuchte von Hackschnitzeln in Sattdampfatmosphäre 45

6.4.3 Bestimmung der spezifischen elektrischen Leistungsaufnahme in der Fa-

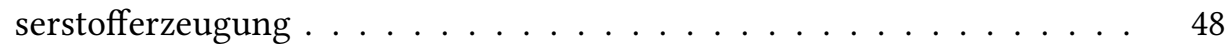

6.4.4 Bestimmung der Feuchte und Konsistenz von Faserstoffen . . . . . . . . 49

6.4.5 Bestimmung der Dispersitätseigenschaften von Faserstoffen mit der Kollektivmethode Luftstrahl-Prüfsiebung . . . . . . . . . . . . . 49

6.4.6 Bestimmung der Dispersitätseigenschaften von Faserstoffen mit der Individualmethode quantitative Bildanalyse . . . . . . . . . . . 51

6.4.7 Mikroskopische Untersuchungen an den Faserstoffen . . . . . . . . . . . 54

6.4.8 Bestimmung der Wärmeleitfähigkeit von Faserstoffen . . . . . . . . . . 54

6.5 Datenverarbeitung und statistische Methoden . . . . . . . . . . . . . . 56

7 Ergebnisse und Diskussion

7.1 Grundlegende Untersuchungen zur Prozessführung in der labortechnischen Fa-

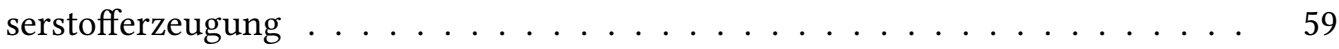

7.1 .1 Einleitung . . . . . . . . . . . . . . . . . . . . 59

7.1.2 Ausgangs-Partikelgröße der Laborhackschnitzel und Ziel-Partikelgröße industrieller Faserstoffe . . . . . . . . . . . . . . . .

7.1.3 Untersuchung grundlegender Parameter der Prozessführung auf ausgewählte Zielgrößen der labortechnischen Faserstofferzeugung . . . . . . 64

7.1.4 Erkenntnisse und Konsequenzen für die weiteren Untersuchungen . . . 69

7.2 Untersuchungen zur Holzfeuchte von Hackschnitzeln in Sattdampfatmosphäre . 71

7.2 .1 Einleitung . . . . . . . . . . . . . . . . 71

7.2.2 Absorption von Wasser in Abhängigkeit des Sattdampfdrucks . . . . . . 72

7.2.3 Holzfeuchte nach Entspannung des Sattdampfdrucks . . . . . . . . . . . 80

7.2.4 Zeitlicher Verlauf der Absorption und Desorption . . . . . . . . . . . . 81

7.3 Untersuchungen zur spezifischen elektrischen Leistungsaufnahme in der Faser-

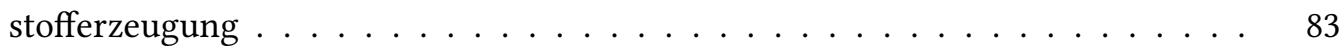

$7.3 .1 \quad$ Einleitung . . . . . . . . . . . . . . . . 83

7.3.2 Spezifische elektrische Leistungsaufnahme im TMP-Verfahren . . . . . . 83

7.3.3 Spezifische elektrische Leistungsaufnahme im RMP-Verfahren . . . . . . 100 
7.4 Untersuchungen zum elektrischen und thermischen Energiebedarf in der Faser-

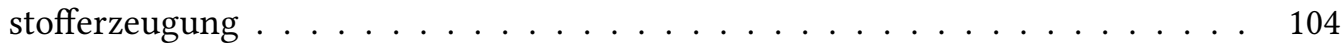

$7.4 .1 \quad$ Einleitung . . . . . . . . . . . . . . . . . . . . . 104

7.4.2 Spezifische elektrische Leistungsaufnahme als Funktion der Temperatur 105

7.4.3 Holzfeuchte und Konsistenz nach dem Aufschluss . . . . . . . . . . . . 109

7.4.4 Elektrischer und thermischer Energiebedarf in der Faserstofferzeugung mit dem RMP- und dem TMP-Verfahren . . . . . . . . . . . . . . . . 111

7.5 Untersuchungen zur Beschaffenheit der Faserstoffe . . . . . . . . . . . . . . . 115

7.5.1 Einleitung . . . . . . . . . . . . . . . . . . . . 115

7.5.2 Dispersitätseigenschaften nach der Kollektivmethode Luftstrahl-Prüfsiebung 115

7.5.3 Dispersitätseigenschaften nach der Individualmethode quantitative Bild-

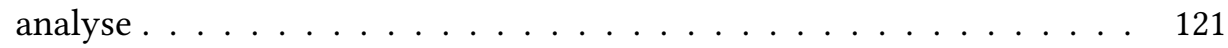

7.5.4 Effizienz in der Faserstofferzeugung . . . . . . . . . . . . . . . 131

7.5.5 Mikroskopische Untersuchungen an den Faserstoffen . . . . . . . . . . 134

7.6 Untersuchungen zur Wärmeleitfähigkeit der Faserstoffe . . . . . . . . . . . . . . 141

7.6 .1 Einführung . . . . . . . . . . . . . . . . . . . 141

7.6.2 Wärmeleitfähigkeit der Faserstoffe . . . . . . . . . . . . . . . 141

7.6.3 Zusammenhänge zwischen Faserstofferzeugung, Dispersitätseigenschaften und der Wärmeleitfähigkeit . . . . . . . . . . . . . . . . 147

8 Abschließende und zusammenfassende Diskussion mit Ausblick 150

8.1 Physikalisch-technologischer Erkenntnisgewinn zur Faserstofferzeugung für die

Herstellung von Holzfaserdämmstoffen . . . . . . . . . . . . . . . 150

8.2 Nutzen von Laborversuchen und Übertragung der Ergebnisse in den industriellen Maßstab . . . . . . . . . . . . . . . . . . . 153

9 Danksagung $\quad 170$

$\begin{array}{ll}10 \text { Anhang } & 171\end{array}$

10.1 R-Codes zur Datenverarbeitung und graphischen Darstellung . . . . . . . 171

10.2 Korrelationen mit der Wärmeleitfähigkeit . . . . . . . . . . . . . . . . . . 175

10.3 Veröffentlichungen . . . . . . . . . . . . . . . . . . 180 


\section{Zusammenfassung}

Die Zielstellung der Arbeit war es, die Faserstofferzeugung für die Herstellung von Holzfaserdämmstoffen zu untersuchen, um zu einem besseren Verständnis der dort stattfindenden physikalischen Vorgänge beizutragen. Für die Bearbeitung der Zielstellung wurden physikalische Experimente zur Faserstofferzeugung im Labormaßstab mit dem TMP-Verfahren (Thermo Mechanical Pulp) und dem RMP-Verfahren (Refiner Mechanical Pulp) sowie den Holzarten Fichte (Picea abies Karst.) und Buche (Fagus sylvatica L.) durchgeführt.

Aus den grundlegenden Untersuchungen zur Prozessführung der labortechnischen Faserstofferzeugung konnte eine Vorgehensweise abgeleitet werden, mit der reproduzierbare Ergebnisse in der Untersuchung von Mahlscheiben erzielt werden. Die Untersuchung der Sorption von Wasserdampf durch Hackschnitzel in Sattdampfatmosphäre mit einem Sattdampfdruck zwischen 1 bar und 9 bar respektive einer Temperatur von $120^{\circ} \mathrm{C}$ bis $180^{\circ} \mathrm{C}$ wurde mittels einer gravimetrischen Messeinrichtung ermittelt. Mit dieser in situ Bestimmung der Holzfeuchte wurde eine vom Sattdampfdruck und der Ausgangs-Holzfeuchte abhängige Absorption von Wasser nachgewiesen, die mit der berechneten Wärmemenge zur Erwärmung der Hackschnitzel assoziiert ist. Die Holzfeuchte nach Entspannung des Sattdampfdrucks war unabhängig von der Höhe des Sattdampfdrucks und stellte sich oberhalb der Ausgangs-Holzfeuchte ein. Der zeitliche Verlauf der Wasseraufnahme und -abgabe erfolgte simultan zum Anstieg und Abfall des Sattdampfdrucks. Die Untersuchungen zur spezifischen elektrischen Leistungsaufnahme im TMP-Verfahren zeigten ferner, dass signifikante Unterschiede bezüglich der Holzarten, der Aufschlusstemperaturen und der Mahlscheibenmuster bestanden. Die Faserstofferzeugung mit der Holzart Buche zog, im Vergleich zur Holzart Fichte, eine geringere spezifische elektrische Leistungsaufnahme nach sich. Mit steigender Aufschlusstemperatur nahm die spezifische elektrische Leistungsaufnahme bei beiden untersuchten Holzarten ab und die Differenzen zwischen den Mahlscheibenmustern wurden geringer. Im RMP-Verfahren konnte die prinzipielle Machbarkeit der Faserstofferzeugung für Holzfaserdämmstoffe mit einem speziell entwickelten Mahlscheibenmuster belegt werden. Aus den Versuchen zur Faserstofferzeugung wurde die Erkenntnis gewonnen, dass neben dem Erweichungsverhalten der Holzarten, insbesondere die Gestaltung der Mahlscheibe mit der Reduzierung der spezifischen elektrischen Leistungsaufnahme einherging. Aus den weiteren Untersuchungen zum elektrischen und thermischen Energiebedarf in der Faserstofferzeugung entstand die Erkenntnis, dass neben dem Sattdampfdruck respektive der Aufschlusstemperatur die spezifische elektrische Leistungsaufnahme in Beziehung mit der Konsistenz der Faserstoffe nach dem Aufschluss steht.

Die Analyse zur Beschaffenheit der Faserstoffe wurde mit der Kollektivmethode LuftstrahlPrüfsiebung und der Individualmethode quantitative Bildanalyse durchgeführt. Mit beiden Methoden konnten Unterschiede in den Dispersitätseigenschaften bezüglich der Holzarten, der Aufschlusstemperaturen und der Mahlscheibenmuster nachgewiesen werden. Sowohl mit der Luftstrahl-Prüfsiebung als auch mit der quantitativen Bildanalyse wurde festgestellt, dass die Feinheit der Partikel mit steigender Aufschlusstemperatur zunimmt. Die Wärmeleitfähigkeit wurde im Zweiplattenverfahren an Faserstoffprobekörpern ermittelt, die mit einer aerodynamischen Vlieslegung gebildet wurden. Korrelationstabellen zur Identifizierung von funktionalen Zusammenhängen zwischen Dispersitätseigenschaften und der Wärmeleitfähigkeit lassen den Schluss zu, dass die Feinheit der faserartigen Partikel in Beziehung mit der Wärmeleitfähigkeit stehen. Insgesamt boten spezielle Mahlscheibenmuster ein hohes Potenzial, um den elektrischen und thermischen Energiebedarf im Prozess der Faserstofferzeugung zu senken und die Beschaffenheit der Faserstoffe für die Herstellung von Holzfaserdämmstoffen zu optimieren. 


\section{Abstract}

The aim of this work was to investigate the process step of pulp production for the manufacture of wood fibre insulation materials in order to contribute to a better understanding of the physical processes taking place. For this purpose, physical experiments on a laboratory scale were carried out in the thermo mechanical pulping process (TMP) and refiner mechanical pulping process (RMP) with spruce (Fagus sylvatica L.) as well as with beech (Fagus sylvatica L.).

From the basic investigations on the process of the pulp production with a laboratory refiner plant, it was possible to derive a method to gain reproducible results in the testing of refiner plates. The procedure applied yielded results that are comparable to those obtained in industrial pulp production. The investigation of the sorption of water vapor by wood chips in saturated steam atmosphere with a pressure between 1 bar and 9 bar respectively a temperature from $120^{\circ} \mathrm{C}$ to $180^{\circ} \mathrm{C}$ was determined by means of a gravimetric measuring device. With this in situ determination the moisture content of the wood was determined as a function of the saturated steam pressure and the initial moisture content of the wood, which was then compared with the calculated amount of steam for heating the wood chips. The wood moisture content after relaxation of the saturated steam pressure was independent of its level and was above the initial wood moisture content. The time course of water uptake and release occurred simultaneously with the rise and decrease of the saturated steam pressure. The investigations on the specific electrical power consumption in the TMP process also showed that significant differences exist with respect to the wood species, pulping temperatures and the refiner plate patterns. Compared to spruce, the pulp production with the beech resulted in a lower specific electrical power consumption. With increasing pulping temperature, the specific electrical power consumption decreased for both species, and the differences between the refiner plate patterns became smaller. For the RMP process the feasibility in principle of producing pulps for wood fiber insulation materials was demonstrated with a with a specially developed refiner plate pattern.

From the pulp production trials, the insight was gained that, in addition to the softening behavior of the wood species, especially the design of the refiner plate correlates with the reduction of the specific electrical power consumption. From the further investigations on the electrical and thermal power consumption in pulp production, it was discovered that the moisture content of the pulp after refining is related to the pulping temperature and the specific electrical power requirement.

The analysis of pulp was carried out using the collective method of air-jet sieving and the individual method of quantitative image analysis. With both methods, significant differences in the dispersity characteristics with respect to the wood species, pulping temperatures, and refiner plate patterns were demonstrated. With both air jet test sieving and quantitative image analysis, it was found, that the fineness of the particles increases with elevated pulping temperature.

The thermal conductivity was determined by the two-plate method on dry pulp samples which were formed with an aerodynamic nonwoven layup. Correlation tables to identify functional relationships between dispersivity properties and thermal conductivity indicated that a high fineness of the fibrous particles is related to a low thermal conductivity. Overall, special refiner plates offered a high potential to reduce the electrical and thermal energy requirements in the pulp production process and to optimize the properties of the pulp for the production of wood fiber insulation materials. 


\section{Abkürzungsverzeichnis}

Alphabetische Auflistung von Einheiten, Sonder- und Formelzeichen

${ }^{\circ} d H \quad$ Grad deutscher Härte

$\rightarrow \infty \quad$ gegen unendlich

" Zoll

A Fläche

$A_{i} \quad$ Partikelfläche

$A_{p} \quad$ durchschnittliche Partikeloberfläche

$c_{H} \quad$ spezifische Wärmekapazität von $\mathrm{Holz}$

$C_{i} \quad$ flächenäquivalenter Durchmesser

$c_{W} \quad$ spezifische Wärmekapazität von Wasser

d Tag

$d \quad$ Dicke

$\Delta T \quad$ Temperaturdifferenz

$\Delta x_{r} \quad$ Partikelausdehnungsintervall

$\eta \quad$ Effizienz

$h \quad$ Stunde

$\mathrm{Hz} \quad$ Hertz

$J \quad$ Joule

K Kelvin

kJ Kilojoule

$k W \quad$ Kilowatt

$k W h \quad$ Kilowattstunde

$l \quad$ Liter

$\lambda \quad$ Wärmeleitfähigkeit

$\lambda_{10} \quad$ Wärmeleitfähigkeit bei einer Probenmitteltemperatur von $10^{\circ} \mathrm{C}$

$\lambda_{90 / 90} \quad 90 \%$ der Messwerte sind mit $90 \%$-iger Wahrscheinlichkeit kleiner oder gleich dem Nennwert der Wärmeleitfähigkeit

$\lambda_{D} \quad$ Nennwert der Wärmeleitfähigkeit

$\lambda_{w \|} \quad$ Wärmeleitung parallel zur Faser

$\lambda_{w \perp} \quad$ Wärmeleitung senkrecht zur Faser

$\mu m \quad$ Mikrometer

$m \quad$ Meter

$M^{\prime} \quad$ Speichermodul

$M^{\prime \prime} \quad$ Verlustmodul 


\begin{tabular}{|c|c|}
\hline$m_{0}$ & Trockenmasse \\
\hline$\dot{M}_{\text {atro }}$ & Massenfluss absolut trocken \\
\hline$m_{D}$ & Dampfmenge \\
\hline$m_{D, Q}$ & Dampfmenge für die Erwärmung \\
\hline$m_{H}$ & Masse Holz \\
\hline$m_{i}$ & Masse Einwaage \\
\hline$m_{s}$ & feuchte Masse der Hackschnitzel \\
\hline$m_{u}$ & feuchte Masse \\
\hline$m_{W}$ & Masse von Wasser \\
\hline$m x_{r}$ & mittlere Partikelausdehnung \\
\hline$m g$ & Miligramm \\
\hline $\min$ & Minute \\
\hline $\min ^{-1}$ & Umdrehungen pro Minute \\
\hline$m l$ & Mililiter \\
\hline$m m$ & Milimeter \\
\hline$n$ & Anzahl/Stichprobenumfang/Wiederholungen \\
\hline$N$ & Grundgesamtheit \\
\hline$P_{e l}$ & elektrische Leistung \\
\hline$P_{N T}$ & elektrische „no-treatment“-Leistung \\
\hline$P_{r e f}$ & elektrische Nettoleistung \\
\hline$P_{\text {spez }}$ & spezifische elektrische Leistungsaufnahme \\
\hline$\dot{Q}$ & Wärmestrom \\
\hline$Q$ & Wärmemenge \\
\hline$q_{r}$ & Verteilungsdichte \\
\hline$Q_{r}$ & Verteilungssumme \\
\hline$Q_{\text {spez }}$ & spezifischer thermischer Energiebedarf \\
\hline$\rho_{w}$ & Rohdichte der trockenen Holzsubstanz \\
\hline$r$ & $\begin{array}{l}\text { Mengenart, Index einer Verteilungsfunktion: } r=0 \text { : Anzahl; } \\
r=2 \text { : Fläche; } r=3 \text { : Masse }\end{array}$ \\
\hline$r$ & Verdampfungsenthalpie \\
\hline$R$ & Korrelationskoeffizient \\
\hline$R^{2}$ & Bestimmtheitsmaß \\
\hline$r_{W}$ & Verdampfungsenthalpie von Wasser \\
\hline$\sigma$ & Standardabweichung \\
\hline $\tan \delta$ & Verlustfaktor \\
\hline $\tan \delta_{\max }$ & Maximum des $\alpha$-Peaks des Verlustfaktors \\
\hline$t$ & Tonne \\
\hline$T_{g}$ & Erweichungstemperatur \\
\hline$u_{d}$ & gemessene Holzfeuchte nach Entspannung des Sattdampfdrucks \\
\hline$u_{i}$ & Ausgangs-Holzfeuchte \\
\hline
\end{tabular}




$\begin{array}{ll}u_{g} & \begin{array}{l}\text { gemessene Holzfeuchte } \\ u_{r}\end{array} \\ V & \text { relative Holzfeuchte } \\ & \text { Volt } \\ W & \text { Watt } \\ W h & \text { Wattstunde } \\ x_{16, r} & \text { Partikelgröße des } 16-\% \text { Perzentils der Verteilungssumme } \\ x_{50, r} & \text { Partikelgröße des Medianwerts der Verteilungssumme } \\ x_{84} & \text { Partikelgröße des } 84 \text {-\% Perzentils der Verteilungssumme } \\ x_{97, r} & \text { Partikelgröße des } 97 \% \text {-Perzentils der Verteilungssumme } \\ x_{E} & \text { geodätische Dicke } \\ x_{F m a x} & \text { maximaler Feret-Durchmesser } \\ x_{i} & \text { Partikel-Formdeskriptor } \\ x_{L G} & \text { geodätische Länge } \\ \bar{x} & \text { arithmetisches Mittel } \\ x_{r} & \text { mittlere Partikelausdehnung } \\ y_{A} & \text { flächenbezogene Gewichtungsfunktion }\end{array}$

\section{Alphabetische Auflistung von Abkürzungen}

$\begin{array}{ll}\text { atro } & \text { absolut trocken } \\ \text { BCTMP } & \text { bleached chemi-thermomechanical pulp } \\ \text { Bikofaser } & \text { Thermoplastische Zweikomponenten-Kunststofffaser } \\ \text { BMEL } & \begin{array}{l}\text { Bundesministerium für Ernährung und Landwirtschaft } \\ \text { bzw. }\end{array} \\ & \text { beziehungsweise } \\ \text { ca. } & \text { circa } \\ \text { CC } & \text { cell corner region, Zellecke } \\ \text { CML } & \text { Primärwand-Mittellamelle-Komplex, compound middle lamella } \\ \text { CMP } & \text { chemi mechanical pulp } \\ \text { CSF } & \text { canadian standard freeness } \\ \text { CTMP } & \text { chemi-thermomechanical pulp } \\ \text { DMA } & \text { Dynamisch-mechanische Analyse } \\ \text { DMTA } & \text { Dynamisch-mechanisch-thermische Analyse } \\ \text { dpi } & \text { dots per inch, Bildpunkte } \\ \text { E } & \text { Elongation } \\ & \\ \text { f. } & \text { auf der folgenden Seite } \\ \text { fa } & \text { faserartig } \\ \text { FB } & \text { Faserbündel } \\ \text { ff. } & \text { auf den nächsten Seiten } \\ \text { FS } & \text { Feinstoff } \\ \text { FSB } & \text { Fasersättigungsbereich }\end{array}$




\begin{tabular}{|c|c|}
\hline $\begin{array}{l}\text { GS } \\
\text { ggf. }\end{array}$ & $\begin{array}{l}\text { Grobstoff } \\
\text { gegebenenfalls }\end{array}$ \\
\hline $\mathrm{HC}$ & high consistency, hohe Konsistenz \\
\hline HDF & high density fibreboard, hochdichte Faserplatte \\
\hline HSD & honestly significant difference, ehrliche Signifikanz \\
\hline IQR & interquartile range, Interquartilabstand \\
\hline K & Konsistenz \\
\hline Karst. & standardisiertes Kürzel für „Hermann Karsten“ \\
\hline $\mathrm{kf}$ & kornförmig \\
\hline $\mathrm{Kf}$ & Kurzfaser \\
\hline $\mathrm{L}$. & standardisiertes Kürzel für „Carl von Linné“ \\
\hline LC & low consistency, niedrige Konsistenz \\
\hline LF & Langfaser \\
\hline LOESS & $\begin{array}{l}\text { locally estimated scatterplot smoothing, } \\
\text { lokal geschätzte Streudiagrammglättung }\end{array}$ \\
\hline $\max$. & maximal \\
\hline MDF & medium density fibreboard, mitteldichte Faserplatte \\
\hline MDI & Diphenyl-methan-diisocyanat \\
\hline MFA & micro fibril angle, Mikrofibrillenwinkel \\
\hline ML & Mittellamelle \\
\hline n.a. & not available, nicht verfügbar \\
\hline NBSK & $\begin{array}{l}\text { northern bleached softwood kraft, } \\
\text { nördliches gebleichtes Nadelholz Kraftpapier }\end{array}$ \\
\hline NIR & near-infrared, Nahinfrarot \\
\hline $\mathrm{P}$ & Primärwand \\
\hline RMP & Refiner Mechanical Pulp, mechanischer Refiner Holzstoff \\
\hline s. & siehe \\
\hline S1 & erste Sekundärwandschicht \\
\hline $\mathrm{S} 2$ & zweite Sekundärwandschicht \\
\hline S3 & dritte Sekundärwandschicht \\
\hline SEL & spezifische elektrische Leistungsaufnahme \\
\hline $\mathrm{T}$ & Tertiärwand \\
\hline TEM & Transmisionselektronenmikroskop \\
\hline TMP & Thermo Mechanical Pulp, thermomechanischer Refiner Holzstoff \\
\hline $\mathrm{u}$ & Holzfeuchte \\
\hline u.a. & unter anderem \\
\hline UDP & Uridindiphosphat \\
\hline
\end{tabular}


vgl. vergleiche

z. B. zum Beispiel 
„My precious.“ J.R.R. Tolkien 


\section{Einleitung}

\subsection{Hintergrund}

Der steigenden Produktionskapazität von Holzfaserdämmstoffen in Deutschland (Mantau 2012) und Europa (Euwid 05.07.2018) steht, gemessen an der Anzahl wissenschaftlicher Veröffentlichungen und geförderter Drittmittelprojekte, im Vergleich zu anderen Holzwerkstoffen wie mitteldichte Faserplatte (MDF) eine vergleichsweise geringe Forschungsaktivität gegenüber. Angesichts des Marktpotenzials, nicht zuletzt durch die Gesetzgebung bezüglich der Energieeinsparung in Gebäuden (Deutscher Bundestag 2020), stellen Holzfaserdämmstoffe nach wie vor ein Nischenprodukt mit einem langsam wachsenden Marktanteil dar (Fachagentur Nachwachsende Rohstoffe e.V. 2020). Aus Sicht der Holzwerkstoffindustrie wird die Marktsituation von synthetischen und anorganischen Dämmstoffen dominiert. Holzfaserdämmstoffe sind für die Verwendung in der Baubranche durch die geringere Verfügbarkeit und die höheren Herstellungskosten gegenüber den Konkurrenzprodukten ökonomisch weniger attraktiv (Pavel \& Blagoeva 2018, S. 11). Trotz deutlich höherer Rohstoffkosten der synthetischen Dämmstoffe können diese Produkte mit teilweise besseren Eigenschaften günstiger angeboten werden (Barth 2019). Zudem bestehen beispielsweise aufgrund der Schwel- bzw. Glimmneigung von Holzfaserdämmstoffen bauordnungsrechtliche Hürden, die einer Erweiterung der Marktanteile zusätzlich im Weg stehen (Steeger et al. 2017).

Hobballah et al. (2018) identifizierten Optimierungspotenziale für Holzfaserdämmstoffe mit mathematischen Methoden und stuften unter anderem die Senkung der Wärmeleitfähigkeit und Herstellungskosten als erforderlich für die Steigerung der Wettbewerbsfähigkeit ein. In der vorliegenden Dissertation wird deshalb die Faserstofferzeugung für die Herstellung von Holzfaserdämmstoffen untersucht, denn solch ein Zerkleinerungsprozess verursacht nach Schönert (2003, S. 300) maßgebliche Kosten in der Produktion. Die Entwicklung und Untersuchung spezieller Mahlscheibenmuster im zentralen Prozessschritt der Faserstofferzeugung bietet eine kostengünstige Optimierungsmöglichkeit, denn es handelt sich hierbei um Verschleißteile, die regelmäßig ausgetauscht werden. Aus der industriellen Herstellung von MDF ist außerdem bekannt, dass durch die Entwicklung von Mahlscheiben die spezifische elektrische Leistungsaufnahme gesenkt und die Produkteigenschaften verbessert werden können (Börste 2008).

Im modernen Trockenverfahren zur Herstellung von Holzfaserdämmstoffen wird zur Faserstofferzeugung MDF-Technologie eingesetzt (Antrag auf Nichtnennung 1998). Vor dem Hintergrund der von MDF grundsätzlich verschiedenen, maßgeblichen Produkteigenschaft Wärmeleitfähigkeit ist es fraglich, ob in der Herstellung von Holzfaserdämmstoffen derzeit das wirtschaftlich und technisch optimale Verfahren zur Faserstofferzeugung eingesetzt wird. Die Eignung von Laubund Nadelhölzern in der MDF-Herstellung und der Einfluss ihrer Morphologie auf die physikalischen Produkteigenschaften sind grundlegend untersucht und deren Verwendung seit längerem Stand der Technik (Roffael et al. 1994a,b, 1995). Über den Einfluss von Mahlscheiben und von Aufschlussbedingungen in der Faserstofferzeugung für die Herstellung von Holzfaserdämmstoffen im TMP-Verfahren (Thermo Mechanical Pulp) mit Buchen- und Fichtenholz lagen zu Beginn der Bearbeitung entweder keine Ergebnisse von systematischen Untersuchungen zur spezifischen 
elektrischen Leistungsaufnahme vor oder es bestanden Wissenslücken bezüglich der Wärmeleitfähigkeit. Das RMP-Verfahren (Refiner Mechanical Pulp) wurde seit den 1960er Jahren in der Papierherstellung eingesetzt und im Zusammenhang mit der Holzfaserdämmstoffherstellung bislang nicht auf die Machbarkeit und Energieeinsparpotenziale hin untersucht. Inwiefern Unterschiede in der Beschaffenheit der Faserstoffe (Faserlänge, Faserfeinheit, Anteile der Partikelklassen, anatomische Unterschiede der Holzarten) in Beziehung zur Wärmeleitfähigkeit stehen, ist nicht gesichert.

\subsection{Zielstellung}

Die Zielstellung der Arbeit umfasst grundlegende physikalische und technologische Fragestellungen zum Einfluss unterschiedlicher Mahlscheibenmuster sowie Aufschlusstemperaturen auf den thermischen und elektrischen Energiebedarf in der Faserstofferzeugung. Das übergeordnete Ziel ist es, den Prozessschritt der Faserstofferzeugung für die Herstellung von Holzfaserdämmstoffen zu untersuchen, um zu einem besseren Verständnis der dort stattfindenden physikalischen Vorgänge beizutragen. Für die Bearbeitung der Zielstellung werden physikalische Experimente zur Faserstofferzeugung im Labormaßstab mit dem TMP-Verfahren (Thermo Mechanical Pulp) und dem RMP-Verfahren (Refiner Mechanical Pulp) sowie den Holzarten Fichte (Picea abies Karst.) und Buche (Fagus sylvatica L.) durchgeführt. Zu Beginn der Arbeit wird der Stand der Wissenschaft und Technik zu Holz als Faserrohstoff, zur Faserstofferzeugung im TMP- und RMP-Verfahren, der Charakterisierung von Faserstoffen sowie zur Herstellung von Holzfaserdämmstoffen zusammengefasst. Aus den hierbei gewonnenen Erkenntnissen wird der Forschungs- und Entwicklungsbedarf des Themas herausgearbeitet. Im speziellen Teil der Arbeit werden dazu experimentelle physikalische und technologische Untersuchungen durchgeführt sowie unterschiedliche Methoden zur Analyse der Beschaffenheit und der Eigenschaften von Faserstoffen angewendet. Die eigenen Untersuchungen sind zu diesem Zweck in sechs Teilaspekte untergliedert.

Teilaspekt 1 widmet sich grundlegenden Untersuchungen zur Prozessführung in der labortechnischen Faserstofferzeugung mit der zentralen Forschungsfrage: Welche Auswirkungen hat die Prozessführung auf ausgewählte Zielgrößen der labortechnischen Faserstofferzeugung?

In Teilaspekt 2 werden Untersuchungen zur Holzfeuchte von Hackschnitzeln in Sattdampfatmosphäre durchgeführt. Hierbei wird eine Antwort auf die Frage gesucht, wie sich die Holzfeuchte in Sattdampfatmosphäre unter erhöhtem Druck und erhöhter Temperatur verhält.

Der Teilaspekt 3 enthält Untersuchungen zur spezifischen elektrischen Leistungsaufnahme in der Faserstofferzeugung. In diesem Zusammenhang wird der Fragestellung nachgegangen, welchen Einfluss unterschiedliche Mahlscheibenmuster auf die spezifische elektrische Leistungsaufnahme im TMP- und RMP-Verfahren haben.

Die Untersuchungen zum elektrischen und thermischen Energiebedarf in der Faserstofferzeugung in Teilaspekt 4 behandeln die Fragestellung, welche Beziehungen zwischen der Holzfeuchte der Hackschnitzel in der Faserstofferzeugung und der Konsistenz der Faserstoffe nach der Faserstofferzeugung sowie dem elektrischen und thermischen Energiebedarf bestehen.

Teilaspekt 5 dient Untersuchungen zur Beschaffenheit der Faserstoffe. Hier lautet die Forschungsfrage, welche Auswirkung die Faserstofferzeugung im TMP- und RMP-Verfahren mit unterschiedlichen Mahlscheibenmustern auf die Beschaffenheit der Faserstoffe hat. Weiterhin stellt sich die Frage, ob funktionale Zusammenhänge zwischen den Dispersitätseigenschaften und der Wärmeleitfähigkeit der Faserstoffe bestehen.

Der abschließende Teilaspekt 6 beinhaltet Untersuchungen zur Wärmeleitfähigkeit der Fa- 
serstoffe. Bezüglich der maßgeblichen Produkteigenschaft von Holzfaserdämmstoffen ist die zentrale Forschungsfrage in diesem Fall, welche Wärmeleitfähigkeit Faserstoffvarianten besitzen, die mit unterschiedlichen Mahlscheibenmustern, Aufschlusstemperaturen und Aufschlussverfahren erzeugt wurden. Den Teilaspekten ist jeweils eine Einleitung vorangestellt, in der die wissenschaftlichen Arbeitsziele formuliert sind. 


\section{Charakterisierung von Holz als Faserrohstoff unter Berücksichtigung der Holzarten Fichte und Buche}

\subsection{Anatomischer Aufbau}

\subsubsection{Strukturmerkmale}

Aus verfahrenstechnischer Sicht bietet der anatomische Aufbau von Holz und dessen Struktur, die als Aggregat aus Holzzellen beschrieben werden kann (Bohnet 2004, S. 14), eine einzigartige Zweckeignung zur produkt- und prozessspezifischen Faserstofferzeugung (Kürschner 1966, S. 34). Im Holz von Bäumen spiegelt sich der für biologische Materialien typische, hierarchisch strukturierte Aufbau in der makroskopischen, mikroskopischen und submikroskopischen Betrachtungsebene wider (Stamm 1964a, S. 3). Synonym zu Holz wird auch von Lignozellulosen gesprochen, um das filigrane Gefüge des Mikro- und Nanoverbunds aus Polysacchariden und Ligninen zu betonen (Faix 2008, S. 47).

Makroskopisch betrachtet ist Holz ein von Gymnospermen (z. B. Pinaceae) und Angiospermen (z. B. Fagaceae) gebildeter poröser, heterogener Zellverbund (Fromm 2013, 33 ff.). In den gemäßigten Klimazonen bildet die periodische Aktivität des Kambiums während des sekundären Dickenwachstums konzentrische Jahresringe aus Früh- und Spätholz aus. Im Stammquerschnitt sind weiterhin Splint und Trockenkern bei der Holzart Fichte (Picea abies Karst.), respektive Splint und Kernholz bei der Holzart Buche (Fagus sylvatica L.) funktionell unterscheidbar (Kollmann 1951a, S. 6).

Mit der Einteilung des Holzgewebes in physiologische und mechanische Aufgaben beginnt die mikroskopische Betrachtungsebene. Allen Holzarten gemein ist die Zelldifferenzierung ausgehend von zwei meristematischen Zelltypen: Den fusiformen Initialen, aus denen die axial angeordneten Zellen hervorgehen, und den Strahlinitialen, die radial angeordnete Zellsysteme ausbilden (Fromm 2013, S. 5). Die geometrische Beschaffenheit der unterschiedlichen Zellarten variiert arttypisch innerhalb des Stammes sowie standortabhängig in Länge, Querschnitt, Durchmesser, Lumen- und Zellwandanteil (Stamm 1964a, S. 4).

Vor dem Hintergrund der Faserstofferzeugung sind in der submikroskopischen Betrachtungsebene der Aufbau des Zellverbunds und die chemisch-physikalischen Eigenschaften der Komponenten von Interesse. Die drei polymeren Hauptkomponenten Zellulose, Hemizellulosen und Lignine bilden hierbei einen porösen Mikro- und Nanoverbund, in dem teilkristalline Zellulosefibrillen in einer Matrix aus amorphen Hemizellulosen und Ligninen organisiert sind (Salmén 2004; Geoffrey 2009, S. 60). Wasser ist ein weiterer Bestandteil der Holzmatrix und hat im hygroskopischen Bereich Einfluss auf die viskoelastischen Eigenschaften des Holzes (Faix 2008, S. 48; Chen et al. 2018). 


\subsubsection{Zusammensetzung und geometrische Variabilität des Holzgewebes}

Die Faserstofferzeugung und die daraus resultierenden, übergeordneten Produkteigenschaften von holzfaserbasierten Werkstoffen sind von der natürlichen Zusammensetzung und geometrischen Variabilität des Holzgewebes abhängig. Dies betrifft insbesondere die Zusammensetzung der Zellarten, die Länge der Zellen sowie deren Dicke bzw. Feinheit. Die Ausbildung der Merkmale ist arttypisch und wird von anatomischen Ausprägungen des Baumes (Jung-, Kern-, Früh-, Spät-, Reaktionsholz, etc.) und der forstwirtschaftlichen Holzproduktion (Forstpflanzenzucht, Waldumtriebszeiten, etc.) beeinflusst (Kucera 1994; Lindstrom 1997). In Abbildung 2.1 sind typische Zellen von Nadel- und Laubhölzern abgebildet, um deren grundlegende Unterschiede zu verdeutlichen. Die Variabilität des Holzgewebes hängt darüber hinaus von der Population (Standort) ab und schwankt zwischen einzelnen Bäumen auch aufgrund der genetischen Varianz (Zobel \& Buijtenen 2012). Grundsätzlich liegt die geometrische Variabilität der Zellarten in Laub- und Nadelhölzern weitgehend normalverteilt vor (Kollmann 1965; Trendelenburg \& Mayer-Wegelin 1955, S. 145). Tabelle 2.1 gibt einen quantitativen Überblick über die Variabilität der geometrischen Eigenschaften, die in den Untersuchungen zur Beschaffenheit der Faserstoffe zur Anwendung kommen.

$\mathbf{a}$

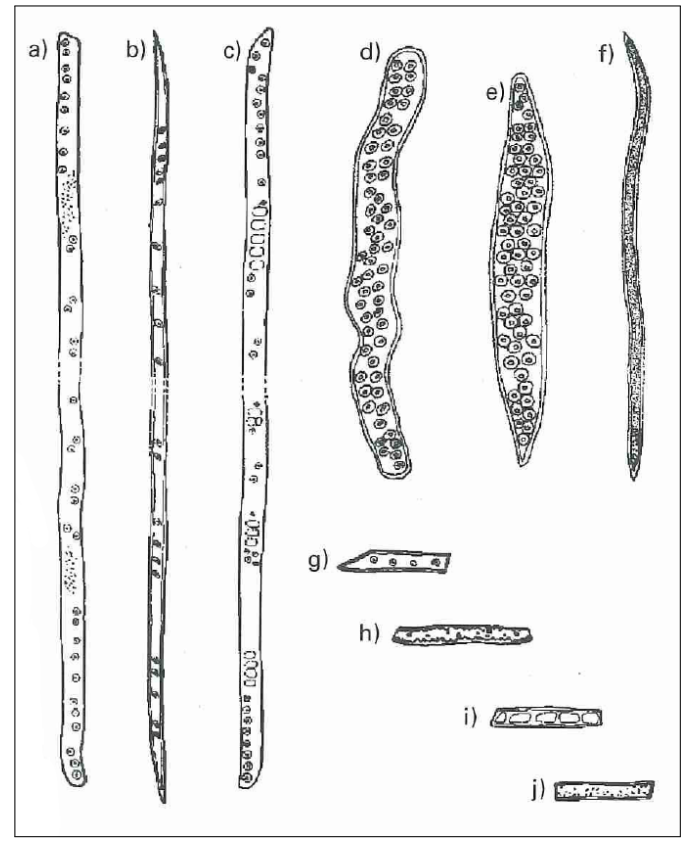

$\boldsymbol{b}$

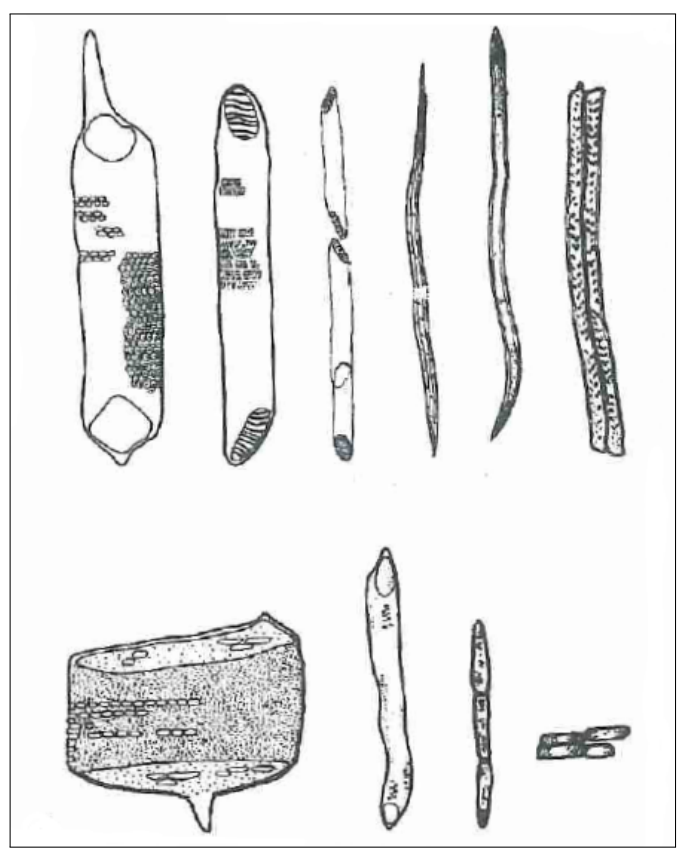

Abbildung 2.1: Darstellung von unterschiedlichen Zellarten des Holzgewebes. a, Typische Nadelholzzellen: a) Frühholztracheide der Fichte; $b$, c) Früh- und Spätholztracheiden der Kiefer; d) Vasizentrische Tracheide; $e, f)$ Gefäßtracheiden; $g$, h) Strahltracheiden der Kiefer ( $g$ ) und Fichte $(h) ; i$, j) Strahlparenchym der Kiefer (i) und Fichte (j). (Geoffrey 2009, S. 49). b, Typische Laubholzzellen: a) Obere Reihe von links nach rechts: Frühholzgefäß Esche, Frühholzgefäß Birke; Gefäß Birke, Libriformfaser Birke, Tracheide Birke, Tracheide Eiche. Untere Reihe von links nach rechts: Früh- und Spätholzgefäße Eiche, Längsparenchym Eiche, Strahlparenchym Birke. (Geoffrey 2009, S. 54). 


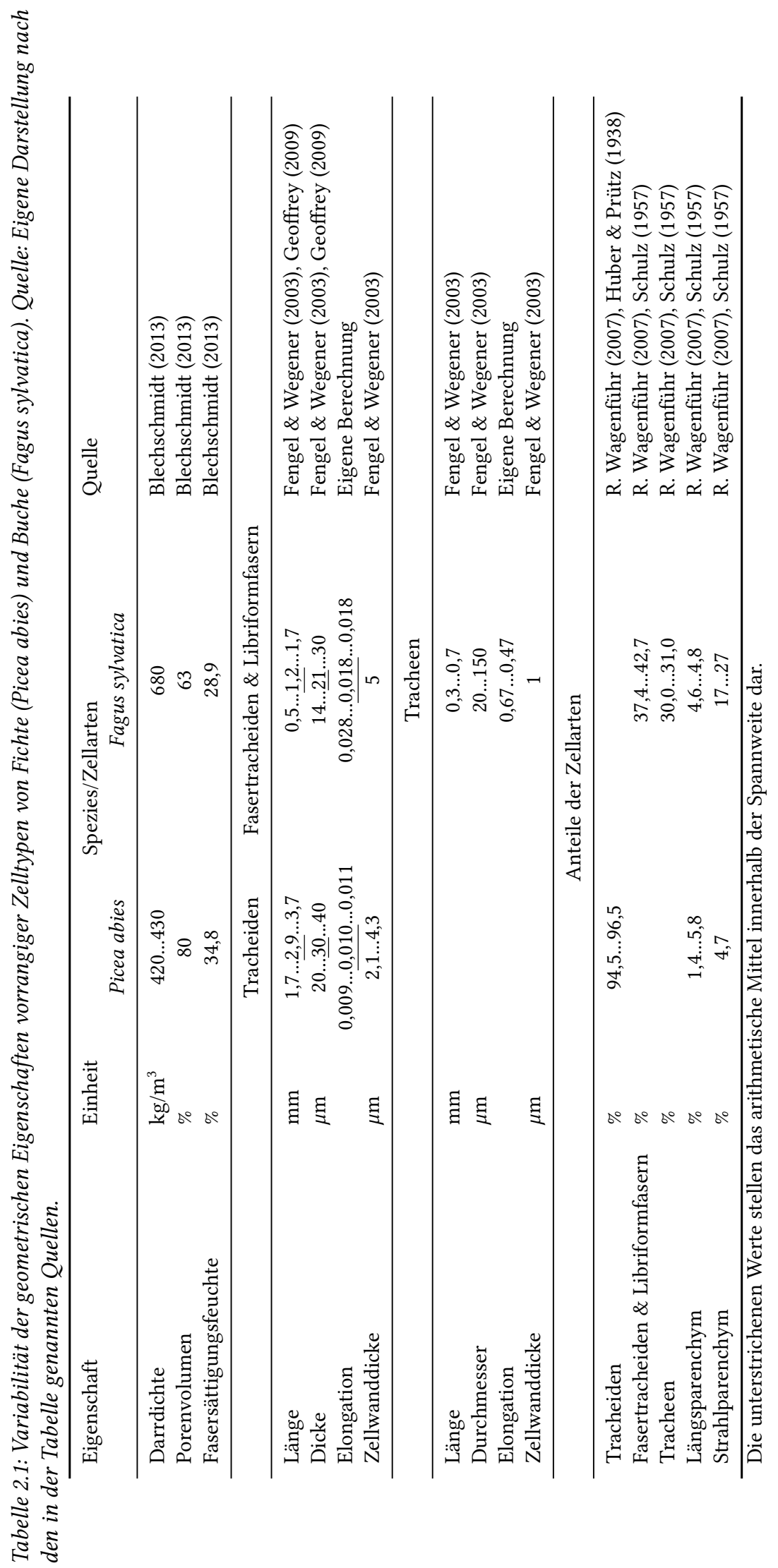




\subsection{Chemisch-struktureller Aufbau}

\subsubsection{Ultrastruktur und chemische Komponenten}

Die Organisation der Ultrastruktur, speziell die Konstitution und das Zusammenwirken der Holzpolymere in der Zellwand und Mittellamelle, sind Gegenstand der Forschung. Das wird insbesondere bei Versuchen deutlich, Holzeigenschaften auf Grundlage der bislang bekannten Eigenschaften und Zusammensetzungen der Einzelkomponenten zu modellieren (Salmén 2015). Ein generell akzeptiertes Modell für die Ultrastruktur der extra- und intrazellulären Matrix ist das in Abbildung 2.2a beispielhaft dargestellte Schichtmodell. Die konzentrisch angeordneten Zellwandschichten divergieren im Mikrofibrillenwinkel (micro fibril angle, MFA), dem Anteil der Holzpolymere und den Inhaltsstoffen (u.a. Fengel \& Wegener 2003, S. 14). In Bezug auf die Faserstofferzeugung wird das Schichtmodell auch für die Zerlegung des Zellverbunds in Abhängigkeit des Verfahrens herangezogen (Abbildung 2.2b). Die interzelluläre Mittellamelle (ML)

a

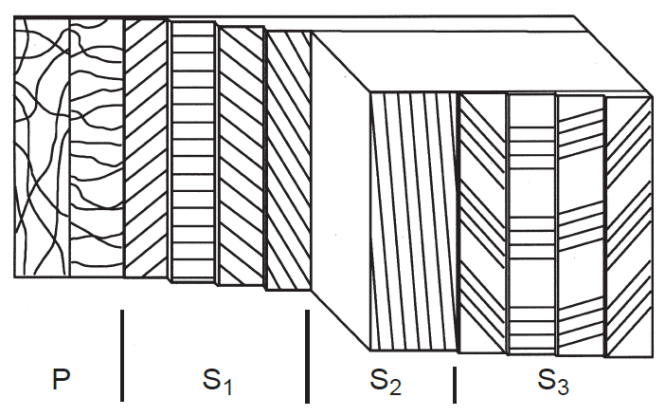

b

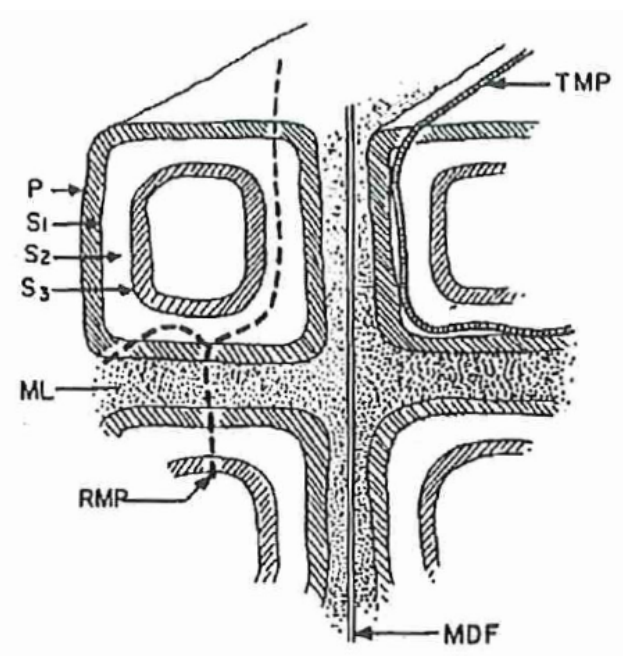

Abbildung 2.2: Darstellung zur Ultrastruktur des Holzes. a, Hypothetisches Modell der Zellwandstruktur mit Orientierung der Mikrofibrillen von Nadelholztracheiden. Blick vom Lumen auf die Zellwand nach Abe \& Funada (2005). b, Zerlegung des Zellverbunds in Abhängigkeit des Verfahrens zur Faserstofferzeugung bzw. der Aufschlusstemperatur nach Franzén (1986). ML: Mittellamelle, P: Primärwand, $S_{1}$ : Erste Sekundärwandschicht, $S_{2}$ : Zweite Sekundärwandschicht, $S_{3}$ : Dritte Sekundärwandschicht. RMP: Refiner mechanical pulp (ca. $\left.95^{\circ} \mathrm{C}\right)$, TMP: Thermomechanical pulp (ca. $\left.135^{\circ} \mathrm{C}\right)$, MDF: Medium density fibreboard (ca. $\left.175^{\circ} \mathrm{C}\right)$.

sorgt im Komplex mit der Primärwand (P) als „,compound middle lamella“ (CML) für die kohäsive Verbindung zwischen benachbarten Zellen (Melelli et al. 2020; R. Wagenführ \& A. Wagenführ 2008, S. 37). Als gesichert gilt, dass die Mittellamelle den höchsten Gehalt an Ligninen aufweist (Stamm 1964a, S. 22; Fengel \& Wegener 2003, S. 14). Weiterhin scheinen die Lignine in der Mittellamelle nicht orientiert zu sein und isotrop vorzuliegen (Salmén et al. 2012). Die Mittellamelle zwischen zwei benachbarten Zellen ist rund 0,5 $\mu \mathrm{m}$ breit (Jayme \& Fengel 1961; Fromm et al. 2003). In der Primärwand nimmt die Anisotropie der Zellwand durch zerstreut orientierte, jedoch quer zur Zellachse ausgerichtete Zellulosefibrillen zu (Fengel \& Wegener 2003, S. 14). Die Primärwand nimmt mit einer Dicke von weniger als $0,1 \mu \mathrm{m}$ einen geringen Anteil an der Zellwand ein (Fengel \& Wegener 2003, S. 17). 
Als weitere Schicht schließt sich die Sekundärwand an die Primärwand an. Die erste Sekundärwandschicht $\left(\mathrm{S}_{1}\right)$ ist mit einem Anteil am Querschnitt von 8-16\% wesentlich dünner, als die dominierende zweite Sekundärwandschicht (S2), die mit einer mittleren Dicke zwischen $1 \mu \mathrm{m}$ bis über $4 \mu \mathrm{m}$ auch den größten Anteil an der Zellwand ausmacht (Fengel \& Stoll 1973). Im Gegensatz zur isotropen Mittellamelle sind die Lignine in der Sekundärwand mit den interfibrillären Mesound Mikroporen assoziiert und entlang des MFA orientiert (Kojiro et al. 2010; Salmén 2015). Der MFA steht weiterhin in Verbindung mit den mechanischen Eigenschaften des Holzes, wobei die Struktur der $S_{1}$ auf Druckfestigkeit und die der $S_{2}$ auf Zugfestigkeit ausgelegt ist (Rowell et al. 2013, S. 48). An der Innenseite der Sekundärwand von Tracheiden, Fasertracheiden und Libriformfasern liegt die von der Zellulosefibrillenanordnung ähnlich zur Primärwand aufgebaute Tertiärwand (T). Die ebenfalls oft erwähnte dritte Sekundärwandschicht $\left(\mathrm{S}_{3}\right)$ kommt in Parenchymzellen einiger Nadelhölzern sowie in den Libriformfasern und Tracheen der Buche vor (Fengel \& Wegener 2003, S. 14). Als weiteren Bestandteil der Mikrostruktur der Zellwand müssen noch die Tüpfel und Interzellulargänge erwähnt werden, die dem Stoffaustausch im Zellverbund dienen (R. Wagenführ \& A. Wagenführ 2008, S. 36).

\section{Zellulose}

Die in der Photosynthese gebildete Zellulosevorstufe (UDP-D-Glucose) wird in den Terminalkomplexen der Plasmamembran, unter Abspaltung eines Wassermoleküls (Anhydro- $\beta$-D-Glucose), über die dimere Grundeinheit An-hydro-Cellobiose zu polymeren Glucanketten verknüpft ( $\beta$-1,4glycosidische Bindung) (Faix 2008, S. 50). Durch die kontrollierte Bewegung der Terminalkomplexe entsteht der geschichtete Aufbau der Zellwand und der MFA (Henriksson \& Lennholm 2009, 94 ff.). Die lineare, ebene Ausrichtung der Glucanketten und Bündelung zu Elementar- und Mikrofibrillen beruht auf der Sesselkonformation sowie den intra- respektive intermolekularen Wasserstoffbrücken (Faix 2008, S. 50). Die kristallinen und amorphen Aggregate der Zellulose bilden interfibrilläre Hohlräume aus, die für Wasser zugänglich sind (Salmén 2004).

\section{Hemizellulosen}

Die Hemizellulosen unterscheiden sich in der Variabilität der Monosaccharide und der Seitenketten (Stevanovic 2016, S. 69). Die Vielfalt an Pentosen, Hexosen, Uronsäuren und Deoxy-Hexosen lässt homo- und heteropolymere Kettenkonfigurationen mit unterschiedlich konfigurierten Seitenketten zu, die vereinfacht nach der Hauptkette, in Xylane und Glucomannane eingeteilt werden. Laubhölzer weisen einen höheren Anteil an Xylanen auf als Nadelhölzer und unterscheiden sich auch in der Konstitution der Glucomannane sowie der Seitenketten (Fengel \& Wegener 2003, S. 106). Die Hemizellulosen binden über Wasserstoffbrücken an die Oberfläche der Mikrofibrillen aus Zellulose (Faix 2008, S. 57). Zwischen Hemizellulosen und Ligninen sorgen kovalente Bindungen und Nebenvalenzen sowie eine räumliche Verschränkung für eine belastbare Struktur (Faix 2008, S. 62). Im Vergleich zur Zellulose sind die Hemizellulosen deutlich hydrophiler. Die chemische und thermische Stabilität ist niedriger als die der Zellulose (Teleman 2009, S. 102).

\section{Lignine}

Die Lignine sind amorphe, phenolische Makromoleküle mit einer komplexen Struktur. Als gesichert gilt, dass die Lignine die Zellwand hydrophobieren, für Druck- und Biegefestigkeit sowie für die Kohäsion im Zellverbund sorgen. Während der Lignifizierung der Zellwand entstehen, aus den Vorstufen 4-Hydroxy-Zimtalkohol, Coniferyalkohol und Sinapinalkohol, die nach Anzahl der Methoxygruppen am Phenylring klassifizierten Monomere: Para-Hydroxy-Phenylpropan (HLignin), Guiacylpropan (G-Lignin) und Syringylpropan (S-Lignin). Nadelhölzer sind hauptsächlich 
G-lignifiziert, Laubhölzer vorwiegend GS-lignifiziert (Faix 2008, S. 60; Henriksson 2009, 121 ff.). Zusätzlich zur Kettenpolymerisation durch Knüpfung von $\beta$-O-4-Bindungen, bestehen innerhalb und zwischen den Makromolekülen weitere kovalente Vernetzungen durch mehrere, eher zufällig verteilte Ether- und Kohlenstoff-Kohlenstoff-Bindungen (Faix 2008, S. 61; Henriksson 2009, 126 ff.).

\section{Pektine}

Es ist bekannt, dass die Primärwand und Mittellamelle sowie die Grenzfläche zwischen Primärwand und Mittellamelle Pektine in teilweise erheblichen Anteilen enthalten (z.B. Zamil \& Geitmann 2017; Stevanovic 2016, S. 78). Allerdings ist nicht geklärt, ob die Pektine, durch niedrige Erweichungstemperaturen und der höchsten Fähigkeit unter den Polysachariden Wasser zu binden, in der Faserstofferzeugung eine Rolle spielen (Teleman 2009, S. 103; Salmén \& Petterson 1995).

\subsubsection{Verteilung der Komponenten im Zellverbund}

Die Verteilung der Komponenten im Zellverbund ist für die Faserstofferzeugung relevant. In diesem Zusammenhang ist von Bedeutung, dass die Verhältnisse der Komponenten über den Querschnitt der Zellwand variieren. Es wird davon ausgegangen, dass die Zerteilung des Zellverbunds an oder innerhalb der von den chemischen Komponenten und Zellwandschichten gebildeten Phasen erfolgt (Abbildung 2.2) (Blechschmidt 2013, S. 58; Bohnet 2004, S. 14). Generell verschieben sich die Verhältnisse von einem hohen Anteil an Lignin in der CML zu einem hohen Anteil an Holozellulosen in der Sekundärwand. Die Ordnung und Packungsdichte der Cellulosefibrillen nimmt von der Mittellamelle zum Lumen hin zu und lässt in den interfibrilären Hohlräumen die sukzessive Einlagerung von Hemizellulosen und Lignin zu (Kürschner 1966, S. 43). Allgemein enthalten Nadelhölzer mehr Lignin als Laubhölzer, letztere weisen dagegen einen höheren Anteil an Hemizellulosen auf (Tabelle 2.2).

Tabelle 2.2: Anteile der chemischen Komponenten in den Holzarten Fichte und Buche (Blechschmidt 2013, S. 58).

\begin{tabular}{ccccccc}
\hline Holzart & $\begin{array}{c}\text { Zellulose } \\
\%\end{array}$ & $\begin{array}{c}\text { Hemizellulosen } \\
\%\end{array}$ & $\begin{array}{c}\text { Hexosane } \\
\%\end{array}$ & $\begin{array}{c}\text { Pentosane } \\
\%\end{array}$ & $\begin{array}{c}\text { Harze, Fette } \\
\%\end{array}$ & $\begin{array}{c}\text { Asche } \\
\%\end{array}$ \\
\hline Fichte & $42 \ldots 47$ & $19 \ldots 24$ & 13 & $9 \ldots 11$ & 2 & $0,23 \ldots 0,77$ \\
Buche & $39 \ldots 53$ & $22 \ldots 25$ & 4 & $18 \ldots 25$ & 2 & $0,4 \ldots 1,2$ \\
\hline
\end{tabular}

Aufgrund der geringen Dicke der Zellwand beziehungsweise der einzelnen Schichten ist eine direkte chemische Analyse und Quantifizierung der Komponenten schwierig. Die nachfolgend erwähnten indirekten analytischen Methoden bieten zumindest Anhaltspunkte zur quantitativen Verteilung der Komponenten (Tabelle 2.3).

Tabelle 2.3: Verteilung von chemischen Komponenten im Zellverbund von Tracheiden in Fichtenholz. Berechnete Werte nach Fengel \& Wegener (2003, S. 228) gemittelt und gerundet.

\begin{tabular}{lccc}
\hline Zellwandschicht & $\begin{array}{c}\text { Zellulose } \\
\%\end{array}$ & $\begin{array}{c}\text { Hemizellulose } \\
\%\end{array}$ & $\begin{array}{c}\text { Lignin } \\
\%\end{array}$ \\
\hline CML & 14 & 27 & 59 \\
S1 & 36 & 36 & 29 \\
S2 + T & 58 & 14 & 27 \\
\hline
\end{tabular}


Transmisionselektronenmikroskopie (TEM) (Abbildung 2.3) und topochemische UV-Mikrospektroskopie können den Einblick in die Struktur und die Lignifizierung der Zellwand ergänzen. Weitere spektroskopische Methoden wie Raman- oder Infrarot-Mikroskopie eignen sich zur Analyse der Zellwandstruktur und -zusammensetzung auf der molekularen Ebene (Hänninen et al. 2011). Diese Methoden sind semiquantitativ oder nicht quantitativ. Untersuchungen an Buchenholz konnten in der Literaturrecherche nicht ermittelt werden.

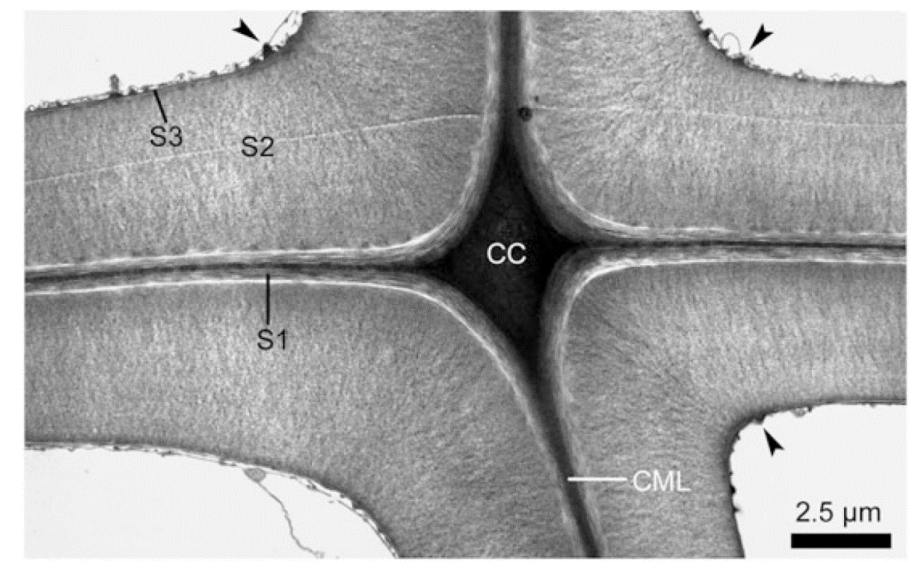

Abbildung 2.3: TEM Mikrofotographie von vier benachbarten Tracheiden der Holzart Tanne (Abies alba). Die Färbung mit Kaliumpermanganat veranschaulicht die Ligninkonzentration der Zellwand, ausgehend von einem hohen Ligninanteil in den Zellecken (cell corner region, CC) und der CML hin zu den Sekundärwandschichten $S_{1}, S_{2}$ und $S_{3}$. Auf der $S_{3}$ weisen die Pfeile auf die Warzenschicht hin. (Koch \& Schmitt 2013).

\subsection{Holzimmanente Eigenschaften}

\subsubsection{Holzfeuchte}

Eine der grundlegenden Eigenschaften des kapillar-porösen Faserrohstoffs Holz ist die Sorption gasförmigen und flüssigen Wassers (Stamm 1964b, 142 ff.). Wasser ist ein wesentlicher Bestandteil des Holzes und insbesondere das hygroskopische Verhalten beeinflusst die physikalischen Materialeigenschaften (Fengel \& Wegener 2003, S. 220; Faix 2008, S. 48). Das Holzgewebe bildet sich in einer mit Wasser gesättigten Umgebung und auch nach Einstellung der anfänglichen physiologischen Funktionen bleibt dieser Zustand in den Zellwänden bestehen (Skaar 1988, S. 20; Fromm 2013, S. 21). Ausgehend vom Splintholz variiert die Holzfeuchte über den Stammquerschnitt nach bekannten Mustern (Trendelenburg \& Mayer-Wegelin 1955, S. 277).

Von prozesstechnischer Bedeutung in der Faserstofferzeugung sind zwei Grenzzustände des Systems Holz-Wasser: Namentlich der hygroskopische Bereich bis zur Fasersättigung (FSB) sowie die maximale Wassersättigung (Niemz \& Sonderegger 2017, S. 85). Innerhalb des hygroskopischen Bereichs stellt sich zeitversetzt die Ausgleichsfeuchte in Abhängigkeit von der Temperatur und dem Wasserdampfpartialdruck (relative Luftfeuchte) ein. Die maximal mögliche Fasersättigung (Tabelle 2.4) wird nach Hoffmeyer et al. (2011) bei konstanter relativer Luftfeuchte von 99,9\% unter atmosphärischen Druck erreicht. Bis zu einer Temperatur von $100^{\circ} \mathrm{C}$ folgen die Sorptionsisothermen dann einem holzartenspezifisch ausgeprägten sigmoidalen Verlauf (Engelund et al. 2013). Hierzu ist anzumerken, dass verschiedene Methoden zur Bestimmung der maximalen Fasersättigung bestehen, die unterschiedliche Werte ergeben.

Verantwortlich für die hygroskopische Ad- und Desorption von Wassermolekülen sind intermole- 
Tabelle 2.4: Grenzzustände des Systems Holz-Wasser in Form der mittleren Holzfeuchte der Holzarten Fichte (Picea abies Karst.) und Buche (Fagus sylvatica L.) nach ${ }^{1}$ Krpan (1954) und ${ }^{2}$ Forest Products Laboratory (2010, S. 4-3).

\begin{tabular}{lll}
\hline Holzart & $\begin{array}{l}\text { Fasersättigung } \\
\%\end{array}$ & $\begin{array}{l}\text { Wassersättigung } \\
\%\end{array}$ \\
\hline Fichte & ${ }^{1} 39$ & ${ }^{2} 152^{*}$ \\
Buche & ${ }^{1} 32$ & ${ }^{2} 82^{* *}$ \\
\hline${ }^{*}$ Annahme: Rohdichte $460 \mathrm{~kg} / \mathrm{m}^{3},{ }^{* *}$ Annahme: Rohdichte $680 \mathrm{~kg} / \mathrm{m}^{3}$
\end{tabular}

kulare Mechanismen im Mikrosystem der Zellwand. Dort sind beispielsweise amorphe, quellbare Bereiche der Zellulose aufgrund des niedrigen chemischen Potenzials für Wasser leicht zugänglich (Chen et al. 2018). Aus der Bindung des Wassers an zugänglichen Hydroxylgruppen folgt das bekannte Quell- und Schwindverhalten des Holzes. An diesem Mechanismus ist auch die Adsorption an Hemizellulose und an deren Grenzflächen zur kristallinen Zellulose beteiligt (Kulasinski et al. 2015). Darüber hinaus hat das gebundene Wasser einen plastifizierenden Effekt auf die Komponenten der Zellwand, insbesondere auf die Hemizellulosen (Kelley et al. 1987). Die Beobachtung, dass bei identischer relativer Luftfeuchte die ebenfalls sigmoidale Desorptionsisotherme stets höher liegt als die Adsorptionsisotherme, wird als Hystereseeffekt bezeichnet (Stamm 1964b, 145 ff.). Die Klärung des Phänomens ist Gegenstand abgeschlossener und aktueller Forschungsarbeiten (Salmén \& P. A. Larsson 2018; Fredriksson \& Thybring 2018; Chen et al. 2020). Es wurden bereits zahlreiche thermodynamische und kinetische Lösungsansätze sowie Transportmodelle untersucht (Merakeb et al. 2009; Hill et al. 2012; Patera et al. 2016).

Oberhalb der Fasersättigung wird flüssiges Wasser im kapillaren Makrosystem der Lumen bis zur Wassersättigung (Tabelle 2.4) aufgenommen. Der maximal mögliche Wassergehalt hängt vom Porenanteil und damit von der Dichte des Holzes ab (Kollmann 1951b, S. 392). Dieses freie Wasser beeinflusst die mechanischen Eigenschaften des Holzes nicht weiter (Niemz \& Sonderegger 2017, S. 132).

\subsubsection{Erweichungsverhalten}

Das rheologische Verhalten von Holz kann als viskoelastisch charakterisiert werden (Becker et al. 1977; Salmén 1981). Das bedeutet, dass neben den physikalischen Einflussgrößen Dichte, Temperatur und Feuchtegehalt hauptsächlich die Dauer der Beanspruchung das Deformationsverhalten beeinflusst (Salmén et al. 1999, S. 35; Baur et al. 2013, S. 37). Für die Faserstofferzeugung ist die Kenntnis des Erweichungsverhaltens von Holz wichtig, um eine energiesparende Prozessführung und qualitativ hochwertige holzfaserbasierte Werkstoffe herzustellen (Hillis \& Rosza 1978).

Aus polymerwissenschaftlicher Sicht stellt die Ultrastruktur des Holzes ein viskoelastisches System im Sinne eines Mehrphasensystems dar, denn der schichtweise Aufbau des Zellverbunds ist geometrisch abgrenzbar. Die Phasen bilden wiederum einen sich stofflich unterscheidenden Werkstoffverbund mit einer Polymermatrix aus Lignin, in dem verstärkende Zellulosefibrillen sowie eigenschaftsverändernde Zusatzstoffe wie Hemizellulosen isotrop und anisotrop verteilt sind. Die Ligninphase wird aufgrund der kovalent vernetzten Polymerstruktur als maßgebendes Durchdringungsnetzwerk verstanden (Fengel \& Wegener 2003, 167 ff.). Auf den intra- und intermolekularen Interaktionen des Netzwerks der Holzpolymere beruhen die multifaktoriellen Zusammenhänge des Erweichungsverhaltens (Smith et al. 2003a, S. 37-65, 2003b, S. 25-33; Baur et al. 2013, $51 \mathrm{ff}$.). 
Im Falle statischer bzw. quasi-statischer Belastungen mit Verformungsgeschwindigkeiten nahe Null besitzt Holz bei kurzzeitigen Belastungen unterhalb der Proportionalitätsgrenze vollständig elastische Festigkeitseigenschaften. Beim Überschreiten der Proportionalitätsgrenze treten irreversible plastische Verformungen in allen Betrachtungsebenen der Holzstruktur auf. Beim Erreichen der Trennfestigkeit führen inter- und intrazelluläre Brüche zum instantanen Versagen der Holzmatrix (Winandy \& Rowell 2013, S. 414-417). Der Ort des Bruches hängt in der Faserstofferzeugung von den Aufschlussbedingungen ab (Abbildung 2.2).

In der mechanischen Faserstofferzeugung bewirken Schubspannungen infolge von Scherung sowie Druckspannungen die Trennung des Zellverbunds, vorrangig in transversaler Richtung (radial, tangential) (Havimo 2009). Solche dynamischen Belastungen mit Verformungsgeschwindigkeiten deutlich größer Null führen zu einem Versagen der Holzmatrix unterhalb der Proportionalitätsgrenze (Aicher \& Christian o. D.). Die einhergehende mechanische Dämpfung durch Deformation des Zellverbunds gilt als Maß für die innere Reibung und wird in Wärme umgewandelt (Bodig \& Jayne 1993, S. 230; Htun et al. 1993, S. 1089).

Im Falle kurzzeitiger Temperatureinwirkungen auf Polymere werden die Veränderungen der mechanischen Eigenschaften als Funktion der Temperatur und/oder Beanspruchungsfrequenz charakterisiert (Baur et al. 2013, S. 111). Die Methode der dynamisch-mechanisch-thermischen Analyse (DMTA) basiert auf harmonischer Belastung einer Probe (Kraft oder Weg) und Messung der Reaktion (Kraft oder Weg) und ist geeignet, den Einfluss der Temperatur getrennt vom zeitlichen Einfluss zu messen (Placet et al. 2007). Dabei beschreibt der Verlustfaktor $\tan \delta$ (Gleichung 2.1) die dynamische Viskoelastizität als dimensionslosen Phasenwinkel (Versatz) zwischen einer Spannung in Form des Speichermodul $M^{\prime}$ und einer Verformung in Form des Verlustmodul $M^{\prime \prime}$.

$$
\tan \delta=\frac{M^{\prime \prime}}{M^{\prime}}
$$

Für einen ideal elastischen Körper nimmt $\tan \delta$ den Wert $0\left(M^{\prime \prime}=0\right.$; $\left.\tan \delta \rightarrow 0\right)$ an und geht für einen ideal viskosen Körper gegen unendlich $\left(M^{\prime} \rightarrow 0\right.$; $\left.\tan \delta \rightarrow \infty\right)$ (DIN EN 6721-1:2019 2019). Das Merkmal für den feuchteabhängigen Erweichungsbereich von Holz ist der $\alpha$-Peak von $\tan \delta$, der als Erweichungstemperatur $T_{g}$ in Anlehnung an die Glasübergangstemperatur von Polymeren definiert ist (Kelley et al. 1987; Placet et al. 2007; Havimo 2009). Bei steigender Beanspruchungsfrequenz weist der Erweichungsbereich Eigenschaften eines dynamischen Glasübergangs auf (Becker et al. 1977; Irvine 1985; Baur et al. 2013, S. 36).

Dieser $\alpha$-Peak kann mit der spezifischen elektrischen Leistungsaufnahme in der mechanischen Faserstofferzeugung in Verbindung gebracht werden, da die hygrothermische Erwärmung mit Sattdampf Einfluss auf die viskoelastischen Festigkeitseigenschaften und das Erweichungsverhalten hat (Blechschmidt et al. 1986). Bei der Erwärmung von Hackschnitzeln mit Sattdampf liegt die Holzfeuchte oberhalb des Fasersättigungsbereichs bis hin zu maximaler Wassersättigung durch Absorption von Kondensat (vgl. Abschnitt 7.2). Die Hemizellulosen erweichen unter wassergesättigten Bedingungen bereits bei $20^{\circ} \mathrm{C}$ und insofern ist die Ligninerweichung in der mechanischen Zerfaserung maßgebend (Back \& Salmén 1982). Die maximalen Werte der $\alpha$-Peaks für den Verlustfaktor tan $\delta_{\max }$ variieren unter wassergesättigten Bedingungen und die entsprechende Erweichungstemperatur $T_{g}$ liegt zwischen $80^{\circ} \mathrm{C}$ und $140^{\circ} \mathrm{C}$ (Tabelle 2.5). Weiteren Quellen zufolge erweichen Nadelhölzer, entsprechend des Erweichungsbereichs ihrer Lignine, bei geringer Beanspruchungsfrequenz von $0,5 \mathrm{~Hz}$ bis $1,0 \mathrm{~Hz}$ bei ca. $92^{\circ} \mathrm{C}$ und Laubhölzer bei ca. $82^{\circ} \mathrm{C}$, abnehmend bis ca. $65^{\circ} \mathrm{C}$ (Olsson \& Salmén 1997; Faix 2008, S. 63). 
Tabelle 2.5: Erweichungstemperaturen der Holzarten Fichte (Picea abies ) und Buche (Fagus sylvatica) in Abhängigkeit von der Beanspruchungsfrequenz. Die Werte wurden unter wassergesättigten Bedingungen ermittelt. Ergänzt nach Havimo (2009).

\begin{tabular}{lllcccl}
\hline Holzart & Methode & Belastung & $\begin{array}{c}\text { Frequenz } \\
\mathrm{Hz}\end{array}$ & $\begin{array}{c}\mathrm{T}_{g} \\
{ }^{\circ} \mathrm{C}\end{array}$ & $\tan \delta_{\max }$ & Quelle \\
\hline Buche & Torsion & transversal & $0,5 \ldots 3$ & 80 & 0,38 & Becker \& Noack (1968) \\
Buche & Biegung & transversal & 1 & 83 & 0,108 & Placet et al. (2007) \\
Fichte & Torsion & transversal & 0,6 & 92 & 0,27 & Höglund et al. (1976) \\
Fichte & Torsion & longitudinal & 2,0 & 95 & 0,14 & Höglund et al. (1976) \\
Fichte & Zug-Druck & transversal & $0,05 \ldots 20$ & $82 \ldots 100$ & $0,14 \ldots 0,17$ & Salmén (1984) \\
Fichte & Torsion & transversal & 350 & $110 \ldots 120$ & 0,30 & Becker et al. (1977) \\
Fichte & Torsion & longitudinal & 770 & $130 \ldots 140$ & 0,17 & Becker et al. (1977) \\
\hline
\end{tabular}

Die Erwärmung führt außerdem zu einem oder mehreren Übergangs- bzw. Erweichungsbereichen, die durch einen Abfall der Elastizitäts- oder Schubmoduln sowie der mechanischen Dämpfung gekennzeichnet sind (Salmén 1984; Kelley et al. 1987). Weiterhin senkt die Protein- und Pektinphase mit einem Anteil von $15 \%$ in der Primärwand die Erweichungstemperatur derselben unter die der ML und Sekundärwand (Salmén \& Petterson 1995; Htun et al. 1993). Ob die Pektinphase in der thermomechanischen Faserstofferzeugung eine Rolle spielt ist fraglich. Dagegen spricht, dass der Zellverbund unter Einwirkung von Pektinase nicht getrennt werden kann (Kürschner 1966, S. 36).

Infolge höherer Beanspruchungsfrequenz nimmt $M^{\prime}$ größere Werte an und der Verlustfaktor $\tan \delta_{\max }$ sowie die Erweichungstemperatur $T_{g}$ steigen an (Abbildung 2.4). Das bedeutet, dass trotz der Ligninerweichung zusätzlich mechanische Energie aufgrund der höheren Beanspruchungsfrequenz zwischen den Mahlscheiben in der mechanischen Zerfaserung aufgewendet werden muss, um den Zellverbund zu defibrillieren (Salmén 1984). Hinzu kommt eine höhere Erweichungstemperatur bei Belastung in tangentialer Richtung, im Vergleich zu radialer Belastung (Placet et al. 2007). Die Erweichungstemperatur steigt um $8 \mathrm{~K}$ in radialer Schnittrichtung und $12 \mathrm{~K}$ in longitudinaler Richtung bei Erhöhung der Beanspruchungsfrequenz um eine Zehnerpotenz (Becker et al. 1977). 
a
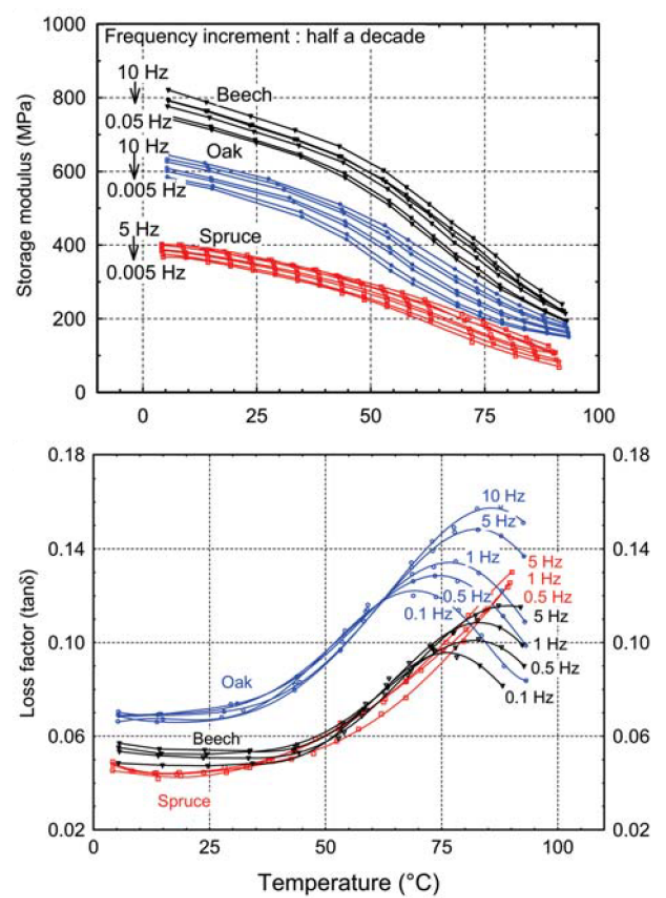

b

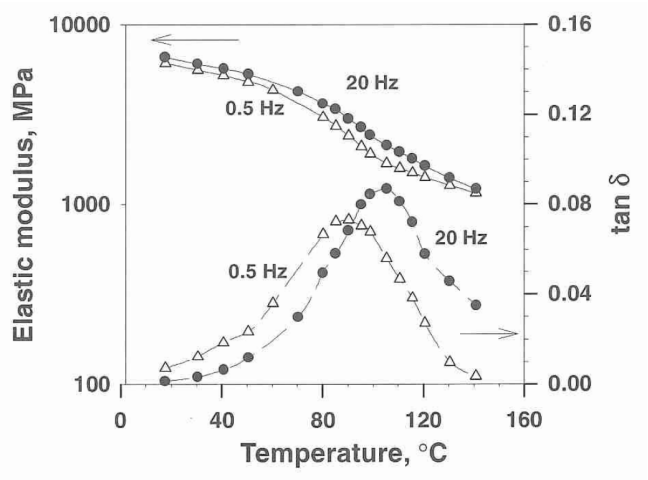

Abbildung 2.4: Viskoelastische Eigenschaften der Holzarten Fichte und Buche in radialer Schnittrichtung als Funktion der Temperatur bis $95^{\circ} \mathrm{C}$. a, Speichermodul (Storage modulus) und Verlustfaktor (Loss factor) (Placet et al. 2007). b, Die viskoelastischen Eigenschaften Elastizitätsmodul (Elastic modulus) und Verlustfaktor (tan $\delta$ ) der Holzart Fichte in axialer Schnittrichtung als Funktion der Temperatur bis $160^{\circ} \mathrm{C}$ (Salmén et al. 1999).

Diese Verschiebung wird durch die Williams-Landel-Ferry (WLF) Gleichung beschrieben und ermöglicht die Berechnung der Zeit-Temperatur-Überlagerung im Bereich der Beanspruchungsfrequenz zwischen den Mahlscheiben im TMP-Verfahren (Salmén 1984; Irvine 1985). Die Beanspruchungsfrequenz durch Druckwechselbeanspruchung liegt in Abhängigkeit der Anzahl der Stege auf der Mahlscheibe und der Rotationsgeschwindigkeit zwischen ca. $3000 \mathrm{~Hz}$ und ca. $32.000 \mathrm{~Hz}$. Irvine (1985) zufolge liegt die optimale Aufschlusstemperatur im TMP-Verfahren zwischen $130^{\circ} \mathrm{C}$ und $135^{\circ} \mathrm{C}$. Unklar ist, wie hier der Zusammenhang zu $\tan \delta$ hergestellt und die Beanspruchungsfrequenz berechnet wurde. Die Interpretationen zum Mechanismus des experimentell ermittelten Erweichungsverhaltens auf submikroskopischer Betrachtungsebene reichen von linearen ZeitTemperatur-Überlagerungen und unspezifischem Verhalten der Holzkomponenten über eine einstufige Erweichung der Lignine bis hin zu zwei getrennten Erweichungsbereichen, die den Hemizellulosen und Ligninen oder den kristallinen und amorphen Bereichen zugeordnet werden (Wert et al. 1984).

\subsubsection{Wärmeleitung}

Holz ist an sich ein schlechter Wärmeleiter (Kollmann 1987). Bei der Wärmeleitung führt ein Temperaturgefälle zum Strömen von Wärme in Form von Strahlung, Leitung und Konvektion. Als Wärmeleitfähigkeit $\lambda$ wird der lineare Wärmestrom $P$ durch ein Material mit der Dicke $d$ über der Fläche $A$ bei einem stationären Temperaturgefälle $\Delta \mathrm{T}$ mit der entsprechenden Einheit 
$\mathrm{WK}^{-1} \mathrm{~m}^{-1}$ bezeichnet und nach Gleichung 2.2 berechnet.

$$
\lambda=\frac{P \cdot d}{A \cdot \Delta T}
$$

Es handelt sich bei der Wärmeleitung um eine temperaturabhängige Stoffkonstante, welche bei $20{ }^{\circ} \mathrm{C}$ für Luft $\lambda=0,025 \mathrm{WK}^{-1} \mathrm{~m}^{-1}$ und für Wasser $\lambda=0,57 \mathrm{WK}^{-1} \mathrm{~m}^{-1}$ beträgt (Gerthsen et al. 2006, 232 f.). Die Wärmeleitfähigkeit des Holzes ist aufgrund der Ultrastruktur der Zellwand richtungs- und feuchteabhängig (Sonderegger et al. 2011). Die trockene Holzsubstanz $\left(\rho_{w}=1.500 \mathrm{~kg} / \mathrm{m}^{3}\right) \mathrm{nimmt}$ bei $20^{\circ} \mathrm{C}$ Werte axial zur Faserorientierung von $\lambda_{w \|}=0,484 \mathrm{WK}^{-1} \mathrm{~m}^{-1}$ und senkrecht zur Faserorientierung von $\lambda_{w \perp}=0,312 \mathrm{WK}^{-1} \mathrm{~m}^{-1}$ an und liegt innerhalb der limitierenden Faktoren Luft und Wasser (Maku 1954).

In Dämmstoffen steht die Reduktion der Wärmeleitung im Vordergrund. In fein verteilten Poren wird der konvektive Anteil der Wärmeleitung vernachlässigbar klein und die geringe Wärmeleitfähigkeit der Luft kommt zur Geltung (Du et al. 2009; Manohar et al. 2012). Der Strahlungsanteil wird in Holzfaserdämmstoffen als vernachlässigbar klein angenommen (Kaemmerlen et al. 2010; Manohar et al. 2012). Damit ist die Wärmeleitfähigkeit hauptsächlich strukturell, also durch die Anordnung der Fasern, deren Dimension und das Netzwerk ihrer Kontaktflächen und Poren bedingt (Kollmann \& Malmquist 1956). Wie in Abbildung 2.5 a ersichtlich ist, wird die Wärmeleitung durch die größere Kontaktfläche der Fasern im Vergleich zu Abbildung $2.5 \mathrm{~b}$ begünstigt.

Mathematisch greifbar, theoretisch begründet und experimentell bestätigt erscheint ein Schichtmodell aus den vier Elementen Holzsubstanz (Zellwand), absorbierte Feuchte, feine Poren (Lumen) und große Poren (Hohlräume zwischen den Fasern), das in Richtung des Wärmestroms in Serie geschaltet einen minimalen Wärmestrom und parallel geschaltet einen maximalen Wärmestrom erklärt. Dieses Modell soll zudem dazu geeignet sein, Unterschiede in der Wärmeleitfähigkeit zwischen Faserlängenverteilungen zu erklären (Kollmann \& Malmquist 1956; Sekino 2016). Zusätzlich scheint auf Grundlage der geometrischen Fasergrößenverteilung eine Optimierung der Wärmeleitfähigkeit durch Vorhersage des Bereichs optimaler Dichte möglich (Manohar et al. 2012).
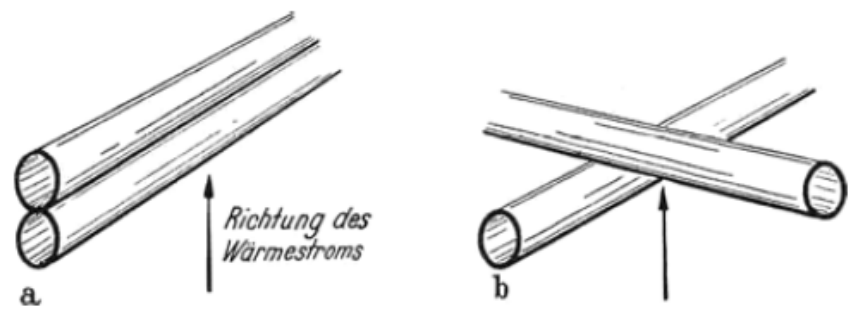

Abbildung 2.5: Schema der Anordnung röhrenförmiger Elemente in Gelegen: a, parallele Anordnung. $b$, sich kreuzende Anordnung. (Kollmann \& Malmquist 1956). 


\section{Technologie der Faserstofferzeugung}

\subsection{Holzvorbereitung}

Die Holzvorbereitung zur Faserstofferzeugung aus Laub- und Nadelholz umfasst Rohstoffauswahl, Rohstoffbeschaffung und Rohstoffaufbereitung. Insbesondere auf die Rohstoffaufbereitung kann vor der Faserstofferzeugung prozesstechnisch Einfluss genommen werden. Das Ausgangsmaterial für die Zellstoff- und Holzstofferzeugung ist, neben Sägewerks-Koppelprodukten wie Hackschnitzel, Spreißel und Schwarten, überwiegend entrindetes Rundholz. Zur Entrindung werden Trommelentrinder oder Einstamm-Rotor-Entrinder (Sägewerk) eingesetzt. Aus Rundholz werden Hackschnitzel mit Profilzerspanern (Sägewerk), Scheibenhackern oder Trommelhackern hergestellt. Die Hackschnitzel werden anschließend sortiert, ggf. zwischengelagert und zum Teil gewaschen. Die Hackertypen haben Einfluss auf die Geometrie und die Größenverteilung der Hackschnitzel (Patt et al. 1981). Die Geometrie und die Größenverteilung beeinflussen speziell im RMP- und TMP-Verfahren wiederum den Durchsatz, die spezifische elektrische Leistungsaufnahme und die Beschaffenheit der erzeugten Faserstoffe (Franzén 1986).

\subsection{Chemischer Aufschluss (Zellstoffherstellung)}

Der chemische Aufschluss zielt auf die Delignifizierung der Holzsubsubstanz ab. Es werden zwei Verfahren von wirtschaftlicher Bedeutung unterschieden. Im sauren Sulfitverfahren wird das Lignin infolge der Sulfonierung mit Bisulfitionen (HSO3-) bei ca. $170^{\circ} \mathrm{C}$ in Lösung gebracht. Die Hydrophilisierung der Ligninfragmente sorgt für die Löslichkeit in Wasser. Ein Teil der Hemizellulosen und der Zellulose wird durch die Reaktion ebenfalls hydrolisiert. Sulfitzellstoffe sind gut bleichbar. Die daraus hergestellten Papiere verfügen über eine geringere Festigkeit gegenüber solchen aus Sulfatzellstoffen. Die Zellstoffausbeute liegt bei $45 \%$ bis $53 \%$. (Fischer \& Bäurich 2013, S. 109 ff.)

Das weltweit dominierende chemische Aufschlussverfahren ist das alkalische Sulfatverfahren. Im Vergleich zum Sulfitverfahren werden damit Nadel- und Laubholzarten (hauptsächlich Kiefer und Eucalyptus) aufgeschlossen. Die Delignifizierung in der Kochflüssigkeit erfolgt durch Hydrogensulfidionen $\left(\mathrm{SH}^{-}\right)$bei ca. $150^{\circ} \mathrm{C}$. Hemizellulosen und monomere Saccharide aus der Zellulose werden ebenfalls teilweise hydrolisiert. Die gegenläufige Ligninkondensation bedingt die dunkle Farbe der Sulfatzellstoffe und erschwert deren Bleiche. Sulfatzellstoffe liefern hohe Festigkeiten. Die Zellstoffausbeute liegt zwischen $42 \%$ und $50 \%$. (Fischer \& Bäurich 2013, S. 130 ff.)

\subsection{Mechanische Faserstofferzeugung}

\subsubsection{Holzschliff}

Das älteste und wirtschaftlich immer noch bedeutsame Prinzip der mechanischen Zerfaserung von Holz ist das Pressen von Rundholz an einen im Wasserbad rotierenden Schleifstein (Vehniäinen 
2008). Im Holzschliff wurden unterschiedliche Verfahren entwickelt, welche heute ausschließlich auf die Papierherstellung begrenzt sind. Durch die Tatsache, dass die Temperatur in der Schleifzone ohne zusätzlich Erwärmung auf über $100^{\circ} \mathrm{C}$ ansteigt, kann die Holzschlifferzeugung als thermomechanischer Prozess charakterisiert werden (Atack \& Pye 1964). Einen Eindruck zum Vorgang der mechanischen Zerfaserung vermittelt Abbildung 3.1. Ein Vergleich unterschiedlicher mechanischer Verfahren hinsichtlich der Aufschlusstemperatur und der spezifischen elektrischen Leistungsaufnahme ist in Tabelle 3.1 gegeben.

Tabelle 3.1: Übersicht mechanischer Verfahren zur Holzstofferzeugung mit Aufschlusstemperatur und spezifischer elektrischer Leistungsaufnahme.

\begin{tabular}{lccl}
\hline Verfahren & $\begin{array}{c}\text { Temperatur } \\
{ }^{\circ} \mathrm{C}\end{array}$ & $\begin{array}{c}\text { SEL } \\
\mathrm{kWh} / \mathrm{t}_{\text {atro }}\end{array}$ & Quelle \\
\hline Holzschliff* $^{*}$ & bis 140 & $1300 \ldots 2100$ & Liimatainen et al. (1999) \\
RMP $^{*}$ & $90 \ldots 110$ & $2100 \ldots 2400$ & Tienvieri et al. (1999) \\
TMP $^{*}$ & $120 \ldots 140$ & $2100 \ldots 2400$ & Tienvieri et al. (1999) \\
TMP für MDF $^{*} 160 \ldots 185$ & $80 \ldots 170$ & Roll (2008) \\
CTMP $^{*}$ & $120 \ldots 140$ & $1000 \ldots 2500$ & Lindholm \& Kurdin (1999) \\
\hline
\end{tabular}

${ }^{*}$ Verfahren der Papierherstellung

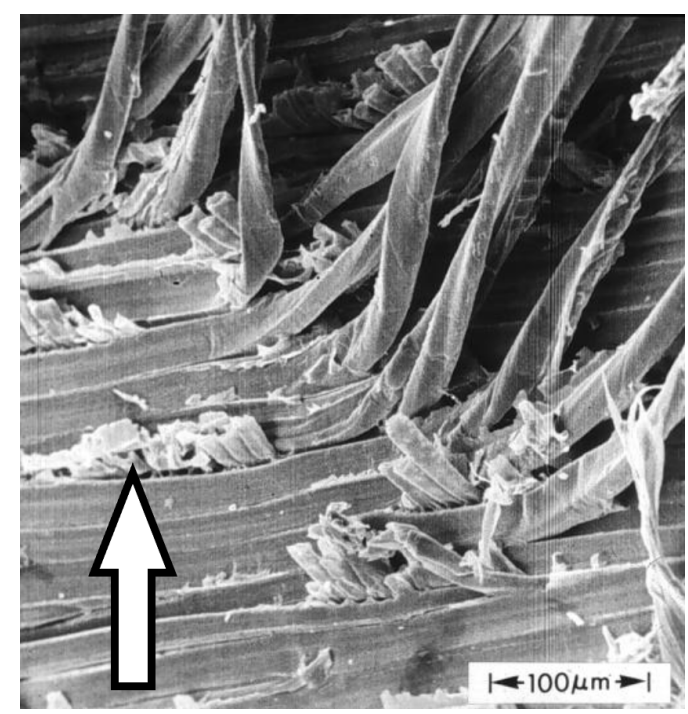

Abbildung 3.1: Rasterelektronenmikroskopische Aufnahme der Holzoberfläche von Fichte (Picea mariana) aus dem Holzschliff. Einzelne Tracheiden werden aus dem Faserverbund separiert. Das Holzstrahlparenchym (Pfeilspitze) kann nicht definiert zerfasert werden. Der Pfeil zeigt die Schliffrichtung an. (Atack1977S.).

\subsubsection{RMP-Verfahren}

Die mechanische Zerfaserung basiert auf der Absorption von Energie durch den Zellverbund infolge dynamischer Druck- und Scherbeanspruchung (vgl. Abschnitt 2.3.2). Diese Funktionsweise wird auch im Refiner Mechanical Pulp Verfahren (RMP-Verfahren) mit Mahlscheiben genutzt. Mit dem RMP-Verfahren wurde die Rohstoffbasis in der Papierindustrie durch nicht für den Holzschliff geeignete Holzsortimente, Sägewerkskoppelprodukte (Hackschnitzel) und Laubhölzer wirtschaftlich vorteilhaft erweitert (Keays \& Leask 1973). Aus den 1950er Jahren sind erste Versuche zur 
Herstellung von Zeitungspapierstoffen mit Doppelscheiben- und Einscheiben-Reibungsmühlen (Refiner) bekannt, die unter Atmosphärendruck und mit offenem Faserauslass betrieben wurden (Carpenter 1989, S. 31; Sorensen 1915; Eberhardt 1960). Diese RMP-Refiner wurden auch mit Schraubenpressen und Extrudern als erste Stufe der Zerfaserung kombiniert (Eberhardt 1955). 1961 wurde, neben zahlreichen weiteren erfolgreichen industriellen Anlagen, ein 48" RMP-Refiner mit einem Durchsatz von 400 t/d installiert (Leask 1968). Im Weiteren wurden solche Anlagen auch mit Druckvorwärmung und anschließender druckloser Zerfaserung betrieben (Peterson \& Dahlquist 1975).

Das RMP-Verfahren wurde vom Thermomechanical Pulp Verfahren (TMP-Verfahren) wegen dessen, für die Papierherstellung besser geeigneten Fasereigenschaften verdrängt und ist heute selten (Vehniäinen 2008). Einzelne Spezialanwendungen sind noch in der Spanplattenherstellung (USA), der Pflanzsubstratherstellung und in der Zerfaserung von Einjahrespflanzen zu finden.

Beim RMP-Verfahren handelt es sich um einen thermomechanischen Prozess, in dem kein zusätzlicher Dampf zur Erwärmung erforderlich ist. Die für die mechanische Zerfaserung eingesetzte elektrische Energie wird teilweise durch Reibungsarbeit zwischen den Mahlscheiben in Wärme umgewandelt und der dabei entstehende Dampf kann zur Erwärmung der Hackschnitzel genutzt werden (Abbildung 3.2) (Asplund 1940b). In Abbildung 6.2 ist die Laborrefineranlage in RMPKonfiguration, mit der die experimentellen Untersuchungen der vorliegenden Arbeit durchgeführt wurden, schematisch dargestellt und erläutert.

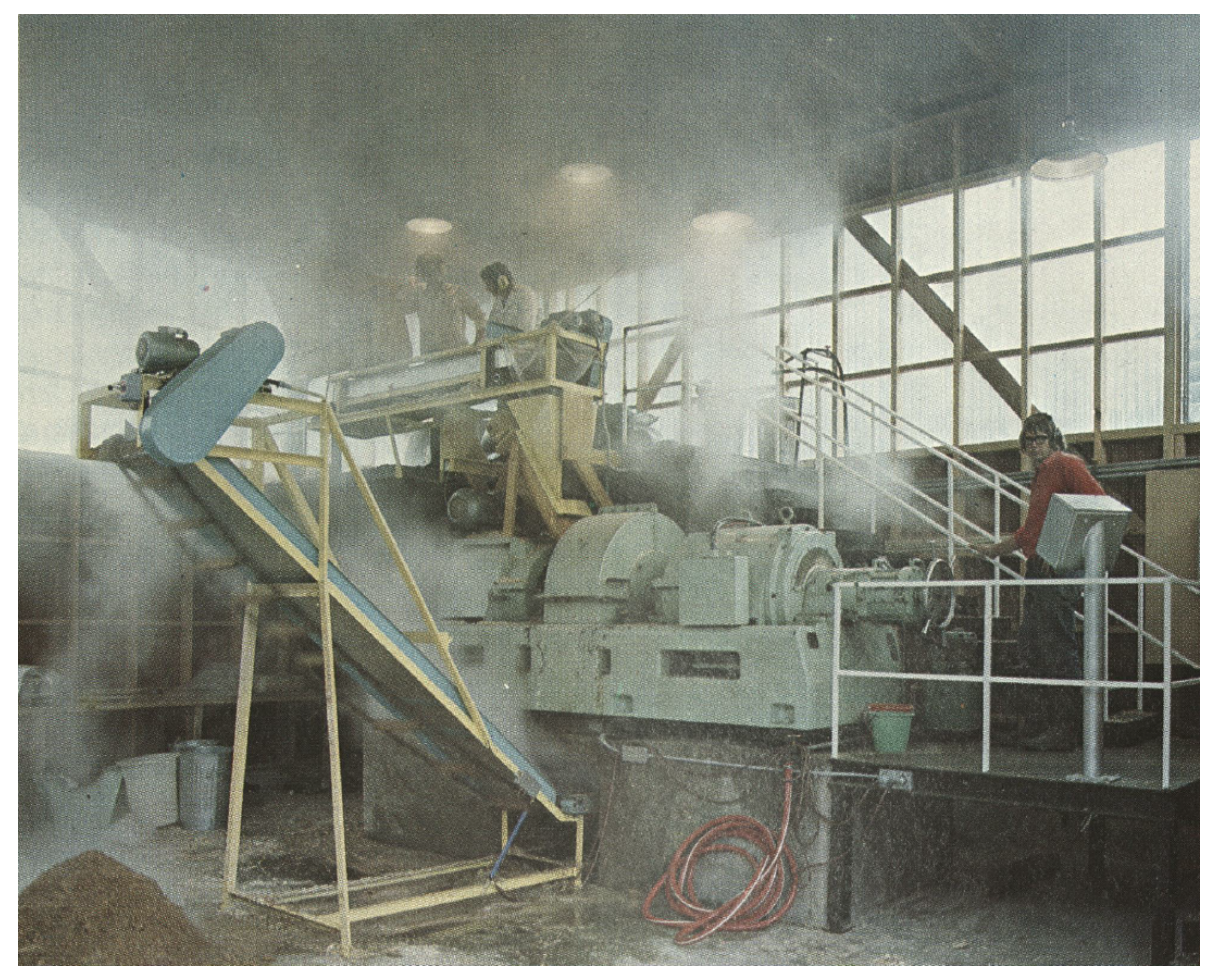

Abbildung 3.2: Versuche mit einer RMP-Laboranlage am Forest Research Institute, Rotorua, Neuseeland. (Anonym 1974). 


\subsubsection{TMP-Verfahren}

Thermomechanical Pulp (TMP) wurde erstmals im Jahr 1934 im industriellen Maßstab hergestellt (Asplund 1940b). Die Anwendung blieb zunächst auf die Herstellung von Isolierplatten, Hartfaserplatten und losen Fasern zur Isolierung (heute: Einblasdämmung) beschränkt. Die Produktionsmenge betrug 1939 in Schweden 120.000 t/a und 100.000 t/a in den USA (Lowgren 1941). Als Rohstoffe wurden Kiefer, Fichte und weitere Nadelhölzer sowie Espe, Ahorn, Pappel und Eiche verwendet. Die Zerfaserung von Weizenstroh und Bagasse war bereits bekannt und im industriellen Einsatz (Lowgren 1941; Asplund 1940b).

Die erste industrielle MDF-Anlage folgte im Jahr 1965 (Lein 1978). Im Jahr 1968 wurde das TMP-Verfahren in die Papierherstellung übertragen (Ahrel \& Bäck 1970). Grundlegende Entwicklungsschritte in der Technologie sind seither nicht bekannt geworden. Verbesserungen wurden in den letzten Jahren in der Prozessführung und -kontrolle und insbesondere im Mahlscheibendesign für die MDF-Produktion erzielt (Ladenburger 2010; Gao 2014). Aus der MDF-Herstellung ist der Einsatz von Laubholz und Einjahrespflanzen bekannt (Deppe \& Ernst 1996, 27 ff.).

Der TMP-Prozess in der MDF- und Holzfaserdämmstoffherstellung unterscheidet sich von dem TMP-Verfahren der Papierherstellung hinsichtlich des Trockenverfahrens in der Mattenund Plattenherstellung. Die Verfahrensschritte zur thermomechanischen Faserstofferzeugung in der Holzwerkstoffherstellung unterscheiden sich nicht wesentlich. In der Holzfaserdämmstoffherstellung werden Hackschnitzel mit atmosphärischem Dampf im Vorkocher auf ca. $90^{\circ} \mathrm{C}$ vorgewärmt und über eine Stopfschnecke in den Druckbehälter (Kocher) der Refineranlage gefördert. In diesem Prozessschritt wird freies Wasser in den Hackschnitzeln und ein beträchtlicher Teil der Extraktstoffe abgequetscht (Sandberg et al. 2020). Je nach Kompressionsgrad erfolgt auch eine mechanische Vorbehandlung.

In Abbildung 3.3 ist ersichtlich, dass die Struktur der Hackschnitzel in der Stopfschnecke verändert wurde. Das Material hat seinen „schnitzeligen“Charakter verloren, ist vorzerkleinert und teilweise aufgefasert worden. Der Einfluss auf die Beschaffenheit der Faserstoffe durch die Beschädigung der Faserstruktur wurde im Kontext der Papierherstellung untersucht (Sabourin 2003). Durch eine mechanische Vorbehandlung sinkt der elektrische Energiebedarf in der Zerfaserung um 10\% bis $30 \%$ (Gorski et al. 2010). 


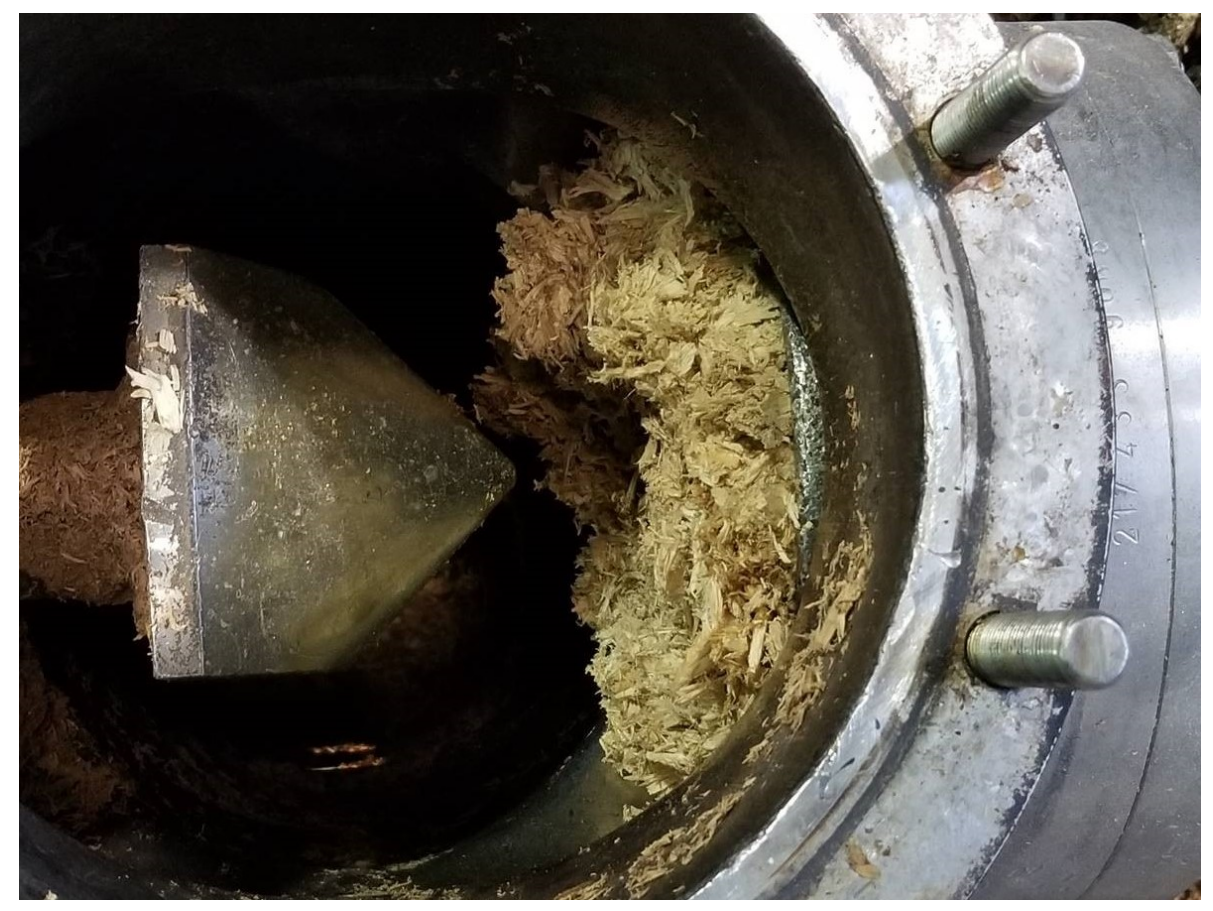

Abbildung 3.3: Blick von oben in den geöffneten Kocher einer kontinuierlich betriebenen Laborrefineranlage (Typ Andritz 12-1CP). Die Hackschnitzel werden von rechts mit der Stopfschnecke in den Druckbehälter gefördert. In der Mitte ist der Kegel des Rückschlagventils zu sehen. Auffällig ist die starke Vorzerkleinerung der Hackschnitzel durch die Stopfschnecke. (IAP Industrieanlagen-Planungsgesellschaft m.b.H.).

Im Kocher werden die Hackschnitzel mit einer Sattdampftemperatur von ca. $150^{\circ} \mathrm{C}$ bis ca. $180^{\circ} \mathrm{C}$ für ca. 3 min bis ca. 6 min erwärmt. Anschließend erfolgt die Zerfaserung typischerweise in einem Einscheibenrefiner (Rotor/Stator) mit einer Kapazität von bis zu $8 t_{\text {atro }} / h$ bei Mahlscheibendurchmessern bis zu 48 ".

Charakteristisch für das TMP-Verfahren in der Holzfaserdämmstoffherstellung ist eine direkte Verbindung zwischen Kocher und Refiner (Tienvieri et al. 1999, S. 160). Die Differenz des Sattdampfdrucks zwischen Kocher und Mahlraum kann geregelt werden und bestimmt die Verweilzeit des Materials zwischen den Mahlscheiben mit. In die Blasleitung werden Additive zur Hydrophobierung sowie Flammschutzmittel dosiert. Anschließend werden die Faserstoffe in einem Stromrohrtrockner getrocknet. In der industriellen Praxis variieren die Parameter der Prozessschritte rohstoff- und herstellerabhängig. Der höhere Gesamtenergieaufwand im TMPVerfahren im Vergleich zu RMP oder Holzschliff wird durch die Dampferzeugung und Trocknung verursacht (Atack 1976; Htun et al. 1993, S. 1088).

In Abbildung 6.1 ist die Laborrefineranlage in TMP-Konfiguration, mit der die experimentellen Untersuchungen der vorliegenden Arbeit durchgeführt wurden, schematisch dargestellt und erläutert.

\subsubsection{CTMP-Verfahren}

Die Anlagen zur Herstellung von Chemi-Thermomechanical Pulp (CTMP) gleichen denen des TMPVerfahrens der Papierherstellung (Petit-Conil et al. 2016, S. 178). Der entscheidende Unterschied ist, dass im CTMP-Verfahren zusätzlich zur thermischen Energie auch chemische Energie für die Erweichung der Holzmatrix eingesetzt wird. Die überwiegende chemische Behandlung ist die 
milde Sulfonierung mit Natriumsulfit bei Nadelholz sowie Natriumsulfit und/oder Natronlauge bei Laubholz (Lindholm \& Kurdin 1999, S. 224). Die Herstellung und Verwendung von CTMP ist in der Holzwerkstoffherstellung nicht von Bedeutung (Roffael et al. 1994a).

\subsection{Mahlscheiben}

\subsubsection{Konstruktionsmerkmale}

Die Mahlscheibe ist im RMP- und TMP-Verfahren das Werkzeug zur Zerfaserung des Holzes. Die produkt- und prozessspezifische Auslegung von Mahlscheiben führt zu einer Verbesserung der Beschaffenheit der Faserstoffe im Sinne der übergeordneten Produkteigenschaften (Deppe \& Ernst 1996, S. 54, 68). Darüber hinaus ist bekannt, dass geeignete Mahlscheibenmuster die spezifische elektrische Leistungsaufnahme in der Faserstofferzeugung für die Herstellung von MDF senken können (Börste 2008).

Die Papierherstellung stellt global den größten Einsatzbereich von Refinern und Mahlscheiben dar. Neben der Faserstofferzeugung im TMP-Verfahren werden Mahlscheiben hier außerdem verwendet, um Rejektstoffe zu behandeln oder die Eigenschaften der Holz- oder Zellstofffasern, wie Flexibilität, Faser-zu-Faser-Bindung und Faserkürzung durch eine Nachmahlung für die entsprechenden Papiersorten vorzubereiten (Annergren \& Hagen 2016). Die Faserstoffe befinden sich dazu in Wassersuspension mit geringem Feststoffanteil (LC-Refining). Im Gegensatz zur Holzwerkstoffindustrie werden Mahlscheiben für die Papierherstellung seit längerem intensiv wissenschaftlich untersucht und weiterentwickelt (Guo et al. 2020). Forschungsergebnisse oder Methoden zur Mahlscheibenentwicklung lassen sich aufgrund der unterschiedlichen Prozess- und Produktspezifikationen nicht auf die Holzfaserdämmstoffherstellung übertragen.

Mahlscheiben für das TMP-Verfahren werden im Formguss-Verfahren hergestellt. Die Laufzeit hängt vom verwendeten Holzsortiment sowie der Effizienz einer ggf. vorhandenen Hackschnitzelwäsche ab und liegt typischerweise zwischen 1000 und 3000 Betriebsstunden. Der Verschleiß erfolgt durch Abrunden und Abtragen der Stege. Mit der Abnutzung nimmt die Faserlänge und die spezifische elektrische Leistungsaufnahme zu. Die Oberflächenhärte wird durch martensitische Legierungen eingestellt ( $0,2 \%-3 \%$ C und $15 \%-30 \%$ Cr) (Tienvieri et al. 1999, S. 189).

Die wesentlichen Konstruktionsmerkmale sind in Abbildung 3.4 dargestellt. Die Vorbrecher zerkleinern das Material grob vor. Anzahl, Winkel, Krümmung und Breite der Stege im Verhältnis zu den Nuten sind die typischen Parameter, um die Mahlscheibe produkt- und prozessspezifisch anzupassen. Über den Stegwinkel kann, ähnlich wie in einer Pumpe, gezielt der Transport des Materials im Mahlspalt und die Verweilzeit zwischen den Mahlscheiben angepasst werden. Die Anzahl der Stege nimmt zur Peripherie der Mahlscheibe im Regelfall zu. Die Oberfläche der Stege wird präzisionsgeschliffen, um den Mahlspalt im Bereich weniger Zehntelmillimeter genau einstellen zu können. An den Befestigungspunkten werden die Ringsegmente auf dem Mahlscheibenhalter befestigt. Die Dämme unterhalb der Stegoberfläche (U-Damm) dienen zur Verstärkung der Stege. Die Dämme auf Höhe der Stege zwingen das Material in den Mahlspalt.

In Abbildung 3.5 sind fünf Mahlscheibentypen abgebildet. Bei Abbildung 3.5 a handelt es sich um einen Mahlscheibentyp, mit dem Tests im RMP-Verfahren von Holzer et al. (1962) durchgeführt wurden. Hierbei handelt es sich um ein bidirektionales Design, das hauptsächlich in der Papierherstellung verwendet wird, aber auch in der MDF- und Holzfaserdämmstoffherstellung verbreitet war. Die Drehrichtung ist nicht vorgegeben und kann reversiert werden. In Abbildung $3.5 \mathrm{~b}$ sind die Stege im Winkel parallel angeordnet. Aus der Rotation des Rotors über dem Stator ergibt sich hieraus ein breites Spektrum an Stegwinkeln. Die Stege müssen durch Korrektur- 


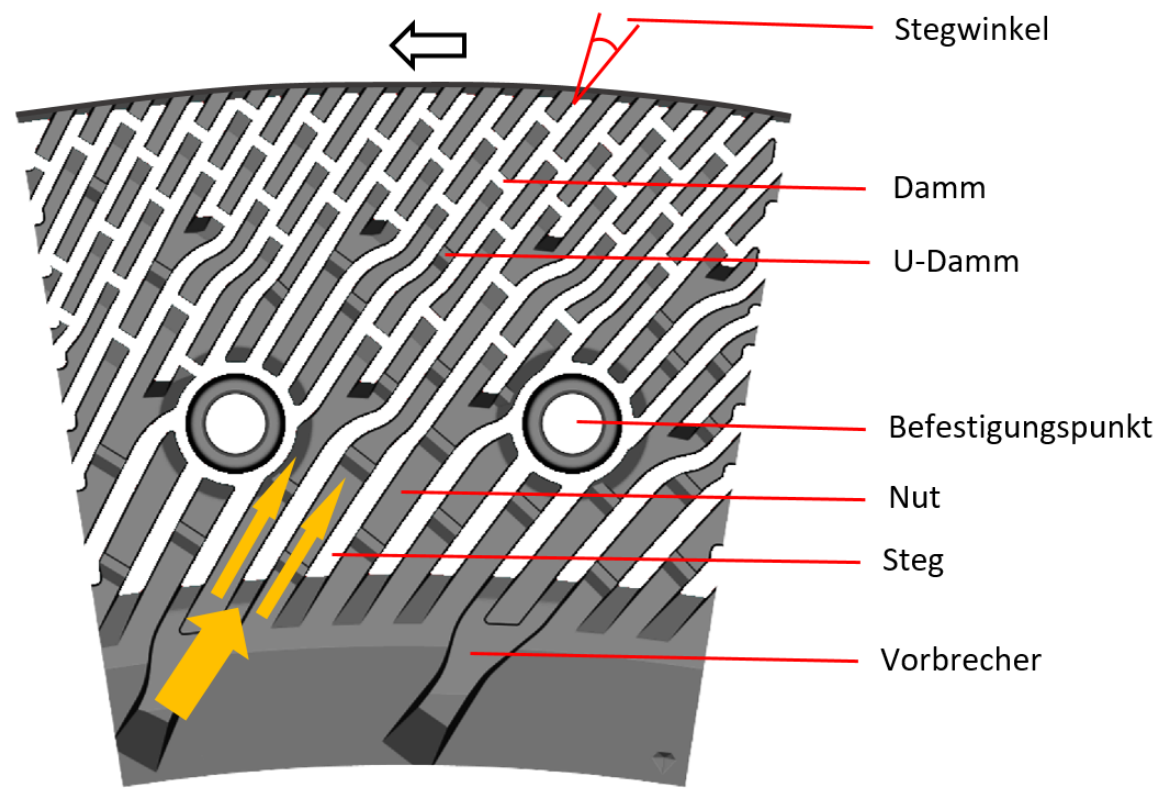

Abbildung 3.4: Die wesentlichen Konstruktionsmerkmale einer Mahlscheibe, dargestellt am Ringsegment eines Rotors. Der Pfeil oberhalb zeigt in Rotationsrichtung. Die Pfeile auf der Mahlbahn stellen den Materialfluss dar. Ergänzt nach T. Michel (2017).

winkel der Kreisform angepasst werden. Das Spiraldesign in Abbildung $3.5 \mathrm{c}$ bietet den Vorteil, dass über die Länge des Steges stets der gleiche Winkel zwischen den Stegen des Rotors und des Stators anliegt. Eine Weiterentwicklung des Spiraldesigns stellt Abbildung $3.5 \mathrm{~d}$ dar. Hierbei handelt es sich um den Stand der Technik im Bereich der MDF-Herstellung. Eine aktuelle TMPMahlscheibe für die Papierherstellung mit Doppelscheibenrefinern in Abbildung 3.5 e weist im Vergleich eine deutlich höhere Anzahl an Stegen auf. Der elektrische Energiebedarf liegt hier rund zehnmal höher als bei MDF. In der Vergangenheit wurden in der Holzfaserdämmstoffherstellung unspezifische Mahlscheiben oder ältere MDF-Typen wie das bidirektionale oder das parallele Design verwendet (persönliche Erfahrung aus Gesprächen, Werksbesichtigungen und Mahlscheibentests mit Holzfaserdämmstoffherstellern). Speziell entwickelte Mahlscheiben für die Holzfaserdämmstoffherstellung sind nicht bekannt.

\subsubsection{Zerfaserungskinetik}

Vom Standpunkt der mechanischen Verfahrenstechnik aus, handelt es sich bei der mechanischen Faserstofferzeugung um einen Zerteilprozess (Zerkleinerung) (Schönert 2003, S. 268). Demzufolge ist die Zerfaserung von Holz das Ergebnis des Überwindens molekularer Bindungskräfte im Zellverbund, welches durch den elektrischen Energieaufwand quantifiziert wird. Eine Hypothese für die Entstehung spezifischer Partikelgrößenverteilungen und -formen lautet: Die Zerfaserung findet in dem, zwischen Rotor und Stator komprimierten, Faserstoffpolster durch Scherbeanspruchung statt (Illikainen et al. 2008, 2010). Die Zerkleinerungskinetik der profilierten Mahlscheibe erzeugt Spannungen im Holz, durch welche die Zerkleinerung (Dispersität) im scheibenförmigen Mahlspalt in radialer Richtung zeitlich zunimmt. Der Abstand zwischen Rotor und Stator hat dabei eine Klassierwirkung, denn lediglich Partikel, die kleiner oder feiner sind als der Mahlspalt, können den Mahlraum verlassen. 
Neben der Zerkleinerungsfunktion besitzen die Mahlscheiben auch eine Transportfunktion. Das zentral aufgegebene Mahlgut wird durch die vom Rotor erzeugte Zentrifugalkraft in Richtung Peripherie befördert. In den Nuten des Stators strömt, durch den zwischen den Mahlscheiben entstehenden Dampf, das Material teilweise in Richtung Zentrum zurück (Atack et al. 1984, 1989; Miles \& May 1990). Die Auswertung hochfrequenter Filmaufnahmen vermittelt eine Vorstellung über die komplexen Partikelbewegungen (Atack et al. 1989; T. S. Fox et al. 1979). Es sind Versuche bekannt, einzelne Aspekte der komplexen Wechselwirkungen in der Zerfaserung im TMP-Verfahren mathematisch zu fassen. Dazu zählt zum Beispiel die Intensität (Kerekes 2015). Auf die Entwicklung von Mahlscheiben für die Holzfaserdämmstoffherstellung sind solche Modelle aufgrund der grundlegend unterschiedlichen Applikationen und der weitgehend unbekannten Zusammenhänge zwischen Rohstoff, Zerfaserungsparametern, Dispersitätseigenschaften und der maßgeblichen Produkteigenschaft Wärmeleitfähigkeit nicht übertragbar.

Nach Antensteiner (2002) und Schönert (2003, S. 365) kann die spezifische elektrische Leistungsaufnahme des Refiner-Antriebmotors in der thermomechanischen Faserstofferzeugung unter Berücksichtigung der folgenden Aspekte berechnet werden. Die vom Motor aufgenommene elektrische Leistung setzt sich wie in Gleichung 3.1 dargestellt zusammen.

$$
P_{r e f}=P_{e l}-P_{N T}
$$

$P_{\text {ref }} \quad$ Elektrische Nettoleistung in $\mathrm{kW}$

$P_{e l} \quad$ Elektrische Leistung des Motors in $\mathrm{kW}$

$P_{N T} \quad$ Elektrische no-treatment Leistung in $\mathrm{kW}$

Die no-treatment Leistung berücksichtigt die mechanischen Verluste (Leerlaufleistung) wenn keine Zerfaserung stattfindet, aber Dampfdruck anliegt. Die ausschlaggebende Messgröße ist die spezifische elektrische Leistungsaufnahme des Refiner-Antriebmotors nach Gleichung 3.2, welche auf den Massenfluss des Mahlguts bezogen ist.

$$
P_{\text {spez }}=\frac{P_{\text {ref }}}{\dot{M}_{\text {atro }}}
$$

$P_{\text {spez }} \quad$ spezifische elektrische Leistungsaufnahme in $\mathrm{Wh} / \mathrm{kg}_{\text {atro }}$

$P_{\text {ref }} \quad$ elektrische Nettoleistung in $\mathrm{W}$

$\dot{M}_{\text {atro }} \quad$ Massenfluss Holz in $\mathrm{kg}_{\text {atro }} / \mathrm{h}$

\subsubsection{Simulation}

Die Entwicklung von Mahlscheiben ist geprägt durch Erfahrung und experimentelle Mahlscheibentests. Für die Simulation zur Entwicklung von Mahlscheiben sind unter anderem folgende Randbedingungen zu beachten: Das Mahlgut Holz stellt im TMP-Verfahren ein Drei-PhasenGemisch aus der festen Phase Holz, der flüssigen Phase Holzfeuchte (Wasser) sowie der dispersen Phase Dampf dar. Die feste Phase Holz ist ein poröser, heterogener, anisotroper, viskoelastischer Mehrschichtenverbund, dessen Struktur und chemische Konstitution holzarten- sowie standortabhängig variiert. Die disperse Phase Dampf ist kompressibel und bewegt sich auf der labilen Sattdampflinie. Außerdem beinhaltet diese Komponente Druck- und Temperaturwechselwirkungen mit der festen und flüssigen Phase. Die interessanten Größen bei der Simulation zur Entwicklung von Mahlscheiben sind die erzeugten Dispersitätseigenschaften der Faserstoffe und die spezifische elektrische Leistungsaufnahme. Hier sind die keine nennenswerten Erfolge durch Simulation von Mahlscheiben bekannt (Eriksson \& Karlstroem 2009; Muhic et al. 2011; Wang et al. 2017; Sandberg et al. 2020; Talebjedi et al. 2020; Tian 2020). 
a

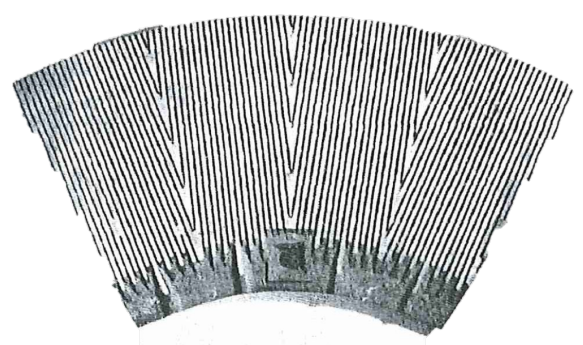

$\boldsymbol{C}$

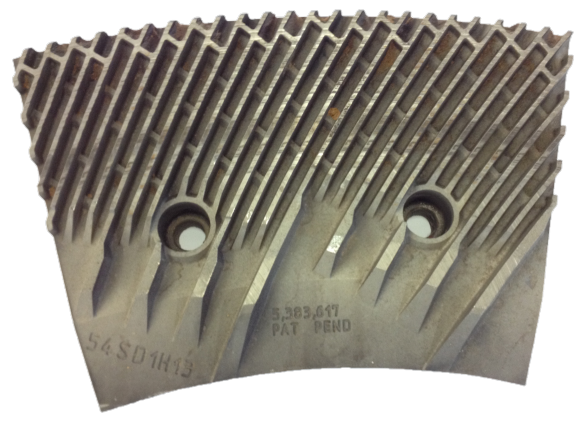

b

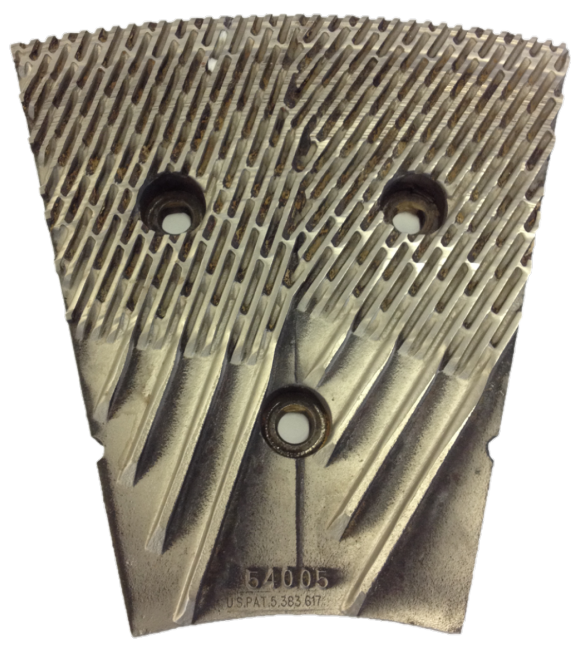

d

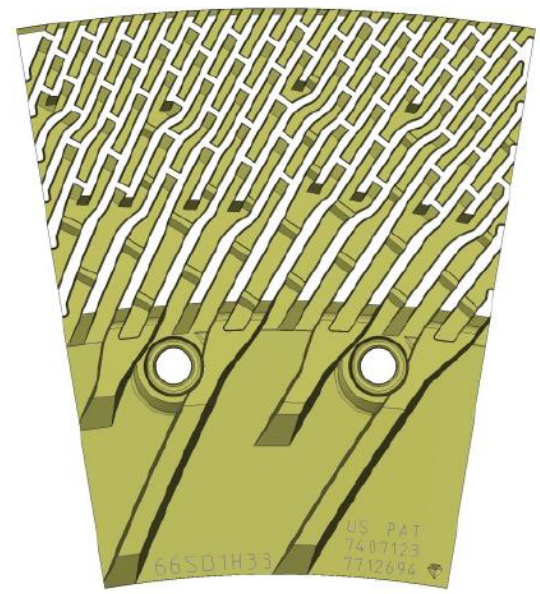

$\boldsymbol{e}$

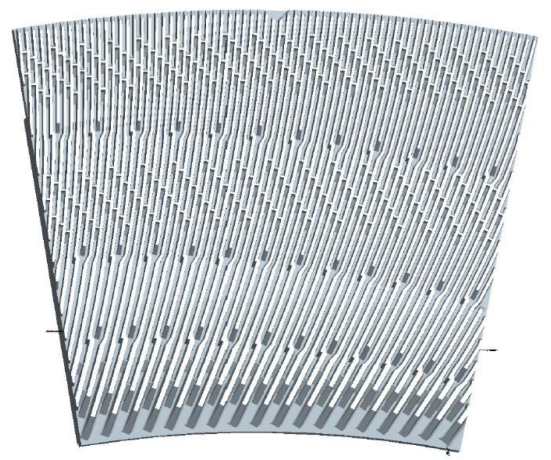

Abbildung 3.5: Übersicht über Entwicklungen im Mahlscheibendesign anhand unterschiedlicher Typen: a, Bidirektionales Design u.a. für das RMP-Verfahren (Holzer et al. 1962). b, Paralleles Design (eigene Aufnahme). c, Spiraldesign (eigene Aufnahme).d, Modernes MDF Design (Gao 2014). e, Modernes TMP Design (Muhic et al. 2011). 


\section{Charakterisierung der Beschaffenheit von Faserstoffen}

\subsection{Zusammensetzung und ausgewählte Eigenschaften}

Im Bereich der Holzwerkstofftechnik wird im Zusammenhang mit der Faserstofferzeugung generell von Holzfasern gesprochen. Der in der Papiertechnik synonym verwendete Begriff Holzstoff deutet auf den nahezu vollständig erhaltenen Ligninanteil hin. Für die Charakterisierung der Beschaffenheit von mechanisch erzeugten Faserstoffen impliziert der Begriff Hochausbeutefaserstoff den vergleichsweise geringen, thermisch indizierten Massenverlust von $4 \%$ bis $6 \%$ im RMP-Verfahren und von $6 \%$ bis $9 \%$ im TMP-Verfahren (Petit-Conil et al. 2016, S. 173). Im Weiteren bezieht die Bezeichnung auch die anatomischen Merkmale der mikroskopischen Betrachtungsebene des Holzgewebes mit ein (vgl. Abschnitt 2.1.2). Die Prozessführung im RMPund TMP-Verfahren beeinflusst zwar die Trennung des Zellverbunds und bestimmt maßgeblich Faserstoffeigenschaften wie die Steifheit sowie die chemische und physikalische Morphologie der Oberfläche (Fernando \& G. Daniel 2008; Salmén et al. 1999, S. 36). Die Zusammensetzung der Faserstoffe ist indessen durch die holzartenspezifische Ausprägung und Variabilität des Holzgewebes größtenteils vorgegeben (Heikkurinen \& Leskelä 1999, 369 ff.).

In einem verfahrenstechnischen Sinn umfasst die Definition des Begriffs Holzfaser (Faserstoff) folglich nach Karnis (1994) \& Forgacs (1963) und Heinemann (2013, S. 96) ein heterogenes Gemisch aus:

- Splitter: Spanförmige Faserbündel, die in der Dicke aus 30 und mehr Zellen bestehen.

- Faserbündel: Aggregate und deren Bruchstücke, die in der Dicke aus 2 bis 30 und mehr Zellen bestehen und teilweise aufgefasert sind sowie Agglomerate.

- Langfaser: Primärpartikel wie Tracheiden, Libriformfasern und Fasertracheiden

- Kurzfaser: Sekundärpartikel aus angeschnittenen, zerteilten oder delaminierten Einzelfasern sowie Parenchym.

- Feinstoff: Fragmente von Einzelfasern, der Mittellamelle und der delaminierten Zellwand, separierte Tüpfel.

Die physikalische Oberflächenmorphologie der Faserstoffe hängt unter anderem mit der Aufschlusstemperatur zusammen. Bei hohen Aufschlusstemperaturen $\left(170^{\circ} \mathrm{C}\right)$ weisen Nadelholztracheiden eine glatte und weitgehend intakte Zellwand auf (Atack 1972). Bei niedrigeren Aufschlusstemperaturen $\left(110^{\circ} \mathrm{C}\right.$ bis $\left.135^{\circ} \mathrm{C}\right)$ sind die Primärwand und die S1-Schicht teilweise vollständig flöckchen- und plättchenförmig abgelöst. Die S2-Schicht delaminiert bändchenförmig, was auch als externe Fibrillierung sichtbar wird (Koran 1970; Fernando \& G. Daniel 2004). In Abbildung 4.1 werden die angesprochenen prinzipiellen morphologischen Eigenschaften mechanischer Faserstoffe im Vergleich mit chemisch aufgeschlossenem Zellstoff dargestellt. 

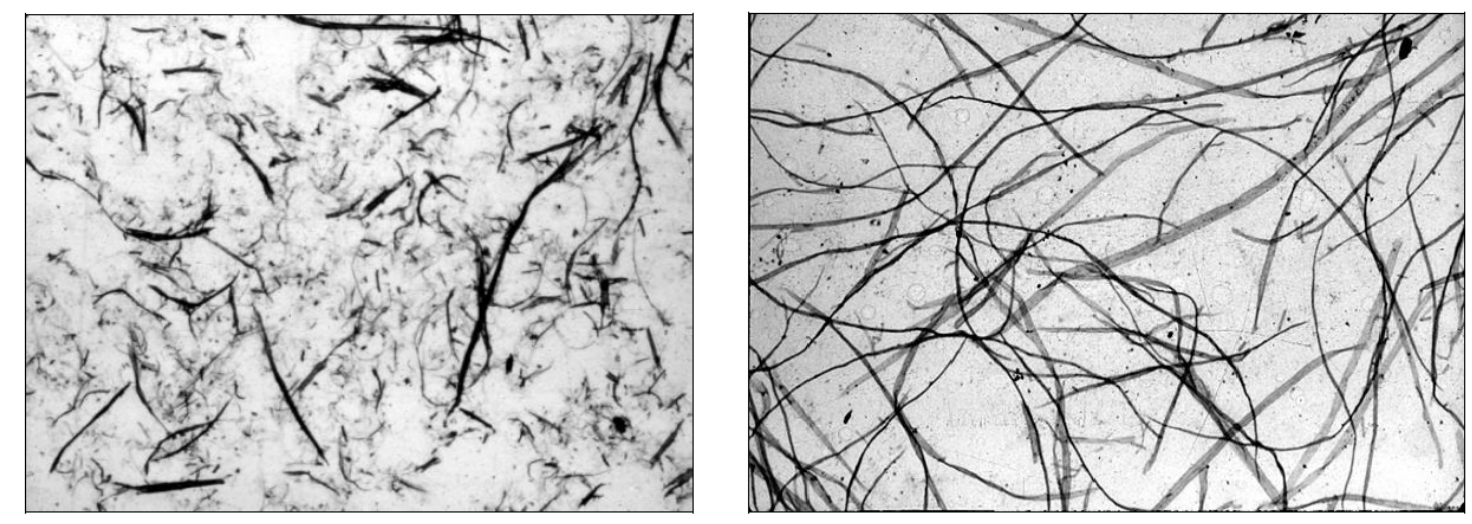

Abbildung 4.1: Mikroskopische Aufnahme für den qualitativen Vergleich von Holzschliff (links) als Beispiel für die mechanische Zerfaserung und Zellstoff (rechts). Im Holzschliff sind zahlreiche Bestandteile der Zellwand als kleinere und feinere Partikel enthalten. (Kerekes 2015).

Eine weitere erwähnenswerte Eigenschaft im Zusammenhang mit der Charakterisierung der Beschaffenheit von Refiner-Faserstoffen ist die Latenz. Damit wird das Phänomen des Verdrehens und Kräuselns der Fasern während der Faserstofferzeugung bezeichnet. Denn diese Verformungen bleiben durch Abkühlen bei hoher Konsistenz erhalten (Htun et al. 1988).

\subsection{Dispersitätseigenschaften und Partikelmesstechnik}

Vom Standpunkt der mechanischen Verfahrenstechnik aus, handelt es sich bei der Faserstofferzeugung um einen Zerteilprozess (s. Abschnitt 3.4.2) (Schönert 2003, S. 268). Die fundamentale Zielgröße bei der Zerkleinerung ist die Partikelgrößenverteilung (Bohnet 2004, S. 9). Das erzeugte Partikelkollektiv (disperses System) ist aus der dispersen Phase der Einzelpartikel und der kontinuierlichen Phase des umgebenden Mediums (im Prozess) zusammengesetzt (Stieß 2009, S. 9). Mit Partikelkollektiv kann im Zusammenhang mit der Herstellung von Holzfaserdämmstoffen folglich das heterogene Gemisch der Faserstoffe überschrieben werden, welches sich in Luft (Trockenverfahren) oder Wasser (Nassverfahren) befindet. Die Charakterisierung disperser Systeme hat unter anderem zum Ziel:

1. Die Auswirkung mechanischer Prozesse anhand der Dispersitätseigenschaften zu beurteilen (Bohnet 2004, S. 4). Im vorliegenden Fall betrifft das die Untersuchungen zur Faserstofferzeugung im RMP- und TMP-Verfahren mit unterschiedlichen Mahlscheiben.

2. Funktionale Zusammenhänge und Abhängigkeiten zwischen Dispersitätseigenschaften und bestimmten Produkteigenschaften im Sinne einer Eigenschaftsfunktion zu identifizieren (Rumpf 1967). Im vorliegenden Fall gilt das für die Untersuchungen zur Wärmeleitfähigkeit der im RMP- und TMP-Verfahren mit unterschiedlichen Mahlscheiben erzeugten Faserstoffe.

Ist bekannt, welche Dispersitätsgrößen die gewünschten Produkteigenschaften ergeben, kann der Prozess darauf eingestellt und optimiert werden. Bei Holzfaserdämmstoffen sind die Dispersitätseigenschaften wissenschaftlich unzureichend untersucht und es ist, abgesehen von der industriellen Praxis, weitgehend unklar, ob die angestrebten Produkteigenschaften mit anderen Rohstoffen und Verfahren energetisch und wirtschaftlich günstiger erreicht werden können. Die Messaufgabe vor dem Hintergrund der Prozessgestaltung besteht darin, relevante Größen und 
ihre Verteilung zur Beschreibung des dispersen Systems zu erkennen. In der Partikelmesstechnik werden hierzu unter anderem grundsätzlich Siebverfahren zur Analyse der Größenverteilung von Partikelkollektiven sowie optische Verfahren für die Größen- und Formanalysen einzelner Partikel unterschieden (Stieß 2009, S. 161).

Aufgrund der Fokussierung auf das Trockenverfahren in der Herstellung von Holzfaserdämmstoffen scheiden Partikelmesssysteme in wässriger Dispersion u.a. wegen der zu erwartenden Entfernung der Latenz und die nicht repräsentative Auflösung von Agglomeraten aus. Hinzu kommt, dass diese Messsysteme aus der Papier- und Zellstoffindustrie nicht bei MDF angewendet werden können (Roffael et al. 1994a; Weber et al. 2014). Für die Charakterisierung von trockenen Faserstoffen in Luft steht eine begrenzte Auswahl an Methoden zur Verfügung, die in der industriellen, wie in der Laborpraxis weder allgemein anerkannt und akzeptiert noch vollständig entwickelt oder standardisiert sind.

Als Trockensiebung kommt die Luftstrahl-Prüfsiebung in der MDF- und Holzfaserdämmstoffherstellung zur gravimetrischen Ermittlung der Größenverteilung zur Anwendung (Ladenburger 2010; Gao 2014). Die unter dem Prüfsieb rotierende Schlitzdüse sorgt durch die hohe Strömungsgeschwindigkeit des eintretenden Luftstrahls für eine hohe Dispergierleistung sowie den pneumatischen Transport des Feinanteils durch die Siebmaschen (Schmidt et al. 2003, S. 146). Die Luftstrahl-Prüfsiebung charakterisiert das Partikelkollektiv in seiner Gesamtheit. Die messbare Dispersitätsgröße ist die geometrische Abmessung der Partikeln mit der Maschenweite des Siebgewebes als Trenngrenze. Schirp et al. (2015) erkannten, dass zwischen der Luftstrahl-Prüfsiebung nach EN ISO 4610:2001 (2002) und optischen Verfahren in der Analyse von MDF-Faserstoffen vermutlich keine Vergleichbarkeit besteht. Krug (2010, S. 117) und Wenderdel \& Krug (2012) wiesen mit dem Luftstrahlsieb eine deutliche Differenzierung von Kiefern-Faserstoffen nach der Verweilzeit, der Aufschlusstemperatur und dem Mahlspalt nach. Unveröffentlichte Ergebnisse aus der Holzwerkstoffindustrie weisen der Luftstrahl-Prüfsiebung eine hohe Präzision durch einen geringen Variationskoeffizienten nach, was den Zeitaufwand der Analyse relativiert. Der Zeitaufwand kann außerdem durch den Einsatz von Prüfmaschinen für beliebig viele Fraktionen erheblich reduziert werden (Wahler 1960; Schmidt \& Coppers 1998). Neben der Luftstrahlsiebung eignet sich auch die trockene Siebfraktionierung mit Plansiebprüfmaschinen und Siebhilfen (Jensen 1975).

Optische Verfahren in Kombination mit quantitativer Bildanalyse bieten als Individualmethode umfangreichere Informationen durch geometrische Abmessungen als Dispersitätsgröße. Als optische Verfahren zur trockenen Charakterisierung von Faserstoffen im TMP-Verfahren sind u.a. die zweidimensional abbildenden Systeme FibreView, FibreCube und FibreShape bekannt. Das System FibreView kommt inline in der MDF-Herstellung zum Einsatz und identifiziert Faserbündel auf der Faservliesoberfläche (Hasener 2013). Das System FibreCube wurde ebenfalls für die inline Fasercharakterisierung entwickelt. Benthien et al. (2014) wiesen damit nach, dass die Fasergröße mit steigender Verweilzeit und Temperatur abnimmt. Mit demselben System untersuchten Benthien et al. (2017) auch die Einflüsse auf die Fasergrößenverteilung und arbeiteten heraus, dass Holzart und Mahlspalt den größten Einfluss haben. Das am Laboreinsatz orientierte FibreShape-System ist ein statisches System, das auch für die Charakterisierung von Faserstoffen für die Holzfaserdämmstoffanalyse eingesetzt wird (Imken et al. 2021). Schirp et al. (2014, 2015) verwendeten eine Anzahl- sowie Flächengewichtung und konnten zeigen, dass das System für die Analyse der Länge, Breite und Konvexität von TMP-Faserstoffen geeignet ist.

In puncto Methodik zur Charakterisierung von Faserstoffen sind die Arbeiten von Wenderdel et al. (2014, 2016b) und Wenderdel et al. (2017) erwähnenswert. Hierin werden Kriterien, ähnlich wie in Karnis (1994) \& Forgacs (1963) zur Klassierung der Partikeltypen Staub, Einzelfaser und Faserbün- 
del mit Unterklassen eingeführt (Abschnitt 4.1). Die Analyse erfolgte mit einer Kombination aus QualScan- und CamSizer-System in wässriger Dispersion mit Volumen-Gewichtung. Weiterhin zeigte Wenderdel (2015), dass die Differenzierung nach Partikeltypen vorteilhaft für die Ermittlung von funktionalen Zusammenhängen und Korrelationen zwischen Dispersitätseigenschaften und bestimmten Produkteigenschaften ist.

\subsection{Mikroskopische Untersuchungen}

In Konsequenz der Faserstofferzeugung nach dem RMP- und TMP-Verfahren ist die Struktur der Zellwand teilweise mechanisch deformiert und intern fibrilliert (Heikkurinen \& Leskelä 1999, S. 407). Diese strukturellen Veränderungen infolge der mechanischen Beanspruchung können durch eine zweifarbige differenzielle Färbung (Simons' stain) sichtbar gemacht werden (Simon 1950). In der Papiertechnik und in der Biotechnologie ist diese Methode anerkannt und verbreitet (Heikkurinen \& Leskelä 1999, S. 407; Meng et al. 2013; Blanchette et al. 1992). Der Mechanismus wird von Yu et al. (1995) folgendermaßen erklärt: Die geringe Molekülgröße des blauen Farbstoffs erzeugt eine graduelle Farbtiefe infolge der Zugänglichkeit der inneren Oberfläche der Zellwand. Überschreitet die Größe der Mikroporen die Molekülgröße des orangenen Farbstoffs $(5 \mathrm{~nm})$, erscheinen diese Regionen aufgrund der höheren Affinität orange. Eine breite Größenverteilung der Mikroporen färbt die Fasern grünlich (Yu et al. 1995). Weiterhin stellten Esteghlalian et al. (2001) fest, dass gebleichte Zellstofffasern orange gefärbt werden, was auf die verbesserte Zugänglichkeit der interfibrilären Hohlräumen zurückzuführen ist. Die Methode scheint sich zur quantitativen Erfassung von Deformation und Fibrillierung von TMP-Faserstoffen zu eignen (Fernando \& G. Daniel 2010). Papiertechnische Untersuchungen an verschiedenen Nadelhölzern und Laubholz (Populus tremuloides) sind bekannt (Akhtar et al. 1995). Die Anwendung an Buchenholz und die Charakterisierung von RMP- sowie TMP-Faserstoffen mit höherer Aufschlusstemperatur sind nicht bekannt. 


\section{Herstellung und ausgewählte Eigenschaften von Holzfaserdämmstoffen}

\subsection{Rohstoffe, Zusatzstoffe, Hilfsstoffe und Klebstoffe}

Den Angaben in den technischen Datenblättern der Holzfaserdämmstoffhersteller zufolge kommt in der Herstellung von Holzfaserdämmstoffen ausschließlich Nadelholz (Kiefer, Fichte, Tanne) zum Einsatz. Dabei wird überwiegend auf Nadelrundholz zurückgegriffen. Von einem Hersteller ist der Einsatz von Sägewerks-Koppelprodukten bekannt. Die Eignung von Fichtenholz für Holzfaserdämmstoffe legen bereits Untersuchungen von Kollmann \& Malmquist (1956) und Richter (1993) nahe. Aus mehreren Forschungsvorhaben ist die Eignung von Laubholzsortimenten für die Herstellung von Holzfaserdämmstoffen bekannt geworden. So stellt Heinrich (2004) fest, dass Faserstoffe aus Buche und Pappel eine vergleichbare Wärmeleitfähigkeit wie Faserstoffe aus Fichte und Kiefer besitzen. Bartholme et al. (2009) und Brombacher (2015) wiesen die Eignung von Buchen-Faserstoffen und daraus hergestellter Dämmstoffe nach. Nach Eichhorn (2017) eignet sich sogar Eichenholz-Faserstoff als Dämmstoff. Die Untersuchungen von Vignon et al. (2016) zeigen die Eignung von Pappel als Rohstoff für die Holzfaserdämmstoffherstellung durch die niedrigere Wärmeleitfähigkeit im Vergleich zu Kiefer und den niedrigeren elektrischen Energieaufwand in der Zerfaserung hin. Barth $(2017,2018)$ weist ebenfalls auf die technologischen und ökonomischen Vorteile der Verwendung von Buchenholz in der Herstellung von Holzfaserdämmstoffen hin.

In der Herstellung von Holzfaserdämmstoffen kommen diverse Zusatzstoffe zur Einstellung bestimmter Produkteigenschaften zum Einsatz. Dazu zählen hauptsächlich Paraffine als Hydrophobierungsmittel und Ammoniumsalze als brandhemmende Stoffe mit einstelligen Prozentanteilen an der Trockenmasse. Darüber hinaus können Holzschutzmittel mit fungizider und insektizider Wirkung enthalten sein. Weitere Hilfsstoffe kommen im Nassverfahren zur chemischen Behandlung des Prozesswassers und als Trennmittel auf den Sieb- und Transportbändern auch im Trockenverfahren zum Einsatz. (Brombacher 2015)

Als Klebstoffe werden im Trockenverfahren zur Herstellung druckfester Holzfaserdämmstoffplatten Polyurethane auf Basis von Diphenyl-methan-diisocyanat (MDI) mit Aushärtungseigenschaften bei niedrigen Temperaturen $\left(<100^{\circ} \mathrm{C}\right)$ eingesetzt. Holzfaserdämmstoffplatten aus dem Nassverfahren können mit einem geringen Anteil an Phenolharzen gebunden sein. Bei den flexiblen Holzfaserdämmstoffmatten kommen thermoplastische Zweikomponenten-Kunstfasern (Bikofasern) zum Einsatz (Barth et al. 2018). Das Polymer des Mantels hat einen niedrigeren Schmelzpunkt $\left(<130^{\circ} \mathrm{C}\right)$ als das Polymer des Kerns $\left(>200^{\circ} \mathrm{C}\right)$. Zur Bindung der Faserstoffe wird der Mantel geschmolzen. Die Kernfaser sorgt durch ihre Stützfunktion dafür, dass die geringe Rohdichte der Matte erhalten bleibt. Im Fall von Buchen-Faserstoffen zeigte Barth (2017), dass die Auswahl der Bikofasern entscheidend für vergleichbare mechanische Eigenschaften zu aus Fichten-Faserstoffen hergestellten Dämmstoffmatten ist. Der Anteil an der Trockenmassse konnte durch prozesstechnische Verbesserungen und Optimierung der Bikofasern mittlerweile von $6 \%$ bis $8 \%$ auf $2 \%$ bis $3 \%$ gesenkt werden. 


\subsection{Spezielle Prozesstechnik}

Neben der bereits in Abschnitt 3.3.3 besprochenen Faserstofferzeugung sind das Nass- und das Trockenverfahren spezifisch in der Herstellung von Holzfaserdämmstoffen. Für beide Verfahren werden die Faserstoffe im TMP-Verfahren erzeugt. Im Nassverfahren kann zusätzlich eine LC-Mahlung zur Anwendung kommen, um die Bindungskräfte zwischen den Holzfasern durch Fibrillierung zu erhöhen. Die Stoffsuspension wird anschließend auf einer Langsiebmaschine, ähnlich wie in der Papierherstellung, zu einem Faservlies geformt (Lampert 1967). Die Rohdichte der damit hergestellten Dämmstoffplatten liegt zwischen $100 \mathrm{~kg} / \mathrm{m}^{3}$ und $300 \mathrm{~kg} / \mathrm{m}^{3}$ bei einer maximalen Dicke von $20 \mathrm{~mm}$. Die Dicke ist durch die notwendige Trocknung der Platten begrenzt. Höhere Dämmstoffdicken werden durch Verleimen mehrerer Schichten erzielt.

Mit der Übertragung des Trockenverfahrens aus der MDF-Herstellung wurde es möglich, Holzfaserdämmstoffplatten kontinuierlich bis zu einer Dicke von $300 \mathrm{~mm}$ sowie flexible Holzfaserdämmstoffmatten herzustellen (Antrag auf Nichtnennung 1998). Die Faservliesbildung erfolgt mittels mechanischer MDF-Streuköpfe oder adaptierten aerodynamisch-mechanischen Vliesbildnern aus der Textilherstellung. Im Gegensatz zur MDF-Herstellung kommt zur Aushärtung der Dämmstoffe Dampf- und/oder Heißluft zur Anwendung. Dadurch kann das poröse Faservlies mit geringer Dichte schnell auf die notwendige Temperatur gebracht werden. Der Pressdruck und die zur Aushärtung notwendige Temperatur sind vergleichsweise niedrig. Kawasaki et al. (1998) bestätigten die Praktikabilität dieser Technologie.

\subsection{Aspekte der Wärmeleitfähigkeit}

In Ergänzung zu Abschnitt 2.3.3 sollen hier produktspezifische Aspekte der Wärmeleitfähigkeit von Holzfaserdämmstoffen angesprochen werden. Im Zusammenhang mit den Produkteigenschaften von Holzfaserdämmstoffen wird weitgehend nicht auf die Normierung eingegangen, denn diese bildet nicht den Stand der Wissenschaft und Technik ab. Einen Überblick über die harmonisierten europäischen Dämmstoffnormen bietet Albrecht (2010). Moderne Holzfaserdämmstoffe werden als Platten, Matten und Einblasdämmung angeboten. Die Rohdichte liegt bei den druckfesten Dämmplatten typischerweise zwischen $120 \mathrm{~kg} / \mathrm{m}^{3}$ und $200 \mathrm{~kg} / \mathrm{m}^{3}$ sowie bei den flexiblen Dämmmatten zwischen $50 \mathrm{~kg} / \mathrm{m}^{3}$ und $60 \mathrm{~kg} / \mathrm{m}^{3}$. Die Wärmeleitfähigkeit $\lambda$ von Holzfaserdämmstoffen wird in $\mathrm{W} /(\mathrm{m} \cdot \mathrm{K})$ angegeben. Hierbei ist zwischen Mess-, Nenn- und Bemessungswerten zu unterscheiden. Die Holzwerkstoffhersteller sind verpflichtet, den Nennwert $\lambda_{\mathrm{D}}$ der Wärmeleitfähigkeit anzugeben (EU 2011). Der Nennwert basiert auf $\lambda_{90 / 90}$-Werten: Das heißt, dass $90 \%$ der Messwerte mit $90 \%$-iger Wahrscheinlichkeit kleiner oder gleich dem Nennwert sind. Der weiterhin angegebene Bemessungswert enthält einen Sicherheitszuschlag für die statistische $\lambda_{90 / 90}$-Unsicherheit $\left(\lambda_{\mathrm{D}}+20 \%\right)$ oder mit technischer Spezifikation $\left(\lambda_{\text {grenz }}+5 \%\right)(\mathrm{DIN} V$ 4108-4:200407 2004). In der vorliegenden Arbeit erfolgt die Auswertung ausschließlich an Messwerten der Wärmeleitfähigkeit mit der europäisch einheitlich geregelten Bezugsfeuchte von $23^{\circ} \mathrm{C} / 50 \%$ relativer Luftfeuchte (siehe Abschnitt 6.4.8). Moderne Holzfaserdämmstoffe weisen Nennwerte von minimal $0,036 \mathrm{~W} /(\mathrm{m} \cdot \mathrm{K})$ bei flexiblen Dämmmatten mit einer Rohdichte von $60 \mathrm{~kg} / \mathrm{m}^{3} \mathrm{bis} \mathrm{hin}$ zu $0,048 \mathrm{~W} /(\mathrm{m} \cdot \mathrm{K})$ bei einer Rohdichte von $270 \mathrm{~kg} / \mathrm{m}^{3}$ auf.

Einflussfaktoren auf die Wärmeleitfähigkeit von Holzfaserdämmstoffen wurden eingehend wissenschaftlich untersucht. Bezüglich der Beschaffenheit der Faserstoffe sind Untersuchungen von Scheiding (2000), F. Michel et al. (2014) und Makas (2016) zu erwähnen, die sich mit der Wärmeleitfähigkeit von Siebfraktionen beschäftigen. Scheiding (2000) zeigte an Fichten-Faserstoff, 
dass die mit einem Schwingsieb erzeugte Feinfraktion (Durchgang Siebmaschenweite 0,355 $\mathrm{mm}$ ) eine niedrigere Wärmeleitfähigkeit aufweist, als der unklassierte Faserstoff. Die Grobfraktion (Rückstand Siebmaschenweite 4,0 mm) wies eine höhere Wärmeleitfähigkeit auf. F. Michel et al. (2014) klassierte u.a. Buchen- und Fichten-Faserstoffe in eine feine (Siebmaschenweite 0,5 mm bis $4,0 \mathrm{~mm}$ ) und eine grobe Sieblinie (Siebmaschenweite $4,0 \mathrm{~mm}$ bis $16 \mathrm{~mm}$ ). Die feine Sieblinie wies bei beiden Holzarten die niedrigste Wärmeleitfähigkeit auf (Abbildung 5.1). Im Gegensatz zu Scheiding (2000) lag auch die grobe Sieblinie niedriger als der unklassierte Faserstoff. Diese Feststellung bestätigt Brombacher (2015, S. 111) für die industriellen Faserstoffe aus Buche, Fichte und Kiefer. Die Angabe der Siebmethode fehlt allerdings. Bei Makas (2016) hingegen weist der unklassierte Faserstoff die niedrigste Wärmeleitfähigkeit auf, gefolgt von der Fein-, Mittelund Grobfraktion. Angaben zur Holzart, Siebmaschenweite oder Siebmethode sind jedoch nicht genannt.

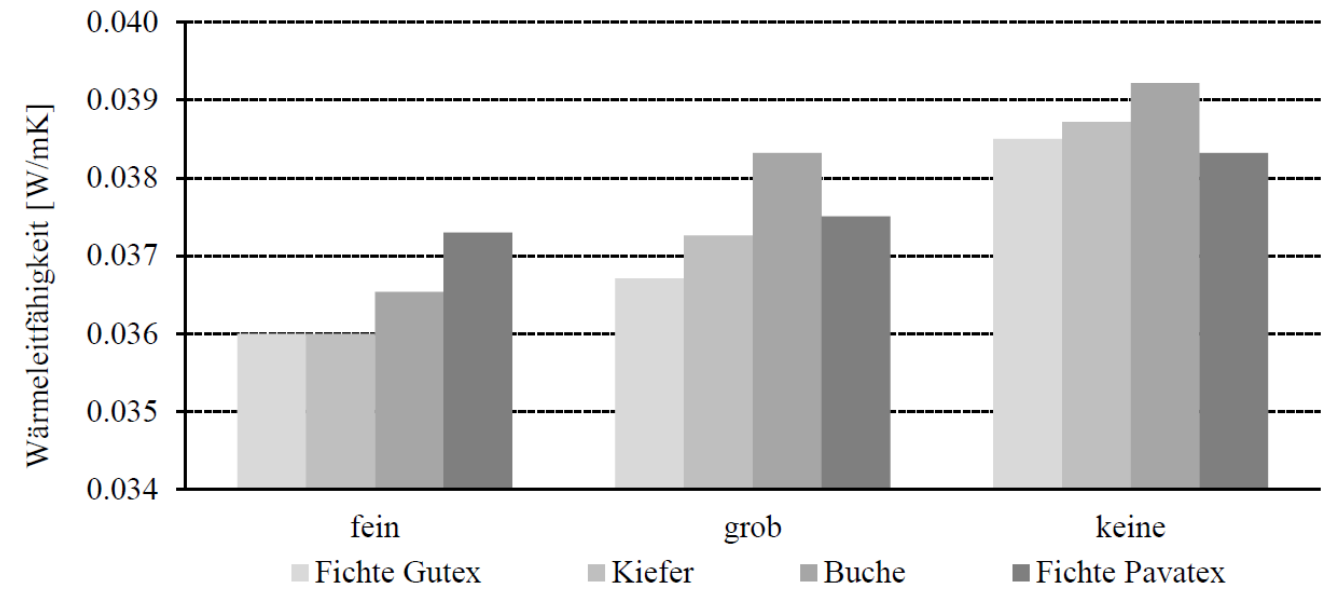

Abbildung 5.1: Einfluss der Klassierung von Faserstoffen auf die Wärmeleitfähigkeit bei einer Rohdichte von $80 \mathrm{~kg} / \mathrm{m}^{3}$. (F. Michel et al. 2014).

Eine weitere Einflussgröße auf die Wärmeleitfähigkeit stellt die Rohdichte dar. Vogel (1999) zufolge liegt das Minimum der Wärmeleitfähigkeit für TMP-Faserstoff bei rund $54 \mathrm{~kg}_{\text {atro }} / \mathrm{m}^{3}$. Brombacher et al. (2012) wiesen einen linearen Zusammenhang $\left(\mathrm{R}^{2}=0,87\right)$ zwischen der Rohdichte und der Wärmeleitfähigkeit von Holzfaserdämmstoffen unabhängig von deren Zusammensetzung oder dem Herstellungsprozess nach. Weiterhin scheint Brombacher et al. (2016) zufolge eine Korrelation $\left(\mathrm{R}^{2}=0,68\right)$ zwischen der Schüttrohdichte und der Wärmeleitfähigkeit von Fichten-Faserstoffen vorzuliegen. Vignon et al. (2016) schlagen anhand ihrer Erkenntnisse zum übergeordneten Einfluss der Rohdichte ein mathematisches Modell zur Berechnung der Wärmeleitfähigkeit als Funktion der Rohdichte vor. Die Ergebnisse stimmen weitgehend mit Makas (2016) überein, der ein Minimum der Wärmeleitfähigkeit zwischen $45 \mathrm{~kg} / \mathrm{m}^{3}$ und $65 \mathrm{~kg} / \mathrm{m}^{3}$ verortet. Untersuchungen von Heinrich (2004) zum Einfluss der Rohdichte von Buchen-Faserstoffen gehen nicht über $33 \mathrm{~kg}_{\text {atro }} / \mathrm{m}^{3}$ hinaus. Brombacher (2015, S. 111) stellte keine Abhängigkeit der Wärmeleitfähigkeit von industriellem Buchen-Faserstoff bei einer Rohdichte zwischen rund $80 \mathrm{~kg} / \mathrm{m}^{3}$ und $110 \mathrm{~kg} / \mathrm{m}^{3}$ fest.

Neben der Beschaffenheit der Faserstoffe und der Rohdichte muss auf die Holzfeuchte als Einflussgröße auf die Wärmeleitfähigkeit eingegangen werden. Heinrich (2004) gibt an, dass der Einfluss der Holzfeuchte auf die Wärmeleitfähigkeit von Faserstoffen gering ist und praktisch keine Unterschiede zwischen Normklima und absolut trockenen Probekörpern festzustellen sind. 
Die umfangreicheren Untersuchungen durch Sonderegger \& Niemz (2012) belegen allerdings einen linearen Zusammenhang der Holzfeuchte im Bereich von $0 \%$ bis $14 \%$ bei einer Probenmitteltemperatur von $10{ }^{\circ} \mathrm{C}\left(\mathrm{R}^{2}=0,91\right), 20^{\circ} \mathrm{C}\left(\mathrm{R}^{2}=0,94\right)$ und $30^{\circ} \mathrm{C}\left(\mathrm{R}^{2}=0,96\right)$. Weiterhin postulieren Ormondroyd et al. (2017) in diesem Zusammenhang eine abnehmende Ausgleichsfeuchte mit steigender Aufschlusstemperatur anhand einer Faserstoff-Mischung aus Fichte, Kiefer und Tanne. 


\section{Material und Methoden}

\subsection{Material}

\subsubsection{Beschreibung des Ausgangsmaterials}

Die Untersuchungen wurden an nicht pathologischem Fichtenholz (Picea abies Karst.) und Buchenholz (Fagus sylvatica L.) aus dem Panger Forst der Bayerischen Staatsforsten (Landkreis Rosenheim, Bayern, 4749'17.5” N 1202'07.4” O) durchgeführt. Der Standort liegt auf einer Höhe von ca. $480 \mathrm{~m}$ und ist geprägt von Hochmoortorf und Moränenmaterial. Das Alter der Bäume lag zwischen minimal ca. 35 Jahren und maximal ca. 67 Jahren. Von den frühsommerlich frisch eingeschlagenen Stämmen wurde der Erd- und Mittelstamm verwendet und manuell entrindet. Für die Hackschnitzelherstellung wurde ein Trommelhacker vom Typ DH 120x400 L-2WV mit Vibrationsförderrinne CV 400N (BRUKS Klöckner GmbH, Hirtscheid, Deutschland) verwendet. Die Hackmesser wurden vor den Untersuchungen geschliffen und mit einer Einstelllehre eingestellt, um eine einwandfreie Hackqualität zu gewährleisten. Die fixen Prozessparameter des Trommelhackers sind in Tabelle 6.1 zusammengefasst. Die Konfiguration des Trommelhackers folgte der Anforderung an die nominelle Hacklänge nach Formel von BRUKS Klöckner GmbH (BRUKS Klöckner GmbH 02.10.2015). Die Lagerdauer der Hackschnitzel zwischen Erzeugung und Zerfaserung war kürzer als sieben Tage (Arbeitsgruppe Sägenebenprodukte (AG SNP) im Ausschuß Forst und Holz 2000).

Tabelle 6.1: Prozessparameter des Trommelhackers BRUKS Klöckner DH 120x400 L-2WV (BRUKS Klöckner $\mathrm{GmbH} 2015)$.

\begin{tabular}{ll}
\hline Vorschubgeschwindigkeit & $27 \mathrm{~m} / \mathrm{min}$ \\
Rotordurchmesser & $450 \mathrm{~mm}$ \\
Rotordrehzahl & $817 \mathrm{~min}^{-1}$ \\
Hackmesseranzahl & 2 \\
Sieblochung & $35 \mathrm{~mm} \mathrm{x} \mathrm{45,} \mathrm{mm}$ \\
Nominelle Hacklänge & $16,5 \mathrm{~mm}$ \\
\hline
\end{tabular}

Anschließend an die Hackschnitzelerzeugung erfolgte eine Sortierung mit einem Trommelsieb. Dabei wurde Feingut (Durchgang durch ein Sieb mit $3 \mathrm{~mm}$ Maschenweite) sowie Grobgut (Rückstand auf einem Sieb mit $20 \mathrm{~mm}$ Maschenweite) abgeschieden.

\subsubsection{Industrielle Zellstoffe, Holzstoffe und Holzfaserdämmstoffe}

Für vergleichende Untersuchungen zur Faserstofferzeugung, zur Beschaffenheit der Faserstoffe, zur Wärmeleitfähigkeit wurden folgende Proben aus der industriellen Produktion beschafft:

- Northern bleached softwood kraft (NBSK), Holzart: Fichte, Hersteller: Zellstoff- und Papierfabrik Rosenthal GmbH. 
- Magnesiumbisulfit-Aufschluss, Nachsortierung, Holzart: Buche $35 \%$, Fichte $65 \%$, Hersteller: Sappi Ehingen $\mathrm{GmbH}$.

- Bleached chemi-thermomechanical pulp (BCTMP), Holzart: Fichte, Hersteller: West Fraser Mills Ltd..

- Holzschliff, ungebleicht, Holzart: Fichte, Hersteller: Kabel Premium Pulp \& Paper GmbH.

- Thermomechanical pulp (TMP), 1. Refinerstufe, Low Retention, High Temperature and High Speed Verfahren (RTS ${ }^{\mathrm{TM}}$, Andritz AG) , Holzart: Fichte, Hersteller: UPM GmbH.

- MDF-Faser, Holzart: Kiefer, Hersteller: Kronospan GmbH.

- HDF-Faser, Holzart: Kiefer, Hersteller: IKEA Industry Poland.

- Zellulosedämmung: Altpapier, Hersteller: Peter Seppele Gesellschaft m.b.H..

- Holzfaserdämmstoff-Faser 1, Holzart: Fichte, Hersteller: Holzwerk Gebr. Schneider GmbH.

- Holzfaserdämmstoff-Faser 2, Holzart: Fichte, Hersteller: GUTEX Holzfaserplattenwerk H. Henselmann GmbH \& Co. KG.

- Holzfaserdämmstoff-Faser 3, Holzart: Kiefer, Hersteller: STEICO SE.

- Holzfaserdämmstoff-Faser 4, Holzart: Kiefer, Hersteller: HOMATHERM GmbH.

- Holzfaserdämmstoff-Matte 1, Holzart: Fichte, Hersteller: Holzwerk Gebr. Schneider GmbH.

- Holzfaserdämmstoff-Matte 2, Holzart: Kiefer, Hersteller: STEICO SE.

Der Magnesiumbisulfit-Aufschluss, der BCTMP-Stoff, der Holzschliff sowie der TMP-Stoff wurden entwässert zur Verfügung gestellt und ungetrocknet präpariert. NBSK wurde als geschnittenes Handmuster zur Verfügung gestellt. Die MDF- und HDF-Fasern sind beleimt und getrocknet aus einer laufenden Produktion entnommen worden. Die Holzfaserdämmstoff-Fasern 1 und 2 sind handelsübliche Einblasdämmung. Die Holzfaserdämmstoff-Fasern 3 und 4 stammen aus der Produktion unterschiedlicher Dämmstoffhersteller und enthalten produktspezifische Zusatzstoffe. Bei der Zellulosedämmung und den Holzfaserdämmstoff-Matten handelt es sich um handelsübliche Produkte diverser Dämmstoffhersteller. Die Materialien wurden von einem Baustoffhändler bezogen.

\subsection{Faserstofferzeugung}

\subsubsection{Spezifikationen der Mahlscheiben}

Die Untersuchungen fanden an sechs unterschiedlichen Mahlscheibenmustern statt. Die Spezifikationen sind in Tabelle 6.2 bis Tabelle 6.8 aufgeführt. Die Mahlscheibenmuster wurden im Rahmen der Dissertation in Zusammenarbeit mit der Andritz AG ausgewählt, entwickelt, konstruiert und gefertigt. Unter den untersuchten Mahlscheiben befinden sich ein bidirektionales Muster (Tabelle 6.2), zwei Spiralen (Tabelle 6.3 und Tabelle 6.4), ein Muster, das dem Stand der Technik in der MDF-Herstellung entspricht (Tabelle 6.5), ein speziell für Holzfaserdämmstoffe 
entwickeltes Muster (Tabelle 6.6 und Tabelle 6.7) und eine Neuentwicklung für das RMP-Verfahren (Tabelle 6.8).

Die Mahlscheiben sind als Ringe mit einen Durchmesser von 12" (305 mm) ausgeführt und verfügen über drei Befestigungspunkte. Vor den Untersuchungen wurden die Mahlscheiben im Laborrefiner mit der Nenndrehzahl und unter Zugabe von Verdünnungswasser plan geschliffen. Dadurch wird Planparallelität der Mahlscheibenoberflächen von Rotor und Stator und die damit zusammenhängende Genauigkeit in der Einstellung des Mahlspalts gewährleistet. Außerdem wurden die Mahlscheiben vor den Untersuchungen zwischen 20 und 50 Betriebsstunden in der Faserstofferzeugung eingefahren. Zum einen wurde dadurch das Verhalten der Mahlscheibenmuster erprobt und zum anderen wurden den Kanten der Stege die anfängliche Schärfe genommen.

Tabelle 6.2: Ausgewählte Spezifikationen des Mahlscheibenmusters 1. Andritz AG, Graz, Österreich.

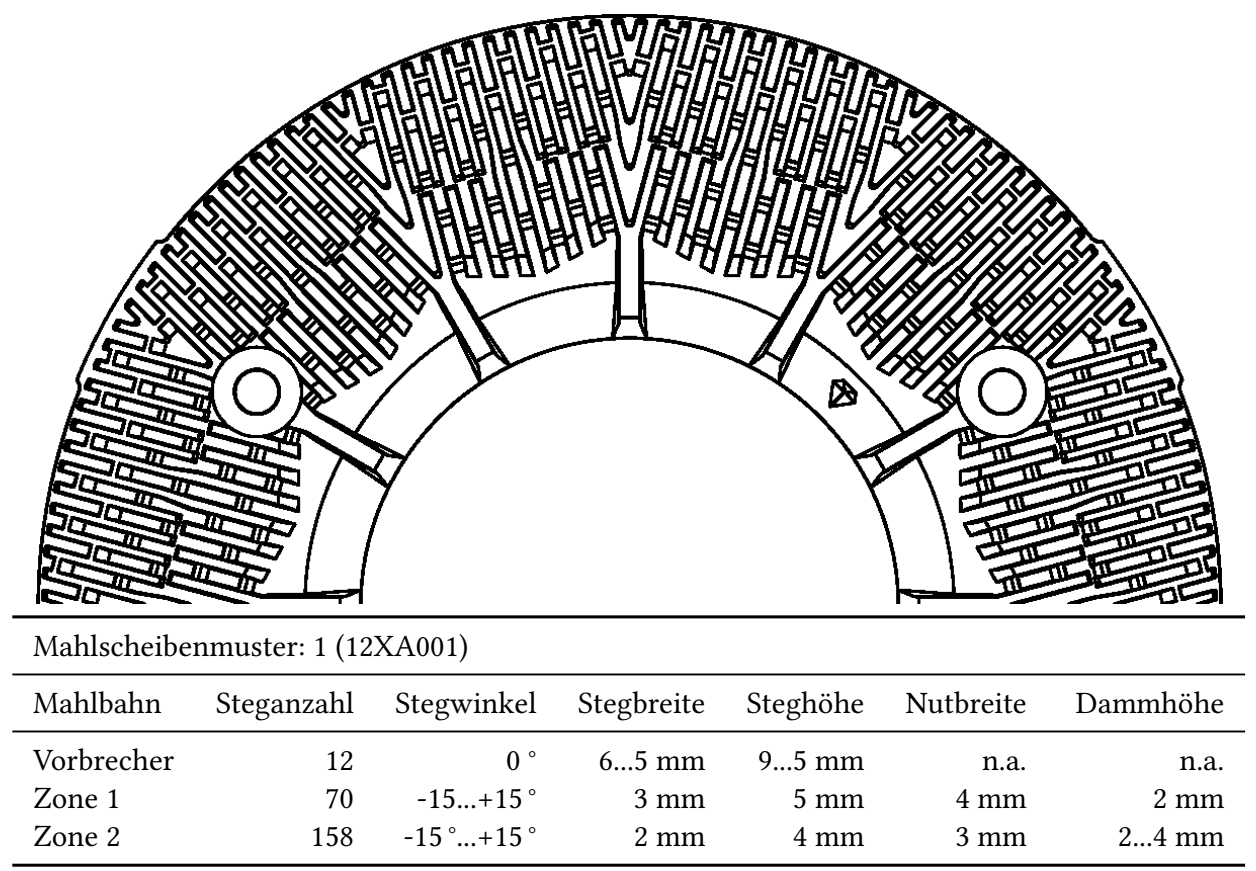


Tabelle 6.3: Ausgewählte Spezifikationen des Mahlscheibenmusters 2. Andritz AG, Graz, Österreich.

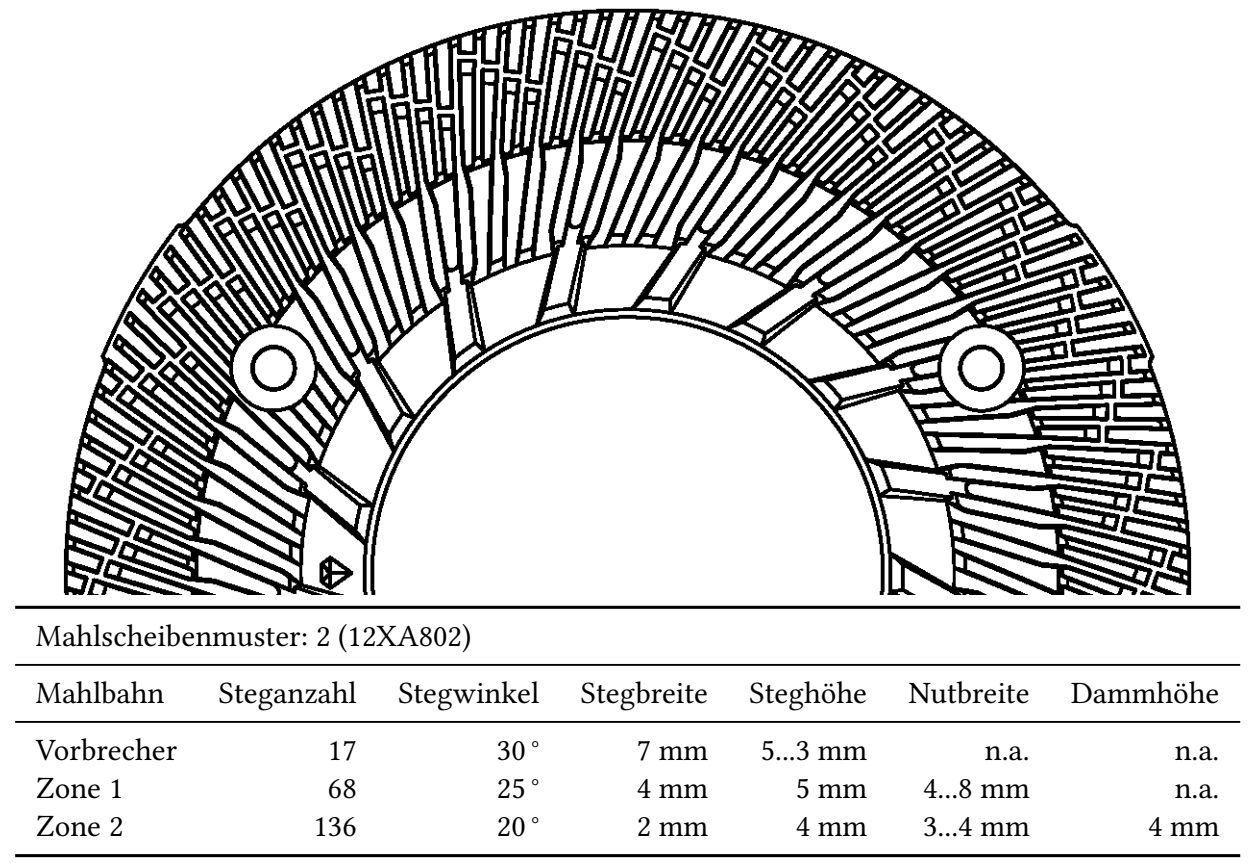

Tabelle 6.4: Ausgewählte Spezifikationen des Mahlscheibenmusters 3. Andritz AG, Graz, Österreich.

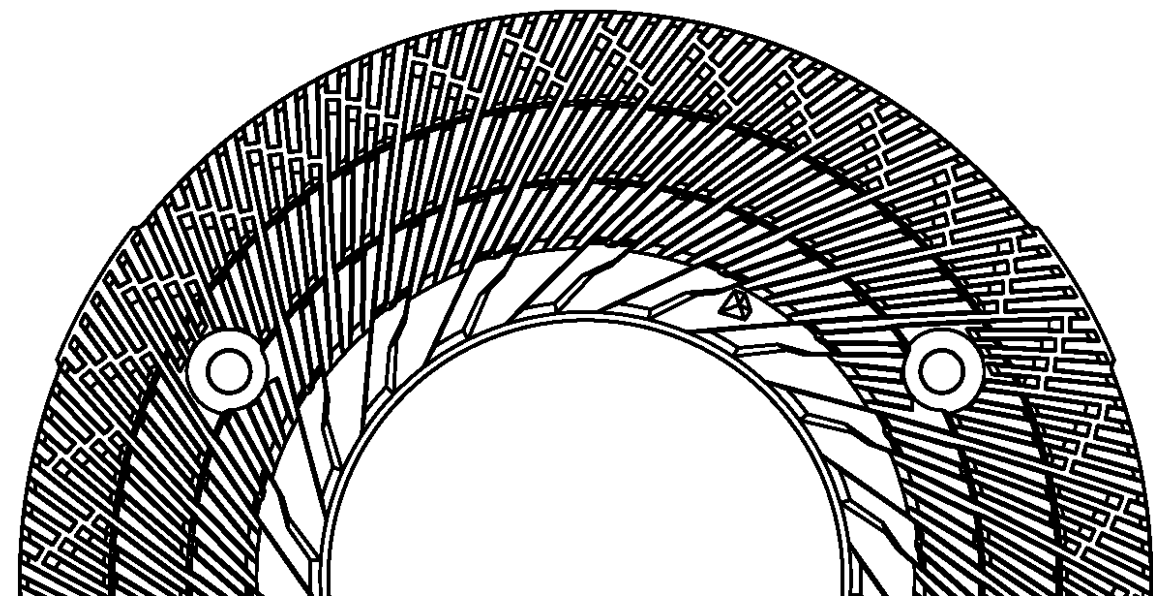

Mahlscheibenmuster: 3 (12XA803)

\begin{tabular}{lrrrrrr}
\hline Mahlbahn & Steganzahl & Stegwinkel & Stegbreite & Steghöhe & Nutbreite & Dammhöhe \\
\hline Vorbrecher & 18 & $30^{\circ}$ & $5 \ldots 10 \mathrm{~mm}$ & $4,5 \ldots 5,5 \mathrm{~mm}$ & n.a. & n.a. \\
Zone 1 & 72 & $30^{\circ}$ & $3 \mathrm{~mm}$ & $6 \mathrm{~mm}$ & $3 \ldots 4 \mathrm{~mm}$ & n.a. \\
Zone 2 & 108 & $36^{\circ}$ & $2,5 \mathrm{~mm}$ & $5 \mathrm{~mm}$ & $2,5 \ldots 3,5 \mathrm{~mm}$ & n.a. \\
Zone 3 & 144 & $44^{\circ}$ & $2 \mathrm{~mm}$ & $4 \mathrm{~mm}$ & $2,5 \ldots 3 \mathrm{~mm}$ & $4 \mathrm{~mm}$ \\
\hline
\end{tabular}


Tabelle 6.5: Ausgewählte Spezifikationen des Mahlscheibenmusters 4. Andritz AG, Graz, Österreich.

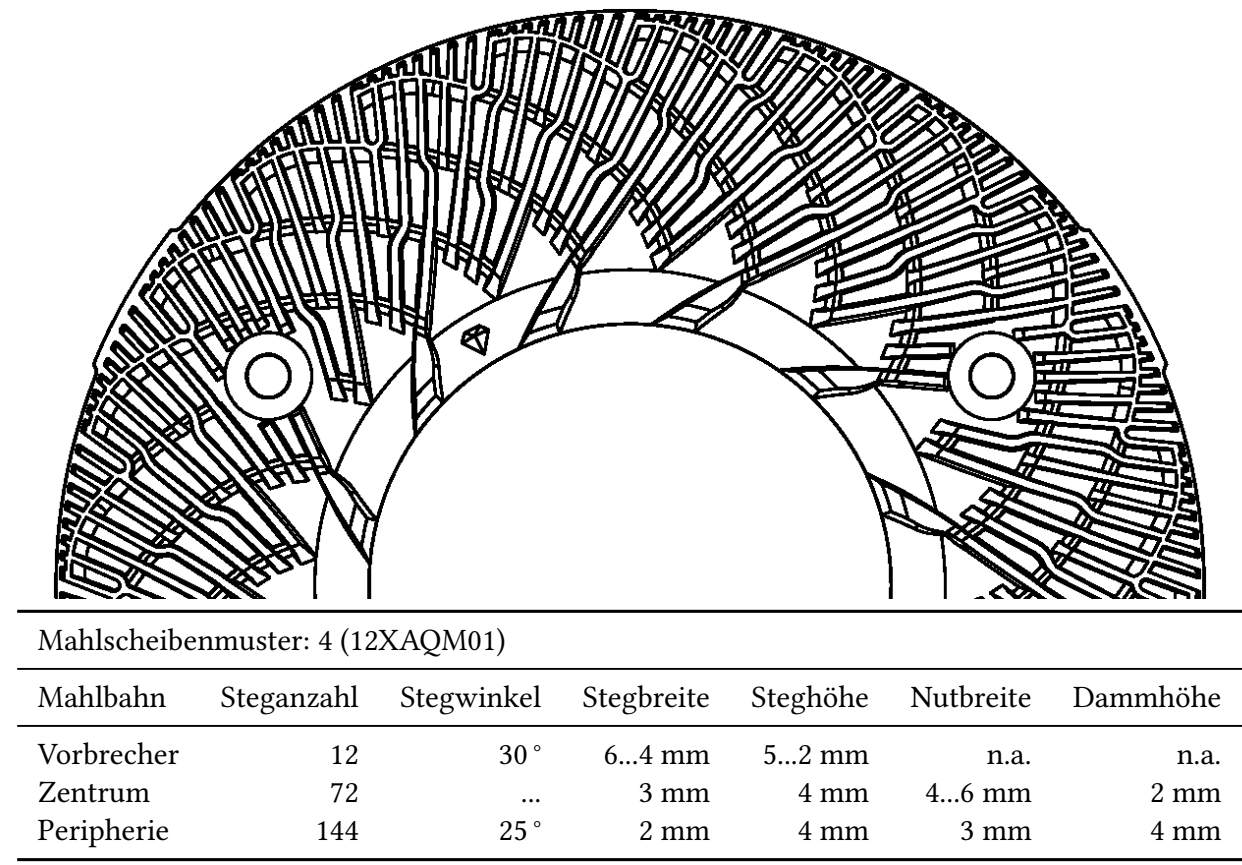

Tabelle 6.6: Ausgewählte Spezifikationen des Rotors des Mahlscheibenmusters 5. Andritz AG, Graz, Österreich.

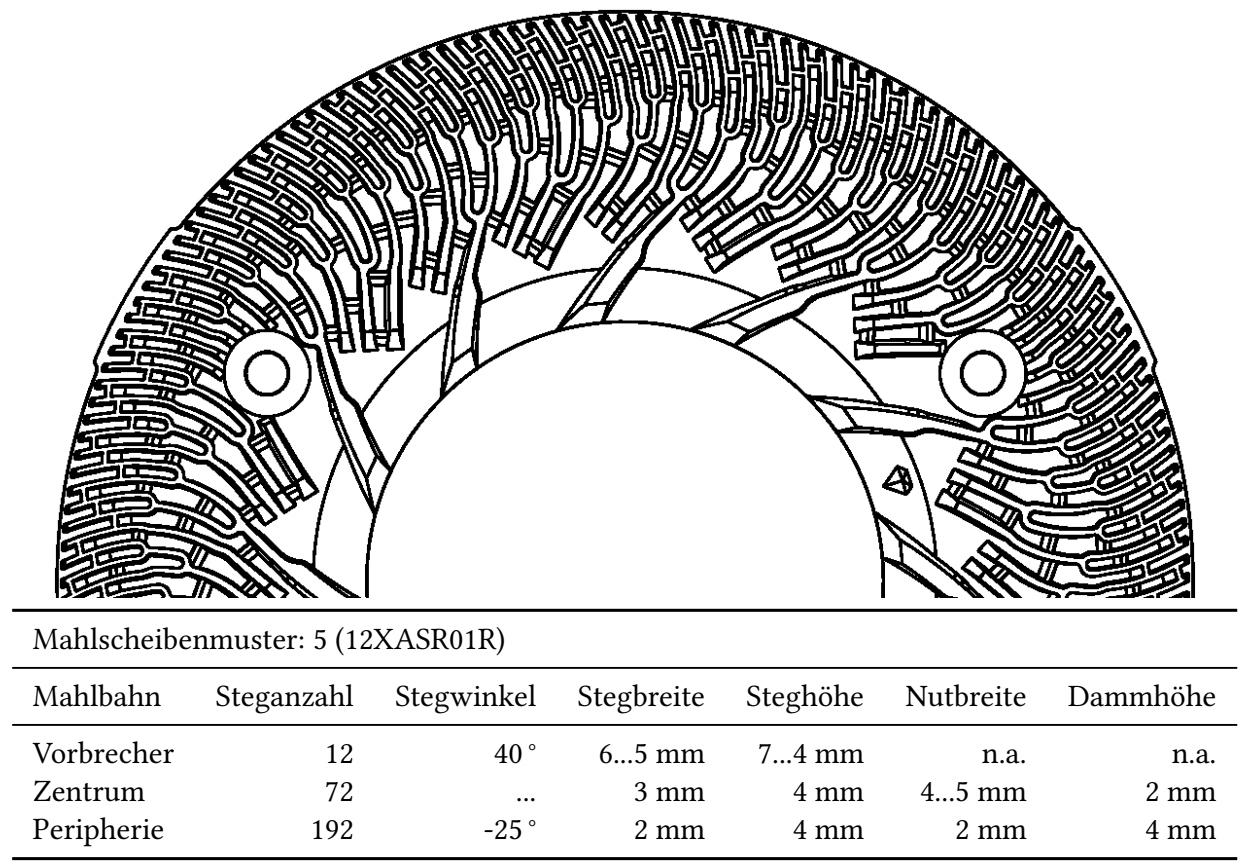


Tabelle 6.7: Ausgewählte Spezifikationen des Stators des Mahlscheibenmusters 5. Andritz AG, Graz, Österreich.

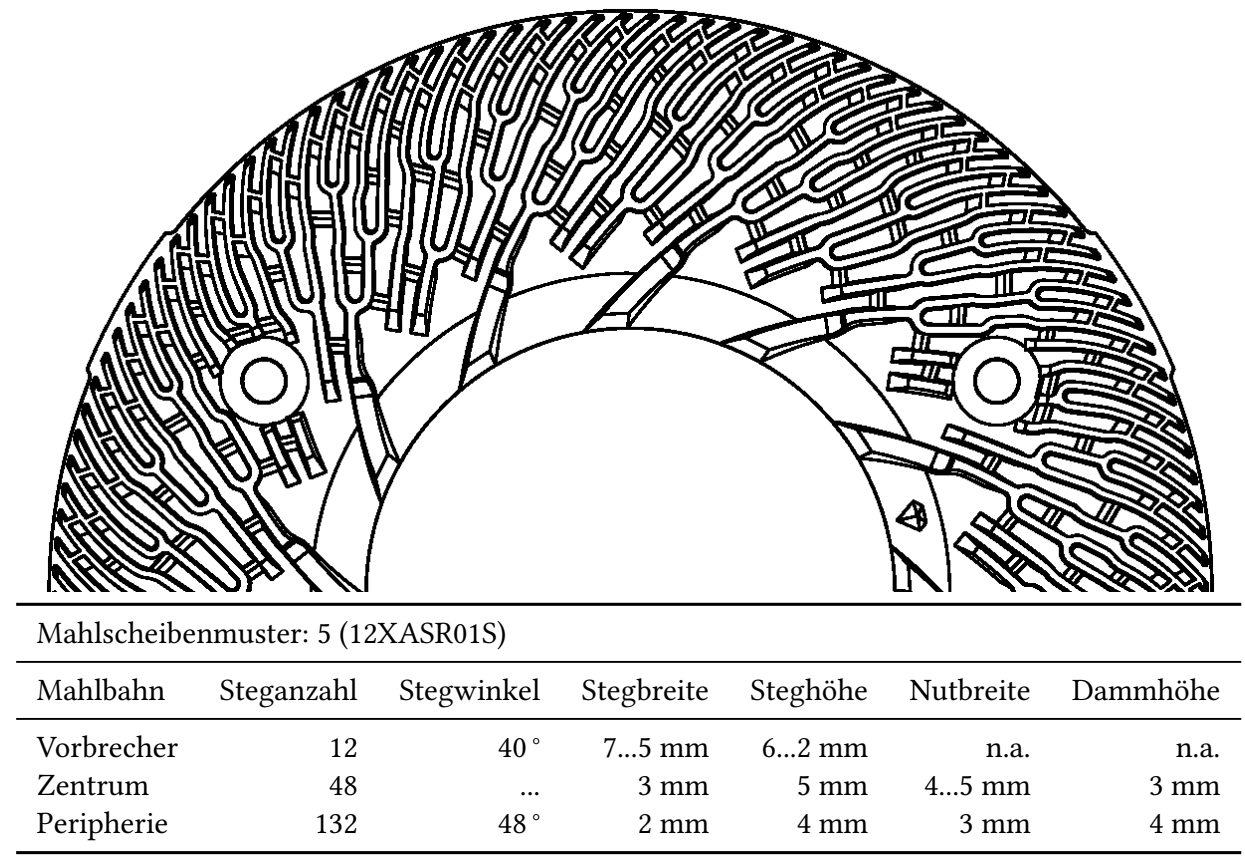

Tabelle 6.8: Ausgewählte Spezifikationen des Mahlscheibenmusters 6. Andritz AG, Graz, Österreich.

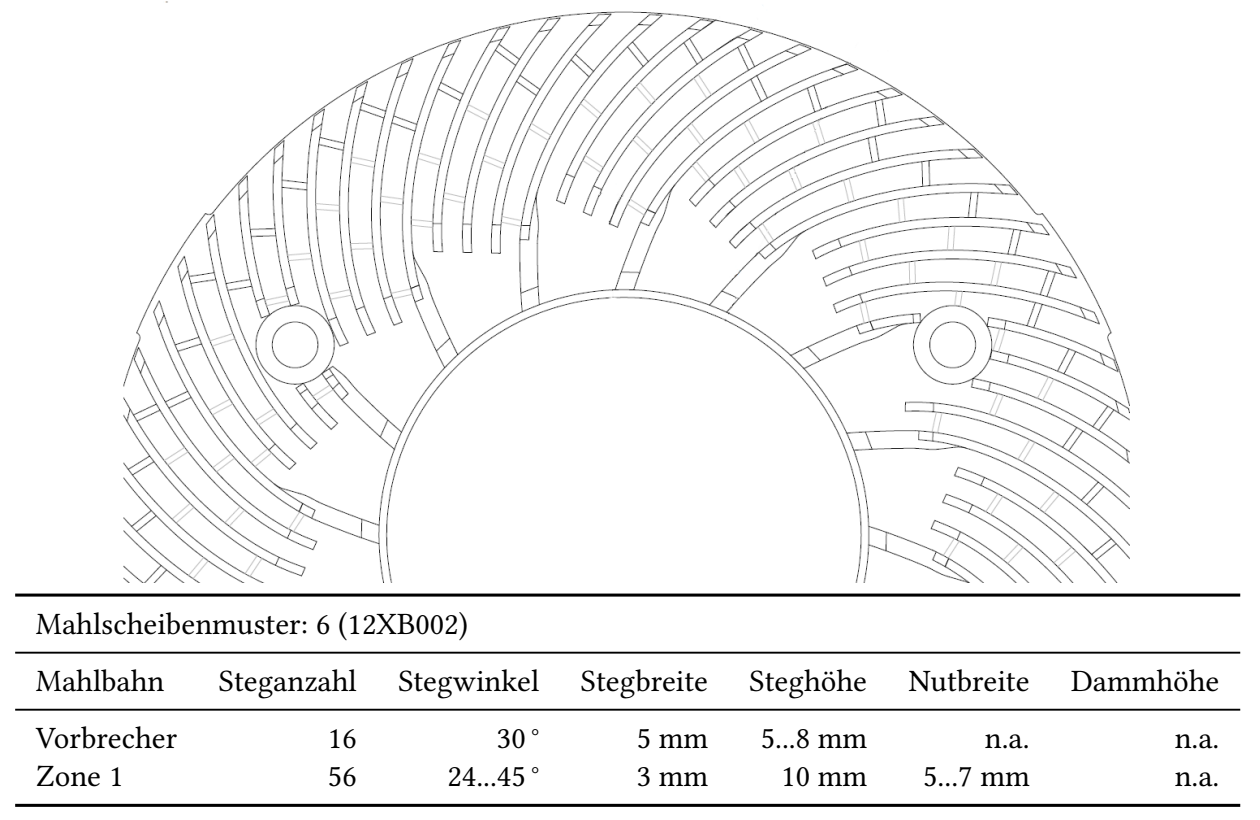

\subsubsection{Konfiguration der Laborrefineranlage im TMP-Verfahren}

Die thermomechanischen Aufschlussversuche fanden im Labor für Holzwerkstofftechnik an der Technischen Hochschule Rosenheim statt. Zum Einsatz kam eine diskontinuierliche 12-1CP Einscheiben-Druckzerfaserungsanlage (Laborrefineranlage) (Andritz AG, Graz, Österreich). Die 
Laborrefineranlage wurde im Jahr 2015 beschafft und vom Verfasser der Dissertation in Betrieb genommen. In Abbildung 6.1 ist die Konfiguration der Anlage im TMP-Verfahren dargestellt. Es kam keine Stopfschnecke oder anderweitige mechanische Vorbehandlung der Hackschnitzel zum Einsatz. Die variablen und fixen Prozessparameter der Laborrefineranlage für das TMP-Verfahren sind in Tabelle 6.9 aufgelistet.

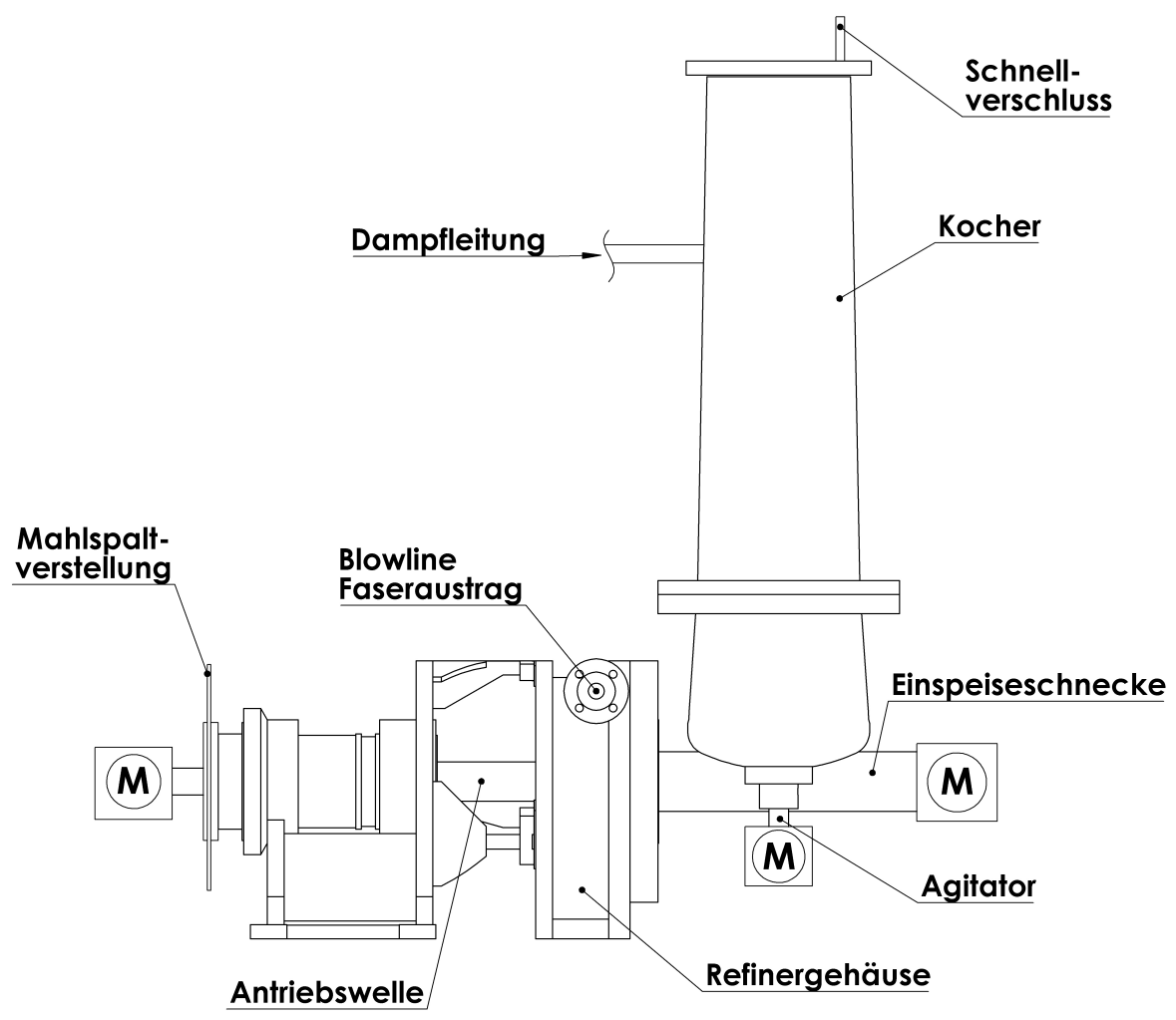

Abbildung 6.1: Schematische Darstellung der Konfiguration der Laborrefineranlage für die Faserstofferzeugung im TMP-Verfahren. Eigene Darstellung.

Vor der Versuchsdurchführung wurden Druckbehälter (Kocher), Refinergehäuse und angrenzende Baugruppen unter Sattdampfdruck auf Betriebstemperatur gebracht. Anschließend wurden die Hackschnitzel über den Schnellverschluss in den Druckbehälter eingefüllt. Die Zerfaserung erfolgte diskontinuierlich (Batch-Verfahren). Der Mahlspalt wurde manuell über ein Handrad mit Ringskala eingestellt. Die Skalenteilung betrug 0,05 mm der Mahlspaltverstellung. Vor der Einstellung wurde der Kontakt der Mahlscheiben als Nullpunkt festgelegt. Die Einstellung erfolgte stets unter Zustellung, um die unkontrollierte Öffnung des Mahlspalts durch Gewindespiel zu unterbinden. Der Faseraustrag erfolgte durch den tangentialen Faserauslass über die Blasleitung (blowline) in einen Zyklon (nicht dargestellt). Unterhalb des Zyklons wurden die Fasern in Behältern aufgefangen. Die Faserproben wurden nach dem Einstellen konstanter Prozessbedingungen entnommen und anschließend in einem Trockenschrank in Abhängigkeit von der Beladung für bis zu $24 \mathrm{~h}$ bei $103^{\circ} \mathrm{C}$ auf eine Feuchte von $5 \%$ bis $8 \%$ getrocknet. 
Tabelle 6.9: Prozessparameter der Laborrefineranlage für das TMP-Verfahren (Andritz AG 2015).

\begin{tabular}{ll}
\hline Variable Prozessparameter & \\
\hline Sattdampfdruck & $1 \ldots 12 \mathrm{bar}$ \\
Aufschlusstemperatur & $120 \ldots 188^{\circ} \mathrm{C}$ \\
Füllmenge max. & 701 \\
Verweilzeit & $0 \ldots 180 \mathrm{~min}$ \\
Förderleistung Einspeiseschnecke & $0 \ldots 100 \%$ \\
Öffnung Blasventil & $0 \ldots 90^{\circ}$ \\
\hline Fixe Prozessparameter & \\
\hline Antriebsleistung, nominell & $54 \mathrm{~kW}$ \\
Drehzahl Refinerwelle & $3000 \mathrm{~min}^{-1}$ \\
Mahlscheibendurchmesser & $12^{\circ} \mathrm{bzw} .305 \mathrm{~mm}$ \\
\hline
\end{tabular}

Den Sattdampf für den Aufschluss lieferte ein Dampferzeuger des Typs DG 200 inklusive Speisewasseraufbereitung (Jumag Dampferzeuger GmbH, Hirschberg an der Bergstraße, Deutschland). Die angewendeten Prozessparameter des Dampferzeugers sind in Tabelle 6.10 aufgeführt. Der alkalische $\mathrm{pH}$-Wert durch Sulfitüberschuss im Speisewasser wird über die Dosierlösung eingestellt. Die Dosierung ist vom Hersteller des Dampferzeugers vorgegeben, um Korrosion im Dampfkessel vorzubeugen.

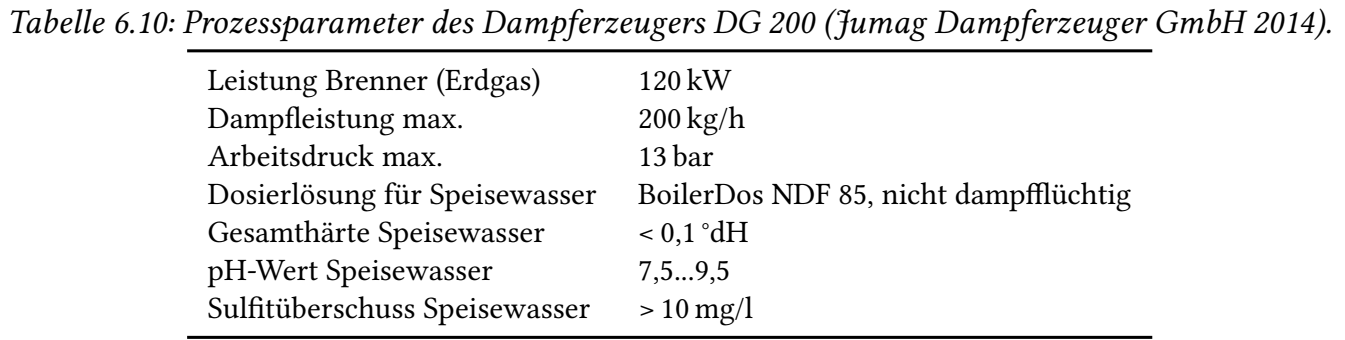

\subsubsection{Konfiguration der Laborrefineranlage im RMP-Verfahren}

Die Untersuchungen zur Faserstofferzeugung im RMP-Verfahren fanden ebenfalls im Labor für Holzwerkstofftechnik an der Technischen Hochschule Rosenheim mit einer 12-1CP Laborrefineranlage (Andritz AG, Graz, Österreich) statt. In Abbildung 6.2 ist die Konfiguration der Anlage im RMP-Verfahren dargestellt. Es kam keine Stopfschnecke oder anderweitige mechanische Vorbehandlung der Hackschnitzel zum Einsatz. Relevante technische Daten sind Tabelle $6.11 \mathrm{zu}$ entnehmen. 


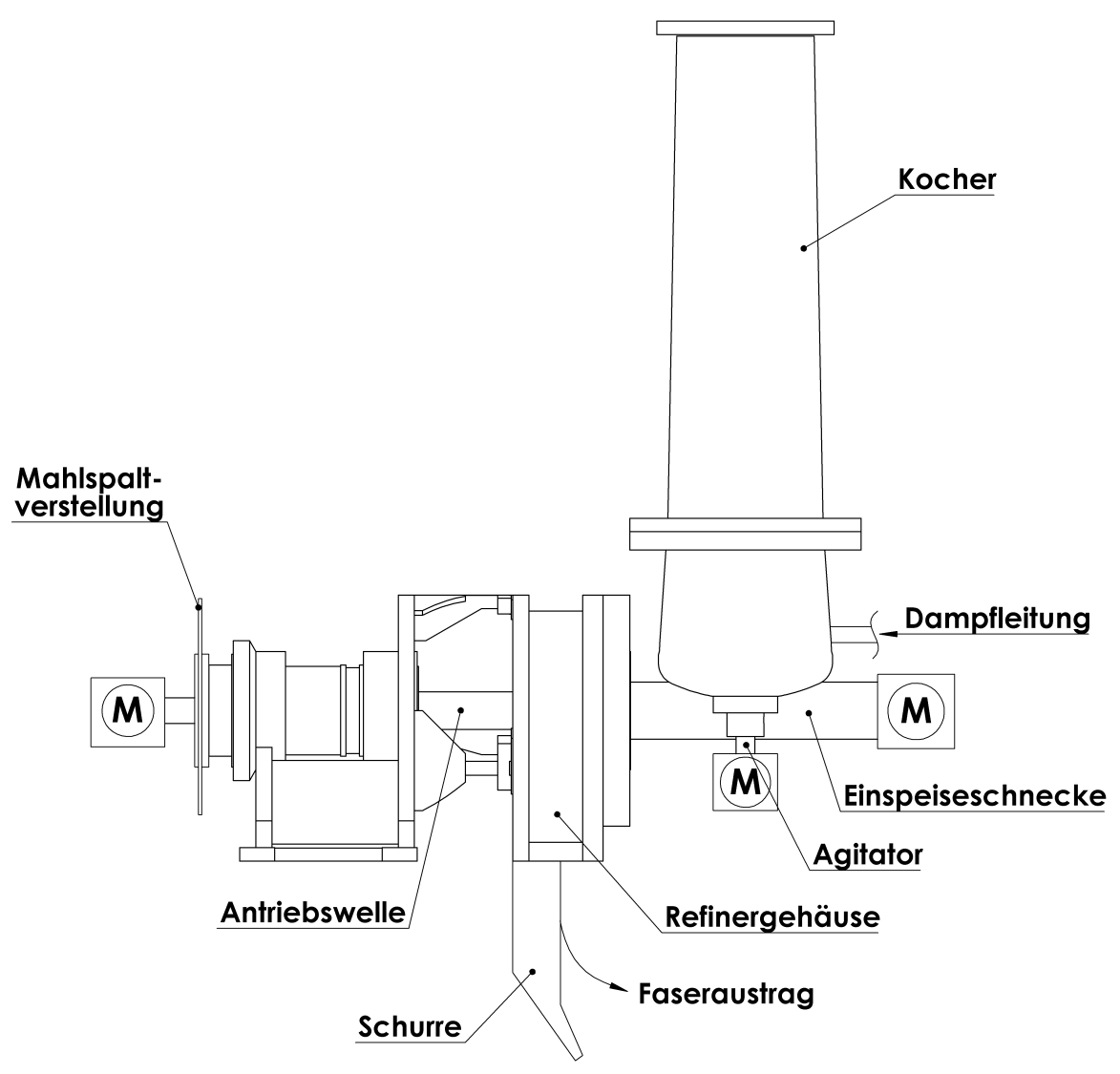

Abbildung 6.2: Schematische Darstellung der Konfiguration der Laborrefineranlage für das RMP-Verfahren.

Die Laborrefineranlage verfügt über Anschlüsse zur Erwärmung von Hackschnitzeln mit Sattdampf ohne Druck in der Einspeiseschnecke und im unteren Teil des Kochers. Die Hackschnitzel wurden von oben in den geöffneten Kocher eingefüllt. Die Faserstofferzeugung erfolgte kontinuierlich. Der Mahlspalt wurde manuell über ein Handrad mit Skala eingestellt. Die Skalenteilung betrug 0,05 mm der Mahlspaltverstellung. Vor der Einstellung wurde der Kontakt der Mahlscheiben als Nullpunkt festgelegt. Die Einstellung des Mahlspalts erfolgte unter Zustellung, um die Öffnung des Mahlspalts durch das Gewindespiel zu unterbinden. Der Faseraustrag erfolgte über ein Schurre an der Unterseite des offenen Refinergehäuses. Die Faserproben für die weiteren Untersuchungen wurden nach dem Einstellen konstanter Prozessbedingungen in Behältern aufgefangen und anschließend in einem Trockenschrank in Abhängigkeit der Beladung für bis zu 24 h bei $103{ }^{\circ} \mathrm{C}$ auf eine Feuchte von $5 \%$ bis $8 \%$ getrocknet. 
Tabelle 6.11: Ausgewählte, relevante technische Daten der Konfiguration der Laborrefineranlage für das RMPVerfahren (Andritz AG 2015).

\begin{tabular}{ll}
\hline Variable Prozessparameter & \\
\hline Aufschlusstemperatur max. & $100{ }^{\circ} \mathrm{C}$ \\
Förderleistung Einspeiseschnecke & $0 \ldots 100 \%$ \\
Verweilzeit & $0 \ldots 180 \mathrm{~min}$ \\
\hline Fixe Prozessparameter & \\
\hline Atmosphärendruck Antriebsleistung, nominell & $54 \mathrm{~kW}$ \\
Drehzahl Refinerwelle & $3000 \mathrm{~min}^{-1}$ \\
Mahlscheibendurchmesser & $12^{\circ} \mathrm{bzw} .305 \mathrm{~mm}$ \\
\hline
\end{tabular}

\subsubsection{Erfassung von Prozessparametern}

Die Datenerfassung von technologischen Parametern der Laborrefineranlage erfolgte mittels Trendanalyse der Software SIMATIC STEP 7 (Siemens AG, München, Deutschland). Erfasst wurden:

- Der Dampfdruck im Kocher mit dem digitalen Drucktransmitter PMP51 (Endress+Hauser GmbH\&Co. KG, Weil am Rhein, Deutschland).

- Die Temperatur im Kocher mittels Temperaturkopftransmitter TMT181-A (Endress+Hauser GmbH\&Co. KG, Weil am Rhein, Deutschland).

- Die Drehzahl der Einspeiseschnecke am Umrichter MICROMASTER 420 (Siemens AG, München, Deutschland).

- Der Mahlscheibenabstand durch den induktiven Analogsensor IA15-30GM-I3 (Pepperl+Fuchs GmbH, Mannheim, Deutschland), in Ergänzung zur Ringskala am Handrad der Mahlspaltverstellung.

- Die Leistungsaufnahme des Refinermotors über den Stromtransformator MAK62/30 (Cewe Instrument $\mathrm{AB}$, Nykoping, Schweden).

Die Rohdaten standen als *.csv-Datei zur Verfügung.

\subsection{Vliesbildung zur Bestimmung der Wärmeleitfähigkeit}

Vor der Vliesbildung wurden die getrockneten Faserstoffe geflufft, um Agglomerate, die während der Ofentrocknung entstanden sind, aufzulösen. Der Fluffer im Labor für Holzwerkstofftechnik an der Hochschule Rosenheim besteht aus einem Ventilator in einem Rohrsystem, indem das Faserstoff-Luft-Gemisch zirkuliert und durch die Rotorblätter des Ventilators vereinzelt wird. Über eine Weiche in dem Rohrsystem kann der Faserstoff über einen Zyklon aus dem Luftstrom abgeschieden und aufgefangen werden.

Zur Bestimmung der Wämeleitfähigkeit der Faserstoffe erfolgte anschließend eine aerodynamische Vliesbildung in einer, im Rahmen der Dissertation entwickelten, Vorrichtung nach Abbildung 6.3. Dazu wurden die Faserstoffe mit einer Venturidüse in den durch den Faserstreuturm zirkulierenden Luftrom eingesaugt. Über die Einblas-, Formierungs- und Vliesbildungszone 
wurde ein gleichmäßiges Faservlies auf einer Fläche von maximal 0,7 x 0,7 $\mathrm{m}^{2}$ gestreut. Die Vlieshöhe betrug maximal $0,6 \mathrm{~m}$. Umfangreiche Vorversuche bestätigten eine deutlich homogene Dichteverteilung über die Fläche der Vliese im Vergleich zu manuell gestreuten Vliesen. Die Reproduzierbarkeit wurde ebenfalls verbessert. Die Faservliesbildung erfolgte ohne Zusatz-, Hilfsoder Klebstoffe.

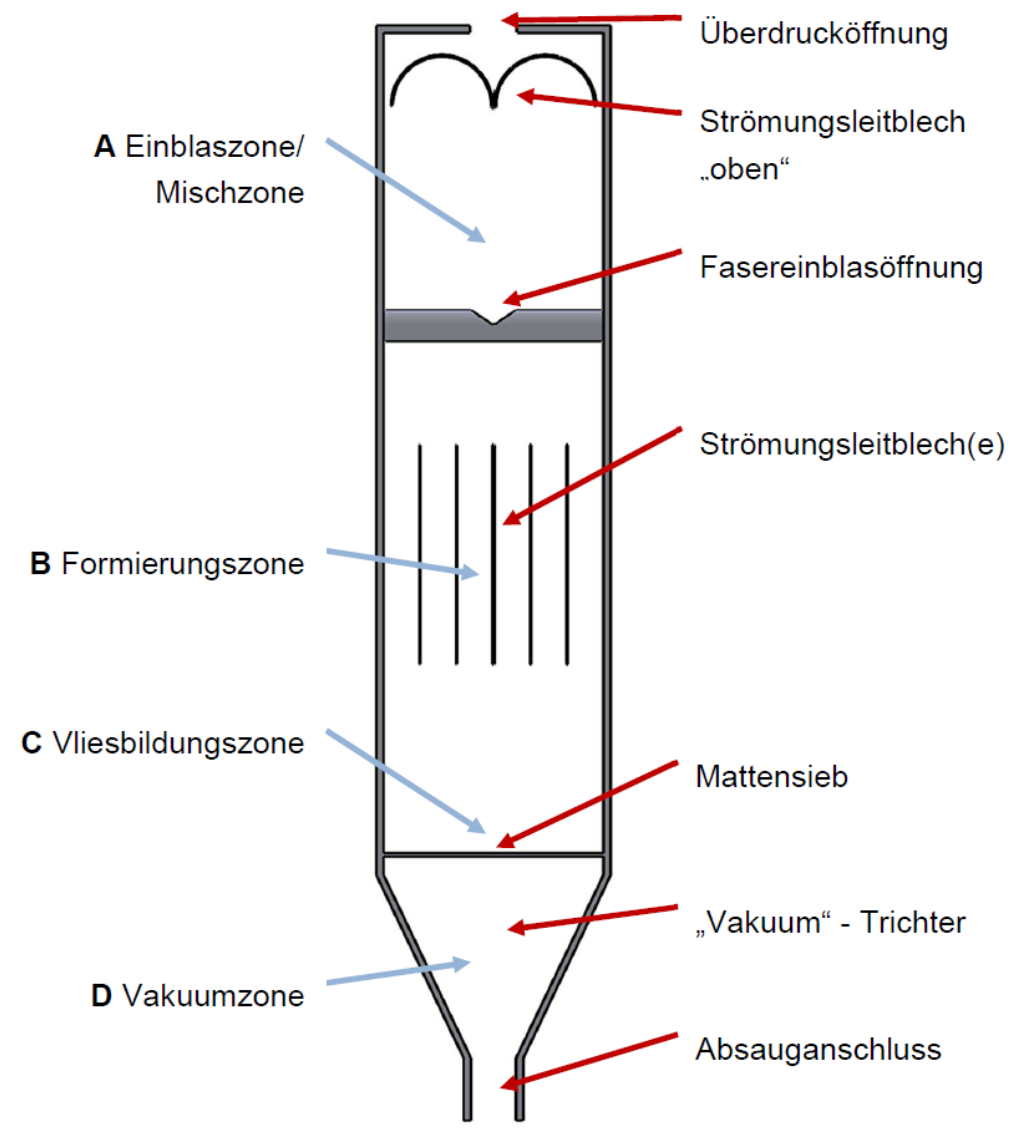

Abbildung 6.3: Schematische Darstellung des Faserstreuturms im Querschnitt.

\subsection{Analytische Methoden}

\subsubsection{Grundlegende Untersuchungen zur Prozessführung in der labortechnischen Faserstofferzeugung}

\section{Bestimmung der Partikelgröße von Hackschnitzeln}

Die Struktur der Hackschnitzel wurde nach dem Prüfverfahren SCAN-CM 40:01 (SCAN-CM 40 2001) an lufttrockenen Hackschnitzeln durchgeführt. Die Prüfsiebung fand auf dem Hackschnitzelsortierer Typ S4019 (Frank-PTI GmbH, Birkenau, Deutschland) mit den Siebeinsätzen $45 \mathrm{~mm}$ Loch, $8 \mathrm{~mm}$ Spalt, $13 \mathrm{~mm}$ Loch, $7 \mathrm{~mm}$ Loch und $3 \mathrm{~mm}$ Loch statt. Die Probemenge umfasste 51. Die Siebzeit betrug 10 min. Die Bestimmung der Partikelgröße fand an drei zufällig ausgewählten Hackschnitzelproben mit Doppelbestimmung statt. 


\section{Bestimmung der Schüttdichte von Hackschnitzeln}

Die Schüttdichte wurde an lufttrockenen Hackschnitzeln volumetrisch bestimmt. Dazu wurden die Hackschnitzel in ein kubisches Gefäß von $0,4 \times 0,4 \times 0,4 \mathrm{~m}^{3}$ geschüttet. Der Überstand wurde mit einer Holzlatte bündig zur Oberkante des Gefäßes entfernt. Anschließend wurde die atro-Masse der Hackschnitzel ermittelt (s. 6.4.2) und damit die Schüttdichte berechnet. Die Bestimmung erfolgte jeweils in dreifacher Wiederholung.

\section{Effekte auf ausgewählte Zielgrößen der Laborrefineranlage}

Zur Identifizierung von Prozessparametern (Faktoren), die einen entscheidenden Effekt auf die Zielgrößen in der labortechnischen Faserstofferzeugung haben, wurde ein zweistufiges faktorielles Screening-Design (Software R) angewendet (Box et al. 2005, S. 177; Grömping 2014). Unter allen möglichen einstellbaren Prozessparametern der Laborrefineranlage wurden quantitative und qualitative Faktoren ausgewählt, die unmittelbar vor, während und nach der Faserstofferzeugung in den Prozess integriert sind. Ein weiteres Auswahlkriterium war, dass die Faktoren in zwei Stufen regel- und kombinierbar sind (Grömping \& J. Fox 2015, S. 16).

Zur Untersuchung grundlegender Effekte auf die Zielgröße Durchsatz wurden die Stufen aus Tabelle 6.12 verwendet. Für die Ermittlung des Durchsatzes der Refineranlage kamen die Pro-

Tabelle 6.12: Stufen der Prozessparameter (Faktoren) für ein zweistufiges faktorielles Screening-Design zur Untersuchung grundlegender Effekte auf die Zielgröße Durchsatz.

\begin{tabular}{llll}
\hline Prozessparameter & Einheit & Stufe -1 & Stufe 1 \\
\hline Holzart & & Buche & Fichte \\
Sattdampfdruck (Temperatur) & bar & $4\left(150^{\circ} \mathrm{C}\right)$ & $8\left(175^{\circ} \mathrm{C}\right)$ \\
Mahlscheibenmuster & & $12 \mathrm{XA802}$ & $12 \mathrm{XASR} 01$ \\
\hline
\end{tabular}

zessparameter aus Tabelle 6.13 zur Anwendung. Die Effekte auf die zwei Zielgrößen spezifische

Tabelle 6.13: Prozessparameter (Faktoren) zur Ermittlung des Durchsatzes der Laborrefineranlage.

\begin{tabular}{ll}
\hline Prozessarameter & Bedingung \\
\hline Holzart & Buche und Fichte \\
Sattdampfdruck (Temperatur) & 4 bar $\left(150^{\circ} \mathrm{C}\right)$ \\
Mahlscheibenmuster & 12 XASR01 \\
Kocherfüllung & $50-601$ \\
Kochzeit & $3 \mathrm{~min}$ \\
Förderleistung Einspeiseschnecke & $33 \%, 66 \%, 99 \%$ \\
\hline
\end{tabular}

elektrische Leistungsaufnahme und Holzfeuchte der Fasern nach dem Aufschluss wurden anhand der Stufen in Tabelle 6.14 untersucht.

\subsubsection{Bestimmung der Holzfeuchte von Hackschnitzeln in Sattdampfatmosphäre}

Die Bestimmung der Holzfeuchte von Hackschnitzeln erfolgte durch Ofentrocknung in Aluminiumschalen mit einem Volumen von 11 bei $103^{\circ} \mathrm{C}$ in dem Trockenschrank UT20 (Heraeus 
Tabelle 6.14: Stufen der Prozessparameter (Faktoren) für ein zweistufiges faktorielles Screening-Design zur Untersuchung grundlegender Effekte auf die zwei Zielgrößen spezifische elektrische Leistungsaufnahme und Holzfeuchte der Fasern nach dem Aufschluss.

\begin{tabular}{llll}
\hline Prozessparameter & Einheit & Stufe -1 & Stufe 1 \\
\hline Holzart & & Fichte & Fichte \\
Holzfeuchte der Hackschnitzel ${ }^{*}$ & $\%$ & 60 & 95 \\
Kochzeit & min & 1 & 10 \\
Sattdampfdruck (Temperatur) & $\mathrm{bar}$ & $3\left(140^{\circ} \mathrm{C}\right)$ & $9\left(180^{\circ} \mathrm{C}\right)$ \\
Förderleistung Einspeiseschnecke & $\%$ & 40 & 100 \\
Mahlspalt & $\mathrm{mm}$ & 0,1 & 0,6 \\
Kocherfüllung & 1 & 15 & 50 \\
Öffnung Blasventil & & $\mathrm{a}$ & $\mathrm{u}$ \\
Drehrichtung Agitator & & rechts & links \\
\hline${ }^{*}$ Bestimmung der Holzfeuchte $\mathrm{n}=6$. & &
\end{tabular}

Instruments) bis zur Massenkonstanz nach Gleichung 6.1.

$$
u=\frac{m_{u}-m_{0}}{m_{0}}
$$

$$
\begin{array}{ll}
u & \text { Holzfeuchte in } \% \\
m_{u} & \text { Feuchte Masse der Hackschnitzel } \\
m_{0} & \text { Trockenmasse der Hackschnitzel }
\end{array}
$$

Zusätzlich wurde die aus der Papierherstellung bekannte Konsistenz nach Gleichung 6.2 berechnet.

$$
k=\frac{m_{0}}{m_{u}} \cdot 100
$$

$$
\begin{array}{ll}
k & \text { Konsistenz in } \% \\
m_{u} & \text { Feuchte Masse der Hackschnitzel } \\
m_{0} & \text { Trockenmasse der Hackschnitzel }
\end{array}
$$

Die Bestimmung der Holzfeuchte von Hackschnitzeln in Sattdampfatmosphäre erfolgte in situ innerhalb des Druckbehälters der Laborrefineranlage. Die untersuchten Varianten sind in Tabelle 6.16 aufgelistet. Die in situ Methode wurde im Rahmen der Dissertation entwickelt. Der Versuchsaufbau ist in Abbildung 6.4 schematisch dargestellt. Die Massenermittlung erfolgte gravimetrisch mit einer mechanischen Waage aus Edelstahl (Artikel-Nr.: 30102, Gastroback GmbH, Hollenstedt, Deutschland). Zur Datenerfassung wurde die Skala der Waage mit einer Digitalkamera durch ein Schauglas in der Wandung des Druckbehälters aufgenommen. Die Auswertung erfolgte videotechnisch. Die Waage befand sich während der Versuchsdurchführung oberhalb der Dampfleitung in den Druckbehälter. Somit konnte ein Wassermitriss aus dem Dampfkessel die Messung nicht beeinflussen. Außerdem wurde oberhalb der Waage ein Rundblech eingesetzt, um herabtropfendes Kondensat abzuleiten (Abbildung 6.4).

$\mathrm{Zu}$ Beginn der Untersuchungen wurden Nulltests mit dem Versuchsaufbau durchgeführt, um den Einfluss von Temperatur und Kondensat auf die Massenermittlung abzuschätzen. Aus den Nullmessungen $(n=8)$ wurde für die Korrektur der Prüfkörpermasse unter Sattdampfdruck ein Wert von $20 \mathrm{~g}$ ermittelt. Dies ist auf Kondensatbildung an der Waagschale zurückzuführen. Die Prüfkörpermasse nach dem Druckabbau muss um $10 \mathrm{~g}$ korrigiert werden. Ein Einfluss der Temperatur auf die Massenermittlung war nicht messbar. 


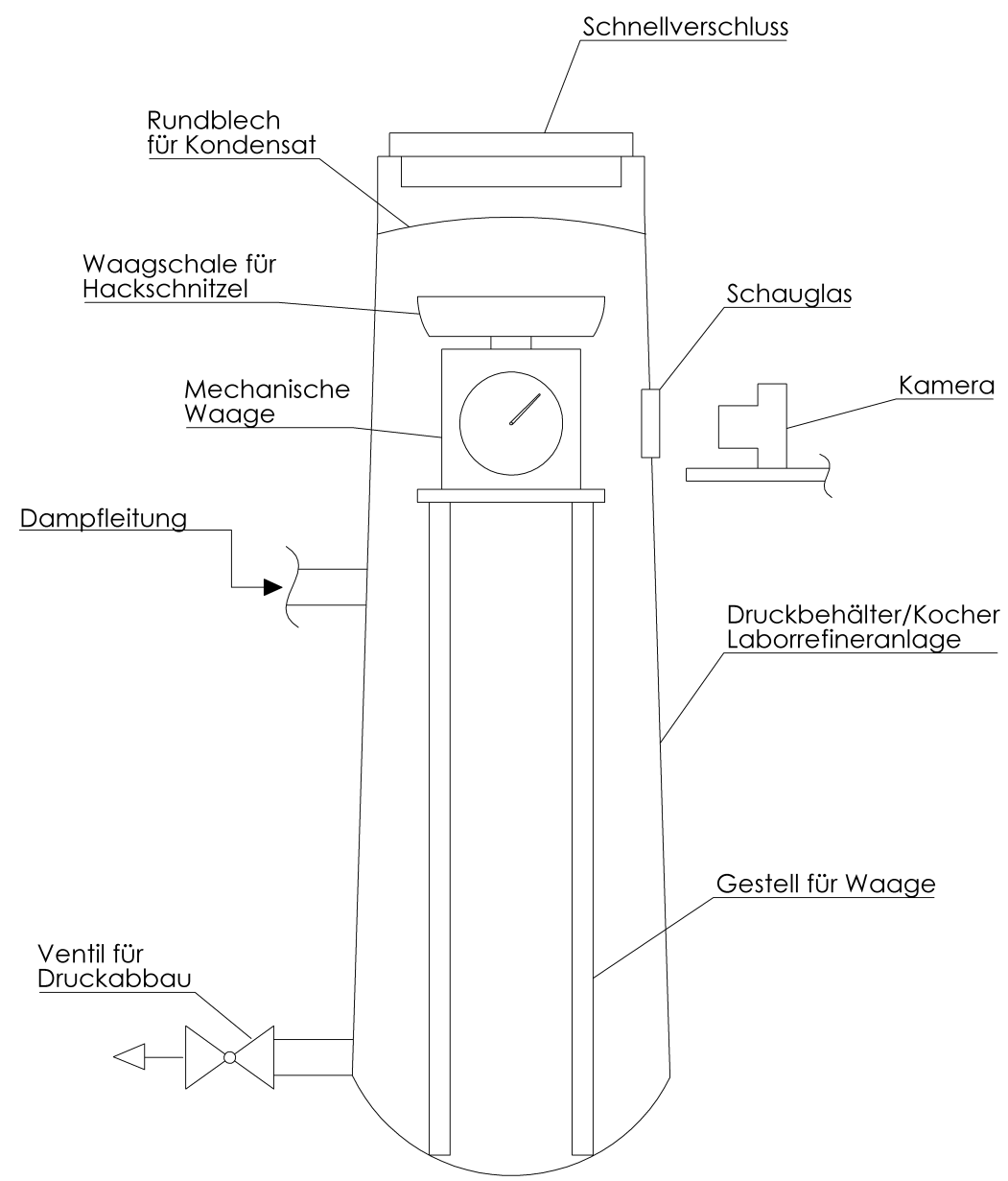

Abbildung 6.4: Schema des Versuchsaufbaus zur in situ Ermittlung der Holzfeuchte von Hackschnitzeln in Sattdampfatmosphäre. Abgebildet ist ein Schnitt durch den Druckbehälter der Laborrefineranlage.

Gegenstand der Untersuchungen waren unbehandelte Hackschnitzel aus Buche (Fagus sylvatica L.) und Fichte (Picea abies Karst.) (Tabelle 6.15). Ein Teil der Fichtenholz-Hackschnitzel wurde für ca. $72 \mathrm{~h}$ wassergelagert, um die Wassersättigung zu erreichen. Vor den Untersuchungen wurden diese Hackschnitzel für ca. $6 \mathrm{~h}$ abgedeckt auf einem Sieb entwässert. Die Holzfeuchte lag im mittleren und nach Wasserlagerung im oberen Bereich der Wassersättigung. Die AnfangsTemperatur der Hackschnitzel betrug ca. $20^{\circ} \mathrm{C}$. Die Hackschnitzel wurden zufällig einer der 10

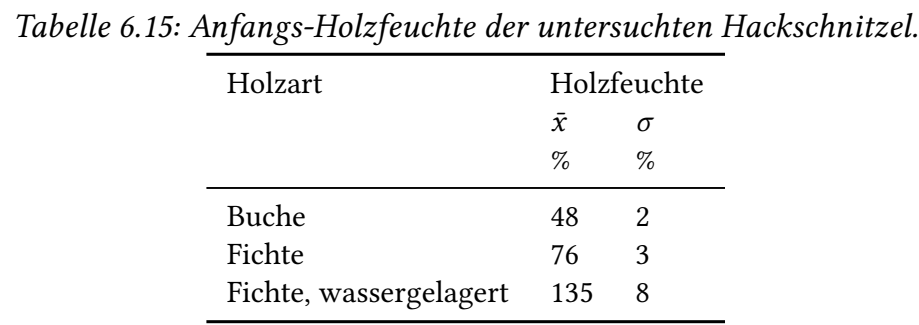

Varianten aus Tabelle 6.16 zugeführt. Die Untersuchungen wurden je Holzart und Variante fünfmal unabhängig wiederholt. 
Tabelle 6.16: Varianten und zugehörige Prozessparameter zur Bestimmung der Holzfeuchte von Hackschnitzeln in Sattdampfatmosphäre.

\begin{tabular}{|c|c|c|c|}
\hline Variante & Bedingungen & & Dauer \\
\hline 1 & Sattdampf & $1 \mathrm{bar}\left(120^{\circ} \mathrm{C}\right)$ & $6 \mathrm{~min}$ \\
\hline 2 & Sattdampf & $2 \operatorname{bar}\left(133^{\circ} \mathrm{C}\right)$ & $6 \mathrm{~min}$ \\
\hline 3 & Sattdampf & $3 \operatorname{bar}\left(143^{\circ} \mathrm{C}\right)$ & $6 \mathrm{~min}$ \\
\hline 4 & Sattdampf & $4 \operatorname{bar}\left(151^{\circ} \mathrm{C}\right)$ & $6 \mathrm{~min}$ \\
\hline 5 & Sattdampf & $5 \operatorname{bar}\left(158^{\circ} \mathrm{C}\right)$ & $6 \min$ \\
\hline 6 & Sattdampf & $6 \operatorname{bar}\left(165^{\circ} \mathrm{C}\right)$ & $6 \min$ \\
\hline 7 & Sattdampf & $7 \operatorname{bar}\left(170^{\circ} \mathrm{C}\right)$ & $6 \min$ \\
\hline 8 & Sattdampf & $8 \operatorname{bar}\left(175^{\circ} \mathrm{C}\right)$ & $6 \min$ \\
\hline 9 & Sattdampf & $9 \operatorname{bar}\left(179^{\circ} \mathrm{C}\right)$ & $6 \mathrm{~min}$ \\
\hline 10 & Sattdampf & 0 bar $\left(100^{\circ} \mathrm{C}\right)$ & * \\
\hline
\end{tabular}

${ }^{*}$ Ventil öffnen und Druckabbau abwarten.

\subsubsection{Bestimmung der spezifischen elektrischen Leistungsaufnahme in der Faserstofferzeugung}

Die Berechnung der spezifischen elektrischen Leistungsaufnahme erfolgt aus der Leistungsaufnahme des Refinermotors nach Gleichung 3.2 in 3.4.2. Im TMP-Verfahren wurden die Varianten der Faserstoffe aus Tabelle 6.18 in vier randomisierten Wiederholungen erzeugt. Die Holzfeuchte der Fichtenholz-Hackschnitzel lag zwischen $109 \%$ und $136 \%$, die der Buchenholz-Hackschnitzel zwischen $61 \%$ und $82 \%(\mathrm{n}=6)$.

Im RMP-Verfahren wurden die Varianten der Faserstoffe aus Tabelle 6.17 in vier randomisierten Wiederholungen erzeugt. Die Versuche wurden in zwei Temperaturstufen unter Atmosphärendruck durchgeführt. In der Temperaturstufe $20^{\circ} \mathrm{C}$ wurden die Hackschnitzel ohne zusätzliche Erwärmung der Zerfaserung zugeführt. Für die Erwärmung der Hackschnitzel auf $100^{\circ} \mathrm{C}$ wurde Sattdampf von unten in die Hackschnitzel eingeleitet (vgl. Abschnitt 6.2.3).

Tabelle 6.17: Prozessparameter zu den Varianten in der Faserstofferzeugung im RMP-Verfahren.

\begin{tabular}{llllll}
\hline Variante & Mahlscheibe & Holzart & Aufschlussbedingungen & Verweilzeit & Mahlspalt \\
\hline 1 & 6 & Fichte & Atmosphärendruck: $20^{\circ} \mathrm{C}$ & n.a. & $0,15 \mathrm{~mm}$ \\
2 & 6 & Fichte & Atmosphärendruck: $100^{\circ} \mathrm{C} *$ & $3 \mathrm{~min}$ & $0,15 \mathrm{~mm}$ \\
3 & 6 & Buche & Atmosphärendruck: $20^{\circ} \mathrm{C}$ & n.a. & $0,15 \mathrm{~mm}$ \\
4 & 6 & Buche & Atmosphärendruck: $100^{\circ} \mathrm{C} *$ & $3 \mathrm{~min}$ & $0,15 \mathrm{~mm}$ \\
\hline
\end{tabular}

${ }^{*}$ Manuelle Einstellung an der Ringskala der Laborrefineranlage.

** Die Erwärmung erfolgte mit Sattdampf unter Atmosphärendruck. 
Tabelle 6.18: Prozessparameter zu den Varianten in der Faserstofferzeugung im TMP-Verfahren.

\begin{tabular}{|c|c|c|c|c|c|}
\hline Variante & Mahlscheibe & Holzart & Aufschlussbedingungen & Verweilzeit & Mahlspalt * \\
\hline 1 & 1 & Fichte & Sattdampf: 2,1 bar $\left(135^{\circ} \mathrm{C}\right)$ & $3 \mathrm{~min}$ & $0,15 \mathrm{~mm}$ \\
\hline 2 & 1 & Fichte & Sattdampf: 3,8 bar $\left(150^{\circ} \mathrm{C}\right)$ & $3 \min$ & $0,15 \mathrm{~mm}$ \\
\hline 3 & 1 & Fichte & Sattdampf: 7,9 bar $\left(175^{\circ} \mathrm{C}\right)$ & $3 \min$ & $0,15 \mathrm{~mm}$ \\
\hline 4 & 1 & Buche & Sattdampf: 2,1 bar $\left(135^{\circ} \mathrm{C}\right)$ & $3 \min$ & $0,15 \mathrm{~mm}$ \\
\hline 5 & 1 & Buche & Sattdampf: 3,8 bar $\left(150^{\circ} \mathrm{C}\right)$ & $3 \mathrm{~min}$ & $0,15 \mathrm{~mm}$ \\
\hline 6 & 1 & Buche & Sattdampf: 7,9 bar $\left(175^{\circ} \mathrm{C}\right)$ & $3 \min$ & $0,15 \mathrm{~mm}$ \\
\hline 7 & 2 & Fichte & Sattdampf: 2,1 bar $\left(135^{\circ} \mathrm{C}\right)$ & $3 \min$ & $0,15 \mathrm{~mm}$ \\
\hline 8 & 2 & Fichte & Sattdampf: 3,8 bar $\left(150^{\circ} \mathrm{C}\right)$ & $3 \min$ & $0,15 \mathrm{~mm}$ \\
\hline 9 & 2 & Fichte & Sattdampf: 7,9 bar $\left(175^{\circ} \mathrm{C}\right)$ & $3 \mathrm{~min}$ & $0,15 \mathrm{~mm}$ \\
\hline 10 & 2 & Buche & Sattdampf: 2,1 bar $\left(135^{\circ} \mathrm{C}\right)$ & $3 \mathrm{~min}$ & $0,15 \mathrm{~mm}$ \\
\hline 11 & 2 & Buche & Sattdampf: 3,8 bar $\left(150^{\circ} \mathrm{C}\right)$ & $3 \mathrm{~min}$ & $0,15 \mathrm{~mm}$ \\
\hline 12 & 2 & Buche & Sattdampf: 7,9 bar $\left(175^{\circ} \mathrm{C}\right)$ & $3 \mathrm{~min}$ & $0,15 \mathrm{~mm}$ \\
\hline 13 & 3 & Fichte & Sattdampf: 2,1 bar $\left(135^{\circ} \mathrm{C}\right)$ & $3 \mathrm{~min}$ & $0,15 \mathrm{~mm}$ \\
\hline 14 & 3 & Fichte & Sattdampf: 3,8 bar $\left(150^{\circ} \mathrm{C}\right)$ & $3 \mathrm{~min}$ & $0,15 \mathrm{~mm}$ \\
\hline 15 & 3 & Fichte & Sattdampf: 7,9 bar $\left(175^{\circ} \mathrm{C}\right)$ & $3 \mathrm{~min}$ & $0,15 \mathrm{~mm}$ \\
\hline 16 & 3 & Buche & Sattdampf: 2,1 bar $\left(135^{\circ} \mathrm{C}\right)$ & $3 \mathrm{~min}$ & $0,15 \mathrm{~mm}$ \\
\hline 17 & 3 & Buche & Sattdampf: 3,8 bar $\left(150^{\circ} \mathrm{C}\right)$ & $3 \mathrm{~min}$ & $0,15 \mathrm{~mm}$ \\
\hline 18 & 3 & Buche & Sattdampf: 7,9 bar $\left(175^{\circ} \mathrm{C}\right)$ & $3 \mathrm{~min}$ & $0,15 \mathrm{~mm}$ \\
\hline 19 & 4 & Fichte & Sattdampf: 2,1 bar $\left(135^{\circ} \mathrm{C}\right)$ & $3 \mathrm{~min}$ & $0,15 \mathrm{~mm}$ \\
\hline 20 & 4 & Fichte & Sattdampf: 3,8 bar $\left(150^{\circ} \mathrm{C}\right)$ & $3 \mathrm{~min}$ & $0,15 \mathrm{~mm}$ \\
\hline 21 & 4 & Fichte & Sattdampf: 7,9 bar $\left(175^{\circ} \mathrm{C}\right)$ & $3 \min$ & $0,15 \mathrm{~mm}$ \\
\hline 22 & 4 & Buche & Sattdampf: 2,1 bar $\left(135^{\circ} \mathrm{C}\right)$ & $3 \mathrm{~min}$ & $0,15 \mathrm{~mm}$ \\
\hline 23 & 4 & Buche & Sattdampf: 3,8 bar $\left(150^{\circ} \mathrm{C}\right)$ & $3 \min$ & $0,15 \mathrm{~mm}$ \\
\hline 24 & 4 & Buche & Sattdampf: 7,9 bar $\left(175^{\circ} \mathrm{C}\right)$ & $3 \min$ & $0,15 \mathrm{~mm}$ \\
\hline 25 & 5 & Fichte & Sattdampf: $2,1 \operatorname{bar}\left(135^{\circ} \mathrm{C}\right)$ & $3 \mathrm{~min}$ & $0,15 \mathrm{~mm}$ \\
\hline 26 & 5 & Fichte & Sattdampf: 3,8 bar $\left(150^{\circ} \mathrm{C}\right)$ & $3 \min$ & $0,15 \mathrm{~mm}$ \\
\hline 27 & 5 & Fichte & Sattdampf: 7,9 bar $\left(175^{\circ} \mathrm{C}\right)$ & $3 \min$ & $0,15 \mathrm{~mm}$ \\
\hline 28 & 5 & Buche & Sattdampf: 2,1 bar $\left(135^{\circ} \mathrm{C}\right)$ & $3 \mathrm{~min}$ & $0,15 \mathrm{~mm}$ \\
\hline 29 & 5 & Buche & Sattdampf: 3,8 bar $\left(150^{\circ} \mathrm{C}\right)$ & $3 \min$ & $0,15 \mathrm{~mm}$ \\
\hline 30 & 5 & Buche & Sattdampf: $7,9 \operatorname{bar}\left(175^{\circ} \mathrm{C}\right)$ & $3 \mathrm{~min}$ & $0,15 \mathrm{~mm}$ \\
\hline
\end{tabular}

* Manuelle Einstellung an der Ringskala der Mahlspaltverstellung.

\subsubsection{Bestimmung der Feuchte und Konsistenz von Faserstoffen}

Zur Bestimmung der Feuchte und Konsistenz von Faserstoffen nach dem Aufschluss wurden die Faserstoffe am Auslass des Zyklons in Aluminiumschalen mit einem Volumen von 11 aufgefangen. Die Bestimmung erfolgte durch Ofentrocknung bei $103^{\circ} \mathrm{C}$ in dem Trockenschrank UT20 (Heraeus Instruments) bis zur Massenkonstanz an drei Proben je Variante (Tabelle 6.18 und Tabelle 6.17). Die Berechnung der Feuchte und Konsistenz erfolgte analog zu den Gleichung 6.1 und Gleichung 6.2 aus Abschnitt 6.4.2.

\subsubsection{Bestimmung der Dispersitätseigenschaften von Faserstoffen mit der Kollektivmethode Luftstrahl-Prüfsiebung}

Die Charakterisierung von Dispersitätseigenschaften am Partikelkollektiv erfolgte durch Luftstrahl-Prüfsiebung der lufttrockenen Holzstoffe. Zum Einsatz kam das Luftstrahlsieb e200LS (Hosokawa Alpine AG, Augsburg) gemeinsam mit dem Messaufnehmer Waage PB602-S (Mettler Toledo, Gießen). 
Es wurden alle Wiederholungen der Faserstoffvarianten aus Tabelle 6.18 und Tabelle 6.17 in Doppelbestimmungen untersucht. Die Probennahme erfolgte manuell aus den getrockneten und geflufften Faserstoffen. Die Probengröße einer Einzelprobe betrug in Anlehnung an DIN 51701 mindestens $0,25 \mathrm{~kg}$, um eine hohe Präzision zu gewährleisten. Die Probenteilung erfolgte manuell in Analyseproben von 5 g. Die Prüfsiebung erfolgte einzeln an sechs Prüfsieben (50 x 200mm) (Haver and Boecker OHG, Oelde) mit den Maschenweiten 125, 315, 600, 1000, 1600 und $2500 \mu \mathrm{m}$. Die Prüfsiebung wurde im Standard-Verfahren durchgeführt (Wägung des Siebrückstandes, beginnend mit der kleinsten Maschenweite). Die siebrelevanten Parameter waren durch den Laborstandard in Anlehnung an EN ISO 4610:2001 (2002) vorgegeben. Die Bestimmung der Dispersitätseigenschaften erfolgte in Doppelbestimmung.

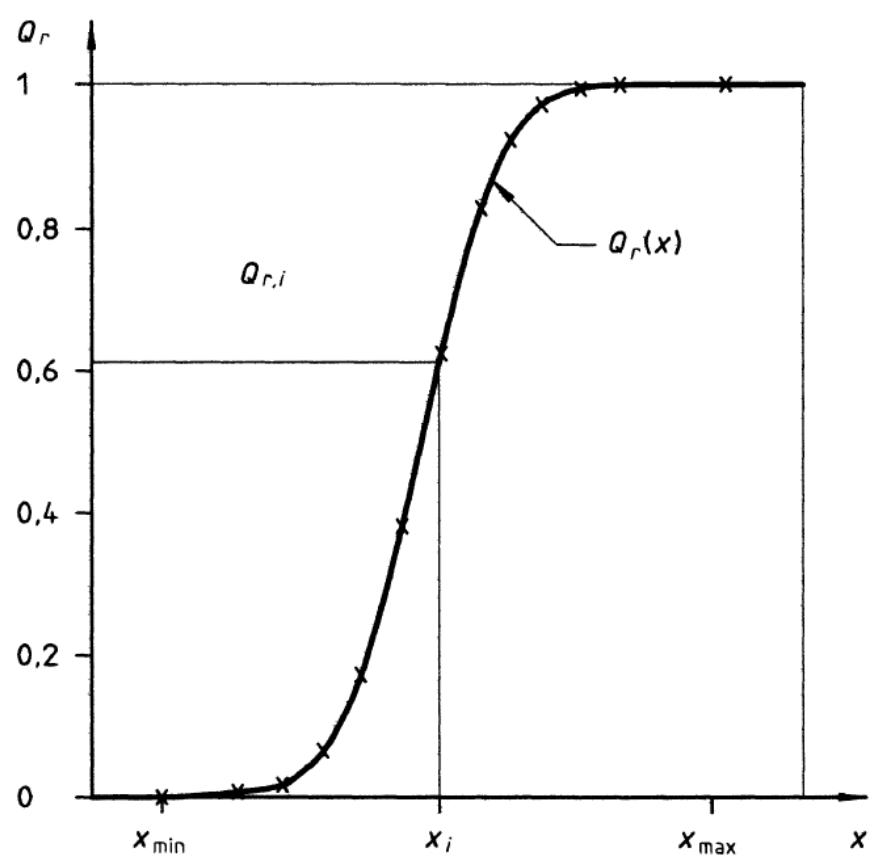

Abbildung 6.5: Die normierte Verteilungssumme $Q_{r}(x)$ erstreckt sich zwischen $0 \%$ und $100 \%$. Feder Punkt der Verteilung $Q_{r}(x)$ gibt die relative Menge von Partikeln kleiner oder gleich $x_{i}$ an (DIN ISO 9276-1:2004-09 2004).

Die Ermittlung der Siebkennlinie erfolgte mit der Methode „Durchgang“ in Bezug auf die Einwaage nach DIN ISO 9276-1:2004-09 (2004) \& DIN ISO 9276-2:2018-09 (2018) (Abbildung 6.5). Daraus folgt eine massenbezogene Gewichtung der Verteilungssumme $\mathbf{Q}_{\mathbf{r}}$ mit der Mengenart Masse $(r=3)$. Die Verbindung der einzelnen Punkte erfolgte in erster Annäherung mit Geraden. Die Darstellung von $Q_{3}$ als relative Summenhäufigkeit ermöglichte die Berechnung von Kennwerten der Partikelgrößenverteilung. Als repräsentative Größe der Faserstoffe wurde der Medianwert $\mathrm{x}_{50,3}$ aus der Verteilungssumme nach Abschnitt 10.1 berechnet (Bohnet 2004, S. 29). Ein weiteres Charakteristikum ist das $97 \%$-Perzentil der Verteilungssumme, welches zur Berechnung der mittlere Partikelausdehnung (Gleichung 6.3) dient und analog zum Medianwert berechnet wurde. Dieser Feinheitskennwert gibt nach Jensen (1975) Auskunft über die Homogenität des Faserstoffes und wird im vorliegenden Fall auf das obere Feinheitsintervall angewendet. Alle Kennwerte nach der Luftstrahl-Prüfsiebung charakterisieren die Partikelgrößenverteilung des Faserstoffes als 
Prüfsiebmaschenweite.

$$
\Delta x_{3}=x_{97,3}-x_{50,3}
$$

$\Delta x_{3} \quad$ Mittlere Partikelausdehnung in $\mu \mathrm{m}$

$x_{97,3}$ Partikelgröße des $97 \%$-Perzentils der Verteilungssumme

$x_{50,3}$ Partikelgröße des Medianwerts der Verteilungssumme

\subsubsection{Bestimmung der Dispersitätseigenschaften von Faserstoffen mit der Individualmethode quantitative Bildanalyse}

Für die statische, quantitative Bildanalyse kam die Software FibreShape Version 6.2.0 (Innovative Sensor Technology IST AG, Ebnat-Kappel, Schweiz) zum Einsatz. Die Aufnahmen der Faserstoffe wurden mit dem Scanner Perfection V800 (SEIKO Epson CORPORATION, Japan) und der Software Silverfast Ai Studio 8 (LaserSoft Imaging AG, Kiel, Deutschland) erzeugt. Die Auflösung wurde auf 2400 dpi eingestellt, wodurch die tatsächliche maximale Auflösung des Scanners nahezu ausgenutzt werden konnte (Schlüter 2017). Die Kantenlänge eines Pixels betrug in dieser Auflösung $10,58 \mu \mathrm{m}$. Die Bildfläche einer Aufnahme maß $12 \times 16 \mathrm{~cm}^{2}$, was der Begrenzung der Dateigröße der FibreShape Software auf 170 MB geschuldet war.

Es wurden alle Wiederholungen der Faserstoffvarianten aus Tabelle 6.18 und Tabelle 6.17 in Doppelbestimmungen untersucht. Die Probennahme erfolgte manuell aus den getrockneten und geflufften Faserstoffen. Die Probengröße einer Einzelprobe betrug in Anlehnung an DIN 51701 mindestens $0,25 \mathrm{~kg}$, um eine hohe Präzision zu gewährleisten. Die Probenteilung erfolgte manuell in Analyseproben von $0,05 \mathrm{~g}$. Die Analyseproben wurden auf drei Glasplatten (3 mm x 200 $\mathrm{mm}$ x $300 \mathrm{~mm})$ mit Prüfsieben ( 50 x $200 \mathrm{~mm}$ ) (Haver and Boecker OHG, Oelde) aufsteigend mit den Siebmaschenweiten $125 \mu \mathrm{m}, 315 \mu \mathrm{m}$ und $630 \mu \mathrm{m}$ vereinzelt, um einen Bildflächenanteil unterhalb des Limits von $3 \%$ sicherzustellen. Die Bildanalyse erfolgte mit der Parameterauswahl in Abbildung 6.6. Zusätzlich wurde in der Software FibreShape die Schattenkorrektur angewendet und ein Schwellwert von 230 manuell ausgewählt.

Die Darstellung der Dispersitätseigenschaften basierte auf den Normen DIN ISO 9276-1:2004-09 (2004), DIN ISO 9276-2:2018-09 (2018) und DIN ISO 9276-6:2012-01 (2012). Die geodätische Länge und geodätische Breite der Fasern wurde mit dem Rechteck-Model (rectangle model) nach Schmid et al. (2020) berechnet. Die Darstellung der Verteilungssumme $\mathrm{Q}_{\mathrm{r}}$ wurde nach Gleichung 6.4 flächenbezogen $(r=2)$ gewichtet. Für die Gewichtung kam die gefüllte Fläche des Partikel (AREAF) nach Schmid et al. (2020) zur Anwendung, die durch die Software FibreShape automatisch ermittelt wurde. Die Funktion ist auf weitere Gewichtungen nach Länge, Dicke etc. anwendbar.

$$
y_{A}=\frac{x_{i} \cdot A_{i}}{\sum_{i=1}^{n} A_{i}}
$$

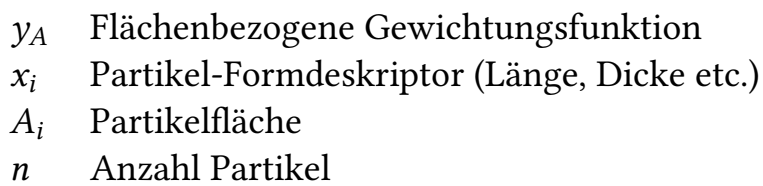

Aus den Rohdaten der Bildanalyse wurden die Partikel anhand von Primär- und Sekundärkriterien in Anlehnung an Wenderdel et al. (2014), Karnis (1994) und Forgacs (1963) in Partikelklassen eingeteilt (Tabellen 6.19 und 6.20). Die Sekundärkriterien für die zweidimensionalen Formdeskriptoren geodätische Länge und geodätische Dicke wurden Tabelle 2.1 entnommen. Das Kriterium der Elongation nach Gleichung 6.5 wurde zur Unterscheidung von faserartigen und kornförmigen 
Partikeln auf den Wert 0,3 festgelegt. Die Kriterien wurden so gewählt, dass die Flächenanteile der einzelnen Klassen in Summe der Gesamtfläche entsprechen.

$$
E=\frac{x_{E}}{x_{L G}}
$$

E Elongation

$x_{E} \quad$ Geodätische Dicke

$x_{L G} \quad$ Geodätische Länge

a

\begin{tabular}{|c|c|c|}
\hline Parameter & Value & \\
\hline Density $[\mathrm{g} / \mathrm{cm} 3]$ & 0.0 & \\
\hline Fill holes & TRUE & $\checkmark$ \\
\hline Kind of material & fibre & \\
\hline Knot punch out & FALSE & $\checkmark$ \\
\hline Max. pixel size[um] & 1000.0 & \\
\hline Size model & rectangle & $\checkmark$ \\
\hline Transparent objects & FALSE & $\checkmark$ \\
\hline Zoom factor & 0.71 & \\
\hline
\end{tabular}

b

\begin{tabular}{|l|l|l|}
\hline Parameter & Minimum & Maximum \\
\hline 1/Convexity & 0.0 & 1.5 \\
\hline 1/Elongation & 0.0 & 100000.0 \\
\hline Angle & 0.0 & 180.0 \\
\hline Aspect ratio & 0.0 & 1.0 \\
\hline Geodesic length & 10.0 & 100000.0 \\
\hline Solidity & 0.0 & 1.0 \\
\hline Thickness & 10.0 & 10000.0 \\
\hline Thickness [pix] & 2 & 100000 \\
\hline Parameter & Value & \\
\hline Closing count & 0 & \\
\hline Measure object with holes & True & \\
\hline
\end{tabular}

Abbildung 6.6: Relevante Parameter der Messmaske Software FibreShape für die quantitative Bildanalyse der Faserstoffe. a, Algorithmus-Parameter. b, Parameter des morphologischen Filters gemäß DIN ISO 9276-6:2012-01 (2012) \& DIN ISO 9276-1:2004-09 (2004) Namenskonvention. 
Tabelle 6.19: Klassifizierung von Partikeln aus Fichtenholz-Faserstoffen anhand zweidimensionaler Formdeskriptoren.

\begin{tabular}{|c|c|c|c|c|}
\hline Partikelklasse & Partikelform & $\begin{array}{l}\text { Primär- } \\
\text { kriterium }\end{array}$ & $\begin{array}{l}\text { Sekundär- } \\
\text { kriterien }\end{array}$ & Beschreibung \\
\hline Faserbündel (FB) & faserartig & $\mathrm{E} \leq 0.3$ & $x_{\mathrm{E}}>43 \mu \mathrm{m}$ & $\begin{array}{l}\text { Faserbündel bestehen in der Dicke } \\
\text { aus mind. } 2 \text { Zellen, Bruchstücke }\end{array}$ \\
\hline Langfaser (LF) & faserartig & $\mathrm{E} \leq 0.3$ & $\begin{array}{l}x_{\mathrm{E}} \leq 43 \mu \mathrm{m} \\
x_{\mathrm{LG}}>1700 \mu \mathrm{m}\end{array}$ & Tracheiden \\
\hline Kurzfaser (KF) & faserartig & $\mathrm{E} \leq 0.3$ & $\begin{array}{l}x_{\mathrm{E}} \leq 43 \mu \mathrm{m} \\
x_{\mathrm{LG}} \leq 1700 \mu \mathrm{m}\end{array}$ & $\begin{array}{l}\text { Bruchstücke von Tracheiden, } \\
\text { Parenchym }\end{array}$ \\
\hline Grobstoff (GS) & kornförmig & $\mathrm{E}>0.3$ & $x_{\mathrm{Fmax}}>50 \mu \mathrm{m}$ & Bruchstücke von Faserbündeln \\
\hline Feinstoff (FS) & kornförmig & $\mathrm{E}>0.3$ & $x_{\mathrm{Fmax}} \leq 50 \mu \mathrm{m}$ & Bruchstücke von Einzelfasern \\
\hline
\end{tabular}

DIN ISO 9276-6 Namenskonvention: E = Elongation, $x_{\mathrm{E}}=$ geodätische Dicke, $x_{\mathrm{LG}}=$ geodätische Länge, $x_{\text {Fmax }}=$ maximaler Feret-Durchmesser

Tabelle 6.20: Klassifizierung von Partikeln aus Buchenholz-Faserstoffen anhand zweidimensionaler Formdeskriptoren.

\begin{tabular}{|c|c|c|c|c|}
\hline Partikelklasse & Partikelform & $\begin{array}{l}\text { Primär- } \\
\text { kriterium }\end{array}$ & $\begin{array}{l}\text { Sekundär- } \\
\text { kriterien }\end{array}$ & Beschreibung \\
\hline Faserbündel (FB) & faserartig & $\mathrm{E} \leq 0.3$ & $x_{\mathrm{E}}>32 \mu \mathrm{m}$ & $\begin{array}{l}\text { Faserbündel bestehen in der Dicke } \\
\text { aus mind. } 2 \text { Zellen, Bruchstücke }\end{array}$ \\
\hline Langfaser (LF) & faserartig & $\mathrm{E} \leq 0.3$ & $\begin{array}{l}x_{\mathrm{E}} \leq 32 \mu \mathrm{m} \\
x_{\mathrm{LG}}>500 \mu \mathrm{m}\end{array}$ & $\begin{array}{l}\text { Fasertracheiden und Libri- } \\
\text { formfasern }\end{array}$ \\
\hline Kurzfaser (KF) & faserartig & $\mathrm{E} \leq 0.3$ & $\begin{array}{l}x_{\mathrm{E}} \leq 32 \mu \mathrm{m} \\
x_{\mathrm{LG}} \leq 500 \mu \mathrm{m}\end{array}$ & $\begin{array}{l}\text { Bruchstücke von Fasertracheiden } \\
\text { und Libriformfasern, Parenchym }\end{array}$ \\
\hline Grobstoff (GS) & kornförmig & $\mathrm{E}>0.3$ & $x_{\text {Fmax }}>50 \mu \mathrm{m}$ & $\begin{array}{l}\text { Bruchstücke von Faserbündeln, } \\
\text { Tracheen }\end{array}$ \\
\hline Feinstoff (FS) & kornförmig & $\mathrm{E}>0.3$ & $x_{\mathrm{Fmax}} \leq 50 \mu \mathrm{m}$ & Bruchstücke von Einzelfasern \\
\hline
\end{tabular}

DIN ISO 9276-6 Namenskonvention: $\mathrm{E}=$ Elongation, $x_{\mathrm{E}}=$ geodätische Dicke, $x_{\mathrm{LG}}=$ geodätische Länge, $x_{\text {Fmax }}=$ maximaler Feret-Durchmesser

Im Weiteren wurden nach Wenderdel (2015) \& Krug (2010) (ursprünglich Jensen (1975)) die mittlere Partikelausdehnung (Gleichung 6.6) und das Partikelausdehnungsintervall (6.7) der Verteilungssumme definiert. Diese Kennwerte stellen ein Maß für die mittlere Ausdehnung einer logarithmisch aufgetragenen Verteilungssumme dar und geben durch die Breite des Ausdehnungsintervalls Auskunft über den Grad der Homogenität eines Partikel-Formdeskriptors.

$$
m x_{r}=\frac{x_{16, r}+x_{50, r}+x_{84, r}}{3}
$$

$m x_{r} \quad$ Mittlere Partikelausdehnung in $\mu \mathrm{m}$

$x_{16, r} \quad$ Partikelgröße des 16 -\% Perzentils der Verteilungssumme

$x_{50, r} \quad$ Partikelgröße des Medianwerts der Verteilungssumme

$x_{84, r} \quad$ Partikelgröße des 84 -\% Perzentils der Verteilungssumme

$$
\Delta x_{r}=x_{84, r}-x_{16, r}
$$

$\Delta x_{r} \quad$ Partikelausdehnungsintervall in $\mu \mathrm{m}$

$x_{16, r} \quad$ Partikelgröße des 16 -\% Perzentils der Verteilungssumme

$x_{84, r}$ Partikelgröße des 84 -\% Perzentils der Verteilungssumme 


\subsubsection{Mikroskopische Untersuchungen an den Faserstoffen}

Für die mikroskopischen Untersuchungen wurden Fasern (ca. $2 \mathrm{~g}$ ) aus dem Spektrum der Holz- und Zellstoffe aus Abschnitt 6.1.2 und der Faserstoffvarianten aus Tabelle 6.18 und Tabelle 6.17 entnommen. Bei den Faserstoffvarianten wurden die Faserstoffe untersucht, mit denen eine Wärmeleitfähigkeitsmessung durchgeführt wurde. Es wurde darauf geachtet, die Dispersitätseigenschaften des Partikelkollektivs für die Mikroskopie zu erhalten. Um die chemischen Oberflächeneigenschaften, die Zugänglichkeit der inneren Struktur und den Grad der Fibrilierung der Fasern sichtbar zu machen, wurde eine zweifarbige differentielle Färbemethode nach Simon (1950) angewendet (Simons' stain). Das Färbungsmittel bestand aus $1 \%$-iger wässriger Lösung mit Chicago Sky Blue 6B (CAS: 2610-05-1, Fisher Scientific GmbH, Schwerte) und 1\%-iger wässriger Lösung mit Direct Yellow 11 (CAS: 1325-37-7, Merck KGaA, Darmstadt), die im Verhältnis 1:1 gemischt wurden. Die Färbungsmittel wurden nicht weiter vorbehandelt.

Die Faserpräparation erfolgte durch die Herstellung einer Faserstoff-Suspension mit einer Konsistenz von $0,05 \%$ in demineralisiertem Wasser. Die Färbungsprozedur begann mit der Präparation der Fasern auf einem Objektträger nach T 401 om-03 (2008). Die fixierten Fasern wurden mit $1 \mathrm{ml}$ bis $2 \mathrm{ml}$ des Färbungsmittels getränkt und anschließend bei $60^{\circ} \mathrm{C}$ ofengetrocknet. Es folgte eine gründliche Spülung mit demineralisiertem Wasser unter einem Deckglas und wiederholter Ofentrockung bei $60^{\circ} \mathrm{C}$.

Für die mikroskopischen Aufnahmen wurde das Mikroskop BX61 mit der Durchlichteinheit BX-UCN und der Lichtquelle U-RFL-T sowie der Software Stream Motion (OLYMPUS EUROPA SE \& CO. KG, Hamburg) verwendet. Folgende Einstellungen wurden am Mikroskop vorgenommen: Lampenintensität 6V, Aperturblende $75 \%$, Objektivvergrößerung $4 \mathrm{x}$, Verstärkung (Weißabgleich) Rot: 1.49 , Grün: 1.00, Blau: 2.23.

\subsubsection{Bestimmung der Wärmeleitfähigkeit von Faserstoffen}

Die Bestimmung der Wärmeleitfähigkeit der Faserstoffe wurde an der Technischen Hochschule Rosenheim im Zweiplattenverfahren durchgeführt. Zum Einsatz kam das WärmeleitfähigkeitMessgerät TLP 900 GX mit der Software Lambda V.2012 (TAURUS Instruments AG, Weimar) nach den Anforderungen der ISO 8302:1991 (1991). Das Gerät verfügt über eine Messkammerhaube mit Temperierung auf die Probenmitteltemperatur, um die Messung gegenüber Umwelteinflüssen und den Wärmeaustausch mit der Umgebungsluft weitgehend zu schützen. Der Aufbau der Messplattenapparatur ist in Abbildung 6.7 schematisch dargestellt. Die Darstellung zeigt auch den typischen Probekörpereinbau für eine Zweiplatten-Messung. Im Falle der vorliegenden Arbeit konnte auf den Einsatz von Kontaktmatten verzichtet werden, da es sich bei den Faserstoffen um ein leicht zu komprimierendes Material handelt und dadurch der vollflächige Kontakt ohne Lufteinschlüsse zu Heiz- und Kühlplatten gewährleistet war. 


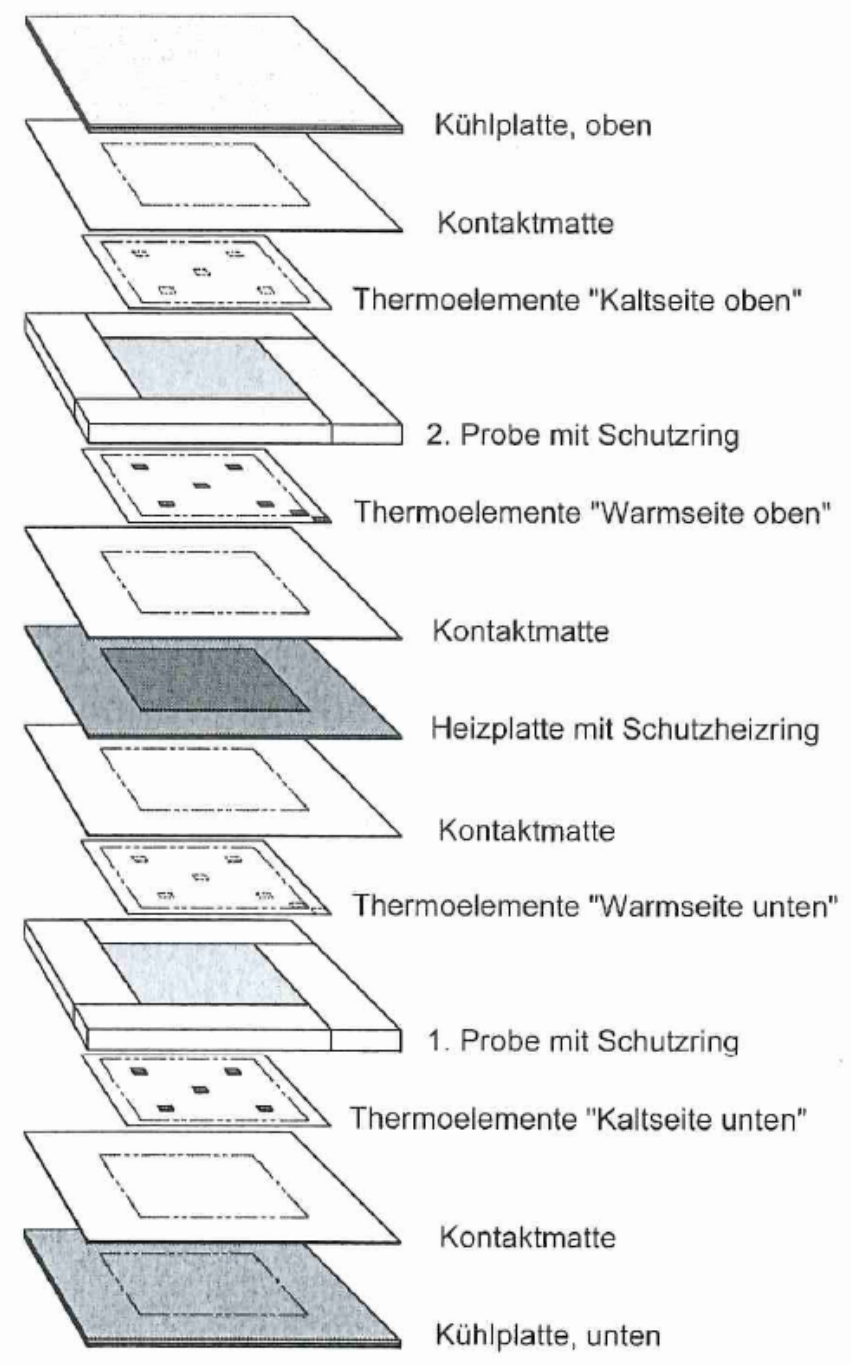

Abbildung 6.7: Aufbau der Messplattenapparatur im Wärmeleitfähigkeit-Messgerät TLP 900 GX. (TAURUS Instruments $A G$ 2018).

Der in der Messung erfasste Wärmestrom wird von der Heizplatte in der Mitte auf einer Fläche von $0,3 \times 0,3 \mathrm{~m}^{2}$ erzeugt und fließt aufgrund einer definierten Temperaturdifferenz senkrecht durch die Probekörper zur oberen und unteren Kühlplatte. Die Messung der Temperaturdifferenz erfolgte durch fünf Thermoelemente auf Trägerfolien je Messebene (Heiz- und Kühlplatte). Durch einen Schutzheizring und eine zusätzliche Probenschutzdämmung wird der Wärmefluss an den Randzonen unterbunden. Die Konstruktion ist so ausgelegt, dass die zugeführte elektrische Leistung über die gesamte Messfläche homogen in Wärme umgewandelt wird. Den Leistungsangaben des Gerätes zufolge liegt die Messunsicherheit im gesamten Messbereich $(0,005 \mathrm{~W} /(\mathrm{m} \cdot \mathrm{K})$ bis $0,5 \mathrm{~W} /(\mathrm{m} \cdot \mathrm{K}))$ typischerweise bei $1 \%$ und die Reproduzierbarkeit liegt bei 0,5\% (TAURUS Instruments AG 2018). Die Wärmeleitfähigkeit wurde an Faserstoff-Probekörpern ohne Zusatz-, Hilfs- oder Klebstoffe durchgeführt (siehe Abschnitt 6.3). Untersucht wurde eine Probe je Variante aus Tabelle 6.18 und aus Tabelle 6.17. Von den vier Wiederholungen wurde diejenige Probe ausgewählt, die dem Mittelwert der spezifischen elektrischen Leistungsaufnahme am nächsten lag.

Die Faservliesstoffe (Abschnitt 6.3) wurden für die Bestimmung der Wärmeleitfähigkeit in einen 
Streurahmen mit der Abmessung 0,6 x 0,6 x 0,05 $\mathrm{m}^{3}$ eingebracht. Die Rohdichte der Probekörper lag bei $50 \mathrm{~kg}_{\mathrm{atro}} / \mathrm{m}^{3}$. Die Feuchte der Faserstoffe wurde vor der Probekörperherstellung mit dem Feuchtebestimmer DLB (KERN \& SOHN GmbH, Balingen-Frommern) bestimmt.

Der Probekörpereinbau erfolgte unter Kraftvorgabe der Schließmechanik, um die Proben mit derselben Dicke zu messen und die Vergleichbarkeit vor dem Hintergrund des Rohdichteinflusses (siehe Abschnitt 5.3) und der direkt in die Berechnung der Wärmeleitfähigkeit eingehenden Probekörperdicke (siehe Abschnitt 2.3.3) zu gewährleisten.

Die Messung der Wärmeleitfähigkeit erfolgte mit $10 \mathrm{~K}$ Temperaturdifferenz zwischen Heiz- und Kühlplatte bei Probenmitteltemperaturen von ca. $10^{\circ} \mathrm{C}, 20^{\circ} \mathrm{C}$ und $30^{\circ} \mathrm{C}$. Die Messungen wurden mit sechs Wiederholungen in jeder Temperaturstufe nach Einstellen des Stabilitätskriteriums von 0,3\% Unterschied zwischen zwei Messungen im Abstand von $1 \mathrm{~h}$ durchgeführt. Jede Messung dauerte somit ca. $24 \mathrm{~h}$. Aus den ermittelten Werten der drei Temperaturstufen wurde eine Regressionsgerade berechnet und die Wärmeleitfähigkeit bei $10^{\circ} \mathrm{C}$ Probenmitteltemperatur $\left(\lambda_{10}\right)$ bestimmt.

\subsection{Datenverarbeitung und statistische Methoden}

Die elektronischen Rohdaten aus unterschiedlichen Formaten (".xml, *.txt, "..csv, *.pdf) sowie die schriftlich im Labornotizbuch festgehaltenen Daten wurden zuerst in MS-Excel importiert und gesammelt, dann in den Arbeitsblättern sortiert und gruppiert und anschließend im *.xlsx-Format gespeichert. Die deskriptive Beschreibung und graphische Darstellung der Ergebnisse erfolgte mit der Software R und der Oberfläche R-Studio (R Core Team 2017). Im Anhang sind die angewendeten Pakete und R-Codes im Sinne der Reproduzierbarkeit der Ergebnisse beispielhaft aufgeführt. In der weiteren statistischen Auswertung zur Identifikation signifikanter Unterschiede zwischen Gruppen wurde als erstes geprüft, ob parametrische oder nicht-parametrische Verfahren angewendet werden mussten. Die ANOVA (Analysis of Variance) zur Varianzanalyse ist ein parametrisches Verfahren, welches voraussetzt, dass die Daten normalverteilt sind und die Varianzen homogen verteilt sind. Auf Normalverteilung wurde bis zu einem Stichprobenumfang von 5000 mittels Shapiro-Wilk-Test geprüft (Yap \& Sim 2011). Die Annahme der Normalverteilung wurde ab einem p-Wert größer 0,05 verworfen. Der Levene-Test kam in der Überprüfung der Varianzhomogenität zum Einsatz (Lim \& Loh 1996). Ergab der Test einen p-Wert größer 0,05 wurde davon ausgegangen, dass Homoskedastizität vorliegt. Waren die Daten sowohl normalverteilt als auch die Homogenität der Varianzen gegeben, konnte als Post-Hoc-Test ein TukeyHSD-Test angewendet werden. Ein adaptierter, robuster TukeyHSD-Test wurde durchgeführt, wenn die Daten zwar normalverteilt, die Varianzen jedoch heterogen verteilt waren (Herberich et al. 2010). Lagen die Daten nicht normalverteilt vor, kam ein Kruskal-Wallis-Test als nicht-parametrisches Verfahren äquivalent zur klassischen ANOVA zur Anwendung. In diesem Fall wurden signifikante Unterschiede mit dem Wilcoxon-Mann-Whitney-Test durch paarweise Vergleiche der Mittelwerte identifiziert.

Die Signifikanzniveaus sind durch die Anzahl Asteriske $\left(^{*}\right)$ gekennzeichnet. Die zugehörige Überschreitungswahrscheinlichkeit ist unter den jeweiligen Abbildungen oder Signifikanztabellen angegeben.

Der Korrelationskoeffizient misst das Ausmaß des Zusammenhangs zwischen zwei Variablen. Ein Wert von 1 zeigt eine starke Beziehung zwischen der abhängigen und unabhängigen Variable an. Ein Wert näher an 0 deutet auf eine schwache Beziehung zwischen den Variablen hin. Die einfache lineare Regression ist eine weitere statistische Methode, um Zusammenhänge zwischen 
Variablen zu untersuchen. Das Bestimmtheitsmaß stellt in einem linearen Regressionsmodell den Anteil der Varianz für die abhängige Variable dar.

a

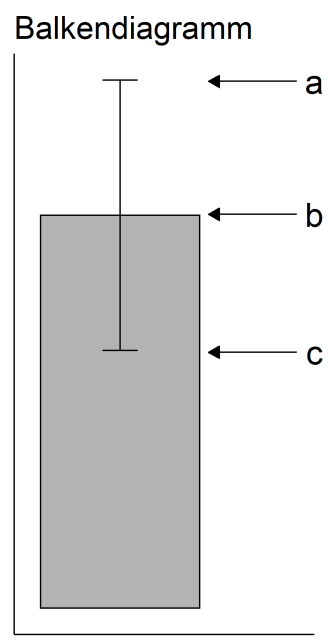

b

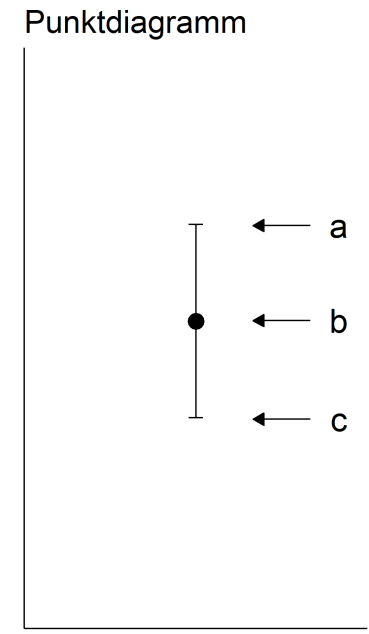

c

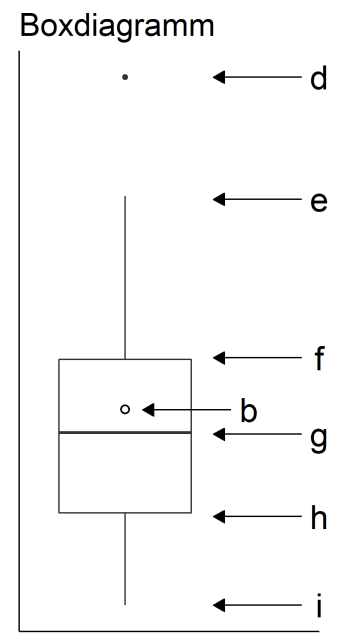

Abbildung 6.8: Statistische Darstellung der Messergebnisse. a, Balkendiagramm. b, Punktdiagramm. c, Boxplot. Statistische Kennwerte: $a$ = arithmetisches Mittel + Standardabweichung; $b=$ arithmetisches Mittel; $c=$ arithmetisches Mittel - Standardabweichung; $d=$ Ausreißer; $e=+1,5 \cdot I Q R ; f=75 \%$-Perzentil; $g=$ Median; $h=25 \%$-Perzentil; $i=-1,5 \cdot I Q R$.

Zur graphischen Darstellung wurden Diagramme nach Abbildung 6.8 erstellt. Dabei wurden aus den gruppierten Daten der Stichprobenumfang, das arithmetische Mittel und die Standardabweichung berechnet. Die Höhe der Balkendiagramme entspricht dem Mittelwert der Gruppe und wird durch die Standardabweichung in Form von Fehlerbalken ergänzt. In den ebenfalls verwendeten gestapelten Balkendiagrammen gibt die Höhe des Balkens den Anteil der Gruppe an der Gesamtheit an. Wurden Mittelwerte als Punktdiagramme dargestellt, so ist der Stichprobenumfang unterhalb der Abbildung angegeben oder die Standardabweichung als Fehlerbalken kenntlich gemacht. In den Boxdiagrammen wurden mehrere statistische Kennwerte in einer Darstellung zusammengefasst. Als Mittelwerte sind der Median und das arithmetische Mittel dargestellt. Der Interquartilabstand (IQR) als Streuungsmaß zwischen dem $25 \%$-Perzentil und dem $75 \%$-Perzentil umfasst $50 \%$ der Messwerte. Die Whisker reichen bis zum 1,5-fachen IQR. Messwerte außerhalb des 1,5-fachen IQR werden als Ausreißer bezeichnet und durch Punkte visualisiert. Zur Darstellung zeitlicher Abläufe wurden Liniendiagramme verwendet. Die Anpassung der Achsenabschnitte, der Achsenbeschriftungen und der Legenden erfolgte nach Bedarf manuell.

Weiterhin kam ein zweistufiges, faktorielles Screening Design in Taguchi L12 Anordnung zur Anwendung, um Effekte auf eine Zielgröße zu untersuchen. Die graphische Darstellung erfolgte in Form von half-normal plots (auch bekannt als Daniel plot) nach C. Daniel (1959) (Abbildung 6.9a). In einem half-normal plot gibt die Plot-Position der Faktoren in Zusammenhang mit dem Signifikanzniveau $\alpha$ Aufschluss über Effekte auf untersuchte Zielgrößen. Grundlage dieser Schätzung ist die Differenz zwischen den Mittelwerten der Zielgröße als Antwort auf die untersuchten zwei Stufen der Faktoren (Grömping 2015). Dabei weichen die Plot-Positionen der „aktiven“Effekte von den auf einer Linie durch den Ursprung liegenden Faktoren ab, da die Effekte 
größer sind, als man von der Größe der zufälligen Variation innerhalb des Experiments erwarten würde (Grömping \& J. Fox 2015). Rückschlüsse zu Interaktionen zwischen einzelnen Effekten sind nicht möglich.
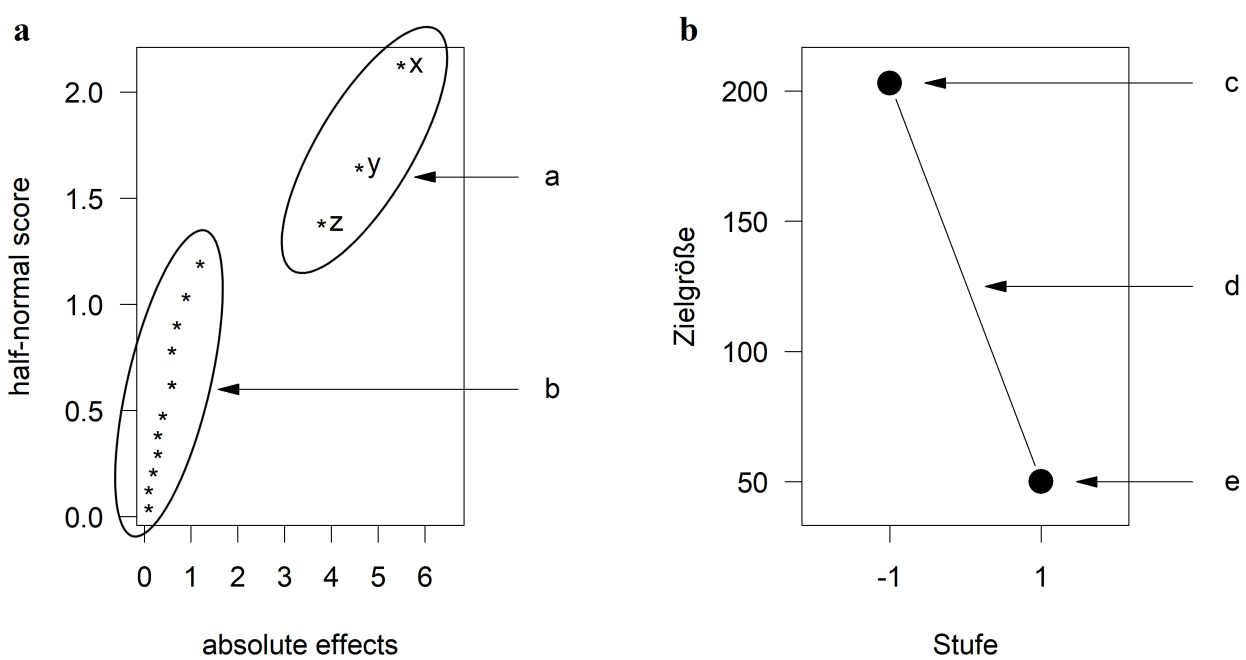

Abbildung 6.9: Statistische Darstellung der Messergebnisse. a, Hal-normal plot. b, Main effects plott. Statistische Kennwerte: $a=$ Haupteffekte; $b=$ Nebeneffekte; $c=$ Auswirkung eines Effekts in der Stufe -1 (z.B. niedrige Drehzahl); $d$ =Verbindungslinie zur Verdeutlichung der Auswirkung; $e=$ Auswirkung eines Effekts in der Stufe 1 (z.B. hohe Drehzahl).

Die Auswirkung der Effekte kann mit main effects plots nach Grömping (2014) dargestellt werden. Hier ist die Differenz zwischen den arithmetischen Mittelwerten der Effekte für die zwei untersuchten Stufen der Faktoren separat dargestellt. Die graphische Auswertung gibt in Ergänzung zum half-normal plot einen Hinweis darauf, in welcher Richtung sich der Effekt auf die Zielgröße auswirkt (Grömping \& J. Fox 2015). Die Anpassung der Achsenabschnitte, der Achsenbeschriftungen und der Legenden erfolgte nach Bedarf manuell. 


\section{Ergebnisse und Diskussion}

\subsection{Grundlegende Untersuchungen zur Prozessführung in der labortechnischen Faserstofferzeugung}

\subsubsection{Einleitung}

Das RMP- und TMP-Verfahren sind dem Teilgebiet des Zerkleinerns der mechanischen Verfahrenstechnik zuzuordnen (Stieß 2009, S. 2). Bedingt durch den hierarchischen Aufbau des Agglomerat-Aggregats Holz (Bohnet 2004, S. 14) stellen der in den Verfahren stattfindende hydrothermische Aufschluss und die mechanische Zerfaserung einen komplexen verfahrenstechnischen Prozess dar. Die Beschaffenheit der erzeugten Faserstoffe unterliegt einer Vielzahl an prozessspezifischen Einflussfaktoren.

Das Ziel der grundlegenden Untersuchungen zur Prozessführung in der labortechnischen Faserstofferzeugung ist es, zuerst die Ausgangs-Partikelgröße der Laborhackschnitzel zu ermitteln und die Frage zu beantworten, welche Ziel-Partikelgröße im Aufschluss erreicht werden soll. Auf Grundlage der ermittelten Anforderungen kann die Prozessführung angepasst werden, denn weder das Zerkleinerungsverhalten noch die Auswirkungen der Prozessparameter auf die Beschaffenheit der Faserstoffe und die Produkteigenschaften der Holzfaserdämmstoffe sind genau vorhersagbar.

Eine weitere Fragestellung betrifft die unbekannten Auswirkungen von Prozessparametern auf die spezifische elektrische Leistungsaufnahme in der labortechnischen Faserstofferzeugung. Die fundamentale Zielgröße in der Untersuchung von Mahlscheiben ist die spezifische elektrische Leistungsaufnahme. Diese wird unter anderem durch die elasto-mechanischen (rheologischen) Eigenschaften der Holzart beeinflusst (Koran 1981). Ein weiterer Bestandteil der grundlegenden Untersuchungen ist die Ermittlung der unbekannten Zielgröße des Durchsatzes der Laborrefineranlage mit den Holzarten Fichte und Buche als Grundlage zur Berechnung der spezifischne elektrischen Leistungsaufnahme. Außerdem werden Effekte auf die Holzfeuchte der Faserstoffe nach dem Aufschluss untersucht, um erste Rückschlüsse zu den Vorgängen der Feuchteaufnahme und -abgabe bei dem Erwärmen von Hackschnitzeln in Sattdampfatmosphäre ziehen zu können. Diese Erkenntnisse sind in der Konsequenz für die Auswahl und Auslegung der Verfahrensschritte erforderlich, um aussagekräftige und reproduzierbare Ergebnisse zu produzieren.

Auf Wissen aus der Industrie- und Laborpraxis zur Untersuchung der Faserstofferzeugung kann nur bedingt zurückgegriffen werden. Der Einfluss von Mahlscheiben und Aufschlussbedingungen auf die spezifische elektrische Leistungsaufnahme, den thermischen Energiebedarf und die maßgebliche Produkteigenschaft Wärmeleitfähigkeit im Zusammenhang mit der Herstellung von Holzfaserdämmstoffen wurde bisher noch nicht im Labormaßstab untersucht. 


\subsubsection{Ausgangs-Partikelgröße der Laborhackschnitzel und Ziel-Partikelgröße industrieller Faserstoffe}

\section{Ausgangs-Partikelgröße und Schüttdichte der Laborhackschnitzel}

Neben der Holzqualität ist die Verteilung der Ausgangs-Partikelgröße der Hackschnitzel ausschlaggebend für die Beschaffenheit der daraus erzeugten Faserstoffe, wie von Hartler (1963) und Behler et al. (1981) beschrieben. Aus der Massenverteilungsdichte der Laborhackschnitzel in Abbildung 7.1 geht hervor, dass die Hackschnitzel beider Holzarten überdimensionierte (F2-Fraktion) und unterdimensionierte (F4-Fraktion) Partikel und Feinanteil (F5-Fraktion) enthalten.
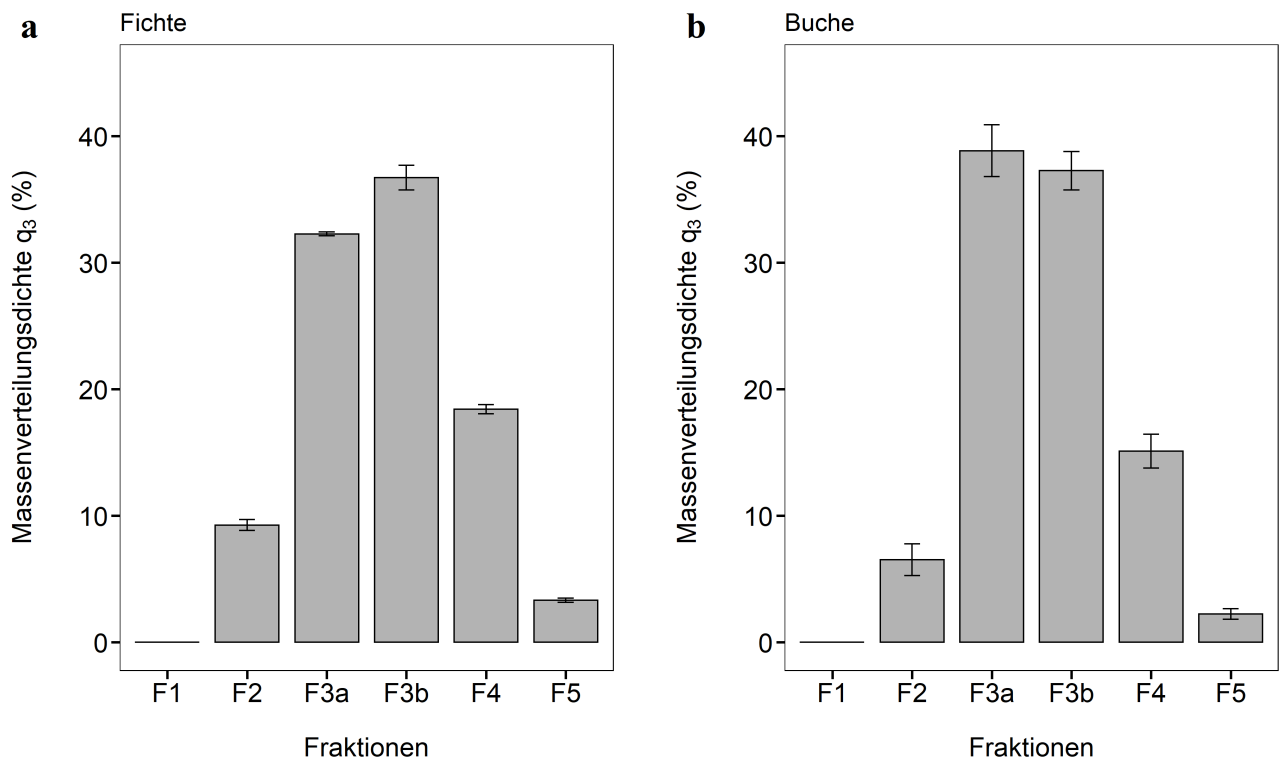

Abbildung 7.1: Ausgangs-Partikelgröße von Laborhackschnitzeln nach dem Trommelhacker: a, FichtenholzHackschnitzel. b, Buchenholz-Hackschnitzel. Siebfraktionierung nach SCAN-CM 40 (2001). N=3 mit n=2.

Ziel der Trommelsiebung mit den Siebmaschenweiten war es, überdimensionierte Partikel und Feinanteil aus den Hackschnitzeln zu entfernen. Mittels Trommelsiebung der frischen Hackschnitzel konnte der Anteil überdimensionierter Partikel (F2-Fraktion) bei beiden Holzarten reduziert werden (Tabelle 7.1). Der Feinanteil (F5-Fraktion) wurde durch die Trommelsiebung fast vollständig entfernt. Bei den Fichten- und Buchenholz-Hackschnitzeln stieg der Anteil der „pin chips" (F4-Fraktion) durch die Trommelsiebung leicht an. Weiterhin ist anzumerken, dass die F3a-Fraktion ebenfalls reduziert wurde und die F3b-Fraktion leicht höher liegt. 
Tabelle 7.1: Istwerte der Hackschnitzel-Gutfraktion nach dem Trommelsieb. Sollwerte zur Erzeugung von TMP nach dem Verband Deutscher Papierfabriken e.V. (2001). N=3 mit $n=3$.

\begin{tabular}{lllllll}
\hline Fraktion & Sieb & Sollwert & \multicolumn{3}{c}{ Istwert } \\
& & & \multicolumn{3}{c}{ Fichte } & \multicolumn{3}{c}{ Buche } \\
& & & $\bar{x}$ & $\sigma$ & $\bar{x}$ & $\sigma$ \\
& & $(\%)$ & $(\%)$ & $(\%)$ & $(\%)$ & $(\%)$ \\
\hline F1 & 45 mm Lochsieb & 0 & 0 & 0 & 0 & 0 \\
F2 & 8 mm Spaltsieb & $<10$ & 6,4 & 2,5 & 2,6 & 0,6 \\
F3a & 13 mm Lochsieb & & 24,1 & 2,8 & 32,8 & 4,9 \\
F3b & 7 mm Lochsieb & & 46,2 & 2,6 & 43,2 & 1,7 \\
F4 & 3 mm Lochsieb & $<6$ & 22,9 & 2,4 & 20,9 & 4,1 \\
F5 & Rest & 0 & 0,3 & 0,0 & 0,5 & 0,0 \\
\hline
\end{tabular}

Die Gutfraktion mit optimalen Abmessungen der Hackschnitzel für den thermomechanischen Aufschluss stellt nach dem Verband Deutscher Papierfabriken e.V. (2001) die F3-Fraktion dar. Allerdings liegt der Anteil der F3-Fraktion im Vergleich zu Scheibenhackern und Profilzerspanern bei Trommelhackern am niedrigsten (Parameswaran et al. 1985). Deppe \& Ernst (1996, S. 48) weisen darauf hin, dass der Feinanteil bei Trommelhackern gegenüber Scheibenhackern oder Profilzerspanern bauartbedingt erhöht ist.

Die Hackschnitzeldimension der Holzart Fichte hat Patt et al. (1980) zufolge erheblichen Einfluss auf die technologischen Eigenschaften von TMP-Papierstoffen. Insbesondere der Splittergehalt liegt demnach beim Einsatz der F5-Fraktion ungewöhnlich hoch. Reißlänge, Berstfestigkeit und Durchreißfestigkeit von Papieren, hergestellt aus der F4-Fraktion, nehmen gegenüber der F3Fraktion deutlich ab. Weiter stellten Patt et al. (1980) fest, dass der Langfaseranteil von FichtenholzTMP aus der F4-Fraktion niedriger und der Mehlstoffanteil höher liegen. Neben den makroskopisch beobachtbaren Partikelgrößen rufen die Zerspanungsarten nach Patt et al. (1981) Faserkürzungen und Zellwandschädigungen in der Mikromorphologie der Hackschnitzel hervor, die sich in den kleineren Partikeln stärker auf die Stoffqualität auswirken. Die bauartbedingt ungünstigeren Schnittwinkel der Trommelhacker, insbesondere beim Messereingriff auf der Oberseite des Hackguts und die Nachzerkleinerung des Rückstands auf dem Hackerauslasssieb sind nachteilig für die Massenverteilungsdichte (Aghakhani et al. 2020).

Zum Zeitpunkt des Verfassens der Dissertation lagen keine Untersuchungen zum Einfluss der Auswirkungen der Hackschnitzeldimension auf die Produkteigenschaften von Holzfaserdämmstoffen vor. Es ist nicht auszuschließen, dass der hohe Anteil der F4-Fraktion in den Buchenund Fichtenholzhackschnitzeln die Dispersitätseigenschaften der daraus hergestellten Holzstoffe beeinflusst. Für den Betrieb der Laborrefineranlage war es jedoch notwendig, die Übergrößen und den (mineralischen) Feinanteil zu entfernen, um die Mahlscheiben zu schonen. Mit Trommelhackern hergestellte Industriehackschnitzel für die Produktion von Holzwerkstoffen besitzen größere nominelle Hackschnitzellängen von $25 \mathrm{~mm}$ bis $35 \mathrm{~mm}$ und mehr. Im Vergleich zu den verwendeten Laborhackschnitzeln mit einer nominellen Hackschnitzellänge von 16mm kann dadurch der Durchsatz erhöht und der elektrische Energiebedarf für die Erzeugung der Hackschnitzel gesenkt werden (Maier 1983).

Die Bestimmung der Schüttdichte der klassierten Hackschnitzel ergab bekannte Werte für die Holzarten Fichte und Buche (Tabelle 7.2) (Deppe \& Ernst 1996, S. 54-57). 
Tabelle 7.2: Schüttdichte der Hackschnitzel-Gutfraktion nach dem Trommelsieb. N=3 mit n=3.

\begin{tabular}{lcc}
\hline Holzart & \multicolumn{3}{c}{ Schüttdichte } \\
& \multicolumn{2}{c}{$\mathrm{kg}_{\text {atro }} / \mathrm{m}^{3}$} \\
& $\bar{x}$ & $\sigma$ \\
\hline Fichtenholz-Hackschnitzel & 154 & 3,08 \\
Buchenholz-Hackschnitzel & 202 & 4,34 \\
\hline
\end{tabular}

\section{Ziel-Partikelgröße und Dispersitätseigenschaften industrieller Faserstoffe}

Einen Überblick über die $50 \%$ - und $97 \%$-Perzentilen der Luftstrahl-Prüfsiebung von MDF-, HDFund Holzfaserdämmstoff-Fasern von vier Holzfaserdämmstoffherstellern gibt Tabelle 7.3. Daraus ist abzuleiten, dass MDF-Fasern die geringsten Prüfsieb-Maschenweiten und damit den höchsten Aufschlussgrad aufweisen. Das 97 \%-Perzentil liegt bei HDF-Fasern höher, was auf einen größeren Anteil an nicht aufgeschlossenen Faserbündeln zurückzuführen ist.

Die Prüfsieb-Maschenweiten des Medianwerts und des $97 \%$-Perzentils der HolzfaserdämmstoffFasern liegen deutlich höher als diejenigen der MDF-Fasern. Außerdem bestehen zwischen den einzelnen Herstellern deutliche Unterschiede. Die größten Prüfsieb-Maschenweiten weisen die Holzfaserdämmstoff-Fasern 1 und 2 auf. Die Holzfaserdämmstoff-Fasern 3 und 4 liegen im Bereich der Prüfsieb-Maschenweiten der HDF-Faser. Die HDF-Faser und insbesondere die Holzfaserdämmstoff-Fasern 1 und 2 sind nach subjektiv-optischen Kriterien als „grob“ zu beschreiben. Die MDF-Faser und die Holzfaserdämmstoff-Faser 4 dagegen als „fein“. Eine Sonderstellung nimmt die Zellulosedämmung durch den hohen Medianwert und das hohe $97 \%$-Perzentil ein. Die Zellulosedämmung enthält zahlreiche voluminöse Agglomerate und nicht aufgeschlossene, blättchenförmige Partikel. Diese Eigenschaften sind auf den trockenen Aufschluss des Altpapiers und die enthaltenen Trockenfestmittel (Stärke) und mineralischen Füllstoffe (Calciumcarbonat und Kaolin) zurückzuführen.

Tabelle 7.3: Dispersitätseigenschaften industrieller Faserstoffe nach der Luftstrahl-Prüfsiebung. $n=2$.

\begin{tabular}{llll}
\hline Produkt & Holzart & \multicolumn{2}{c}{ Prüfsieb-Maschenweite } \\
& & $\begin{array}{l}\bar{x}_{50,3} \\
\mu \mathrm{m}\end{array}$ & $\begin{array}{l}\bar{x}_{97,3} \\
\mu \mathrm{m}\end{array}$ \\
\hline MDF-Faser & Kiefer & 117 & 791 \\
HDF-Faser & Kiefer & 237 & 1496 \\
Zellulosedämmung & Altpapier & 746 & 1933 \\
Holzfaserdämmstoff-Faser 1 & Fichte & 497 & 2493 \\
Holzfaserdämmstoff-Faser 2 & Fichte & 209 & 1823 \\
Holzfaserdämmstoff-Faser 3 & Kiefer & 312 & 1209 \\
Holzfaserdämmstoff-Faser 4 & Kiefer & 291 & 1042 \\
\hline
\end{tabular}

Die quantitative Bildanalyse ergänzt die Erkenntnisse zur Größenverteilung des Partikelkollektivs aus der Luftstrahl-Prüfsiebung mit Formfaktoren individueller Partikel. Für die Analyse der Ziel-Partikelgröße wurde die geodätische Länge und das Partikelform-Verhältnis mit dem Unterscheidungskriterium Elongation nach Gleichung 6.5 gewählt.

Die $10 \%$-Perzentilen der geodätischen Länge differieren zwischen den Holzstoffen unwesentlich. Die $50 \%$ - und $97 \%$-Perzentilen der geodätischen Länge spiegeln hingegen die in der Luftstrahl-Prüfsiebung ermittelten Abstufungen der einzelnen Holzstoffe wider. Den Holzstoffen, welche eine feinere Partikelgrößenverteilung in der Luftstrahl-Prüfsiebung aufweisen, kann 
eine geringere geodätische Länge in der quantitativen Bildanalyse nachgewiesen werden. Die Holzfaserdämmstoff-Fasern 1 bis 3 weisen insbesondere in der $97 \%$-Perzentile eine hohe geodätische Länge auf. Die Holzfaserdämmstoff-Faser 4 gleicht dagegen der MDF-Faser in Bezug auf geodätische Länge. Bei der Betrachtung des Partikelform-Verhältnisses fällt auf, dass die MDF-, HDF- und die Holzfaserdämmstoff-Faser 4 einen höheren Anteil an längeren, faserartigen Partikeln als die restlichen Faserstoffe aufweisen. Ein Erklärungsansatz hierfür ist ein höherer Aufschlussgrad dieser Faserstoffe, der weniger Faserbündel und Bruchstücke mit sich bringt. Die Zellulosedämmung weißt die kürzesten Längen der untersuchten Faserstoffe auf. Die Zellulosedämmung kann aufgrund der zahlreichen voluminösen Agglomerate und nicht aufgeschlossenen, blättchenförmigen Partikeln mit der angewendeten Methode nicht aussagekräftig charakterisiert werden.

Tabelle 7.4: Ziel-Partikelgröße (anzahlgewichtete Dispersitätseigenschaften) industrieller Faserstoffe nach der quantitativen Bildanalyse. $n=3$.

\begin{tabular}{llllll}
\hline Produkt & Holzart & \multicolumn{3}{l}{ Geodätische Länge } & Partikelform-Verhältnis \\
& & $\bar{x}_{10,2}$ & $\bar{x}_{50,2}$ & $\bar{x}_{97,2}$ & kornförmig $(\mathrm{E}>0,3) /$ \\
& & $\mu \mathrm{m}$ & $\mu \mathrm{m}$ & $\mu \mathrm{m}$ & faserartig $(\mathrm{E} \leq 0,3)$ \\
\hline MDF-Faser & Kiefer & 36 & 113 & 2901 & 1,14 \\
HDF-Faser & Kiefer & 40 & 198 & 3213 & 1,47 \\
Zellulosedämmung & Altpapier & 27 & 61 & 1038 & 4,16 \\
Holzfaserdämmstoff-Faser 1 & Fichte & 43 & 610 & 4930 & 0,38 \\
Holzfaserdämmstoff-Faser 2 & Fichte & 45 & 252 & 4060 & 0,61 \\
Holzfaserdämmstoff-Faser 3 & Kiefer & 49 & 434 & 4001 & 0,45 \\
Holzfaserdämmstoff-Faser 4 & Kiefer & 45 & 114 & 2801 & 1,29 \\
\hline E = Elongation & & & & &
\end{tabular}

Bezüglich der Zielstellung der vorliegenden Arbeit ist festzuhalten, dass die Dispersitätseigenschaften der industriellen Faserstoffe, die zur Herstellung von Dämmstoffen mit vergleichbaren Produkteigenschaften verwendet werden, kein einheitliches Bild ergeben. Die Unterschiede sind zu groß, als dass ein bewährter Stand der Technik daraus abgeleitet werden könnte. Die weiter unten in dieser Arbeit ermittelte Wärmeleitfähigkeit industrieller Faserstoffe und Dämmstoffe in Tabelle 7.16 verstärkt diesen Eindruck. Auf Grundlage dieser Erkenntnisse wird in den weiteren Untersuchungen die Beschaffenheit der labortechnischen Faserstoffe genauer untersucht. In der labortechnischen Faserstofferzeugung wird versucht, die Bandbreite sowohl der industriellen Holzfaserdämmstoff-Fasern als auch der MDF-Fasern bezüglich deren Dispersitätseigenschaften abzubilden. 


\subsubsection{Untersuchung grundlegender Parameter der Prozessführung auf ausgewählte Zielgrößen der labortechnischen Faserstofferzeugung}

\section{Zielgröße Durchsatz der Laborrefineranlage}

Für die Berechnung der spezifischen elektrischen Leistungsaufnahme ist die Ermittlung des Durchsatzes grundlegend. Nur durch eine genaue Ermittlung des Massenflusses des Holzes ist es möglich, hierzu präzise Aussagen zu treffen. Nach Abbildung 7.2 ist die Holzart maßgeblich für den Durchsatz. Das Mahlscheibenmuster und der Sattdampfdruck haben einen untergeordneten Effekt. Der Einfluss der Holzart ist durch den Dichteunterschied zwischen den untersuchten Holzarten Fichte und Buche zu erklären.

In Abbildung 7.3 ist der Durchsatz für die Holzarten Fichte und Buche dargestellt. Die linea-

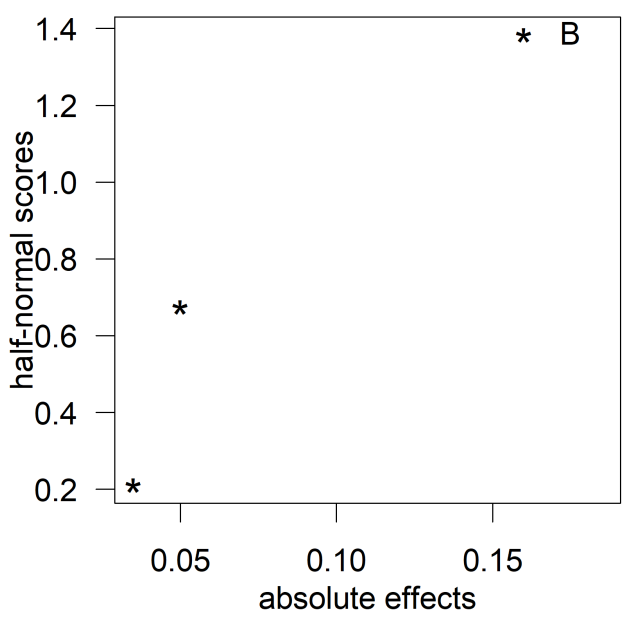

Abbildung 7.2: Effekte auf den Durchsatz der Laborrefineranlage. Half-normal plot für die Zielgröße Durchsatz. Den größten Effekt auf den Durchsatz hat die Holzart, markiert mit B, $\alpha=0,1$. 2-stufiges, faktorielles ScreeningDesign.

re Regression zeigt, dass der Durchsatz der Laborrefineranlage mit hoher Wahrscheinlichkeit linear von der Förderleistung der Einspeiseschnecke abhängt. Durch die ebenfalls dargestellte Geradengleichung ist es möglich, den Durchsatz der Laborrefineranlage in Abhängigkeit der Förderleistung der Einspeiseschnecke zu berechnen. Damit ist eine Voraussetzung erfüllt, um die spezifische elektrische Leistungsaufnahme in der Faserstofferzeugung mit den Holzarten Fichte und Buche zu bestimmen.

\section{Zielgröße spezifische elektrische Leistungsaufnahme des Refinermotors im TMP-Verfahren}

Eine weitere Voraussetzung für die Berechnung der spezifischen elektrischen Leistungsaufnahme ist die Ermittlung der Leerlaufleistung und der „no treatment“-Leistung $\mathrm{P}_{\mathrm{NT}}$ (Abschnitt 3.4.2). Die „no treatment"-Leistung unterscheidet sich von der Leerlaufleistung durch die Anwesenheit eines Fördermediums wie Wasser oder Dampf. Bei der Laborrefineranlage ist keine Veränderung 

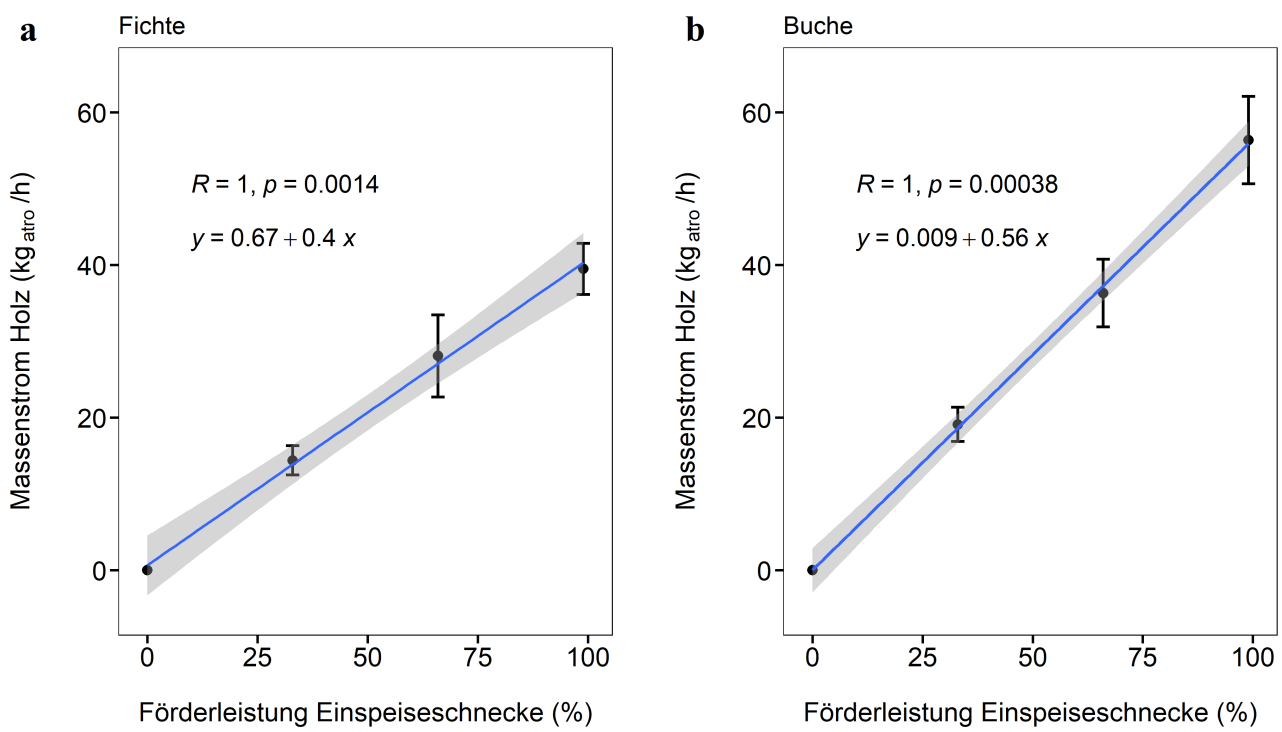

Abbildung 7.3: Ermittlung des Durchsatzes der Laborrefineranlage: a, Durchsatz mit der Holzart Fichte. b, Durchsatz mit der Holzart Buche. Die lineare Regression liefert die Geradengleichung zur Berechnung des Durchsatzes bei einer beliebig gewählten Förderleistung der Einspeiseschnecke. $n=12$.

von $\mathrm{P}_{\mathrm{NT}}$ mit steigendem Sattdampfdruck festzustellen (Abbildung 7.4). Unregelmäßigkeiten im Verlauf von $\mathrm{P}_{\mathrm{NT}}$ zwischen den Zeitpunkten 10:44:00 und 10:46:00 sind auf Kondensatbildung innerhalb der Refineranlage zurückzuführen. Das Kondensat gelangt zwischen die Mahlscheiben und ruft die Schwankungen hervor. Die Leerlaufleistung beträgt im Mittel der regelungsbedingten Schwankungen 13,07 kW. Im Folgenden wird zur Berechnung der spezifischen elektrischen Leistungsaufnahme ein Wert von 13,1 kW herangezogen.

Bei der Untersuchung der physikalisch-technologischen Eigenschaften von Mahlscheiben ist die spezifische elektrische Leistungsaufnahme des Refinermotors eine wichtige Zielgröße. Zum einen ist es wichtig, in der Versuchsplanung und -durchführung unerwünschte Einflüsse auf die spezifische elektrische Leistungsaufnahme zu vermeiden. Zum anderen wird anhand dieser Zielgröße der Einfluss des Mahlscheibenmusters auf den elektrischen Energiebedarf untersucht. In Abbildung 7.5 a ist dargestellt, dass unabhängig vom Mahlscheibentyp der Mahlspalt maßgeblich für die spezifische elektrische Leistungsaufnahme der Laborrefineranlage ist (alle untersuchten Faktoren sind in Tabelle 6.14 aufgelistet). Diese Erkenntnis stimmt mit den Ergebnissen von Kerekes (2010) überein. Demzufolge wird durch die Verringerung des Mahlspalts die Kraft auf die Fasern im Mahlspalt erhöht (Abbildung 7.5b). Im Gegensatz zu Krug (2010, 113 ff.) wirkte sich die Kochzeit (1 min und $10 \mathrm{~min}$ ) nicht entscheidend auf die spezifische elektrische Leistungsaufnahme aus.

Die Effekte Hackschnitzelfeuchte und Aufschlusstemperatur treten nicht signifikant hervor. Es könnte sein, dass eine niedrigere Hackschnitzelfeuchte zu einer geringeren spezifischen elektrischen Leistungsaufnahme führt. Darauf deutet Abbildung 7.5c hin. Der Wassergehalt in den Hackschnitzeln kann einen Einfluss auf die spezifische elektrische Leistungsaufnahme haben. So geht Atack \& Wood (1973) zufolge die spezifische elektrische Leistungsaufnahme von $1876 \mathrm{kWh} / \mathrm{t}$ auf $1488 \mathrm{kWh} / \mathrm{t}$ zurück, wenn die Konsistenz (Gleichung 6.2) vor dem Refiner von 13,3\% auf $24 \%$ erhöht wird.

Die Untersuchungen von Asplund (1940a) und Krug (2010, S. 113) bestätigen den hier sichtbaren 
a

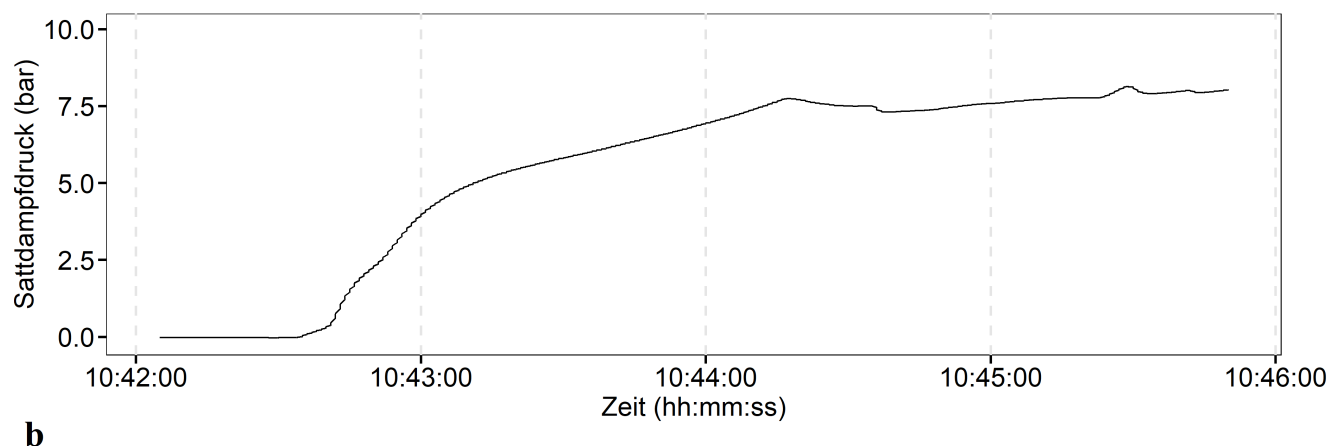

b

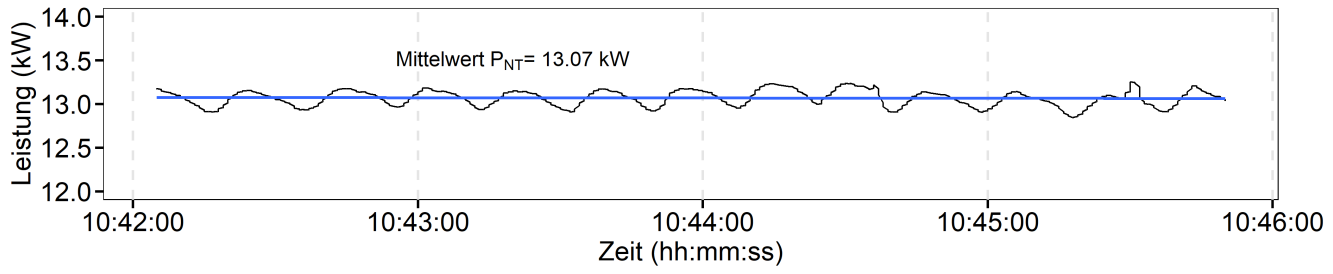

Abbildung 7.4: Ermittlung der „no treatment“-Leistung $P_{N T}$ der Laborrefineranlage: a, Zeitlicher Verlauf des Sattdampfdrucks. b, Zeitlicher Verlauf der „no treatment“-Leistung $P_{N T}$. Der Anstieg des Sattdampfdrucks wirkt sich nicht auf $P_{N T}$ aus. Der Mittelwert von $P_{N T}$ liegt bei 13,07 kW. Die Leistungsaufnahme schwankt regelungsbedingt.

Trend für den Einfluss der Aufschlusstemperatur (Abbildung 7.5d). Untersuchungen zum Einfluss niedriger Aufschlusstemperaturen und zur drucklosen Zerfaserung mit Nadel- und Laubholz im Zusammenhang mit speziellen Mahlscheiben und Einscheibenrefinern sind nicht bekannt. Der Durchsatz dürfte im Falle der spezifischen elektrischen Leistungsaufnahme keinen Effekt haben (Abbildung 7.5e).

\section{Zielgröße Holzfeuchte der Faserstoffe nach dem Aufschluss}

Im vorherigen Abschnitt wurde festgestellt, dass die Hackschnitzelfeuchte keinen signifikanten Einfluss auf die spezifische elektrische Leistungsaufnahme hatte. Die Holzfeuchte lag bei den Untersuchungen bei $60 \%$ und $95 \%$. Durch einen größeren Unterschied zwischen der Holzfeuchte könnte der Effekt auf die spezifische elektrische Leistungsaufnahme größer werden. Insbesondere, wenn sich die Holzfeuchte dem Fasersättigungsbereich oder der Wassersättigung annähert. Inwiefern die Holzfeuchte allerdings von der Sattdampfatmosphäre im Kocher des Refiners beeinflusst wird ist nicht bekannt. Deshalb wurde, auch vor dem Hintergrund des thermischen Energiebedarfs im TMP-Verfahren sowie der anschließenden Trocknung der Faserstoffe, mit einem zweistufigen Screening-Design untersucht, welche Prozessgröße im TMP-Verfahren einen entscheidenden Effekt auf die Holzfeuchte der Fasern nach dem Aufschluss hat.

Es konnte festgestellt werden, dass unter den untersuchten Prozessparametern (Tabelle 6.14) die Sattdampftemperatur den stärksten Einfluss auf die Holzfeuchte der Faserstoffe nach dem Aufschluss hat (Abbildung 7.6a). Bei beiden Sattdampf-Druckstufen liegt die Holzfeuchte der Faserstoffe nach dem Aufschluss über der Holzfeuchte der Hackschnitzel vor dem Aufschluss. Der höhere Wert für die Holzfeuchte der Faserstoffe nach dem Aufschluss mit 8 bar deutet darauf hin, dass die Hackschnitzel unter höherem Sattdampfdruck mehr Wasser enthalten (Abbildung 


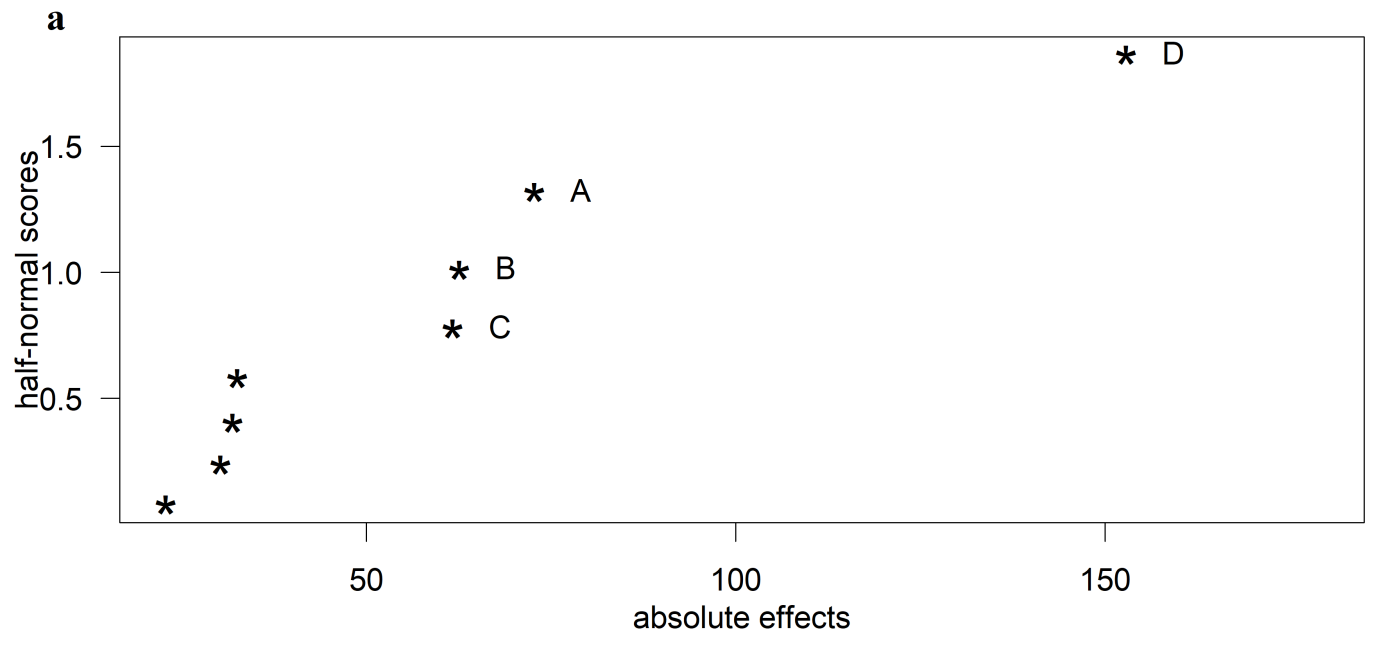

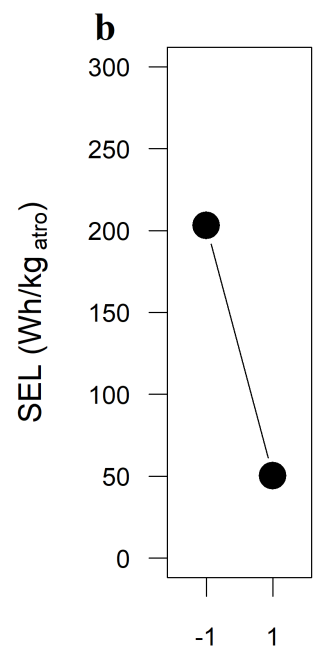

Stufe

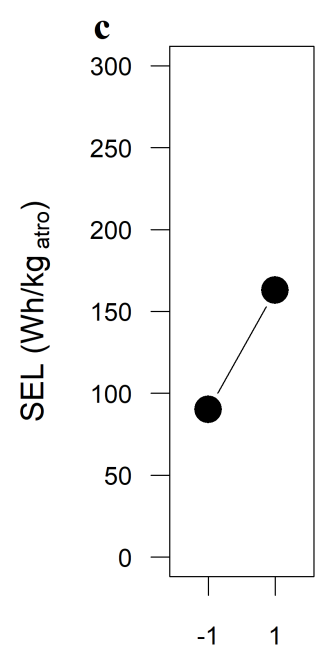

Stufe

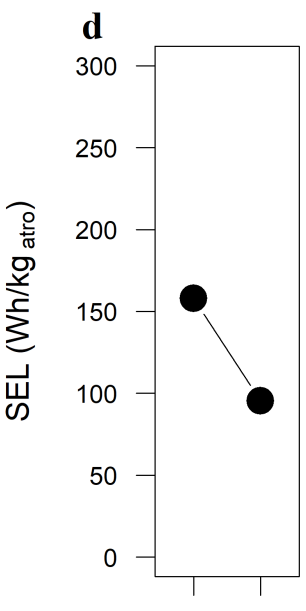

$-1$

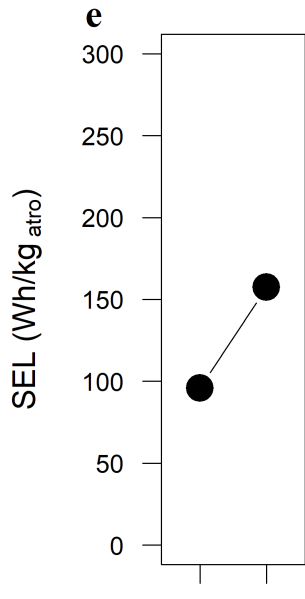

$\begin{array}{ll}-1 & 1\end{array}$

Stufe

Abbildung 7.5: Effekte auf die spezifische elektrische Leistungsaufnahme: a, Half-normal plot für die Zielgröße spezifische elektrische Leistungsaufnahme (SEL). A = Hackschnitzelfeuchte; $B=$ Aufschlusstemperatur; $C=$ Durchsatz; $D=$ Mahlspalt. Den größten Effekt zeigt der Mahlspalt (D), $\alpha=0,1$. b, Main effect plot für den Haupteffekt Mahlspalt(D): Eine Verringerung des Mahlspalts führt zu einer höheren Leistungsaufnahme. c-e, Main effect plots für die Nebeneffekte, die ab einem Signifikanzniveau von $\alpha=0,4$ sichtbar werden. c, Eine niedrigere Hackschnitzelfeuchte (A) könnte die spezifische elektrische Leistungsaufnahme senken. $\boldsymbol{d}$, Die Aufschlusstemperatur (B) hat aufgrund der geringen Differenz der Mittelwerte einen untergeordneten Effekt. e, Die Variation des Durchsatzes $(C)$ hat ebenfalls einen untergeordneten Effekt. Zweistufiges, faktorielles Screening-Design in Taguchi L12 Anordnung.

7.6b). Tiefgreifendere Erklärungsansätze sind im Anschluss an die Untersuchungen im Abschnitt 7.2 formuliert.

Die Holzfeuchte der Hackschnitzel vor dem Aufschluss scheint keinen maßgeblichen Effekt auf 

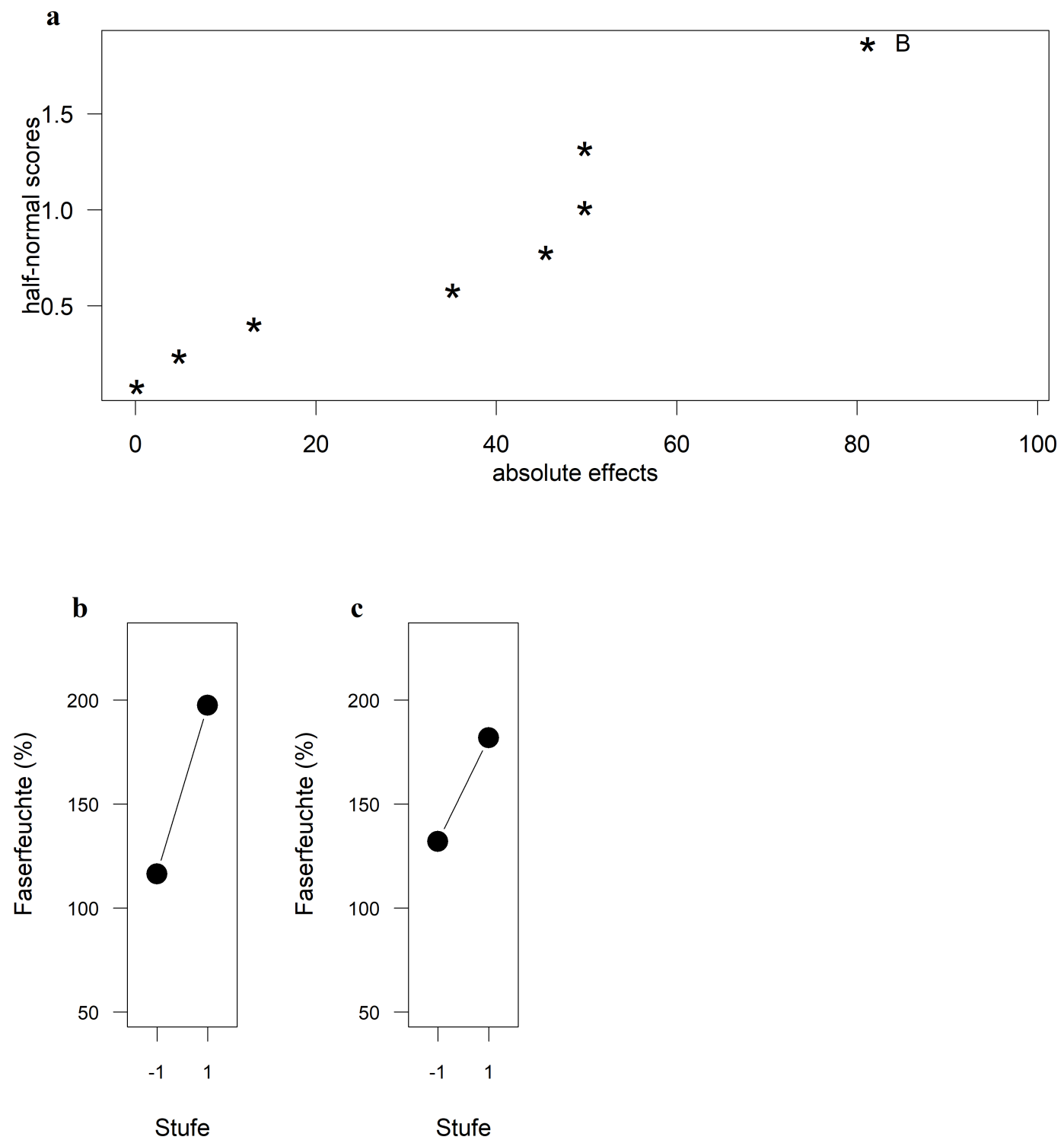

Abbildung 7.6: Effekte auf die Holzfeuchte der Fasern nach dem TMP-Aufschluss: a, Half-normal plot für die Zielgröße Faserfeuchte. Den größten Effekt hat die Sattdampftemperatur (B), $\alpha=10 \%$. b, Main effect plot für den Haupteffekt Sattdampftemperatur: Eine Erhöhung der Sattdampftemperatur zieht eine höhere Faserfeuchte nach dem Aufschluss nach sich. c, Die Hackschnitzelfeuchte vor dem Refiner könnte ebenfalls einen Effekt auf die Faserfeuchte nach dem Aufschluss haben, $\alpha=40 \%$. Zweistufiges, faktorielles Screening-Design in Taguchi L12 Anordnung.

die Holzfeuchte der Fasern nach dem Aufschluss zu haben (Abbildung 7.6c). Allerdings muss hierbei die eingeschränkte Aussagekraft des zweistufigen faktoriellen Screening-Designs und die große Standardabweichung berücksichtigt werden. Eine Vergleichmäßigung der Holzfeuchte, wie in Deppe \& Ernst (1996, S. 52) angesprochen, ist nicht erkennbar.

Die Holzfeuchte der Fasern nach dem TMP-Aufschluss ist mit einfachen Mitteln messbar. Die 
Holzfeuchte der Hackschnitzel in Sattdampfatmosphäre ist nicht bekannt. Sie ist nicht ohne weiteres zu ermitteln, da Druck und Temperatur den Einsatz von Messgeräten erschweren. Theoretisch kann die Holzfeuchte der Hackschnitzel unter Sattdampfdruck über den Dampfbedarf zur Erwärmung berechnet werden. In Abschnitt 7.2 wird die Holzfeuchte in Sattdampfatmosphäre experimentell ermittelt und dem berechneten Dampfbedarf gegenübergestellt.

\subsubsection{Erkenntnisse und Konsequenzen für die weiteren Untersuchungen}

Die in den vorhergehenden Abschnitten gewonnenen Erkenntnisse zur Charakterisierung der labortechnischen Faserstofferzeugung im TMP-Verfahren sind von Bedeutung für die Auswahl und Auslegung der Verfahrensschritte in den weiteren Untersuchungen.

Die Laborhackschnitzel enthalten nach dem Trommelhacker überdimensionierte Hackschnitzel und Feinanteil. Vorversuche zeigten, dass insbesondere die überdimensionierten Hackschnitzel die thermomechanische Faserstofferzeugung beeinträchtigen, da deren Zuführung in den Mahlspalt zu Leistungsspitzen führt. Nach dem Trommelsieb enthielt die Gutfraktion keine der ganz großen überdimensionierten Hackschnitzel mehr und der (mineralische) Feinanteil wurde ebenfalls entfernt. Die Schüttdichte der Laborhackschnitzel war mit der von industriellen Hackschnitzeln aus der MDF-Produktion vergleichbar.

Die Analyse von Dispersitätseigenschaften industrieller Faserstoffe ermöglichte die Quantifizierung der Ziel-Partikelgrößen. Überraschend ist, dass die Holzfaserdämmstoff-Fasern untereinander hohe Differenzen sowohl in der gravimetrischen Luftstrahl-Prüfsiebung als auch in der quantitativen Bildanalyse aufwiesen. Das Spektrum reichte von „feiner“ MDF-Qualität mit kurzen Faserlängen und geringem kornförmig-zu-faserartig-Verhältnis bis zu „groben“ Fasern mit großer Faserbündellänge sowie hohem Verhältnis der kornförmigen und faserartigen Partikel. Vorversuche haben gezeigt, dass mit der Laborrefineranlage Faserstoffe mit vergleichbaren Eigenschaften hergestellt werden können. Die geodätische Länge der Faserbündel aus den industriellen Faserstoffen wird dabei unter vergleichbaren Aufschlussbedingungen nicht vollumfänglich erreicht. Dies ist auf die kleinere Ausgangs-Partikelgröße der Laborhackschnitzel im Vergleich zu industriellen Hackschnitzeln aus der Holzwerkstoffherstellung zurückzuführen.

Bezüglich des Durchsatzes der Laborrefineranlage ist festzuhalten, dass für den Mahlscheibentyp und den Sattdampfdruck kein Effekt festzustellen ist. Somit können die für die Holzarten Buche und Fichte ermittelten Durchsätze zur Berechnung der spezifischen elektrischen Leistungsaufnahme herangezogen werden. Die weiteren Untersuchungen mit Mahlscheiben und beiden Holzarten werden auf Basis dieser Ergebnisse vergleichbar.

Ferner wurde für die Berechnung der spezifischen elektrischen Leistungsaufnahme die Leerlaufleistung des Hauptmotors der Laborrefineranlage ermittelt. Die Messung der „no treatment“-Leistung zeigte, dass der Sattdampfdruck keinen Einfluss die spezifische elektrische Leistungsaufnahme hat.

In den weiteren Untersuchungen zur Faserstofferzeugung mit unterschiedlichen Mahlscheiben muss der Mahlspalt über alle Versuche konstant gehalten werden. Eine Vergleichbarkeit ist sonst nicht gegeben. Dies bestätigen Vorversuche, bei denen der Mahlspalt variiert wurde sowie Untersuchungen von Kerekes (2015). Die Untersuchungen zeigen, dass die Hackschnitzelfeuchte in einem geringen Prozentanteil variieren kann ohne die spezifische elektrische Leistungsaufnahme zu beeinflussen. In den weiteren Versuchen sollte außerdem der Durchsatz konstant gehalten werden, da ein Einfluss auf die spezifische elektrische Leistungsaufnahme nicht ausgeschlossen werden kann. Die Kochzeit zeigte keinen Effekt auf die Leistungsaufnahme und kann in Übereinstimmung mit Deppe \& Ernst (1996, S. 54) und Brombacher et al. (2016) konstant bei 3 min 
gehalten werden.

Die Sattdampftemperatur kann und sollte variiert werden, um den Einfluss der Mahlscheibe und der Holzart auf die Beschaffenheit sowie die physikalisch-technologischen Eigenschaften der Holzstoffe zu untersuchen. In diesem Zusammenhang kommt der Untersuchung der Holzfeuchte der Hackschnitzel während des Aufschlusses und der Holzfeuchte der Fasern nach dem Aufschluss besondere Bedeutung zu (siehe folgender Abschnitt). 


\subsection{Untersuchungen zur Holzfeuchte von Hackschnitzeln in Sattdampfatmosphäre}

\subsubsection{Einleitung}

Im TMP-Verfahren wird Sattdampf zur Erwärmung von Hackschnitzeln eingesetzt. Folglich sind Holz-Wasser-Wechselwirkungen in der Faserstofferzeugung zu beachten. Insbesondere ist die Sorption von Wasserdampf durch die Hackschnitzel in Präsenz der Sattdampfatmosphäre zu erwarten. Darauf weisen auch Malkov et al. (2002) hin. Weitere Zusammenhänge der Holzfeuchte mit dem Erweichungsverhalten von Holz in der Faserstofferzeugung sind in Abschnitt 2.3.2 beschrieben. Ausgang der Untersuchungen zur Holzfeuchte war die Beobachtung, dass auf den Hackschnitzeln während des thermischen Aufschlusses ein Kondensationsfilm aus Wasser sichtbar ist (Abbildung 7.7a). Nach Entspannung des Sattdampfdrucks ist der Kondensationsfilm auf der Oberfläche von Buchenholz-Hackschnitzeln nicht mehr sichtbar (Abbildung 7.7b). Das Phänomen ist auch bei Fichtenholz-Hackschnitzeln zu beobachten.

a

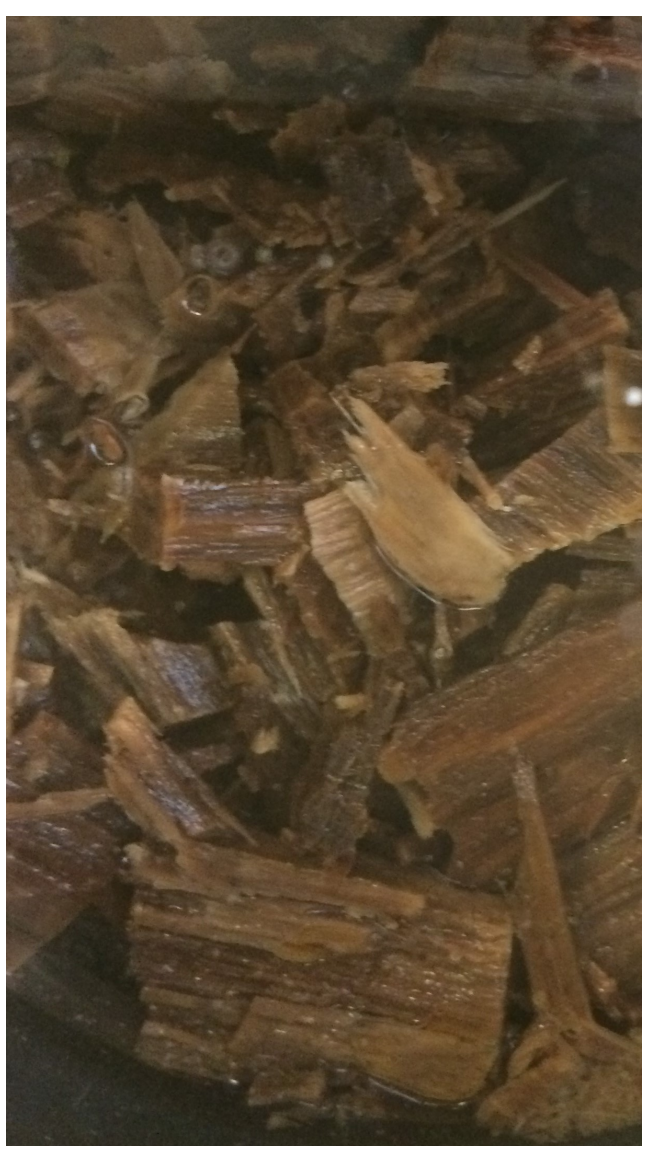

b

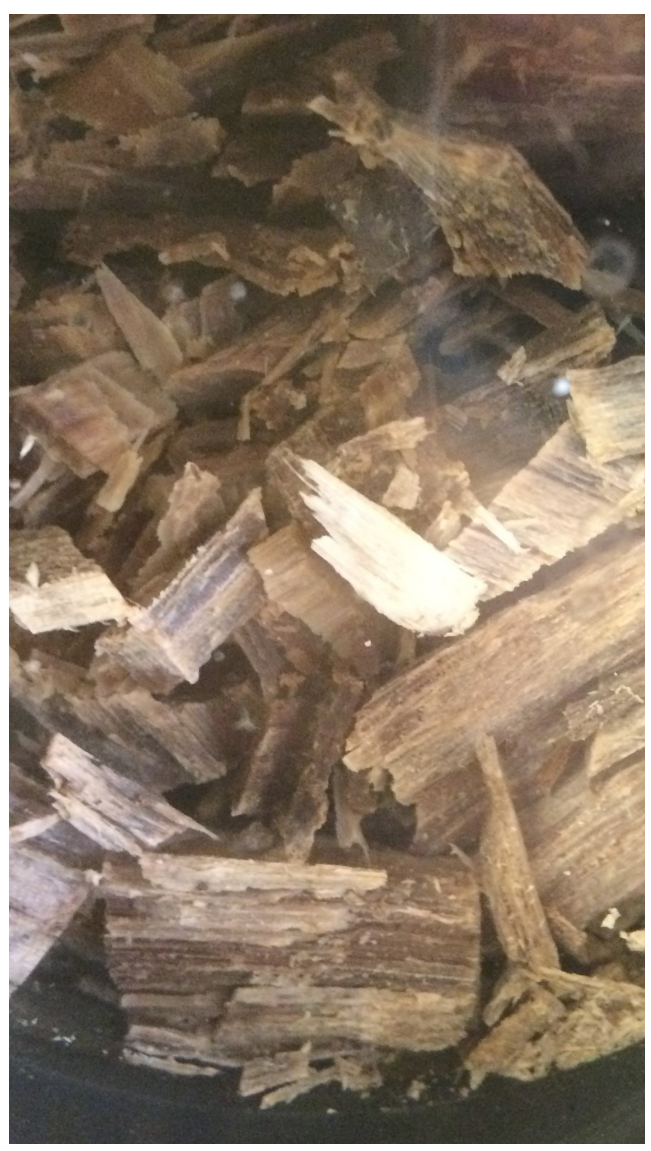

Abbildung 7.7: Blick durch ein Schauglas in den Druckbehälter des Laborrefiners. a, Zu sehen ist ein Kondensationsfilm auf der Oberfläche von Buchenholz-Hackschnitzeln in Sattdampfatmosphäre bei 9 bar. b, Zu sehen sind die selben Hackschnitzel nachdem der Sattdampfdruck abgebaut wurde. Im Vergleich zu a ist der Kondensationsfilm auf der Oberfläche nicht mehr sichtbar. 
Nach Blechschmidt (2013, S. 75) sollte die Holzfeuchte vor der mechanischen Faserstofferzeugung über dem Fasersättigungsbereich liegen. Die Berechnungen von Asplund (1940b) führen zu dem Ergebnis, dass die Hackschnitzel nach der Erwärmung in Sattdampfatmosphäre im TMPVerfahren wassergesättigt sind. Hartler (1977) untersuchte den Einfluss der Ausgangs-Holzfeuchte zwischen $10 \%$ und $60 \%$ auf die Faserqualität zur Papierherstellung und wies nach, dass trockenere Hackschnitzel einen höheren Splittergehalt mit sich bringen. Die Ausgleichsfeuchte und das Adund Desorptionsverhalten von Holz ist im hygroskopischen Bereich für Temperaturen bis $160^{\circ} \mathrm{C}$ untersucht worden (Kauman 1956; Noack 1959; Strickler 1968; Engelhardt 1979; Ishikawa et al. 2004). Ebenfalls ist der simultane Massenverlust als Folge der thermischen Beanspruchung bekannt (Lenth \& Kamke 2001). Allerdings ist der hygroskopische Bereich für das TMP- und RMP-Verfahren nicht von Interesse. Durch den Einsatz von Frischholz liegt die Holzfeuchte hier typischerweise oberhalb des Fasersättigungsbereichs.

Zum Einfluss der Ausgangs-Holzfeuchte auf den thermischen Energiebedarf in der Faserstofferzeugung nach dem RMP- und TMP-Verfahren und in der anschließenden Trocknung der Faserstoffe fehlen wissenschaftliche Untersuchungen. Die in situ Ermittlung der Holzfeuchte von Hackschnitzeln in Sattdampfatmosphäre hat zum Ziel, einen ersten Beitrag zum Einfluss der Prozessführung auf die Holzfeuchte vor dem Hintergrund der Faserstofferzeugung für Holzfaserdämmstoffe zu leisten. Dafür wurde ein Versuchsaufbau mit einer gravimetrischen Messeinrichtung entwickelt (Abbildung 6.4). Das Experiment soll Aufschluss darüber geben, wie die Holzfeuchte in der Sattdampfatmosphäre mit der Ausgangs-Holzfeuchte und dem Dampfverbrauch zusammenhängt. Nicht zuletzt sind auch die Holzfeuchte nach Entspannung des Sattdampfdrucks und der zeitliche Verlauf der Wasseraufnahme und -abgabe der Hackschnitzel von Interesse.

\subsubsection{Absorption von Wasser in Abhängigkeit des Sattdampfdrucks}

Die zur Erwärmung von Hackschnitzeln benötigte Wärmemenge wurde nach Gleichung 7.1 berechnet. Ist die Wärmemenge bekannt, kann die entsprechende Dampfmenge für die Erwärmung der Hackschnitzel mit der Gleichung 7.2 ermittelt werden. Unter isobaren thermischen Zustandsgrößen ist die im Dampf enthaltene Wärmemenge proportional der Hackschnitzelmasse und der zu erzielenden Temperaturdifferenz. Der Wärmeübergang erfolgt durch die freigesetzte Verdampfungsenthalpie bei der Kondensation des Dampfes auf der zugänglichen äußeren und inneren Oberfläche der Hackschnitzel. Das Prinzip der Wärmeübertragung durch Kondensation wird in Brännvall (2009, S. 140) durch den Ausgleich der Wasserdampfpartialdruckdifferenz zwischen dem Sattdampf und der Luft in den Hackschnitzeln beschrieben. Zusätzlich werden Luftanteile durch thermische Ausdehnung ausgetrieben. Der Sattdampf, der in die Lumen eindringt, kondensiert und die Lumen füllen sich mit Wasser.

$$
Q=\Delta T\left(m_{H} \cdot c_{H}+m_{W} \cdot c_{W}\right)
$$

$Q \quad$ Wärmemenge in $J$

$\Delta T \quad$ Temperaturdifferenz

$m_{H} \quad$ Masse Holz

$c_{H} \quad$ Spezifische Wärmekapazität Holz

$m_{W} \quad$ Masse Wasser

$c_{W} \quad$ Spezifische Wärmekapazität Wasser

$$
m_{D, Q}=\frac{Q}{r}
$$




$$
\begin{array}{ll}
m_{D, Q} & \text { Dampfmenge für die Erwärmung in } \mathrm{kg} \\
Q & \text { Wärmemenge } \\
r & \text { Verdampfungsenthalpie }
\end{array}
$$

In Abbildung 7.8a ist die Dampfmenge für die Erwärmung von drei Ausgangs-Holzfeuchten über dem Sattdampfdruck aufgetragen. Die Dampfmenge ist von der Ausgangs-Holzfeuchte abhängig, da Wasser eine wesentlich höhere spezifische Wärmekapazität besitzt, als die wasserfreie Holzsubstanz. Die spezifische Verdampfungsenthalpie ist druckabhängig, deshalb ist der Kurvenverlauf degressiv. In Abbildung 7.8b ist die prozentuale Massenzunahme dargestellt, die sich bei einer vollständigen Absorption der berechneten Dampfmenge infolge von Kondensation ergibt. Die Massenzunahme spiegelt sich in der Holzfeuchte wider und wird im weiteren Verlauf der Ergebnisdarstellung herangezogen. Die Annahme von isobaren thermischen Zustandsgrößen entspricht einem kontinuierlichen Prozess und wurde näherungsweise auf den diskontinuierlichen labortechnischen Prozess angewendet. Ferner wird angenommen, dass sich der lineare Verlauf der spezifischen Wärmekapazität der trockenen Holzsubsubstanz zwischen $0^{\circ} \mathrm{C}$ und $100^{\circ} \mathrm{C}$, wie von Radmanović et al. (2014) dargestellt, auch über $100^{\circ} \mathrm{C}$ fortsetzt. Von Interesse ist, ob die zur

a

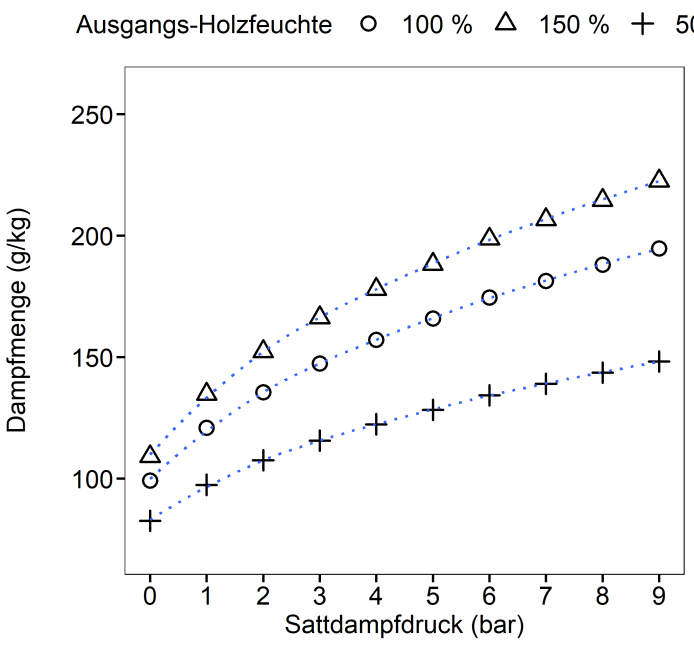

b
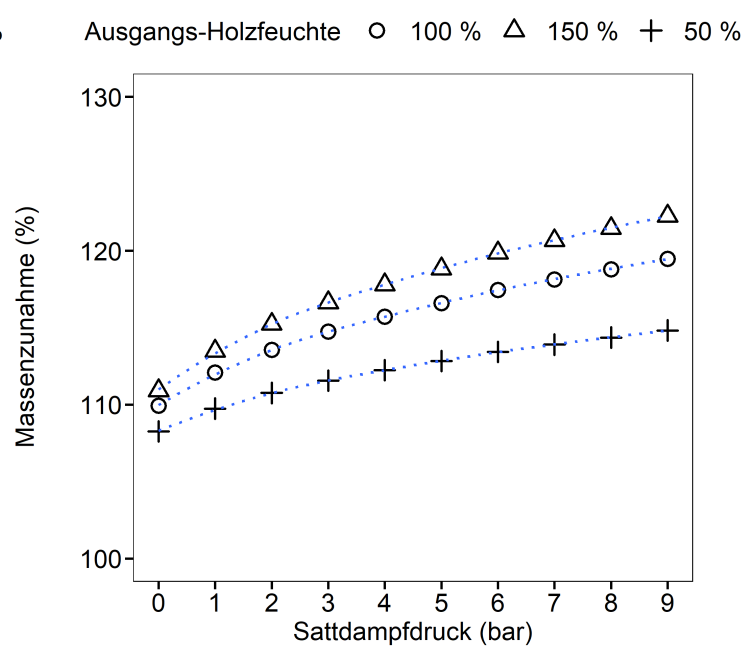

Abbildung 7.8: Theoretische Betrachtungen zur Holzfeuchte in Sattdampfatmosphäre: a, Darstellung der Dampfmenge um $1 \mathrm{~kg}$ Holz mit einer Ausgangs-Holzfeuchte von 50\%, $100 \%$ und $150 \%$ auf die Temperatur des entsprechenden Sattdampfdrucks zu erwärmen. $\boldsymbol{b}$, Darstellung der Massenzunahme durch die vollständige Absorption der in a berechneten Dampfmenge. Annahmen: isobare Zustandsgrößen, $c_{H}=1,4 \mathrm{~kJ} /(\mathrm{kg} \cdot \mathrm{K})$ (Radmanović et al. 2014), $c_{W}=4,2 \mathrm{~kJ} /(\mathrm{kg} \cdot \mathrm{K}), T_{0}=20^{\circ} \mathrm{C}$, Sättigungstabelle für Wasser aus VDI e.V. (2013).

Erwärmung der Hackschnitzel notwendige Dampfmenge tatsächlich absorbiert wird. Deshalb wird in Abbildung 7.9 die in situ ermittelte Dampfmenge durch Massenzunahme infolge von Absorption des kondensierten Sattdampfs dargestellt. Damit kann der Versuch unternommen werden, den Verlauf mit der berechneten Dampfmenge nach Gleichung 7.3 zu vergleichen.

$$
m_{D}=\frac{\left(m_{s}-m_{i}\right) \cdot 1000 \mathrm{~g} / \mathrm{kg}}{m_{i}}
$$


$m_{D} \quad$ Dampfmenge in $\mathrm{g} / \mathrm{kg}$

$m_{s} \quad$ Masse der Hackschnitzel in Sattdampfatmosphäre

$m_{i} \quad$ Einwaage der Hackschnitzel mit der Ausgangs-Holzfeuchte

In diesem Vergleich wird deutlich, dass die in situ ermittelten Werte für die Dampfmenge im Mittel mit dem berechneten Verlauf qualitativ assoziiert sind. Der quantitative Vergleich bei einem Sattdampfdruck von 1 bar und 9 bar zeigt, dass die in situ Werte innerhalb der berechneten Grenzwerte mit einer Ausgangs-Holzfeuchte von $50 \%$ und $150 \%$ liegen. Dies ist ein erstes Indiz dafür, dass die Menge an Sattdampf von den Hackschnitzeln absorbiert wird, die der übertragenen Wärmemenge entspricht. In der Abbildung wird außerdem eine breite Streuung der Messwerte, insbesondere bei der Holzart Buche sichtbar. Dies ist der Ablesegenauigkeit der mechanischen Waage geschuldet. Eine höhere Zahl an Wiederholungen würde die Lage der Mittelwerte im Sinne eines glatten Verlaufs einer Ausgleichskurve wie in Abbildung 7.8 vermutlich ermöglichen.

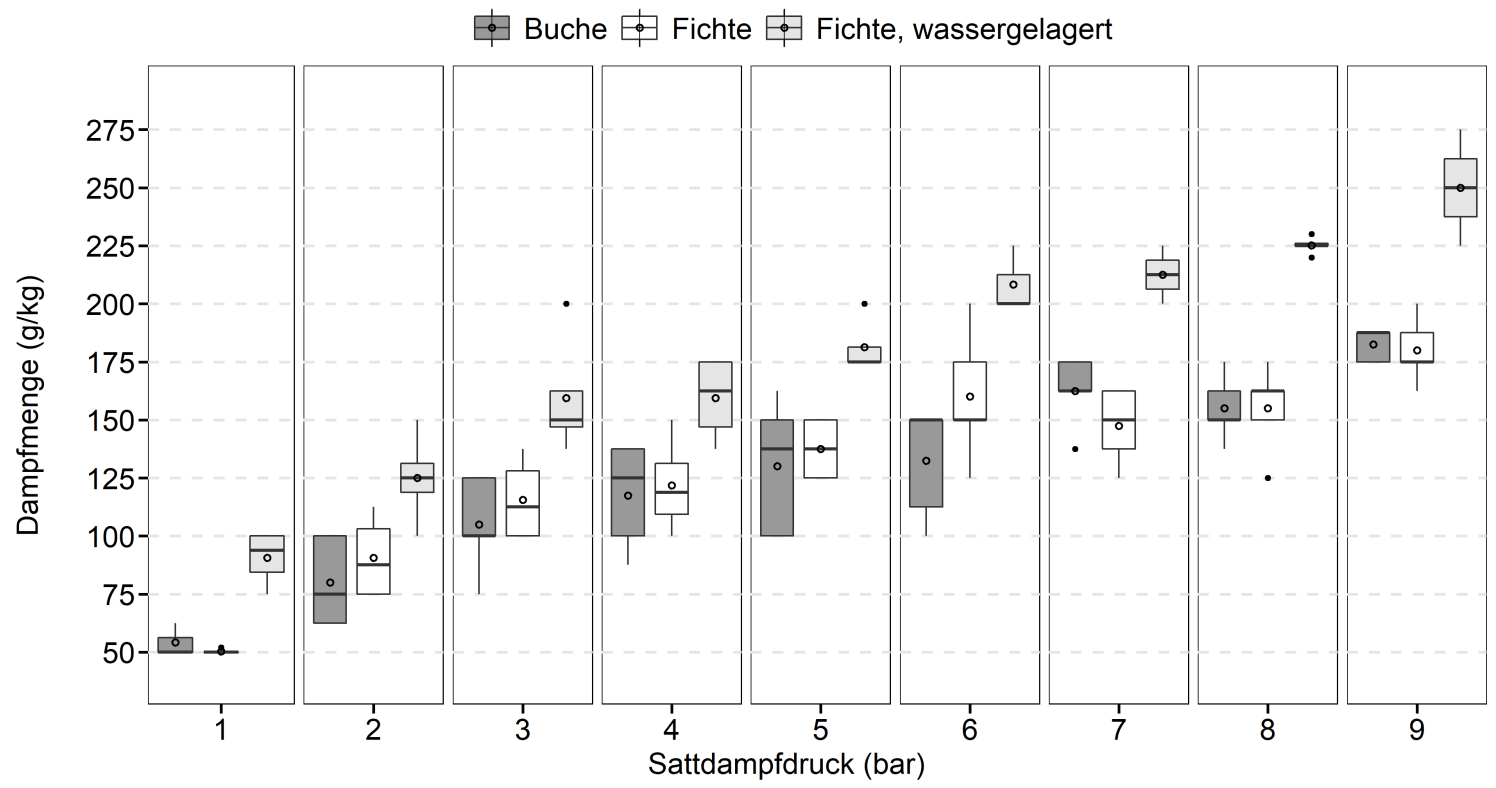

Abbildung 7.9: Darstellung der in situ Dampfmenge durch Massenzunahme infolge absorbierten Sattdampfs. Untersuchung an Buchen- und Fichtenholz-Hackschnitzeln mit unterschiedlicher Ausgangs-Holzfeuchte. Die Druckstufen wurden unabhängig voneinander untersucht. $n=5$.

Die Erwärmung der Hackschnitzel in Sattdampfatmosphäre geht mit einer Zunahme der Masse einher, die in Abbildung 7.10 dargestellt ist. Die Massenzunahme wurde nach Gleichung 7.4 berechnet.

$$
m=\frac{m_{s}}{m_{i}} \cdot 100
$$

$m \quad$ Massenzunahme in \%

$m_{s} \quad$ Masse der Hackschnitzel in Sattdampfatmosphäre

$m_{i} \quad$ Einwaage der Hackschnitzel mit Ausgangs-Holzfeuchte 
Mit steigendem Sattdampfdruck nimmt die Masse der Hackschnitzel wie bereits gezeigt zu. Die Unterschiede zwischen den arithmetischen Mittelwerten der einzelnen Holzarten und unterschiedlichen Ausgangs-Holzfeuchte differieren allerdings nicht signifikant. Die maximale Massenzunahme zwischen 1 bar und 9 bar liegt bei rund $25 \%$, ähnlich wie in Abbildung 7.8b. Weiterhin spricht die Massenzunahme in Verbindung mit dem degressiven Verlauf ebenfalls für einen Zusammenhang zwischen notwendiger Dampfmenge zur Erwärmung und absorbiertem Kondensat. Die ermittelte Massenzunahme liegt rund $10 \%$ bis $20 \%$ niedriger als die in den Untersuchungen von Ishikawa et al. (2004) im Bereich der hygroskopischen Ausgleichsfeuchte ermittelten Massenzunahme (Abschnitt 7.2). Diese Untersuchungen wurden an Sicheltanne (Cryptomeria japonica D. Don) mit einer Ausgangs-Holzfeuchte von $0 \%$ und einer Verweilzeit in Sattdampfatmosphäre von $1 \mathrm{~h}$ durchgeführt. In den damit erzielten Ergebnissen zeigt sich ebenfalls eine degressive Massenzunahme, ähnlich zu Abbildung 7.9. Die in Asplund (1940b) angegebene Massenzunahme von $140 \%$ bis $150 \%$ bei 8 bar liegt deutlich höher, als die in den vorliegenden Untersuchungen ermittelte Massenzunahme. Auch in dieser Abbildung 7.10 zeigt sich eine breite Streuung der Messwerte, bedingt durch den Versuchsaufbau und die verwendete Messtechnik.

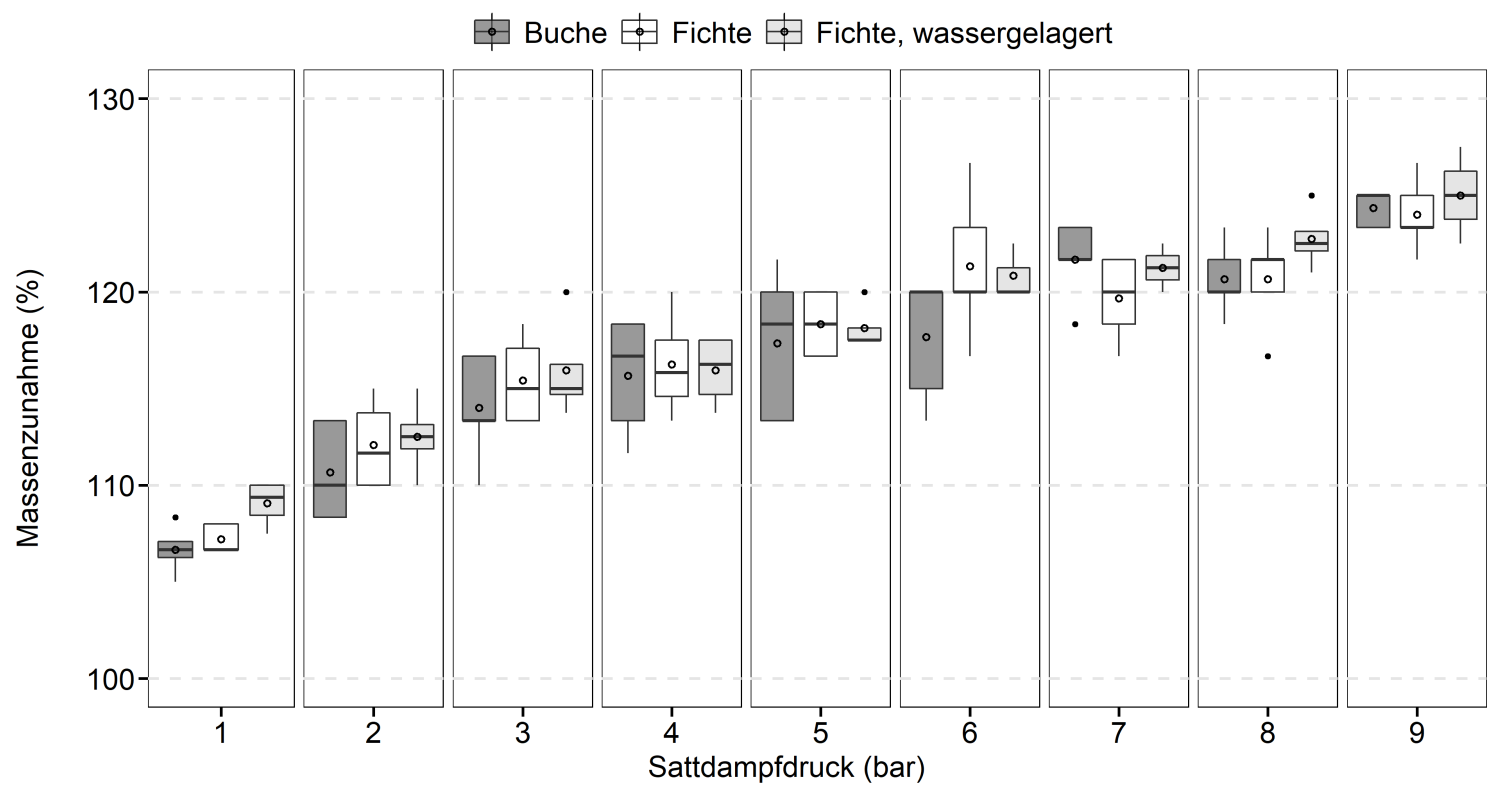

Abbildung 7.10: Änderung der Probenmasse unter Variation des Sattdampfdrucks. Untersuchung der Wasserdampf-Absorption von Buchen- und Fichtenholz-Hackschnitzeln mit unterschiedlicher AusgangsHolzfeuchte. Die Druckstufen wurden unabhängig voneinander untersucht. Dauer: 6 min, n=5.

Anhand des Untersuchungsmaterials wird in Abbildung 7.11 berechnete Dampfmenge für die Erwärmung der Hackschnitzel der mittleren tatsächlichen Massenzunahme gegenübergestellt. Im Rahmen der Messungenauigkeit wird der theoretische degressive Verlauf der Massenzunahme über dem Sattdampfdruck auch hier bestätigt. In den Abbildungen $7.11 \mathrm{a}$ und $\mathrm{b}$ fällt die niedrige Massenzunahme bei 1 bar auf. In Abbildung c liegt die gemessene Massenzunahme bei niedrigem Sattdampfdruck gleichermaßen niedrig. Ein Erklärungsversuch hierfür könnte sein, dass in diesen Fällen die dem Sattdampfdruck entsprechende Temperatur in den Hackschnitzeln nicht in dem untersuchten Zeitraum erreicht wurde. Nach Lashkov et al. (2001) wird flüssiges Wasser im kapillar-porösen System der Hackschnitzel mit einer Geschwindigkeit von 8,33 $10^{-6} \mathrm{~m} / \mathrm{s}$ transpor- 
tiert. Dieser, angesichts einer halben Hackschnitzeldicke von wenigen Millimetern, sehr hohen Geschwindigkeit wirkt der spezifische hydraulische Widerstand im inneren der Hackschnitzel entgegen. Zusätzlich kann die Absorption im kapillar-porösen System der Holzart Fichte durch Tüpfelverschluss und bei der Holzart Buche durch Verthyllung der Tracheen reduziert sein (Bonsen 1991). Dadurch dringt der Dampf oder das Kondensat nicht in den kompletten Porenanteil im Holz ein und die Wärmeübertragung wird verzögert. Die hohe Ausgangs-Holzfeuchte der wassergelagerten Fichtenholz-Hackschnitzel in Abbildung 7.11c trägt vermutlich durch freies Wasser im kapillar-porösen System auch zur Verzögerung bei der Massenzunahme bei. Das Phänomen wird anhand der Ergebnisse in Abschnitt 7.2.4 weiter diskutiert.

Bei dem bisher erarbeiteten Erkenntnisgewinn ist zu beachten, dass keine Korrektur für den Mas-

○ Masse Dampf, berechnet $\Delta$ Massenzunahme, gemessen
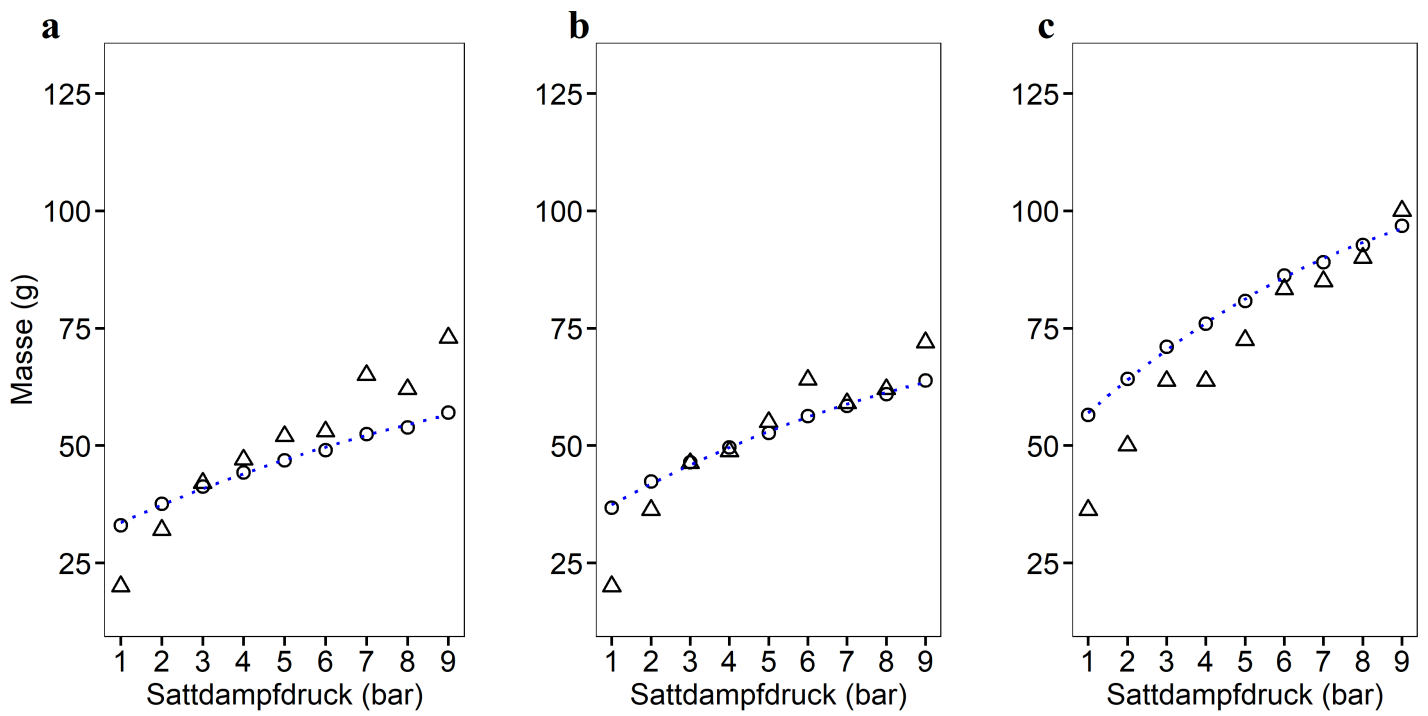

Abbildung 7.11: Vergleich der, aus den Versuchsergebnissen berechneten, theoretisch benötigten Dampfmenge zur Erwärmung der Hackschnitzel, mit der gemessenen Massenzunahme der Hackschnitzel infolge von Absorption von Dampf. a, Buchenholz-Hackschnitzel, Ausgangsholzfeuchte $48 \%$, Einwaage $300 \mathrm{~g}, n=5$. b. FichtenholzHackschnitzel, Ausgangsholzfeuchte $76 \%$, Einwaage $300 \mathrm{~g}, n=5$. c, Fichtenholz-Hackschnitzel, wassergelagert, Ausgangsholzfeuchte $135 \%$, Einwaage $400 \mathrm{~g}, n=5$.

senverlust infolge thermischer Zersetzung oder Abgabe flüchtiger organischer Verbindungen erfolgte, wie in Fengel (1966) oder Košík et al. (1968) beschrieben. Während die amorphen, quellbaren Bereiche der Zellulose in dem untersuchten Temperaturbereich relativ unverändert bleiben sollten, ist der Massenverlust hauptsächlich durch den Abbau von Hemizellulose bedingt (Sandermann \& Augustin 1964; Gellerstedt 2009, S. 50). Dieses Phänomen tritt bei hoher Temperatur in Verbindung mit hoher Holzfeuchte verstärkt auf und kann zur Reduzierung der Sorptionsfähigkeit im TMP-Verfahren beitragen (Lenth \& Kamke 2001). Die Effekte können grundsätzlich zu dem degressiven Verlauf der Massenzunahme ab ca. 3 bar Sattdampfdruck beitragen (Engelhardt 1979; Ishikawa et al. 2004).

Unter inerten Bedingungen beträgt der Massenverlust nach Košík et al. (1968) bei der Holzart Buche (Fagus sylvatica) infolge einer Temperatursteigerungsrate von $5{ }^{\circ} \mathrm{C}$ in der Minute ca. $8 \%$ bei $150^{\circ} \mathrm{C}$ und ca. $10 \%$ bei $20{ }^{\circ} \mathrm{C}$. Für die Holzart Fichte (Picea abies) wurde von Fengel (1966) 
ein Massenverlust infolge einer Heizzeit von $24 \mathrm{~h}$ von ca. $2 \%$ bei $120^{\circ} \mathrm{C}$, ca. $7 \%$ bei $150{ }^{\circ} \mathrm{C}$ und ca. $15 \%$ bei $200^{\circ} \mathrm{C}$ festgestellt. Der Massenverlust steigt bei beiden Holzarten mit zunehmender Temperatur exponentiell an.

Im TMP-Verfahren mit einer Sattdampftemperatur von $183^{\circ} \mathrm{C}$ liegt der Massenverlust bei der Holzart Kiefer nach 2 min bei ca. $6 \%$, nach 4 min bei ca. $9 \%$ und nach nach 6 min bei ca. $11 \%$ (Asplund 1949). Hierbei muss berücksichtigt werden, dass die Holzart Kiefer den höchsten Extraktstoffgehalt unter den einheimischen Holzarten aufweist (Björklund \& Nilvebrant 2009). Aktuellere Untersuchungen durch Ishikawa et al. (2004) am Kernholz der Sicheltanne (Cryptomeria japonica) in Sattdampfatmosphäre ergaben Massenverluste von ca. $0 \%$ bei $120^{\circ} \mathrm{C}$, ca. $2 \%$ bei $140{ }^{\circ} \mathrm{C}$ und ca. $5 \%$ bei $160^{\circ} \mathrm{C}$ bei einer Verweilzeit von $1 \mathrm{~h}$. Kernholz enthält generell einen höheren Anteil an Extraktstoffen als das Splintholz (Hillis 1971). Die Ausbeuteverluste liegen aufgrund der thermischen Beanspruchung des Holzes im RMP-Verfahren zwischen $4 \%$ und $6 \%$ sowie im TMPVerfahren zwischen $6 \%$ und $9 \%$ (Belgacem \& Pizzi 2016, S. 173).

Aus der ermittelten Massenzunahme wird die gemessene Holzfeuchte $u_{g}$ ohne thermische Massenverluste oder reduzierte Sorptionsfähigkeit nach Gleichung 7.5 berechnet.

$$
u_{g}=\frac{m_{s}-m_{0}}{m_{0}} \cdot 100
$$

$u_{g} \quad$ gemessene Holzfeuchte in Sattdampfatmosphäre in \%

$m_{s} \quad$ Feuchte Masse der Hackschnitzel in Sattdampfatmosphäre

$m_{0} \quad$ Trockenmasse der Hackschnitzel

Aus der Darstellung der Ergebnisse in Abbildung 7.12 wird deutlich, dass die Massenzunahme durch Wasserdampf-Absorption eine Zunahme der gemessenen Holzfeuchte mit steigendem Sattdampfdruck mit sich bringt. Bemerkenswert ist, dass die wassergelagerten FichtenholzHackschnitzel mit einer Ausgangs-Holzfeuchte von 135\% eine Erhöhung der gemessenen Holzfeuchte um ca. $40 \%$ aufweisen. Der kondensierte Wasserdampf führt hier zu einer Holzfeuchte oberhalb der maximalen Wassersättigung (Stamm 1964b, S. 145, 153-154). Die Fichten- und Buchenholz-Hackschnitzel mit niedrigerer Ausgangs-Holzfeuchte nähern sich mit steigendem Sattdampfdruck der maximalen Wassersättigung an.

Die Hackschnitzel mit geringerer Ausgangs-Holzfeuchte erreichen in der Sattdampfatmosphäre nach 6 min nicht das Niveau der wassergelagerten Fichtenholz-Hackschnitzel. Ob es sich bei der Massenzunahme, beziehungsweise der Steigerung der gemessenen Holzfeuchte lediglich um Oberflächen-Kondensation handelt, kann anhand der Beobachtung in Abbildung 7.13 diskutiert werden. Die hier dargestellte relative Änderung der gemessenen Holzfeuchte $u_{r}$ (ohne thermische Massenverluste oder reduzierte Sorptionsfähigkeit) wurde folgenderweise berechnet:

$$
u_{r}=\frac{u_{s}}{u_{i}}
$$

$$
\begin{array}{ll}
u_{r} & \text { relative Holzfeuchteänderung in \% } \\
u_{g} & \text { gemessene Holzfeuchte in Sattdampfatmosphäre } \\
u_{i} & \text { Ausgangs-Holzfeuchte }
\end{array}
$$

Die relative Holzfeuchteänderung stellt das Verhältnis der gemessenen Holzfeuchte in Sattdampfatmosphäre $u_{s}$ und der Ausgangs-Holzfeuchte der Hackschnitzel $u_{i}$ dar. Daraus folgt, dass die Hackschnitzel mit geringerer Ausgangs-Holzfeuchte im Verhältnis mehr Wasserdampf absorbieren, als die Hackschnitzel mit höherer Ausgangs-Holzfeuchte. Das bereits in den Hackschnitzeln enthaltene Wasser begrenzt somit die Wasseraufnahme in Form der Differenz zwischen 


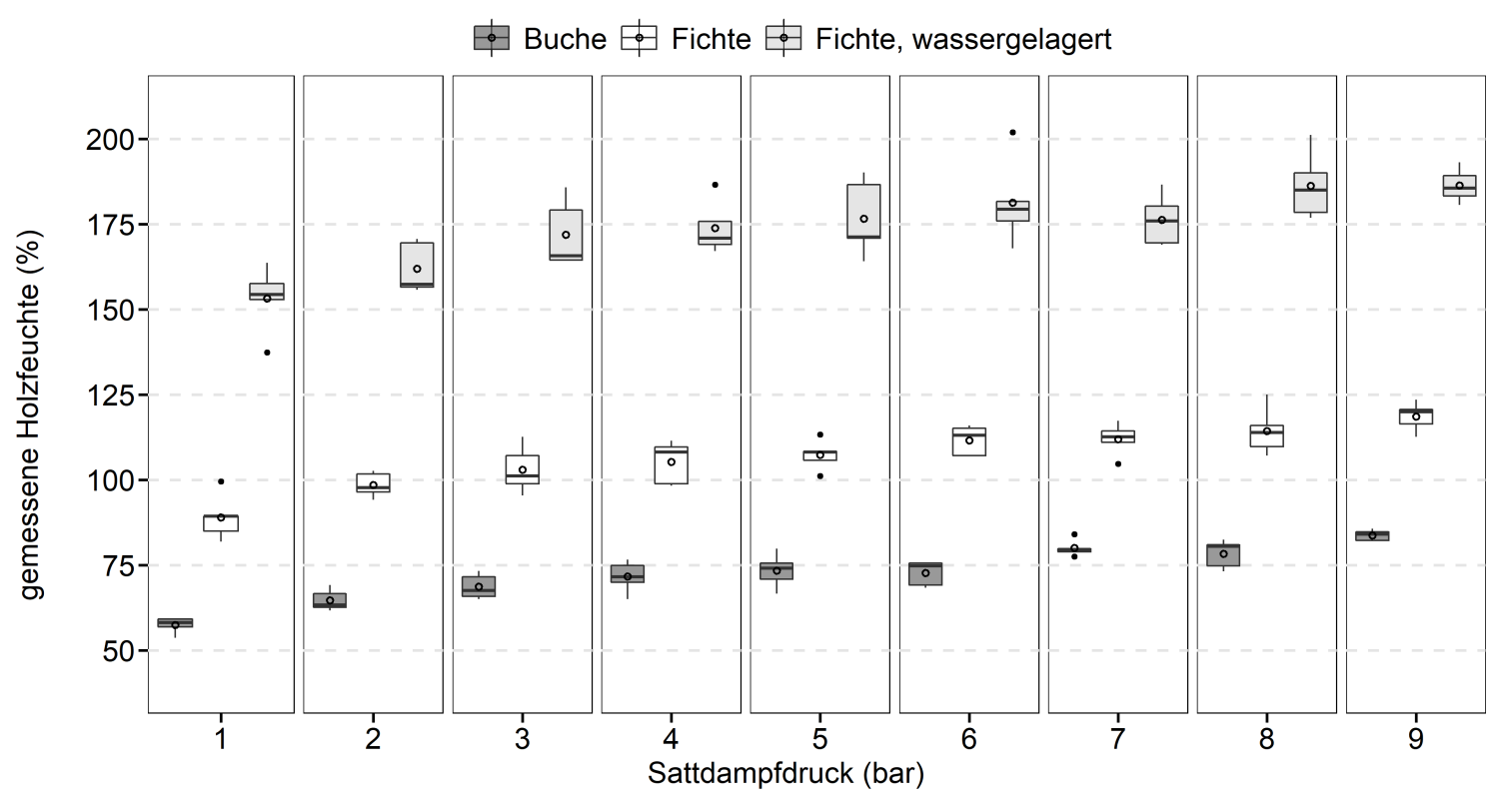

Abbildung 7.12: Änderung der gemessenen Holzfeuchte unter Variation des Sattdampfdrucks. Untersuchung der Wasserdampf-Absorption von Buchen- und Fichtenholz-Hackschnitzeln mit unterschiedlicher AusgangsHolzfeuchte. Die Druckstufen wurden unabhängig voneinander untersucht. Dauer: 6 min, n=5.

Ausgangs-Holzfeuchte und gemessener Holzfeuchte in Sattdampfatmosphäre. Das Phänomen der Massenzunahme kann nicht allein durch Kondensation auf der Oberfläche der Hackschnitzel, wie die Beobachtung in Abbildung 7.7a zeigt, erklärt werden. Die Massenzunahme muss auch durch Kondensation und Transport von Wasser im inneren der Hackschnitzel stattfinden, denn hier begrenzt die vorhandene Holzfeuchte die Massenzunahme. Es handelt sich deshalb bei dem untersuchten Phänomen um Absorption in Abgrenzung zu Adsorption im hygroskopischen Bereich der Holzfeuchte.

Hartler (1977) zufolge eignen sich auch Hackschnitzel mit einer Holzfeuchte unterhalb des Fasersättigungsbereichs für die Herstellung von Holzstoffen für die Papierherstellung im TMP-Verfahren. Voraussetzung dafür ist allerdings, dass die Hackschnitzel vor dem Kontakt mit den Mahlscheiben wassergesättigt sind. 


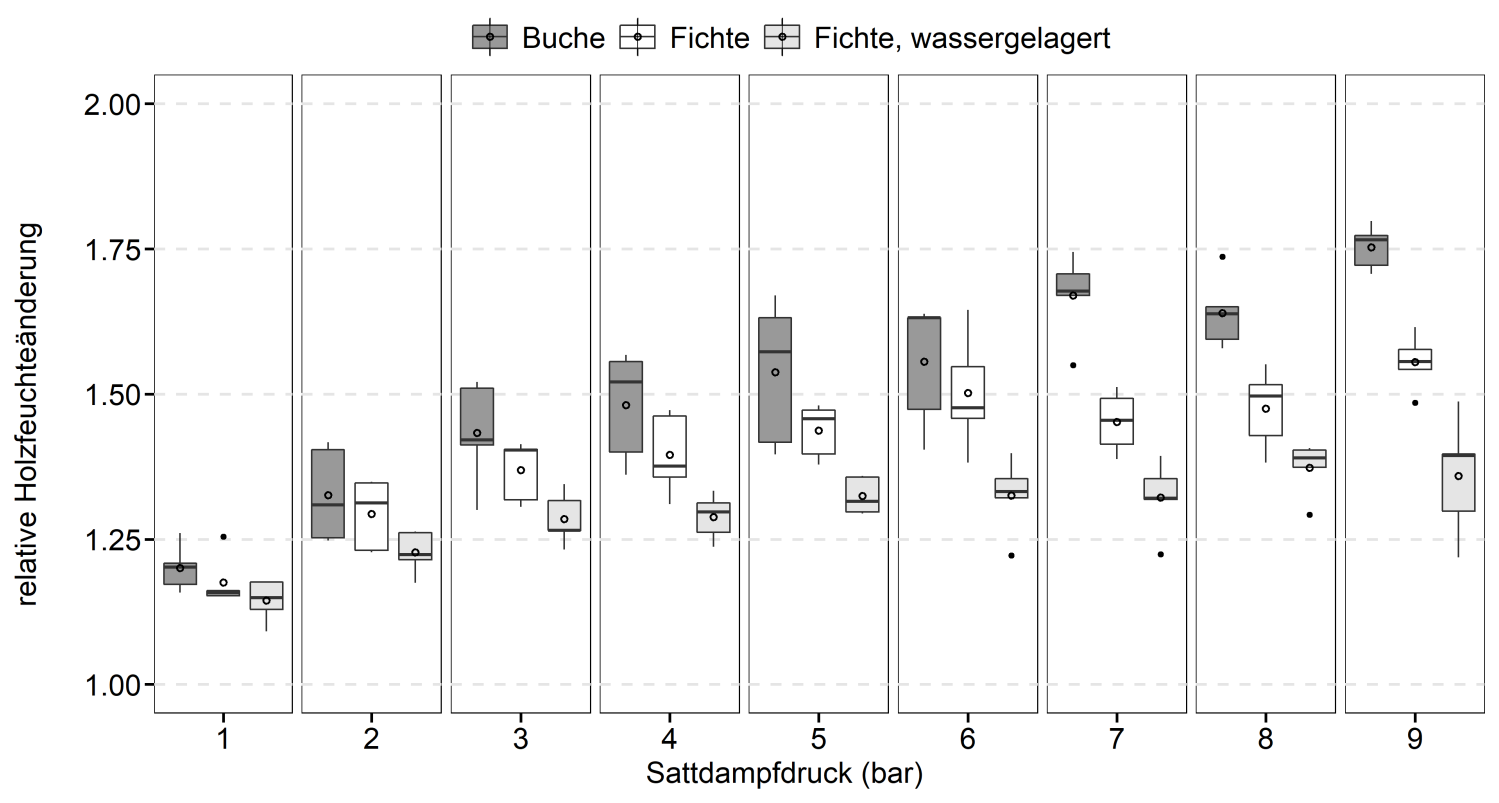

Abbildung 7.13: Relative Holzfeuchteänderung von Buchen- und Fichtenholzhackschnitzeln mit unterschiedlicher Ausgangs-Holzfeuchte im Verhältnis zur gemessenen Holzfeuchte unter Variation des Sattdampfdrucks. Die Druckstufen wurden unabhängig voneinander untersucht. Dauer: 6 min, $n=5$.

Die in situ Konsistenz der Hackschnitzel ist in Tabelle 7.5 aufgelistet. Auffällig ist die hohe Konsistenz der Fichten- und insbesondere der Buchenholz-Hackschnitzel mit niedriger AusgangsHolzfeuchte. Lediglich die wassergelagerten Fichtenholz-Hackschnitzel liegen im Bereich der von Kerekes (2015) bezüglich des RMP- und TMP-Verfahrens der Papierherstellung angegebenen Konsistenz von ca. 30 \%. Über den Einfluss einer hohen Konsistenz auf die spezifische elektrische Leistungsaufnahme sind keine Veröffentlichungen bekannt. Auch die daraus resultierende Beschaffenheit der Faserstoffe sowie deren Eignung für die Herstellung von Holzwerkstoffen wurde bisher nicht untersucht.

\begin{tabular}{|c|c|c|c|c|c|c|}
\hline \multirow{3}{*}{$\begin{array}{l}\text { Sattdampf- } \\
\text { druck }\end{array}$} & \multicolumn{6}{|c|}{ Konsistenz } \\
\hline & \multicolumn{2}{|c|}{ Buche } & \multicolumn{2}{|c|}{ Fichte } & \multicolumn{2}{|c|}{ Fichte, wassergelagert } \\
\hline & $\bar{x}$ & $\sigma$ & $\bar{x}$ & $\sigma$ & $\bar{x}$ & $\sigma$ \\
\hline bar & $\%$ & $\%$ & $\%$ & $\%$ & $\%$ & $\%$ \\
\hline 1 & 60 & 1,4 & 50 & 0,8 & 39 & 0,7 \\
\hline 2 & 57 & 1,0 & 48 & 1,0 & 38 & 1,0 \\
\hline 3 & 56 & 1,1 & 47 & 1,5 & 37 & 1,2 \\
\hline 4 & 55 & 1,3 & 46 & 1,2 & 37 & 0,9 \\
\hline 5 & 55 & 1,4 & 46 & 0,8 & 36 & 1,3 \\
\hline 6 & 55 & 1,0 & 45 & 1,1 & 36 & 1,4 \\
\hline 7 & 53 & 0,6 & 45 & 1,6 & 36 & 0,9 \\
\hline 8 & 53 & 1,1 & 44 & 1,3 & 35 & 1,1 \\
\hline 9 & 51 & 0,4 & 43 & 0,8 & 35 & 0,5 \\
\hline
\end{tabular}

Zur in situ Feuchtemessung von Hackschnitzeln in Sattdampfatmosphäre wurden im Rahmen des Promotionsvorhabens außerdem Untersuchungen mit der Methode der Near-Infrared- 
Spektroskopie (NIR-Spektroskopie) durchgeführt. Im NIR-Spektrum ist über die Änderung der Absorptionsintensitäten und Verschiebung der Peakbande von Wasser der Wassergehalt des reflektierenden Materials messbar (Ding et al. 2005). Die Ergebnisse aus diesen Untersuchungen wurden von den an den Untersuchungen beteiligten Unternehmen nicht zur Veröffentlichung freigegeben.

\subsubsection{Holzfeuchte nach Entspannung des Sattdampfdrucks}

Der Begriff Desorption ist im Zusammenhang mit der Ausgleichs-Holzfeuchte im hygroskopischen Bereich unter atmosphärischen Bedingungen und relativer Luftfeuchte definiert als die Umkehr der Adsorption (Stamm 1964c, S. 133). In dem vorliegenden Fall wird der Begriff Desorption allgemeiner verwendet, um das Verdampfen von freiem und gebundenem Wasser aus den Hackschnitzeln und Faserstoffen zu beschreiben. Schließlich ist nicht geklärt, wie der Sattdampfdruck und erhöhte Temperaturen die Ausgleichs-Holzfeuchte beeinflussen. In Stamm (1964c, S. 152-154) finden sich Hinweise darauf, dass die Ausgleichs-Holzfeuchte durch thermische Beanspruchung höher liegen kann.

Die gemessene Holzfeuchte nach Entspannung des Sattdampfdrucks $u_{d}$ wurde nach Gleichung 7.6 ohne thermische Massenverluste oder reduzierte Sorptionsfähigkeit berechnet. Auf die Faserstofferzeugung folgt im TMP- und RMP-Verfahren folgt der Prozessschritt der Trocknung. Dabei nimmt der Sattdampfdruck nach dem Refiner über die Blowline in den Stromrohrtrockner bis auf den atmosphärischen Druck ab. Die Desorption von Wasser nach Entspannung des Sattdampfdrucks ist deshalb auch bezüglich des thermischen Energiebedarfs in der Faserstoff-Trocknung von Interesse.

$$
u_{d}=\frac{m_{d, 0}}{m_{0}} \cdot 100
$$

$u_{d} \quad$ Holzfeuchte nach Entspannung des Sattdampfdrucks \%

$m_{d} \quad$ Feuchte Masse nach Entspannung des Sattdampfdrucks

$m_{0} \quad$ Trockenmasse

Hierbei ist festzuhalten, dass die Holzfeuchte nach Entspannung des Sattdampfdrucks unabhängig von der Höhe des Sattdampfdrucks vor der Entspannung ist, wie Abbildung 7.14 veranschaulicht. Dies kann daran festgemacht werden, dass die gemessene Holzfeuchte über alle Druckstufen im Rahmen der Messungenauigkeit die selben Werte annimmt. Die gemessene Holzfeuchte nach Entspannung des Sattdampfdrucks liegt dabei deutlich höher als die AusgangsHolzfeuchte der Hackschnitzel. Das von den Hackschnitzeln absorbierte Wasser verdampft folglich nicht wieder vollständig. Dieser Effekt könnte mit der thermischen Behandlung der Hackschnitzel in der Sattdampfatmosphäre zusammenhängen. Der steile Anstieg der Sorptionsisothermen der Ausgleichsfeuchte bei hohem relativem Dampfdruck (rel. Luftfeuchte) deutet darauf hin, dass die Ultrastruktur des Holzes hierbei für die Wasserdampfadsorption zugänglicher wird. Der thermische Einfluss des Sattdampfs kann diesen Effekt verstärken, indem beispielsweise Hemizellulosen abgebaut und Hydroxylgruppen frei werden.

Weiterhin kann die Beobachtung aus Abbildung 7.6 c, dass die Holzfeuchte der Hackschnitzel vor dem Aufschluss einen maßgeblichen Effekt auf die Holzfeuchte der Fasern nach dem Aufschluss zu haben scheint, bestätigt werden. Inwiefern die gemessene Holzfeuchte nach Entspannung des Sattdampfdrucks mit der tatsächlichen Feuchte der Faserstoffe nach dem Aufschluss zusammenhängt bleibt offen. Diese Thematik wird in Abschnitt 7.4 weiter untersucht. 


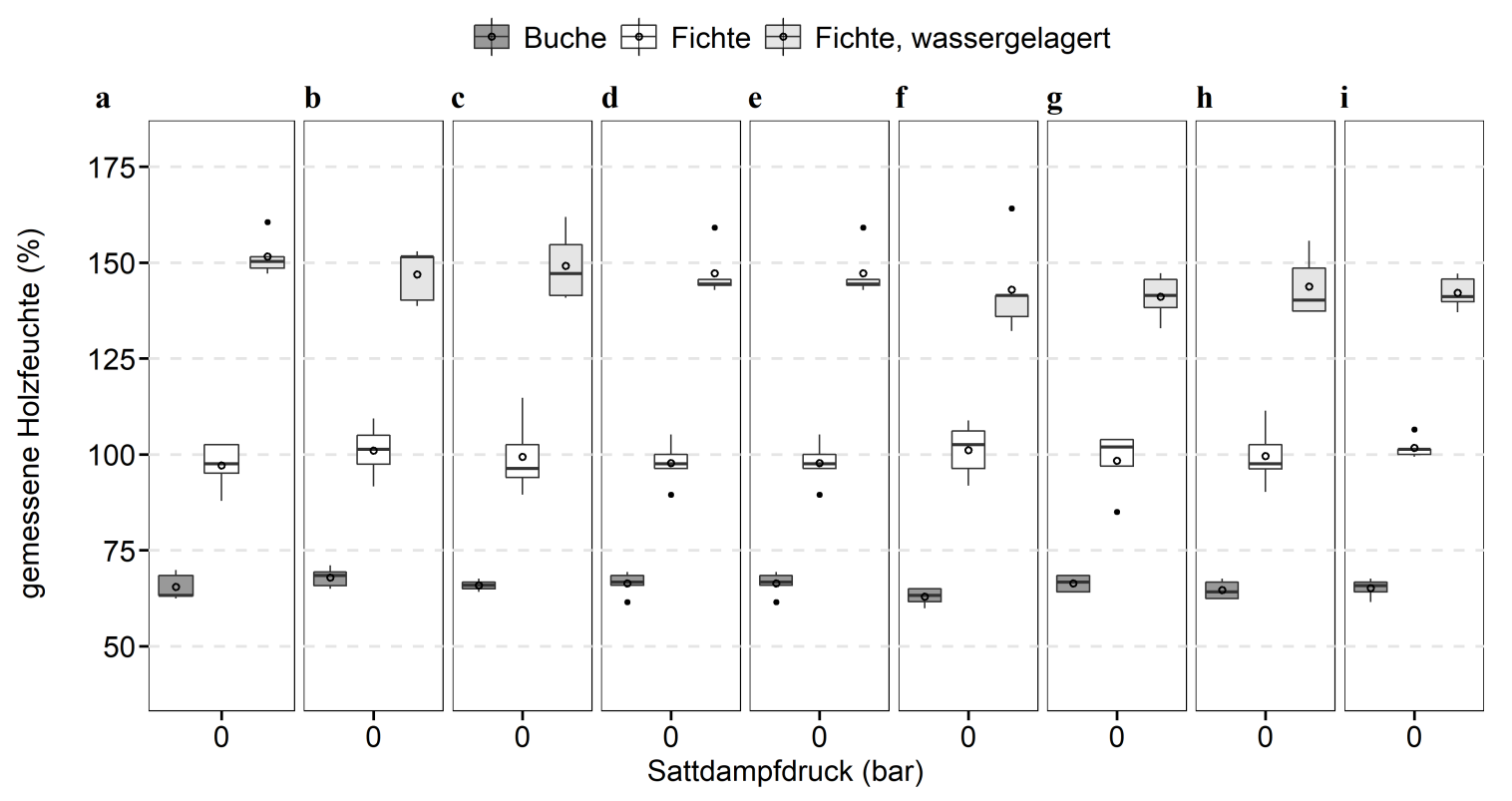

Abbildung 7.14: Gemessene Holzfeuchte infolge der Desorption von Buchen- und Fichtenholz-Hackschnitzeln mit unterschiedlicher Ausgangs-Holzfeuchte nach Entspannung des Sattdampfdrucks. Die Druckstufen wurden unabhängig voneinander untersucht. Der Sattdampfdruck vor der Entspannung betrug: a, 1 bar; b, 2 bar; , 3 bar; $\boldsymbol{d}, 4$ bar; e, 5 bar; $\boldsymbol{f}, 6$ bar; $\mathbf{g}, 7$ bar; $\boldsymbol{h}, 8$ bar; $\boldsymbol{i}, 9$ bar; $n=5$.

\subsubsection{Zeitlicher Verlauf der Absorption und Desorption}

Aus dem in Abbildung 7.15 beispielhaft dargestellten zeitlichen Verlauf der Feuchtezunahme bei einem Sattdampfdruck von 9 bar lassen sich folgende Erkenntnisse ableiten. Die Massenzunahme und -abnahme erfolgen ohne messbare Verzögerung simultan mit dem Verlauf des Sattdampfdrucks. Die Massenzunahme flacht ab, wenn der Solldruck das Sattdampfs erreicht ist. $\mathrm{Ab}$ diesem Zeitpunkt absorbieren die Hackschnitzel keinen Wasserdampf mehr. Über den Zeitraum vom Erreichen des Solldrucks bis zur Entspannung des Sattdampfdrucks ist keine weitere $\mathrm{Zu}$ - oder Abnahme der gemessenen Holzfeuchte messbar, die nicht auf eine Druckschwankung zurückzuführen ist. Diese Druckschwankungen treten regelungs- und anlagenbedingt auf. Um die Genauigkeit der Untersuchungen zu verbessern, wäre ein kleineres Druckbehältervolumen sinnvoll. Weiterhin ist im Rahmen der Messungenauigkeit kein Massenverlust des Holzes feststellbar, der nicht durch zusätzliche Absorption ausgeglichen würde. Außerdem kann ein untergeordneter Einfluss der Kochzeit auf die Konsistenz, wie in Abbildung $7.2 \mathrm{c}$ dargestellt, hiermit bestätigt werden.

Die Erkenntnisse sind ein Hinweis darauf, dass die Erwärmung der Hackschnitzel innerhalb weniger Sekunden erfolgt, vorausgesetzt es steht eine ausreichende Menge an Sattdampf zur Verfügung. Die Geschwindigkeit kann auch mit dem hydrostatischen Druck des Sattdampfs erklärt werden, der alle zugänglichen Volumen im Holz simultan füllt. Mit zunehmender Dicke der Hackschnitzel steigt vermutlich der Anteil der Wärmeleitung zur Erwärmung des Kerns der Hackschnitzel, da der Dampf hier nicht penetrieren kann. Die Ausgangs-Partikelgröße aus Abschnitt 7.1.2 hat vermutlich einen erheblichen Einfluss auf die Geschwindigkeit der Erwärmung und damit letztendlich auch auf die Konsistenz vor dem Aufschluss. 

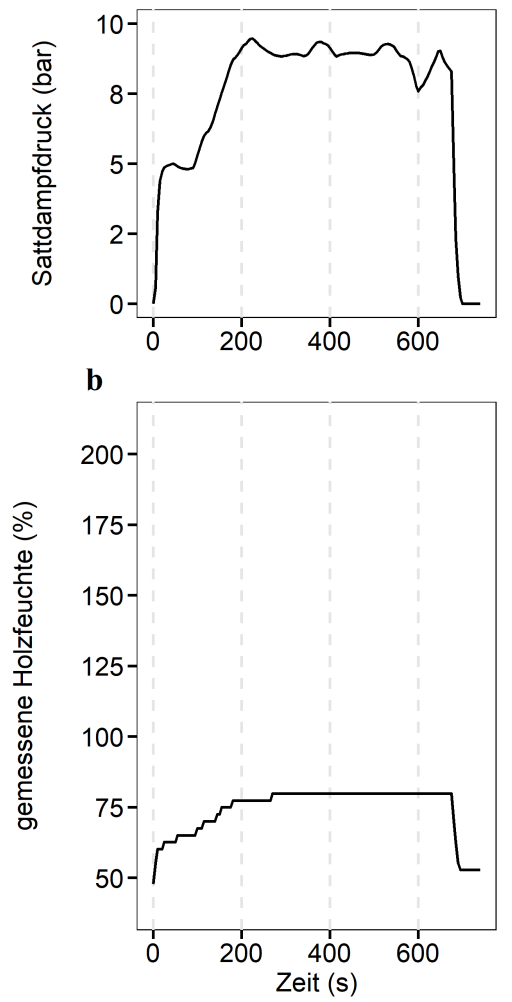

c

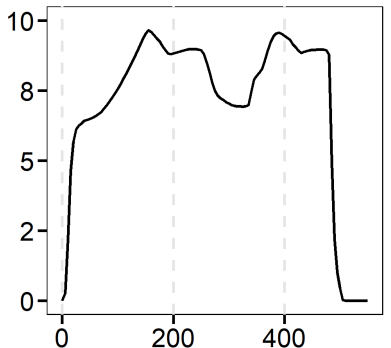

d

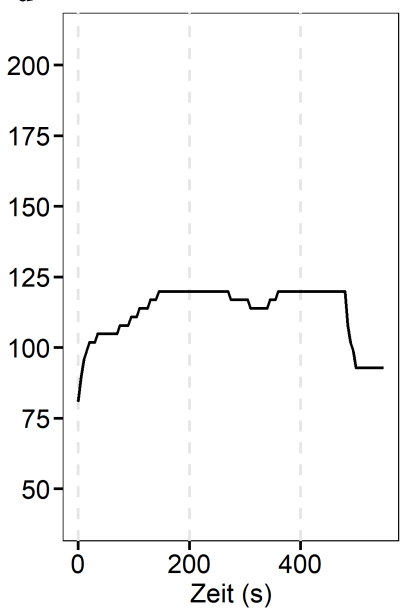

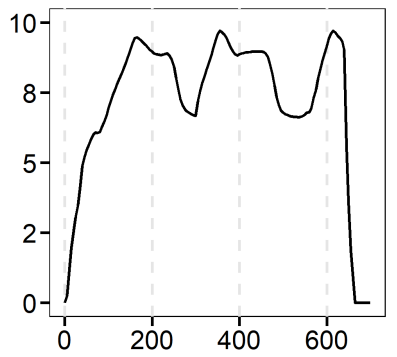

f

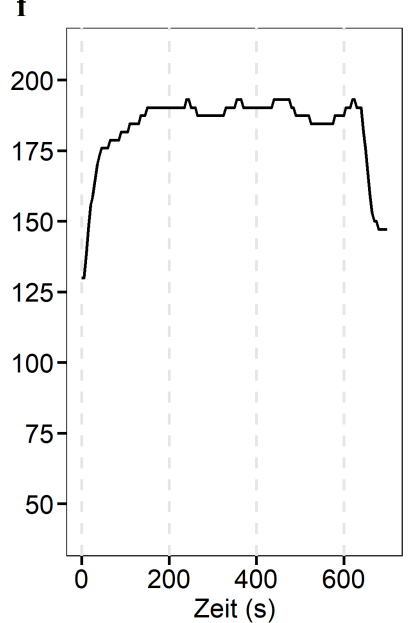

Abbildung 7.15: Zeitlicher Verlauf der Absorption und Desorption von Hackschnitzeln in Sattdampfatmosphäre. Zeitlicher Verlauf des Sattdampfdrucks (Sollwert 9 bar): a, Buchenholz-Hackschnitzel. c, FichtenholzHackschnitzel. e, wassergelagerte Fichtenholz-Hackschnitzel. Zeitlicher Verlauf der Ab- und Desorption: b, Buchenholz-Hackschnitzel. d, Fichtenholz-Hackschnitzel. f, wassergelagerte Fichtenholz-Hackschnitzel. $n=1$.

Rückblickend auf die in diesem Kapitel gesammelten Ergebnisse, muss davon ausgegangen werden, dass die Erwärmung der Hackschnitzel in ähnlicher Geschwindigkeit erfolgt, wie die Massenzunahme. Dabei sollte berücksichtigt werden, dass eine hohe Ausgangs-Holzfeuchte die Erwärmung verzögern kann. Weitere genauere und umfangreichere Untersuchungen zum Einfluss der Ausgangs-Holzfeuchte der Hackschnitzel auf die Hackschnitzelerwärmung, auf die Beschaffenheit der Faserstoffe und auf die spezifische elektrische Leistungsaufnahme sind notwendig. 


\subsection{Untersuchungen zur spezifischen elektrischen Leistungsaufnahme in der Faserstofferzeugung}

\subsubsection{Einleitung}

Aus der Papierherstellung sind zahlreiche wissenschaftliche Untersuchungen zu Mahlscheiben und der spezifischen elektrischen Leistungsaufnahme in der mechanischen Faserstofferzeugung im Zusammenhang mit Stoff- und Produkteigenschaften bekannt (u.a. Leask 1973; Miles \& May 1990; Muhic et al. 2011). In der MDF-Herstellung finden Mahlscheibentests in Zusammenarbeit mit den Mahlscheibenherstellern statt. Eine geringe Zahl an Untersuchungen zum Einsatz von Mahlscheiben in der MDF-Herstellung ist bekannt (Börste 2008; Roll 2010a,b; Ladenburger 2010; Gao 2014). Weitere Untersuchungen zur Faserstofferzeugung für die MDF-Herstellung beschränken sich auf Untersuchungen des Einflusses der Holzart und der Aufschlussbedingungen Sattdampfdruck bzw. Temperatur, Verweilzeit und Mahlspalt auf die Beschaffenheit der Faserstoffe und die Eigenschaften der daraus hergestellten MDF (u.a. Roffael et al. 1994a; Schneider et al. 2000; Neimsuwan et al. 2008; Ayrilmis et al. 2017; Benthien et al. 2014).

Im Zusammenhang mit Holzfaserdämmstoffen wurde das TMP-Verfahren bislang einmal wissenschaftlich untersucht (Brombacher 2015). Im Fokus der labortechnischen Untersuchungen standen hierbei die Auswirkungen der Holzarten Kiefer und Buche unter Variation der Aufschlussbedingungen Temperatur bzw. Sattdampfdruck, Verweilzeit und Mahlspalt auf die Wärmeleitfähigkeit der Faserstoffe (F. Michel et al. 2014; Brombacher et al. 2016). Das Ziel der vorliegenden Untersuchungen ist es deshalb, experimentell zu ermitteln, welchen Einfluss unterschiedliche Mahlscheibenmuster unter Variation der Sattdampftemperatur und der Holzarten Buche und Fichte auf die spezifische elektrische Leistungsaufnahme haben. Vor dem Hintergrund der Produktund Prozessoptimierung und der Senkung von Herstellungskosten ist dabei die Reduzierung des elektrischen und insbesondere des thermischen Energiebedarfs von Interesse. Deshalb wurde neben dem TMP-Verfahren auch die Machbarkeit des RMP-Verfahrens untersucht.

Den Untersuchungen zur spezifischen elektrischen Leistungsaufnahme in der Faserstofferzeugung gingen eine Reihe von Vorversuchen voraus, die der Methodenentwicklung für den Vergleich von Mahlscheibenmustern im labortechnischen Maßstab anhand der spezifischen elektrischen Leistungsaufnahme dienten. Als zielführend hat sich dabei herausgestellt, dass die Parameter Verweilzeit, Holzfeuchte, Durchsatz und vor allem der Mahlspalt als fixe Parameter und die Parameter Holzart, Mahlscheibenmuster und Sattdampftemperatur variabel untersucht werden (vgl. Abschnitt 7.1.4). Die Vorversuche machten außerdem deutlich, dass die TMP-Mahlscheibenmuster nicht für das RMP-Verfahren geeignet sind. Aus diesem Grund wurde ein spezielles Mahlscheibenmuster für das RMP-Verfahren entwickelt.

\subsubsection{Spezifische elektrische Leistungsaufnahme im TMP-Verfahren}

Im TMP-Verfahren wurden fünf Mahlscheibenmuster mit den Holzarten Fichte und Buche in jeweils drei Druck- bzw. Temperaturstufen untersucht. Die Prozessparameter der untersuchten Varianten sind in Tabelle 6.18 aufgelistet. Das Hauptergebnis ist das arithmetische Mittel der SEL aus den Wiederholungen der Aufschlussversuche. In Tabelle 7.6 ist ein Überblick über die Mittelwerte gegeben. Die ergänzend enthaltene Standardabweichung zeigt die starken Schwankungen der spezifischen elektrischen Leistungsaufnahme an, die im weiteren Verlauf der Ergebnisdarstellung vertiefend betrachtet werden. 
Tabelle 7.6: Auflistung des arithmetischen Mittels und der Standardabweichung der ermittelten spezifischen elektrischen Leistungsaufnahme (SEL) im TMP-Verfahren.

\begin{tabular}{|c|c|c|c|c|c|}
\hline Variante & Mahlscheibe & Holzart & Aufschlussbedingungen & $\begin{array}{l}\mathrm{SEL} \\
\bar{x} \\
\mathrm{Wh} / \mathrm{kg}_{\text {atro }}\end{array}$ & $\begin{array}{l}\sigma \\
\mathrm{Wh} / \mathrm{kg}_{\text {atro }}\end{array}$ \\
\hline 1 & 1 & Fichte & Sattdampf: 2,1 bar $\left(135^{\circ} \mathrm{C}\right)$ & 489 & 176 \\
\hline 2 & 1 & Fichte & Sattdampf: 3,8 bar $\left(150^{\circ} \mathrm{C}\right)$ & 261 & 195 \\
\hline 3 & 1 & Fichte & Sattdampf: 7,9 bar $\left(175^{\circ} \mathrm{C}\right)$ & 160 & 141 \\
\hline 4 & 1 & Buche & Sattdampf: 2,1 bar $\left(135^{\circ} \mathrm{C}\right)$ & 315 & 253 \\
\hline 5 & 1 & Buche & Sattdampf: 3,8 bar $\left(150^{\circ} \mathrm{C}\right)$ & 192 & 116 \\
\hline 6 & 1 & Buche & Sattdampf: 7,9 bar $\left(175^{\circ} \mathrm{C}\right)$ & 158 & 162 \\
\hline 7 & 2 & Fichte & Sattdampf: 2,1 bar $\left(135^{\circ} \mathrm{C}\right)$ & 493 & 416 \\
\hline 8 & 2 & Fichte & Sattdampf: 3,8 bar $\left(150^{\circ} \mathrm{C}\right)$ & 139 & 51 \\
\hline 9 & 2 & Fichte & Sattdampf: 7,9 bar $\left(175^{\circ} \mathrm{C}\right)$ & 112 & 57 \\
\hline 10 & 2 & Buche & Sattdampf: 2,1 bar $\left(135^{\circ} \mathrm{C}\right)$ & 150 & 63 \\
\hline 11 & 2 & Buche & Sattdampf: 3,8 bar $\left(150^{\circ} \mathrm{C}\right)$ & 138 & 62 \\
\hline 12 & 2 & Buche & Sattdampf: 7,9 bar $\left(175^{\circ} \mathrm{C}\right)$ & 97 & 61 \\
\hline 13 & 3 & Fichte & Sattdampf: 2,1 bar $\left(135^{\circ} \mathrm{C}\right)$ & 178 & 82 \\
\hline 14 & 3 & Fichte & Sattdampf: 3,8 bar $\left(150^{\circ} \mathrm{C}\right)$ & 124 & 43 \\
\hline 15 & 3 & Fichte & Sattdampf: 7,9 bar $\left(175^{\circ} \mathrm{C}\right)$ & 98 & 47 \\
\hline 16 & 3 & Buche & Sattdampf: 2,1 bar $\left(135^{\circ} \mathrm{C}\right)$ & 142 & 53 \\
\hline 17 & 3 & Buche & Sattdampf: 3,8 bar $\left(150^{\circ} \mathrm{C}\right)$ & 123 & 51 \\
\hline 18 & 3 & Buche & Sattdampf: 7,9 bar $\left(175^{\circ} \mathrm{C}\right)$ & 75 & 41 \\
\hline 19 & 4 & Fichte & Sattdampf: 2,1 bar $\left(135^{\circ} \mathrm{C}\right)$ & 526 & 453 \\
\hline 20 & 4 & Fichte & Sattdampf: 3,8 bar $\left(150^{\circ} \mathrm{C}\right)$ & 282 & 248 \\
\hline 21 & 4 & Fichte & Sattdampf: 7,9 bar $\left(175^{\circ} \mathrm{C}\right)$ & 136 & 121 \\
\hline 22 & 4 & Buche & Sattdampf: 2,1 bar $\left(135^{\circ} \mathrm{C}\right)$ & 218 & 135 \\
\hline 23 & 4 & Buche & Sattdampf: 3,8 bar $\left(150^{\circ} \mathrm{C}\right)$ & 124 & 61 \\
\hline 24 & 4 & Buche & Sattdampf: 7,9 bar $\left(175^{\circ} \mathrm{C}\right)$ & 101 & 70 \\
\hline 25 & 5 & Fichte & Sattdampf: 2,1 bar $\left(135^{\circ} \mathrm{C}\right)$ & 395 & 282 \\
\hline 26 & 5 & Fichte & Sattdampf: 3,8 bar $\left(150^{\circ} \mathrm{C}\right)$ & 157 & 121 \\
\hline 27 & 5 & Fichte & Sattdampf: 7,9 bar $\left(175^{\circ} \mathrm{C}\right)$ & 124 & 56 \\
\hline 28 & 5 & Buche & Sattdampf: 2,1 bar $\left(135^{\circ} \mathrm{C}\right)$ & 268 & 140 \\
\hline 29 & 5 & Buche & Sattdampf: 3,8 bar $\left(150^{\circ} \mathrm{C}\right)$ & 147 & 76 \\
\hline 30 & 5 & Buche & Sattdampf: 7,9 bar $\left(175^{\circ} \mathrm{C}\right)$ & 104 & 94 \\
\hline
\end{tabular}

${ }^{*}$ Manuelle Einstellung an der Ringskala der Mahlspaltverstellung 

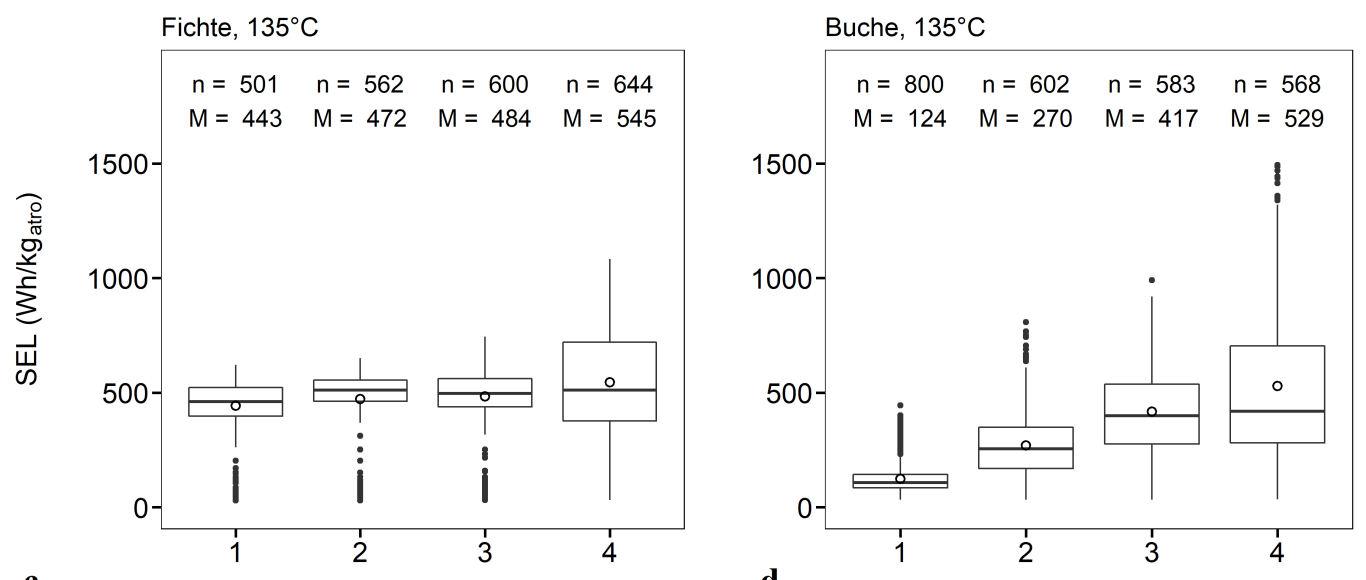

d
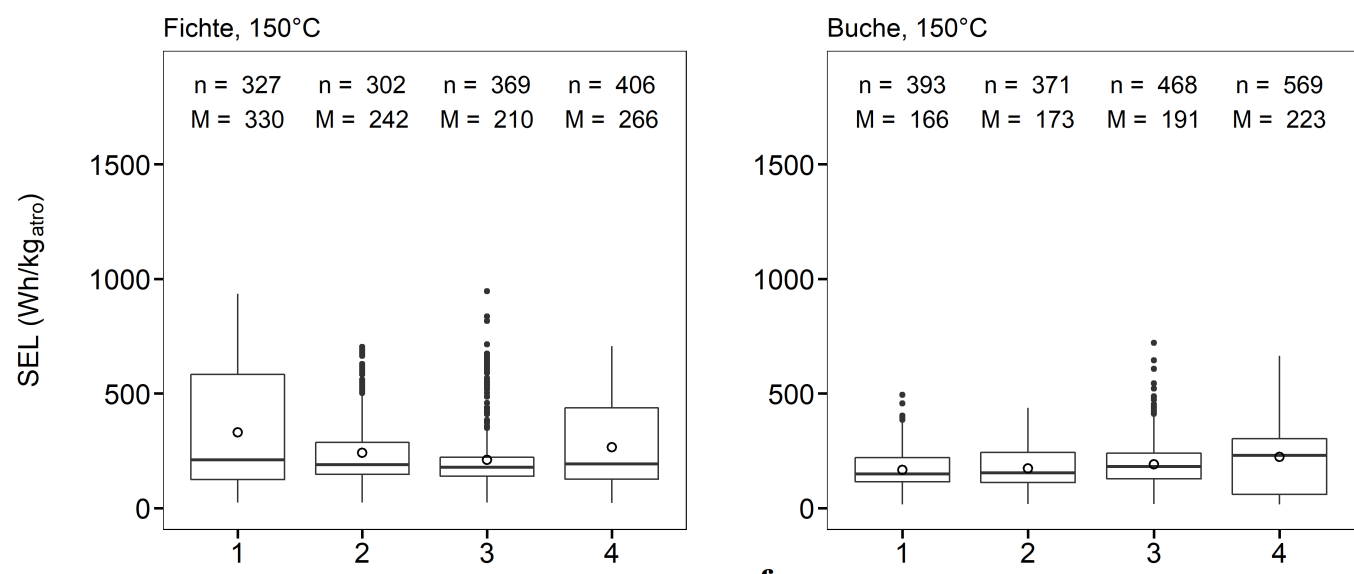

Fichte, $175^{\circ} \mathrm{C}$

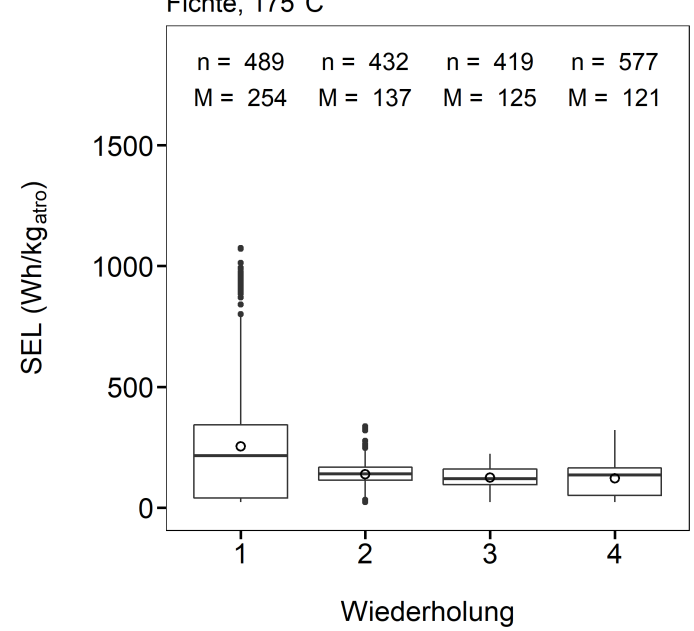

f

Buche, $175^{\circ} \mathrm{C}$

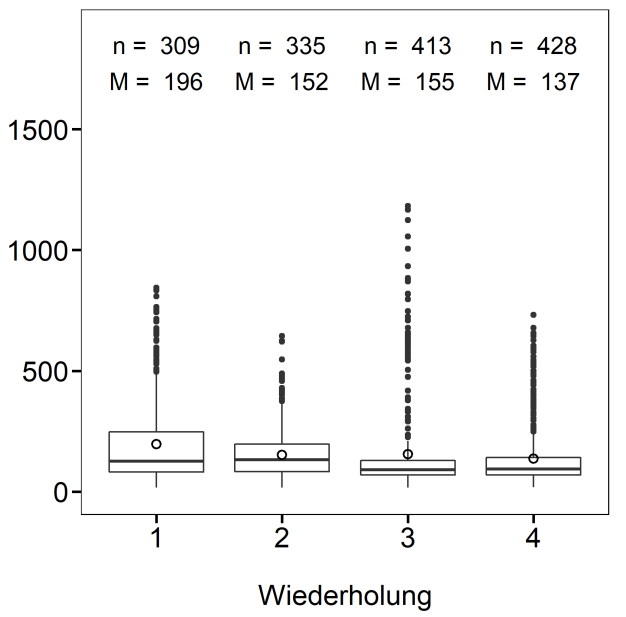

Abbildung 7.16: Darstellung der spezifischen elektrischen Leistungsaufnahme (SEL) aus den Wiederholungen mit dem Mahlscheibenmuster 1. a, c, e: In der linken Spalte sind die Wiederholungen mit der Holzart Fichte aufsteigend nach der Sattdampftemperatur dargestellt. $\boldsymbol{b}, \boldsymbol{d}, \boldsymbol{f}$ : In der rechten Spalte sind die Wiederholungen mit der Holzart Buche aufsteigend nach der Sattdampftemperatur dargestellt. n: Anzahl der Messpunkte, M: Arithmetisches Mittel der SEL. 


\section{Mahlscheibenmuster 1}

Bei dem Mahlscheibenmuster 1 handelt es sich um ein bidirektionales Design (Tabelle 6.2). Die Drehrichtung ist nicht vorgegeben. Bei Abrundung der Stege ist es deshalb möglich die Drehrichtung zu reversieren. Dieser Mahlscheibentyp ist heute noch in der LC-Mahlung der Papierherstellung und in seltenen Fällen in der MDF- oder Holzfaserdämmstoffherstellung anzutreffen. In der Holzwerkstoffherstellung gilt dieses Design als überholt. In Abbildung 7.16 sind die einzelnen Wiederholungen der Untersuchungen mit dem Mahlscheibenmuster dargestellt. Bei der Holzart Fichte fallen die hohe Leistungsaufnahme und die Ausreißer nach unten in Abbildung 7.16a auf. Darüber hinaus sind in Abbildung 7.16c die starken Leistungsschwankungen markant, die durch die Interquartilabstände (IQR), Whisker und Ausreißer angezeigt werden. Der hohe Mittelwert von Wiederholung 1 in Abbildung 7.16e ist ebenso wie die Unterschiede in Abbildung 7.16b vermutlich auf Bedienfehler in der Einstellung des Mahlspalts zurückzuführen. Ins Auge fallen zudem die Ausreißer und die hohe Leistungsaufnahme in Abbildung 7.16b.

$\mathbf{a}$

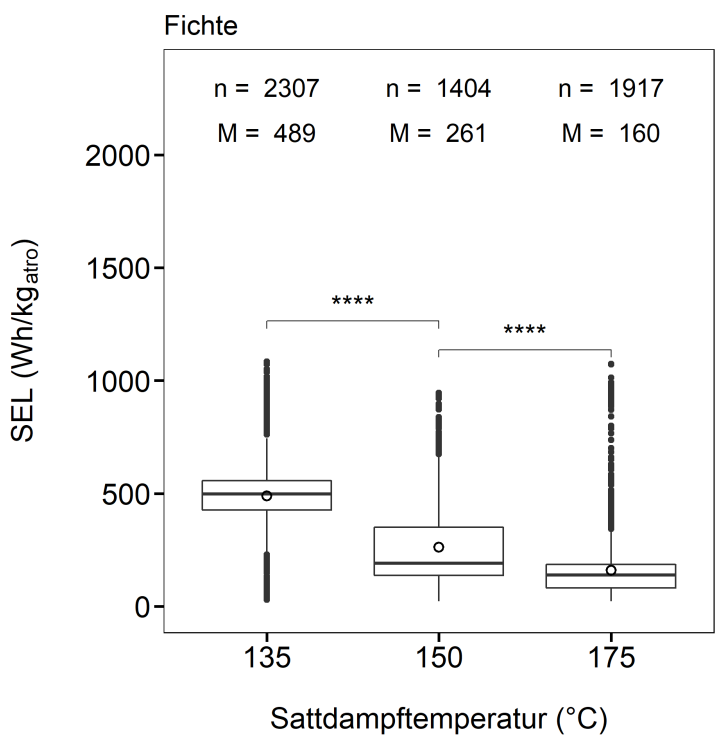

b

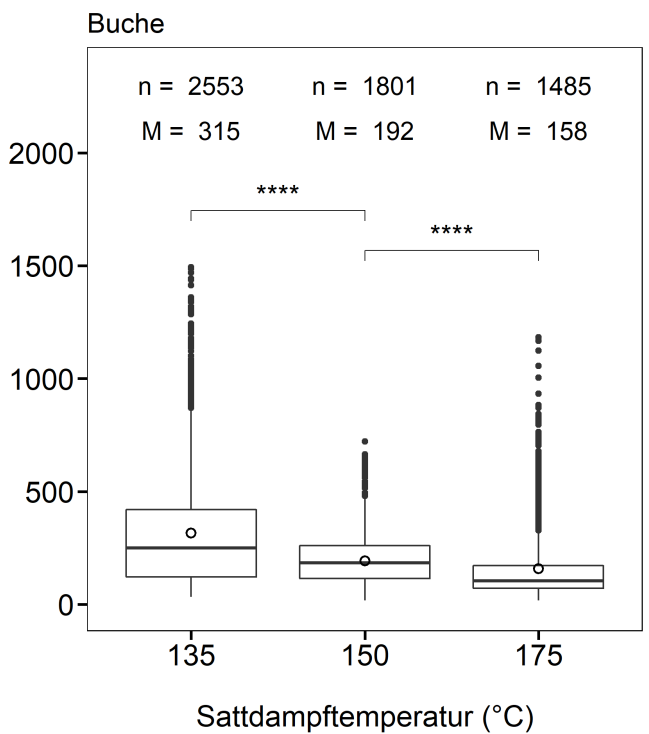

Abbildung 7.17: Darstellung des arithmetischen Mittelwerts der spezifischen elektrischen Leistungsaufnahme (SEL) über der Sattdampftemperatur aus den Versuchen mit dem Mahlscheibenmuster 1. a, Holzart Fichte. b, Holzart Buche. n: Anzahl der Messpunkte, M: Arithmetisches Mittel der SEL. N=4, Kruskall-Wallis-Test Signifikanzcode: $0,0001^{* * *} ; 0,001^{* * *} ; 0,01^{* *} ; 0,05^{*}$.

Der Vergleich des arithmetischen Mittels in Abbildung 7.17 zeigt den bereits beschriebenen Zusammenhang zwischen steigender Aufschlusstemperatur und abnehmender SEL. Auffällig ist bei der Sattdampftemperatur von $135^{\circ} \mathrm{C}$ die deutlich geringere mittlere Leistungsaufnahme bei der Holzart Buche (Abbildung 7.17b), bei gleichzeitig deutlich ausgeprägteren Leistungsspitzen im Vergleich zur Holzart Fichte (Abbildung 7.17a). Bei der Sattdampftemperatur von $175^{\circ} \mathrm{C}$ ist kein Unterschied zwischen den Holzarten festzustellen. 

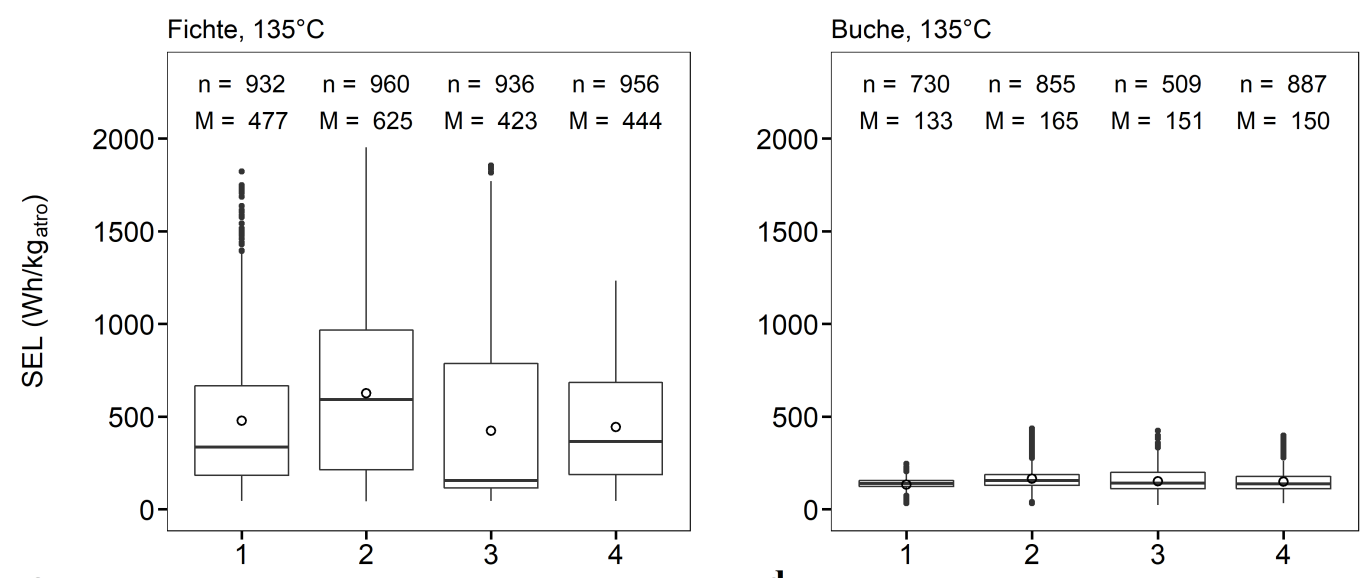

Fichte, $150^{\circ} \mathrm{C}$

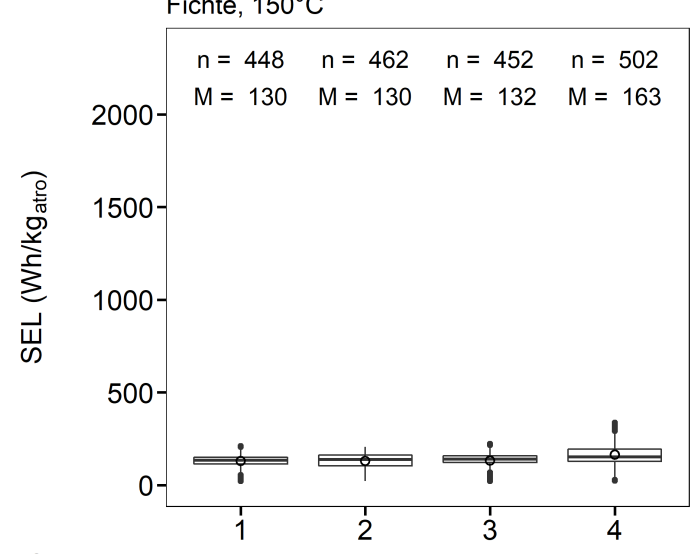

Buche, $150^{\circ} \mathrm{C}$

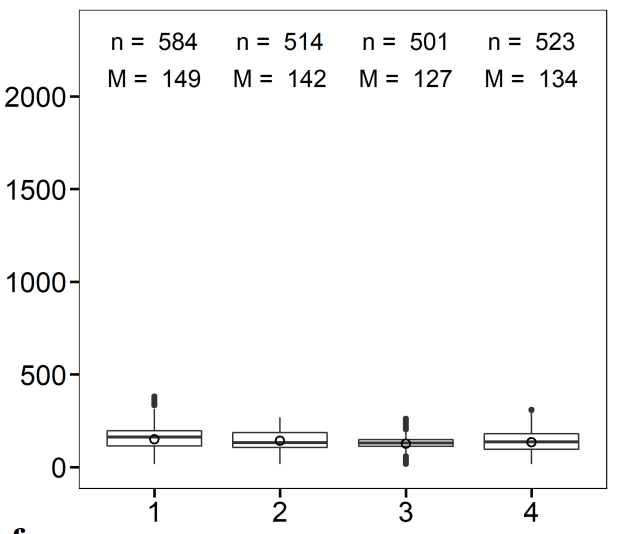

f

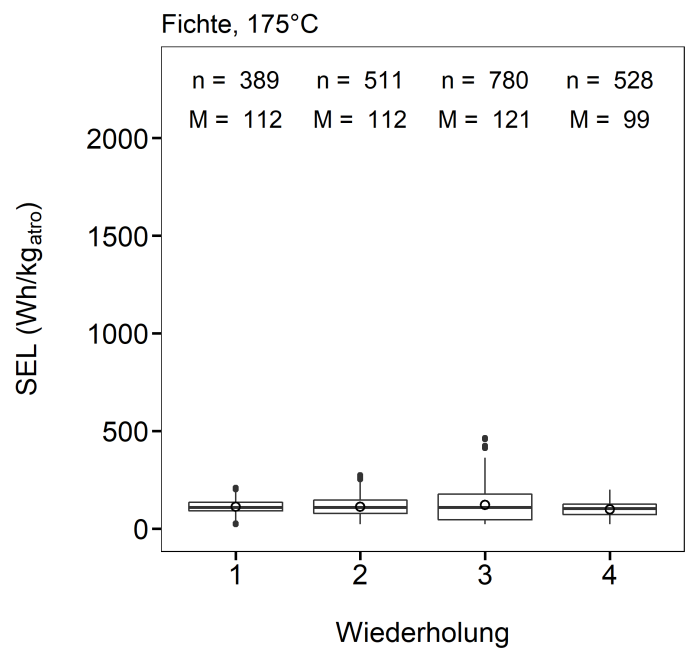

Abbildung 7.18: Darstellung der spezifischen elektrischen Leistungsaufnahme (SEL) aus den Wiederholungen mit dem Mahlscheibenmuster 2. a, c, e: In der linken Spalte sind die Wiederholungen mit der Holzart Fichte aufsteigend nach der Sattdampftemperatur dargestellt. $\boldsymbol{b}, \boldsymbol{d}, \boldsymbol{f}$ : In der rechten Spalte sind die Wiederholungen mit der Holzart Buche aufsteigend nach der Sattdampftemperatur dargestellt. n: Anzahl der Messpunkte, M: Arithmetisches Mittel der SEL. 


\section{Mahlscheibenmuster 2}

Charakteristisch für den Typ 2 (Tabelle 6.3) ist die in zwei Zonen geteilte Mahlbahn und der geringe Stegwinkel von $20^{\circ}$ bis $30^{\circ}$. Die innere Zone verfügt über eine relativ geringe Anzahl an Stegen und die Stegbreite ist mit $4 \mathrm{~mm}$ vergleichsweise breit. Ebenso wie die Nutbreite, die von innen nach außen von $4 \mathrm{~mm}$ auf $8 \mathrm{~mm}$ zunimmt. Die Dämme sind in Zone 2 tangential angeordnet. Es handelt sich um ein Spiraldesign mit geringer Krümmung der Stege. In der Darstellung der Wiederholungen in Abbildung 7.18 ist ein deutlicher Unterschied zwischen den Holzarten Fichte und Buche im Aufschluss bei einer Sattdampftemperatur von $135^{\circ} \mathrm{C}$ (Abbildung 7.18a und b) sichtbar. Die großen IQR und die Whisker bis $2000 \mathrm{Wh} / \mathrm{kg}_{\text {atro }}$ in Abbildung 7.18a weisen darauf hin, dass dieses Mahlscheibenmuster nicht für die Zerfaserung von Fichte bei niedrigen Sattdampftemperaturen geeignet ist. Die weiteren Wiederholungen zeigen ein recht einheitliches Bild mit niedriger SEL.

a

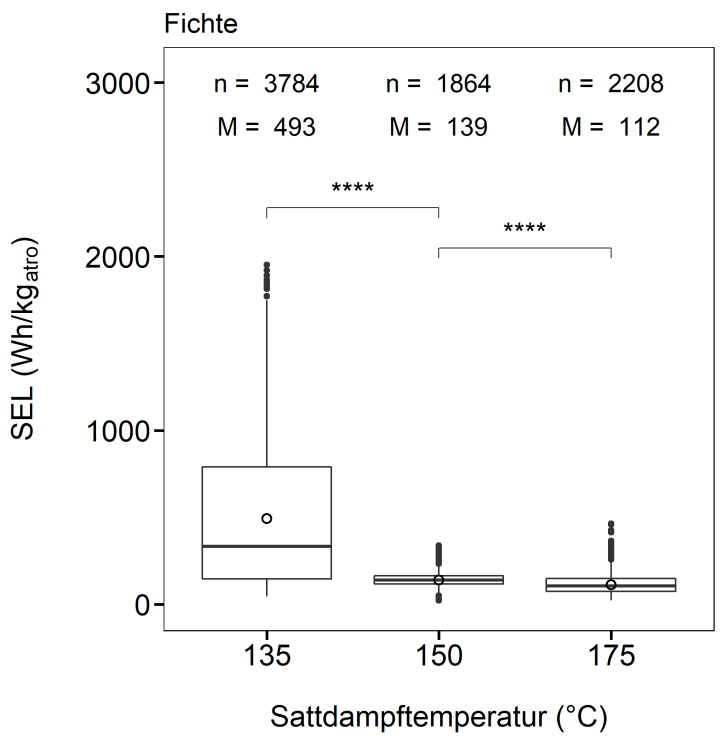

b

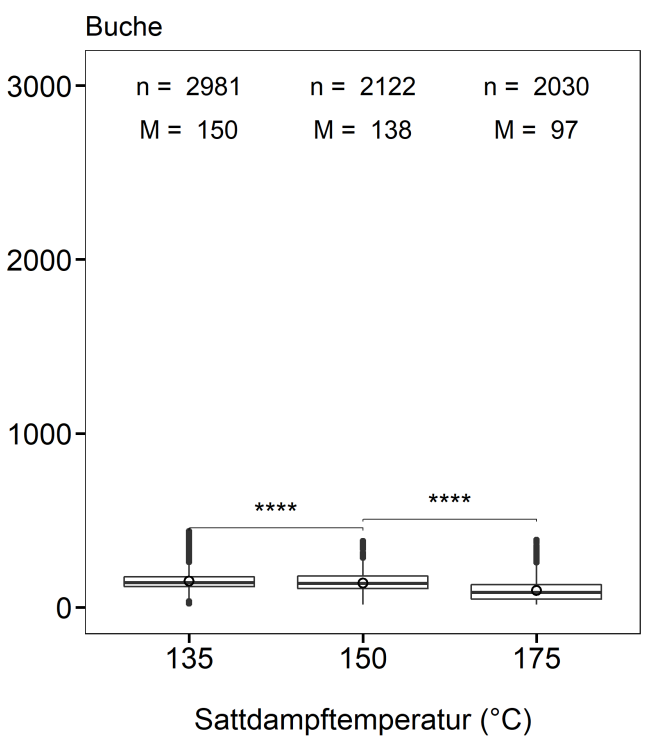

Abbildung 7.19: Darstellung des arithmetischen Mittelwerts der spezifischen elektrischen Leistungsaufnahme (SEL) über der Sattdampftemperatur aus den Versuchen mit dem Mahlscheibenmuster 2. a, Holzart Fichte. b, Holzart Buche. n: Anzahl der Messpunkte, M: Arithmetisches Mittel der SEL. N=4, Kruskall-Wallis-Test Signifikanzcode: $0,0001^{* * * *} ; 0,001^{* * *} ; 0,01^{* *} ; 0,05^{*}$.

Diese Beobachtungen werden auch durch den Vergleich der Mittelwerte in Abbildung 7.19 bestätigt. Hier ist wieder eine Abnahme der spezifischen elektrischen Leistungsaufnahme mit steigender Aufschlusstemperatur zu beobachten. Die Unterschiede zwischen den Temperaturstufen sind signifikant und belaufen sich zwischen $150^{\circ} \mathrm{C}$ und $175^{\circ} \mathrm{C}$ auf rund $20 \%$ bei der Holzart Fichte und auf rund $30 \%$ bei der Holzart Buche. Zwischen $135^{\circ} \mathrm{C}$ und $150^{\circ} \mathrm{C}$ besteht ein Unterschied von rund $8 \%$ bei der Holzart Buche. Die ermittelte spezifische elektrische Leistungsaufnahme bei $175^{\circ} \mathrm{C}$ liegt am unteren Ende der typischerweise in der MDF-Herstellung auftretenden Werte (Gao 2014). 



d

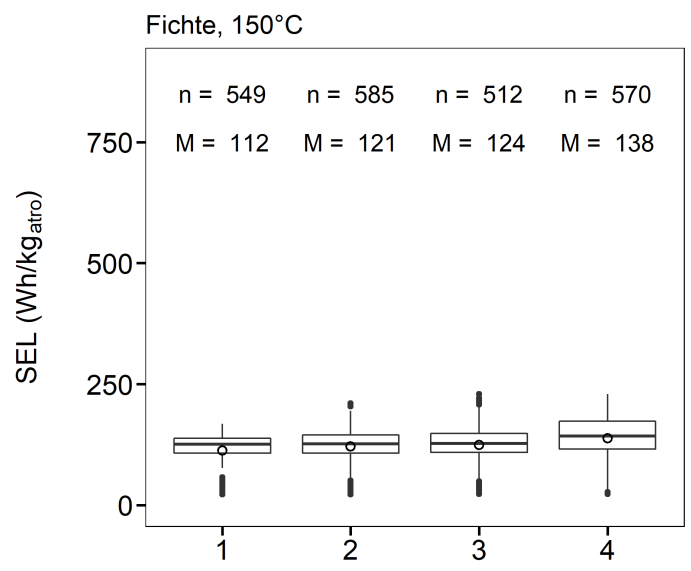

Buche, $150^{\circ} \mathrm{C}$

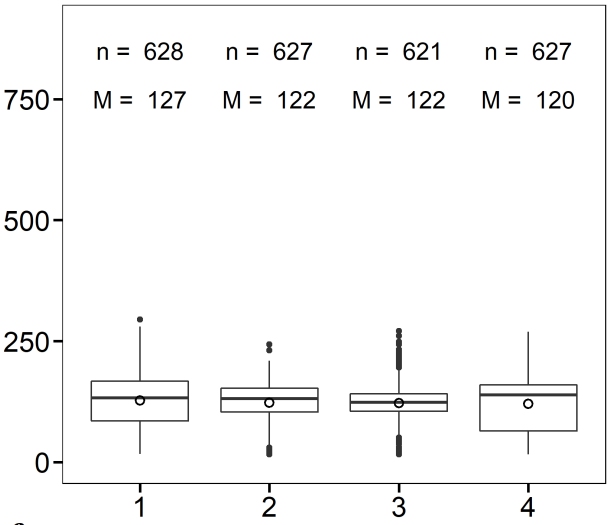

Fichte, $175^{\circ} \mathrm{C}$

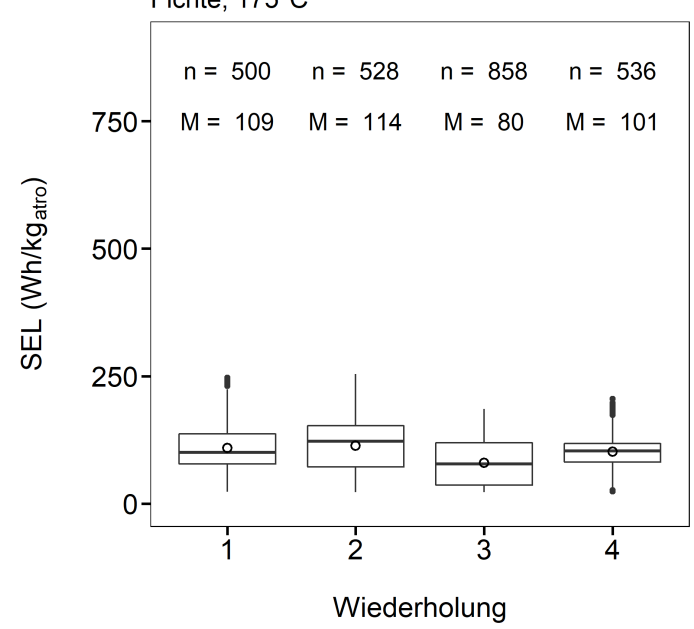

f

Buche, $175^{\circ} \mathrm{C}$

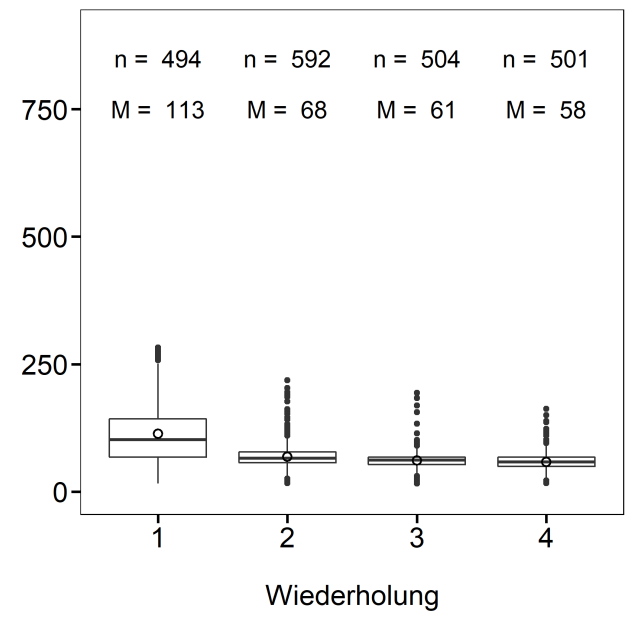

Abbildung 7.20: Darstellung der spezifischen elektrischen Leistungsaufnahme (SEL) aus den Wiederholungen mit dem Mahlscheibenmuster 3. a, c, e: In der linken Spalte sind die Wiederholungen mit der Holzart Fichte aufsteigend nach der Sattdampftemperatur dargestellt. $\boldsymbol{b}, \boldsymbol{d}, \boldsymbol{f}$ : In der rechten Spalte sind die Wiederholungen mit der Holzart Buche aufsteigend nach der Sattdampftemperatur dargestellt. n: Anzahl der Messpunkte, M: Arithmetisches Mittel der SEL. 


\section{Mahlscheibenmuster 3}

Das Mahlscheibenmuster 3 (Tabelle 6.4) zeichnet sich durch drei Zonen mit einer zur Peripherie hin steigenden Anzahl an Stegen aus. Charakteristisch ist die umlaufende Unterbrechung der Stege zwischen den Zonen 1 und 2 sowie den Zonen 2 und 3. Ein weiteres Merkmal ist die hohe Anzahl an Vorbrechern, welche zudem am Übergang zur inneren Zone relativ breit ausgeführt sind. Dämme unterhalb der Steghöhe sind nicht vorhanden. In der äußeren Zone 3 sind Dämme tangential angeordnet. Es handelt sich, wie beim Mahlscheibenmuster 2, um ein Spiraldesign, allerdings mit steilerem Stegwinkel. In der Betrachtung der Wiederholungen, dargestellt in Abbildung 7.20 fällt das recht einheitliche Verhalten in allen Temperaturstufen und bei beiden Holzarten auf. Im Vergleich zu den bereits beschriebenen Mahlscheibenmustern kann festgehalten werden, dass die Leistungsspitzen und -schwankungen geringer ausfallen. Die höhere spezifische elektrische Leistungsaufnahme der Wiederholung 1 in Abbildung 7.20f (Buche $175^{\circ} \mathrm{C}$ ) zeigt die Herausforderung in der Versuchsdurchführung auf. Wird der Mahlspalt (unabsichtlich) geringfügig enger eingestellt steigt die spezifische elektrische Leistungsaufnahme an.

$\mathbf{a}$

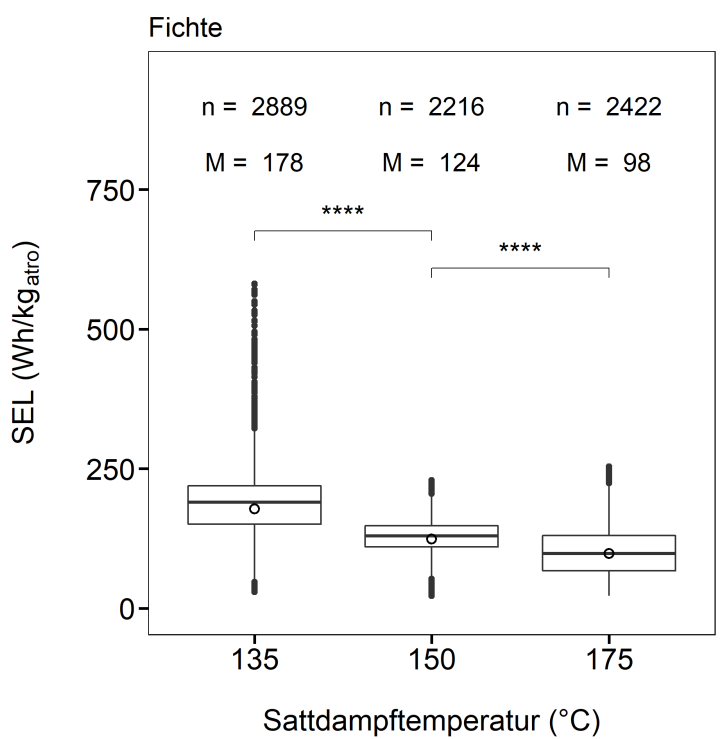

b

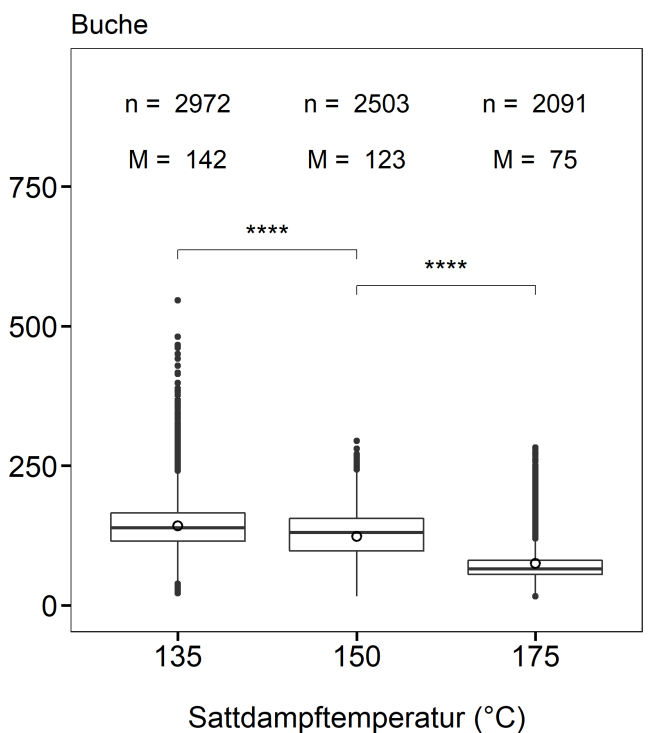

Abbildung 7.21: Darstellung des arithmetischen Mittelwerts der spezifischen elektrischen Leistungsaufnahme (SEL) über der Sattdampftemperatur aus den Versuchen mit dem Mahlscheibenmuster 3. a, Holzart Fichte. b, Holzart Buche. n: Anzahl der Messpunkte, M: Arithmetisches Mittel der SEL. N=4, Kruskall-Wallis-Test Signifikanzcode: $0,0001^{* * * *} ; 0,001^{* * *} ; 0,01^{* *} ; 0,05^{*}$.

Der Vergleich des arithmetischen Mittelwerts in Abbildung 7.21 ergab weitere Erkenntnisse. Es zeigt sich, dass die Zerfaserung von Fichtenholz bei $135^{\circ} \mathrm{C}$ rund $20 \%$ mehr elektrische Energie erfordert, als die Faserstofferzeugung mit der Holzart Buche. Bei einer Sattdampftemperatur von $150{ }^{\circ} \mathrm{C}$ ist kein Unterschied festzustellen. Und bei $175^{\circ} \mathrm{C}$ liegt die Zerfaserungsleistung mit der Holzart Buche rund $24 \%$ unter der mit der Holzart Fichte. Hervorzuheben ist die niedrige spezifische elektrische Leistungsaufnahme in der Faserstofferzeugung mit der Holzart Buche bei einer Sattdampftemperatur von $175^{\circ} \mathrm{C}$. 


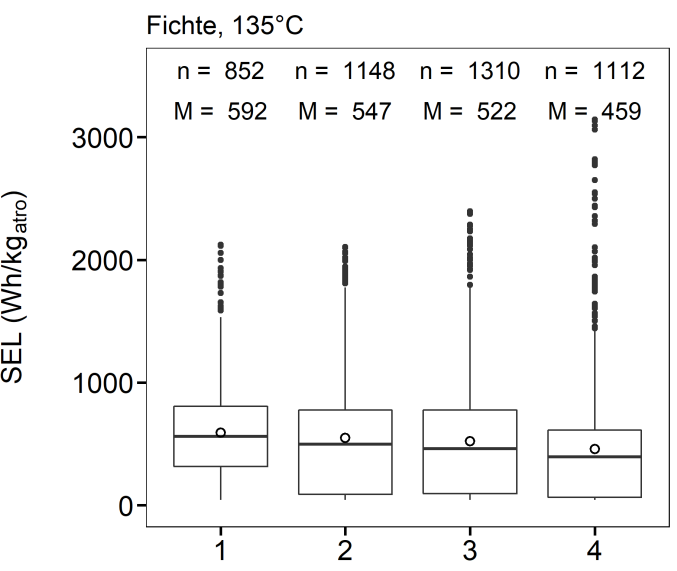

c

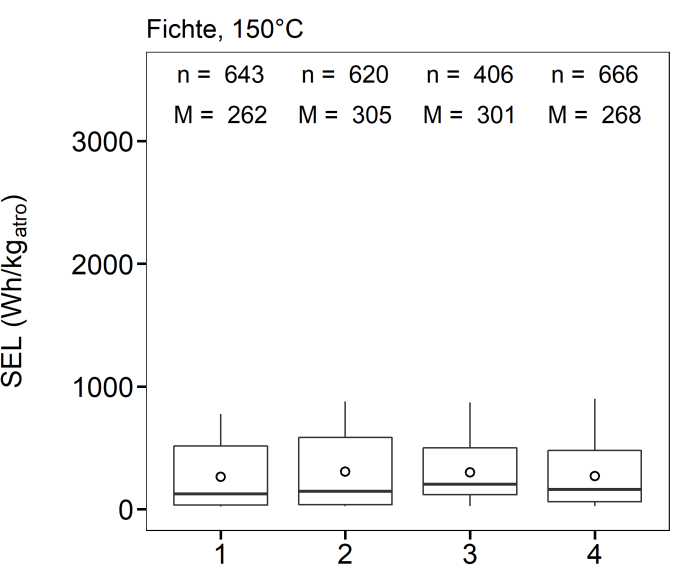

e

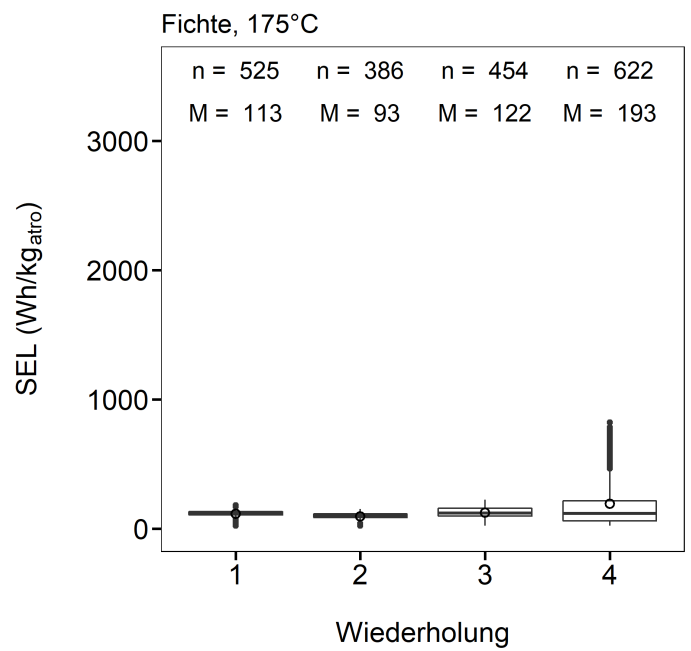

b

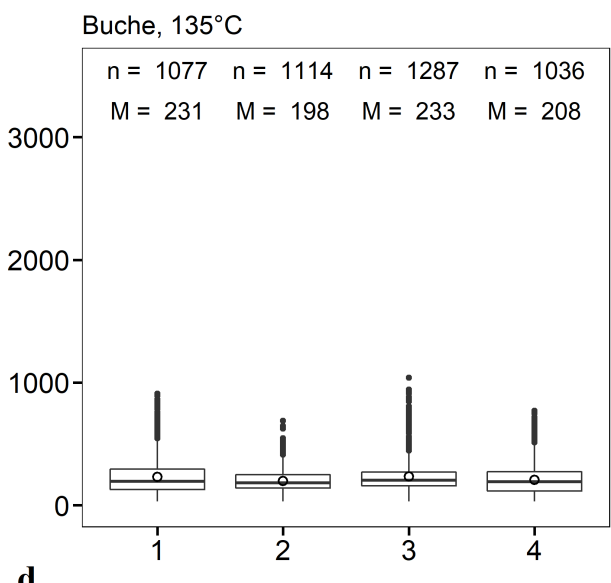

d

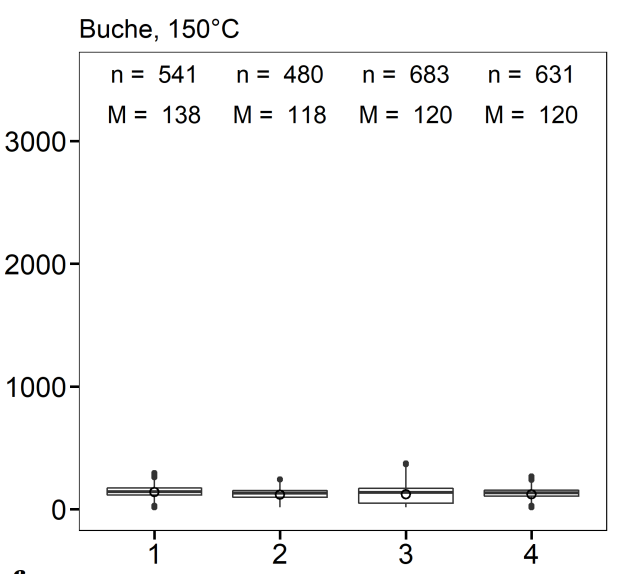

f

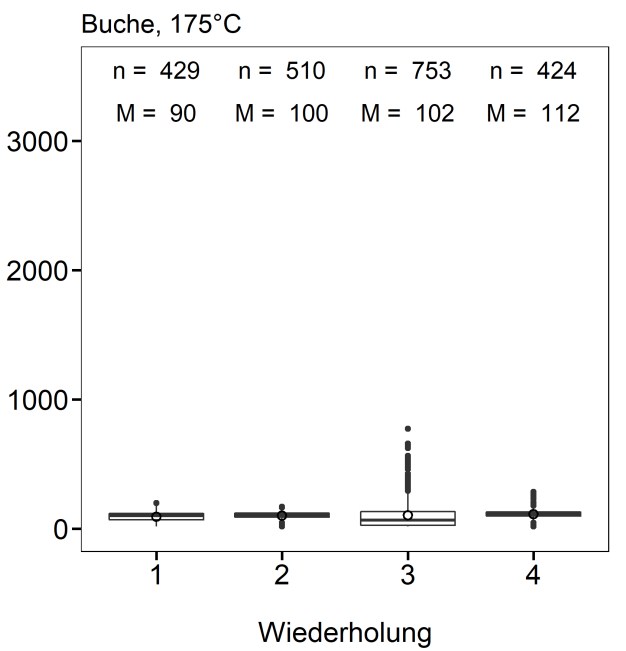

Abbildung 7.22: Darstellung der spezifischen elektrischen Leistungsaufnahme (SEL) aus den Wiederholungen mit dem Mahlscheibenmuster 4. a, c, e: In der linken Spalte sind die Wiederholungen mit der Holzart Fichte aufsteigend nach der Sattdampftemperatur dargestellt. $\boldsymbol{b}, \boldsymbol{d}, \boldsymbol{f}:$ In der rechten Spalte sind die Wiederholungen mit der Holzart Buche aufsteigend nach der Sattdampftemperatur dargestellt. n: Anzahl der Messpunkte, M: Arithmetisches Mittel der SEL. 


\section{Mahlscheibenmuster 4}

Bei dem Mahlscheibenmuster 4 (Tabelle 6.5) handelt es sich um ein Design, das dem Stand der Technik in der MDF-Herstellung entspricht. Das Mahlscheibenmuster wurde vom industriellen Maßstab in den Labormaßstab verkleinert. Die Stege sind spiralförmig angeordnet, wobei die Krümmung leicht in Rotationsrichtung zeigt. Die Anzahl der Stege nimmt zur Peripherie hin zu und der Stegwinkel nimmt gleichzeitig ab. In den Nuten sind drei U-Dämme und ein Damm in der Peripherie ebenfalls spiralförmig entgegen der Rotationsrichtung angeordnet. In Abbildung 7.22e,f wird sichtbar, dass die Mahlscheibe im für MDF üblichen hohen Temperaturbereich eine relativ geringe spezifische elektrische Leistungsaufnahme erzielt. Die hohen Ausreißern der Wiederholungen 3 respektive 4 sind auffällig. Bei niedrigeren Temperaturen sind dagegen starke Leistungsschwankungen sichtbar. Die Leistungsspitzen liegen bei $135^{\circ} \mathrm{C}$ (Abbildung 7.22a) zwischen 2000 und $3000 \mathrm{Wh} / \mathrm{kg}_{\text {atro }}$ (!). Mit der Holzart Buche zeigt das Mahlscheibenmuster eine relativ gleichmäßige SEL.

a

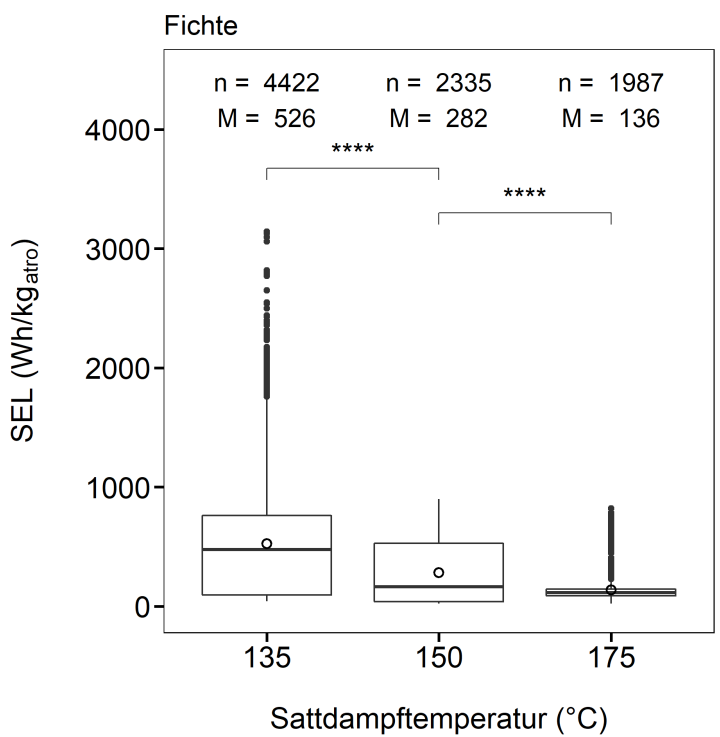

b

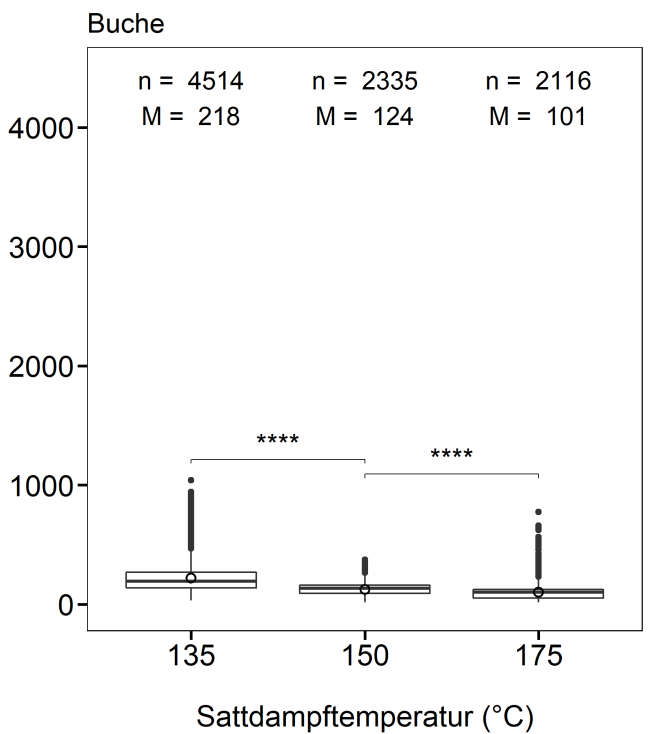

Abbildung 7.23: Darstellung des arithmetischen Mittelwerts der spezifischen elektrischen Leistungsaufnahme (SEL) über der Sattdampftemperatur aus den Versuchen mit dem Mahlscheibenmuster 4. a, Holzart Fichte. b, Holzart Buche. n: Anzahl der Messpunkte, M: Arithmetisches Mittel der SEL. N=4, Kruskall-Wallis-Test Signifikanzcode: $0,0001^{* * *} ; 0,001^{* * *} ; 0,01^{* *} ; 0,05^{*}$.

Der Vergleich der arithmetischen Mittelwerte der spezifischen elektrischen Leistungsaufnahme in Abbildung 7.23 bestätigt die oben beschriebenen Beobachtungen. Das Mahlscheibenmuster scheint für niedrigere Sattdampftemperaturen mit der Holzart Fichte nicht geeignet zu sein. Darüber hinaus ist die Temperaturabhängigkeit bei der Holzart Fichte stark ausgeprägt. Auch bei der Holzart Buche sind die Unterschiede zwischen den Sattdampftemperaturen vergleichsweise stark ausgeprägt. Die Werte bei $175^{\circ} \mathrm{C}$ mit Fichte sind mit den Werten von Gao (2014) aus der industriellen MDF-Produktion vergleichbar. 



Buche, $150^{\circ} \mathrm{C}$

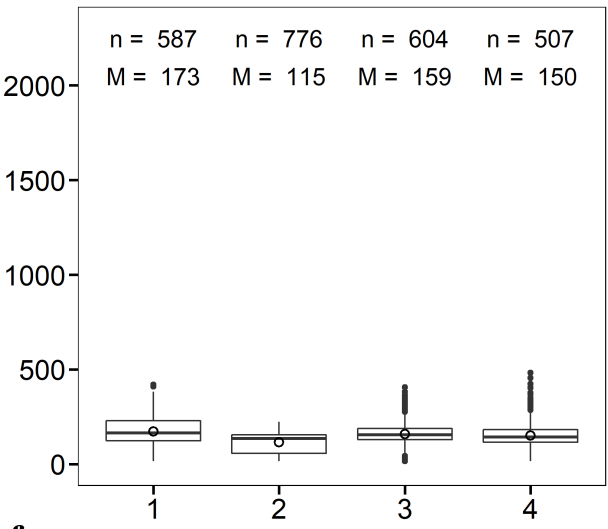

Fichte, $175^{\circ} \mathrm{C}$

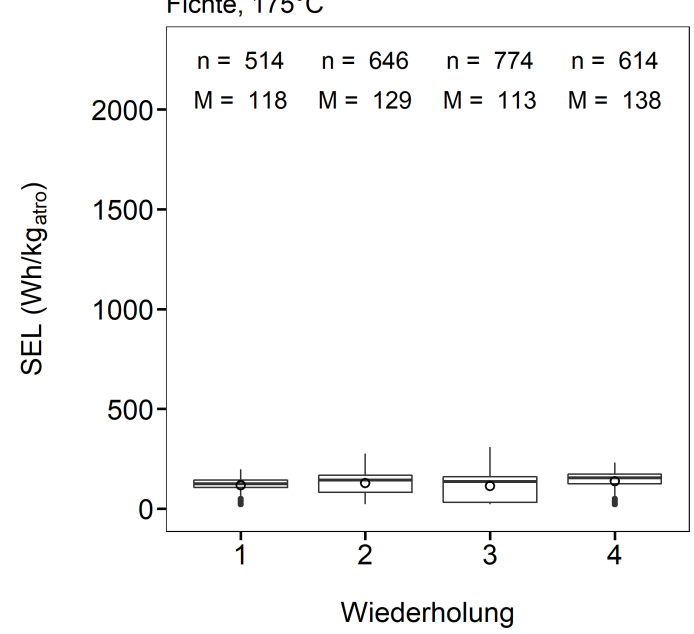

$\mathbf{f}$

Buche, $175^{\circ} \mathrm{C}$

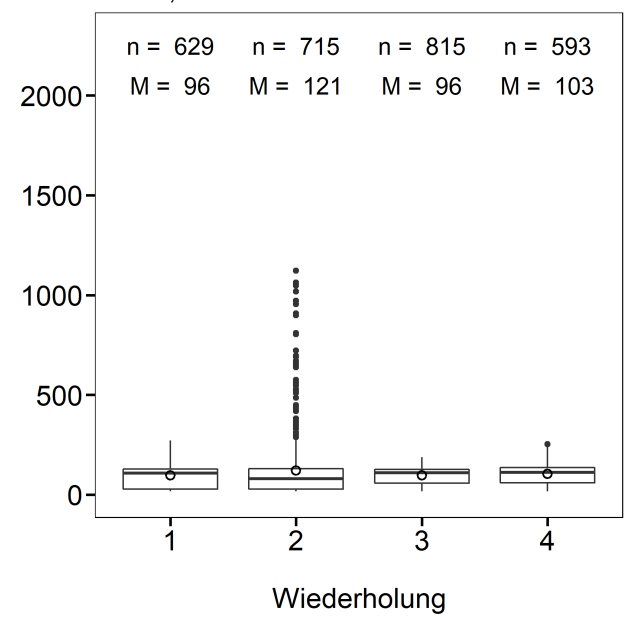

Abbildung 7.24: Darstellung der spezifischen elektrischen Leistungsaufnahme (SEL) aus den Wiederholungen mit dem Mahlscheibenmuster 5. a, c, e: In der linken Spalte sind die Wiederholungen mit der Holzart Fichte aufsteigend nach der Sattdampftemperatur dargestellt. $\boldsymbol{b}, \boldsymbol{d}, \boldsymbol{f}$ : In der rechten Spalte sind die Wiederholungen mit der Holzart Buche aufsteigend nach der Sattdampftemperatur dargestellt. n: Anzahl der Messpunkte, M: Arithmetisches Mittel der SEL. 


\section{Mahlscheibenmuster 5}

Das Mahlscheibenmuster 5 (Tabelle 6.6 und Tabelle 6.7) ist eine Weiterentwicklung des Mahlscheibenmusters 4 . Es zeichnet sich durch einen unterschiedlich gestalteten Rotor und Stator aus. Die Stege des Rotors sind stark entgegen der Laufrichtung gekrümmt. An der Peripherie liegt die Steganzahl gegenüber den anderen untersuchten Mahlscheibenmustern deutlich höher. Die Krümmung auf dem Stator ist der Krümmung auf dem Rotor entgegengesetzt. Gleichzeitig ist die Steganzahl auf dem Stator reduziert. Im eingebauten Zustand zeigen die Stege von Rotor und Stator in dieselbe Richtung. Das Mahlscheibenmuster wurde speziell für die Holzfaserdämmstoffherstellung entwickelt. In Abbildung 7.24 zeigen die Wiederholungen innerhalb der Holzarten ein relativ einheitliches Bild. Ein Erklärungsansatz für die Ausreißer der Wiederholung 2 in Abbildung 7.24c und Wiederholung 2 in Abbildung 7.24f sind Schwankungen in der Größenverteilung oder eine Verunreinigung der Hackschnitzel. Möglicherweise sind die Leistungsspitzen auch auf Resonanzen der Laborrefineranlage zurückzuführen.

$\mathbf{a}$

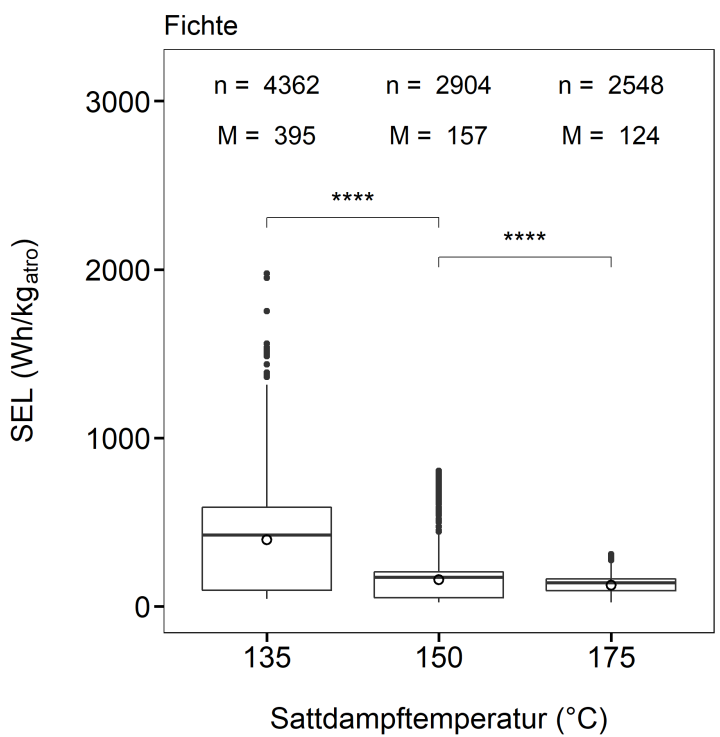

b

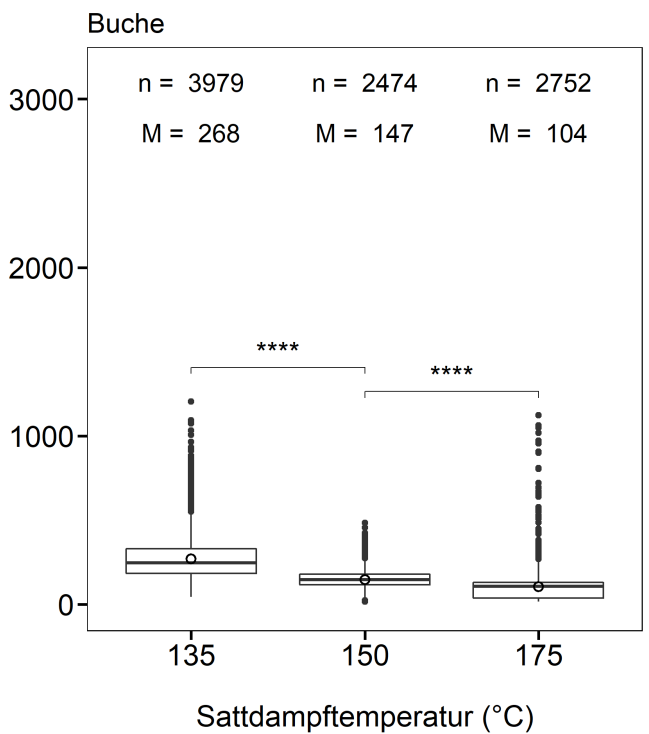

Abbildung 7.25: Darstellung des arithmetischen Mittelwerts der spezifischen elektrischen Leistungsaufnahme (SEL) über der Sattdampftemperatur aus den Versuchen mit dem Mahlscheibenmuster 5. a, Holzart Fichte. b, Holzart Buche. n: Anzahl der Messpunkte, M: Arithmetisches Mittel der SEL. N=4, Kruskall-Wallis-Test Signifikanzcode: $0,0001^{* * * *} ; 0,001^{* * *} ; 0,01^{* *} ; 0,05^{*}$.

Die arithmetischen Mittelwerte der spezifischen elektrischen Leistungsaufnahme in Abbildung 7.25 zeigen eine vergleichsweise starke Temperaturabhängigkeit. Zwischen $135^{\circ} \mathrm{C}$ und $150^{\circ} \mathrm{C}$ besteht bei der Holzart Fichte ein Unterschied von rund $60 \%$ und bei der Holzart Buche von rund $55 \%$. Die Temperaturabhängigkeit geht zwischen $150^{\circ} \mathrm{C}$ und $175^{\circ} \mathrm{C}$ auf rund $21 \%$ bei der Holzart Fichte und auf rund $29 \%$ bei der Holzart Buche zurück. Besonders laufruhig zeigt sich die Faserstofferzeugung mit der Holzart Fichte bei $175^{\circ} \mathrm{C}$. 


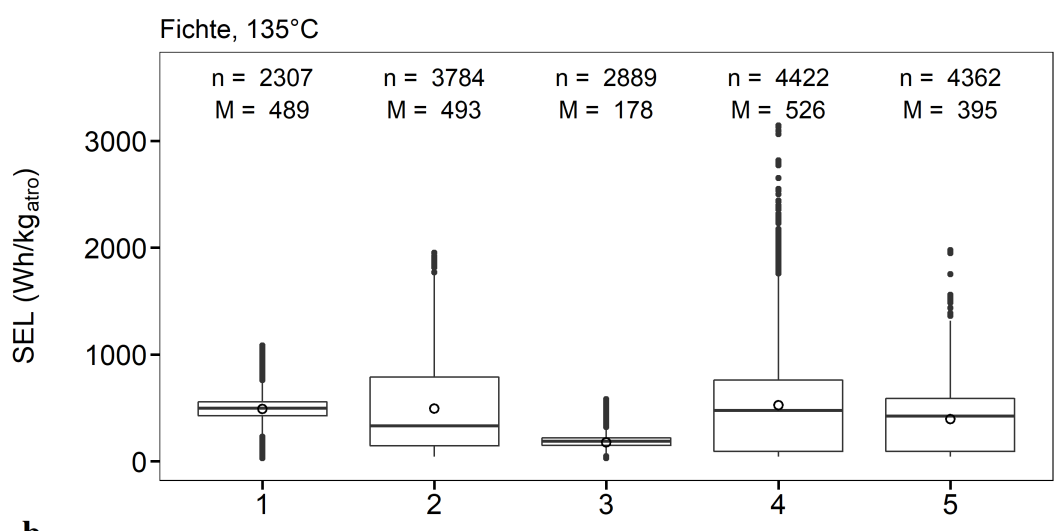

b

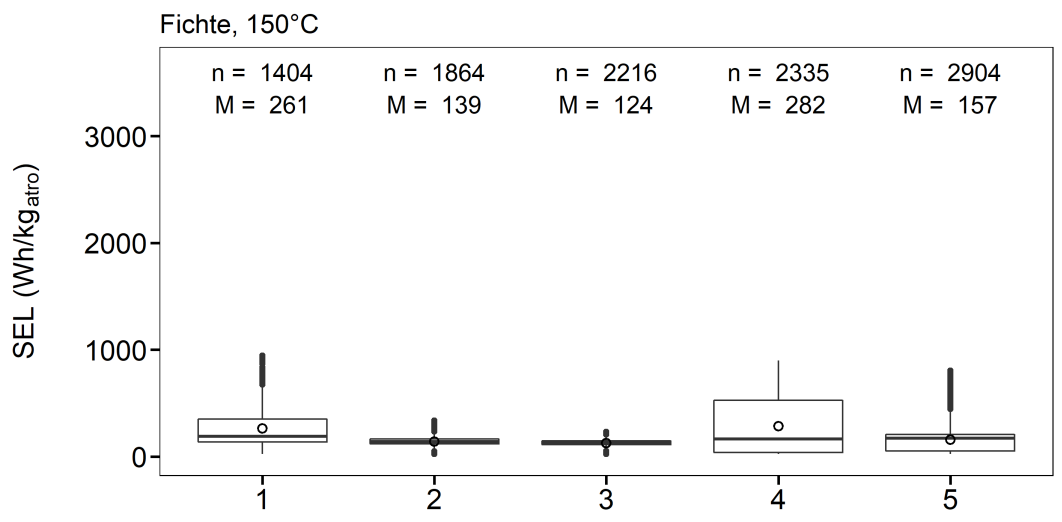

c

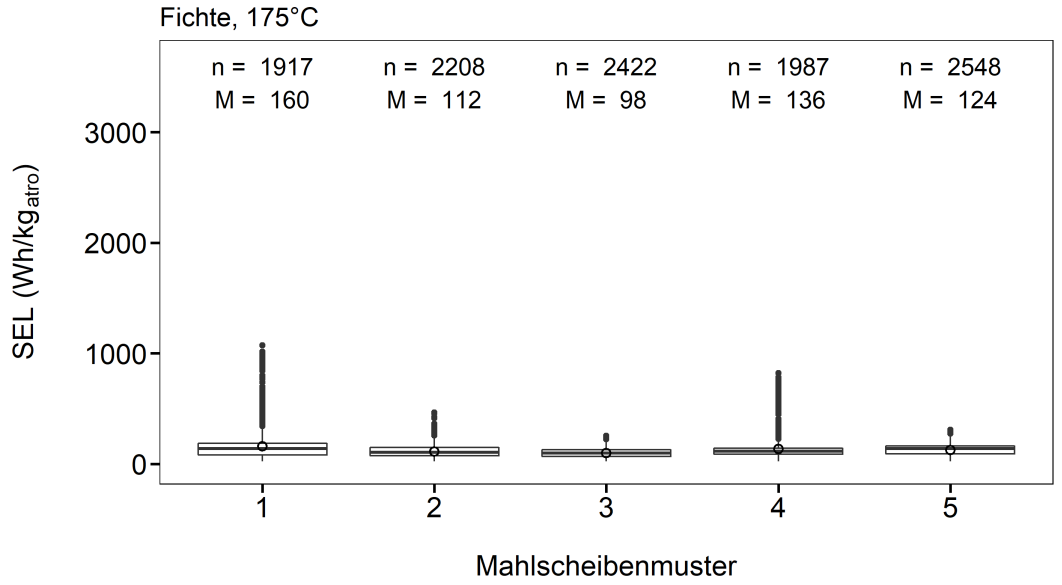

Abbildung 7.26: Vergleich der spezifischen elektrischen Leistungsaufnahme (SEL) in der Faserstofferzeugung mit der Holzart Fichte innerhalb der Aufschlusstemperaturen: a, $135^{\circ} \mathrm{C} ; \boldsymbol{b}, 150^{\circ} \mathrm{C} ; \boldsymbol{c}, 175^{\circ} \mathrm{C} . n$ : Anzahl der Messpunkte, M: Arithmetisches Mittel der SEL. 
Tabelle 7.7: Statistische Auswertung in Ergänzung zu Abbildung 7.26 (Faserstofferzeugung mit der Holzart Fichte). Die Asteriske indizieren das Signifikanzniveau.

\begin{tabular}{|c|c|c|c|c|c|c|c|c|c|c|c|c|}
\hline \multirow[b]{2}{*}{ M. } & \multicolumn{4}{|c|}{$135^{\circ} \mathrm{C}$} & \multicolumn{4}{|c|}{$150^{\circ} \mathrm{C}$} & \multicolumn{4}{|c|}{$175^{\circ} \mathrm{C}$} \\
\hline & 1 & 2 & 3 & 4 & 1 & 2 & 3 & 4 & 1 & 2 & 3 & 4 \\
\hline 2 & $* * * *$ & & & & $* * * *$ & & & & $* * * *$ & & & \\
\hline 3 & $* * * *$ & $* * * *$ & & & $* * * *$ & $* * * *$ & & & $* * * *$ & $* * * *$ & & \\
\hline 4 & ns & ns & $* * * *$ & & $* * * *$ & $* * * *$ & $* * * *$ & & $* * * *$ & $* * * *$ & $* * * *$ & \\
\hline 5 & $* * * *$ & $* * * *$ & $* * * *$ & $* * * *$ & $* * * *$ & $* * * *$ & $* * * *$ & $* * * *$ & $* * * *$ & $* * * *$ & $* * * *$ & $* * * *$ \\
\hline
\end{tabular}

\section{Vergleich der Mahlscheibenmuster}

In Abbildung 7.26 und in Abbildung 7.27 sind die Ergebnisse zur spezifischen elektrischen Leistungsaufnahme der untersuchten Mahlscheibenmuster vergleichend innerhalb der Temperaturstufen dargestellt. Aus der Betrachtung der Darstellungen geht hervor, dass die verschiedenen Mahlscheibenmuster grundsätzlich charakteristische Unterschiede in der Faserstofferzeugung zeigen.

In Abbildung 7.26a wird deutlich, dass mit der Holzart Fichte in der Sattdampftemperatur von $135^{\circ} \mathrm{C}$ im Vergleich zu den anderen Aufschlusstemperaturen die höchste spezifische elektrische Leistungsaufnahme sowie die größten Schwankungen und Leistungsspitzen auftreten. Ein Erklärungsansatz hierfür wäre das Erweichungsverhalten des Fichtenholzes. Den Untersuchungen von Asplund (1940a) und Krug (2010) zufolge, führt das Erweichungsverhalten im TMP-Verfahren erst ab Aufschlusstemperaturen von über $170^{\circ} \mathrm{C}$ zu einem deutlichen Rückgang der SEL. Im vorliegenden Fall muss allerdings darauf hingewiesen werden, dass die Konstruktion der Mahlscheibe ebenfalls einen entscheidenden Einfluss auf die spezifische elektrische Leistungsaufnahme hat. So zeigt das Mahlscheibenmuster 2 zwar eine hohe SEL, aber eine relativ geringe Streuung. Das Mahlscheibenmuster 3 weist hingegen eine vergleichsweise sehr geringe spezifische elektrische Leistungsaufnahme und Streuung auf. Die Ergebnisse werden vor dem Hintergrund des Erweichungsverhaltens von Holz in Abschnitt 7.4.2 ausführlicher betrachtet. Negativ fällt in Abbildung 7.26a das Mahlscheibenmuster 4 mit der insgesamt höchsten ermittelten spezifischen elektrischen Leistungsaufnahme und den höchsten Ausreißern auf. Die für einen direkten Vergleich ungünstige Skalierung Ordinate ist dieser Tatsache geschuldet. Mit steigender Aufschlusstemperatur werden die Unterschiede zwischen den Mahlscheibenmustern geringer. Es bleibt aber festzuhalten, dass mit den Mahlscheibenmustern 2 und 3 in den Aufschlusstemperaturen $150^{\circ} \mathrm{C}$ und $175^{\circ} \mathrm{C}$ die niedrigste spezifische elektrische Leistungsaufnahme ermittelt wurde. Das Mahlscheibenmuster 4 scheint ebenfalls wie das Mahlscheibenmuster 5 bei hohen Sattdampftemperaturen besser zu funktionieren. Das bidirektionale Mahlscheibenmuster 1 weist die höchste spezifische elektrische Leistungsaufnahme bei $175^{\circ} \mathrm{C}$ auf.

Bezüglich der Holzart Buche liegt die spezifische elektrische Leistungsaufnahme allgemein niedriger als bei der Faserstofferzeugung mit der Holzart Fichte. Die Unterschiede zwischen den Mahlscheibenmustern bezüglich der spezifischen elektrischen Leistungsaufnahme fallen geringer aus und auch die IQR zeigen ein gleichmäßigeres Bild. Hier ist ebenfalls das Mahlscheibenmuster 3 hervorzuheben, mit dem in allen drei Temperaturstufen die niedrigste spezifische elektrische Leistungsaufnahme und die geringste Leistungsschwankung ermittelt werden konnte. Mit dieser Mahlscheibe konnte insgesamt die geringste spezifische elektrische Leistungsaufnahme mit einer 
Aufschlusstemperatur von $175^{\circ} \mathrm{C}$ erzielt werden. Das bidirektionale Mahlscheibenmuster 1 weist bei der Holzart Buche die höchste spezifische elektrische Leistungsaufnahme und die größten Leistungsspitzen auf.

a

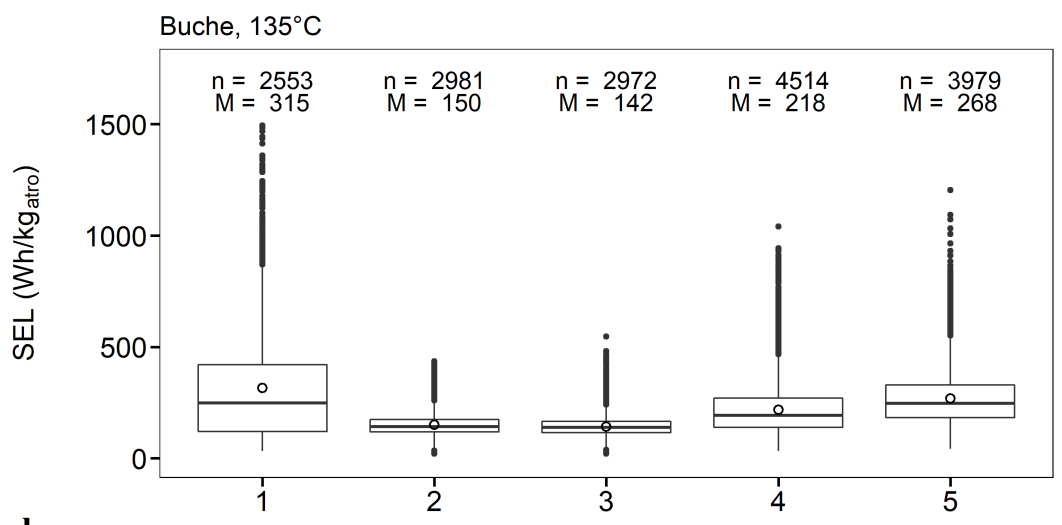

b

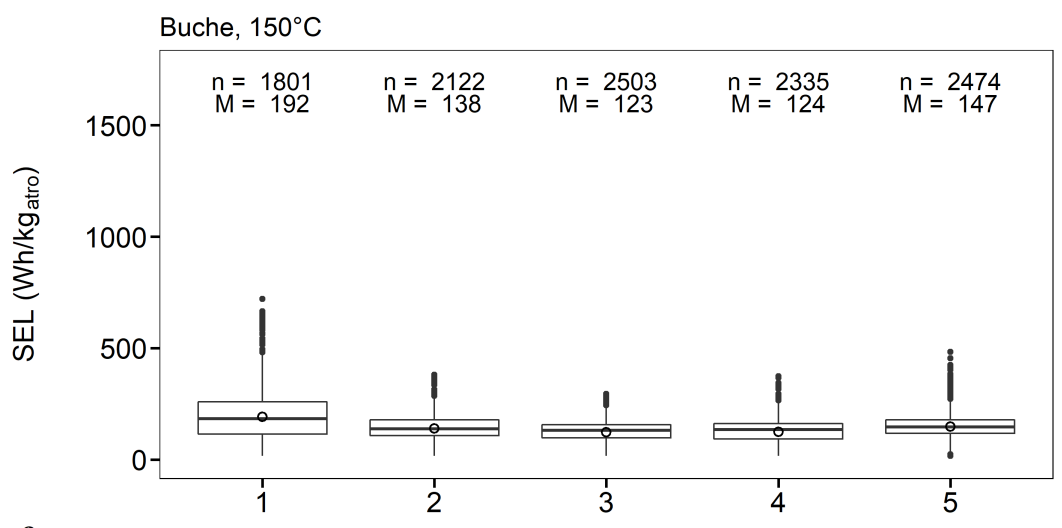

c

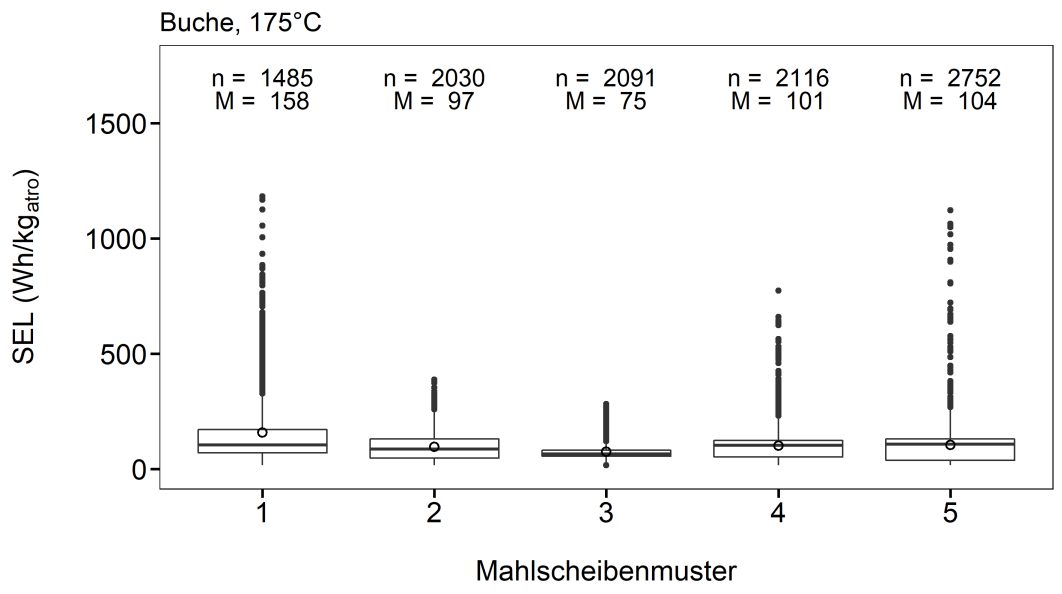

Abbildung 7.27: Vergleich der spezifischen elektrischen Leistungsaufnahme (SEL) in der Faserstofferzeugung mit der Holzart Buche innerhalb der Aufschlusstemperaturen: a, $135^{\circ} \mathrm{C} ; \boldsymbol{b}, 150^{\circ} \mathrm{C}$; c, $175^{\circ} \mathrm{C}$. n: Anzahl der Messpunkte, M: Arithmetisches Mittel der SEL. 
Tabelle 7.8: Statistische Auswertung in Ergänzung zu Abbildung 7.27 (Faserstofferzeugung mit der Holzart Fichte). Die Asteriske indizieren das Signifikanzniveau.

\begin{tabular}{|c|c|c|c|c|c|c|c|c|c|c|c|c|}
\hline \multirow[b]{2}{*}{ M. } & \multicolumn{4}{|c|}{$135^{\circ} \mathrm{C}$} & \multicolumn{4}{|c|}{$150{ }^{\circ} \mathrm{C}$} & \multicolumn{4}{|c|}{$175^{\circ} \mathrm{C}$} \\
\hline & 1 & 2 & 3 & 4 & 1 & 2 & 3 & 4 & 1 & 2 & 3 & 4 \\
\hline 2 & $* * * *$ & & & & $* * * *$ & & & & $* * * *$ & & & \\
\hline 3 & $* * * *$ & $* * * *$ & & & $* * * *$ & $* * * *$ & & & $* * * *$ & $* * * *$ & & \\
\hline 4 & $* * * *$ & $* * * *$ & $* * * *$ & & $* * * *$ & $* * * *$ & $* *$ & & $* * * *$ & $* *$ & $* * * *$ & \\
\hline 5 & ns & $* * * *$ & $* * * *$ & $* * * *$ & $* * * *$ & $* * *$ & $* * * *$ & $* * * *$ & $* * * *$ & ns & $* * * *$ & ns \\
\hline
\end{tabular}

In der statistischen Auswertung des Mahlscheibenvergleichs mit der Holzart Fichte in Tabelle 7.7 wurden zwei Mahlscheiben-Paarungen (1-4 und 2-4) identifiziert, bei denen keine signifikanten Differenzen zwischen den zentralen Tendenzen der Grundgesamtheit bestehen. In Tabelle 7.8 besteht bezüglich der Holzart Buche eine nicht signifikante Paarung in der Temperaturstufe $135^{\circ} \mathrm{C}$ (1-5) und zwei Paarungen in der Temperaturstufe $175^{\circ} \mathrm{C}$ (2-5 und 4-5). Dies ist insofern bemerkenswert, als dass der Stichprobenumfang sehr hoch ist. Hier stellt sich die Frage, ob die Beschaffenheit der erzeugten Faserstoffe Unterschiede aufweist.

Das Design des Mahlscheibenmusters 3 unterscheidet sich von den anderen untersuchten Mahlscheiben, durch die umlaufende Unterbrechung der Stege zwischen den Zonen auf der Mahlbahn (Tabelle 6.4). Zudem besitzt das Muster relativ viele Vorbrecher. Ein Erklärungsansatz für die geringe spezifische elektrische Leistungsaufnahme und die Laufruhe ist, dass die umlaufende Unterbrechung der Bildung einer Stagnationszone bzw. eines Temperaturmaximums auf der Mahlbahn entgegenwirken. Atack et al. (1989) zeigten an einem bidirektionalem Mahlscheibenmuster im TMP-Verfahren der Papierherstellung, dass mit einer umlaufenden Unterbrechung der Stege im Bereich des Temperaturmaximums die Faserstoffqualität bei gleicher spezifischer elektrischer Leistungsaufnahme verbessert wird. Darüber hinaus trug diese Spezifikation dazu bei, die Schwankungen und Leistungsspitzen insbesondere unter hoher Last zu reduzieren. Dadurch konnte der Durchsatz erhöht werden.

Das Phänomen des Temperaturmaximums wird u.a. von Atack et al. (1984), Miles \& May (1990) und Roll $(2010 \mathrm{a}, \mathrm{b})$ beschrieben und konnte bei industriellen Mahlscheibentests im Rahmen der vorliegenden Arbeit ebenfalls beobachtet werden (Abbildung 7.28). Die Bedeutung des Temperaturprofils auf der Mahlbahn wird auch von Fredrikson \& Paltakari (2020) für das TMP-Verfahren der Papierherstellung beschrieben. Demnach hat der Mahlspalt einen geringen bis keinen Einfluss auf das Temperaturprofil der Mahlbahn. Den Widerstand für den Dampffluss bewirken hauptsächlich die Mahlscheiben. Eine Temperaturmessung im Mahlspalt kann nach Fredrikson \& Paltakari (2020) zur Prozesskontrolle eingesetzt werden. In den vorliegenden Ergebnissen wurde die Ausbildung des Temperaturmaximums nicht weiter untersucht. Beim Wechsel der Mahlscheiben wurde jedoch festgestellt, dass die Ausbildung des typischen dunkel gefärbten Rings auf der Mahlbahn bei dem Mahlscheibenmuster 12XA803 deutlich geringer ausfällt. 


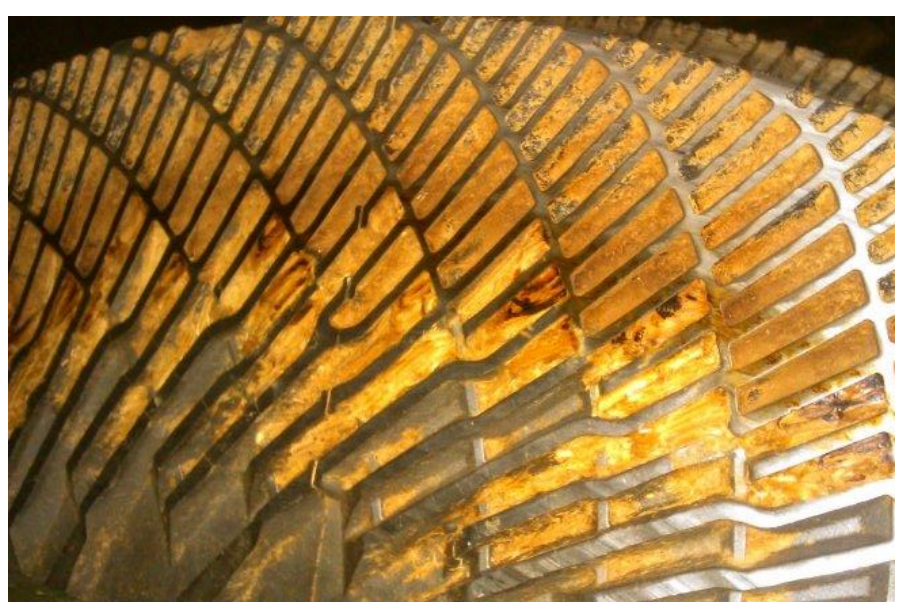

Abbildung 7.28: Aufnahme der Mahlbahn nach einem Mahlscheibentest im industriellen Maßstab. Es handelt sich um eine Variante des Mahlscheibenmusters 2. Zu sehen ist ein dunkel gefärbter Ring etwa in der Mitte der Mahlbahn. Die dunkle Färbung lässt auf ein Temperaturmaximum in diesem Bereich schließen, welches eine Karbonisierung des Faserstoffs hervorruft.

Mit dem labortechnischen Batch-Verfahren waren die Zeiträume für die Datenerfassung in der Faserstofferzeugung auf ca. 5 min mit einem durchgesetzten Hackschnitzelvolumen von ca. 201 Hackschnitzelvolumen begrenzt. Vor diesem Hintergrund muss die Aussagekraft der Untersuchungen diskutiert werden. Ein Verschleiß der Mahlscheiben, wie er nach hunderten von Betriebsstunden auftritt, konnte nicht untersucht werden. Wie in Gao (2014) beschrieben, bleibt die Faserqualität allerdings auch nach vielen Betriebsstunden vergleichbar. Ebenda zufolge ist die Entwicklung der spezifischen elektrischen Leistungsaufnahme über die Zeit vom Mahlscheibenmuster abhängig. Insofern sind die Ergebnisse in der vorliegenden Arbeit untereinander vergleichbar, da die Mahlscheiben aus den gleichen Legierung bestanden und in dem selben Verschleißzustand untersucht wurden. Darüber hinaus wurde der Mahlspalt in allen Versuchen gleich gehalten. Leistungsschwankungen und Leistungsspitzen treten auch im Betrieb industrieller Refiner auf (Eriksen et al. 2006). Interessant ist die Beobachtung, dass mit dem Mahlscheibendesign hierauf Einfluss genommen werden kann. Aus den vorliegenden Untersuchungen sind in diesem Kontext die Mahlscheibenmuster 2 und 3 in allen Temperaturbereichen und bei beiden Holzarten und das Muster 5 bei der Holzart Fichte und der Sattdampftemperatur $175^{\circ} \mathrm{C}$ erwähnenswert.

In Abschnitt 7.1.3 wurde der Mahlspalt als Haupteffekt auf die spezifische elektrische Leistungsaufnahme identifiziert. Die geringe Wiederholgenauigkeit in den Untersuchungen zur spezifischen elektrischen Leistungsaufnahme ist auf eine fehlerbehaftete Einstellung des Mahlspalts zurückzuführen. Anscheinend unterliegt der Mahlspalt im labortechnischen Batch-Verfahren trotz genauer manueller Einstellung an der Ringskala gewissen Schwankungen. Diese können auch durch die thermische Längenänderung der Refinerantriebswelle bedingt sein. Ähnliche Beobachtungen machten Wenderdel et al. (2016a, S. 8). Weiterhin sind Schwankungen in der Holzfeuchte, Größenverteilung und Schüttdichte der Hackschnitzel nicht auszuschließen. 


\subsubsection{Spezifische elektrische Leistungsaufnahme im RMP-Verfahren}

Im RMP-Verfahren wurde die Machbarkeit einer Faserstofferzeugung unter Atmosphärendruck für die Herstellung von Holzfaserdämmstoffen untersucht. Dieses Verfahren bietet den Vorteil, dass gegenüber dem TMP-Verfahren Einsparungen im thermischen Energiebedarf zu erwarten sind. Durch Verzicht auf die Erwärmung der Hackschnitzel in Sattdampfdruck wird der Dampfbedarf reduziert und es erfolgt kein Anstieg der Holzfeuchte im Kocher (vgl. Abschnitt 7.2.2). Es ist weiterhin zu erwarten, dass die Wärmeentwicklung zwischen den Mahlscheiben einen Trocknungseffekt auf die Faserstoffe hat und somit die Trocknerbeladung reduziert wird. Kerekes (2015) beziffert den Energiebedarf für das RMP-Verfahren in der Papierherstellung auf 6,4 GJ/t im Vergleich zu 7,0 GJ/t für die Faserstofferzeugung im TMP-Verfahren.

Die Versuche im RMP-Verfahren wurden in zwei Temperaturstufen unter Atmosphärendruck durchgeführt. Die Ergebnisse zur spezifischen elektrischen Leistungsaufnahme sind in Tabelle 7.9 aufgelistet. Hier wird ein deutlicher Unterschied zwischen den Holzarten Fichte und Buche sichtbar, auf den im Folgenden näher eingegangen wird.

Tabelle 7.9: Auflistung des arithmetischen Mittels und der Standardabweichung der ermittelten spezifischen elektrischen Leistungsaufnahme (SEL) im RMP-Verfahren.

\begin{tabular}{|c|c|c|c|c|c|}
\hline Variante & Mahlscheibe & Holzart & Aufschlussbedingungen & $\begin{array}{l}\mathrm{SEL} \\
\bar{x} \\
\mathrm{Wh} / \mathrm{kg}_{\text {atro }}\end{array}$ & $\begin{array}{l}\sigma \\
\mathrm{Wh} / \mathrm{kg}_{\text {atro }}\end{array}$ \\
\hline 1 & 6 & Fichte & Atmosphärendruck: $20^{\circ} \mathrm{C}$ & 520 & 286 \\
\hline 2 & 6 & Fichte & Atmosphärendruck: $100^{\circ} \mathrm{C}^{* *}$ & 465 & 303 \\
\hline 3 & 6 & Buche & Atmosphärendruck: $20^{\circ} \mathrm{C}$ & 219 & 82 \\
\hline 4 & 6 & Buche & Atmosphärendruck: $100^{\circ} \mathrm{C}^{* *}$ & 215 & 113 \\
\hline
\end{tabular}

\section{Mahlscheibenmuster 6}

Für die Zerfaserung nach dem RMP-Verfahren wurde das Mahlscheibenmuster 6 (Tabelle 6.8) untersucht, das speziell für das RMP-Verfahren entwickelt wurde. Das Mahlscheibenmuster unterscheidet sich insbesondere durch hohe Stege und zur Peripherie hin breiter werdende Nuten von den TMP-Mahlscheibenmustern. Es handelt sich grundsätzlich um ein für das RMP-Verfahren entwickeltes Spiraldesign. Der Stegwinkel nimmt zur Peripherie hin auf bis zu $45^{\circ} \mathrm{zu}$. Dies begünstigt den Materialtransport durch den Mahlspalt und verkürzt dadurch die Beanspruchungszeit auf der Mahlbahn. Bereits Leask (1987, 93 ff.) weist darauf hin, dass Mahlscheiben für das TMP-Verfahren nicht für das RMP-Verfahren geeignet sind und umgekehrt. Vorversuche im Rahmen der vorliegenden Arbeit zeigten, dass mit TMP-Mahlscheibenmustern der Durchsatz im RMP-Verfahren stark reduziert war. Eine zentrale Anforderung in der Entwicklung war deshalb die Vorgabe, den selben Durchsatz wie mit den Mahlscheibenmustern im TMP-Verfahren zu erzielen. Dieses erste Ziel wurde erreicht.

In Abbildung 7.29 zeigen die Wiederholungen mit und ohne Erwärmung der Hackschnitzel, dass die Zerfaserung unter Atmosphärendruck funktioniert. Der hohe IQR und die Whisker deuten bei der Holzart Fichte auf starke Schwankungen in der spezifischen elektrischen Leistungsaufnahme hin. Bei der Holzart Buche treten diese Schwankungen in geringerem Maße auf. Aus Abbildung 7.30 geht hervor, dass zwischen beiden Temperaturstufen bei der Holzart Fichte ein 
a

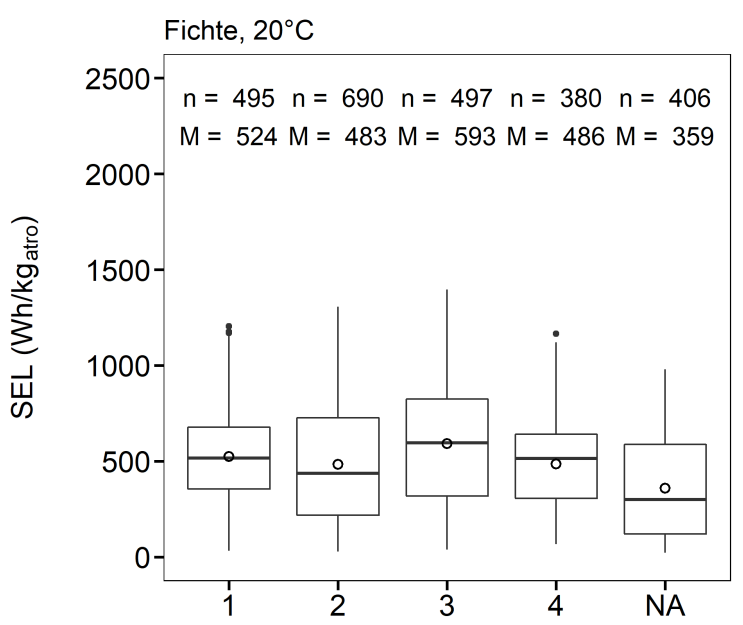

c

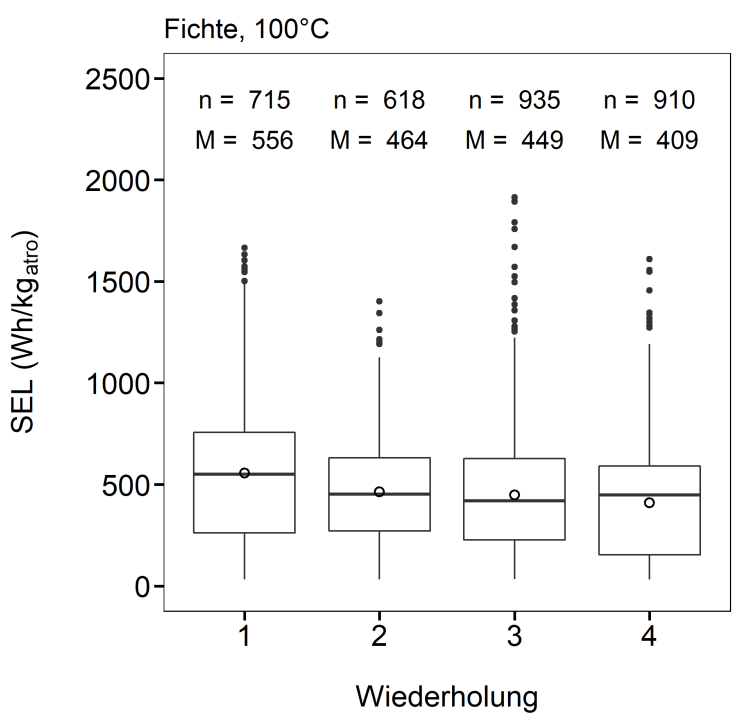

$\mathbf{b}$

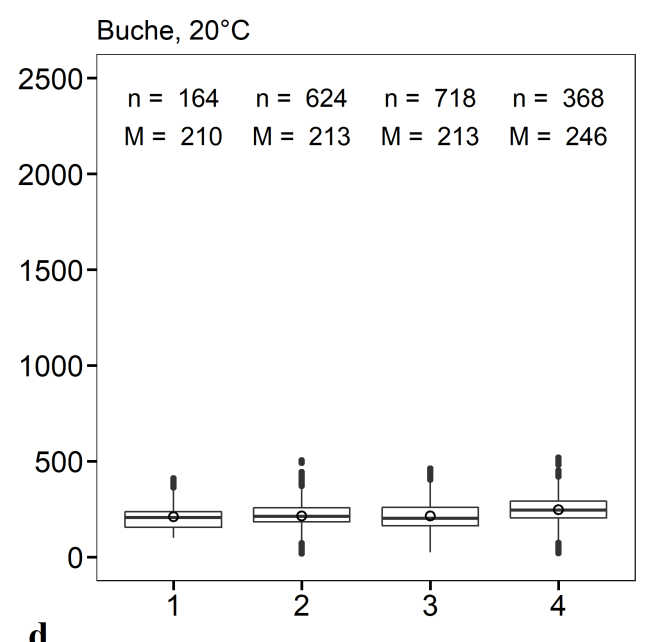

d

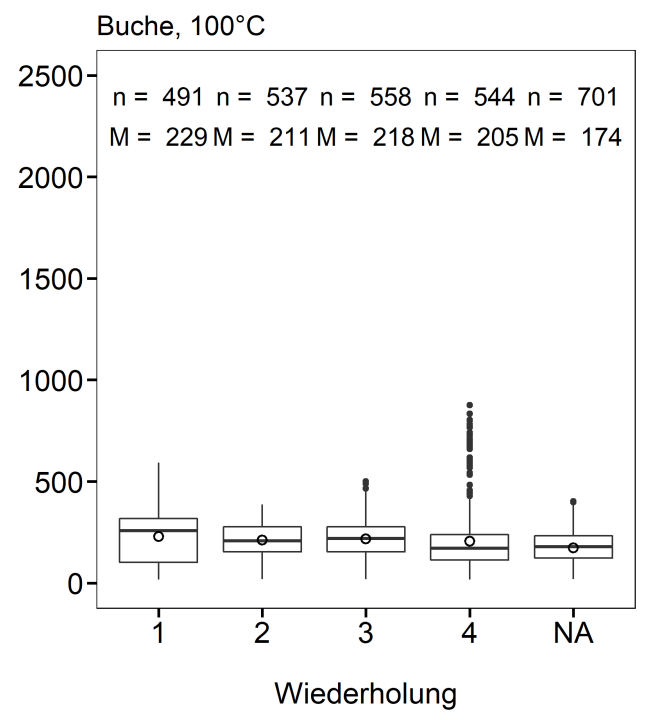

Abbildung 7.29: Darstellung der spezifischen elektrischen Leistungsaufnahme (SEL) aus den Wiederholungen mit dem Mahlscheibenmuster 6. a, c: In der linken Spalte sind die Wiederholungen mit der Holzart Fichte aufsteigend nach der Sattdampftemperatur dargestellt. $\boldsymbol{b}, \boldsymbol{d}:$ In der rechten Spalte sind die Wiederholungen mit der Holzart Buche aufsteigend nach der Sattdampftemperatur dargestellt. n: Anzahl der Messpunkte, M: Arithmetisches Mittel der SEL.

Unterschied von $11 \%$ und bei der Holzart Buche lediglich eine geringe Signifikanz bezüglich der spezifischen elektrischen Leistungsaufnahme besteht. Die spezifische elektrische Leistungsaufnahme im RMP-Verfahren liegt im Vergleich zum TMP-Verfahren im Bereich der Aufschlusstemperatur von $135^{\circ} \mathrm{C}$ und damit niedriger als erwartet.

Die relativ breite Streuung der spezifischen elektrischen Leistungsaufnahme bei der Holzart Fichte kann mit den Beobachtungen in Abbildung 7.31 erklärt werden. Die Darstellung in Abbildung 7.31a zeigt starke periodische Schwankungen der Leistung des Hauptmotors der Laborrefineranlage. Die Schwankungen sind vermutlich auf eine ungleichmäßige Hackschnitzelzuführung durch die Einspeiseschnecke in den Mahlspalt zurückzuführen. Ähnliche Phänomene wurden 
a

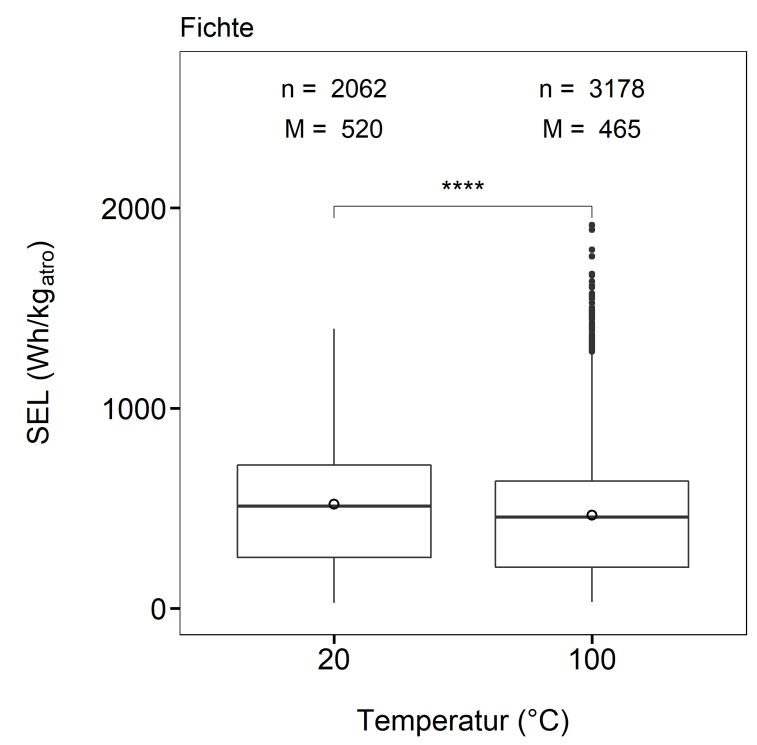

b

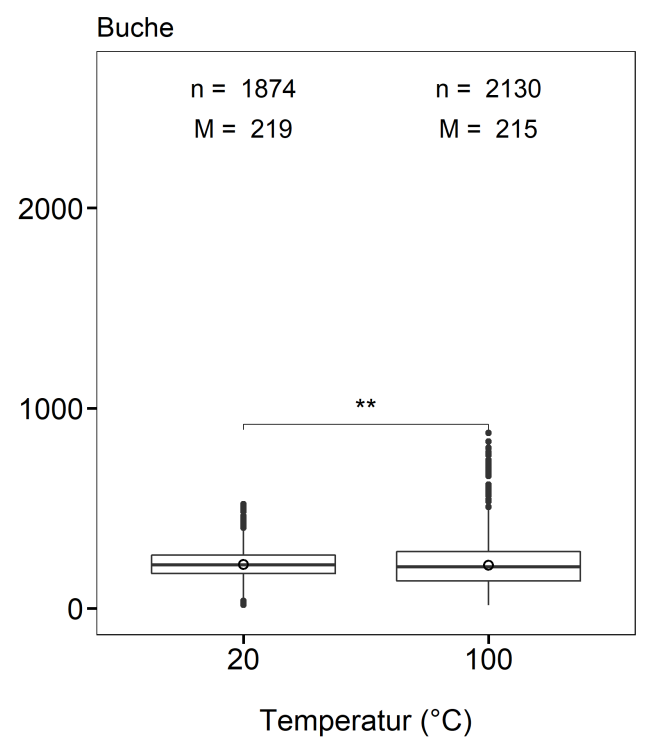

Abbildung 7.30: Darstellung des arithmetischen Mittelwerts der spezifischen elektrischen Leistungsaufnahme (SEL) über der Sattdampftemperatur aus den Versuchen mit dem Mahlscheibenmuster 6. a, Holzart Fichte. b, Holzart Buche. n: Anzahl der Messpunkte, M: Arithmetisches Mittel der SEL. N=4, Kruskall-Wallis-Test Signifikanzcode: $0,0001^{* * * *} ; 0,001^{* * *} ; 0,01^{* *} ; 0,05{ }^{*}$.

bereits von Neill \& Beath (1963) beschrieben und durch eine gleichmäßigere Hackschnitzelzuführung verbessert. Der in Abbildung 7.31b dargestellte Temperaturanstieg im Kocher der Laborrefineranlage wird durch die Dampfentwicklung zwischen den Mahlscheiben hervorgerufen. Atack \& Stationwalla (1975) stellten bei einer vergleichbaren Leistungsaufnahme eine Temperatur von $110^{\circ} \mathrm{C}$ in der Peripherie der Mahlscheibe fest. Die Konsistenz der Hackschnitzel lag dabei durch die Zugabe von Verdünnungswasser mit ca. $20 \%$ deutlich niedriger. Die Untersuchungen von Peterson et al. (1973) und Dana et al. (1975) ergaben, dass der zwischen den Mahlscheiben entstehende Dampf für die „Eigendruckbeaufschlagung“ der Hackschnitzelvorwärmung genutzt werden kann.

Bei der Holzart Buche sind die Leistungsschwankungen nicht so stark ausgeprägt wie mit der Holzart Fichte (Abbildung 7.32a). Der Temperaturanstieg ist allerdings in der selben Höhe wie bei der Holzart Fichte zu beobachten (Abbildung 7.32b).

Die Faserstofferzeugung im RMP-Verfahren war anspruchsvoll, da die Auslegung der Laborrefineranlage zwar den Betrieb im RMP-Verfahren ermöglicht, aber insbesondere die Hackschnitzelbehandlung und -zuführung auf das TMP-Verfahren ausgelegt ist. Weitere Untersuchungen sind notwendig, um die Faserstofferzeugung im RMP-Verfahren mit angepassten Anlagenkomponenten und weiterentwickelten Mahlscheiben zu optimieren (Holzer et al. 1962). Prinzipiell ist es möglich, den Schwierigkeiten mit einer zweiten Zerfaserungsstufe zu begegnen, wie von Pearson (1990) vorgeschlagen. Demzufolge soll die spezifische elektrische Leistungsaufnahme um $6 \%$ reduziert und die Standzeit der Mahlscheiben um 30 \% erhöht werden. Es sind auch Kombinationen von Erwärmung unter Sattdampfdruck und Zerfaserung unter Atmosphärendruck durch Peterson et al. (1973) erfolgreich untersucht worden. Auch die Vorbehandlung mit Natriumsulfit bzw. einer Kombination von Natriumsulfit und Natriumhydroxid bei Laubhölzern wurde beispielsweise von 
Fichte, $20^{\circ} \mathrm{C}$

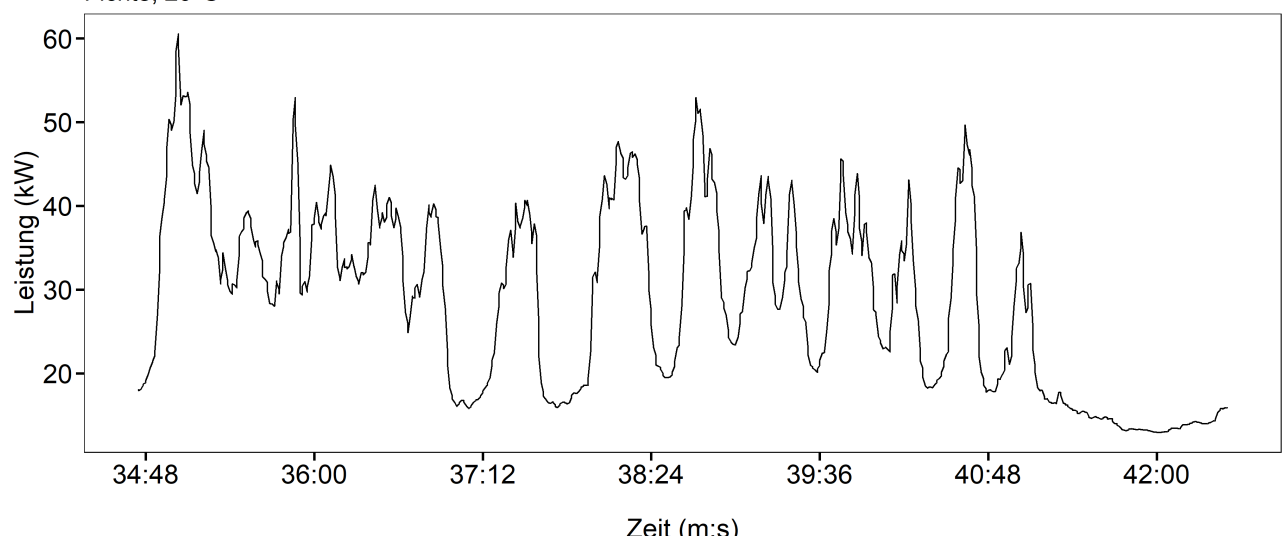

b

Fichte, $20^{\circ} \mathrm{C}$

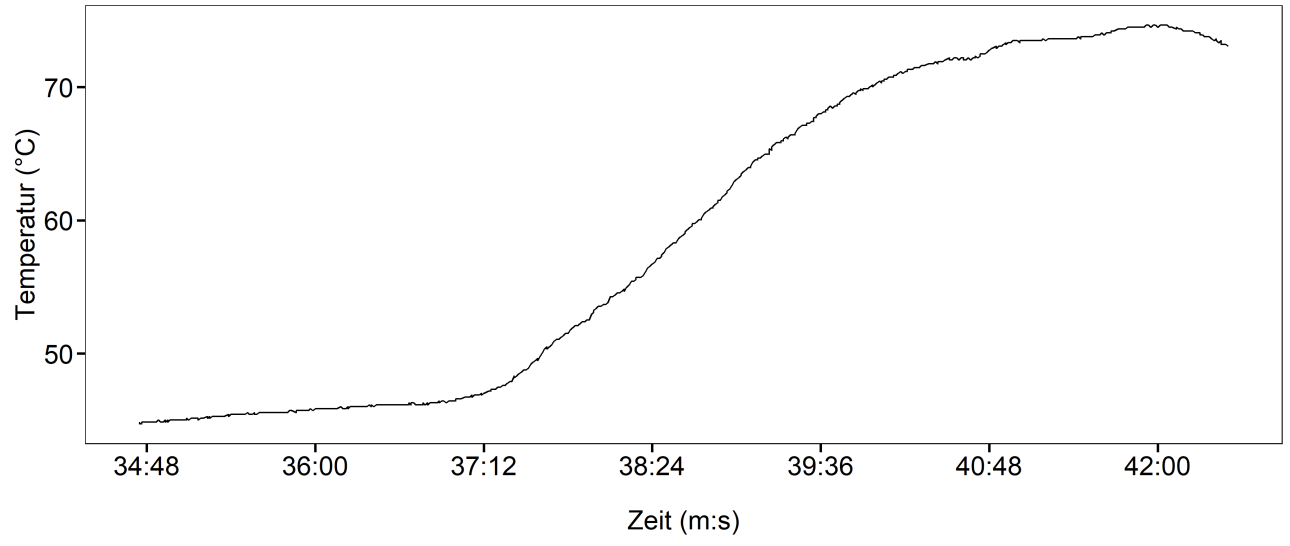

Abbildung 7.31: Technologische Beobachtungen in der Zerfaserung von Fichtenholz-Hackschnitzeln nach dem RMP-Verfahren. a, Darstellung der periodischen Schwankungen der elektrischen Leistung des Hauptmotors der Laborrefineranlage. $\boldsymbol{b}$, Temperaturanstieg in den Hackschnitzeln während der Zerfaserung mit einer Ausgangstemperatur von $20^{\circ} \mathrm{C}$.

Keays \& Leask (1973) untersucht und ist als CMP-Verfahren bekannt. 


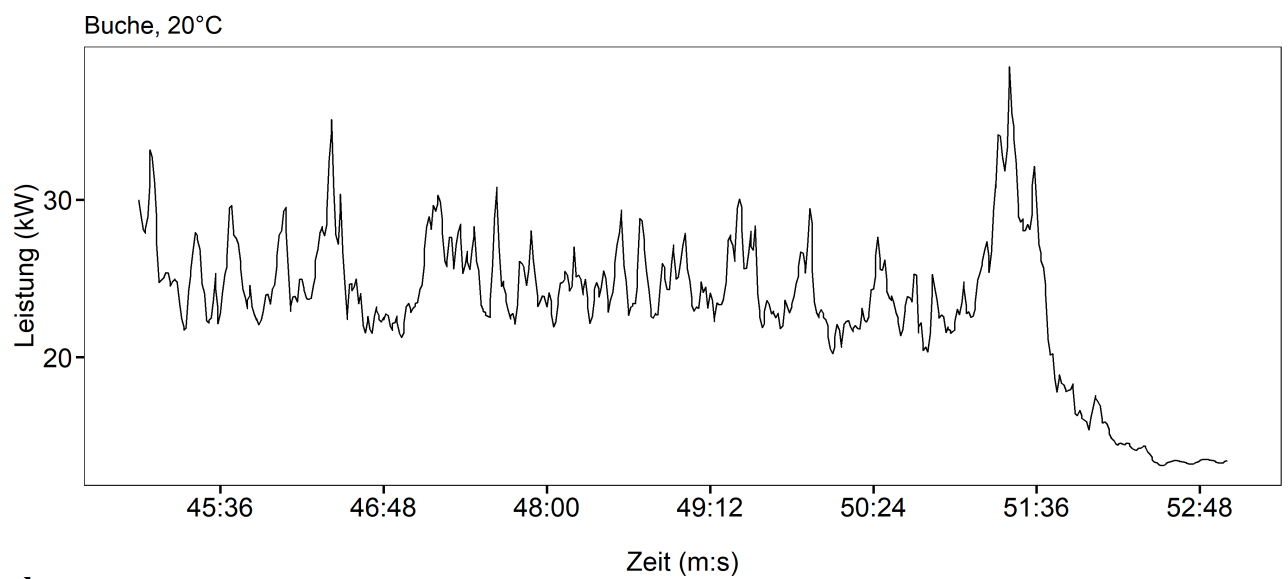

b

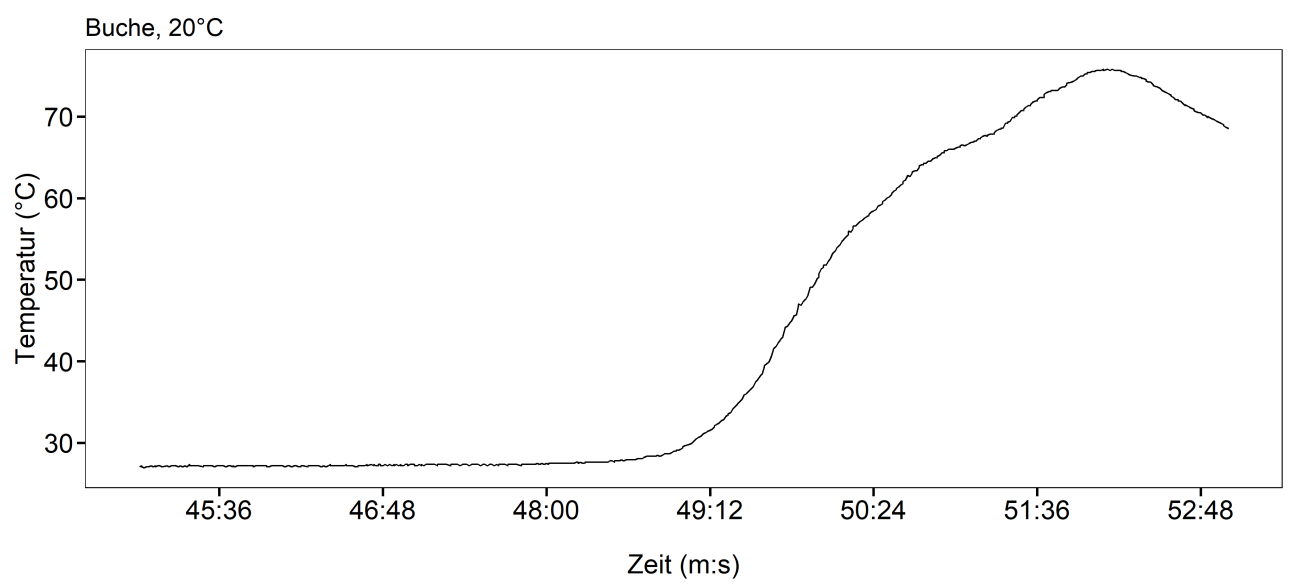

Abbildung 7.32: Technologische Beobachtungen in der Zerfaserung von Buchenholz-Hackschnitzeln nach dem RMP-Verfahren. a, Darstellung der periodischen Schwankungen der elektrischen Leistung. b, Temperaturanstieg in den Hackschnitzeln während der Zerfaserung mit einer Ausgangstemperatur von $20^{\circ} \mathrm{C}$.

\subsection{Untersuchungen zum elektrischen und thermischen Energiebedarf in der Faserstofferzeugung}

\subsubsection{Einleitung}

In Ergänzung zu den Untersuchungen der Holzfeuchte von Hackschnitzeln in Sattdampfatmosphäre als auch der spezifischen elektrischen Leistungsaufnahme werden im folgenden Abschnitt weitere technologische Teilaspekte der Faserstofferzeugung betrachtet. Die Faserstofferzeugung für Holzwerkstoffe ist ein energieaufwändiger Prozess. Bezüglich der HolzfaserdämmstoffProduktion ist durch Braun (2014) bekannt, dass die Faserstofferzeugung einen Anteil zwischen $32 \%$ und $44 \%$ am elektrischen Energiebedarf der gesamten Produktion hat. Zusätzlich trägt die Dampferzeugung für das TMP-Verfahren mit $16 \%$ bis $23 \%$ und der Stromrohrtrockner mit rund $74 \%$ zum thermischen Energiebedarf der gesamten Produktion bei. Der Anteil der elektrischen Energie am gesamten Energiebedarf der Produktion kann folglich auf rund $10 \%$ bis $20 \%$ beziffert werden (Braun 2014).

Hier offenbart sich ein entscheidender Nachteil der Holzfaserdämmstoffe gegenüber den syntheti- 
schen und mineralischen Wettbewerbern: Das Erwärmen und Trocknen des Holzbestandteils Wasser mit seiner hohen spezifischen Wärmekapazität. Der Argumentation, dass in Holzwerkstoffwerken ausreichend und quasi kostenfrei thermische Energie zur Verfügung steht, muss entgegengehalten werden, dass die Erzeugung von thermischer Energie aus Reststoffen oder Koppelprodukten Wartungs-, Instandhaltungs- und Betriebs- sowie Investitionskosten verursacht. Die Kosten für eine Kilowattstunde Dampf belaufen sich, Schätzungen aus der MDF-Produktion zufolge, auf ein Viertel bis ein Drittel der Kosten für die selbe Energieeinheit Strom. Reicht die im Werk aus eigenen Reststoffen bereitgestellte thermische Energie nicht aus, muss außerdem auf fossile Energieträger zurückgegriffen oder auch Biomasse als Brennstoff zugekauft werden.

Das Ziel der weiteren energietechnischen Untersuchungen der Faserstofferzeugung ist es, den Stand der Wissenschaft und Technik zum Erweichungsbereich von Fichten- und Buchenholz anhand der Untersuchungen zur spezifischen elektrischen Leistungsaufnahme aus den Mahlscheibentests zu überprüfen. Darüber hinaus ist es von wissenschaftlichem und wirtschaftlichem Interesse, die Untersuchungen zur Holzfeuchte in Sattdampfatmosphäre (s. Abschnitt 7.2) mit Messungen zur Konsistenz nach der Zerfaserung in Beziehung zu dem elektrischen und thermischen Energiebedarf in der Faserstofferzeugung zu setzen.

\subsubsection{Spezifische elektrische Leistungsaufnahme als Funktion der Temperatur}

Die Untersuchungen von Asplund (1940a) mit Laubholz (Birke, Espe) und Nadelholz (Kiefer, Fichte) zur Abhängigkeit der spezifischen elektrischen Leistungsaufnahme von der Aufschlusstemperatur werden auch heute noch als Grundlage für die Prozessführung im TMP-Verfahren der Holzwerkstoffherstellung herangezogen (Wenderdel et al. 2013). Dabei sollte beachtet werden, dass die Untersuchungen seinerzeit mit der ursprünglichen Entwicklung Asplunds durchgeführt wurden: Einer Art Hammermühle in einem Druckbehälter (Asplund 1940a). Die Zerkleinerungskinetik dieses Defibrators unterscheidet sich von modernen Refineranlage allerdings u.a. durch die Beanspruchungszeit der Faserstoffe zwischen 4 min und 13 min, wie in Muguet et al. (2012) beschrieben, gegenüber ca. 0,5 s auf der Mahlbahn moderner Refiner, wie Murton et al. (2002) postulierten.

Die Ergebnisse aus Abschnitt 7.3 sollen deshalb dazu genutzt werden, die spezifische elektrische Leistungsaufnahme als Funktion der Temperatur in Abhängigkeit der untersuchten Mahlscheibenmuster darzustellen. Dies soll zu einem besseren Verständnis des Erweichungsverhaltens von Fichten- und Buchenholz im TMP-Verfahren beitragen. In Abbildung 7.33 ist die spezifische elektrische Leistungsaufnahme für die Faserstofferzeugung mit der Holzart Fichte über der Aufschlusstemperatur aufgetragen. In Abbildung 7.33a zeigt die polynomiale Regression, die alle Mahlscheibenmuster sowie das RMP- und TMP-Verfahren umfasst, den von Asplund beschriebenen Zusammenhang. Diese negative Korrelation zwischen spezifischer elektrischer Leistungsaufnahme und Sattdampftemperatur wurde auch von Solala et al. (2014), mit der Weiterentwicklung des ursprünglichen Asplund-Defibrators (wing defibrator) und der Holzart Fichte (Picea abies) zwischen $130^{\circ} \mathrm{C}$ und $170^{\circ} \mathrm{C}$, bestätigt. Im Gegensatz zu Asplund war ein deutlicher Rückgang der spezifischen elektrischen Leistungsaufnahme hier bereits ab $130^{\circ} \mathrm{C}$ zu beobachten. Im Weiteren weisen die Ergebnisse von Krug (2010) mit Kiefernholz darauf hin, dass diese Beziehung auch bei Temperaturen zwischen $170^{\circ} \mathrm{C}$ und $200^{\circ} \mathrm{C}$ und einer längeren Verweilzeit besteht. Auf Basis der Ergebnisse der vorliegenden Arbeit kann der Versuch unternommen werden, den Stand der Wissenschaft und Technik differenzierter zu betrachten und zu ergänzen. In Abbildung 7.33a wird deutlich, dass der oben beschriebene Zusammenhang zwischen Aufschlusstemperatur und spezifischer elektrischer Leistungsaufnahme im TMP-Verfahren prinzipiell gültig ist. Die 
Abnahme der spezifischen elektrischen Leistungsaufnahme hängt aber auch mit dem verwendeten Mahlscheibenmuster zusammen und scheint mit den Beobachtungen von Solala et al. (2014) übereinzustimmen. Das betrifft insbesondere die niedrigeren Aufschlusstemperaturen $135^{\circ} \mathrm{C}$ und $150^{\circ} \mathrm{C}$. Bei einer Sattdampftemperatur von $175^{\circ} \mathrm{C}$ liegen die Werte der Mahlscheibenmuster bezüglich der spezifischen elektrischen Leistungsaufnahme dicht zusammen. Der Trend im RMP-Verfahren liegt überraschenderweise umgekehrt. Vom Einfluss der Mahlscheibenmuster und der Vergleichbarkeit der Beschaffenheit der erzeugten Faserstoffe abgesehen, macht es den Anschein, als dass die spezifische elektrische Leistungsaufnahme bei der Faserstofferzeugung ohne Erwärmung durch Sattdampfdruck $\left(20^{\circ} \mathrm{C}\right)$ niedriger liegt. Gleichzeitig wird in Abbildung 7.33b deutlich, dass die Gestaltung der Mahlscheibe einen Einfluss auf die Steigung der Temperaturabhängigkeit hat. Die Mahlscheibenmuster 2 und 5 verursachen zwischen $135^{\circ} \mathrm{C}$ und $150^{\circ} \mathrm{C}$ die stärkste Reduzierung der spezifischen elektrischen Leistungsaufnahme.

a

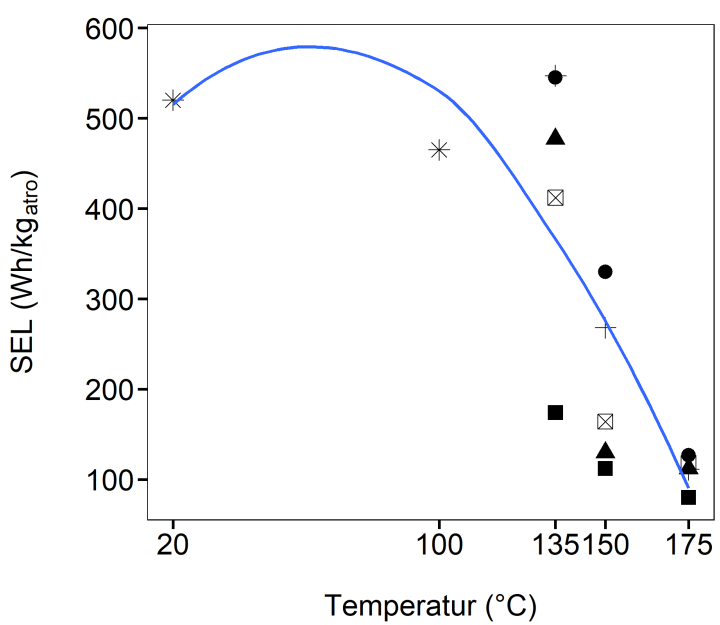

b

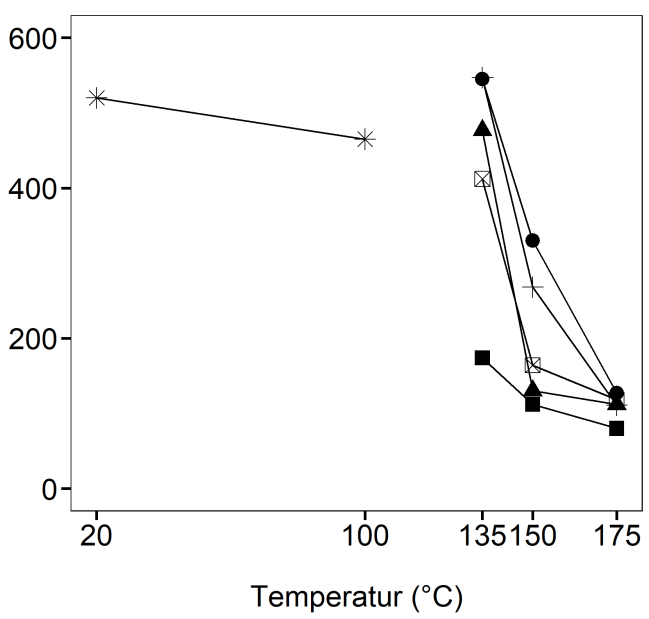

1 - $3 \otimes 5$

- $2+4 * 6$

Abbildung 7.33: Spezifische elektrische Leistungsaufnahme als Funktion der Temperatur in der mechanischen Faserstofferzeugung mit der Holzart Fichte. Temperaturbereich $20^{\circ} \mathrm{C}$ bis $100^{\circ} \mathrm{C}$ : RMP-Verfahren. Temperaturbereich $135^{\circ} \mathrm{C}$ bis $175^{\circ}$ : TMP-Verfahren. a, Polynomiale Regression nach Methode LOESS. b, Verbindung der Messpunkte zur Kenntlichmachung des Einflusses des Mahlscheibenmusters. $n=3-4$.

Mit der Holzart Buche verhält es sich ähnlich wie mit der Holzart Fichte, wie Abbildung 7.34 veranschaulicht. Die beschriebene Temperaturabhängigkeit und der Einfluss der Mahlscheibenmuster kann auch hier festgestellt werden (Abbildung 7.34a). Allerdings weisen die Ergebnisse auf größere Unterschiede bezüglich der spezifischen elektrischen Leistungsaufnahme bei höheren Aufschlusstemperaturen hin. In Abbildung 7.34b weisen die Mahlscheibenmuster 1, 4 und 5 eine überproportionale Abnahme der spezifischen elektrischen Leistungsaufnahme zwischen $135^{\circ} \mathrm{C}$ und $150^{\circ} \mathrm{C}$ auf. Die Ergebnisse belegen weiterhin die generell niedrigere spezifische elektrische Leistungsaufnahme mit der Holzart Buche im Vergleich zur Holzart Fichte. Dies betrifft auch die absolute Reduzierung der spezifischen elektrischen Leistungsaufnahme im Zusammenhang mit der Aufschlusstemperatur, die hier niedriger ausfällt. Einen Erklärungsansatz für dieses Phänomen bietet der höhere Methoxygehalt von Buchenlignin. Damit einher geht eine geringere Anzahl 
kondensierter Bindungen im Lignin, der unterhalb dem der Nadelhölzer liegt (Olsson \& Salmén 1997). Laubhölzer weisen außerdem einen höheren Anteil an Xylanen als Nadelhölzer auf und unterscheiden sich auch in der Konstitution der Gluco-/Mannane sowie der Seitenketten, wodurch die niedrigere spezifische elektrische Leistungsaufnahme auch begründet werden kann (Fengel \& Wegener 2003, S. 106). Im Gegensatz zur Faserstofferzeugung im RMP-Verfahren mit der Holzart Fichte weisen die Ergebnisse in Abbildung 7.34b auf eine geringfügige Reduzierung der spezifischen elektrischen Leistungsaufnahme durch die Erwärmung der Buchenholz-Hackschnitzel mit Sattdampf hin.

a

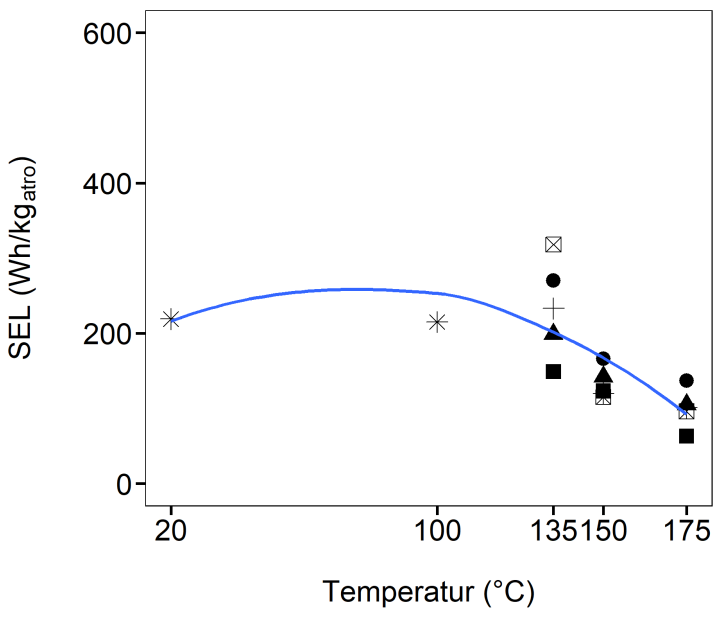

b

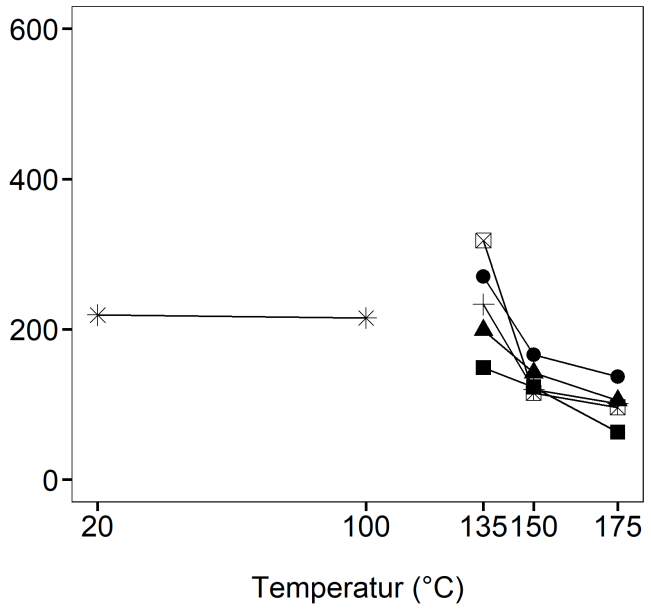

- 1 - $3 \otimes 5$

வ $2+4 * 6$

Abbildung 7.34: Spezifische elektrische Leistungsaufnahme als Funktion der Temperatur in der mechanischen Faserstofferzeugung mit der Holzart Buche. Temperaturbereich $20^{\circ} \mathrm{C}$ bis $100^{\circ} \mathrm{C}$ : RMP-Verfahren. Temperaturbereich $135^{\circ} \mathrm{C}$ bis $175^{\circ}$ : TMP-Verfahren. a, Polynomiale Regression nach Methode LOESS. b, Verbindung der Messpunkte zur Kenntlichmachung des Einflusses des Mahlscheibenmusters. $n=3-4$.

Wie in Abschnitt 2.3.2 beschrieben, liegen der untersuchte Temperaturbereich sowie die Holzfeuchte im TMP-Verfahren weit über dem Glasübergangsbereich von Hemizellulose und den amorphen Bereiche der Zellulose. Das kann auch für das RMP-Verfahren angenommen werden. Folglich spielt das Lignin als steifste verbleibende Komponente im Zellverbund eine bedeutende Rolle für den elektrischen Energiebedarf in der Zerfaserung. Der „Knick“ der polynomialen Regression bei ca. $100^{\circ} \mathrm{C}$ (Abbildungen 7.33a und 7.34a) könnte auf die beginnende Ligninerweichung zwischen 100 und $135^{\circ} \mathrm{C}$ hinweisen. Diese Beobachtung stimmt mit der Angabe von $120^{\circ} \mathrm{C}$ nach Becker et al. (1977) und Blechschmidt et al. (1986), respektive $120^{\circ} \mathrm{C}$ bis $135^{\circ} \mathrm{C}$ nach Atack (1972) überein. Allerdings wurden in der vorliegenden Arbeit keine Messungen in diesem Temperaturbereich durchgeführt. Back \& Salmén (1982) stellten einen sigmoidalen Verlauf der spezifischen elektrischen Leistungsaufnahme zwischen $100^{\circ} \mathrm{C}$ und $190^{\circ} \mathrm{C}$ fest, dessen größte Steigung im Temperaturbereich zwischen ungefähr $130^{\circ} \mathrm{C}$ und ungefähr $170^{\circ} \mathrm{C}$ liegt. Diese Beobachtungen werden durch Koran (1981) insofern bestätigt, als dass der Energieaufwand zur Trennung des Zellverbunds bei Temperaturen über $170^{\circ} \mathrm{C}$ vernachlässigbar klein wird. Dies könnte auch die geringen Differenzen in der spezifischen elektrischen Leistungsaufnahme zwischen allen 
Mahlscheibenmustern bei $175^{\circ} \mathrm{C}$ erklären (s. Abbildung 7.33). Die vorliegenden Untersuchungen passen, insbesondere durch die polynomiale Regression in Abbildung 7.34a und Abbildung 7.33a, in das Muster der beschriebenen Literaturstellen. Es konnte allerdings ein erheblicher Einfluss der Mahlscheiben auf den Verlauf und die Höhe der spezifischen elektrischen Leistungsaufnahme nachgewiesen werden.

Die vorliegenden Untersuchungen bestätigen zudem die Temperaturabhängigkeit der spezifischen elektrischen Leistungsaufnahme. Die in Abschnitt 2.3.2 zusammengetragenen Erkenntnisse aus der Grundlagenforschung zum Erweichungsverhalten liefern allerdings keine weiteren schlüssigen Erklärungen oder Anwendungen für die beobachteten Phänomene in der Faserstofferzeugung. Durch die experimentelle Untersuchung konnte kein Zusammenhang zwischen M', tan $\delta$ und $T_{g}$ und der spezifischen elektrischen Leistungsaufnahme im RMP- oder TMP-Verfahren gefunden werden. Die Methoden der DMA oder DMTA spiegeln nicht die tatsächlichen Prozessbedingungen (Sattdampfdruck, Temperatur, Holzfeuchte, Beanspruchungsart, -frequenz und - zeit) im RMPoder TMP-Verfahren wider.

$\mathrm{Zu}$ dem in der Literatur angegebenen Beanspruchungsfrequenzbereich der Mahlscheiben konnte im Rahmen der Literaturrecherche der vorliegenden Dissertation keine Gleichung zur Berechnung derselben gefunden werden. Von Eriksen et al. (2006) sind Messungen der Beanspruchungsfrequenz in einem Twin-Refiner bekannt, die in etwa älteren Literaturangaben entsprechen. Daraus kann Gleichung 7.7 für die Berechnung der Beanspruchungsfrequenz abgeleitet werden:

$$
f=\frac{N}{n}
$$

$f \quad$ Beanspruchungsfrequenz in $\mathrm{Hz}$

$N \quad$ Anzahl der Stege in einem bestimmten Bereich der Mahlbahn des Rotors

$n \quad$ Umdrehungen des Rotors pro Sekunde

Mit Gleichung 7.7 wurde die Frequenz der untersuchten Mahlscheiben berechnet. Der Theorie zufolge müsste eine höhere Beanspruchungsfrequenz bei derselben Aufschlusstemperatur eine höhere spezifische elektrische Leistungsaufnahme nach sich ziehen. Das konnte nicht beobachtet werden, wie Abbildung 7.35 belegt. Dagegen sprechen auch Untersuchungen von Ferguson (1997), die zeigen, dass sich durch eine höhere Rotationsgeschwindigkeit der Mahlscheiben die spezifische elektrische Leistungsaufnahme senken lässt. In der mechanischen Faserstofferzeugung geschehen viele Prozesse gleichzeitig, sodass es schwierig ist, einzelne Aspekte, wie die Beanspruchungsfrequenz zu charakterisieren, wie auch Salmén et al. (1999, 36 f.) anmerken. Die Gestaltungsmerkmale einer Mahlscheibe, wie Stegwinkel oder -krümmung sowie Anzahl und Position der Dämme, scheinen ausschlaggebender zu sein. 

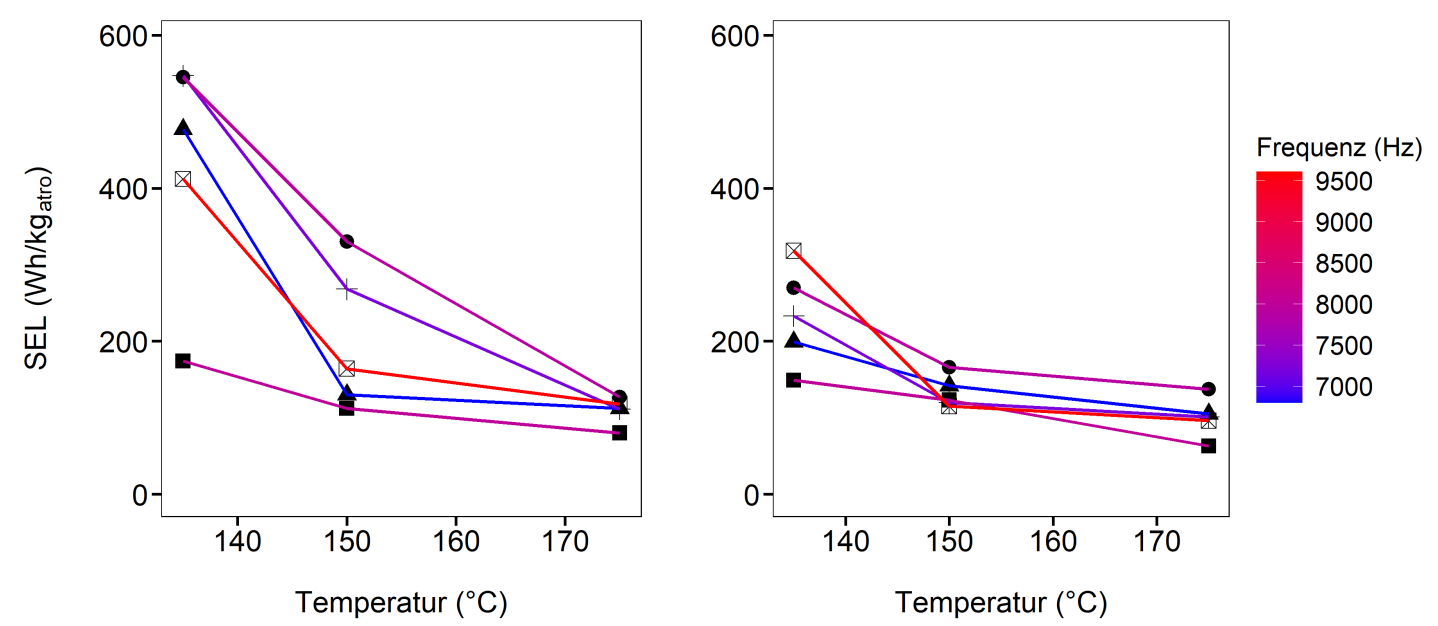

Mahlscheibenmuster

- 1 - $3 \otimes 5$

$2+4 * 6$

Abbildung 7.35: Zusammenhang zwischen spezifischer elektrischer Leistungsaufnahme (SEL), Aufschlusstemperatur und Beanspruchungsfrequenz unterschiedlicher Mahlscheibenmuster. Aufschluss mit dem TMP-Verfahren. a, Holzart Fichte. b, Holzart Buche. $n=4$.

\subsubsection{Holzfeuchte und Konsistenz nach dem Aufschluss}

Über die Untersuchung zur Holzfeuchte von Hackschnitzeln in Sattdampfatmosphäre (vgl. Abschnitt 7.2) hinaus ist die Feuchte und Konsistenz der Faserstoffe nach dem Aufschluss vor dem Hintergrund des elektrischen und thermischen Energiebedarfs in der Faserstofferzeugung von Interesse. Es ist nicht bekannt, in welcher Beziehung die Aufschlussbedingungen und die spezifische elektrische Leistungsaufnahme mit der Holzfeuchte bzw. der Konsistenz stehen und ob in der Prozessführung möglicherweise darauf Einfluss genommen werden kann. Zu diesem Zweck wurde in den Untersuchungen zur spezifischen elektrischen Leistungsaufnahme in der Faserstofferzeugung (vgl. Abschnitt 7.3) ebenfalls die Konsistenz der Faserstoffe nach dem Aufschluss erfasst. Die Probennahme erfolgte unmittelbar am unteren Auslass des Zyklons der Laborrefineranlage, in welchem die Faserstoffe vom Dampf getrennt werden.

Die Ergebnisse der Konsistenzmessungen sind in den Abbildungen 7.36 und 7.37 zusammenfassend dargestellt. Wird die Konsistenz über der Aufschlusstemperatur aufgetragen, zeigt die polynomiale Regression in Abbildung 7.36a, dass der Anteil der Trockenmasse im RMP-Verfahren, trotz Erwärmung der Hackschnitzel mit Sattdampf bis ca. $100^{\circ} \mathrm{C}$, zunimmt. Weiter im TMP-Verfahren bis $175^{\circ} \mathrm{C}$ weist die Regression eine kontinuierliche negative Steigung auf. Das bedeutet, dass die Wassersättigung bzw. der Wasseranteil zunimmt. In Abbildung 7.33 zeigt die spezifische elektrische Leistungsaufnahme einen ähnlichen Verlauf. Das Phänomen kann, zumindest für den Temperaturbereich von $100^{\circ} \mathrm{C}$ bis $175^{\circ} \mathrm{C}$, durch eine Abhängigkeit der Konsistenz von der spezifischen elektrischen Leistungsaufnahme erklärt werden. Die Zerfaserung bei niedrigeren Temperaturen erfordert mehr elektrische Energie, da das Material steifer ist und größere Mengen an mechanischer Energie absorbiert (vgl. Abschnitt 2.3.2). Die Reibungsarbeit zwischen den Mahlscheiben führt zu einer stärkeren Erwärmung des Materials und die Holzfeuchte verdampft teilweise. Über die Dampfentwicklung zwischen den Mahlscheiben berichteten u.a. Peterson et al. 
(1973) und Dana et al. (1975). Dieser Erklärungsansatz wird auch durch die Beobachtung gestützt, dass beispielsweise das Mahlscheibenmuster 1 jeweils die höchste Konsistenz und die höchste spezifische elektrische Leistungsaufnahme sowie das Mahlscheibenmuster 3 jeweils die niedrigste Konsistenz und die niedrigste spezifische elektrische Leistungsaufnahme aufweisen.

Deutlich wird der Effekt in Abbildung 7.36b. Hier stellen die gestrichelten Linien den Bereich der Konsistenz der Hackschnitzel vor dem Aufschluss dar. Die Balken geben die berechnete Konsistenz der Hackschnitzel in Sattdampfatmosphäre an (vgl. Abschnitt 7.2). Je höher die Aufschlusstemperatur und je geringer die spezifische elektrische Leistungsaufnahme, desto niedriger ist die Konsistenz der erzeugten Faserstoffe direkt nach dem Aufschluss. Das bedeutet, dass die Aufschlusstemperatur und die Mahlscheiben einen direkten Einfluss auf die Konsistenz und damit auf den thermischen Energiebedarf in der Faserstoff-Trocknung haben.

a

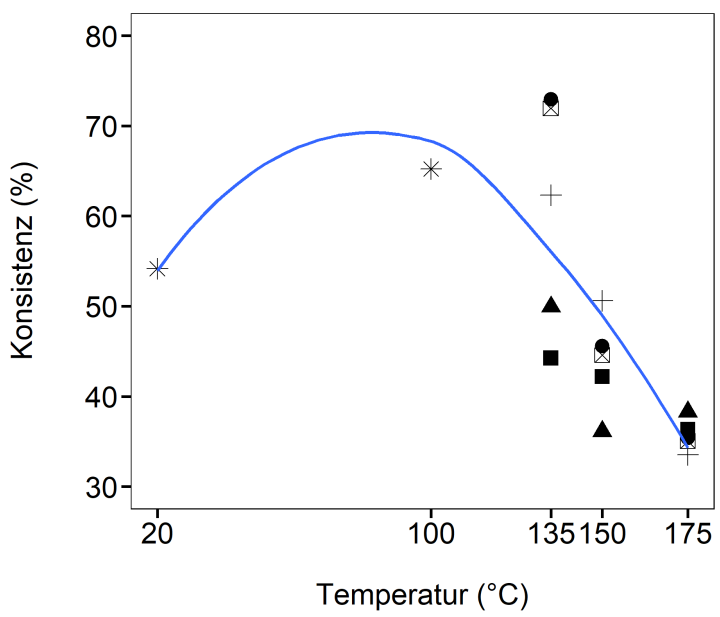

b

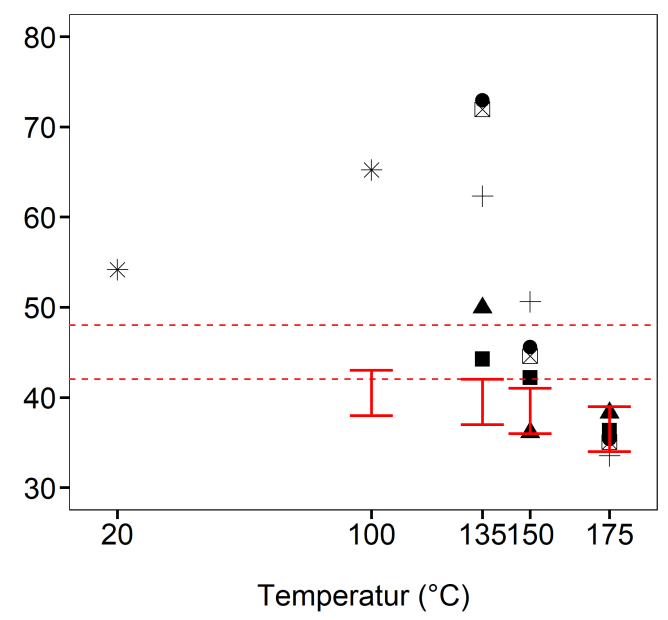

- 1 - $3 \otimes 5$

Abbildung 7.36: Arithmetische Mittelwerte der Konsistenz der Faserstoffe aus Fichte nach dem Aufschluss im RMP- $\left(20^{\circ} \mathrm{C}\right.$ bis $\left.100^{\circ} \mathrm{C}\right)$ und TMP-Verfahren $\left(135^{\circ} \mathrm{C}\right.$ bis $\left.175^{\circ} \mathrm{C}\right)$ mit unterschiedlichen Mahlscheiben. a, Polynomiale Regression nach Methode LOESS. $\boldsymbol{b}$, Die gestrichelten Linien markieren die Standardabweichung der Konsistenz der Hackschnitzel vor dem Einfüllen in den Kocher. Die Balken zeigen die Konsistenz der Hackschnitzel infolge der Erwärmung in Sattdampfatmosphäre vor der Zerfaserung an. $n=12$.

Bei der Holzart Buche sind die Zusammenhänge ähnlich wie bei der Holzart Fichte (vgl. Abbildung 7.37). Aufgrund der höheren Rohdichte des Buchenholzes liegen die Anfangs-Konsistenz und die Konsistenz der Faserstoffe vergleichsweise höher. Der Argumentation mit der Holzart Fichte folgend, führt die niedrigere spezifische elektrische Leistungsaufnahme bei der Holzart Buche zu geringeren Differenzen zwischen der berechneten und der gemessenen Konsistenz in Abbildung 7.37b. Die offenkundigen Abweichungen der Werte bei $150^{\circ} \mathrm{C}$, die teilweise höher liegen als bei $135^{\circ} \mathrm{C}$, sind auf das Anlagenkonzept zurückzuführen. Obwohl die Messungen reproduzierbar sind muss berücksichtigt werden, das die Probennahme unterhalb des Zyklons nicht die tatsächliche Konsistenz nach dem Aufschluss widerspiegelt. Über den Zyklon entweicht der hauptsächliche Anteil des Dampfes und die Faserstoffe geben hier ebenfalls Feuchte ab. 
a

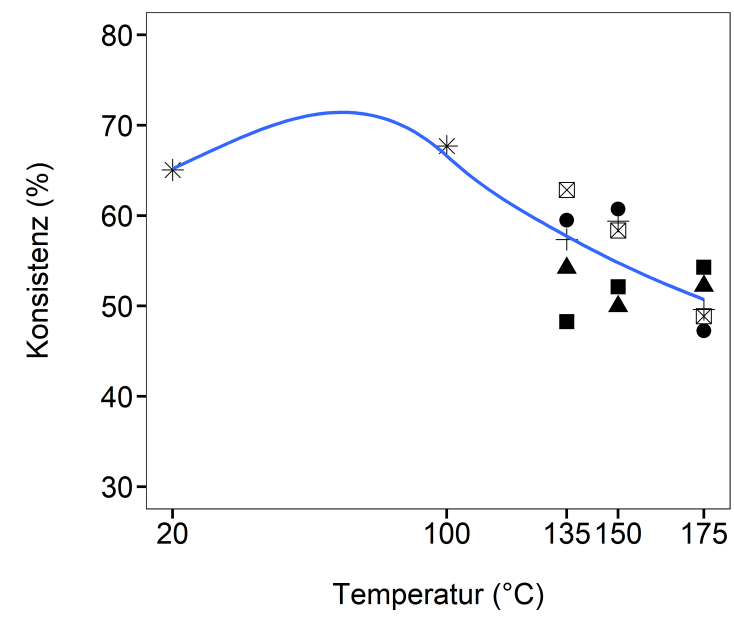

b

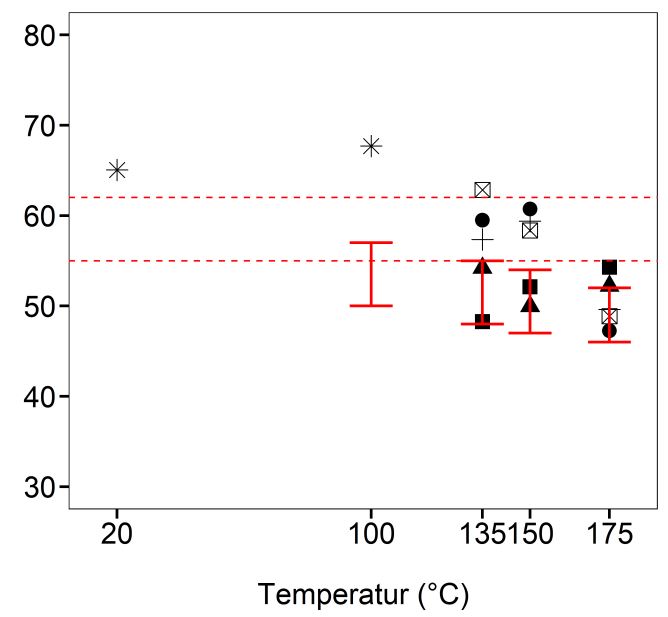

- 1 - $3 \otimes 5$

- $2+4 * 6$

Abbildung 7.37: Arithmetische Mittelwerte der Konsistenz der Faserstoffe aus Buche nach dem Aufschluss im RMP- $\left(20^{\circ} \mathrm{C}\right.$ bis $\left.100^{\circ} \mathrm{C}\right)$ und TMP-Verfahren $\left(135^{\circ} \mathrm{C}\right.$ bis $\left.175^{\circ} \mathrm{C}\right)$ mit unterschiedlichen Mahlscheiben. a, Polynomiale Regression nach Methode LOESS. b, Die gestrichelten Linien markieren die Standardabweichung der Konsistenz der Hackschnitzel vor dem Aufschluss. Die Balken stehen für die, der Sattdampftemperatur infolge der Hackschnitzelerwärmung entsprechende Konsistenz. $n=12$.

Für das TMP-Verfahren in der Papierherstellung gibt Kerekes (2015) eine Konsistenz unmittelbar vor der Zerfaserung zwischen $30 \%$ und $40 \%$ für Nadelhölzer an. Diese Werte werden typischerweise durch Zugabe von Verdünnungswasser eingestellt. Atack \& Wood (1973) und Miles \& May (1990) stellten fest, dass mit steigender Konsistenz bis $25 \%$ der elektrische Energiebedarf in der Zerfaserung abnimmt. Aus der Herstellung von MDF oder Holzfaserdämmstoffen sind keine Zahlen zur Konsistenz bekannt. Es ist davon auszugehen, dass hier die Konsistenz der Hackschnitzel unmittelbar vor der Zerfaserung von der Ausgangs-Holzfeuchte und der Sattdampftemperatur bestimmt wird. Inwiefern eine höhere Konsistenz vor der Zerfaserung Einfluss auf die spezifische elektrische Leistungsaufnahme und die Beschaffenheit der Faserstoffe im RMP- und TMP-Verfahren für die Holzfaserdämmstoffherstellung hat, ist ebenfalls nicht bekannt. Dies zu untersuchen ist die Aufgabe im folgenden Abschnitt.

\subsubsection{Elektrischer und thermischer Energiebedarf in der Faserstofferzeugung mit dem RMP- und dem TMP-Verfahren}

Zum Abschluss der technologischen Untersuchungen der Faserstofferzeugung steht die energietechnische Betrachtung des RMP- und des TMP-Verfahrens im Fokus. Von Interesse ist hierbei, wie sich die Anteile der elektrischen und der thermischen Energie in den bisherigen Untersuchungen zusammensetzen. Das System für die energietechnischen Betrachtungen wird hierzu auf die Hackschnitzelerwärmung mit Sattdampf, die Zerfaserung zwischen den Mahlscheiben und die Trocknung der Faserstoffe ausgehend von der Konsistenz nach dem Aufschluss begrenzt. Weiterhin werden Wärmeverluste und Wirkungsgrade nicht in die Betrachtungen einbezogen. Es wird von einem möglichst idealen System ausgegangen, um grundlegende Zusammenhänge ohne den 
Einfluss von Prozesstechnik erkennen zu können. Die Berechnung des Bedarfs an elektrischer und thermischer Energie in der Faserstofferzeugung umfasst folglich nur grundlegende physikalische Zusammenhänge der Erwärmung, Zerfaserung und Trocknung des Holzes.

Weitere Annahmen zur Berechnung des Energiebedarfs basieren auf den durchgeführten Untersuchungen im Labormaßstab sowie persönlichen Erfahrungen aus der MDF-Produktion und umfassen:

- Die mittlere spezifische elektrische Leistungsaufnahme aus den Untersuchungen in Abschnitt 7.3.

- Die mittlere Ausgangs-Holzfeuchte der Hackschnitzel aus den Untersuchungen in Abschnitt 7.3.

- Die Berechnung der Erwärmung der Hackschnitzel mit Sattdampf aus den Untersuchungen in Abschnitt 7.2.

- Die Trocknung der Faserstoffe auf eine Holzfeuchte von $10 \%$.

- Die Verdampfungswärme des Wassers mit $2260 \mathrm{~kJ} / \mathrm{kg}$ bei einer Temperatur von $100^{\circ} \mathrm{C}$.

Die Berechnung des spezifischen thermischen Energiebedarfs in der Trocknung der Faserstoffe erfolgte nach Gleichung 7.8:

$$
Q_{s p e z}=\frac{m_{W} \cdot r_{W}}{m_{0}}
$$

$$
\begin{array}{ll}
Q_{s p e z} & \text { Spezifischer thermischer Energiebedarf in } \mathrm{Wh} / \mathrm{kg} \\
m_{W} & \text { Masse des zu verdampfenden Wassers } \\
r_{W} & \text { Verdampfungsenthalpie von Wasser } \\
m_{0} & \text { Trockenmasse des Holzes als Bezugsgröße }
\end{array}
$$

In Tabelle 7.10 sind die Ergebnisse nach thermischem, elektrischem und gesamtem Energiebedarf getrennt aufgelistet. Die Berechnungen bestätigen die Beobachtungen und Erklärungsansätze aus den vorhergehenden Abschnitten. Bemerkenswert sind die gegenläufigen thermischen und elektrischen Anteile am Gesamtenergiebedarf mit steigender Aufschlusstemperatur (s. Abbildung 7.10). Die Holzart Buche weist in dem untersuchten Temperaturbereich generell einen deutlich niedrigeren Gesamtenergiebedarf auf. Mit der Holzart Buche wurde der höchste Gesamtenergiebedarf mit dem Mahlscheibenmuster 5 bei $135^{\circ} \mathrm{C}$ ermittelt $(636 \mathrm{Wh} / \mathrm{kg})$. Für die Holzart Fichte ebenfalls bei $135^{\circ} \mathrm{C}$ mit dem Muster $2(945 \mathrm{Wh} / \mathrm{kg})$. Die niedrigsten Werte für die Holzart Buche lieferten die Kombinationen Mahlscheibenmuster $4 / 150^{\circ} \mathrm{C}(482 \mathrm{Wh} / \mathrm{kg})$ und Mahlscheibenmuster $5 / 150^{\circ} \mathrm{C}(484 \mathrm{Wh} / \mathrm{kg})$. Mit der Holzart Fichte sind die energetisch günstigsten Kombinationen Mahlscheibenmuster $3 / 150^{\circ} \mathrm{C}(662 \mathrm{Wh} / \mathrm{kg})$ und Mahlscheibenmuster $5 / 150^{\circ} \mathrm{C}(699 \mathrm{Wh} / \mathrm{kg})$. 
Tabelle 7.10: Abschätzung des thermischen, elektrischen und gesamten Energiebedarfs in der Faserstofferzeugung nach dem TMP- und RMP-Verfahren.

\begin{tabular}{|c|c|c|c|c|c|c|c|c|}
\hline Var. & Mahlsch. & Holzart & $\begin{array}{l}\text { Temp. } \\
{ }^{\circ} \mathrm{C}\end{array}$ & $\begin{array}{l}\text { thermisch } \\
\mathrm{Wh} / \mathrm{kg}_{\text {atro }}\end{array}$ & $\begin{array}{l}\text { elektrisch } \\
\mathrm{Wh} / \mathrm{kg}_{\text {atro }}\end{array}$ & $\begin{array}{l}\text { Energiebedarf } \\
\text { gesamt } \\
\text { Wh/ } / \mathrm{kg}_{\text {atro }}\end{array}$ & $\begin{array}{l}\text { thermisch } \\
\%\end{array}$ & $\begin{array}{l}\text { elektrisch } \\
\%\end{array}$ \\
\hline \multicolumn{9}{|c|}{ TMP-Verfahren } \\
\hline 1 & 1 & Fichte & 135 & 322 & 545 & 867 & 37 & 63 \\
\hline 2 & 1 & Fichte & 150 & 528 & 330 & 858 & 62 & 38 \\
\hline 3 & 1 & Fichte & 175 & 637 & 127 & 764 & 83 & 17 \\
\hline 4 & 1 & Buche & 135 & 339 & 270 & 609 & 56 & 44 \\
\hline 5 & 1 & Buche & 150 & 354 & 166 & 520 & 68 & 32 \\
\hline 6 & 1 & Buche & 175 & 469 & 137 & 606 & 77 & 23 \\
\hline 7 & 2 & Fichte & 135 & 468 & 477 & 945 & 50 & 50 \\
\hline 8 & 2 & Fichte & 150 & 588 & 130 & 718 & 82 & 18 \\
\hline 9 & 2 & Fichte & 175 & 618 & 112 & 730 & 85 & 15 \\
\hline 10 & 2 & Buche & 135 & 373 & 199 & 572 & 65 & 35 \\
\hline 11 & 2 & Buche & 150 & 422 & 142 & 564 & 75 & 25 \\
\hline 12 & 2 & Buche & 175 & 437 & 105 & 542 & 81 & 19 \\
\hline 13 & 3 & Fichte & 135 & 504 & 174 & 678 & 74 & 26 \\
\hline 14 & 3 & Fichte & 150 & 550 & 112 & 662 & 83 & 17 \\
\hline 15 & 3 & Fichte & 175 & 631 & 80 & 711 & 89 & 11 \\
\hline 16 & 3 & Buche & 135 & 410 & 149 & 559 & 73 & 27 \\
\hline 17 & 3 & Buche & 150 & 408 & 123 & 531 & 77 & 23 \\
\hline 18 & 3 & Buche & 175 & 424 & 63 & 487 & 87 & 13 \\
\hline 19 & 4 & Fichte & 135 & 389 & 547 & 936 & 42 & 58 \\
\hline 20 & 4 & Fichte & 150 & 496 & 268 & 764 & 65 & 35 \\
\hline 21 & 4 & Fichte & 175 & 649 & 111 & 760 & 85 & 15 \\
\hline 22 & 4 & Buche & 135 & 353 & 233 & 586 & 60 & 40 \\
\hline 23 & 4 & Buche & 150 & 362 & 120 & 482 & 75 & 25 \\
\hline 24 & 4 & Buche & 175 & 454 & 101 & 555 & 82 & 18 \\
\hline 25 & 5 & Fichte & 135 & 328 & 412 & 740 & 44 & 56 \\
\hline 26 & 5 & Fichte & 150 & 535 & 164 & 699 & 77 & 23 \\
\hline 27 & 5 & Fichte & 175 & 639 & 118 & 757 & 84 & 16 \\
\hline 28 & 5 & Buche & 135 & 318 & 318 & 636 & 50 & 50 \\
\hline 29 & 5 & Buche & 150 & 369 & 115 & 484 & 76 & 24 \\
\hline 30 & 5 & Buche & 175 & 459 & 96 & 555 & 83 & 17 \\
\hline \multicolumn{9}{|c|}{ RMP-Verfahren } \\
\hline 1 & 6 & Fichte & 20 & 123 & 464 & 587 & 21 & 79 \\
\hline 2 & 6 & Fichte & 100 & 310 & 421 & 731 & 42 & 58 \\
\hline 3 & 6 & Buche & 20 & 90 & 235 & 325 & 29 & 71 \\
\hline 4 & 6 & Buche & 100 & 247 & 215 & 462 & 51 & 49 \\
\hline
\end{tabular}

Var. $=$ Variante, Mahlsch. $=$ Mahlscheibenmuster, Temp. $=$ Aufschlusstemperatur

Im RMP-Verfahren zeigen die Varianten ohne Hackschnitzelerwärmung mit Sattdampf die günstigsten Werte im Hinblick auf den Gesamtenergiebedarf. Durch die Erwärmung der Hackschnitzel mit Sattdampf bei $100^{\circ} \mathrm{C}$ liegt der Gesamtenergiebedarf im Bereich der Spannweite des TMPVerfahrens. Dies gilt für die Holzarten Buche und Fichte gleichermaßen. Zu beachten ist in diesem Zusammenhang, dass im RMP-Verfahren eine Reduzierung und Vereinfachung der Prozessschritte möglich ist. Im RMP-Verfahren ist keine energie- und wartungsintensive Stopfschnecke notwendig. Es fällt kein Quetschwasser an, das aufbereitet und geklärt werden muss. Hochdruck- 
Dampferzeugung und Druckbehälter sind in diesem Fall nicht erforderlich. Weiterhin fällt der Dampfverlust durch den Transport der Faserstoffe in der Blasleitung weg. Insbesondere in der Holzfaserdämmstoffherstellung mit relativ niedrigen Durchsätzen ist der Einsatz eines energiesparenden Bandtrockners mit Wärmerückgewinnung anstelle eines elektrisch und thermisch aufwendigen Stromrohrtrockners denkbar. Insgesamt sind dadurch geringere Investitions- und Betriebskosten zu erwarten.

Temperaturabhängige Ausbeuteverluste wurden nicht untersucht. Wie in Abschnitt 7.2 bereits diskutiert, steigt mit höherer Aufschlusstemperatur und Verweilzeit der thermisch bedingte Massenverlust an. Insbesondere bei Laubhölzern verläuft Back \& Salmén (1982) zufolge, der Ausbeuteverlust rascher und bei niedrigeren Temperaturen. Laut Back \& S. A. Larsson (1972) bestehen die wirtschaftlichen Vorteile einer niedrigeren Aufschlusstemperatur in einer höheren Ausbeute, niedrigerem Dampfverbrauch und geringerer Abwasserbelastung. Ebenda weisen außerdem darauf hin, dass niedrigere Aufschlusstemperaturen flexiblere Fasern erzeugen, die allerdings eine erhöhte Wasseraufnahme und Dickenquellung mit sich bringen. Dies wird allgemein durch die Freilegung der S2-Schicht der Zellwand begründet.

$\mathbf{a}$

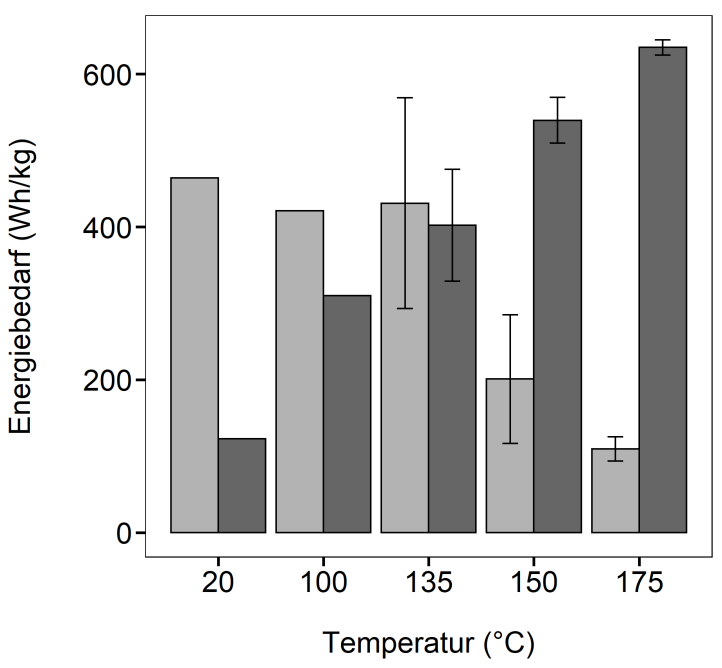

b

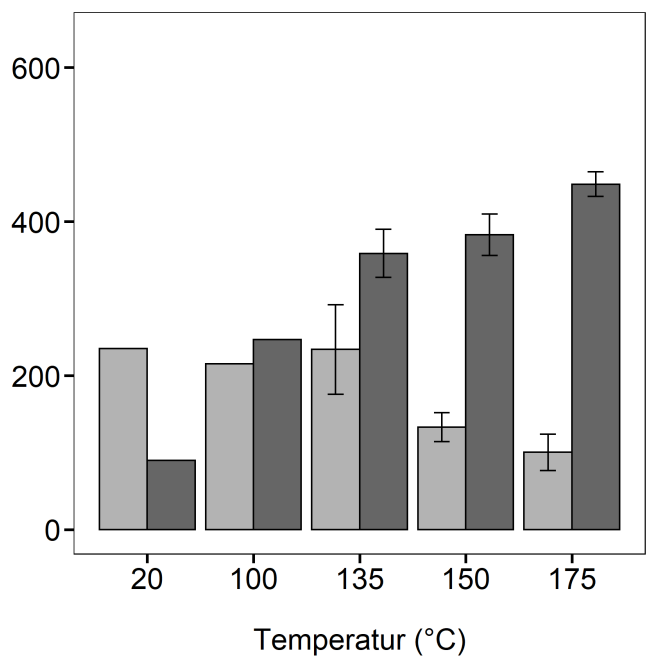

Energie $\square$ elektrisch $\square$ thermisch

Abbildung 7.38: Verhältnis des elektrischen und thermischen Energiebedarfs der Faserstofferzeugung im RMPVerfahren $\left(20^{\circ} \mathrm{C}\right.$ bis $\left.100^{\circ} \mathrm{C}\right)$ und TMP-Verfahren $\left(135^{\circ} \mathrm{C}\right.$ bis $\left.175^{\circ} \mathrm{C}\right)$. Der thermische Energiebedarf enthält den Energiebedarf für die Erwärmung der Hackschnitzel und für die Trocknung der Faserstoffe. Die Schwankungen um die Mittelwerte werden durch die Mahlscheibenmuster verursacht. a, Holzart Fichte. b, Holzart Buche.

In Abbildung 7.38 sind die Ergebnisse aus Tabelle 7.10 zusammenfassend übersichtlich dargestellt. Die auffällige Reduzierung des thermischen Energiebedarfs bei $20^{\circ} \mathrm{C}$ ist auf die hier fehlende Erwärmung der Hackschnitzel mit Frischdampf zurückzuführen. Der Bedarf an thermischer Energie wird allein durch die Trocknung der Faserstoffe verursacht. Zusätzlich kann der zwischen den Mahlscheiben entstehende und in den Kocher zurückströmende Dampf zur Erwärmung der Hackschnitzel genutzt werden. 


\subsection{Untersuchungen zur Beschaffenheit der Faserstoffe}

\subsubsection{Einleitung}

Vor dem Hintergrund des in der Holzfaserdämmstoffproduktion eingesetzten Trockenverfahrens wurden zur Untersuchung der Beschaffenheit der Faserstoffe zwei trockene Analysemethoden ausgewählt. Die Kollektivmethode der Luftstrahl-Prüfsiebung und die Individualmethode der quantitativen Bildanalyse. Die Verfahren erlauben unterschiedliche Erkenntnisse bezüglich der Dispersitätseigenschaften der Faserstoffe. Die Luftstrahl-Prüfsiebung eignet sich zur Analyse des gesamten Partikelkollektivs nach der Mengenart Masse. Die quantitative Bildanalyse hingegen ermöglicht Aussagen über Formfaktoren individueller Partikel mit verschiedenen Gewichtungen. Für die Charakterisierung der trockenen Faserstoffe steht allgemein eine geringe Auswahl an Methoden zur Verfügung, die außerdem in der Holzwerkstoffindustrie, wie auch in der Laborpraxis weder allgemein akzeptiert noch vollständig entwickelt oder standardisiert sind. Aus der Papierherstellung sind standardisierte Methoden zur Ermittlung charakteristischer Kennwerte für die Beschaffenheit von in Wasser suspendierten Faserstoffen bekannt (z.B. Schopper Riegler, canadian standard freeness (CSF)), die in Beziehung mit bestimmten Produkteigenschaften stehen. In der Holzforschung zu MDF und in der Holzwerkstoffindustrie ist weitgehend bekannt und untersucht, dass der Rohstoff Holz und die Beschaffenheit der Faserstoffe Produktionsprozesse und Produkteigenschaften beeinflussen. Die entscheidende Produkteigenschaft der Holzfaserdämmstoffe, die Wärmeleitfähigkeit, wurde dahingehend noch nicht umfassend untersucht. Es ist weitgehend unbekannt, welche Charakteristika Faserstoffe besitzen sollten, um eine möglichst niedrige Wärmeleitfähigkeit zu erzielen. Wie die Untersuchung von industriellen Holzfaserdämmstoffen in einer ersten Annäherung gezeigt hat, zeigen sich deutliche Unterschiede in den Dispersitätseigenschaften (Tabelle 7.4 und Tabelle 7.3).

Das Ziel der Untersuchungen zur Beschaffenheit der Faserstoffe ist zum einen die Auswirkung der Faserstofferzeugung im RMP- und TMP-Verfahren mit unterschiedlichen Mahlscheiben zu ermitteln. Zum anderen soll die Analyse dazu dienen, funktionale Zusammenhänge und Abhängigkeiten zwischen Dispersitätseigenschaften und der Wärmeleitfähigkeit der erzeugten Faserstoffe sichtbar zu machen. Im einfachsten Fall kann eine bestimmte charakteristische Partikelgröße, z. B. der Medianwert maßgeblich sein. Eventuell hängen bestimmte Eigenschaften der Verteilungsfunktion oder der Partikelklassen mit der Wärmeleitfähigkeit zusammen. Dies wird im Abschnitt 7.6 näher betrachtet.

\subsubsection{Dispersitätseigenschaften nach der Kollektivmethode Luftstrahl-Prüfsiebung}

Der Fokus der Charakterisierung der Faserstoffe mit der Luftstrahl-Prüfsiebung liegt auf den Auswirkungen der Mahlscheiben und Aufschlusstemperaturen auf die Faserstoffe. Die Kollektivmethode vermittelt einen umfassenden Eindruck über die Wirksamkeit und Leistungsfähigkeit in der mechanischen Zerfaserung, durch die Erfassung sämtlicher Partikel des Kollektivs. Die Probenmasse liegt um eine Zehnerpotenz höher als in der Methode der quantitativen Bildanalyse, was insbesondere in der Erfassung zahlenmäßig geringer Faserbündel und Agglomerate mit vergleichsweise hoher Masse vorteilhaft ist. Die Methode der Luftstrahl-Prüfsiebung scheint für die Erfassung dieser voluminösen und verzweigten Partikel und Agglomerate im Vergleich zur Bildanalyse (Abschnitt 7.5.3) besser geeignet zu sein.

In Tabelle 7.11 ist der Medianwert $\mathrm{x}_{50,3}$ als eine repräsentative Größe der Faserstoffe angegeben (Bohnet 2004, S. 29). Ein weiteres Charakteristikum ist das $97 \%$-Perzentil $\left(\mathrm{x}_{97,3}\right)$, das auch zur 
Tabelle 7.11: Dispersitätseigenschaften der Faserstoffe nach der Kollektivmethode Luftstrahl-Prüfsiebung.

\begin{tabular}{|c|c|c|c|c|c|c|c|c|}
\hline Var. & Mahlsch. & Holzart & Temp. & $\begin{array}{l}\bar{x}_{50,3} \\
\mu \mathrm{m}\end{array}$ & $\begin{array}{l}\sigma \\
\mu \mathrm{m}\end{array}$ & $\begin{array}{l}\bar{x}_{97,3} \\
\mu \mathrm{m}\end{array}$ & $\begin{array}{l}\sigma \\
\mu \mathrm{m}\end{array}$ & $\begin{array}{l}\text { Partikel- } \\
\text { ausdehnung } \\
\mu \mathrm{m}\end{array}$ \\
\hline
\end{tabular}

\begin{tabular}{|c|c|c|c|c|c|c|c|c|}
\hline \multicolumn{9}{|c|}{ TMP-Verfahren } \\
\hline 1 & 1 & Fichte & 135 & 153 & 8 & 1359 & 249 & 1205 \\
\hline 2 & 1 & Fichte & 150 & 269 & 38 & 1309 & 249 & 1040 \\
\hline 3 & 1 & Fichte & 175 & 270 & 21 & 1377 & 247 & 1107 \\
\hline 4 & 1 & Buche & 135 & 200 & 0 & 742 & 278 & 542 \\
\hline 5 & 1 & Buche & 150 & * & * & 1299 & 626 & * \\
\hline 6 & 1 & Buche & 175 & * & * & 927 & 435 & * \\
\hline 7 & 2 & Fichte & 135 & 363 & 71 & 1532 & 417 & 1195 \\
\hline 8 & 2 & Fichte & 150 & 671 & 74 & 2428 & 59 & 1757 \\
\hline 9 & 2 & Fichte & 175 & 340 & 71 & 1434 & 66 & 1094 \\
\hline 10 & 2 & Buche & 135 & 378 & 34 & 1427 & 36 & 1049 \\
\hline 11 & 2 & Buche & 150 & 148 & 0 & 1030 & 391 & 882 \\
\hline 12 & 2 & Buche & 175 & * & * & 743 & 247 & * \\
\hline 13 & 3 & Fichte & 135 & 768 & 22 & 2399 & 42 & 1631 \\
\hline 14 & 3 & Fichte & 150 & 679 & 53 & 2401 & 0 & 1722 \\
\hline 15 & 3 & Fichte & 175 & 438 & 71 & 1930 & 271 & 1492 \\
\hline 16 & 3 & Buche & 135 & 376 & 36 & 1484 & 74 & 1108 \\
\hline 17 & 3 & Buche & 150 & 160 & 29 & 1110 & 310 & 950 \\
\hline 18 & 3 & Buche & 175 & 127 & 0 & 923 & 263 & 795 \\
\hline 19 & 4 & Fichte & 135 & 397 & 88 & 1444 & 185 & 1046 \\
\hline 20 & 4 & Fichte & 150 & 317 & 56 & 1429 & 338 & 1112 \\
\hline 21 & 4 & Fichte & 175 & 415 & 45 & 1674 & 143 & 1260 \\
\hline 22 & 4 & Buche & 135 & 268 & 23 & 970 & 17 & 702 \\
\hline 23 & 4 & Buche & 150 & 230 & 20 & 1276 & 72 & 1046 \\
\hline 24 & 4 & Buche & 175 & 137 & 4 & 602 & 8 & 465 \\
\hline 25 & 5 & Fichte & 135 & 206 & 27 & 995 & 205 & 789 \\
\hline 26 & 5 & Fichte & 150 & 399 & 49 & 1761 & 217 & 1362 \\
\hline 27 & 5 & Fichte & 175 & 290 & 22 & 1073 & 135 & 783 \\
\hline 28 & 5 & Buche & 135 & 271 & 43 & 1306 & 453 & 1035 \\
\hline 29 & 5 & Buche & 150 & 174 & 35 & 965 & 25 & 791 \\
\hline 30 & 5 & Buche & 175 & 129 & 0 & 623 & 9 & 495 \\
\hline
\end{tabular}

\begin{tabular}{lllllllll}
\hline \multicolumn{1}{l}{ RMP-Verfahren } & & & & & & \\
\hline 1 & 6 & Fichte & 20 & 484 & 32 & 1656 & 134 & 1172 \\
2 & 6 & Fichte & 100 & 532 & 104 & 1888 & 289 & 1356 \\
3 & 6 & Buche & 20 & 406 & 46 & 1610 & 142 & 1205 \\
4 & 6 & Buche & 100 & 420 & 54 & 1702 & 273 & 1281 \\
\hline
\end{tabular}

${ }^{*}$ Verteilungssumme beginnt oberhalb von $\mathrm{x}_{50,3}$

Var. $=$ Variante, Mahlsch. $=$ Mahlscheibenmuster, Temp. $=$ Aufschlusstemperatur

Berechnung der Partikelausdehnung dient. Dieser Feinheitskennwert wird im vorliegenden Fall für ein oberes Feinheitsintervall formuliert (Gleichung 6.3). Die Ergebnisse der mittels LuftstrahlPrüfsiebung ermittelten Kennwerte decken die Größenverteilung der Faserstoffe aus Tabelle 7.3 ab. Folglich kann festgehalten werden, dass die Dispersitätseigenschaften der labortechnisch hergestellten Faserstoffe mit solchen aus industrieller Produktion vergleichbar sind.

Aus den Verteilungssummen in Abbildung 7.39 lassen sich weitere Charakteristika ablesen. Es 
ist ersichtlich, dass die Verteilungen in Abhängigkeit der Holzart, der Aufschlusstemperatur und der Mahlscheibenmuster variieren. Die Abhängigkeit der Dispersitätseigenschaften von den Mahlscheibenmustern tritt besonders deutlich in der Aufschlusstemperatur $135^{\circ} \mathrm{C}$ hervor. Ferner sind die Spezifikationen der Mahlscheiben in den erzeugten Dispersitätseigenschaften sichtbar Insbesondere in den Abbildungen $\mathrm{a}, \mathrm{b}$ und $\mathrm{c}$ sind Paarungen der Mahlscheibenmuster 2 und 3 sowie 4 und 5 erkennbar. Dies ist durch die grundsätzliche Ähnlichkeit in der Konstruktion dieser Paarungen erklärbar. Anhand der Schnittpunkte der Verteilungssummen mit den Perzentilen $\mathrm{x}_{50,3}$ und $\mathrm{x}_{97,3}$ wird weiterhin deutlich, dass der Einfluss der Mahlscheiben mit steigender Aufschlusstemperatur abnimmt. Dieser Effekt passt zu der Beobachtung aus Abbildung 7.33 und Abbildung 7.34. Dort wurde festgestellt, dass die Unterschiede in der spezifischen elektrischen Leistungsaufnahme zwischen den Mahlscheibenmustern mit steigender Aufschlusstemperatur geringer werden. Die grundsätzlich feinere Verteilung der Buchenholz-Faserstoffe ist an dem hohen Durchgang durch die Siebmaschenweite $125 \mu \mathrm{m}$ erkennbar. Dieser Zustand kann mit den anatomischen Merkmalen dieser Holzart erklärt werden (Tabelle 2.1). Dabei kommt die stärker differenzierte Gewebestruktur des Buchenholzes mit geringeren Anteilen an faserartigen und kürzeren faserartigen Zellen zum Tragen.

Zur Dispersitätseigenschaft Feinheit ist anzumerken, dass das Luftstrahlsieb grundsätzlich prädestiniert für die Analyse feindisperser Stoffe mit Korngrößen zwischen $20 \mu \mathrm{m}$ und $100 \mu \mathrm{m}$ ist (Batel 1957; Menck 1994). Durch die Dispergierwirkung des Luftstrahls lassen sich die Haftkräfte zwischen den Partikeln überwinden (Schmidt 1967). Eigene Voruntersuchungen und die Untersuchungen durch Schirp et al. (2015) zeigen, dass mit Siebmaschenweiten unter $125 \mu \mathrm{m}(32 \mu \mathrm{m}$ und $63 \mu \mathrm{m}$ ) die Aussagekraft der Verteilungssumme im unteren Feinheitsintervall verbessert werden kann. Die vorliegenden Untersuchungen werden durch eine Bildanalyse ergänzt, weshalb auf den Einsatz kleinerer Maschenweiten zugunsten einer feineren Abstufung bei größeren Maschenweiten verzichtet wurde. 

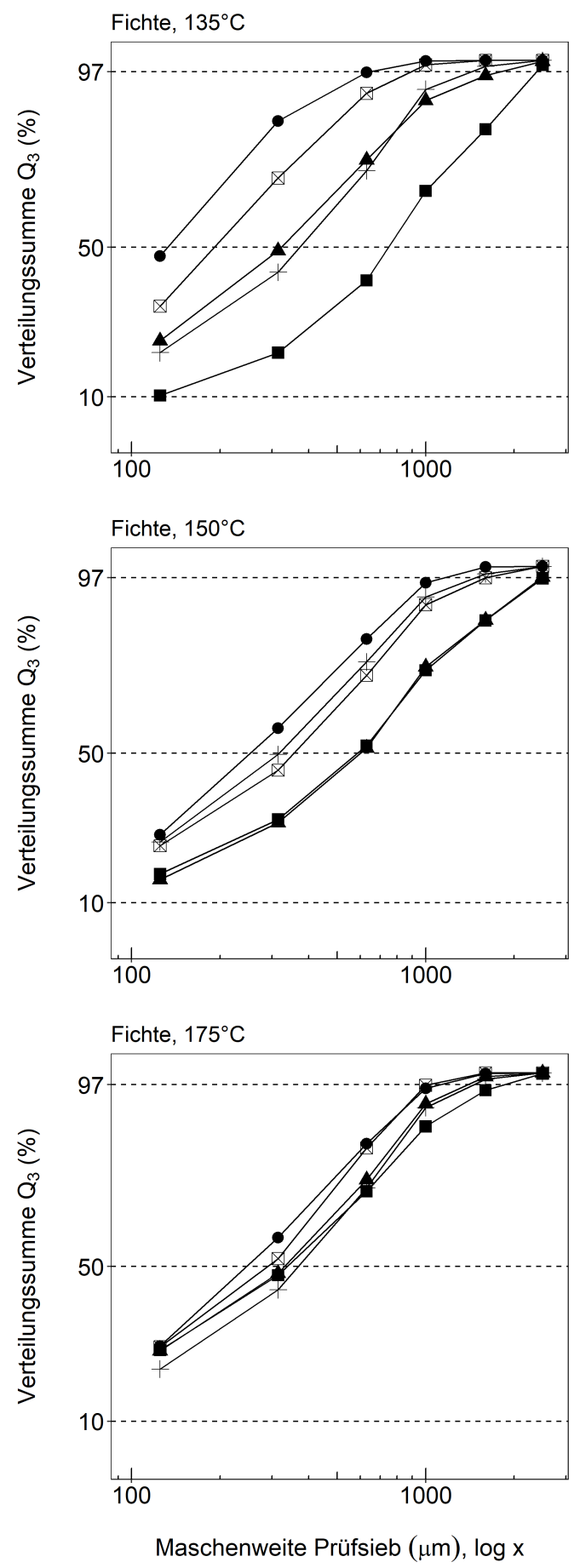

b

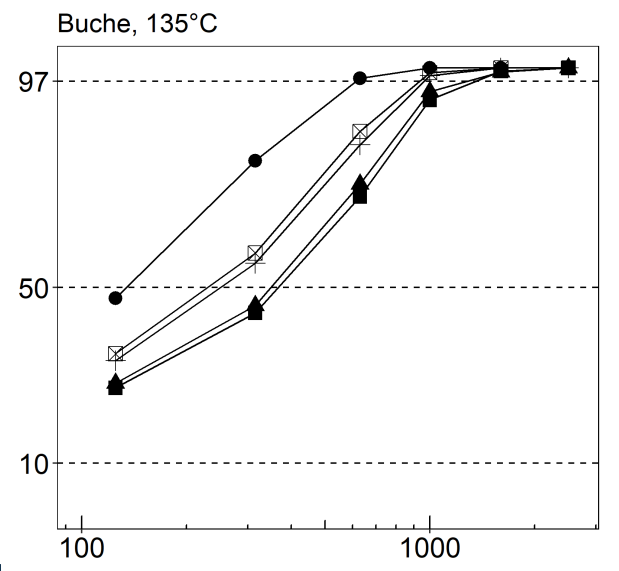

d

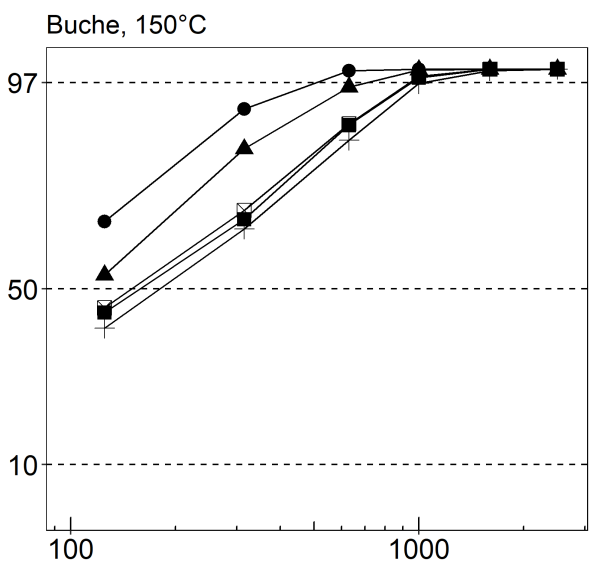

f

Buche, $175^{\circ} \mathrm{C}$

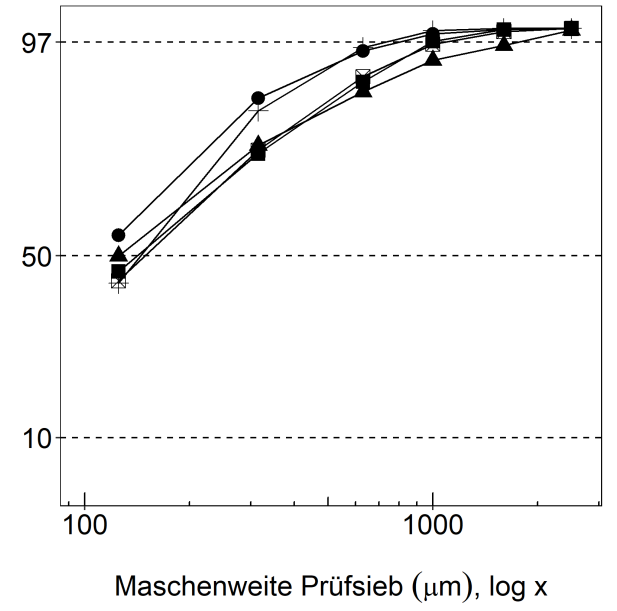

Maschenweite Prüfsieb $(\mu \mathrm{m}), \log x$

Mahlscheibenmuster $\bullet 1$ \ 2 - $3+4 \otimes 5$

Abbildung 7.39: Vergleichende Darstellung der massengewichteten Verteilungssumme $Q_{3}$ der mit unterschiedlichen Mahlscheiben im TMP-Verfahren erzeugten Faserstoffen. a, c, e: In der linken Spalte sind die Verteilungssummen der Fichtenholz-Faserstoffe aufsteigend nach der Sattdampftemperatur dargestellt. $\boldsymbol{b}, \boldsymbol{d}, \boldsymbol{f}:$ In der rechten Spalte sind die Verteilungssummen der Buchenholz-Faserstoffe aufsteigend nach der Sattdampftemperatur dargestellt. Doppelbestimmung. 
Die teilweise deutlichen Unterschiede zwischen den Holzarten und Aufschlussbedingungen sind bei der Faserstofferzeugung im RMP-Verfahren nicht zu beobachten. Davon abgesehen, dass hier nur ein Mahlscheibenmuster untersucht werden konnte, liegen die Verteilungssummen in Abhängigkeit der Aufschlusstemperaturen und der Holzarten recht ähnlich. Die Dispersitätseigenschaften der RMP-Faserstoffe liegen im Bereich der TMP-Faserstoffe mit der Aufschlusstemperatur $135^{\circ} \mathrm{C}$.

a

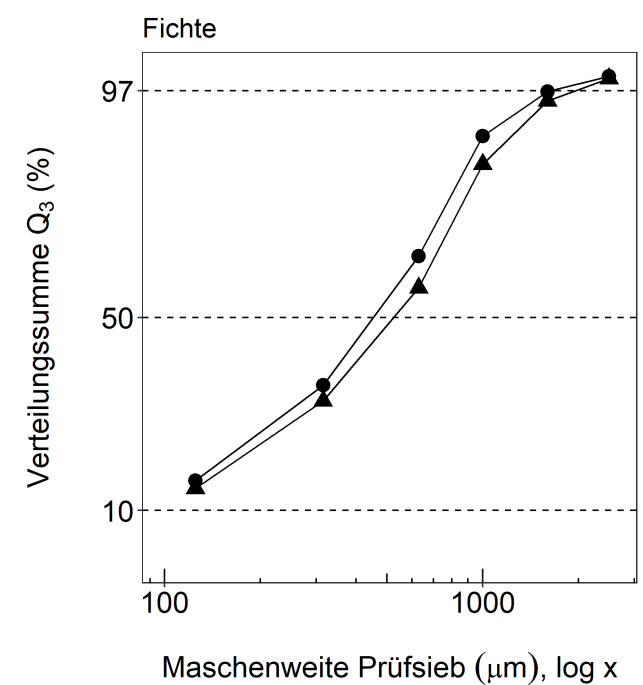

b

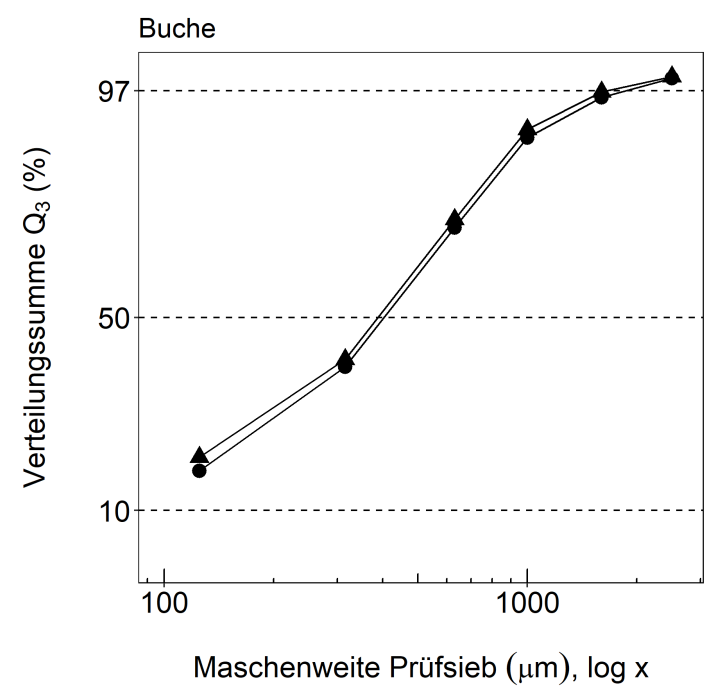

Aufschlusstemperatur $\quad 20^{\circ} \mathrm{C} \Delta 100{ }^{\circ} \mathrm{C}$

Abbildung 7.40: Vergleichende Darstellung der massengewichteten Verteilungssumme $Q_{3}$ der mit dem Mahlscheibenmuster 6 im RMP-Verfahren erzeugten Faserstoffen. a, Verteilungssummen der Fichtenholz-Faserstoffe. $\boldsymbol{b}$, Verteilungssummen Buchenholz-Faserstoffe. Doppelbestimmung.

Weitergehend führt die Betrachtung des Medianwerts in Abbildung 7.41 zu Erkenntnissen über Zusammenhänge zwischen Mahlscheibenmuster, spezifischer elektrischer Leistungsaufnahme und Feinheit der Faserstoffe. Hier zeigt sich die, in Abbildung 7.39 bereits beobachtete, Abhängigkeit der Dispersitätseigenschaften von dem Mahlscheibenmuster und der Aufschlusstemperatur. Die Spreizung des Medianwerts und der spezifischen elektrischen Leistungsaufnahme nimmt bei der Holzart Fichte mit steigender Aufschlusstemperatur zu. Das Mahlscheibenmuster 3 sticht hierbei mit den höchsten Medianwerten und den niedrigsten Werten für die spezifische elektrische Leistungsaufnahme heraus. Eine hohe spezifische elektrische Leistungsaufnahme führt bei der Holzart Fichte durchgängig zu kleineren Medianwerten. Bei der Holzart Buche scheint die Temperatur einen höheren Einfluss auf die Feinheit der Faserstoffe zu haben. Ein Erklärungsansatz hierfür ist die geringere Erweichungstemperatur des Buchenholzes. Der generelle Einfluss der Aufschlusstemperatur auf die Feinheit der Faserstoffe wird u.a. durch Brombacher et al. (2016) bestätigt. Bemerkenswert ist weiterhin, dass die Gruppierung der Aufschlusstemperatur mittels der Verbindungslinien in Abbildung 7.41a anzeigt, dass die Spannweite der Medianwerte $\mathrm{x}_{50,3}$ der Gruppe A $\left(175^{\circ} \mathrm{C}\right)$ sowie der Gruppe B $\left(150^{\circ} \mathrm{C}\right)$ innerhalb der Spannweite der Gruppe $\mathrm{C}\left(135^{\circ} \mathrm{C}\right)$ liegen. Bei der Holzart Buche ist das nicht der Fall (Abbildung 7.41b).

Die Faserstofferzeugung im RMP-Verfahren ist in Abbildung 7.41 durch die Mahlscheibe 6 repräsentiert. Bei der Holzart Fichte liegen die Medianwerte bezüglich der Siebmaschenweite 
innerhalb der Spannweiten der Gruppen A, B und C. Das ist vielversprechend in Bezug auf die Eignung des Verfahrens für die Herstellung von Holzfaserdämmstoffen. Bei der Holzart Buche hingegen liegen die Medianwerte relativ hoch. Die Aussagekraft von Regressionsanalysen wurde überprüft, ist jedoch durch die nur teilweise vorliegende Normalverteilung stark eingeschränkt.

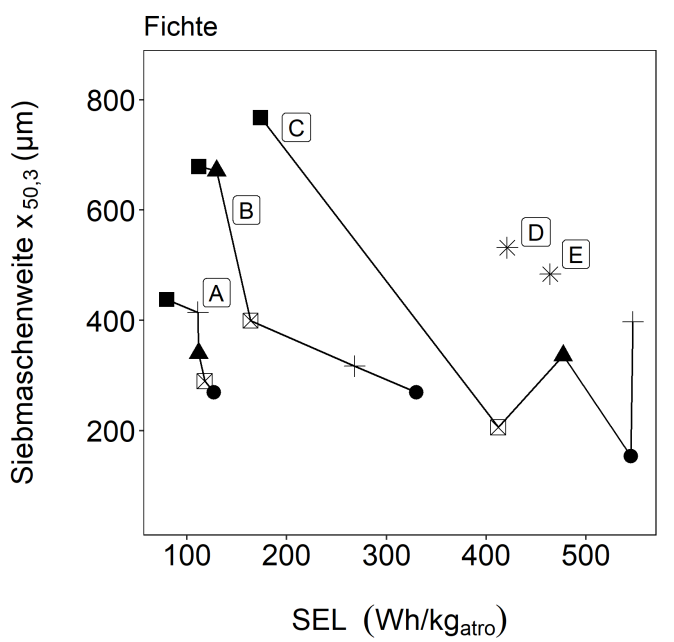

b

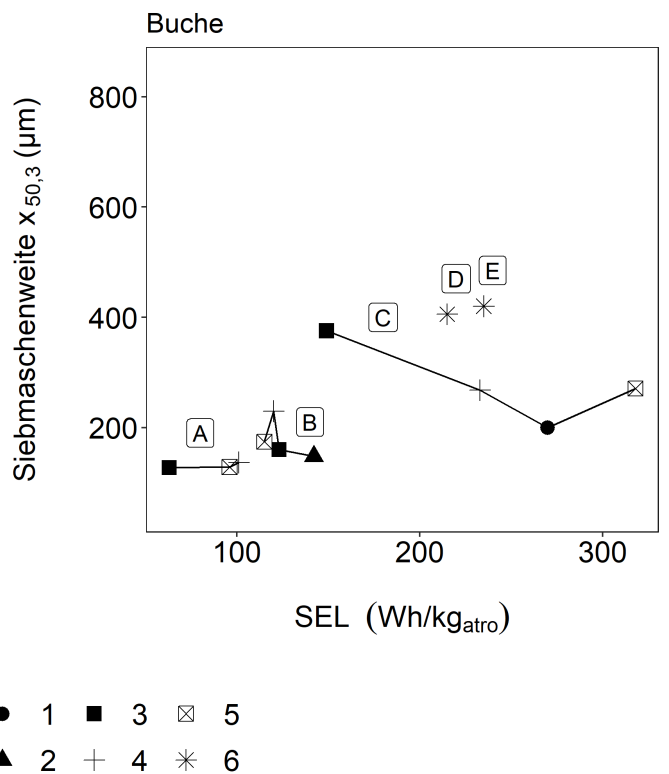

Abbildung 7.41: Vergleichende Darstellung des massengewichteten Medianwerts der Siebmaschenweite $x_{50,3}$ nach der Luftstrahl-Prüfsiebung über der spezifischen elektrischen Leistungsaufnahme (SEL) in Abhängigkeit der Mahlscheibenmuster und Aufschlusstemperatur. a, Holzart Fichte. b, Holzart Buche. Gruppierung nach der Aufschlusstemperatur über Verbindungslinien: Gruppe A, $175^{\circ} \mathrm{C}$; Gruppe B, $150^{\circ} \mathrm{C}$; Gruppe C, $135^{\circ} \mathrm{C}$; Gruppe $D, 100^{\circ} \mathrm{C}$; Gruppe $\mathrm{E}, 20^{\circ} \mathrm{C}$.

In Ergänzung zu den bereits beschriebenen Charakteristika bietet die Partikelausdehnung einen weiteren Einblick in die Beschaffenheit der Faserstoffe (Abbildung 7.42). Die Partikelausdehnung ist ein Maß für die Homogenität der Größenverteilung der Faserstoffe. Anzumerken ist in diesem Zusammenhang, dass das Mahlscheibenmuster 3 mit der Holzart Fichte eine vergleichsweise heterogene Größenverteilung und das Mahlscheibenmuster 5 eine vergleichsweise homogene Größenverteilung erzeugen. Interessant ist auch die relativ breite Streuung der Partikelausdehnung bei niedriger spezifischer elektrischer Leistungsaufnahme im Vergleich zur geringen Spannweite der Medianwerte bei niedriger spezifischer elektrischer Leistungsaufnahme in Abbildung 7.41a. Dies ist ein Indiz dafür, dass mit der Holzart Fichte bei niedriger spezifischer elektrischer Leistungsaufnahme (und hoher Aufschlusstemperatur) eher eine heterogene Größenverteilung erzeugt wird. Bei der Holzart Buche nimmt dagegen mit geringer spezifischer elektrischer Leistungsaufnahme die Heterogenität eher ab. 
a

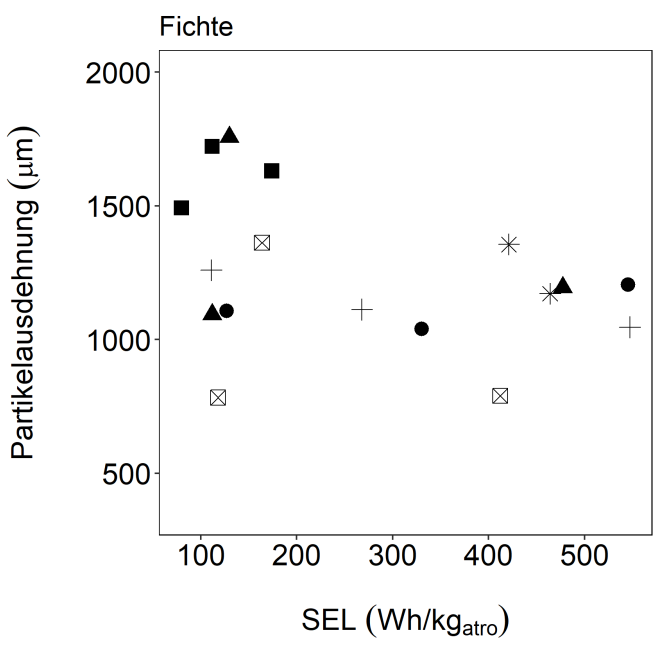

b

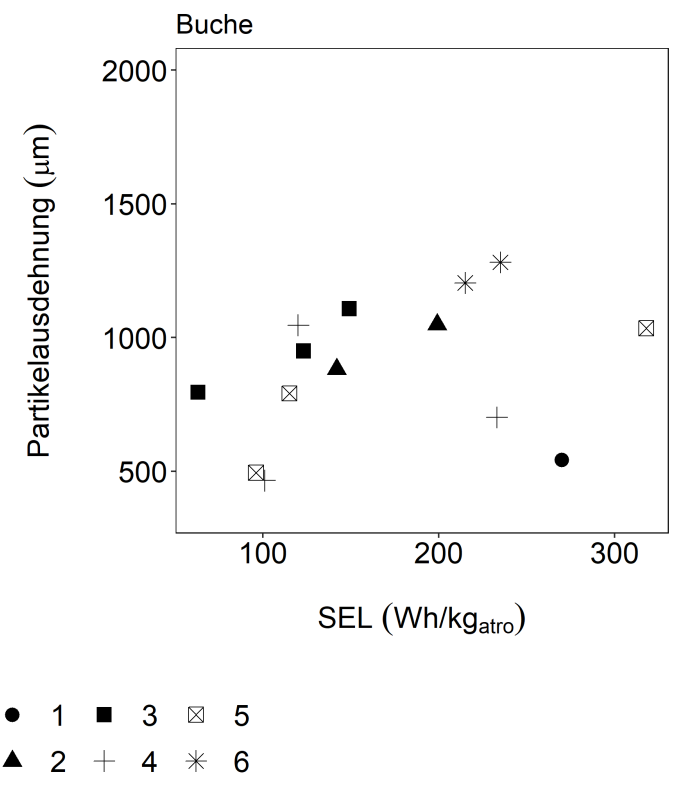

Abbildung 7.42: Vergleichende Darstellung der Partikelausdehnung über der spezifischen elektrischen Leistungsaufnahme (SEL) im TMP-Verfahren. a, Fichtenholz-Faserstoffe. b, Buchenholz-Faserstoffe.

Bei der übergeordneten Betrachtung der Luftstrahl-Prüfsiebung müssen abschließend Einflüsse auf die Ergebnisse und Fehlerquellen diskutiert werden. Unter der Annahme, dass die Stichprobenauswahl und die Probenteilung mit größter Sorgfalt durchgeführt wurden, können Abweichungen durch undispergierte agglomerierte feine Partikel verursacht werden, die als Grobgut ausgetragen werden (DIN ISO 9276-2:2018-09 2018). Eine weitere mögliche Fehlerquelle besteht durch den Abrieb der Faserstoffe infolge des dispergierenden Luftstrahls. Vorversuche zur Ermittlung von Siebrückstandszeitkurven zeigten, dass mit Faserstoffen die anfänglich starke Abnahme des Siebrückstands nicht regelmäßig konstant wird. Es könnte also zutreffen, dass z.B. die verzweigten, nicht vollständig separierten Faserbündel, die in der Breite aus mindestens zwei Zellen bestehen, abriebempfindlich sind. Dies erschwert die Siebdauerbestimmung durch das Abbruchkriterium nach DIN 66165-1:2016-08 (2016) oder Coppers (2002).

\subsubsection{Dispersitätseigenschaften nach der Individualmethode quantitative Bildanalyse}

In Ergänzung zur Luftstrahlsiebanalyse bietet die quantitative Bildanalyse als Individualmethode die Möglichkeit, weitere Informationen über die Partikelgrößenverteilung und insbesondere die Partikelform zu ermitteln. In der Auswertung und Darstellung der Ergebnisse wird deshalb neben Untersuchungen zu den Auswirkungen der Mahlscheiben auf die Faserstoffe auch versucht, funktionelle Zusammenhänge zwischen den Dispersitätseigenschaften und der Wärmeleitfähigkeit der Faserstoffe zu identifizieren. Zur Identifizierung ebendieser Zusammenhänge wurde die Methode von Wenderdel et al. (2014, 2016b, 2017) zur Bildung von Partikelklassen adaptiert, die für die Untersuchung von MDF entwickelt wurde und dort brauchbare Ergebnisse lieferte. Zu diesem Zweck erfolgte eine flächenbezogene Gewichtung der Verteilungssumme. Die Partikel wurden in Klassen anhand der im TMP- und RMP-Verfahren typischerweise entstehenden Partikelformen und den anatomischen Merkmalen des Fichten- und Buchenholzes eingeteilt. Dadurch sollen 


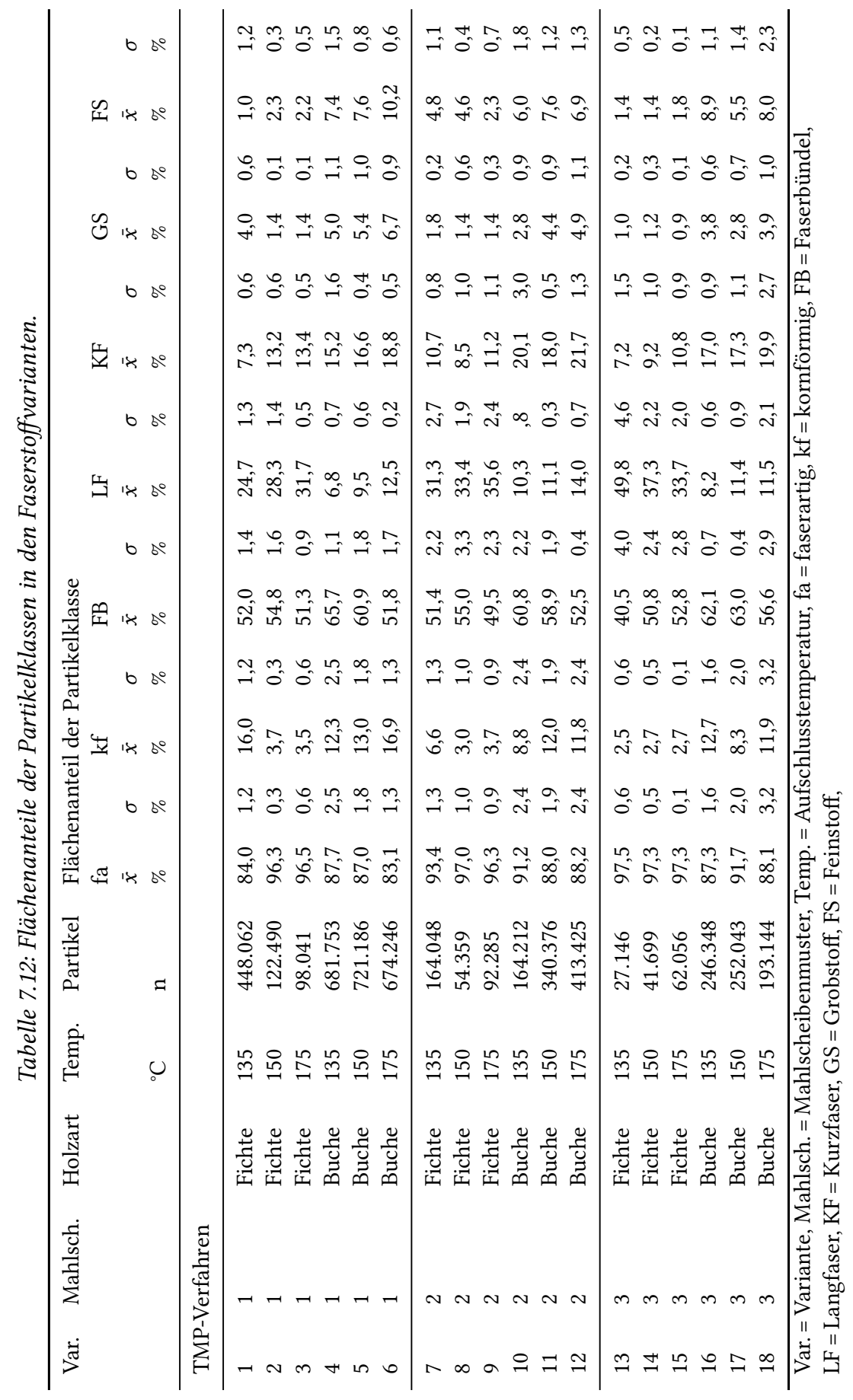




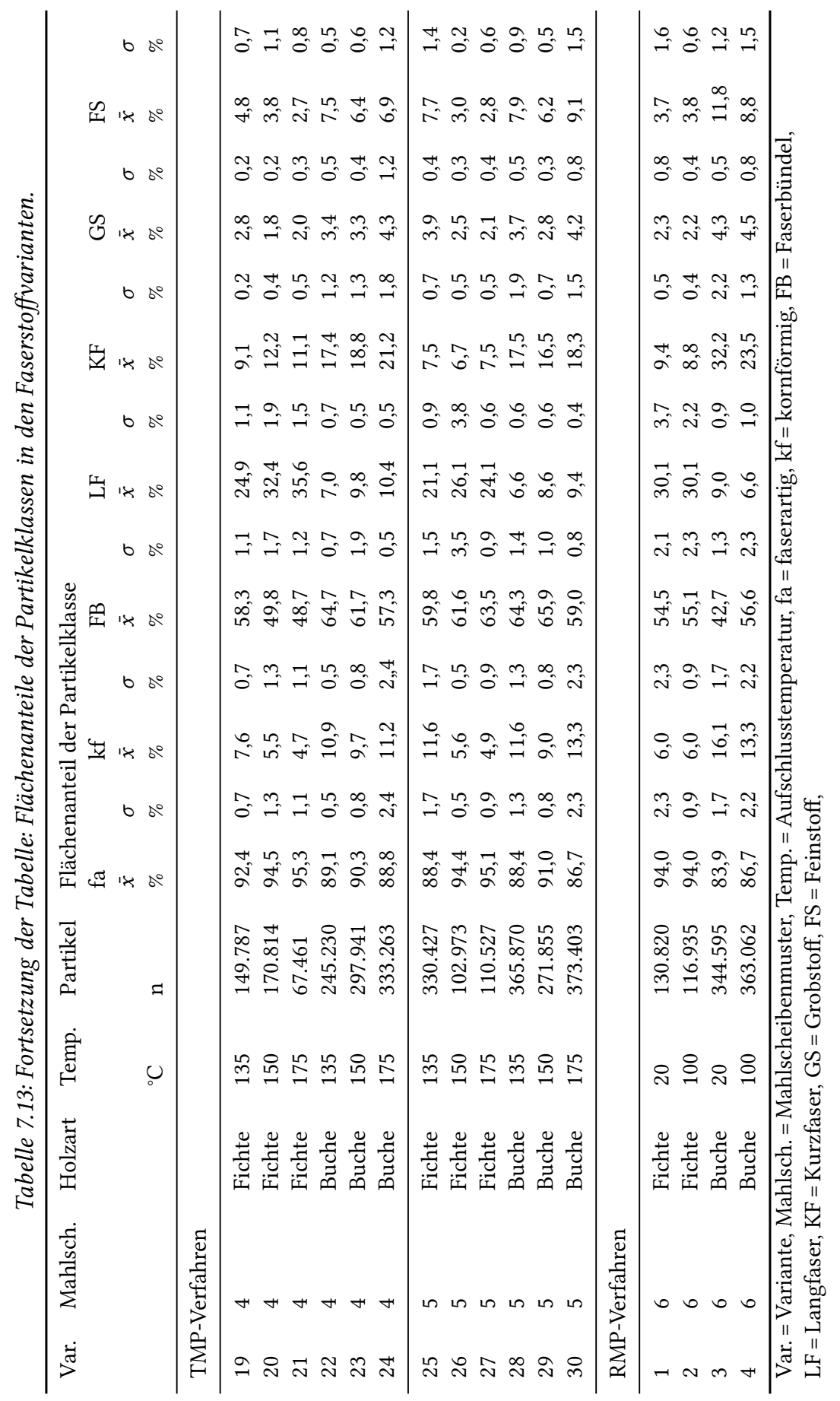


Unterschiede zwischen den Dispersitätseigenschaften der einzelnen Faserstoffe sichtbar gemacht werden. In Tabelle 7.12 und Tabelle 7.13 sind die Werte zu den Flächenanteilen der Partikelklassen der Faserstoffvarianten enthalten.

$\mathbf{a}$

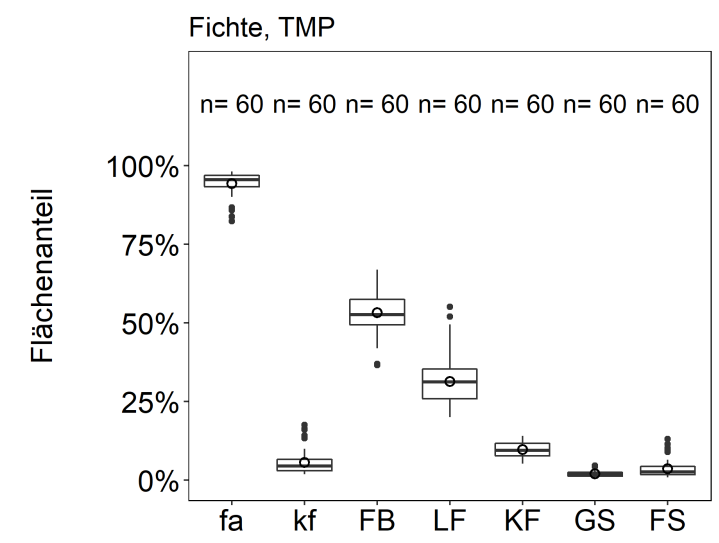

c

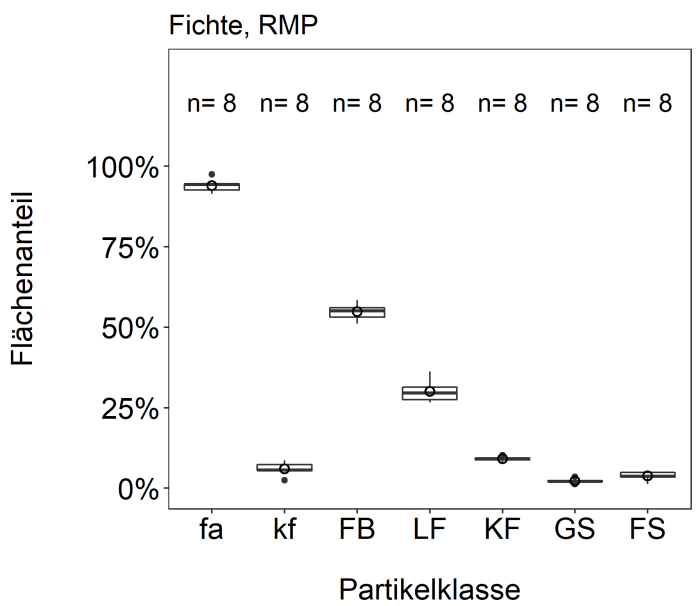

b

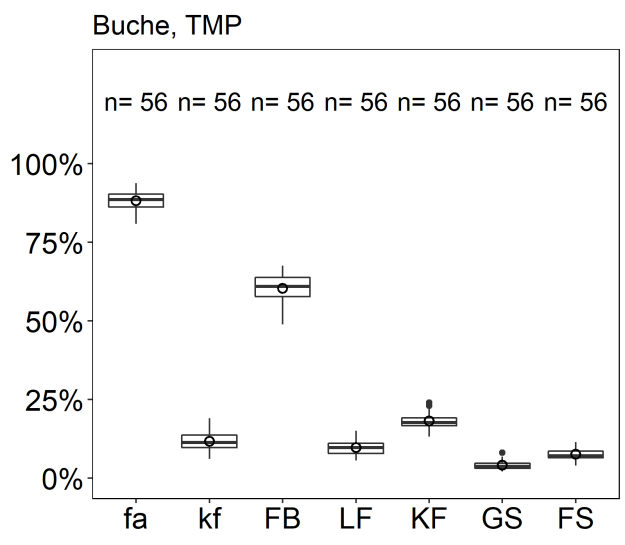

d

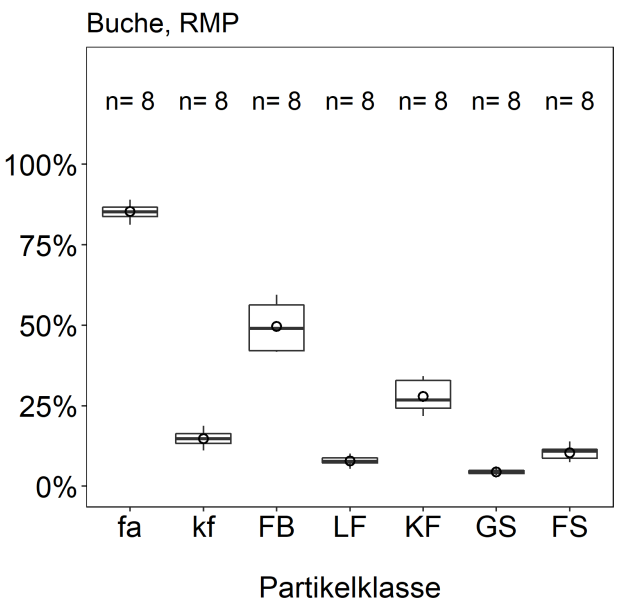

Abbildung 7.43: Flächenanteile der Partikelklassen. a, Holzart Fichte, TMP-Verfahren. b, Holzart Buche, TMPVerfahren. c, Holzart Fichte, RMP-Verfahren. d, Holzart Buche, RMP-Verfahren. Abkürzungen: fa = faserartig, $k f=$ kornförmig, $F B=$ Faserbündel, LF = Langfaser, KF = Kurzfaser, GS = Grobstoff, FS = Feinstoff.

Der Übersichtlichkeit halber sind in Abbildung 7.43 die Flächenanteile der Partikelklassen aus Tabelle 7.12 und Tabelle 7.13 als Boxplots, ohne Unterteilung nach Mahlscheiben oder Aufschlusstemperatur, dargestellt. Daraus geht hervor, dass die faserartigen Partikel (fa) erwartungsgemäß den größten Flächenanteil besitzen. Die weitere Unterteilung in Faserbündel (FB), Langfasern (LF) und Kurzfasern (KF) zeigt, dass die Klasse der Faserbündel bei beiden Holzarten sowie im TMPund RMP-Verfahren den größten Flächenanteil besitzt. Den zweithöchsten Flächenanteil nehmen bei der Holzart Fichte die Langfasern ein, die aus individuellen, intakten Tracheiden bestehen sollten. Diese Klasse ist bei der Holzart Buche nicht vorrangig vertreten, was auf den geringen Anteil der Fasertracheiden und Libriformfasern zurückzuführen ist. Eine weitere Erklärung hierfür könnte sein, dass das angewendete Sekundärkriterium der minimalen und maximalen Faserlänge für die Holzart Buche nicht passend gewählt wurde. Jedenfalls übersteigt in dieser Darstellung der Flächenanteil der Kurzfasern bzw. Faserbruchstücke jenen der Langfasern. 
Es wird außerdem deutlich, dass der Flächenanteil der kornförmigen Partikel (kf) mit einem hohen Dicke-zu-Länge-Verhältnis ( $\mathrm{E}>0,3)$ bei der Holzart Buche höher, als bei der Holzart Fichte ist. Eine weitere Differenzierung dieser Klasse in Grobstoff (GS) und Feinstoff (FS) anhand des größten Durchmessers der ausgefüllten Fläche (maximaler Feret-Durchmesser) zeigt, dass der Anteil an Partikeln $<50 \mu \mathrm{m}$ und $>50 \mu \mathrm{m}$ recht ähnlich ist. Hierzu muss angemerkt werden, dass die Verhältnisse der flächenbezogenen Gewichtung unterliegen. Anzahlgewichtet sind die kornförmigen Partikel deutlich präsenter. Die Darstellung umfasst sämtliche Faserstoffvarianten und abgesehen von den Partikelklassen FB und LF bei der Holzart Fichte im TMP-Verfahren (Abbildung 7.43a) und der Partikelklassen FB und KF bei der Holzart Buche im RMP-Verfahren (Abbildung 7.43d), sind die Unterschiede zwischen den Mahlscheibenmustern und Aufschlusstemperaturen überraschenderweise eher als gering einzustufen.

Ein differenzierteres Bild ergibt sich aus Abbildung 7.44. Hier sind die Flächenanteile der Partikelklassen als gestapelte Balkendiagramme dargestellt. Die Lage der Mittelwerte der einzelnen Partikelklassen unterscheidet sich überwiegend signifikant, wie aus Tabelle 7.14 und Tabelle 7.15 hervorgeht. Das ist durch den großen Umfang der Stichproben auch zu erwarten. Ähnlich wie die Medianwerte bei der Luftstrahl-Prüfsiebung treten auch in der Bildanalyse die größten Unterschiede bei der Holzart Fichte und der Aufschlusstemperatur $135^{\circ} \mathrm{C}$ auf. Hier sticht die Mahlscheibe 3 mit einem vergleichsweise hohen Langfaseranteil bei geringem Kurzfaser-, kornförmigen und Faserbündelanteil heraus. Bezüglich des Mahlscheibenmusters 5 ist anzumerken, dass der Faserbündelanteil bei der Holzart Fichte und den Aufschlusstemperaturen $150^{\circ} \mathrm{C}$ und $175^{\circ} \mathrm{C}$ relativ hoch ist. Die Konstruktion der Mahlscheibe zielt durch die Krümmung des Rotors (Tabelle 6.6) darauf ab, lange, dünne Faserbündel zu erzeugen. Dies bestätigt die Annahme, dass der Rotor einen größeren Einfluss auf die Fasererzeugung hat als der Stator. Die Untersuchungen von Alahautala et al. (2004) zur Faserstoffdichte im Mahlspalt mittels optischer Messungen, untermauern diese Beobachtung. Demnach transportiert der Rotor hauptsächlich die Faserstoffe in Richtung Peripherie und es findet keine gleichmäßige Verteilung zwischen Rotor und Stator statt.

Die Flächenanteile der RMP-Faserstoffe aus der Holzart Fichte in Abbildung 7.45a weisen praktisch keine Unterschiede zwischen den beiden Temperaturen auf. Bei der Holzart Buche in Abbildung 7.45b sind dagegen weniger Faserbündel zu Lasten hauptsächlich höherer Anteile an Kurzfasern und kornförmiger Partikeln zu finden. Wenderdel et al. (2016a, S. 28) kamen zu ähnlichen Ergebnissen. Dies könnte ein Hinweis auf den niedrigeren Erweichungsbereich der Holzart Buche sein, der, wie weiter oben ausgeführt, bereits bei $80^{\circ} \mathrm{C}$ bis $90^{\circ} \mathrm{C}$ beginnt. Allerdings entsteht hierbei ein Anteil von annähernd $50 \%$ an kurzfaserigen und kornförmigen Partikeln. 

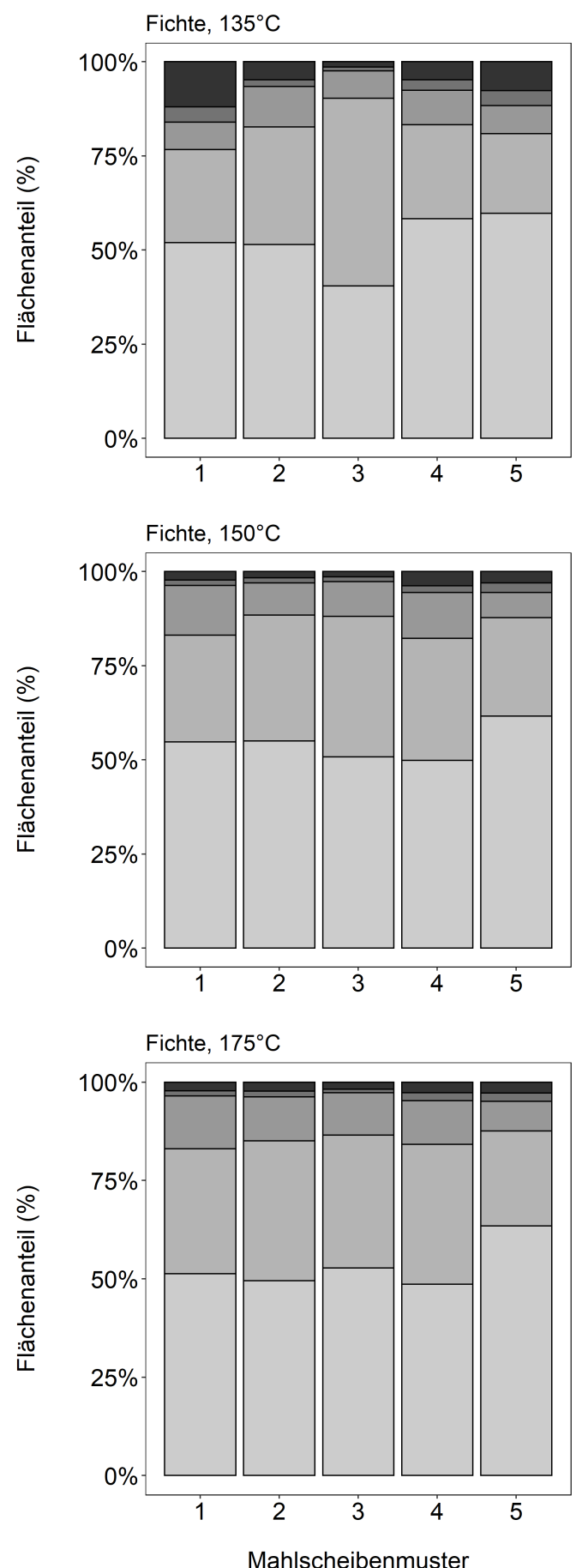

b

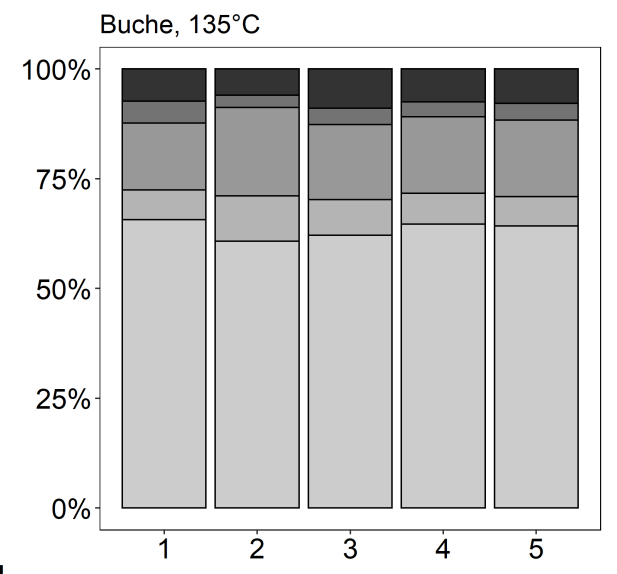

d

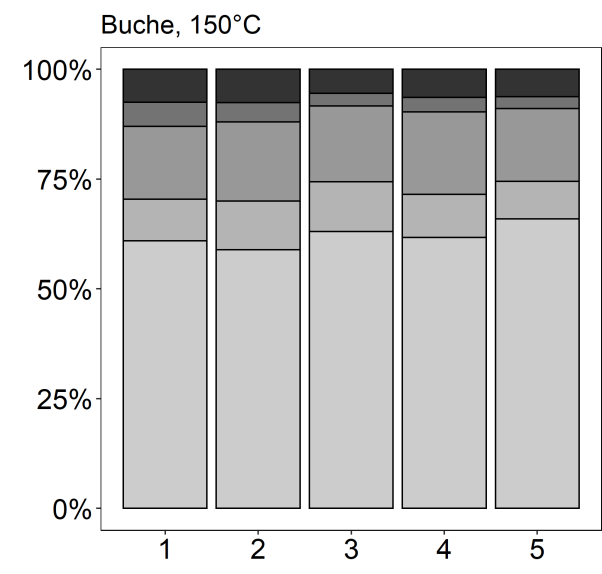

f

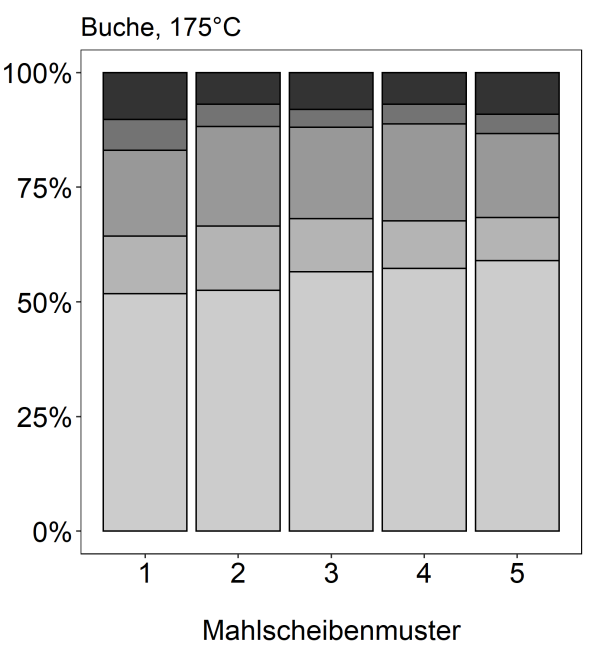

Klasse $\square$ Feinstoff $\square$ Grobstoff $\square$ Kurzfaser $\square$ Langfaser $\square$ Faserbündel

Abbildung 7.44: Flächenanteile der Partikelklassen im TMP-Verfahren. a, c,e, Faserstoffe aus der Holzart Fichte, aufsteigend nach Aufschlusstemperatur sortiert. b, d,e, Faserstoffe aus der Holzart Buche, aufsteigend nach Aufschlusstemperatur sortiert. 
Tabelle 7.14: Statistische Auswertung in Ergänzung zu den Abbildungen 7.44a,c,e (Faserstofferzeugung mit der Holzart Fichte). Die Asteriske indizieren das Signifikanzniveau.

\begin{tabular}{llllllllllllll}
\hline & \multicolumn{3}{c}{$135{ }^{\circ} \mathrm{C}$} & & \multicolumn{3}{c}{$150{ }^{\circ} \mathrm{C}$} & & \multicolumn{5}{c}{$17{ }^{\circ} \mathrm{C}$} \\
\hline M. & 1 & 2 & 3 & 4 & 1 & 2 & 3 & 4 & 1 & 2 & 3 & 4 \\
2 & $* * * *$ & & & & $* * * *$ & & & & $* * * *$ & & & \\
3 & $* * * *$ & $* * * *$ & & & $* * * *$ & $* * * *$ & & & $* * * *$ & $* * * *$ & & \\
4 & $* * * *$ & $* * * *$ & $* * * *$ & & $* * * *$ & $* * * *$ & $* * * *$ & & $* * * *$ & $* * * *$ & $* * * *$ & \\
5 & $* * * *$ & $* * * *$ & $* * * *$ & $* * * *$ & ns & $* * * *$ & $* * * *$ & $* * * *$ & $* * * *$ & $* * * *$ & $* * * *$ & $* * * *$ \\
\hline
\end{tabular}

Signifikanz: $0,0001^{* * * *} ; 0,001^{* * *} ; 0,01^{* *} ; 0,05^{*} ;>0,05 \mathrm{~ns}$ (nicht signifikant) M. = Mahlscheibenmuster

Tabelle 7.15: Statistische Auswertung in Ergänzung zu den Abbildungen 7.44b,d,f (Faserstofferzeugung mit der Holzart Buche). Die Asteriske indizieren das Signifikanzniveau.

\begin{tabular}{llllllllllllll}
\hline & \multicolumn{3}{c}{$135{ }^{\circ} \mathrm{C}$} & & \multicolumn{5}{c}{$150{ }^{\circ} \mathrm{C}$} & & \multicolumn{3}{c}{$175{ }^{\circ} \mathrm{C}$} \\
\hline M. & 1 & 2 & 3 & 4 & 1 & 2 & 3 & 4 & 1 & 2 & 3 & 4 \\
2 & $* * * *$ & & & & $* * * *$ & & & & $* * * *$ & & & \\
3 & $* * * *$ & $* * * *$ & & & $* * * *$ & $* * * *$ & & & ns & $* * * *$ & & \\
4 & $* * * *$ & $* * * *$ & $* * * *$ & & $* * * *$ & $* * * *$ & $* * * *$ & & $* * * *$ & $* * * *$ & $* * * *$ & \\
5 & $* * * *$ & $* * * *$ & $* * * *$ & $* * * *$ & $* * * *$ & $* * * *$ & $* * * *$ & $* * * *$ & $* * * *$ & $* * * *$ & $* * * *$ & $* * * *$ \\
\hline
\end{tabular}

Signifikanz: $0,0001^{* * * *} ; 0,001^{* * *} ; 0,01^{* *} ; 0,05^{*} ;>0,05 \mathrm{~ns}$ (nicht signifikant) M. = Mahlscheibenmuster

a

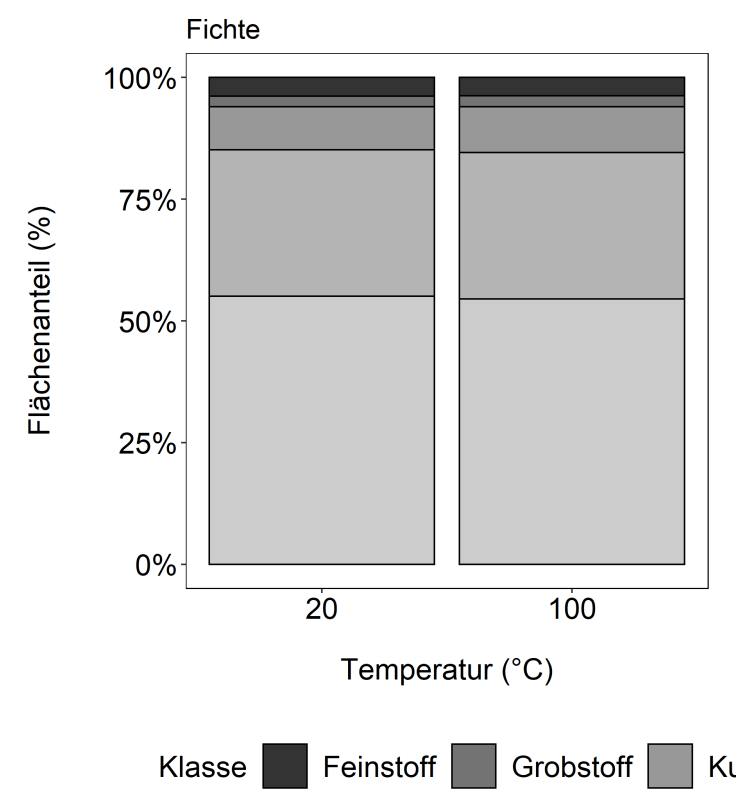

b

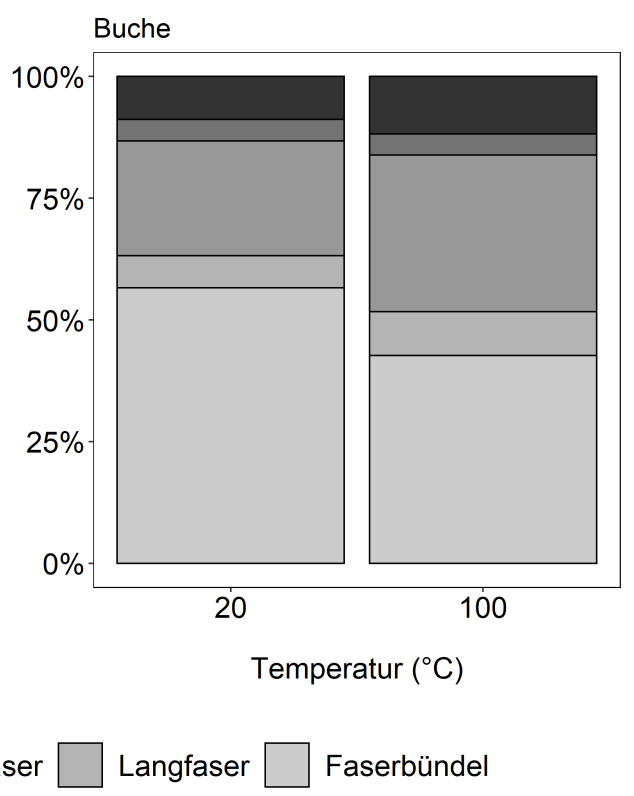

Abbildung 7.45: Flächenanteile der Partikelklassen im RMP-Verfahren. a, Faserstoffe aus der Holzart Fichte. b, Faserstoffe aus der Holzart Buche. Mahlscheibenmuster 6. 
Abgesehen von den Buchen-Faserstoffen überwiegt bei allen Mahlscheiben und Aufschlusstemperaturen die Partikelklasse der Faserbündel bei den Flächenanteilen. Angesichts der eher geringen Unterschiede in den Flächenanteilen der anderen Partikelklassen kann deshalb vermutet werden, dass die Faserbündel einen Einfluss auf die maßgebliche Produkteigenschaft Wärmeleitfähigkeit haben. Im Gegensatz zu den anderen Partikelklassen sind die Faserbündel nicht in der Länge und Dicke definiert. Eine genauere Betrachtung durch weitere Differenzierung der Verteilungssummen innerhalb dieser Partikelklasse soll im Weiteren dazu dienen, funktionelle Zusammenhänge aufzudecken. In Abbildung 7.46 ist beispielhaft die Verteilungssumme der Elongation der Faserbündel dargestellt. Daran wird deutlich, dass innerhalb der Klassen eine Größenverteilung der Partikel durch die Länge und die Dicke vorliegt. Diese Erkenntnis dient im Weiteren zur Berechnung zusätzlicher Kennwerte.

Den Untersuchungen von Wenderdel (2015, S. 88) zufolge bestehen Faserbündel in Faserstoffen aus der Holzart Kiefer (Pinus sylvestris) vor allem aus Frühholz. Die Einzelfasern bestehen zum größten Teil aus Spätholz. Mott et al. (2002) kommen nach Untersuchungen der Holzart Kiefer (Pinus taeda) allerdings zu dem Schluss, dass vor allem das Frühholz im Feinanteil von MDF-Faserstoff zu finden ist. Wenderdel et al. (2016a) erklären den höheren Feinstoffanteil bei Buchen-Faserstoffen durch die Zerkleinerung von Gefäßen im TMP-Verfahren.

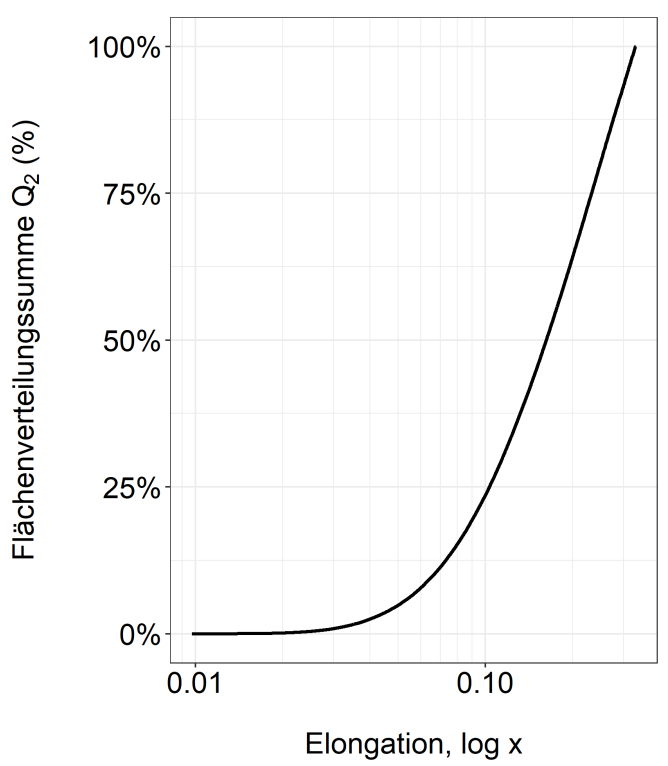

Abbildung 7.46: Beispielhafte Darstellung einer Verteilung innerhalb einer Partikelklasse anhand der flächengewichteten Verteilungssumme $Q_{2}$ der Elongation in der Partikelklasse Faserbündel (Mahlscheibe 1, Holzart Fichte, $\left.135^{\circ} \mathrm{C}\right)$.

Bei der Untersuchung der Effekte von Mahlscheiben und Aufschlusstemperaturen auf die Beschaffenheit von Faserstoffen wurde die natürliche geometrische Variabilität der Zellarten berücksichtigt. Die Anatomie und Morphologie der Holzarten ist prozesstechnisch von Bedeutung und hat Einfluss auf die daraus hergestellten Produkte. In der Holzwerkstoffindustrie sind Laubhölzer für die Produktion von MDF seit längerer Zeit Stand der Technik (Roffael et al. 1994a). Der Einsatz von Fichtenholz in der Holzfaserdämmstoffherstellung ist Stand der Technik. Die Frage, inwiefern sich Buchenholz, dessen Struktur und Anatomie divergiert, vom physikalisch-technologischen 
Standpunkt aus für die Herstellung von Holzfaserdämmstoffen eignet, wird durch die Untersuchungen in Abschnitt 7.6 beantwortet. Dort wird ebenfalls abschließend auf Zusammenhänge zwischen der Beschaffenheit der Faserstoffe und der Wärmeleitfähigkeit eingegangen.

Bei der übergeordneten Betrachtung der quantitativen Bildanalyse sollen an dieser Stelle Einflüsse und Fehlerquellen diskutiert werden. Die FibreShape-Software ist nicht in der Lage, über Kreuz liegende Partikel zu identifizieren und zu analysieren. Solche Agglomerate lassen sich mit der angewendeten Präparationsmethode nicht vermeiden und stellen allgemein eine Problematik in der Analyse von Faserstoffen dar, wie auch Benthien et al. (2017) anmerken. Der morphologische Filterparameter „Convexity“ (ISO 9276-6: Solidität) beschreibt das Verhältnis zwischen der Fläche der konvexen Hülle (Ummantelung) und der Projektionsfläche eines Partikels. Dadurch wird der Filterparameter 1/Convexity „mächtig,, in der Selektion von Partikelformen (vgl. Abbildung 6.6). Mit steigenden Maximalwerten werden zunehmend über Kreuz liegende Fasern und in der Präparation nicht aufgelöste Agglomerate in die Auswertung einbezogen. Mit steigenden Minimalwerten werden kornförmige sowie nicht gekrümmte, kompakte Partikel aus der Analyse ausgeschlossen. In Abbildung 7.47 wird die Problematik veranschaulicht.

Bei der quantitativen Bildanalyse wird in der Regel immer ein Kompromiss zwischen einer möglichst detaillierten Abbildung der Partikel durch eine geringe Pixelgröße und der Notwendigkeit, eine statistisch relevante Partikelzahl zu erfassen, eingegangen. Das in der vorliegenden Arbeit angewendete Analyseverfahren unterscheidet sich von Untersuchungen mit dem FibreShape-System nach Imken et al. (2021), Plinke et al. (2016) und Schirp et al. (2015) durch die höhere Auflösung von 2400 dpi (gegenüber 1200 dpi) und die Verwendung von Glasplatten für die Faserstoff-Präparation (gegenüber Overhead-Folie). Durch die höhere Auflösung konnte die Pixelgröße in den Untersuchungen der vorliegenden Arbeit auf 10,6 $\mu \mathrm{m}$ halbiert und somit die Erfassung der kornförmigen und kurzfaserigen Partikeln sowie der Feinheit verbessert werden. Insbesondere liegt die mittlere Feinheit einzelner Tracheiden der Holzart Fichte bei $30 \mu \mathrm{m}$ und die mittlere Feinheit von Libriformfasern bei $21 \mu \mathrm{m}$ (vgl. Tabelle 2.1). Zudem wurde durch die Verwendung von Glasplatten der Einfluss von Defekten in der Overhead-Folie, wie Lunker, Dichteunterschiede und Schattenbildung, vermieden. Voruntersuchungen und Nullmessungen zeigten, dass diese Defekte in der Analyse relevant sind, da sie als Holzpartikel erfasst werden.

Bezüglich der Varianz bzw. Wiederholgenauigkeit ist darauf zu achten, den Bildflächenanteil der Partikel konstant zu halten, denn bei Bildflächenanteilen über $3 \%$ beginnt die Messung „schief“ zu werden. Weitere mögliche Fehlerquellen bei der Analyse eines Einzelbildes sind in DIN ISO 9276-6:2012-01 (2012) aufgeführt. An dieser Stelle sei noch darauf hingewiesen, dass der Schwellwert für die Graustufen innerhalb des Bildes nur manuell ausgewählt werden konnte und daher für alle Messungen gleich gehalten wurde. Durch den Schwellwert wird die Kontur auf der einen Seite des Grenzwertes als Hintergrund betrachtet und somit von der Erfassung ausgeschlossen. Das bedeutet, dass nur durch die Einstellung des Schwellwertes erheblich Einfluss auf die Charakterisierung von Dispersitätseigenschaften genommen wird. Ohne Informationen zu den Algorithmus-Parametern, den morphologischen Parametern und dem Schwellwert sind Ergebnisse aus der quantitativen Bildanalyse nicht vergleichbar.

Die flächenbezogene Gewichtung wurde einer anzahl- und volumenbezogenen Gewichtung vorgezogen. Nach DIN ISO 9276-6:2012-01 (2012) bietet die Projektionsfläche einen robusten zweidimensionalen Parameter, der direkt bestimmt werden kann. Die der quantitativen Bildanalyse als individuelle Zählmethode angemessene Gewichtung ist die anzahlbezogene Gewichtung. Hierbei werden allerdings die kornförmigen Partikel überrepräsentiert und führen regelmäßig zu einer bimodalen Häufigkeitsverteilung. Das macht die Analyse anhand von Verteilungssummen ungenau. Eine volumenbezogene Gewichtung erfordert eine dreidimensionale Erfassung der 
a

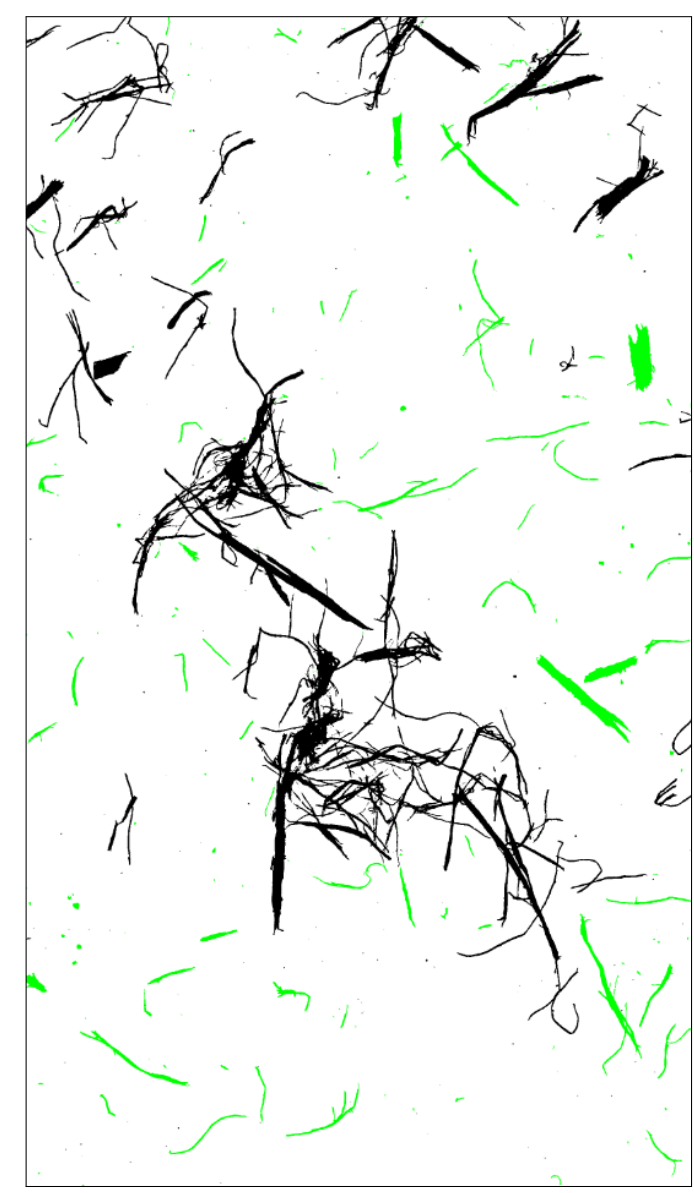

b

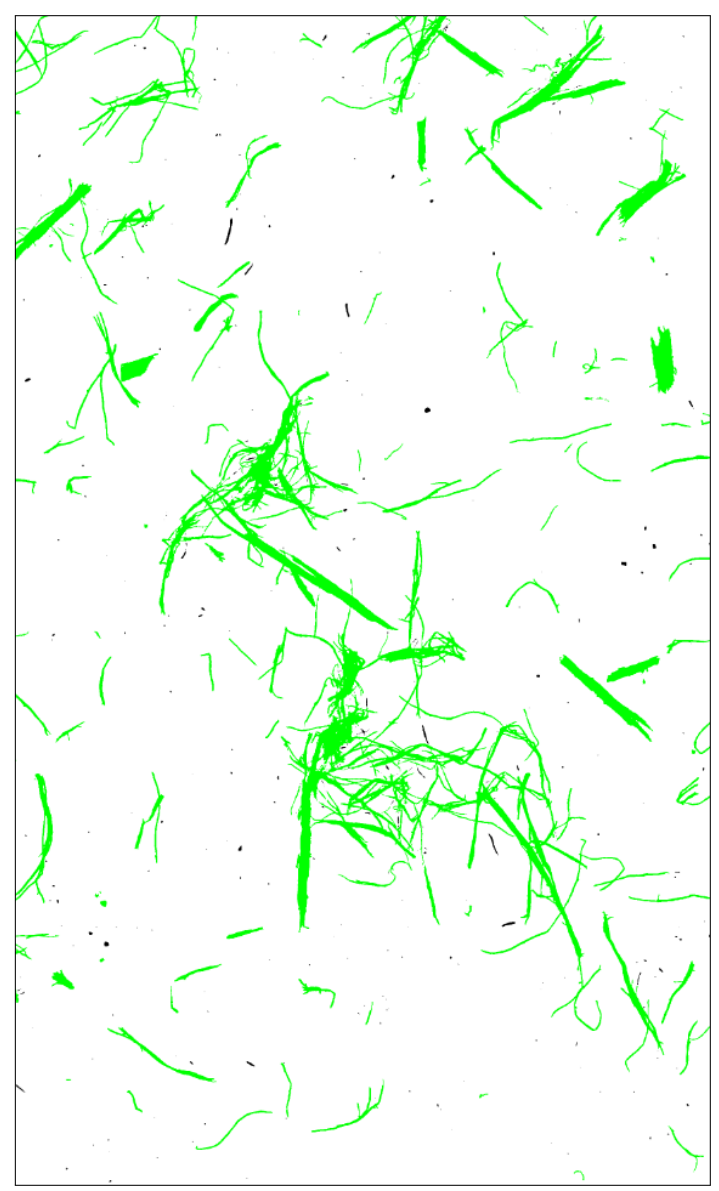

Abbildung 7.47: Ausschnitte aus einer, mit der FibreShape-Software analysierten, Aufnahme eines Fichtenfaserstoffes. Die grüngefärbten Fasern wurden durch die FibreShape-Software akzeptiert und die schwarzgefärbten nicht. a, Auswahl Filterparameter 1/Convexity: Minimum: 0, Maximum: 1,5. Die Auswahl selektiert über Kreuz liegende Partikel weitgehend. b, Auswahl Filterparameter 1/Convexity: Minimum: 1, Maximum: 2,5. Das große, nicht repräsentative Agglomerat wird beispielsweise als ein Partikel erfasst und verzerrt die Analyse. Kein Maßstab verfügbar.

Partikel. Die Software FibreShape berechnet zwar aus den zweidimensionalen Bilddaten das Volumen über ein Ellipsoid-Modell. Diese Vorgehensweise ist allerdings fehlerbehaftet. Außerdem werden dadurch wiederum die Faserbündel überproportional gewichtet, denn diese weisen keinen kreisrunden Querschnitt auf.

Auch die Prozessführung hat einen Einfluss auf die Dispersitätseigenschaften der Faserstoffe. Der Differenzdruck beeinflusst unter anderem die Verweilzeit der Faserstoffe im Mahlspalt (Miles \& May 1990). In der Versuchsdurchführung wurde darauf geachtet, dass der Druck im Mahlraum durch die manuelle Bedienung des Blasventils knapp unter dem Druck des Kochers gehalten wurde. Ek et al. (2009, S. 82) und Brombacher et al. (2016) stellten weiterhin fest, dass bei einer Verweilzeit der Hackschnitzel im Kocher von 2 min die geringsten Feinheiten und Längen in der Partikelgrößenverteilung beobachtet werden. Wohingegen bei $3 \mathrm{~min}$ bis $4 \mathrm{~min}$ und $6 \mathrm{~min}$ Verweilzeit im Kocher kein wesentlicher Unterschied bestand. 


\subsubsection{Effizienz in der Faserstofferzeugung}

Über die Charakterisierung von Dispersitätseigenschaften hinaus bietet die Methode der quantitativen Bildanalyse eine Möglichkeit, die Effizienz in der Faserstofferzeugung zu berechnen und vergleichend darzustellen. Nach Lamb (1960) kann die Erzeugung von Faserstoffen als eine Vergrößerung der Oberfläche des Holzes erachtet werden. Die Effizienz der Mahlscheiben ist folglich ein Maß dafür, wie viel elektrische Energie für die Vergrößerung der Oberfläche des Holzes aufgebracht wird. Für die Berechnung der Oberfläche des Holzes bzw. der Fasern bietet sich die gefüllte Fläche der Partikel (AREAF nach Schmid et al. (2020)) aus der Bildanalyse an. Damit wird der flächenäquivalente Durchmesser (CircDiam nach Schmid et al. (2020)) einer Kugel mit gleicher Projektionsfläche wie die des Partikels berechnet. Nach Leschonski (1984) und DIN ISO 9276-6:2012-01 (2012) wird dieser 2D-Deskriptor als idealer Normalisierungsparameter (Größenparameter zur Normalisierung von Formdeskriptoren) beschrieben.

Die Berechnung der Effizienz erfolgt nach Gleichung 7.10 mit dem Quotienten aus flächenequivalentem Durchmesser und Partikelanzahl als durchschnittliche Partikeloberfläche nach Gleichung 7.9.

$$
A_{p}=\frac{\sum_{i=1}^{n} C_{i}}{n}
$$

$A_{p} \quad$ durchschnittliche Partikeloberfläche

$C_{i} \quad$ flächenäquivalenter Durchmesser der Partikel

$n \quad$ Anzahl der Partikel

$$
\eta=\frac{A_{p}}{P_{\text {spez }}}
$$

$\eta \quad$ Effizienz der Mahlscheibenmuster in der Faserstofferzeugung

$A_{p} \quad$ durchschnittliche Partikeloberfläche

$P_{\text {spez }} \quad$ spezifische elektrische Leistungsaufnahme (SEL)

In Abbildung 7.48 ist die Effizienz der Mahlscheibenmuster in den einzelnen Aufschlussvarianten vergleichend dargestellt. Der Erkenntnisgewinn liegt in der Feststellung, dass die Effizienz der Faserstofferzeugung von der Konstruktion der Mahlscheiben, der Aufschlusstemperatur und der verwendeten Holzart abhängt. Pearson (1990, S. 19) fügt dem noch einen Einfluss des Durchsatzes und der Konsistenz hinzu. Die Faserstofferzeugung mit der Holzart Buche ist, gemessen an der Oberflächenvergrößerung zum Energieeinsatz, generell weniger Effizient als die Faserstofferzeugung mit der Holzart Fichte. Dies kann als Hinweis angesehen werden, dass die Mahlscheibenmuster für die Zerfaserung von Buchenholz weiter optimiert werden können. Hinweise für die Optimierung bietet die Konstruktion des Mahlscheibenmusters 2 bei $135^{\circ} \mathrm{C}$ und die Konstruktion des Mahlscheibenmusters 3 bei $175^{\circ} \mathrm{C}$.

In der Effizienz ragt bezüglich der Holzart Fichte das Mahlscheibenmuster 3 heraus. Interessanterweise nimmt die Effizienz hier mit steigender Aufschlusstemperatur ab. Diese Beobachtung passt zu dem ermittelten hohen Langfaser-Flächenanteil aus Abbildung 7.44. Anscheinend ist das Mahlscheibenmuster 3 sowohl für die Faserstofferzeugung bei niedrigen als auch bei hohen Aufschlusstemperaturen geeignet. Einen Erklärungsansatz für dieses Verhalten bietet die bereits diskutierte umlaufende Unterbrechung der Stege zur Entschärfung des Temperaturmaximums auf der Mahlbahn (Atack et al. 1989). Dem Einsatzgebiet in der MDF-Herstellung gemäß weist das Mahlscheibenmuster 4 eine hohe Effizienz in dem dort üblichen Temperaturbereich auf. Im RMP-Verfahren ist die Effizienz grundsätzlich noch relativ gering. Hier sind weitere Entwicklungsschritte basierend auf diesem erstmalig getesteten Mahlscheibenmuster notwendig. 
Leißner (2015, S. 9) zufolge weisen Zerkleinerungsprozesse im Allgemeinen einen geringen energietechnischen Wirkungsgrad auf. Die vorliegenden Untersuchungen belegen, dass über die Prozessführung und die Konstruktion von Mahlscheiben die Effizienz der Zerfaserung gesteigert werden kann. Für weitere Untersuchungen zu diesem Sachverhalt ist zu empfehlen, die Methode der quantitativen Bildanalyse dahingehend anzupassen, sodass eine massenbezogene Berechnung der Effizienz ermöglicht wird. Im vorliegenden Fall konnte die Vergrößerung der Oberfläche nicht sicher der Gesamtmasse der Partikel zugeordnet werden und es musste auf die Partikelanzahl zurückgegriffen werden. 


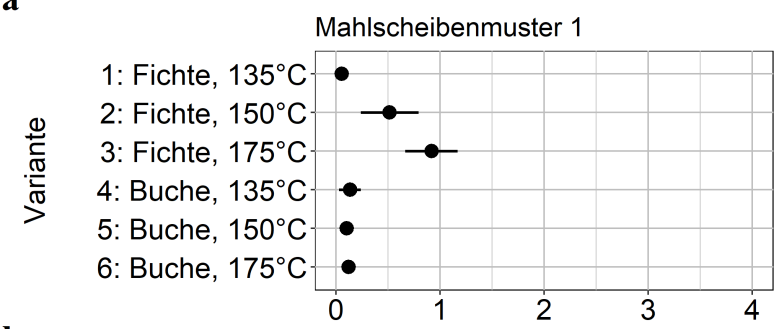

b

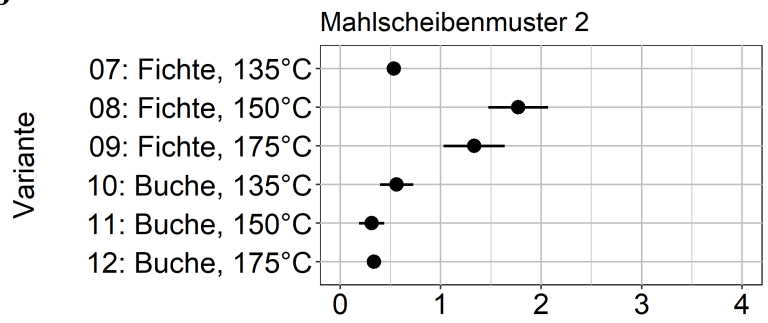

c

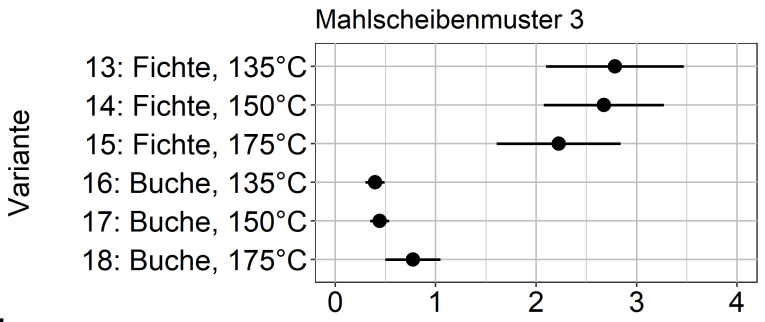

d

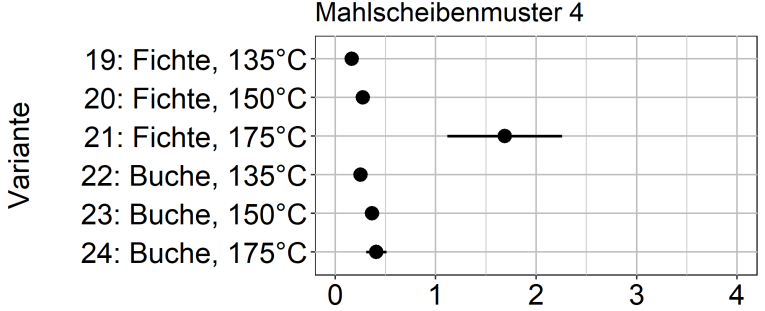

e

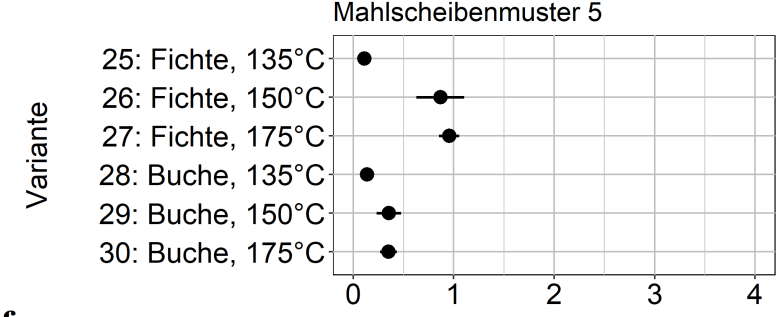

f

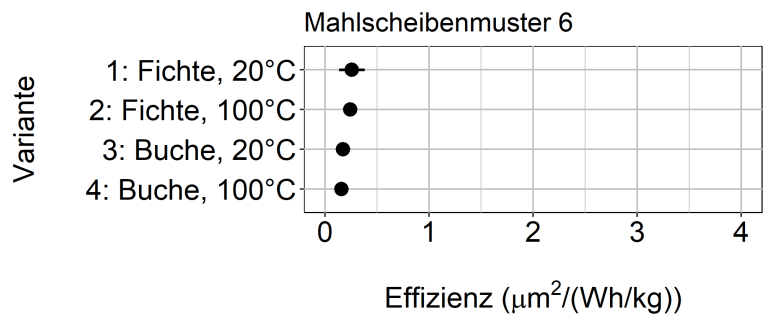

Abbildung 7.48: Vergleichende Darstellung der Effizienz der Mahlscheibenmuster in der Faserstofferzeugung, gemessen an der durchschnittlichen Partikeloberfläche. Dargestellt ist die Lage des arithmetischen Mittels innerhalb der Standardabweichung. TMP-Verfahren: a, Mahlscheibenmuster 1. b, Mahlscheibenmuster 2. c, Mahlscheibenmuster 3. d, Mahlscheibenmuster 4. e, Mahlscheibenmuster 5. RMP-Verfahren: f, Mahlscheibenmuster 6. $n=4$. 


\subsubsection{Mikroskopische Untersuchungen an den Faserstoffen}

Abschließend zu den Untersuchungen zur Beschaffenheit von Faserstoffen soll ein qualitativer Vergleich die Ausführungen ergänzen und die theoretischen Betrachtungen veranschaulichen. Im Folgenden sind mikroskopische Aufnahmen der labortechnisch erzeugten TMP- und RMPFaserstoffe gemeinsam mit Holzschliff, Holzfaserdämmstoffen, MDF- und Zellstoffen dargestellt. Durch einen Vergleich sollen die bisherigen Untersuchungen in den Kontext der industriellen Faserstofferzeugung eingeordnet werden. Dazu sind die wichtigsten, in den Bildausschnitten sichtbaren, qualitativen Merkmale der Faserstoffe in den Bildunterschriften knapp wiedergegeben. Um die chemischen Oberflächeneigenschaften, die Zugänglichkeit der inneren Struktur und den Grad der Fibrilierung der Fasern sichtbar zu machen, wurde eine zweifarbige differentielle Färbemethode nach Simon (1950) angewendet (Simons' stain, siehe Abschnitt 4.3). Den Ergebnissen von Kwok et al. (2017) folgend, wurde anstelle des nicht mehr verfügbaren Farbstoffs Direct Orange 15 der gleichwertige Farbstoff Direct Yellow 11 verwendet. Auf Grundlage der Untersuchungen von Fernando \& G. Daniel (2010) zur Quantifizierung mechanischer Beanspruchungen wurde die Färbemethode auf die Faserstoffe der vorliegenden Arbeiten angewendet, um mögliche Unterschiede zwischen den Mahlscheibenmustern und Aufschlusstemperaturen zu identifizieren. In diesem Zusammenhang muss zuerst festgehalten werden, dass die Färbemethode auch für die Färbung von Buchen-Faserstoffen geeignet ist, was bislang nicht beschrieben wurde.

In Ergänzung zur manuellen Quantifizierung nach Fernando \& G. Daniel (2010) konnte in Vorversuchen gezeigt werden, dass eine quantitative Auswertung mittels Bildanalyse möglich ist und dadurch eine größere Anzahl Partikel erfasst wird. Zum einen bietet die Software FibreShape die Möglichkeit, Farbwerte zu analysieren, zum anderen eignet sich auch frei verfügbare Software wie ImageJ zur Quantifizierung der Farbanteile. Für die vorliegende Arbeit wurden allerdings durch die Bildanalyse keine wesentlichen neuen Erkenntnisse zur Beschaffenheit der Faserstoffe gefunden. Im Hinblick auf die Zielsetzung wurden diese Ergebnisse nicht in die Ausarbeitung aufgenommen. Letztendlich bietet die Färbung dennoch einen guten Kontrast für das Mikroskopieren. Eine weitere interessante Färbemethode zur lichtmikroskopischen Charakterisierung von Faserstoffen beschreiben Srebotnik \& Messner (1994). Damit könnten Ligninanteile auf der Oberfläche der Fasern sichtbar gemacht werden.

Der mikroskopische Vergleich von labortechnischen Faserstoffen mit industriellen Papierstoffen in Abbildung 7.49 ist aufschlussreich hinsichtlich der qualitativen Beschaffenheit. In den labortechnischen Faserstoffen sind, im Vergleich zu den Papierstoffen, praktisch keine desintegrierten und fibrillierten Bestandteile sichtbar. Insbesondere im Vergleich mit dem TMP-Papierstoff aus der 1. Refinerstufe (Abbildung 7.49 d) wird deutlich, dass die Zellstruktur der labortechnischen Faserstoffe im Gegensatz zu dem Papierstoff intakt ist. In den Papierstoffen ist die Primärwand und die $S_{1}$-Schicht teilweise vollständig flöckchen- und plättchenförmig abgelöst. Die $S_{2}$-Schicht delaminiert bändchenförmig und wird als externe Fibrillierung sichtbar (Koran 1970; Fernando \& G. Daniel 2004). Allerdings ist eine gewisse Latenz durch das Verdrehen und Kräuseln der Fasern sichtbar. Die spezifische elektrische Leistungsaufnahme zur Herstellung des Papierstoffes lag bei rund $660 \mathrm{kWh} / \mathrm{t}_{\text {atro }}$, die der labortechnischen Faserstoffe zwischen rund $180 \mathrm{Wh} / \mathrm{kg}_{\text {atro }}$ und $490 \mathrm{Wh} / \mathrm{kg}_{\text {atro }}$.

In Abbildung 7.50 werden die anatomischen Unterschiede zwischen den Holzarten Fichte und Buche sichtbar. Die faserartigen Bestandteile der Buche sind feiner und kürzer als die der Fichte. Zudem sind die Tracheen in der Holzart Buche zahlreich vertreten. Insbesondere in Abbildung 7.50a und Abbildung 7.50b ist die Latenz durch gekrümmte Fasern und agglomerierte Fibrillen gekennzeichnet. Ein Vergleich der labortechnischen Faserstoffe mit industriellen Fa- 
serstoffen im selben Aufschlusstemperatur-Bereich in Abbildung 7.51 macht deutlich, dass sich die Morphologie der industriellen Faserstoffe, abgesehen von der Holzart, nicht wesentlich von den labortechnischen unterscheidet. Zwischen der Aufschlusstemperatur von $135^{\circ} \mathrm{C}$ und $175^{\circ} \mathrm{C}$ bestehen markante Unterschiede in den Buchen-Faserstoffen, wie aus Abbildung 7.52 hervorgeht. Bei der niedrigeren Aufschlusstemperatur treten vermehrt Bruchstücke auf und die Tracheen werden zerkleinert. Die RMP-Faserstoffe in Abbildung 7.53 unterscheiden sich deutlich in ihrer Morphologie von den TMP-Faserstoffen. Es sind hauptsächlich kurze, unregelmäßige und teils stark aufgefaserte Faserbündel sowie geknickte, gebrochene und gekrümmte Fasern sichtbar. Die Faserbündel erscheinen aufgrund der geringen Lichtdurchlässigkeit relativ dick zu sein. 
a

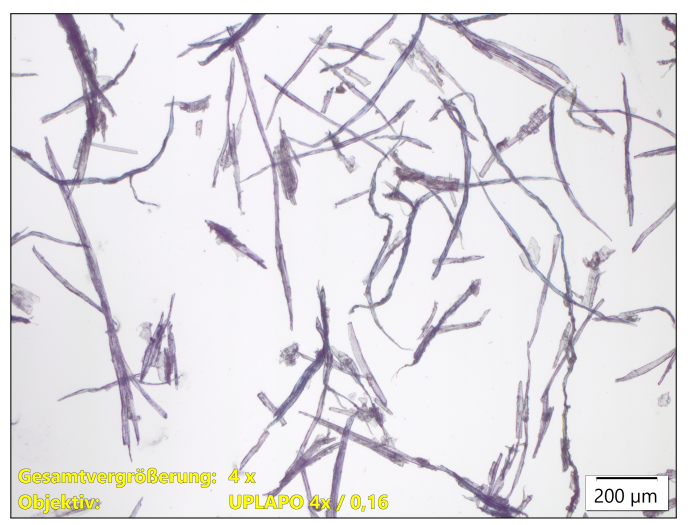

c

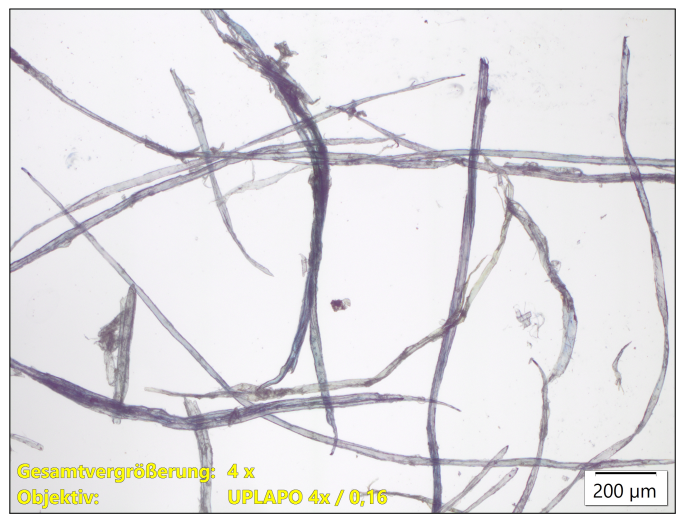

e

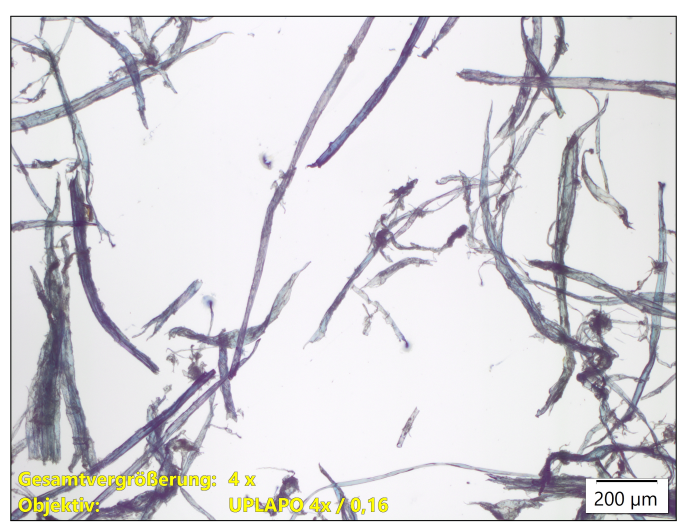

b

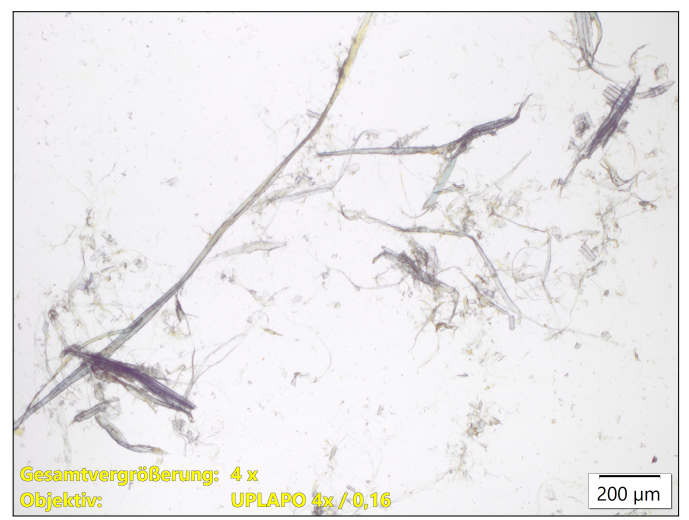

d

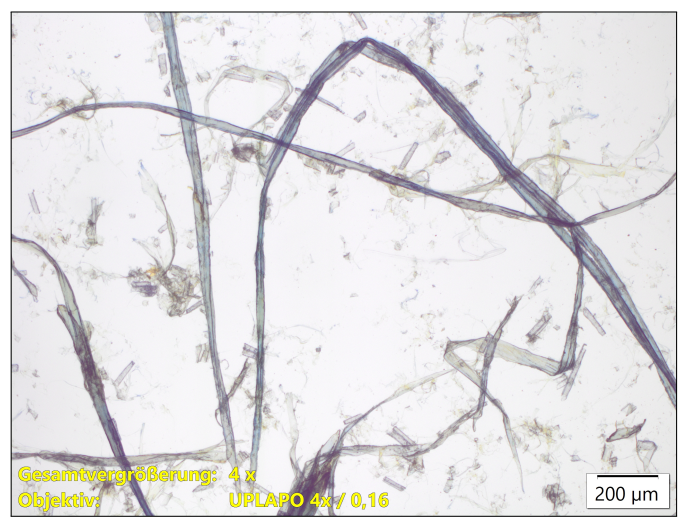

$f$

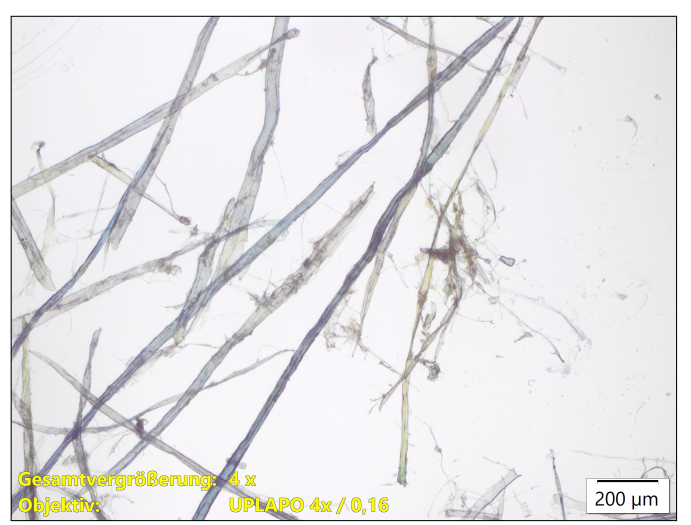

Abbildung 7.49: Mikroskopische Aufnahmen von Faserstoffen: Qualitativer Vergleich labortechnisch erzeugter Faserstoffe mit industriell erzeugten Faserstoffen aus der Papierproduktion. a, Mahlscheibenmuster 1, Fichte, $135^{\circ} \mathrm{C}$ : Zahlreiche Bruchstücke von Tracheiden und Faserbündeln. b, Holzschliff, ungebleicht, Fichte: Stark desintegrierte und fibrilierte Tracheiden und Bruchstücke. c, Mahlscheibenmuster 3, Fichte, $135^{\circ} \mathrm{C}$ : Relativ intakte Tracheiden. $\boldsymbol{d}$, TMP 1. Refinerstufe, $145^{\circ} \mathrm{C}$ Fichte: Intakte und delaminierte, fibrilierte Tracheiden.e, Mahlscheibenmuster 5, Fichte, $135^{\circ} \mathrm{C}$ : Fibrillierte Faserbündel und Agglomerate. f, BCTMP, Fichte: Relativ intakte Tracheiden und Feinstoff. Die Aufnahmen liegen in hoher Auflösung vor. Zur detaillierten Betrachtung eignet sich die Vergrößerungsfunktion des PDF-Readers. 
a

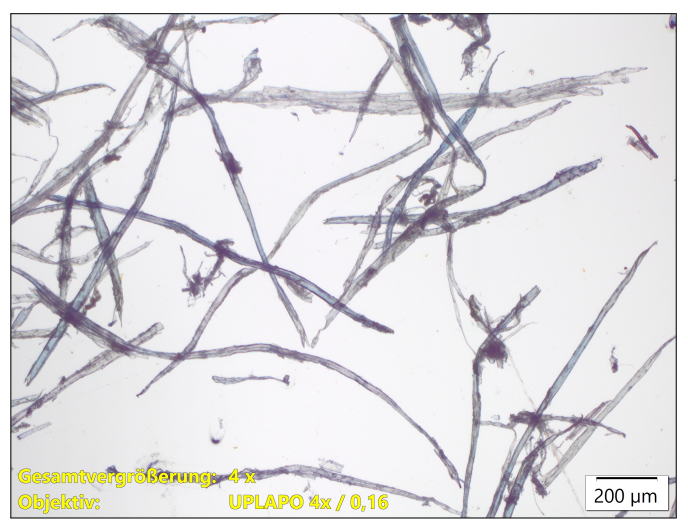

C

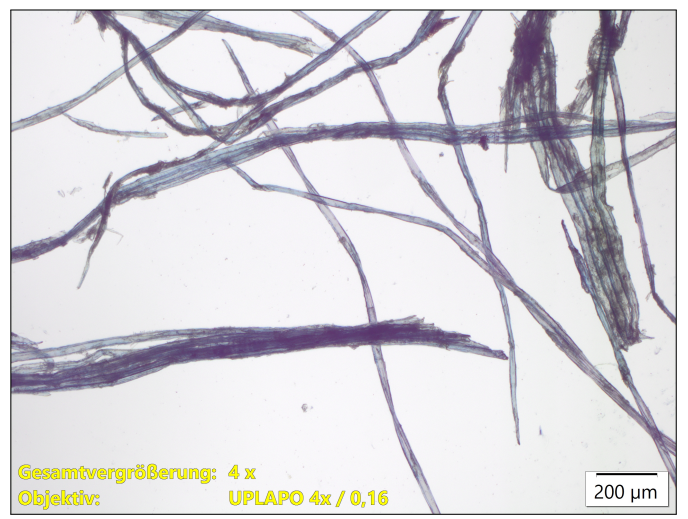

e

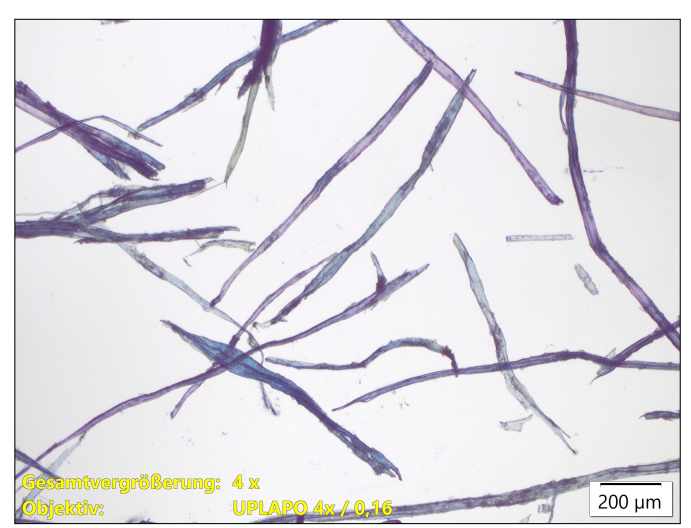

b

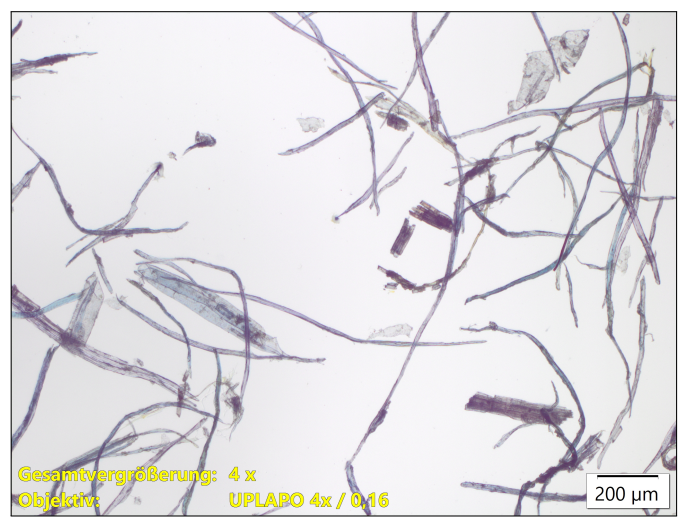

$\boldsymbol{d}$

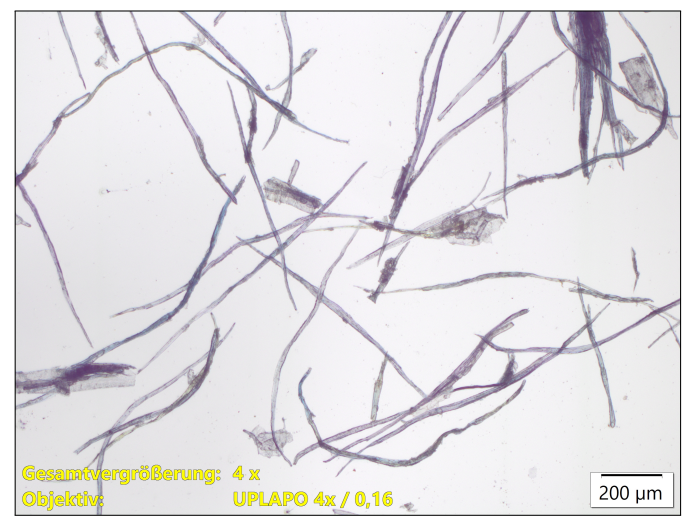

$f$

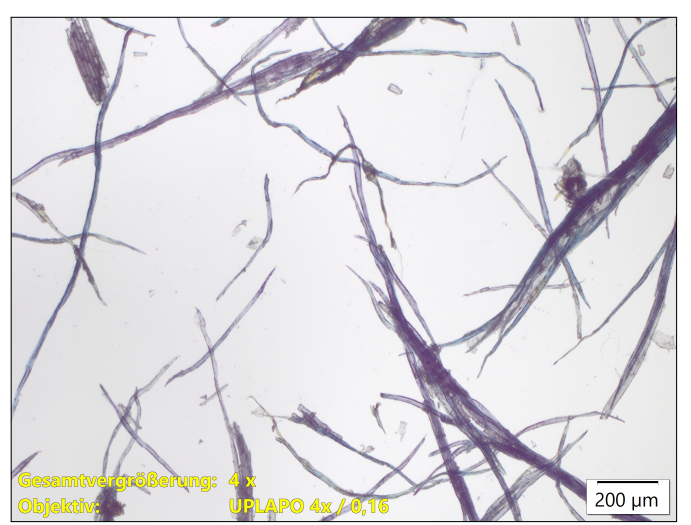

Abbildung 7.50: Mikroskopische Aufnahmen von Faserstoffen: Qualitativer Vergleich labortechnisch erzeugter Faserstoffe aus Fichten- und Buchenholz. a, Mahlscheibenmuster 1, Fichte, $150^{\circ} \mathrm{C}$ : Teilweise desintegrierte Tracheiden, Bruchstücke und Agglomerate aus Fibrillen. b, Mahlscheibenmuster 1, Buche, $150^{\circ} \mathrm{C}$ : Bruchstücke von Parenchym und Tracheen sowie intakte Fasern. c, Mahlscheibenmuster 3, Fichte, $150^{\circ} \mathrm{C}$ : Faserbündel und Tracheiden. d, Mahlscheibenmuster 3, Buche, $150^{\circ} \mathrm{C}$ : Relativ intakte Fasern sowie Tracheen. e, Mahlscheibenmuster 5, Fichte, $150^{\circ} \mathrm{C}$ : Teilweise angeschnittene Tracheiden.f, Mahlscheibenmuster 5, Buche, $150^{\circ} \mathrm{C}$ : Feine Bruchstücke und Faserbündel. Die Aufnahmen liegen in hoher Auflösung vor. Zur detaillierten Betrachtung eignet sich die Vergrößerungsfunktion des PDF-Readers. 
a

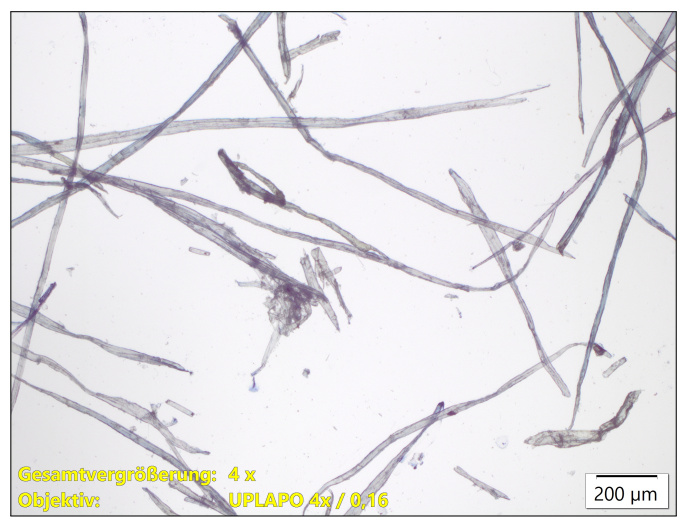

c

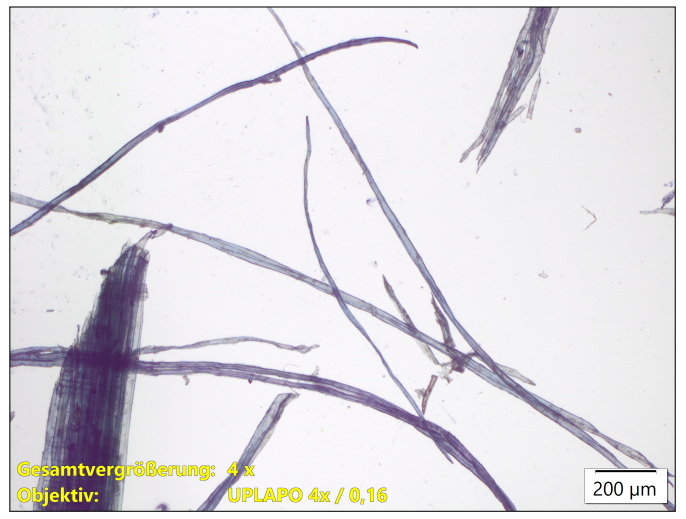

e

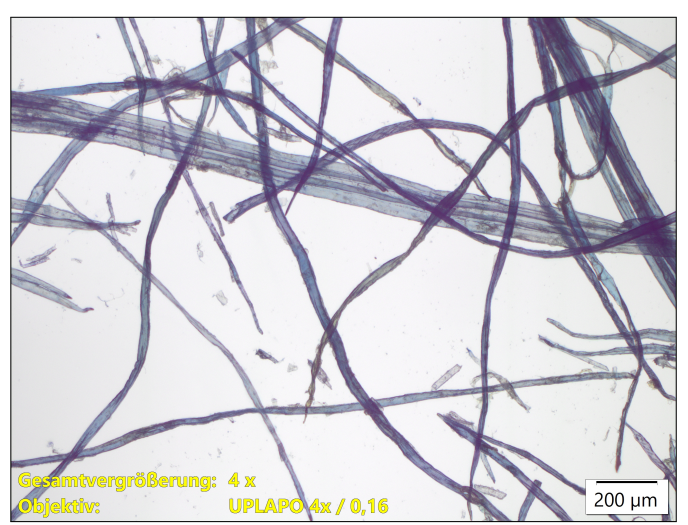

b

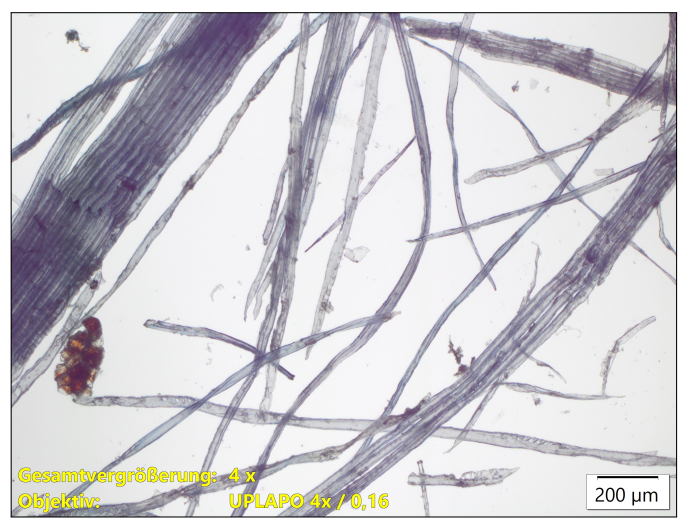

d

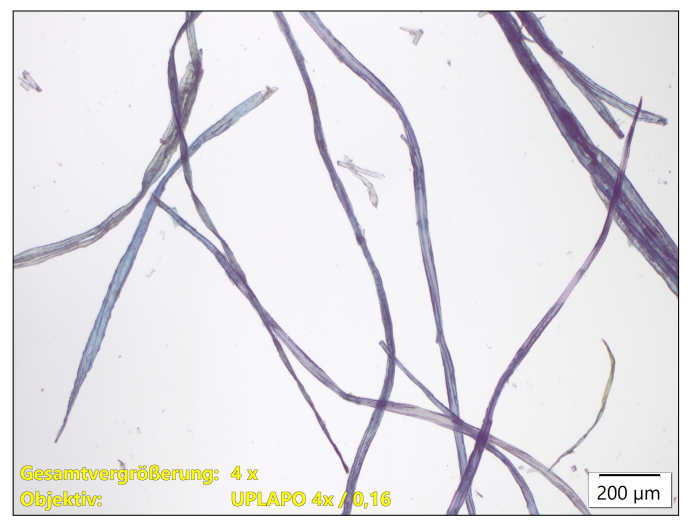

$f$

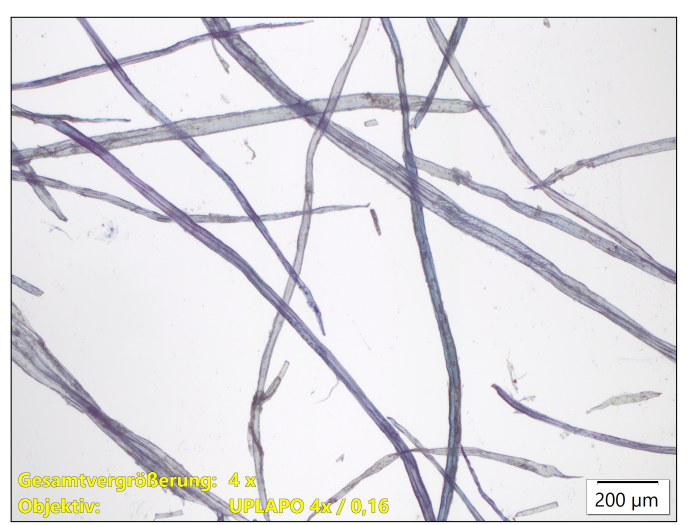

Abbildung 7.51: Mikroskopische Aufnahmen von Faserstoffen: Qualitativer Vergleich labortechnisch erzeugter Faserstoffe mit industriellen Holzfaserdämmstoff- und MDF-Faserstoffen. a, Mahlscheibenmuster 1, Fichte, $175^{\circ} \mathrm{C}$ : Teilweise desintegrierte Tracheiden, Bruchstücke und Agglomerate aus Fibrillen. Die blassblaue Färbung weist auf eine relativ starke mechanische Beanspruchung hin. b, Holzfaserdämmstoff, Kiefer: Dicke Faserbündel, separierte Tracheiden und Bruchstücke. c, Mahlscheibenmuster 3, Fichte, $175^{\circ} \mathrm{C}$ : Faserbündel, separierte Tracheiden und vereinzelte Bruchstücke. d, Holzfaserdämmstoff, Fichte: Faserbündel, separierte Tracheiden und vereinzelte Bruchstücke. e, Mahlscheibenmuster 5, Fichte, $175^{\circ} \mathrm{C}$ : Faserbündel, separierte Tracheiden und Bruchstücke.f, MDF, Kiefer: Schmale Faserbündel, separierte Tracheiden und Bruchstücke. Die Aufnahmen liegen in hoher Auflösung vor. Zur detaillierten Betrachtung eignet sich die Vergrößerungsfunktion des PDF-Readers. 
$\mathbf{a}$

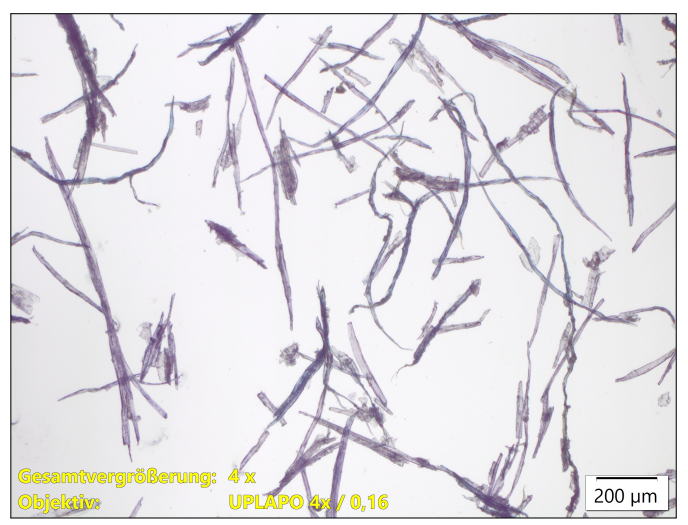

$\boldsymbol{C}$

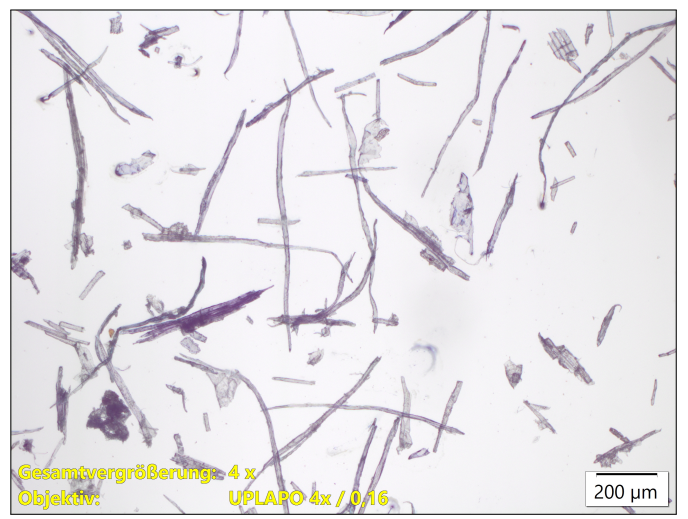

e

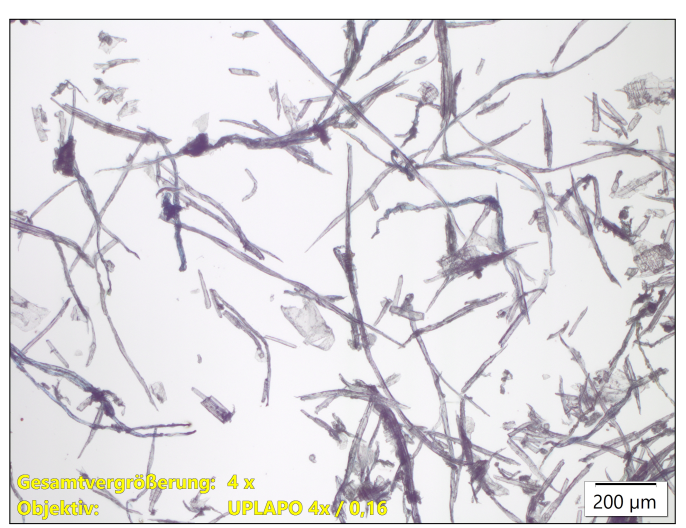

.

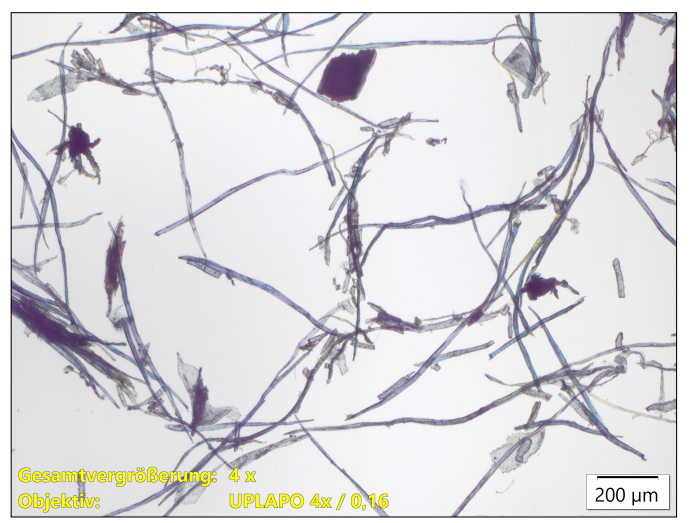

$d$

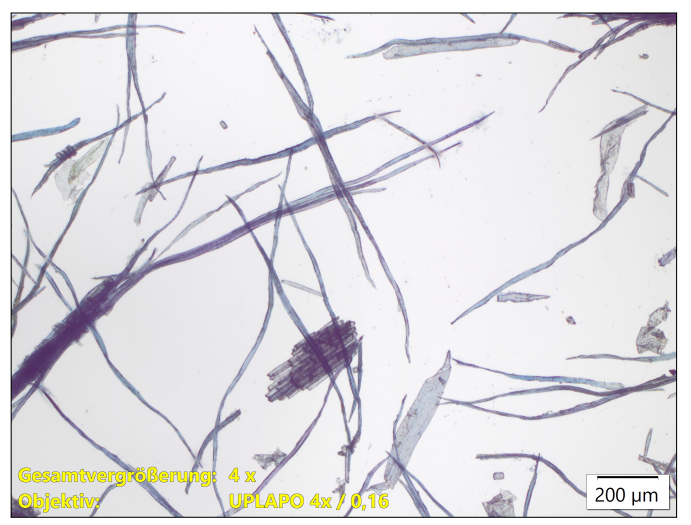

$f$

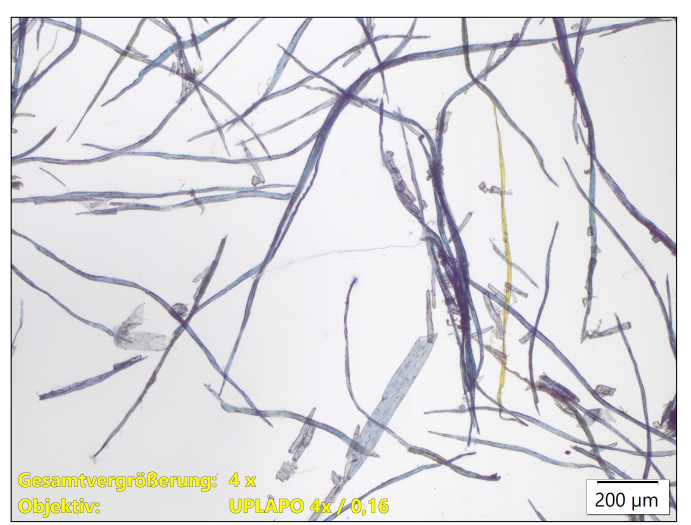

Abbildung 7.52: Mikroskopische Aufnahmen von Faserstoffen: Qualitativer Vergleich labortechnisch erzeugter Faserstoffe aus Buchenholz. a, Mahlscheibenmuster 1, Buche, $135^{\circ} \mathrm{C}$ : Zahlreiche Bruchstücke sowie verdrillte Fasern. b, Mahlscheibenmuster 1, Buche, $175^{\circ} \mathrm{C}$ : Zahlreiche dickere und an den Fasern agglomerierte Bruchstücke. c, Mahlscheibenmuster 3, Buche, $135^{\circ} \mathrm{C}$ : Überwiegend Bruchstücke. d, Mahlscheibenmuster 3, Buche, $175^{\circ} \mathrm{C}$ : Intakte Fasern und Tracheen neben größeren Bruchstücken. e, Mahlscheibenmuster 5, Buche, $135^{\circ} \mathrm{C}$ : Gehacktes von der Buche.f, Mahlscheibenmuster 5, Buche, $175^{\circ} \mathrm{C}$ : Intakte Fasern und Tracheen neben kleineren Bruchstücken. Die Aufnahmen liegen in hoher Auflösung vor. Zur detaillierten Betrachtung eignet sich die Vergrößerungsfunktion des PDF-Readers. 
a

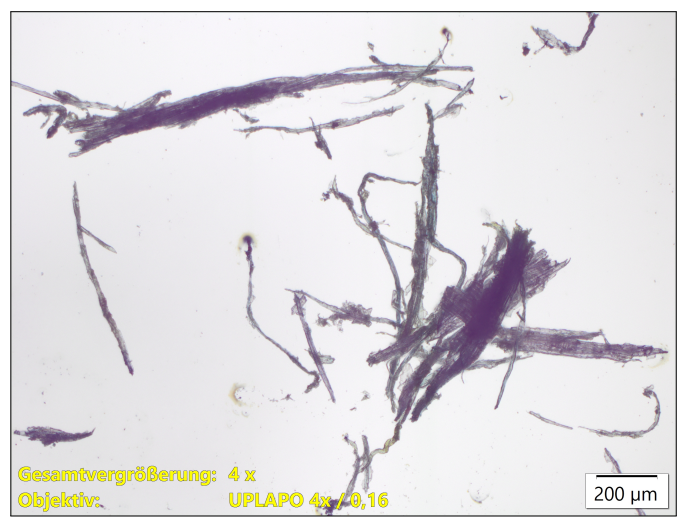

c

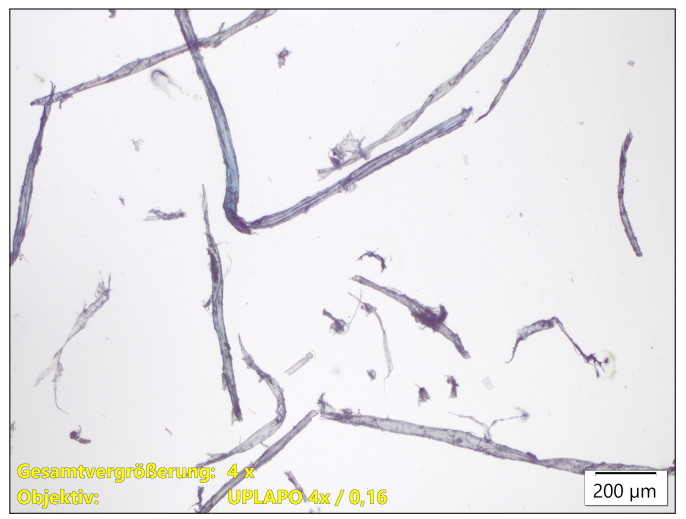

e

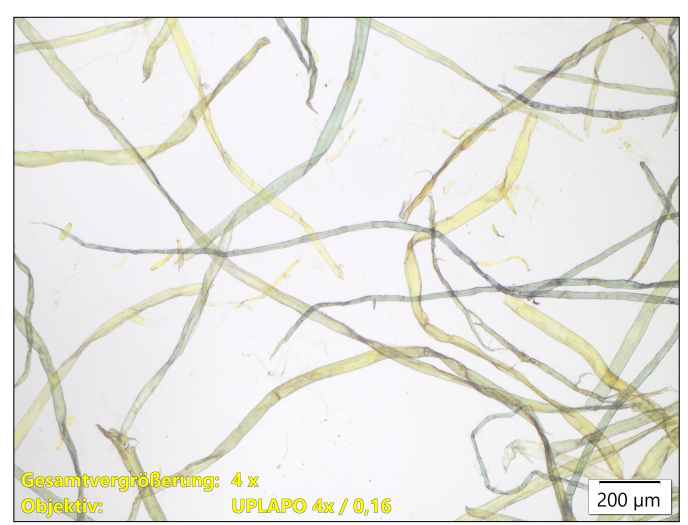

b

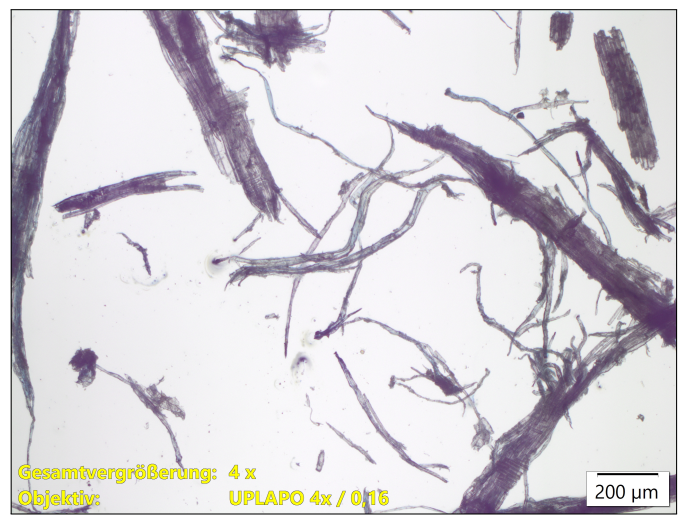

d

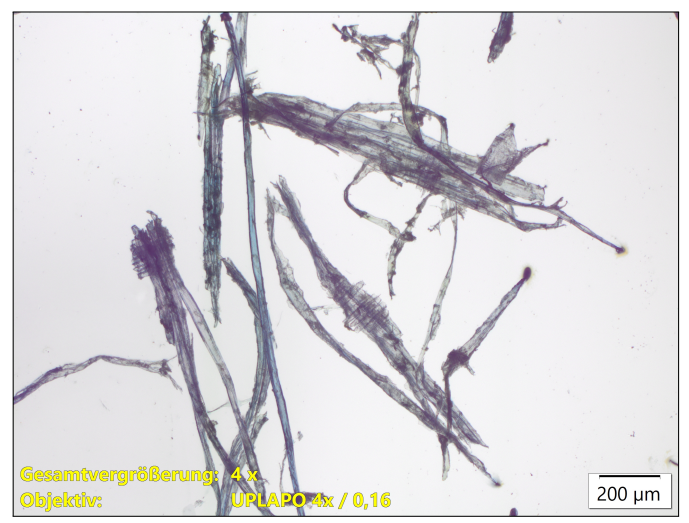

$f$

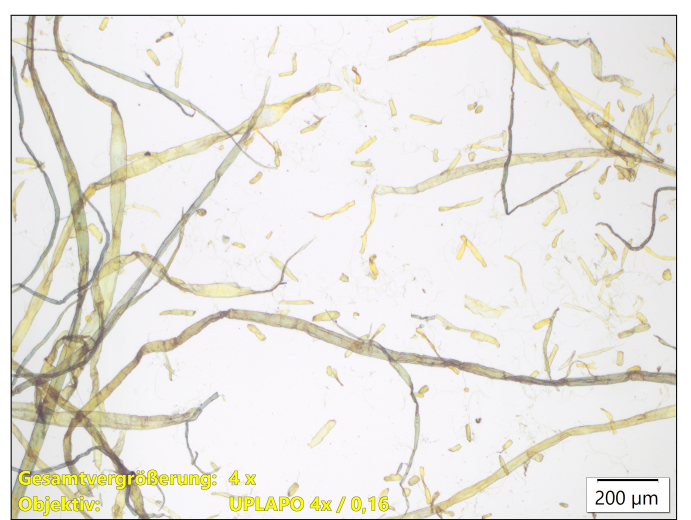

Abbildung 7.53: Mikroskopische Aufnahmen von Faserstoffen: Qualitativer Vergleich labortechnisch erzeugter Faserstoffe im RMP-Verfahren sowie mikroskopische Aufnahmen von Zellstoffen. a, Mahlscheibenmuster 6, Buche, $20^{\circ} \mathrm{C}$ : Kurze, unregelmäßige Faserbündel und Agglomerate. b, Mahlscheibenmuster 6, Buche, $100^{\circ} \mathrm{C}$ : Unregelmäßige, aufgefaserte Faserbündel und Agglomerate. c, Mahlscheibenmuster 6, Fichte, $20^{\circ} \mathrm{C}$ : Geknickte, gebrochene Tracheiden und Bruchstücke. d, Mahlscheibenmuster 6, Fichte, $100^{\circ} \mathrm{C}$ : Kurze, angeschnittene, aufgefaserte Faserbündel. e, NBSK, Fichte: Bändchenförmige, kollabierte Tracheiden. Die gelbe Färbung ist auf die mechanische Beanspruchung in der LC-Mahlung zurückzuführen.f, Sulfit-Zellstoff, Nachsortierung, Buche $35 \%$, Fichte 65 \%: Bändchenförmige, kollabierte Tracheiden neben Buchenfasern und zahlreichen Bruchstücken. Die gelbe Färbung ist auf die mechanische Beanspruchung in der LC-Mahlung zurückzuführen. Die Aufnahmen liegen in hoher Auflösung vor. Zur detaillierten Betrachtung eignet sich die Vergrößerungsfunktion des PDFReaders. 


\subsection{Untersuchungen zur Wärmeleitfähigkeit der Faserstoffe}

\subsubsection{Einführung}

Die Wärmeleitfähigkeit ist die maßgebenden Produkteigenschaft von Holzfaserdämmstoffen. Die Untersuchungen zur Faserstofferzeugung und der Beschaffenheit der Faserstoffe sind für sich genommen zwar erkenntnisreich, ohne die Ermittlung der Wärmeleitfähigkeit allerdings nicht weiter zielführend. Mit der Ermittlung der Wärmeleitfähigkeit geht die bislang weitgehend ungeklärte Frage einher, welche Charakteristika Faserstoffe besitzen sollten, um eine möglichst niedrige Wärmeleitfähigkeit zu erzielen.

Das Ziel der Untersuchungen zur Wärmeleitfähigkeit ist deshalb, die Eigenschaften der Faserstoffvarianten, die mit unterschiedlichen Mahlscheibenmustern und Aufschlusstemperaturen erzeugt wurden, zu ermitteln. Dies gilt insbesondere für die niedrigen Aufschlusstemperaturen und das RMP-Verfahren. Zusätzlich wird der Versuch unternommen, Beziehungen zwischen technologischen Faktoren in der Prozessführung sowie den Dispersitätseigenschaften der Faserstoffe mit der Wärmeleitfähigkeit aufzudecken.

\subsubsection{Wärmeleitfähigkeit der Faserstoffe}

$\mathrm{Zu}$ Beginn der Untersuchungen wurde die Frage beantwortet, welche Wärmeleitfähigkeit industriell erzeugte Faserstoffe und Holzfaserdämmstoffe besitzen. Zusammen mit der Bestimmung der Ausgangs-Partikelgröße der Hackschnitzel und der Ziel-Partikelgröße der industriellen Faserstoffe (Abschnitt 7.1.2) sind diese Untersuchungen grundlegend für die labortechnische Faserstofferzeugung. In Tabelle 7.16 sind die Ergebnisse der Wärmeleitfähigkeit-Messung industrieller Faserund Holzfaserdämmstoffe aufgelistet. Die niedrigste Wärmeleitfähigkeit besitzt die MDF-Faser. Den höchsten Wert weist die Holzfaserdämmstoff-Faser 1 auf.

Tabelle 7.16: Wärmeleitfähigkeit ausgewählter industrieller Holzfaserdämmstoffe.

\begin{tabular}{lllll}
\hline Bezeichnung & Holzart & $\begin{array}{l}\text { Wärmeleit- } \\
\text { fähigkeit } \lambda_{10}{ }^{*} \\
\mathrm{~W} /(\mathrm{m} \cdot \mathrm{K})\end{array}$ & $\begin{array}{l}\text { Erweiterte } \\
\text { Messunsicherheit } \\
\mathrm{W} /(\mathrm{m} \cdot \mathrm{K})\end{array}$ & $\begin{array}{l}\text { Nennwert des } \\
\text { Herstellers } \\
\mathrm{W} /(\mathrm{m} \cdot \mathrm{K})\end{array}$ \\
\hline MDF-Faser & Kiefer & 0,0348 & 0,0007 & n.a. \\
Holzfaserdämmstoff-Faser 1 & Fichte & 0,0383 & 0,0007 & n.a. \\
Holzfaserdämmstoff-Faser 3 & Kiefer & 0,0363 & 0,0007 & $0,038^{* *}$ \\
Holzfaserdämmstoff-Faser 4 & Kiefer & 0,0352 & 0,0007 & $0,038^{* *}$ \\
Zellulosedämmung & Altpapier & 0,0374 & 0,0007 & 0,038 \\
Holzfaserdämmstoff-Matte 1 & Fichte & 0,0356 & 0,0007 & 0,038 \\
Holzfaserdämmstoff-Matte 2 & Kiefer & 0,0373 & 0,0007 & 0,038 \\
\hline${ }^{*}$ Feuchtekorrektur nach Normklima: $23^{\circ} \mathrm{C} / 50 \%$. \\
${ }^{* *}$ Einblasdämmung: Nennwert bezogen auf Rohdichte von $35 \mathrm{~kg} / \mathrm{m}^{3} \mathrm{bis} 45 \mathrm{~kg} / \mathrm{m}^{3}$
\end{tabular}

Die Wärmeleitfähigkeit wurde an, aus Faserstoffen gebildeten, Vliesen mit einer Rohdichte von $50 \mathrm{~kg}$ atro $/ \mathrm{m}^{3}$, ermittelt. Durch die enthaltene Feuchte ist ein Einfluss auf die Wärmeleitfähigkeit zu erwarten (vgl. Abschnitt 5.3). Die notwendigen Ressourcen und Einrichtungen vorausgesetzt, ist es grundsätzlich empfehlenswert, die Wärmeleitfähigkeit an darrtrockenen Probekörpern zu bestimmen und die Werte anschließend auf Normklima umzurechnen. Entsprechende Vorgaben sind in der EN ISO 10456:2007 + AC:2009 (2010) beschrieben. Die Problematik, die bei einer Wärmeleitfähigkeit-Messung an feuchten Probekörpern auftritt, hängt mit den Probenmitteltemperaturen $10^{\circ} \mathrm{C}, 20^{\circ} \mathrm{C}$ und $30^{\circ} \mathrm{C}$ zusammen. An diesen Punkten stellt sich eine 
unterschiedliche Ausgleichsfeuchte im Vergleich zum Einbauzustand der Probe ein. Insbesondere die Desorption kann zu Tauwasserkondensation an der Kühlplatte führen und einen Feuchtegradienten über den Probekörperquerschnitt hervorrufen. Dadurch kann die Messung ungenau und die Vergleichbarkeit beeinträchtigt werden. Im vorliegenden Fall handelt es sich allerdings nicht um eine industrielle Qualitätskontrolle, sondern es geht darum, Unterschiede in der Wärmeleitfähigkeit zwischen den Holzarten, Mahlscheibenmustern, Aufschlusstemperaturen und Aufschlussverfahren herauszuarbeiten. Zu diesem Zweck wurde eine Feuchtekorrektur angewendet, um die Vergleichbarkeit der Ergebnisse zu verbessern.

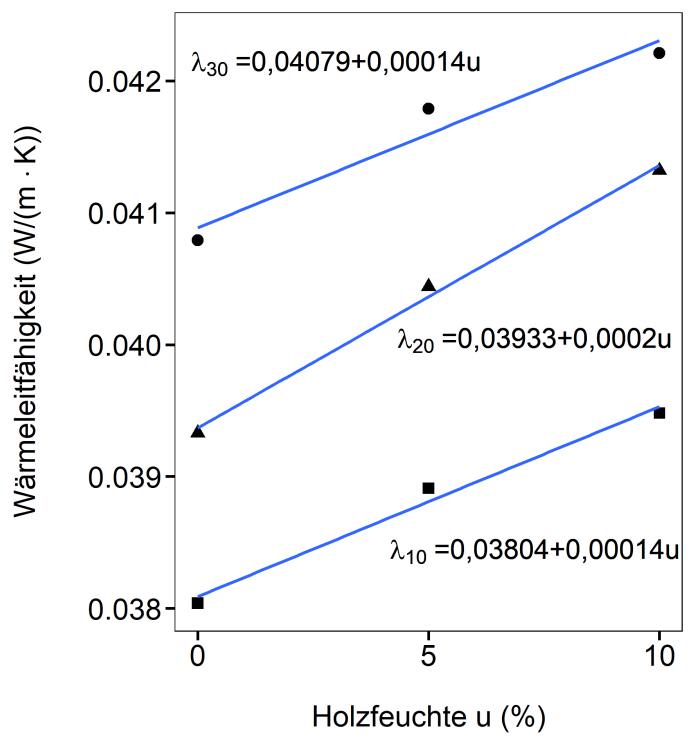

Abbildung 7.54: Ermittlung einer Geradengleichung der Wärmeleitfähigkeit von Fichten-Faserstoff bei Probenmitteltemperaturen von $10^{\circ} \mathrm{C}\left(\lambda_{10}\right), 20^{\circ} \mathrm{C}\left(\lambda_{20}\right)$ und $30^{\circ} \mathrm{C}\left(\lambda_{30}\right)$ in Abhängigkeit von der Holzfeuchte bei einer Rohdichte von $50 \mathrm{~kg}_{\text {atro }} / \mathrm{kg}$ mittels linearer Regression.

Aus Wärmeleitfähigkeit-Messungen an Probekörpern aus Fichten-Faserstoffen mit $50 \mathrm{~kg}$ atro $/ \mathrm{m}^{3}$ und einer Holzfeuchte von $0 \%, 5 \%$ und $10 \%$ konnte eine Geradengleichung ermittelt werden (Abbildung 7.54). Die lineare Regression liefert für die Probenmitteltemperaturen $10^{\circ} \mathrm{C}, 20^{\circ} \mathrm{C}$ und $30^{\circ} \mathrm{C}$ eine Änderung der Wärmeleitfähigkeit zwischen $3,7 \%$ und $5,1 \%$ pro $1 \%$ Holzfeuchteänderung. Sonderegger \& Niemz (2012) fanden für Holzfaserdämmstoff-Platten aus dem Nassverfahren mit einer Rohdichte von $130 \mathrm{~kg}$ atro $/ \mathrm{m}^{3}$ eine Änderung der Wärmeleitfähigkeit zwischen $5,7 \%$ und $6,0 \%$ pro $1 \%$ Holzfeuchteänderung für die Probenmitteltemperaturen $10^{\circ} \mathrm{C}, 20^{\circ} \mathrm{C}$ und $30^{\circ} \mathrm{C}$. Die Holzart ist bei Sonderegger \& Niemz (2012) nicht angegeben. Für die Ergebnisse der vorliegenden Arbeit erfolgt die Feuchtekorrektur daher mit dem Mittelwert der eigenen Messung als einer Änderung der Wärmeleitfähigkeit von $4,4 \%$ pro $1 \%$ Holzfeuchteänderung. Damit wurde die Feuchtedifferenz zwischen den Probekörpern auf die europäisch einheitlich geregelte Bezugsfeuchte von $23^{\circ} \mathrm{C} / 50 \%$ relative Luftfeuchte normiert. Die Ausgleichsfeuchte der Holzarten Buche und Fichte in dem Normklima wurde Böhner (1996) entnommen.

In Ergänzung zur Theorie der Wärmeleitung in Faserstoffen (Abschnitt 2.3.3) sind in Abbildung 7.55 Querschnitte von Holzfaserdämmstoffen dargestellt. Ein qualitativer Vergleich dient zur Einordnung der Ergebnisse zu den Wärmeleitfähigkeit-Messungen dahingehend, dass durch 
die labortechnische, aerodynamische Faservliesbildung (Abbildung 7.55 a und b) scheinbar die Faserorientierung senkrecht zur Mattenebene stärker ausgeprägt ist, als bei den industriellen Holzfaserdämmstoff-Matten. Insbesondere die industrielle Holzfaserdämmstoff-Matte 1 in Abbildung $7.55 \mathrm{c}$ weist überwiegend in Mattenebene orientierte Fasern auf. Dies könnte zu dem niedrigen Messwert der Wärmeleitfähigkeit von $\lambda_{10}=0,0356 \mathrm{~W} /(\mathrm{m} \cdot \mathrm{K})$ beitragen.

a

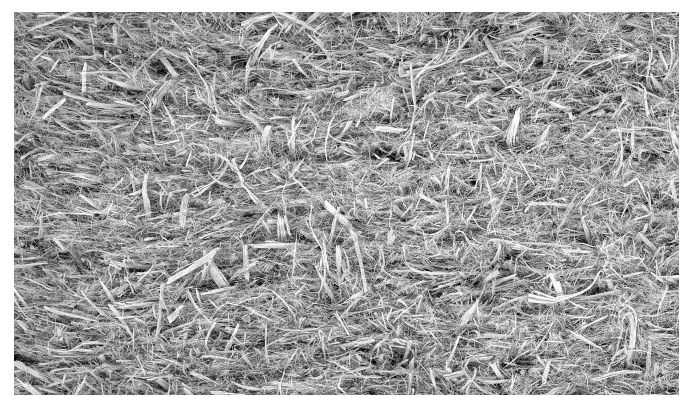

C

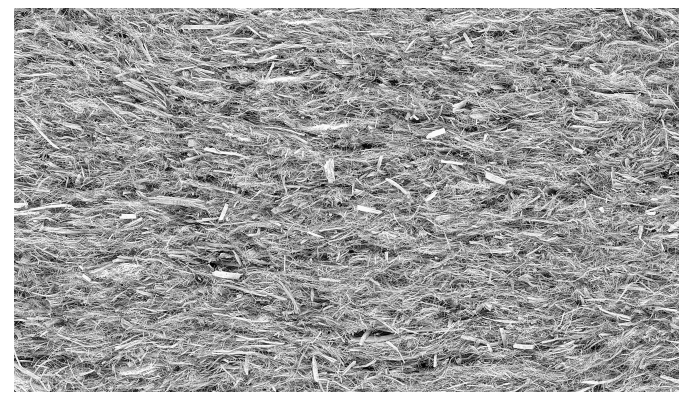

b

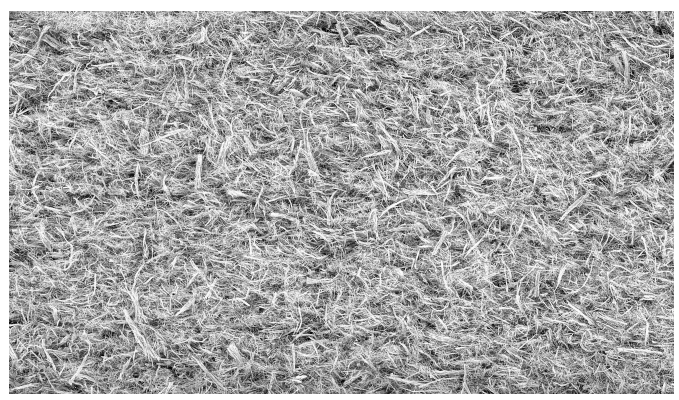

$\boldsymbol{d}$

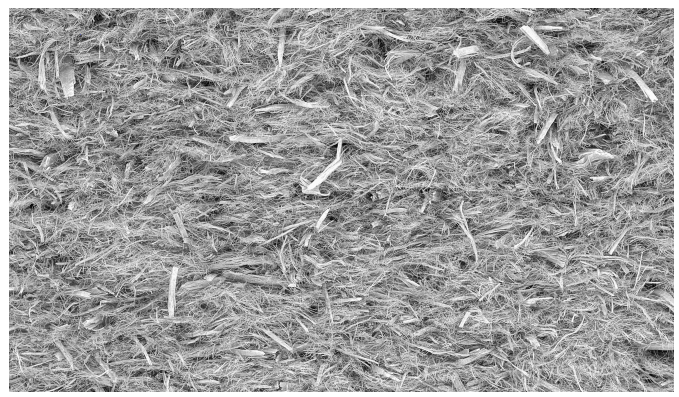

Abbildung 7.55: Qualitativer Vergleich der Faserorientierung im Querschnitt labortechnischer und industrieller Holzfaserdämmstoffe. a, labortechnisch hergestellte Holzfaserdämmstoff-Matte aus Fichten-Faserstoff, Rohdichte $50 \mathrm{~kg}_{\text {atro }} / \mathrm{m}^{3}$. b. labortechnisch hergestellte Holzfaserdämmstoff-Matte aus Buchen-Faserstoff, Rohdichte $50 \mathrm{~kg}_{\text {atro }} / \mathrm{m}^{3}$. c, industrielle Holzfaserdämmstoff-Matte 1 aus Kiefern-Faserstoff, Rohdichte $60 \mathrm{~kg}_{\text {atro }} / \mathrm{m}^{3}$. d, industrielle Holzfaserdämmstoff-Matte 2 aus Kiefern-Faserstoff, Rohdichte $50 \mathrm{~kg}_{\text {atro }} / \mathrm{m}^{3}$.

Die Ergebnisse der Wärmeleitfähigkeit-Messungen sind in Tabelle 7.17 aufgelistet. Teilweise wiesen die Faserstoffe eine höhere Schüttdichte als die für die Untersuchungen erforderliche Rohdichte auf. Um die Vergleichbarkeit zwischen den Messungen zu gewähren, wurden diese Faserstoffe nicht in die Auswertung mit einbezogen. In der Tabelle ist zusätzlich noch die erweiterte Messunsicherheit (Erweiterungsfaktor 2) als größte wahrscheinliche Messabweichung des Wertes der Feuchtekorrektur angegeben, da die Wärmeleitfähigkeit-Messungen ohne Wiederholung durchgeführt wurden. Der Wert wurde der Messabweichungsanalyse des Herstellers entnommen (TAURUS Instruments AG 2018). 
Tabelle 7.17: Wärmeleitfähigkeit der Faserstoffe mit Feuchtekorrektur nach Normklima.

\begin{tabular}{|c|c|c|c|c|c|c|}
\hline \multirow[t]{2}{*}{ Var. } & \multirow[t]{2}{*}{ Mahlscheibe } & \multirow[t]{2}{*}{ Holzart } & \multirow{2}{*}{$\begin{array}{l}\text { Temp. } \\
{ }^{\circ} \mathrm{C}\end{array}$} & \multicolumn{2}{|c|}{ Wärmeleitfähigkeit } & \multirow{2}{*}{$\begin{array}{l}\text { Erweiterte } \\
\text { Messunsicherheit } \\
\mathrm{W} /(\mathrm{m} \cdot \mathrm{K})\end{array}$} \\
\hline & & & & $\begin{array}{l}\text { Messwert } \\
\lambda_{10} \\
\mathrm{~W} /(\mathrm{m} \cdot \mathrm{K})\end{array}$ & $\begin{array}{l}\text { Feuchtekorrektur* } \\
\lambda_{10} \\
\mathrm{~W} /(\mathrm{m} \cdot \mathrm{K})\end{array}$ & \\
\hline \multicolumn{7}{|c|}{ TMP-Verfahren } \\
\hline 1 & 1 & Fichte & 135 & $* *$ & $* *$ & $* *$ \\
\hline 2 & 1 & Fichte & 150 & 0,0367 & 0,0375 & 0,0008 \\
\hline 3 & 1 & Fichte & 175 & 0,0348 & 0,0346 & 0,0007 \\
\hline 4 & 1 & Buche & 135 & $* *$ & $* *$ & $* *$ \\
\hline 5 & 1 & Buche & 150 & $* *$ & $* *$ & $* *$ \\
\hline 6 & 1 & Buche & 175 & $* *$ & $* *$ & $* *$ \\
\hline 7 & 2 & Fichte & 135 & 0,0370 & 0,0373 & 0,0007 \\
\hline 8 & 2 & Fichte & 150 & 0,0347 & 0,0347 & 0,0007 \\
\hline 9 & 2 & Fichte & 175 & 0,0337 & 0,0338 & 0,0007 \\
\hline 10 & 2 & Buche & 135 & 0,0384 & 0,0387 & 0,0008 \\
\hline 11 & 2 & Buche & 150 & 0,0368 & 0,0373 & 0,0007 \\
\hline 12 & 2 & Buche & 175 & 0,0348 & 0,0350 & 0,0007 \\
\hline 13 & 3 & Fichte & 135 & 0,0360 & 0,0363 & 0,0007 \\
\hline 14 & 3 & Fichte & 150 & 0,0357 & 0,0354 & 0,0007 \\
\hline 15 & 3 & Fichte & 175 & 0,0341 & 0,0344 & 0,0007 \\
\hline 16 & 3 & Buche & 135 & 0,0381 & 0,0384 & 0,0008 \\
\hline 17 & 3 & Buche & 150 & 0,0358 & 0,0366 & 0,0007 \\
\hline 18 & 3 & Buche & 175 & 0,0348 & 0,0342 & 0,0007 \\
\hline 19 & 4 & Fichte & 135 & 0,0365 & 0,0367 & 0,0007 \\
\hline 20 & 4 & Fichte & 150 & 0,0355 & 0,0358 & 0,0007 \\
\hline 21 & 4 & Fichte & 175 & 0,0353 & 0,0350 & 0,0007 \\
\hline 22 & 4 & Buche & 135 & 0,0398 & 0,0405 & 0,0008 \\
\hline 23 & 4 & Buche & 150 & 0,0370 & 0,0370 & 0,0007 \\
\hline 24 & 4 & Buche & 175 & 0,0344 & 0,0347 & 0,0007 \\
\hline 25 & 5 & Fichte & 135 & 0,0362 & 0,0360 & 0,0007 \\
\hline 26 & 5 & Fichte & 150 & 0,0345 & 0,0348 & 0,0007 \\
\hline 27 & 5 & Fichte & 175 & 0,0332 & 0,0333 & 0,0007 \\
\hline 28 & 5 & Buche & 135 & 0,0407 & 0,0411 & 0,0008 \\
\hline 29 & 5 & Buche & 150 & 0,0365 & 0,0373 & 0,0007 \\
\hline 30 & 5 & Buche & 175 & 0,0351 & 0,0356 & 0,0007 \\
\hline \multicolumn{7}{|c|}{ RMP-Verfahren } \\
\hline 1 & 6 & Fichte & 20 & 0,0383 & 0,0378 & 0,0008 \\
\hline 2 & 6 & Fichte & 100 & 0,0368 & 0,0368 & 0,0007 \\
\hline 3 & 6 & Buche & 20 & 0,0432 & 0,0421 & 0,0008 \\
\hline 4 & 6 & Buche & 100 & $* *$ & $* *$ & $* *$ \\
\hline
\end{tabular}

Die Werte der Wärmeleitfähigkeit nach Feuchtekorrektur sind in Abbildung 7.56 übersichtlich dargestellt und ermöglichen somit einen Vergleich und die Diskussion der Ergebnisse. Aus Abbildung 7.56 geht hervor, dass die Wärmeleitfähigkeit mit steigender Aufschlusstemperatur generell abnimmt. Das gilt für die Holzarten Buche und Fichte als auch für das TMP- und RMP-Verfahren gleichermaßen. Festzuhalten ist die niedrige Wärmeleitfähigkeit der FichtenholzFaserstoffe in den niedrigen Aufschlusstemperaturen $135^{\circ} \mathrm{C}$ und $150^{\circ} \mathrm{C}$. Das trifft auch für die 
Buchenholz-Faserstoffe zu, die mit den Mahlscheibenmustern 2 und 3 und einer Aufschlusstemperatur von $150^{\circ} \mathrm{C}$ erzeugt wurden. Diese Faserstoffe sind mit den industriellen Faser- und Dämmstoffen in Tabelle 7.16 vergleichbar und müssten aller Voraussicht nach einen Nennwert von $\lambda_{D}=0,038 \mathrm{~W} /(\mathrm{m} \cdot \mathrm{K})$ erfüllen. Weiterhin konnte in den Untersuchungen bestätigt werden, dass Buchenholz-Faserstoffe in den Aufschlusstemperaturen von $150^{\circ} \mathrm{C}$ und $175^{\circ} \mathrm{C}$ vergleichbare Werte wie die Fichtenholz-Faserstoffe liefern. Lediglich mit der Aufschlusstemperatur von $135^{\circ} \mathrm{C}$ liegt die Wärmeleitfähigkeit mit der untersuchten Rohdichte im Vergleich mit den anderen Aufschlusstemperaturen zu hoch. Als Ergebnis der Untersuchungen ist somit festzuhalten, dass auch mit niedrigen Aufschlusstemperaturen leistungsfähige Holzfaserdämmstoffe mit einer niedrigen Wärmeleitfähigkeit hergestellt werden können.

Erste Versuche im RMP-Verfahren sprechen grundsätzlich dafür, dass mit diesem Verfahren ebenfalls Holzfaserdämmstoffe herstellbar sind. Die Wärmeleitfähigkeitwerte für die FichtenFaserstoffe sind vielversprechend. Es scheint möglich zu sein, Faserstoffe auch ohne Erwärmung der Hackschnitzel mit Sattdampf zu erzeugen, die vergleichbare Werte zum TMP-Verfahren liefern. Allerdings gilt das im Rahmen der vorliegenden Untersuchungen nicht für die Holzart Buche. In den vorliegenden Untersuchungen wurde die Wärmeleitfähigkeit an Faserstoffen ohne Zusatz-, Hilfs- und Klebstoffen ermittelt. Dies hätte den Umfang der Arbeit gesprengt. Inwiefern die Zugabe von Klebstoffen die Wärmeleitfähigkeit beeinflusst ist nicht bekannt. Die Ergebnisse von Lee et al. (2019) zum Einsatz von Melamin-Harnstoff-Formaldehyd-Harz (MUF), Phenol-Formaldehyd-Harz (PF), emulgierbarem MDI (eMDI) und Latex-Harz mit einem Bindemittelanteil von $35 \%$ (!) legen nahe, dass zumindest die Art des Klebstoffes keinen Einfluss auf die Wärmeleitfähigkeit hat. Brombacher et al. (2016) untersuchten ebenfalls die Wärmeleitfähigkeit an Faserstoffen (Holzart nicht angegeben). Im Gegensatz zu den Ergebnissen der vorliegenden Arbeit fanden die Autoren weder eine gesicherte $\mathrm{Zu}$ - noch eine Abnahme der Wärmeleitfähigkeit mit Aufschlusstemperaturen von rund $150^{\circ} \mathrm{C}, 175^{\circ} \mathrm{C}, 190^{\circ} \mathrm{C}$ und $205^{\circ} \mathrm{C}$. 
a

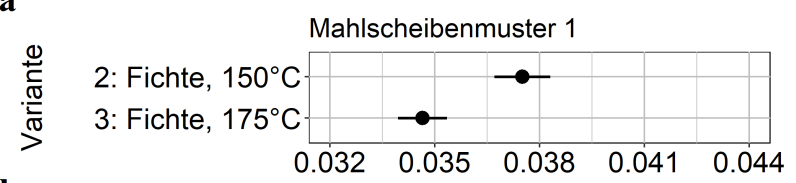

b

Mahlscheibenmuster 2

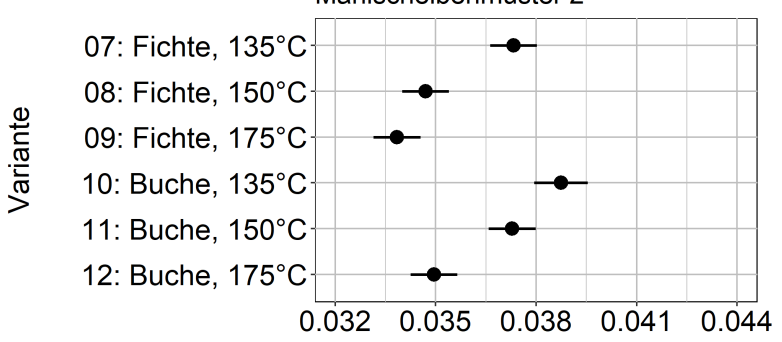

Mahlscheibenmuster 3

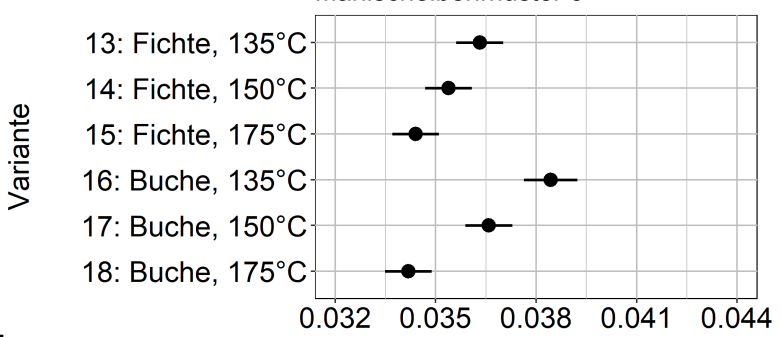

d

Mahlscheibenmuster 4

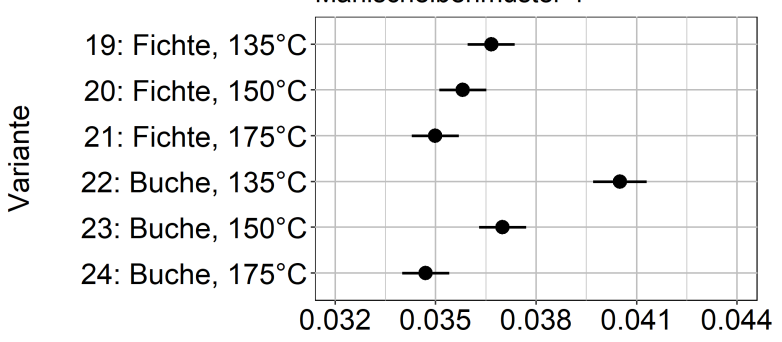

e

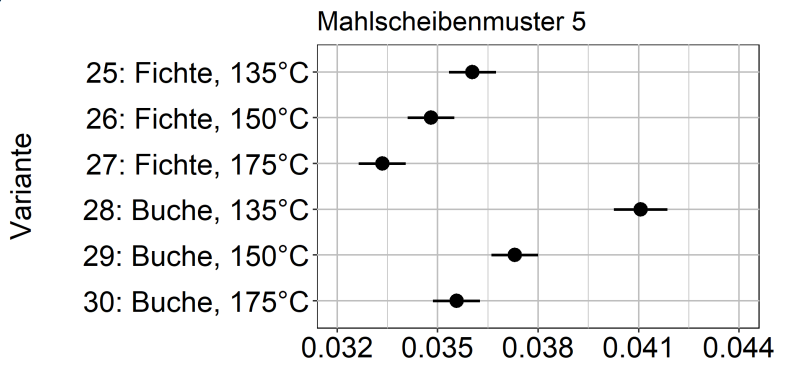

f

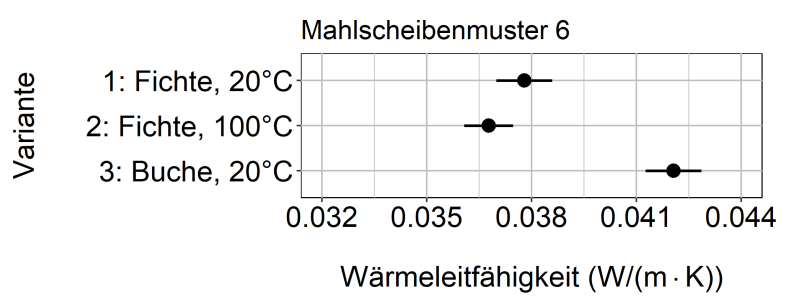

Abbildung 7.56: Vergleichende Darstellung der Wärmeleitfähigkeit der Faserstoffe nach Feuchtekorrektur. Die Fehlerbalken zeigen die erweiterte Messunsicherheit an. TMP-Verfahren: a, Mahlscheibenmuster 1. b, Mahlscheibenmuster 2. c, Mahlscheibenmuster 3. d, Mahlscheibenmuster 4. e, Mahlscheibenmuster 5. RMPVerfahren: $\boldsymbol{f}$, Mahlscheibenmuster 6. 


\subsubsection{Zusammenhänge zwischen Faserstofferzeugung, Dispersitätseigenschaften und der Wärmeleitfähigkeit}

Es wurde eine Korrelations-Matrix erstellt, um insgesamt 27 Parameter von 29 Faserstoffen, an denen die Wärmeleitfähigkeit ermittelt wurde, zu untersuchen. In den, in Abschnitt 10.2 enthaltenen, Tabellen 10.1, 10.2, 10.3, 10.5, 10.4 und 10.6 wurden die weiter oben beschriebene Beschaffenheit der Faserstoffe berücksichtigt und darüber hinaus weitere Parameter untersucht. Neben den hierbei flächengewichteten Dispersitätseigenschaften wurde in Voruntersuchungen auch eine Auswahl an anzahlbezogener Gewichtung untersucht, die keine wesentlichen Unterschiede ergab.

\section{Technologische Faktoren der Prozessführung}

Unter den technologischen Faktoren der Prozessführung besitzen die Aufschlusstemperatur und die spezifische elektrische Leistungsaufnahme mit $-0,72$ und 0,81 bei der Holzart Fichte respektive $-0,81$ und 0,93 bei der Holzart Buche hohe Korrelationskoeffizienten. Der Zusammenhang mit der Temperatur geht aus Abbildung 7.56 hervor. In Abbildung 7.57 ist eine lineare Regression mit Bestimmtheitsmaß dargestellt. Hierin ist nicht dargestellt, aber zu beachten, dass eine niedrige spezifische elektrische Leistungsaufnahme durch das Mahlscheibenmuster und eine hohe Aufschlusstemperatur hervorgerufen wird. Bei der Holzart Fichte sind die Unterschiede zwischen den Mahlscheibenmustern deutlicher ausgeprägt, als bei der Holzart Buche. Dadurch kann das niedrigere Bestimmheitsmaß von $\mathrm{R}^{2}=0,065$ begründet werden (Tabelle 10.1 und Tabelle 10.2).

a

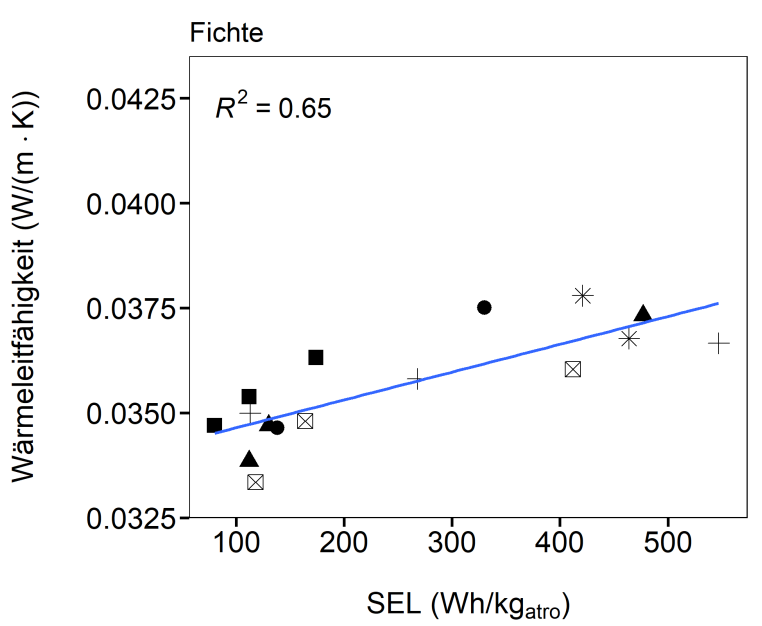

$\mathbf{b}$

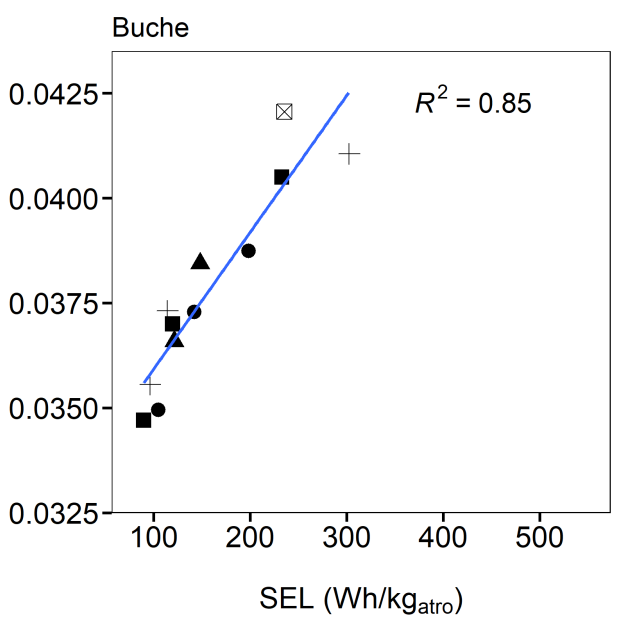

Mahlscheibenmuster

- 1 - $3 \otimes 5$

$2+4 * 6$

Abbildung 7.57: Zusammenhang zwischen der Wärmeleitfähigkeit und der spezifischen elektrischen Leistungsaufnahme (SEL). a, Holzart Fichte. b, Holzart Buche.

Aus Abbildung 7.57 lassen sich weitere Erkenntnisse ziehen. Der Übersichtlichkeit halber sind keine Informationen zu den Aufschlusstemperaturen abgebildet. Der Darstellung zufolge, wurde die niedrigste Wärmeleitfähigkeit von $0,0332 \mathrm{~W} /(\mathrm{m} \cdot \mathrm{K})$ bei der Holzart Fichte mit dem 
Mahlscheibenmuster 5 und der Aufschlusstemperatur $175^{\circ} \mathrm{C}$ erzielt. Die spezifische elektrische Leistungsaufnahme lag bei $118 \mathrm{Wh} / \mathrm{kg}_{\text {atro }}$. Die geringste spezifische elektrische Leistungsaufnahme von $80 \mathrm{Wh} / \mathrm{kg}_{\text {atro }}$ wurde mit dem Mahlscheibenmuster 3 bei einer Wärmeleitfähigkeit von $0,0347 \mathrm{~W} /(\mathrm{m} \cdot \mathrm{K})$ gemessen. Bei der Holzart Buche wurde die niedrigste Wärmeleitfähigkeit von $0,0347 \mathrm{~W} /(\mathrm{m} \cdot \mathrm{K})$ mit einer spezifischen elektrischen Leistungsaufnahme von $90 \mathrm{Wh} / \mathrm{kg}$ atro $\mathrm{mit}$ dem Mahlscheibenmuster 3 erzielt. Die Mahlscheibenmuster 1 und 4 erzielten ebenfalls niedrige Werte.

\section{Beschaffenheit der Faserstoffe nach der Kollektivmethode Luftstrahl-Prüfsiebung}

Die Ergebnisse aus den Untersuchungen mit der Luftstrahl-Prüfsiebung wurden genutzt, um die ermittelten Dispersitätseigenschaften auf Korrelationen mit der Wärmeleitfähigkeit hin zu untersuchen. Bezüglich der Holzart Fichte wurden keine signifikanten Korrelationskoeffizienten identifiziert. Im Gegensatz zur Holzart Buche. Hier wurde der höchste Korrelationskoeffizient dem 97 \%-Perzentil der Verteilungssumme zugeordnet (Tabelle 10.2). Einen ähnlich guten Wert erreicht auch die Partikelausdehnung der Buchen-Faserstoffe. Die Darstellung des Zusammenhangs in Abbildung 7.58 zeigt allerdings, dass diese Beobachtung kritisch betrachtet werden muss, da das Bestimmtheitsmaß bei der Holzart Buche $\mathrm{R}^{2}=0,48$ beträgt. Lediglich das Mahlscheibenmuster 4 zeigt einen linearen Zusammenhang zwischen der Wärmeleitfähigkeit und dem 97 \%-Perzentil auf, da dessen Werte nah an der Regressionsgeraden liegen. Bei der Holzart Fichte sind keine linearen Zusammenhänge zu erkennen.

a

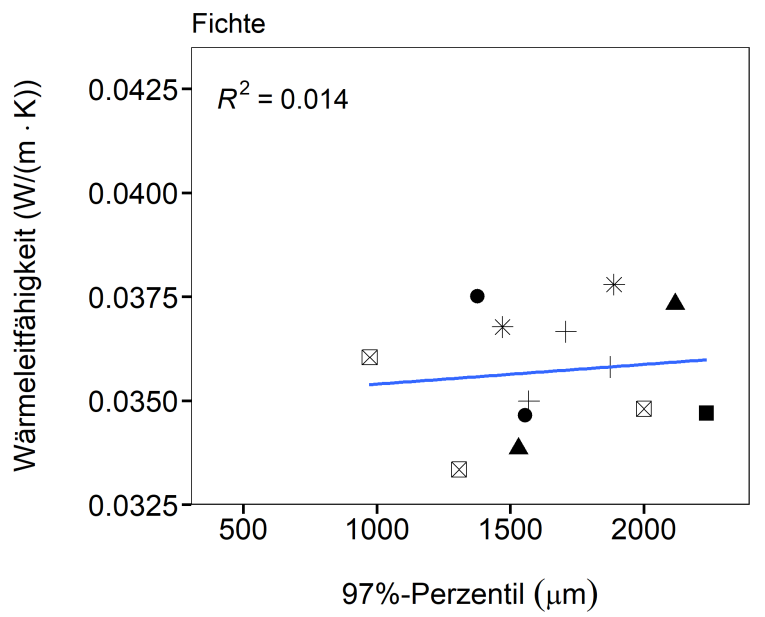

b

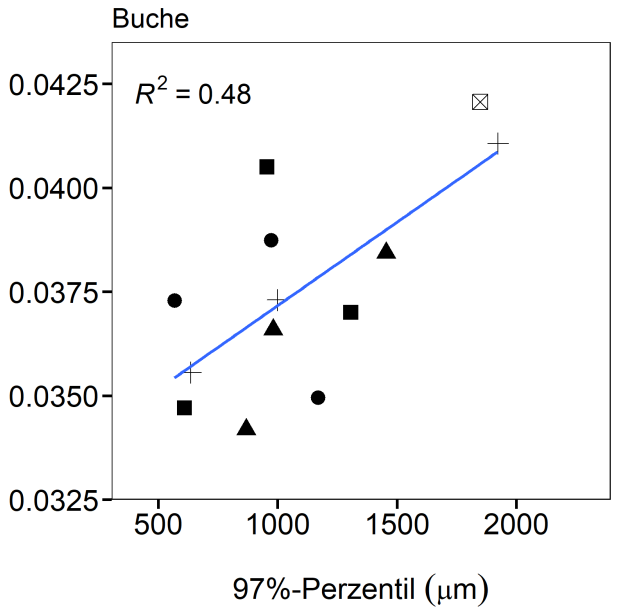

Mahlscheibenmuster

- 1 - $3 \otimes 5$

- $2+4 * 6$

Abbildung 7.58: Zusammenhang zwischen der Wärmeleitfähigkeit und der 97\%-Perzentile. a, Holzart Fichte. b, Holzart Buche.

\section{Beschaffenheit der Faserstoffe nach der Individualmethode quantitative Bildanalyse}

Die umfangreichen Informationen aus den Untersuchungen zur Beschaffenheit der Faserstoffe nach der Individualmethode quantitative Bildanalyse sollen dazu genutzt werden, um weitere 
Korrelationen zu finden. Bezüglich der Holzart Fichte muss festgehalten werden, dass keine nennenswerten Zusammenhänge zwischen den untersuchten Dispersitätseigenschaften und der Wärmeleitfähigkeit aufgedeckt wurden. Lediglich Korrelationskoeffizienten mit mittlerem Signifikanzniveau finden sich zwischen spezifischer elektrischer Leistungsaufnahme und Feinstoffanteil sowie den Flächenanteilen der faserartigen und kornförmigen Partikel. Es scheint, als ob die angewendete Methode nicht dazu geeignet ist hier Zusammenhänge zwischen geometrischen Partikeleigenschaften und der Wärmeleitfähigkeit herzustellen. Gründe dafür könnten sein, dass eine nicht repräsentative Auswahl an Partikeln erfasst oder die Auswahl der morphologischen Parameter die Informationen zu stark gefiltert haben. Es wäre auch möglich, dass systematische Fehler übersehen wurden. Weiterhin muss angemerkt werden, dass die Werte der Wärmeleitfähigkeit bei der Holzart Fichte eventuell zu dicht beieinander liegen, um signifikante Unterschiede zu identifizieren.

Abschließend bleibt anzumerken, dass letztendlich das durch die Faserstoffe ausgebildete dreidimensionale Netzwerk entscheidend für die Wärmeleitfähigkeit ist. Immerhin ist die Luft der Isolator im Dämmstoff. Je kleiner und feiner verteilt die Luftporen in einem Dämmstoff sind, desto geringer wird die Wärmeleitfähigkeit, wie der Vergleich mit Polyurethanschäumen oder extrudiertem Polystyrol zeigt. In diesem Zusammenhang ist die Untersuchung von Tran et al. (2013) interessant. Die Autoren beschreiben hierin eine Methode, um mittels $\boldsymbol{\mu}$-CT-Aufnahmen die Ausbildung von Poren in Holzfaserdämmstoffen zu untersuchen. In dieser Veröffentlichung liegt der Fokus zwar auf mechanischen Eigenschaften, es wäre jedoch vor dem Hintergrund der Ergebnisse aus der vorliegenden Arbeit anzuraten, funktionale Zusammenhänge zwischen Dispersitätseigenschaften und der Wärmeleitfähigkeit auf diesem Weg zu untersuchen. 


\title{
8 Abschließende und zusammenfassende Diskussion mit Ausblick
}

\author{
8.1 Physikalisch-technologischer Erkenntnisgewinn zur \\ Faserstofferzeugung für die Herstellung von \\ Holzfaserdämmstoffen
}

Die Auswahl der Holzarten Fichte und Buche ist begründet durch den Fokus der Untersuchungen auf eine Nadel- und eine Laubholzart, die ein hohes Verwendungspotenzial durch eine hohe Verfügbarkeit besitzt. Mit der Holzart Fichte wurde die nach Varhimo \& Tuovinen (1999) am besten für das TMP-Verfahren geeignete Spezies untersucht, die darüber hinaus den Stand der Technik in der Holzfaserdämmstoffherstellung repräsentiert. Die Holzart Buche stellt Knauf \& Frühwald (2020) zufolge, mengenmäßig die wichtigste Laubholzsorte dar. Die divergierenden anatomischen Merkmale der Holzarten wurden außerdem dazu verwendet Charakteristika der Faserstoffe zu identifizieren, die in Beziehung mit der Faserstofferzeugung und Wärmeleitfähigkeit stehen. In diesem Zusammenhang muss die Einstufung von Buchenholzstäuben durch die Deutsche Forschungsgemeinschaft (2019) als karzinogen wirkend erwähnt werden. Obwohl das verursachende krebserzeugende Prinzip nicht identifiziert ist, muss der Einsatz von Buchenholz in der Holzfaserdämmstoffherstellung überprüft werden.

Darüber hinaus ist zu diskutieren, dass die vorliegende Arbeit keine weiteren normativen Untersuchungen zu Fragestellungen des Brandschutzes, der Wasseraufnahme sowie der mechanischen Eigenschaften von aus Fichten- und Buchenholz-Faserstoffen hergestellten Dämmstoffen umfasst. Insbesondere der Wasseraufnahme sollte, durch die Ausweitung auf die bislang wenig untersuchte und eingesetzte Holzart Buche und die niedrigen Aufschlusstemperaturen im TMP- und RMP-Verfahren, Beachtung in weiteren Forschungsvorhaben geschenkt werden. Die Ergebnisse von Schneider et al. (2000) zeigen, dass die Dickenquellung und Wasseraufnahme von MDF aus Fichtenholz-Faserstoffen mit einer Aufschlusstemperatur von $140^{\circ} \mathrm{C}$, gegenüber einer Aufschlusstemperatur von $180^{\circ} \mathrm{C}$, deutlich erhöht ist. Durch eine erhöhte Wasseraufnahmefähigkeit könnte Brombacher et al. (2012) zufolge, die Wärmeleitfähigkeit erhöht und die Witterungsbeständigkeit von Holzfaserdämmstoffen herabgesetzt sein.

Die labortechnischen Untersuchungen zum Einsatz von unterschiedlichen Mahlscheiben im TMPund RMP-Verfahren zeigen, dass Mahlscheibentests im Labormaßstab reproduzierbare Ergebnisse liefern. Voraussetzung dafür ist, dass grundlegende Effekte der Prozessführung auf die untersuchten Zielgrößen bekannt sind und in der Faserstofferzeugung berücksichtigt werden. Aus den Erkenntnissen zu den identifizierten Effekte konnten Konsequenzen für die weiteren Untersuchungen gezogen werden. Beispielsweise bedarf der Haupteffekt der Größe des Mahlspalts besonderer Beachtung bei der Versuchsdurchführung. Die im Rahmen der vorliegenden Arbeit entwickelte Vorgehensweise kann für weitere Untersuchungen herangezogen werden. Die labortechnisch erzeugten Faserstoffe sind mit industriell hergestellten Faserstoffen hinsichtlich der spezifischen elektrischen Leistungsaufnahme, der Dispersitätseigenschaften sowie der Wärmeleitfähigkeit 
vergleichbar. Das angewendete Batch-Verfahren erwies sich als nicht nachteilig. Allerdings könnte ein kontinuierlicher Prozess mit einer Prozesszeit von mehr als 15 min möglicherweise die Wiederholgenauigkeit verbessern.

Ferner kann als Erkenntnisgewinn bezüglich der Forschungsfrage zum Sorptionsverhalten von Fichten- und Buchenholz-Hackschnitzeln in Sattdampfatmosphäre festgehalten werden, dass die Massenzunahme mit der benötigten Dampfmenge assoziiert ist, die zur Erwärmung der Hackschnitzel benötigt wird. Die sich größtenteils durch die Absorption kondensierten Sattdampfs einstellende gemessene Holzfeuchte bzw. Konsistenz hängt durch die hohe spezifische Wärmekapazität von Wasser maßgeblich mit der Ausgangs-Holzfeuchte zusammen. Im Prozess der Faserstofferzeugung ist es folglich möglich, die Holzfeuchte im Prozess der Faserstofferzeugung gezielt über die Ausgangs-Holzfeuchte zu steuern. Eine niedrige Ausgangs-Holzfeuchte sollte zu einem geringeren Dampfverbrauch und einer geringeren Trocknerbeladung führen. Bei niedrigen Aufschlusstemperaturen und ohne Sattdampfdruck liegt die Konsistenz der Faserstoffe nach dem Aufschluss unter der Ausgangs-Holzfeuchte. Zu den Auswirkungen der Holzfeuchte auf die spezifische elektrische Leistungsaufnahme und die Beschaffenheit der Faserstoffe sind weitere Untersuchungen notwendig. Hierzu bieten sich Versuche mit Kalamitäts- und Käferholz oder Holz mit Trockenschäden an. Weiterhin konnte zur Entwässerung der Hackschnitzel durch die Stopfschnecke vor dem Druckbehälter des Refiners Forschungs- und Entwicklungsbedarf identifiziert werden. Die Holzfeuchte nach Entspannung des Sattdampfdrucks stellt sich auf ein einheitliches Niveau oberhalb der Ausgangs-Holzfeuchte der Hackschnitzel ein und ist unabhängig von dem untersuchten Sattdampfdruck. Das während der Erwärmung absorbierte Kondensat verdampft folglich nicht wieder vollständig. Die Beobachtungen zur Geschwindigkeit der Massenzunahme der Hackschnitzel lassen den Schluss zu, dass die Erwärmung unter labortechnischen Bedingungen ohne nennenswerte Verzögerung erfolgt. Der hydrostatische Sattdampfdruck sorgt für eine simultane Erwärmung der zugänglichen Volumina und Oberflächen. Begrenzend auf die Geschwindigkeit der Erwärmung können sich der hydraulische Widerstand und die Dicke der Hackschnitzel auswirken, infolge deren der Anteil an Wärmeleitung durch die Holzsubstanz ansteigt. Weitere Untersuchungen, welche das TMP- und RMP-Verfahren sowie deren Rohstoffe berücksichtigen, können dazu beitragen, diese Prozesse energietechnisch zu optimieren.

In Teilaspekt 3 wurden zur Faserstofferzeugung fünf Mahlscheibenmuster im TMP-Verfahren und ein Mahlscheibenmuster im RMP-Verfahren untersucht. Hierbei ist festzuhalten, dass zu Beginn der Untersuchungen kein bewährter Stand der Technik zu erkennen war und keine wissenschaftlichen Veröffentlichungen zu Mahlscheiben für Holzfaserdämmstoffe bekannt waren. Die Auswahlkriterien wurden deshalb breit gefächert, um die Leistungsfähigkeit unterschiedlicher Mahlscheibenmuster zu untersuchen. In Bezug auf die Forschungsfrage lassen sich aus dem Vergleich der Mahlscheibenmuster folgende Erkenntnisse ziehen. Es bestehen überwiegend signifikante Unterschiede in der spezifischen elektrischen Leistungsaufnahme der Mahlscheibenmuster. Diese Unterschiede nehmen mit steigender Aufschlusstemperatur ab. Zwischen den Mahlscheibenmustern bestehen weiterhin deutliche Unterschiede bezüglich der Leistungsschwankungen und der Leistungsspitzen. Hierzu konnte mit einem Mahlscheibenmuster festgestellt werden, dass eine Unterbrechung der Stege zur Vermeidung eines Temperaturmaximums auf der Mahlbahn und für die Strömung des zwischen den Mahlscheiben entstehenden Dampfes vorteilhaft ist. Insgesamt weist dieses Mahlscheibenmuster auch die geringste spezifische elektrische Leistungsaufnahme und die höchste Laufruhe auf. Generell lag bei der Holzart Buche die spezifische elektrische Leistungsaufnahme niedriger im Vergleich zur Holzart Fichte. $\mathrm{Zu}$ diesem Punkt ist anzumerken, dass insbesondere die Entwicklung von Mahlscheiben für die Faserstoffherstellung mit Laubholzsortimenten ein Optimierungspotenzial besitzt. 
Ergänzend konnte bezüglich der Faserstofferzeugung für die Herstellung von Holzfaserdämmstoffen gezeigt werden, dass gegenüber dem Stand der Technik auch geeignete Faserstoffe bei niedrigeren Aufschlusstemperaturen erzeugt werden können, die eine geeignete Beschaffenheit für Holzfaserdämmstoffe aufweisen. Auch die Machbarkeit des RMP-Verfahrens kann zumindest für die Holzart Fichte bestätigt werden. Hier besteht weiterer Forschungs- und Entwicklungsbedarf bezüglich der Mahlscheiben, der Prozessführung und der Prozesstechnik.

In weiteren Untersuchungen zum elektrischen und thermischen Energiebedarf konnten Antworten auf die Fragestellung gefunden werden, welche Beziehungen zwischen der Holzfeuchte der Hackschnitzel und der Konsistenz Faserstoffe mit dem Energiebedarf in der Faserstofferzeugung bestehen. Die Abhängigkeit der spezifischen elektrischen Leistungsaufnahme von der Aufschlusstemperatur ist bei den Mahlscheibenmustern unterschiedlich ausgeprägt. Grundsätzlich nimmt die spezifische elektrische Leistungsaufnahme mit steigender Aufschlusstemperatur ab. Dieses Phänomen ist somit nicht ausschließlich auf das Erweichungsverhalten der Holzarten zurückzuführen. Es konnte gezeigt werden, dass die Höhe der Abnahme der spezifischen elektrischen Leistungsaufnahme über der Aufschlusstemperatur maßgeblich durch das Mahlscheibenmuster hervorgerufen wird. Damit muss dass Modell von Asplund (1940a) überdacht werden. Darüber hinaus konnte anhand des Quotienten aus der durchschnittlichen Partikeloberfläche und der spezifischen elektrischen Leistungsaufnahme die Effizienz der Mahlscheibenmuster in der Faserstofferzeugung berechnet werden. Bezüglich der Zerkleinerungskinetik konnte kein Zusammenhang zwischen der Beanspruchungsfrequenz und der spezifischen elektrischen Leistungsaufnahme gefunden werden. Möglicherweise sind in der Konstruktion von Mahlscheiben andere Parameter, wie beispielsweise der Stegwinkel, ausschlaggebender. Diesbezüglich muss auch angemerkt werden, dass die Theorie zur Zerkleinerungskinetik von Mahlscheiben ausschließlich vor dem Hintergrund der Papierherstellung erstellt wurde und deshalb die Übertragung auf die Holzfaserdämmstoffherstellung erschwert ist.

Die Untersuchungen zum thermischen und elektrischen Energiebedarf zeigen weiterhin, dass mit einer Reduzierung des Anteils an thermischer Energie der Anteil an elektrischer Energie ansteigt und umgekehrt. Der Gesamtenergiebedarf liegt im TMP-Verfahren mit der Aufschlusstemperatur $175^{\circ} \mathrm{C}$ tendenziell am niedrigsten. Insgesamt legen die Ergebnisse nahe, dass eine Reduzierung des Gesamtenergiebedarfs mit dem RMP-Verfahren möglich ist, wenn der zwischen den Mahlscheiben entstehende und in den Kocher zurückströmende Dampf effektiv für die Erwärmung der Hackschnitzel verwendet wird. Die dadurch trockeneren Faserstoffe verursachen eine geringere Trocknerbeladung. Somit können in diesem Verfahren der prozesstechnisch aufwendige sowie investitions- und wartungsintensive thermische Energiebedarf für die Dampferzeugung und die Trocknung deutlich reduziert werden. Um diese Aussagen zu überprüfen sind weitere Untersuchungen notwendig.

In der Charakterisierung der Beschaffenheit von Faserstoffen für die Holzfaserdämmstoffherstellung erwiesen sich die Kollektivmethode Luftstrahl-Prüfsiebung als auch die Individualmethode quantitative Bildanalyse gleichermaßen als geeignet, um Antworten auf die Eingangs formulierte Forschungsfrage zu finden. Mit beiden Methoden lassen sich die Unterschiede in den Dispersitätseigenschaften der Faserstoffe ermitteln. Insbesondere mit der Luftstrahl-Prüfsiebung konnte festgestellt werden, dass die Unterschiede bezüglich der Dispersitätseigenschaften mit steigender Aufschlusstemperatur kleiner werden. Abschließende Erkenntnisse zu funktionalen Zusammenhängen zwischen der Beschaffenheit der Faserstoffe und der Wärmeleitfähigkeit konnten mit den angewendeten analytischen und statistischen Methoden nicht herausgearbeitet werden. Mit der Luftstrahl-Prüfsiebung und der quantitativen Bildanalyse wurden keine signifikanten Korrelationen bei der Untersuchung von Fichtenholz-Faserstoffen gefunden. Die signifikanten 
Korrelationen bezüglich der Buchenholz-Faserstoffe deuten darauf hin, dass hauptsächlich die Dicke bzw. Feinheit der faserartigen Partikel und Faserbündel die Wärmeleitfähigkeit beeinflussen. In der mikroskopischen Untersuchung der Faserstoffe wurden Unterschiede in der Beschaffenheit zwischen den Holzarten und Aufschlusstemperaturen sichtbar. Der Vergleich der labortechnischen Faserstoffe mit industriellen TMP-Stoffen, Holzschliff und Zellstoffen zeigte darüber hinaus typische Eigenschaften, wie beispielsweise die immanente Latenz der Holzfaserdämmstoff-Faserstoffe, auf.

Bezüglich des abschließenden Teilaspekts der Wärmeleitfähigkeit ist als Ergebnis festzuhalten, dass die Gestaltung der Mahlscheiben, die Aufschlusstemperatur und die Holzarten einen Einfluss haben. Generell führt eine hohe Aufschlusstemperatur zu niedrigeren Werten bei der Wärmeleitfähigkeit. $\mathrm{Zu}$ dieser Beobachtung ist allerdings zu ergänzen, dass auch mit niedrigen Aufschlusstemperaturen vergleichbare Werte mit den industriell hergestellten Faserstoffen erzielt werden können. Darüber hinaus wurden mit den labortechnisch hergestellten Fichtenund Buchenholz-Faserstoffen deutlich niedrigere Werte für die Wärmeleitfähigkeit unter vergleichbaren Bedingungen gemessen. Zudem konnte gezeigt werden, dass niedrige Werte der Wärmeleitfähigkeit mit einer vergleichsweise niedrigen spezifischen elektrischen Leistungsaufnahme erzeugt werden können. Auch die ersten Untersuchungen zum RMP-Verfahren sind mit der Holzart Fichte vielversprechend. Hier konnte ebenfalls ein vergleichbarer Wert für die Wärmeleitfähigkeit ermittelt werden. Für weitere Untersuchungen zu funktionalen $\mathrm{Zu}$ sammenhängen zwischen der Beschaffenheit der Faserstoffe und der Wärmeleitfähigkeit sei abschließend auf die Methode der Mikro-Computertomographie hingewiesen, mit der die Poren des dreidimensionalen Netzwerks der Partikel innerhalb des Faservlieses untersucht werden kann.

\subsection{Nutzen von Laborversuchen und Übertragung der Ergebnisse in den industriellen Maßstab}

Die Erkenntnisse aus der vorliegenden Arbeit zeigen, dass Versuche zur Faserstofferzeugung im Labormaßstab durchaus zielführend sein können. In der Entwicklung von Mahlscheiben sind nach wie vor Tests notwendig, da eine Simulation der Zielgrößen bisher nicht umsetzbar ist. Im industriellen Maßstab können Mahlscheibentests hohe Kosten verursachen. Laborversuche sind ein Mittel, um Versuchskosten gering zu halten und bieten außerdem die Möglichkeit, empirische Daten zum Theorieabgleich zu generieren. Die definierte Rohstoffauswahl und die in einem weiten Bereich variierbare Prozessführung erlauben es, den Einfluss der Mahlscheibenmuster und der Prozessführung auf die Beschaffenheit der Faserstoffe gezielt zu untersuchen und Energiesparpotenziale zu identifizieren. Als nachteilig ist die kurze Laufzeit der Versuche im Labormaßstab zu sehen, die nicht den Verschleiß der Mahlscheiben im industriellen Einsatz widerspiegeln. Trotzdem erleichtert der Erkenntnisgewinn die Entscheidungsfindung für die industrielle Anwendung und bietet der Wissenschaft neue Einblicke in ein bislang wenig beachtetes Themenfeld der holzbasierten Biökonomie.

Mit den vorliegenden Untersuchungen wurden im Rahmen des Forschungsvorhabens „Entwicklung von Mahlscheiben zur Erzeugung spezifischer Fasergeometrien für Faserverbundwerkstoffe mit geringer Dichte“ (BMEL, FKZ: 22012216) Mahlscheibenmuster entwickelt und im industriellen Maßstab getestet. In die Test waren nationale und internationale Holzfaserdämmstoffhersteller eingebunden. Im Rahmen der Tests wurden weitere Anpassungen an den Mahlscheibenmustern in Abhängigkeit der individuellen Anforderungen vorgenommen. Die speziell für Holzfaserdämmstoffe entwickelten Mahlscheibenmuster sind im industriellen Einsatz. Auf eine Auswertung der 
industriellen Mahlscheibentests im Rahmen der vorliegenden Arbeit wurde verzichtet, da die Vergleichbarkeit zwischen unterschiedlichen Anlagen, Rohstoffen und der Prozessführung nicht gegeben ist. 


\section{Literatur}

Abe, H. \& R. Funada (2005). „Review - The Orientation of Cellulose Microfibrils in the Cell Walls of Tracheids in Conifers: A model based on observations by field emission-scanning electron microscopy“. In: IAWA fournal 26.2, S. 161-174.

Aghakhani, M., A. Khazaeian, A. Rafighi \& F. Scholz (2020). „Evaluation of some effective parameters on energy consumption in drum chipper". In: fournal of Wood and Forest Science and Technology 26.4, S. 1-15.

Ahrel, I. \& I. Bäck (1970). „Thermomechanical pulp at Rockhammar“. In: EUCEPA, Proceedings of Symposium of Mechanical Pulp 83. Oslo, Norway, S. 83-91.

Aicher, S. \& Z. Christian (o. D.). „Ermüdungsverhalten von Holz und geklebten Holzbauteilen“. In: Internationales Holzbau-Forum 2015, Forum Holzbau, S. 1-18.

Akhtar, M., R. A. Blanchette \& T. A. Burnes (1995). „Using Simons Stain to Predict Energy Savings During Biomechanical Pulping“. In: Wood and Fiber Science 27.5, S. 258-264.

Alahautala, T., E. Lassila, R. Hernberg, E. Härkönen \& P. Vuorio (2004). „Optical measurement of pulp quantity in a rotating disc refiner". In: Measurement Science and Technology 15.11, S. 2256-2262.

Albrecht, W. (2010). „Neue Wärmeleitfähigkeitsstufen und Leistungsfähigkeit neuer Dämmstoffe“. In: Bauphysik 32.2, S. 110-117.

Andritz AG (2015). Montage-, Betriebs- und Wartungsanleitung Laboranlage 12-1CP FH Rosenheim.

Annergren, G. \& N. Hagen (2016). „Industrial Beating/Refining“. In: Paper Chemistry and Technology. Hrsg. von M. Ek, G. Gellerstedt \& G. Henriksson. Pulp and Paper Chemistry and Technology. Berlin: De Gruyter, S. 121-135.

Anonym (1974). „Refiner Mechanical Pulping: Production of Mathematical Model“. In: What's New in Forest Research 20.

Antensteiner, P. (2002). „Up-date of Current Low Consistency Refining Theories“. Dissertation. Graz: University of Graz.

Antrag auf Nichtnennung (1998). „Verfahren und Vorrichtung zur Herstellung biologisch abbaubarer Dämmplatten“. Patent. Veröffentlichungs-Nr.: DE 000019635410 A1.

Arbeitsgruppe Sägenebenprodukte (AG SNP) im Ausschuß Forst und Holz (2000). Richtlinie: Qualitätsanforderungen der Zellstoff- und Papierindustrie in Deutschland an die Struktur von Hackschnitzeln zur Erzeugung von Zellstoff (Sulfit-/Sulfatzellstoff) und thermomechanischem Holzstoff (TMP). Hrsg. von Verband Deutscher Papierfabriken e.V. Bonn.

Asplund, A. (1940a). „Die Defibrator-Methode und ihre Anwendungsgebiete: 1. Teil“. In: Wochenblatt für Papierfabrikation 45, S. 590-595.

- (1940b). „Die Defibrator-Methode und Ihre Anwendungsgebiete: 2. Teil: Schluß zu Nr. 45, S. 590“. In: Wochenblatt für Papierfabrikation 46, S. 607-611.

- (1949). „Wallboardindustriens bakvattenfraga“. In: Svensk Papperstidning 52.3, S. 79-86.

Atack, D. (1972). „On the characterization of pressurized refiner mechanical pulps“. In: Svensk Papperstidning 75.3, S. 89-94.

- (1976). „Mechanical pulp - conserver of our forests“. In: Appita 30.2, S. 155-160. 
Atack, D. \& I. T. Pye (1964). „The Measurement of Grinding Zone Temeperature“. In: Pulp \& Paper Magazine of Canada, T363-376.

Atack, D. \& M. I. Stationwalla (1975). „On the measurement of temperature and pressure in the refining zone of an open discharge refiner". In: Transactions of the Technical Section, 1975 International Mechanical Pulping Conference. Hrsg. von Canadian Pulp and Paper Association. Bd. 1, S. 71-76.

Atack, D., M. I. Stationwalla, E. Huusari, P. Ahlqvist, J. Fontebasso \& M. Perkola (1989). „HighSpeed photography of pulp flow patterns in a 5 MW pressurized refiner“. In: Paperi ja puu 69, S. 280-293.

Atack, D., M. I. Stationwalla \& A. Karnis (1984). „What happens in refining“. In: Pulp \& Paper Canada 85.12, S. 119-124.

Atack, D. \& P. N. Wood (1973). „On the high consistency operation of large double rotating disc refiners“. In: Svensk Papperstidning 76.17, S. 639-644.

Ayrilmis, N., J. T. Benthien \& M. Ohlmeyer (2017). „Effect of wood species, digester conditions, and defibrator disc distance on wettability of fiberboard“. In: fournal of Wood Science 63.3, S. 248-252.

Back, E. L. \& S. A. Larsson (1972). „Increased pulp yield as means of reducing the BOD of hardboard mill effluent“. In: Svensk Papperstidning 75.18, S. 723-731.

Back, E. L. \& L. Salmén (1982). „Glass transitions of wood components hold implications for molding and pulping processes". In: Tappi fournal 65.7, S. 107-110.

Barth, S. (2017). „Fasergeometrien für Faserverbundwerkstoffe mit geringer Dichte“. In: Vortrag im 12. Holzwerkstoffkolloquium, Institut für Holztechnologie, Dresden.

- (2018). „Möglichkeiten zur Optimierung der Herstellung von Holzfaserdämmstoffen“. In: Vortrag im 3. Kooperationsforum Holz als neuer Werkstoff, Bayern Innovativ, Regensburg.

- (2019). „Bindemitteleinsatz und Mahlplattenentwicklung in der Herstellung von Holzfaserdämmstoffen “. In: Vortrag im 13. Holzwerkstoffkolloquium, Institut für Holztechnologie, Dresden.

Barth, S., J. Vega, C. Fuentealba \& A. Michanickl (2018). „Fiber Insulation Materials from Eucalyptus Bark Fibers - First Results“. In: Pro Ligno 14.4, S. 3-8.

Bartholme, M., G. Avramidis, W. Viöl \& A. Kharazipour (2009). „Herstellung von organisch gebundenen Holzfaser-Dämmplatten aus Buchenholz“. In: Holztechnologie 50.1, S. 23-26.

Batel, W. (1957). „Kritische Betrachtungen zur Teilchengrößenbestimmung durch Siebanalyse, Windsichten, Sedimentieren und den Blain-Test“. In: Chemie - Ingenieur - Technik 29.9, S. 581.589.

Baur, E., S. Brinkmann, T. A. Osswald, N. Rudolph \& E. Schmachtenberg, Hrsg. (2013). Sächtling Kunststoff Taschenbuch. München: Carl Hanser Verlag.

Becker, H., H. Höglund \& G. Tistad (1977). „Frequency and temperature in chip refining“. In: Paperi ja puu 3, S. 123-130.

Becker, H. \& D. Noack (1968). „Studies on dynamic torsional viscoelasticity of wood“. In: Wood Science and Technology 2.3, S. 213-230.

Behler, H., N. Parameswaran \& R. Patt (1981). „Zur Qualität von Nadelrestholzhackschnitzeln für die Faserstoffherstellung. Mitt. I. Einfluß der Holzbeschaffenheit“. In: Wochenblatt für Papierfabrikation 109.

Belgacem, N. \& A. Pizzi, Hrsg. (2016). Lignocellulosic fibers and wood handbook: Renewable materials for today's environment. Salem, Massachusetts: Scrivener Publishing \& Wiley.

Benthien, J. T., C. Bähnisch, S. Heldner \& M. Ohlmeyer (2014). „Effect of fiber size distribution on medium-density fiberboard properties caused by varied steaming time and temperature of defibration process". In: Wood and Fiber Science 46.2, S. 175-185. 
Benthien, J. T., S. Heldner \& M. Ohlmeyer (2017). „Investigation of the interrelations between defibration conditions, fiber size and medium-density fiberboard (MDF) properties". In: European Journal of Wood and Wood Products 75.2, S. 215-232.

Björklund, J. M. \& N.-O. Nilvebrant (2009). „Wood Extractives“. In: Wood Chemistry and Technology. Hrsg. von M. Ek, G. Gellerstedt \& G. Henriksson. Pulp and Paper Chemistry and Technology. Berlin: De Gruyter, S. 148-170.

Blanchette, R. A., M. Akhtar \& M. C. Attridge (1992). „Using Simons stain to evaluate fiber characteristics of biomechanical pulps“. In: Tappi fournal 75.11, S. 121-124.

Blechschmidt, J. (2013). „Rohstoffe der Papiererzeugung“. In: Taschenbuch der Papiertechnik. Hrsg. von J. Blechschmidt. München: Carl Hanser Verlag, S. 52-60.

Blechschmidt, J., P. Engert \& M. Stephan (1986). „The glass transition of wood from the viewpoint of mechanical pulping“. In: Wood Science and Technology 20, S. 263-272.

Bodig, J. \& B. A. Jayne (1993). Mechanics of wood and wood composites. Malabar: Krieger Publishing.

Böhner, G. (1996). „Überlegungen und Ergänzungen zum „Keylwerth-Diagramm”“. In: Holz als Roh- und Werkstoff 54, S. 73-79.

Bohnet, M., Hrsg. (2004). Mechanische Verfahrenstechnik. Weinheim: Wiley-VCH.

Bonsen, K. J. M. (1991). „Gefässverschluss-Mechanismen in Laubbäumen“. In: Vierteljahrsschrift der Naturforschenden Gesellschaft in Zürich 136.1, S. 13-50.

Börste, G. (2008). „New ways of energy savings in the wet fiber preparation process“. In: Vortrag in 6. Europäisches Holzwerkstoff-Symposium, Hannover.

Box, G. E. P., J. S. Hunter \& W. G. Hunter (2005). Statistics for Experimenters. Wiley series in probability and statistics. Hoboken, NJ: Wiley-Interscience.

Brännvall, E. (2009). „Pulping Technology“. In: Pulping Chemistry and Technology. Hrsg. von M. Ek, G. Gellerstedt \& G. Henriksson. Pulp and Paper Chemistry and Technology. Berlin: De Gruyter, S. $121-147$.

Braun, P. (2014). „Untersuchungen zum Energieverbrauch in der Herstellung von Holzfaserdämmplatten“. Bachelorthesis. Rosenheim: Technische Hochschule Rosenheim.

Brombacher, V. (2015). „Untersuchungen zur Optimierung von Holzfaserdämmstoffen in Abhängigkeit von Rohstoff, Herstellverfahren und Aufschlussbedingungen“. Dissertation. Zürich: Eidgenössische Technische Hochschule.

Brombacher, V., M. Franco, D. Krug, M. Torres \& P. Niemz (2016). „Untersuchungen zur Optimierung von Holzfaserdämmstoffen in Abhängigkeit von den Aufschlussbedingungen“. In: Bauphysik 38.5, S. 298-308.

Brombacher, V., F. Michel, P. Niemz \& T. Volkmer (2012). „Untersuchungen zu Wärmeleitfähigkeit und Feuchteverhalten von Holzfaserplatten und Materialkombinationen“. In: Bauphysik 34.4, S. $157-169$.

BRUKS Klöckner GmbH (2015). Betriebsanleitung Trommelhacker DH $120 \times 400$ L-2WV.

- (2.10.2015). Auslegung von Trommelhackmaschinen. E-mail vom 02.10.2015.

Carpenter, C. H. (1989). The history of mechanical pulping. Montgomery, Alabama, USA: Walker Printing Company,

Chen, M., B. Coasne, R. Guyer, D. Derome \& J. Carmeliet (2018). „Role of hydrogen bonding in hysteresis observed in sorption-induced swelling of soft nanoporous polymers". In: Nature communications 9.1, S. 3507.

Chen, M., B. Coasne, R. Guyer, D. Derome \& J. Carmeliet (2020). „A Poromechanical Model for Sorption Hysteresis in Nanoporous Polymers". In: The fournal of Physical Chemistry B 124.39, S. 8690-8703. 
Coppers, M. (2002). „Einsatz der Luft- und Flüssigkeitsstrahl-Präzisionssiebe in Labor und Technikum“. In: Aufbereitungstechnik 43.9, S. 40-47.

Dana, H. R., W. D. May, K. B. Miles \& B. G. Newman (1975). „A study of steam flow and selfpressurization in chip refiners". In: Technical Papers of the International Mechanical Pulping Conference.

Daniel, C. (1959). „Use of Half-Normal Plots in Interpreting Factorial Two-Level Experiments“. In: Technometrics 1.4, S. 311-341.

Deppe, H.-J. \& K. Ernst (1996). MDF - Mitteldichte Faserplatten. Leinfelden-Echterdingen: DRWVerlag Weinbrenner GmbH \& Co.

Deutsche Forschungsgemeinschaft, Hrsg. (2019). MAK- und BAT-Werte-Liste 2019: Maximale Arbeitsplatzkonzentrationen und Biologische Arbeitsstofftoleranzwerte : Ständige Senatskommission zur Prüfung gesundheitsschädlicher Arbeitsstoffe. Bonn.

Deutscher Bundestag (2020). Gesetz zur Einsparung von Energie und zur Nutzung erneuerbarer Energien zur Wärme- und Kälteerzeugung in Gebäuden (Gebäudeenergiegesetz - GEG).

DIN 66165-1:2016-08 (2016). Partikelgrößenanalyse - Siebanalyse - Teil 1: Grundlagen. Berlin: Beuth Verlag.

DIN EN 6721-1:2019 (2019). Kunststoffe - Bestimmung dynamisch-mechanischer Eigenschaften Teil 1: Allgemeine Grundlagen. Berlin: Beuth Verlag.

DIN ISO 9276-1:2004-09 (2004). Darstellung der Ergebnisse von Partikelgrößenanalysen - Teil 1: Grafische Darstellung. Berlin: Beuth Verlag.

DIN ISO 9276-2:2018-09 (2018). Darstellung der Ergebnisse von Partikelgrößenanalysen - Teil 2: Berechnung von mittleren Partikelgrößen / -durchmessern und Momenten aus Partikelgrößenverteilungen. Berlin: Beuth Verlag.

DIN ISO 9276-6:2012-01 (2012). Darstellung der Ergebnisse von Partikelgrößenanalysen - Teil 6: Deskriptive und quantitative Darstellung der Form und Morphologie von Partikeln. Berlin: Beuth Verlag.

DIN V 4108-4:2004-07 (2004). Wärmeschutz und Energie-Einsparung in Gebäuden - Teil 4: Wärmeund feuchtetechnische Bemessungswerte. Berlin: Beuth Verlag.

Ding, F., M. Benaoudia, P. Bédard, R. Lanquette, C. Lejeune \& P. Gagné (2005). „Wood chip physical quality definition and measurement". In: Pulp \& Paper Canada 106.2, T25-T30.

Du, N., J. Fan, H. Wu \& W. Sun (2009). „Optimal porosity distribution of fibrous insulation“. In: International fournal of Heat and Mass Transfer 52.19-20, S. 4350-4357.

Eberhardt, L. (1955). „New and revolutionary Method for production of mechanical pulp: Groundwood from wood chips“. In: Paper Trade fournal 37, S. 26-28.

- (1960). „Method of Producing Wood Pulp“. Patent. Veröffentlichungs-Nr.: 2,947,655.

Eichhorn, S. (2017). „Entwicklung neuartiger mit Polyurethan gebundener Holzfaserdämmstoffe mit geringer Rohdichte“. Dissertation. Göttingen: Georg-August-Universität Göttingen.

Ek, M., G. Gellerstedt \& G. Henriksson, Hrsg. (2009). Pulping Chemistry and Technology. 1. Aufl. Bd. Bd. 2. Pulp and Paper Chemistry and Technology. Berlin: De Gruyter.

EN ISO 10456:2007 + AC:2009 (2010). Baustoffe und Bauprodukte - Wärme- und feuchtetechnische Eigenschaften - Tabellierte Bemessungswerte und Verfahren zur Bestimmung der wärmeschutztechnischen Nenn- und Bemessungswerte. Berlin: Beuth Verlag.

EN ISO 4610:2001 (2002). Kunstoffe - Vinylchlorid-Homo- und Copolymerisate. Siebanalyse mit der Luftstrahl-Siebmaschine. Berlin.

Engelhardt, F. (1979). „Untersuchungen über die Wasserdampfsorption durch Buchenholz im Temperaturbereich von 110 bis $170^{\circ} C^{\prime \prime}$. In: Holz als Roh- und Werkstoff 37.3, S. 99-112. 
Engelund, E. T., L. Thygesen, S. Svensson \& C. A. S. Hill (2013). „A critical discussion of the physics of wood-water interactions". In: Wood Science and Technology 47.1, S. 141-161.

Eriksen, O., Ø. Gregersen \& P.-A. Krogstad (2006). „Pressure and vibration in the refining zone of a TMP refiner - influence of the fibre flow". In: Nordic Pulp \& Paper Research fournal 21.1, S. $90-98$.

Eriksson, K. \& A. Karlstroem (2009). „Modeling approaches for critical process limitations in the operation of thermomechanical pulp refiners“. In: Nordic Pulp \& Paper Research fournal 24.2, S. 231-238.

Esteghlalian, A. R., M. Bilodeau, S. D. Mansfield \& J. N. Saddler (2001). „Do enzymatic hydrolyzability and Simons' stain reflect the changes in the accessibility of lignocellulosic substrates to cellulase enzymes?" In: Biotechnology progress 17.6, S. 1049-1054.

EU (2011). Verordnung (EU) Nr. 305/2011 des Europäischen Parlaments und des Rates vom 9. März 2011 zur Festlegung harmonisierter Bedingungen für die Vermarktung von Bauprodukten und zur Aufhebung der Richtlinie 89/106/EWG des Rates: EU-Bauproduktenverordnung (BauPVO).

Euwid (5.07.2018). „Europäische Holzwerkstoffproduktion ist im vergangenen Jahr um 3,0 \% gestiegen: Überproportionale Zuwächse bei Sperrholz, OSB und Dämmplatten“. In: EUWID Holz- und Holzwerkstoffe 92.27, S. 1, 11.

Fachagentur Nachwachsende Rohstoffe e.V., Hrsg. (2020). Basisdaten Biobasierte Produkte 2021.

Faix, O. (2008). „Chemie des Holzes“. In: Taschenbuch der Holztechnik. Hrsg. von A. Wagenführ \& F. Scholz. München: Carl Hanser Verlag, S. 47-74.

Fengel, D. (1966). „Über die Veränderung des Holzes und seiner Kompnenten im Temperaturbereich bis $200^{\circ} \mathrm{C}$ - Erste Mitteilung: Heiß- und Kaltwasserextrakte von thermisch behandeltem Fichtenholz". In: Holz als Roh- und Werkstoff 24.1, S. 9-14.

Fengel, D. \& M. Stoll (1973). „Über die Veränderungen des Zellquerschnitts, der Dicke der Zellwand und der Wandschichten von Fichtenholz-Tracheiden innerhalb eines Jahrringes“. In: Holzforschung 27.1, S. 1-7.

Fengel, D. \& G. Wegener (2003). Wood: Chemistry, ultrastructure, reactions. Reprint der Orig.-Ausg. (ehem. de Gruyter). Remagen: Verlag Kessel.

Ferguson, K. H. (1997). „TMP Technologies Focus on Reducing Energy Use“. In: Pulp \& Paper 71.6, S. 99-102.

Fernando, D. \& G. Daniel (2004). „Micro-morphological observations on spruce TMP fibre fractions with emphasis on fibre cell wall fibrillation and splitting“. In: Nordic Pulp \& Paper Research fournal 19.3, S. 278-285.

- (2008). „Exploring Scots pine fibre development mechanisms during TMP processing: Impact of cell wall ultrastructure (morphological and topochemical) on negative behaviour". In: Holzforschung 62.5, S. 597-607.

- (2010). „Characterization of spruce thermomechanical pulps at the fiber cell wall level: a method for quantitatively assessing pulp fiber development using Simons stain“. In: Tappi fournal 9 , S. 47-55.

Fischer, K. \& C. Bäurich (2013). „Chemischer Aufschluss von Holz“. In: Taschenbuch der Papiertechnik. Hrsg. von J. Blechschmidt. München: Carl Hanser Verlag, S. 109-165.

Forest Products Laboratory (2010). Wood Handbook - Wood as an engineering material: General Technical Report FPL-GTR-190. Madison, WI: U. S. Department of Agriculture, Forest Service, Forest Products Laboratory.

Forgacs, O. L. (1963). „The characterization of mechanical pulps“. In: Pulp \& Paper Magazine of Canada Convention Issue, T86-T116. 
Fox, T. S., R. S. Brodkey \& A. H. Nissan (1979). „High-speed photography of stock transport in a disk refiner". In: Tappi fournal 62.3, S. 55-58.

Franzén, R. (1986). „General and selective upgrading of mechanical pulps“. In: Nordic Pulp \& Paper Research fournal 3/1986, S. 4-13.

Fredrikson, A. \& J. Paltakari (2020). „Maximizing pulp output and quality through measurement of plate gap temperature in high-consistency refining“. In: BioResources 15.2, S. 2258-2278.

Fredriksson, M. \& E. E. Thybring (2018). „Scanning or desorption isotherms? Characterising sorption hysteresis of wood“. In: Cellulose 25.8, S. 4477-4485.

Fromm, J. (2013). „Xylem Development in Trees: From Cambial Divisions to Mature Wood Cells“. In: Cellular Aspects of Wood Formation. Hrsg. von J. Fromm. Plant Cell Monographs. Berlin, Heidelberg: Springer, S. 3-39.

Fromm, J., B. Rockel, S. Lautner, E. Windeisen \& G. Wanner (2003). „Lignin distribution in wood cell walls determined by TEM and backscattered SEM techniques“. In: fournal of Structural Biology 143.1, S. 77-84.

Gao, X. (2014). „Untersuchung zur Optimierung der Mahlscheiben im MDF-Werk“. Masterthesis. Rosenheim: Technische Hochschule Rosenheim.

Gellerstedt, G. (2009). „Mechanical Pulping Chemistry“. In: Pulping Chemistry and Technology. Hrsg. von M. Ek, G. Gellerstedt \& G. Henriksson. Pulp and Paper Chemistry and Technology. Berlin: De Gruyter, S. 35-56.

Geoffrey, D. (2009). „Wood and Fibre Morphology“. In: Wood Chemistry and Technology. Hrsg. von M. Ek, G. Gellerstedt \& G. Henriksson. Pulp and Paper Chemistry and Technology. Berlin: De Gruyter, S. 46-70.

Gerthsen, C., H. Vogel \& D. Meschede, Hrsg. (2006). Gerthsen Physik. 23. Aufl. Berlin, Heidelberg: Springer.

Gorski, D., J. Hill, P. Engstrand \& L. Johansson (2010). „Review: Reduction of energy consumption in TMP refining through mechanical pre-treatment of wood chips“. In: Nordic Pulp \& Paper Research fournal 25.2, S. 156-161.

Grömping, U. (2014). „R Package FrF2 for Creating and Analyzing Fractional Factorial 2-Level Designs“. In: Fournal of Statistical Software 56.1.

- (2015). „Augmented Half Normal Effects Plots in the Presence of a Few Error Degrees of Freedom“. In: Quality and Reliability Engineering International 31.7, S. 1185-1196.

Grömping, U. \& J. Fox (2015). R Commander Plugin for (industrial) Design of Experiments. http://prof.beuthhochschule.de/groemping/DoE, 2014-08-28 19:56:17.

Guo, X., J. Dong, H. Liu, C. Duan, R. Yang \& K. Qi (2020). „Effect of Combined Refining Plates with Different Bar Angles on Paper Properties during Mixed Pulp Refining“'. In: fournal of Korean Wood Science and Technology 48.5, S. 581-590.

Hänninen, T., E. Kontturi \& T. Vuorinen (2011). „Distribution of lignin and its coniferyl alcohol and coniferyl aldehyde groups in Picea abies and Pinus sylvestris as observed by Raman imaging“. In: Phytochemistry 72.14-15, S. 1889-1895.

Hartler, N. (1963). Some Studies on the Quality of Wood Chips for Pulp Production. Stockholm.

- (1977). „Influence of Chip Moisture in Mechanical Pulping“. In: Proceedings Volume 1 Session 1 "Raw Materials and their Handling". Hrsg. von The Finnish Paper Engineers' Association \& The Finnish Pulp and Paper Research Institute, 6:1-6:19.

Hasener, J. (2013). „Inline Fasercharakterisierung , kein Traum sondern Realität - erste Praxiserfahrungen." In: 3. GreCon-Holzwerkstoffsymposium, Magdeburg.

Havimo, M. (2009). „A literature-based study on the loss tangent of wood in connection with mechanical pulping“. In: Wood Science and Technology 43.7-8, S. 627-642. 
Heikkurinen, A. \& L. Leskelä (1999). „The character and properties of mechanical pulps“. In: Mechanical pulping. Hrsg. von J. Sundholm. Papermaking Science and Technology. Helsinki: Fapet Oy, S. 395-413.

Heinemann, S. (2013). „Aufbereitung des Holzstoffes - Sortierung und Rejektbehandlung“. In: Taschenbuch der Papiertechnik. Hrsg. von J. Blechschmidt. München: Carl Hanser Verlag, S. 9698.

Heinrich, H. (2004). Abschlussbericht zur Prüfung der Dämmeigenschaften von TMP-Faserstoffen unterschiedlicher Holzarten. Technische Universität Kaiserslautern.

Henriksson, G. (2009). „Lignin“. In: Wood Chemistry and Technology. Hrsg. von M. Ek, G. Gellerstedt \& G. Henriksson. Pulp and Paper Chemistry and Technology. Berlin: De Gruyter, S. 121-145.

Henriksson, G. \& H. Lennholm (2009). „Cellulose and Carbohydrate Chemistry“. In: Wood Chemistry and Technology. Hrsg. von M. Ek, G. Gellerstedt \& G. Henriksson. Pulp and Paper Chemistry and Technology. Berlin: De Gruyter, S. 72-99.

Herberich, E., J. Sikorski \& T. Hothorn (2010). „A robust procedure for comparing multiple means under heteroscedasticity in unbalanced designs". In: PloS one 5.3, e9788.

Hill, C. A. S., B. A. Keating, Z. Jalaludin \& E. Mahrdt (2012). „A rheological description of the water vapour sorption kinetics behaviour of wood invoking a model using a canonical assembly of Kelvin-Voigt elements and a possible link with sorption hysteresis“. In: Holzforschung 66.1.

Hillis, W. E. (1971). „Distribution, properties and formation of some wood extractives“. In: Wood Science and Technology 5.4, S. 272-289.

Hillis, W. E. \& A. N. Rosza (1978). „The Softening Temperatures of Wood“. In: Holzforschung 32.2, S. 68-73.

Hobballah, M. H., A. Ndiaye, F. Michaud \& M. Irle (2018). „Formulating preliminary design optimization problems using expert knowledge: Application to wood-based insulating materials“. In: Expert Systems with Applications 92, S. 95-105.

Hoffmeyer, P., E. T. Engelund \& L. Thygesen (2011). „Equilibrium moisture content (EMC) in Norway spruce during the first and second desorptions“. In: Holzforschung 65.6, S. 875-882.

Höglund, H., U. Sohlin \& G. Tistad (1976). „Physical properties of wood in relation to chip refining“. In: Tappi fournal 59.6, S. 144-147.

Holzer, W. F., J. T. Henderson, W. B. West \& K. F. Byington (1962). „,The development and production of disk-refined groundwood pulp“. In: Tappi fournal 45.3, S. 208-213.

Htun, M., P. Engstrand \& L. Salmén (1988). „The implication of lignin softening on latency removal of mechanical and chemimechanical pulps“. In: fournal of Pulp and Paper Science 14.5, J109-J113.

Htun, M., L. Salmén \& L. Eriksson (1993). „A better understanding of wood as a material - a way to increased energy efficiency when making Mechanical Pulps?" In: Energy Efficiency in Progress Technology. Hrsg. von P. A. Pilavachi. London \& New York: Elsevier \& Elsevier Applied Science, S. 1086-1095.

Huber, B. \& G. Prütz (1938). „Über den Anteil von Fasern, Gefäßen und Parenchym am Aufbau verschiedener Hölzer“. In: Holz als Roh- und Werkstoff 10.1, S. 377-381.

Illikainen, M., E. Härkönen \& J. Niinimäki (2010). „Studies on the mechanisms of TMP refining Volume fraction of pulp in the inner refining zone“. In: Paperi ja puu 92.3, S. 28-31.

Illikainen, M., E. Härkönen, M. Ullmar \& J. Niinimäki (2008). „Disruptive shear stress in spruce and pine TMP pulps“. In: Paperi ja puu 90.1, S. 47-52.

Imken, A. A. P., B. Plinke \& C. Mai (2021). „Characterisation of hardwood fibres used for wood fibre insulation boards (WFIB)“. In: European fournal of Wood and Wood Products 79, S. 915-924.

Irvine, G. M. (1985). „The significance of the glass transition of lignin in thermomechanical pulping“. In: Wood Science and Technology 19, S. 139-149. 
Ishikawa, A., N. Kuroda \& A. Kato (2004). „In situ measurement of wood moisture content in high-temperature steam". In: Journal of Wood Science 50.1, S. 7-14.

ISO 8302:1991 (1991). Thermal insulation - Determination of steady-state thermal resistance and related properties - Guarded hot plate apparatus. Berlin: Beuth Verlag.

Jayme, G. \& D. Fengel (1961). „Beitrag zur Kenntnis des Feinbaus der Frühholztracheiden“. In: Holz als Roh- und Werkstoff 19.2, S. 50-55.

Jensen, U. (1975). „Technisches Anleitungsblatt für die Holzindustrie 1975/2: Charakterisierung der Feinheit von Holzfaserstoff durch trockene Siebfraktionierung“. In: Holztechnologie 16.3, S. $182-184$.

Jumag Dampferzeuger GmbH (2014). Betriebsanleitung Baureihe DG 160 - DG 560.

Kaemmerlen, A., F. Asllanej, H. Sallée, D. Baillis \& G. Jeandel (2010). „Transient modeling of combined conduction and radiation in wood fibre insulation and comparison with experimental data". In: International Journal of Thermal Sciences 49.11, S. 2169-2176.

Karnis, A. (1994). „The mechanism of fibre development in mechanical pulping“. In: fournal of Pulp and Paper Science 20.10, J280-J288.

Kauman, W. G. (1956). „Equilibrium moisture content relations and drying control in superheated steam drying". In: Forest Products fournal 6, S. 328-332.

Kawasaki, T., M. Zhang \& S. Kawai (1998). „Manufacture and properties of ultra-low-density fiberboard“. In: Journal of Wood Science 44.5, S. 354-360.

Keays, J. L. \& R. A. Leask (1973). „Refiner Mechanical Pulp - Past Present and Potential“. In: Paper Trade fournal 35, S. 20-23.

Kelley, S. S., T. G. Rials \& W. G. Glasser (1987). „Relaxation behaviour of the amorphous components of wood“. In: Journal of Materials Science 22.2, S. 617-624.

Kerekes, R. J. (2010). „Energy and Forces in Refining“. In: fournal of Pulp and Paper Science 36.1-2.

- (2015). „Perspectives on high and low consistency refining in mechanical pulping“. In: BioResources 10.4, S. 8795-8811.

Knauf, M. \& A. Frühwald (2020). Broschüre Laubholz-Produktmärkte aus technisch-wirtschaftlicher und marktstruktureller Sicht. Hrsg. von Fachagentur Nachwachsende Rohstoffe e.V.

Koch, G. \& U. Schmitt (2013). „Topochemical and Electron Microscopic Analyses on the Lignification of Individual Cell Wall Layers During Wood Formation and Secondary Changes". In: Cellular Aspects of Wood Formation. Hrsg. von J. Fromm. Plant Cell Monographs. Berlin, Heidelberg: Springer, S. 41-69.

Kojiro, K., T. Miki, H. Sugimoto, M. Nakajima \& K. Kanayama (2010). „Micropores and mesopores in the cell wall of dry wood". In: fournal of Wood Science 56.2, S. 107-111.

Kollmann, F. (1951a). „Anatomie und Pathologie des Holzes“. In: Technologie des Holzes und der Holzwerkstoffe. Hrsg. von F. Kollmann. Springer, S. 1-109.

- (1951b). „Physik des Holzes“. In: Technologie des Holzes und der Holzwerkstoffe. Hrsg. von F. Kollmann. Springer, S. 327-574.

- (1965). „Die Bedeutung der Gaußschen Normalverteilung für Struktur, Sorption und Rheologie von Holz“. In: Holz als Roh- und Werkstoff 23.5, S. 165-173.

- (1987). „Poren und Porigkeit in Hölzern“. In: Holz als Roh- und Werkstoff 45.1, S. 1-9.

Kollmann, F. \& L. Malmquist (1956). „Über die Wärmeleitzahl von Holz und Holzwerkstoffen“. In: Holz als Roh- und Werkstoff 14.6, S. 201-204.

Koran, Z. (1970). „Surface structure of thermomechanical pulp fibers studied by electron microscopy". In: Wood and Fiber Science 2.3, S. 247-258.

- (1981). „Energy Consumption in Mechanical Fibre Separation as a Function of Temperature“. In: Pulp \& Paper Canada 82.6, TR40-TR44. 
Košík, M., M. Dandárová \& R. Domanský (1968). „Pyrolyse des Buchenholzes bei niedrigen Temperaturen IX.: Chemische Veränderungen im Buchenholz während der Pyrolyse“. In: Holzforschung Holzverwendung 21, S. 40-43.

Krpan, J. von (1954). „Untersuchungen über den Fasersättigungspunkt des Buchen-, Eichen-, Tannen- und Fichtenholzes“. In: Holz als Roh- und Werkstoff 12.3, S. 84-91.

Krug, D. (2010). „Einfluss der Faserstoff-Aufschlussbedingungen und des Bindemittels auf die Eigenschaften von mitteldichten Faserplatten (MDF) für eine Verwendung im Feucht- und Außenbereich“. Dissertation. Universität Hamburg.

Kucera, B. (1994). „A hypothesis relating current annual height increment to juvenile wood formation in norway spruce". In: Wood and Fiber Science 26.1, S. 152-167.

Kulasinski, K., R. Guyer, D. Derome \& J. Carmeliet (2015). „Water Adsorption in Wood MicrofibrilHemicellulose System: Role of the Crystalline-Amorphous Interface“. In: Biomacromolecules 16.9, S. 2972-2978.

Kürschner, K. (1966). Chemie des Holzes. 2. Auflage. Berlin: Technischer Verlag Herbert Cram.

Kwok, T. T., D. N. Fogg, M. J. Realff \& A. S. Bommarius (2017). „Applying Direct Yellow 11 to a modified Simons' staining assay“. In: Cellulose 24.6, S. 2367-2373.

Ladenburger, C. (2010). „Untersuchungen zur Optimierung der Faserherstellung einer MDFProduktionslinie“. Diplomarbeit. Hochschule Rosenheim.

Lamb, G. E. R. (1960). „The Efficiency of Mechanical Pulping Processes“. In: Tappi fournal 43.11, S. $989-944$.

Lampert, H. (1967). Faserplatten: Rohstoffe, Herstellungsverfahren, Eigenschaften. 3. Auflage. Leipzig: Fachbuchverlag.

Lashkov, V. A., E. I. Levashko \& R. G. Safin (2001). „Heating of Production Wood Chips in a Saturated-Steam Medium“. In: Journal of Engineering Physics and Thermophysics 74.1, S. 112118.

Leask, R. A. (1968). „Developments in refiner groundwood“. In: Paper Technology 9.6, S. 489-497.

- (1973). „The status of refiner mechanical pulping“. In: Svensk Papperstidning 17, S. 631-638.

- (1987). „Refiner Plates“. In: Pulp and paper manufacture. Hrsg. von O. V. Ingruber, M. J. Kocurek \& F. Stevens. Atlanta: Joint Textbook Committee of the Paper Industry, S. 93-97.

Lee, M., S.-M. Lee, E.-C. Kang \& D.-W. Son (2019). „Combustibility and Characteristics of WoodFiber Insulation Boards Prepared with Four Different Adhesives“. In: BioResources 14.3, S. 63166330.

Lein, F. (1978). „Tendenzen der MDF-Plattenerzeugung“. In: Holz als Roh- und Werkstoff 26.10, S. 379-382.

Leißner, T. (2015). „Beitrag zur Kennzeichnung von Aufschluss- und Trennerfolg am Beispiel der Magnetscheidung“. Dissertation. Freiberg: Technische Universität Bergakademie Freiberg.

Lenth, C. A. \& F. A. Kamke (2001). „Equilibrium moisture content of wood in high-temperature pressurized environments". In: Wood and Fiber Science 33.1, S. 104-118.

Leschonski, K. (1984). „Representation and Evaluation of Particle Size Analysis Data“. In: Particle \& Particle Systems Characterization 1.1-4, S. 89-95.

Liimatainen, H., P. Haikkala, M. Lucander, R. Karojärvi \& O. Tuovinen (1999). „Grinding and Pressure Grinding“. In: Mechanical pulping. Hrsg. von J. Sundholm. Papermaking Science and Technology. Helsinki: Fapet Oy, S. 107-158.

Lim, T.-S. \& W.-Y. Loh (1996). „A comparison of tests of equality of variances“. In: Computational Statistics \& Data Analysis 22, S. 287-301.

Lindholm, C.-A. \& J. A. Kurdin (1999). „Chemimechanical Pulping“. In: Mechanical pulping. Hrsg. von J. Sundholm. Papermaking Science and Technology. Helsinki: Fapet Oy, S. 223-248. 
Lindstrom, H. (1997). „Fiber length, tracheid diameter, and latewood percentage in norway spruce: development from pith outwards". In: Wood and Fiber Science 29.1, S. 21-34.

Lowgren, U. (1941). „Asplund Defibrating Process“. In: Paper Trade fournal, S. 29-32.

Maier, G. (1983). „Spanungstechnische Grundlagen der Hackschnitzelerzeugung in Trommelhackern“. In: European fournal of Wood and Wood Products 41.10, S. 437-439.

Makas, M. (2016). „Leichte Faserplatten und Dämmung“. In: Tagungsband der Fachtagung Technologie des Holzes und der Holzwerkstoffe. Hrsg. von Verein zur Förderung holzbiologischer und holztechnischer Forschung e. V. Göttingen: Selbstverlag.

Maku, T. (1954). „Studies on the Heat Conduction in Wood“. In: Wood Research 13, S. 1-80.

Malkov, S., V. Kuzmin, V. Baltakhinov \& P. Tikka (2002). „Efficiency of chip presteaming - result of heating and air escape processes". In: Nordic Pulp \& Paper Research fournal 17.4, S. 420-426.

Manohar, K., G. S. Kochhar \& D. W. Yarbrough (2012). „Heat Transfer Across Opaque Fibers“. In: Journal of Heat Transfer 134.7, S. 072601-1-072601-8.

Mantau, U. (2012). Standorte der Holzwirtschaft, Holzrohstoffmonitoring, Holzwerkstoffindustrie Kapazitätsentwicklung und Holzrohstoffnutzung im fahr 2010. Universität Hamburg, Zentrum Holzwirtschaft. Arbeitsbereich Ökonomie der Holz- und Forswirtschaft,Hamburg.

Melelli, A., O. Arnould, J. Beaugrand \& A. Bourmaud (2020). „The middle lamella of plant fibers used as composite reinforcement: investigation by atomic force microscopy“. In: Molecules 25.3, S. 632 .

Menck, D. (1994). „Das LPS 200 MC - Eine neuartige Analysensiebmaschine für den Fein- und Feinstbereich". In: Aufbereitungstechnik 35.7, S. 359-366.

Meng, X., M. Foston, J. Leisen, J. DeMartini, C. E. Wyman \& A. J. Ragauskas (2013). „Determination of porosity of lignocellulosic biomass before and after pretreatment by using Simons' stain and NMR techniques“. In: Bioresource technology 144, S. 467-476.

Merakeb, S., F. Dubois \& C. Petit (2009). „Modeling of the sorption hysteresis for wood“. In: Wood Science and Technology 43.7-8, S. 575-589.

Michel, F., V. Brombacher \& P. Niemz (2014). „Optimization of fiber board properties“. In: Annals of Warsaw University of Life Sciences - SGGW Forestry and Wood Technology 86, S. 180-188.

Michel, T. (2017). MDF Refiner Plates Design Theory. Andritz AG.

Miles, K. \& W. D. May (1990). „The Flow of Pulp in Chip Refiners“. In: fournal of Pulp and Paper Science 16.2, J63-J72.

Mott, L., L. H. Groom \& S. Shaler (2002). „Mechanical Properties of Individual Southern Pine Fibers. Part 11. Comparison of Earlywood and Latewood Fibers with Respect to Tree Height and Juvenility". In: Wood and Fiber Science 34.2, S. 221-237.

Muguet, M. d. S., J. L. Colodette \& A.-S. Jääskeläinen (2012). „Alkaline Peroxide Mechanical Pulping of Novel Brazilian Eucalyptus Hybrids“. In: BioResources 7.3, S. 3823-3836.

Muhic, D., J.-P. Huhtanen, L. Sundström, C. Sandberg, M. Ullmar, P. Vuorio \& P. Engstrand (2011). „Energy efficiency in double disc refining - influence of intensity by segment design“. In: Nordic Pulp \& Paper Research fournal 26.3, S. 224-230.

Murton, K., G. Duffy \& S. Corson (2002). Pulp residence time influence on refining intensity and pulp quality. In: 56th Appita Annual Conference, Rotorua, New Zealand 18-20 March 2002: Proceedings. Appita Inc.. S. 185.

Neill, M. T. \& L. R. Beath (1963). „Supergroundwood: its manufature from chips and use as sole newsprint furnish“. In: Pulp \& Paper Magazine of Canada 64, T299-T312.

Neimsuwan, T., S. Wang \& X. Philip Ye (2008). „Effects of refining steam pressure on the properties of loblolly pine (Pinus taeda L.) fibers“. In: Holzforschung 62.5, S. 155. 
Niemz, P. \& W. Sonderegger (2017). „Verhalten von Holz und Holzwerkstoffen gegenüber Feuchte“. In: Holzphysik. München: Carl Hanser Verlag, S. 69-132.

Noack, D. (1959). „Beitrag zur Sorption des Holzes im Bereich höherer Temperaturen und Wasserdampfdrücke“. In: Holz als Roh- und Werkstoff 17.5, S. 205-212.

Olsson, A.-M. \& L. Salmén (1997). „The effect of lignin compostition on the viscoelastic properties of wood“. In: Nordic Pulp \& Paper Research fournal 12.3, S. 140-144.

Ormondroyd, G. A., S. K. Källbom, S. F. Curling, B. K. Stefanowski, B. K. Segerholm, M. E. P. Wålinder \& D. Jones (2017). ,Water sorption, surface structure and surface energy characteristics of wood composite fibres refined at different pressures". In: Wood Material Science \& Engineering 12.4, S. 203-210.

Parameswaran, N., H. J. Dengg \& R. Patt (1985). „Restholzhackschnitzel für die Herstellung von TMP und Zellstoff". In: Holz als Roh- und Werkstoff 43.1, S. 1-8.

Patera, A., H. Derluyn, D. Derome \& J. Carmeliet (2016). „Influence of sorption hysteresis on moisture transport in wood". In: Wood Science and Technology 50.2, S. 259-283.

Patt, R., N. Parameswaran, J. Kruse \& H. Behler (1981). „Zur Qualität von Nadelrestholzhackschnitzeln für die Faserstoffherstellung: 2. Mitt. Einfluß der Hackschnitzelherstellung auf die Struktur und Eigenschaften von Faserstoffen“. In: Wochenblatt für Papierfabrikation 109, S. 919-926.

Patt, R., M. Welte \& H. Burkhart (1980). „Untersuchungen über den Einfluß der Beschaffenheit von Industrierestholz auf die Eigenschaften von TMP und Zellstoffen“. In: Papier 34.10A, S. V1-V9.

Pavel, C. C. \& D. T. Blagoeva (2018). Competitive landscape of the EU's insulation materials industry for energy-efficient buildings. Hrsg. von European Commission Joint Research Centre.

Pearson, A. J. (1990). A unified theory of refining. Bd. 6. Pulp and Paper Technology Series. Atlanta: TAPPI.

Peterson, V. \& G. Dahlquist (1975). „Refining of preheated chips. The effect of some process variables on pulp quality“. In: International Mechanical Pulping Conference San Francisco, USA, Vol. I, S. 11-15.

Peterson, V., G. Dahlquist \& B. Engström (1973). „Single-stage refining of preheated chips at SCA in Sweden“. In: Pulp and Paper International 15, S. 1973.

Petit-Conil, M., M. Lecourt \& M. Meyer (2016). „High-yield pulps: an interesting concept for producing lignocellulosic fibers“. In: Lignocellulosic fibers and wood handbook. Hrsg. von N. Belgacem \& A. Pizzi. Salem, Massachusetts: Scrivener Publishing \& Wiley, S. 157-206.

Placet, V., J. Passard \& P. Perré (2007). „Viscoelastic properties of green wood across the grain measured by harmonic tests in the range $0-95^{\circ} \mathrm{C}$ : Hardwood vs. softwood and normal wood vs. reaction wood“. In: Holzforschung 61.5, S. 548-557.

Plinke, B., J. T. Benthien, A. Krause, K. C. Krause, A. Schirp \& L. Teuber (2016). „Optische Größenvermessung von Holzpartikeln für die WPC-Herstellung: Vergleich dreier Messverfahren". In: Holztechnologie 57.4, S. 43-50.

$\mathrm{R}$ Core Team (2017). R: A language and environment for statistical computing. Wien: R Foundation for Statistical Computing.

Radmanović, K., I. Đukić \& S. Pervan (2014). „Specific Heat Capacity of Wood“. In: Drvna industrija 65.2, S. $151-157$.

Richter, C. (1993). „Neues Verfahren zur Herstellung von Dämmstoffen niedriger Dichte aus Holz und Einjahrespflanzen“. In: Holz als Roh- und Werkstoff 51.4, S. 235-239.

Roffael, E., B. Dix, G. Bär \& R. Bayer (1994a). „Über die Eignung von thermo-mechanischem und chemo-thermo-mechanischem Holzstoff (TMP und CTMP) aus Buchen- und Kiefernholz für die Herstellung von mitteldichten Faserplatten (MDF): Teil 1: Aufschluss des Holzes und Eigenschaften der Faserstoffe“. In: European fournal of Wood and Wood Products 52.4, S. 239-246. 
Roffael, E., B. Dix, G. Bär \& R. Bayer (1994b). „Über die Eignung von thermo-mechanischem und chemo-thermo-mechanischem Holzstoff (TMP und CTMP) aus Buchen- und Kiefernholz für die Herstellung von mitteldichten Faserplatten (MDF): Teil 2: Eigenschaften von MDF aus Buchen-Faserstoff“. In: European fournal of Wood and Wood Products 52.5, S. 293-298.

- (1995). „Über die Eignung von thermo-mechanischem (TMP) und chemo-thermomechanischem (CTMP) Holzstoff aus Buchen- und Kiefernholz für die Herstellung von mitteldichten Faserplatten (MDF): Teil 3: Eigenschaften von aus Kiefern-Faserstoff hergestellten MDF“. In: European fournal of Wood and Wood Products 53.1, S. 8-11.

Roll, H. (2008). „Refiner Layout, Dimensioning, Practical Experience“. In: 6th European Wood-Based Panel Symposium.

- (2010a). „Optimierung des Faseraufschlusses im Refiner: Energierelevante Aspekte der Refinerscheibentechnologie bei der MDF-Herstellung unter Berücksichtigung der Faserqualität“. In: Holz-Zentralblatt 2010.16, S. 423-424.

- (2010b). „Optimierung des Faseraufschlusses im Refiner: Energierelevante Aspekte der Refinerscheibentechnologie bei der MDF-Herstellung unter Berücksichtigung der Faserqualität“. In: Holz-Zentralblatt 2010.17, S. 443-444.

Rowell, R. M., R. Pettersen \& M. A. Tschabalala (2013). „Cell Wall Chemistry“. In: Handbook of wood chemistry and wood composites. Hrsg. von R. M. Rowell. Boca Raton: Taylor \& Francis \& CRC Press, S. 33-74.

Rumpf, H. (1967). „Über die Eigenschaften von Nutzstäuben“. In: Staub, Reinhaltung der Luft 27.1, S. 3-13.

Sabourin, M. (2003). „Effect of increasing wood chip defibration on thermomechanical refining efficiency“. In: International Mechanical Pulping Conference, Quebec City, 2003, S. 163-170.

Salmén, L. (1981). „The fundamentals of energy consumption during viscoelastic and plastic deformation of wood“. In: Presented at the 1981 International mechanical pulping conference, EUCEPA, Oslo.

- (1984). „Viscoelastic properties of in situ lignin under water-saturated conditions“. In: fournal of Materials Science 19, S. 3090-3096.

- (2004). „Micromechanical understanding of the cell-wall structure“. In: Comptes rendus biologies 327.9-10, S. 873-880.

- (2015). „Wood morphology and properties from molecular perspectives“. In: Annals of Forest Science 72.6, S. 679-684.

Salmén, L. \& P. A. Larsson (2018). „On the origin of sorption hysteresis in cellulosic materials“. In: Carbohydrate Polymers 182, S. 15-20.

Salmén, L., M. Lucander, E. Härkönen \& J. Sundholm (1999). „Fundamentals of mechanical pulping“. In: Mechanical pulping. Hrsg. von J. Sundholm. Papermaking Science and Technology. Helsinki: Fapet Oy, S. 35-65.

Salmén, L., A.-M. Olsson, J. S. Stevanic, J. Simonovic \& K. Radotic (2012). „Structural organisation of the wood polymers in the wood fibre structure." In: BioResources 7.1, S. 521-532.

Salmén, L. \& B. Petterson (1995). „The Primary Wall; Important for Fibre Separation in Mechanical Pulping“. In: Cellulose Chemistry and Technology 29.3, S. 331-337.

Sandberg, C., J. Hill \& M. Jackson (2020). „On the development of the refiner mechanical pulping process - a review“. In: Nordic Pulp \& Paper Research fournal 35.1, S. 1-17.

Sandermann, S. \& H. Augustin (1964). „Chemische Untersuchungen über die thermische Zersetzung von Holz - Dritte Mitteilung: Chemische Untersuchung des Zersetzungsablaufs“. In: Holz als Roh- und Werkstoff 22, S. 377-386.

SCAN-CM 40 (2001). Wood chips for pulp production. Size distribution. Stockholm. 
Scheiding, W. (2000). „Untersuchungen zur Wärmeleitfähigkeit von Holzfaserstoff“. In: Holz als Roh- und Werkstoff 58.3, S. 177-181.

Schirp, A., M. Mannheim \& B. Plinke (2014). „Influence of refiner fibre quality and fibre modification treatments on properties of injection-moulded beech wood-plastic composites". In: Composites: Part A 61, S. 245-257.

Schirp, A., B. Plinke \& D. Napolow (2015). „Effectiveness of organic and inorganic pigments for mass colouration of thermo-mechanical pulp used in wood-plastic composites". In: Holz als Roh- und Werkstoff 73.1, S. 5-16.

Schlüter, S. (2017). Optimum Resolution \& Sharpening Settings for Epson Scanners.

Schmid, H., G. Schmid, I. Schmid-Götte \& C. Buda (2020). X-Shape Manual 6.2.2.

Schmidt, P. (1967). „Probleme der Feinabsiebung“. In: Aufbereitungstechnik 6, S. 322-324.

Schmidt, P. \& M. Coppers (1998). „Erfahrungen mit dem schnellen Rhewum-Mehrflächen-Präzisions luftstrahlsieb MPS“. In: Aufbereitungstechnik 39.7, S. 343-347.

Schmidt, P., R. Körber \& M. Coppers (2003). Sieben und Siebmaschinen: Grundlagen und Anwendung. Weinheim: VCH.

Schneider, T., E. Roffael \& B. Dix (2000). „Einfluß von Holzaufschlußverfahren (TMP-, CTMPVerfahren) und Aufschlußbedingungen auf die physikalisch-technologischen Eigenschaften von mitteldichten Faserplatten (MDF)“. In: Holz als Roh- und Werkstoff 58, S. 123-124.

Schönert, K. (2003). „Zerteilprozesse“. In: Handbuch der mechanischen Verfahrenstechnik. Hrsg. von H. Schubert. Weinheim: Wiley-VCH, S. 298-382.

Schulz, H. (1957). „Der Anteil der einzelnen Zellarten an dem Holz der Rotbuche“. In: Holz als Roh- und Werkstoff 15.3, S. 113-118.

Sekino, N. (2016). „Density dependence in the thermal conductivity of cellulose fiber mats and wood shavings mats: Investigation of the apparent thermal conductivity of coarse pores". In: Journal of Wood Science 62.1, S. 20-26.

Simon, F. L. (1950). „A stain for use in the microscopy of beaten fibers“. In: Tappi fournal 33.7, S. 312-314.

Skaar, C. (1988). „Wood moisture and the Environment“. In: Wood-water relations. Hrsg. von T. E. Timell \& C. Skaar. Springer Series in Wood Science. Berlin, Heidelberg: Springer, S. 1-45.

Smith, I., E. Landis \& M. Gong (2003a). „Mechanical behaviour of wood“. In: Fracture and fatigue in wood. Hrsg. von I. Smith, E. Landis \& M. Gong. Chichester: John Wiley \& Sons, S. 37-65.

- (2003b). „Structure and properties of wood“. In: Fracture and fatigue in wood. Hrsg. von I. Smith, E. Landis \& M. Gong. Chichester: John Wiley \& Sons, S. 7-35.

Solala, I., T. Antikainen, M. Reza, L.-S. Johansson, M. Hughes \& T. Vuorinen (2014). „Spruce fiber properties after high-temperature thermomechanical pulping (HT-TMP)“. In: Holzforschung 68.2 .

Sonderegger, W., S. Hering \& P. Niemz (2011). „Thermal behaviour of Norway spruce and European beech in and between the principal anatomical directions". In: Holzforschung 65.3, S. 333.

Sonderegger, W. \& P. Niemz (2012). „Thermal and moisture flux in soft fibreboards“. In: European fournal of Wood and Wood Products 70.1-3, S. 25-35.

Sorensen, J. S. (1915). „Attrition Mill“. Patent. Veröffentlichungs-Nr.: 1,146,032.

Srebotnik, E. \& K. Messner (1994). „A Simple Method That Uses Differential Staining and Light Microscopy To Assess the Selectivity of Wood Delignification by White Rot Fungi“. In: Applied and Environmental Microbiology 60.4, S. 1383-1386.

Stamm, A. J. (1964a). „Microscopic Structure of Wood“. In: Wood and Cellulose Science. Hrsg. von A. J. Stamm. New York: The Ronald Press Company, S. 3-33. 
Stamm, A. J. (1964b). „Sorption of Water Vapor by Cellulosic Materials“. In: Wood and Cellulose Science. Hrsg. von A. J. Stamm. New York: The Ronald Press Company, S. 142-165.

- (1964c). „The Phenomenon of Adsorption“. In: Wood and Cellulose Science. Hrsg. von A. J. Stamm. New York: The Ronald Press Company, S. 132-141.

Steeger, F., J. Küppers \& S. Brunkhorst (2017). „Schwel- und Brandverhalten von Wärmedämmverbundsystemen aus Holzfaserdämmstoffen“. In: Bautechnik 94.6, S. 361-367.

Stevanovic, T. (2016). „Chemical Composition and Properties of Wood“. In: Lignocellulosic fibers and wood handbook. Hrsg. von N. Belgacem \& A. Pizzi. Salem, Massachusetts: Scrivener Publishing \& Wiley, S. 49-106.

Stieß, M. (2009). Mechanische Verfahrenstechnik - Partikeltechnologie 1. 3. Auflage. Berlin, Heidelberg: Springer.

Strickler, M. D. (1968). „High temperature moisture relations of grand fir“. In: Forest Products Journal 18.4, S. 69-75.

T 401 om-03 (2008). Fiber analysis of paper and paperboard.

Talebjedi, B., A. Khosravi, T. Laukkanen, H. Holmberg, E. Vakkilainen \& S. Syri (2020). „Energy modeling of a refiner in thermo-mechanical pulping process using ANFIS method". In: Energies 13.19, S. 5113.

TAURUS Instruments AG (2018). Betriebsanleitung TLP 900 (G)X.

Teleman, A. (2009). „Hemicelluloses and Pectins“. In: Wood Chemistry and Technology. Hrsg. von M. Ek, G. Gellerstedt \& G. Henriksson. Pulp and Paper Chemistry and Technology. Berlin: De Gruyter, S. 102-120.

Tian, H. (2020). „Stochastic multi-objective economic model predictive control of two-stage high consistency Mechanical Pulping Processes“. Dissertation. The University Of British Columbia.

Tienvieri, T., E. Huusari, J. Sundholm, V. Petteri, J. Kortelainen, H. Nystedt \& A. Artamo (1999). „Thermomechanical pulping“. In: Mechanical pulping. Hrsg. von J. Sundholm. Papermaking Science and Technology. Helsinki: Fapet Oy, S. 196-221.

Tran, H., P. Doumalin, C. Delisée, J. C. Dupre, J. Malvestio \& A. Germaneau (2013). „3D mechanical analysis of low-density wood-based fiberboards by X-ray microcomputed tomography and Digital Volume Correlation“. In: Journal of Materials Science 48.8, S. 3198-3212.

Trendelenburg, R. \& H. Mayer-Wegelin (1955). Das Holz als Rohstoff. 2. Aufl. München: Carl Hanser Verlag.

Varhimo, A. \& O. Tuovinen (1999). „Raw materials“. In: Mechanical pulping. Hrsg. von J. Sundholm. Papermaking Science and Technology. Helsinki: Fapet Oy, S. 67-104.

VDI e.V., Hrsg. (2013). VDI-Wärmeatlas. 11. Auflage. Springer Reference. Berlin: Springer.

Vehniäinen, A. (2008). „Single fiber properties - a key to the characteristics defibration patterns from wood to paper fibers". Dissertation. Helsinki: Helsinki University of Technology.

Verband Deutscher Papierfabriken e.V., Hrsg. (2001). Qualitätsbestimmungen für Hackschnitzel zur Erzeugung von thermomechanischem Holzstoff (TMP) und Zellstoff (Sulfat/Sulfit).

Vignon, P., M. H. Hobballah, H. Tran, J. Moreau, C. Delisée, M. Lecourt, R. Belalia \& T. Sanguina (2016). „Thermal insulating materials made up of poplar wood fibres“. In: 2nd Conference on Engineered Wood Products Based on Poplar/Willow Wood CEWPPW2. Hrsg. von J. van Acker. Ghent: University Press, S. 37-50.

Vogel, K. (1999). „Die Eignung von Holz als Wärmedämmstoff: vergleichende Untersuchungen von Spänen und Fasern". Dissertation. Ludwig-Maximilian-Universität München.

Wagenführ, R. (2007). Holzatlas. 6. Aufl. Leipzig: Carl Hanser Verlag.

Wagenführ, R. \& A. Wagenführ (2008). „Anatomie des Holzes“. In: Taschenbuch der Holztechnik. Hrsg. von A. Wagenführ \& F. Scholz. München: Carl Hanser Verlag, S. 14-47. 
Wahler, W. (1960). „Eine neue Prüfsiebmaschine nach dem Luftstrahlprinzip für beliebig viele Fraktionen“. In: Tonindustrie-Zeitung und Keramische Rundschau 84.4, S. 93-95.

Wang, P., J. Yang \& J. Wang (2017). „Computer simulation of refining process of a high consistency disc refiner based on CFD“. In: AIP Conference Proceedings 1864, 020079.

Weber, A., A. Raphtel \& U. Sonntag (2014). Echtzeit-Charakterisierung von Holzpartikeln für die Qualitätssicherung und Prozessoptimierung bei der Herstellung von Holzpartikelwerkstoffen in der Holzwerkstoffindustrie - Quick Wood-Particle Size. Hrsg. von Institut für Holztechnologie gGmbH. Dresden.

Wenderdel, C. (2015). „Herstellung mehrschichtiger Faserplatten im Trockenverfahren mit kartonähnlichen Eigenschaften“. Dissertation. Eidgenössische Technische Hochschule Zürich.

Wenderdel, C., E. Hesse, D. Krug, A. Hänsel \& P. Niemz (2013). „Influence of surface roughness of wood fibres on properties of medium density fibreboards". In: Pro Ligno 9.4, S. 423-429.

Wenderdel, C. \& D. Krug (2012). „Untersuchung zum Einfluss der Aufschlussbedingungen auf die morphologische Ausprägung von aus Kiefernholz hergestelltem TMP-Faserstoff". In: European fournal of Wood and Wood Products 70.1-3, S. 85-89.

Wenderdel, C., D. Krug, H. Bartsch \& M. Hielscher (2016a). Untersuchung der Faser-BindemittelWechselwirkung zur Herstellung von MDF aus Laubholz: Schlussbericht FKZ 22022211 (11NR222).

Wenderdel, C., A. Weber, M. Hielscher, M. Pfaff \& U. Sonntag (2016b). „Spezielle Methoden zur morphologischen Charakterisierung lignocelluloser Faserstoffe". In: Holztechnologie 57.3, S. 1622.

Wenderdel, C., A. Weber, M. Pfaff, U. Sonntag \& T. Theumer (2014). „Spezielle Methoden zur morphologischen Charakterisierung lignocelluloser Faserstoffe: Teil 1: Stand der Technik und theoretische Ableitung einer Partikelklassifizierung“. In: Holztechnologie 55.6, S. 12-19.

Wenderdel, C., A. Weber, M. Hielscher \& D. Krug (2017). „Spezielle Methoden zur morphologischen Charakterisierung lignocelluloser Faserstoffe: Teil 3: Einfluss der Fasermorphologie auf die Eigenschaften daraus erzeugter MDF“. In: Holztechnologie 58.1, S. 5-10.

Wert, C. A., M. Weller \& D. Caulfield (1984). „Dynamic loss properties of wood“. In: fournal of Applied Physics 56.9, S. 2453-2458.

Winandy, J. E. \& R. M. Rowell (2013). „Chemistry of wood strength“. In: Handbook of wood chemistry and wood composites. Hrsg. von R. M. Rowell. Boca Raton: Taylor \& Francis \& CRC Press, S. 413-452.

Yap, B. W. \& C. H. Sim (2011). „Comparisons of various types of normality tests“. In: fournal of Statistical Computation and Simulation 81.12, S. 2141-2155.

Yu, X., J. Minor \& R. Atalla (1995). „Mechanism of action of Simons’ stain“. In: Tappi fournal 78.6, S. $175-180$.

Zamil, M. S. \& A. Geitmann (2017). „The middle lamella-more than a glue“. In: Physical biology 14.1, S. 015004.

Zobel, B. J. \& J. P. Buijtenen (2012). Wood variation: Its causes and control. Springer Series in Wood Science. Springer. 


\section{Danksagung}

Die Dissertation entstand, neben meiner wissenschaftlichen Vollzeittätigkeit in den Laboren für Holzwerkstofftechnik sowie Kleb- und Presstechnik an der Technischen Hochschule Rosenheim, als externes Promotionsvorhaben in der Abteilung Holzbiologie und Holzprodukte an der Georg-August-Universität Göttingen. Das begleitende Forschungsvorhaben wurde durch das Bundesministerium für Ernährung und Landwirtschaft über den Projektträger Fachagentur Nachwachsende Rohstoffe e.V. finanziell gefördert. Für die konstruktive, vertrauensvolle Zusammenarbeit insbesondere in der SARS-CoV2-Pandemie möchte ich mich an dieser Stelle bei allen Beteiligten ausdrücklich bedanken.

Bei Herrn Prof. Dr. Carsten Mai möchte ich mich für das Interesse an dem Thema, die Unterstützung in fachlichen Fragen und die Chance als Externer promoviert zu werden bedanken. Herrn Prof. Dr. Andreas Michanickl möchte ich meinen Dank für sein Vertrauen, seine Unterstützung sowie die fachliche und persönliche Förderung aussprechen. Die Arbeit wurde auch durch die Bereitstellung von Informationen und Material durch institutionelle und industrielle Partner gefördert. Für die unkomplizierte, zuvorkommende Zusammenarbeit sowie die Hilfsbereitschaft und Unterstützung in technischen Fragen bedanke ich mich bei Herrn Jörg Dahringer (Trevira $\mathrm{GmbH}$ ), Herrn Felix Hermann (Bayerische Staatsforsten), Herrn Peter Mraz (Andritz AG) und Herrn Tobias Michel (Andritz AG). Mein Dank gilt auch meiner Kollegin Frau Mara Schumacher für ihre Unterstützung beim Experimentieren mit der Laborrefineranlage und die Faseranalyse mit dem Luftstrahlsieb. Herzlich bedanken möchte ich mich auch bei meinem Kollegen Herrn Thomas Schmid für die Unterstützung beim Umbauen, Reparieren und Experimentieren mit der Laborrefineranlage. Meinem Kollegen Herrn Siegfried Lechner gilt mein Dank für die anpackende Problemlösung in praktischen und organisatorischen Fragen. Die gemeinsamen Holzfällungen behalte ich in freudiger Erinnerung. Den Kollegen aus der gemeinsamen Arbeitsgruppe Holz- und Kunstofftechnik Frau Sabine Hummel, Herrn Sebastian Wiedl, Herrn Frederik Obermaier sowie Herrn Prof. Dr. Michael Schemme und Herrn Prof. Dr. Peter Karlinger danke ich für den fachlichen Austausch und die konstruktive Zusammenarbeit. Den Studierenden Frau Katja Bachmann, Frau Janine Bösing, Herrn Johannes Mai und Herrn Yannick Sattler danke ich für ihre Hilfe bei der Literaturbeschaffung und der Durchführung praktischer Arbeiten.

Nicht genug danken kann ich meiner Ehefrau Stephanie Barth für ihre selbstlose Unterstützung in allen Fragen, ihrer Hingabe für Paul und Annika während meiner häufigen Abwesenheit und ganz besonders für den Mut und die Entscheidung das hier durchzuziehen. 


\section{Anhang}

\subsection{R-Codes zur Datenverarbeitung und graphischen Darstellung}

Daten einlesen, filtern, sortieren (packages $\{$ base $\}$, $\{$ readxl\}):



Varianzanalyse (ANOVA) (package \{stats\}):

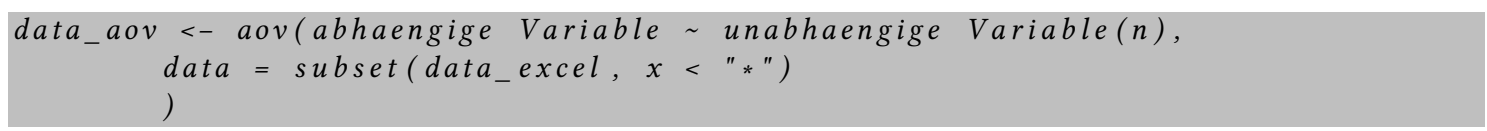

Auf Normalverteilung überprüfen mit Shapiro-Wilk-Test (package \{stats\}) und Levene-Test (package $\{c a r\}):$

shapiro.test (resid(data_aov))

leveneTest (data_aov)

Normalverteilung und Homogenität der Varianzen gegeben: TukeyHSD-Test (package \{stats\})

TukeyHSD (data_aov)

Keine Normalverteilung gegeben: Kruskal-Wallis-Test (package \{stats\})

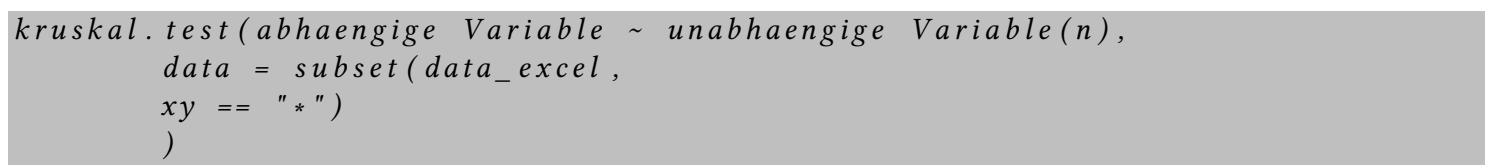

Paarweiser Wilcox (Mann-Whitney-U-Test, package \{ggpubr\}) zum Identifizieren signifikanter Unterschiede:

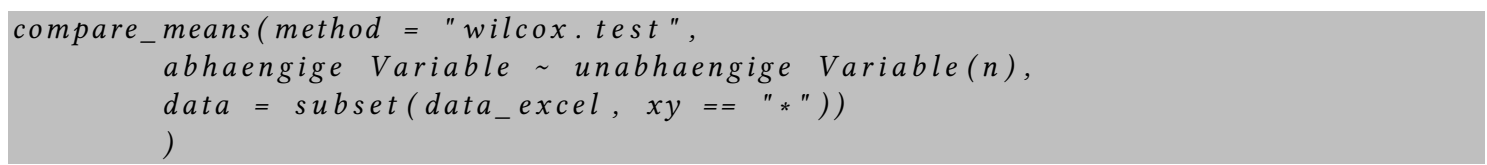

Bereitstellung für die grafische Darstellung (package \{base\}):

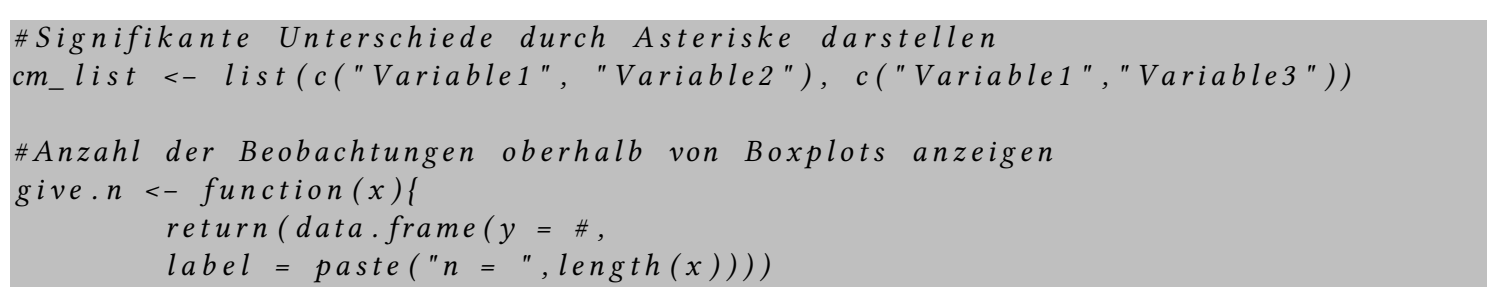




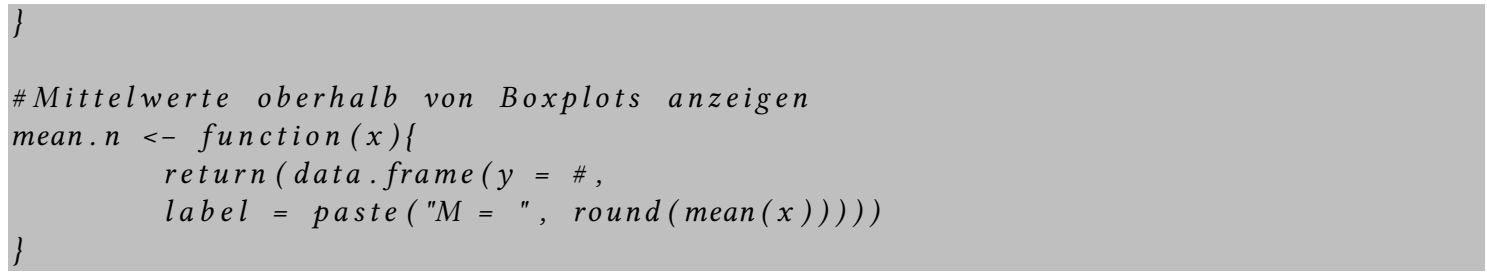

Punkt- und Balkendiagrammen und Boxplots (packages: \{ggplot2\}, \{ggpubr\}, \{ggraph\}, \{ggrepel\}, \{plyr\}, \{tidyr\}, \{gridExtra\}, \{grDevices\}, \{scales\}, \{lattice\})

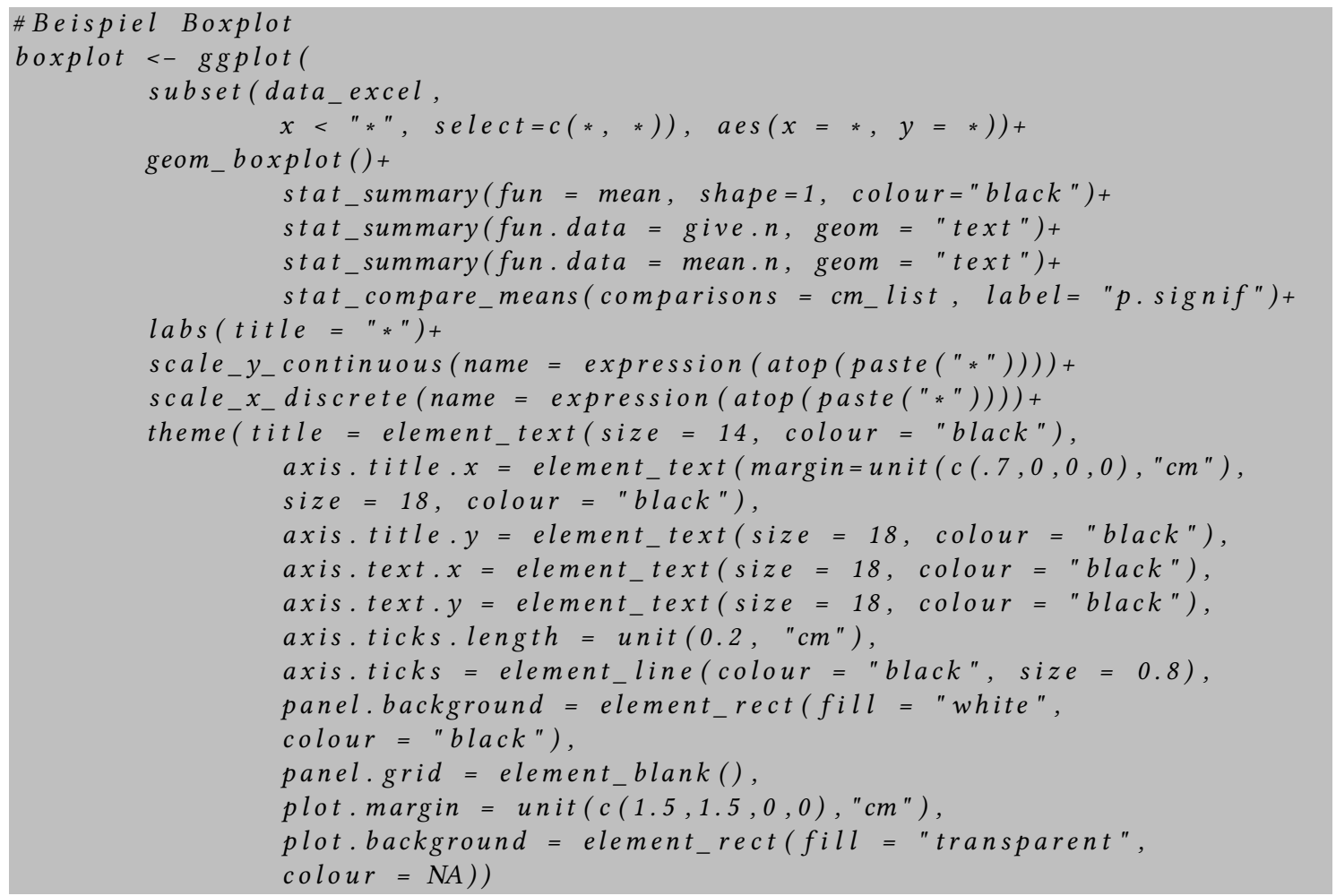

Beschriftung und Legende (packages: \{grid\}, \{gtable\}:

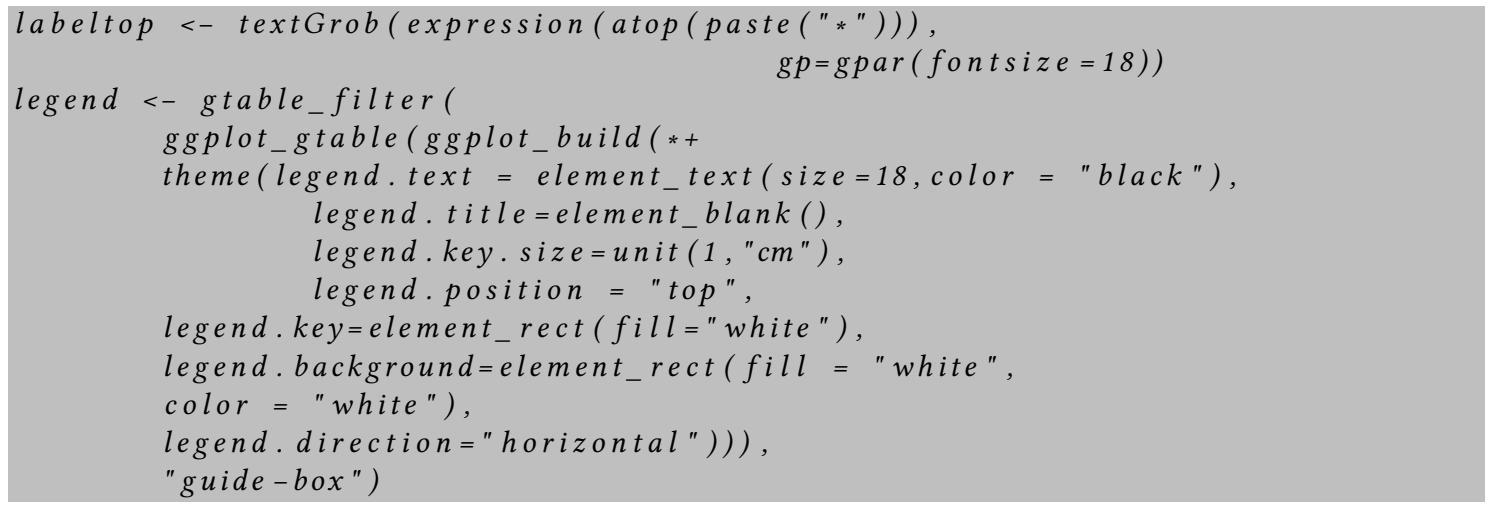

Grafikherstellung aus einzelnen und mehreren Plots, Beschriftung, Legenden und Indizes (package \{cowplot\}):

plot $<-p l o t_{-}$grid(labels $=c("$ auto" $)$, label_fontfamily = "serif",
label_size= 23, vjust=1, boxplot $1+$




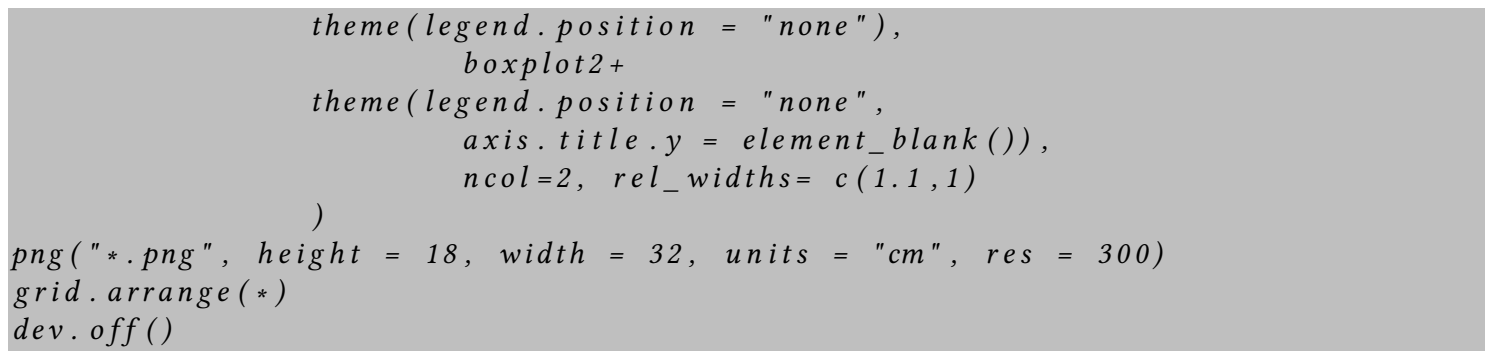

\section{Half-normal plot (Danielplot) (package $\{$ DoE.base\}):}

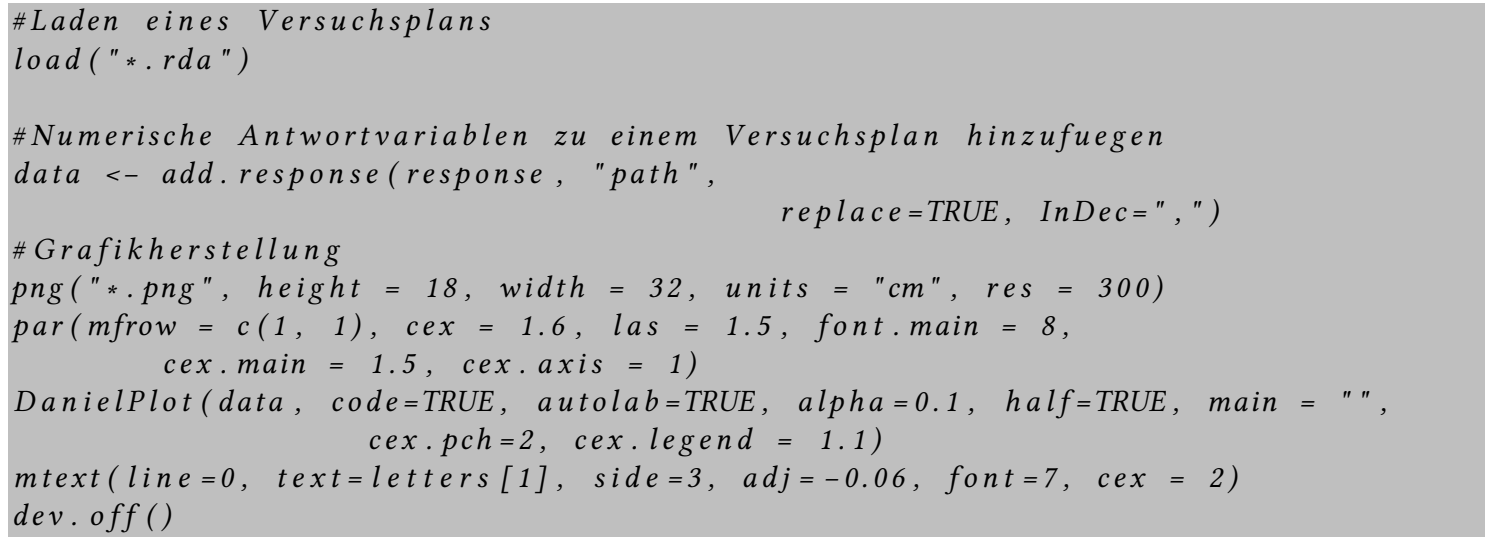

Main-effects plot (package $\{$ RcmdrPlugin.DoE\}):

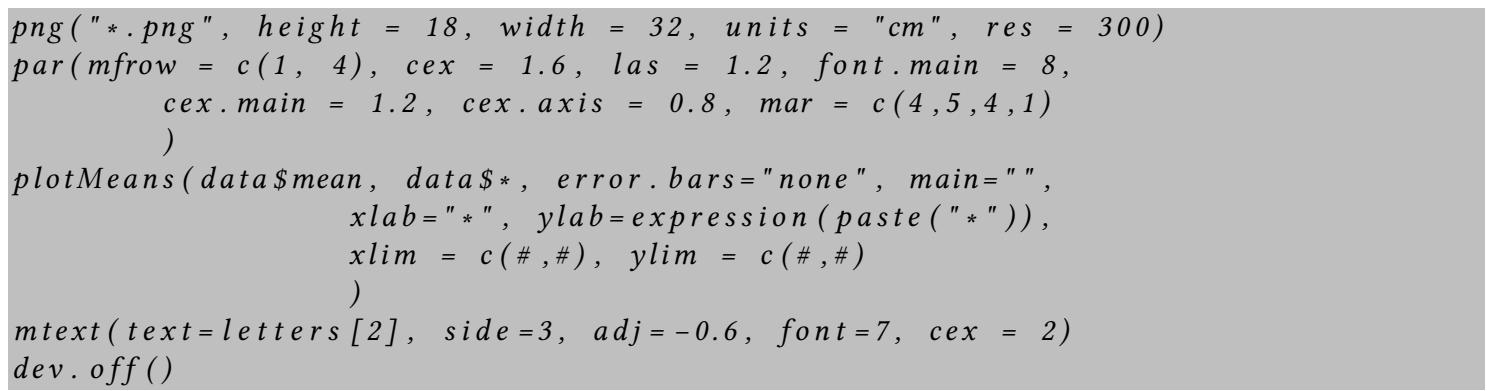

Korrelationstabellen (packages \{corrplot\}, \{xtable\}):

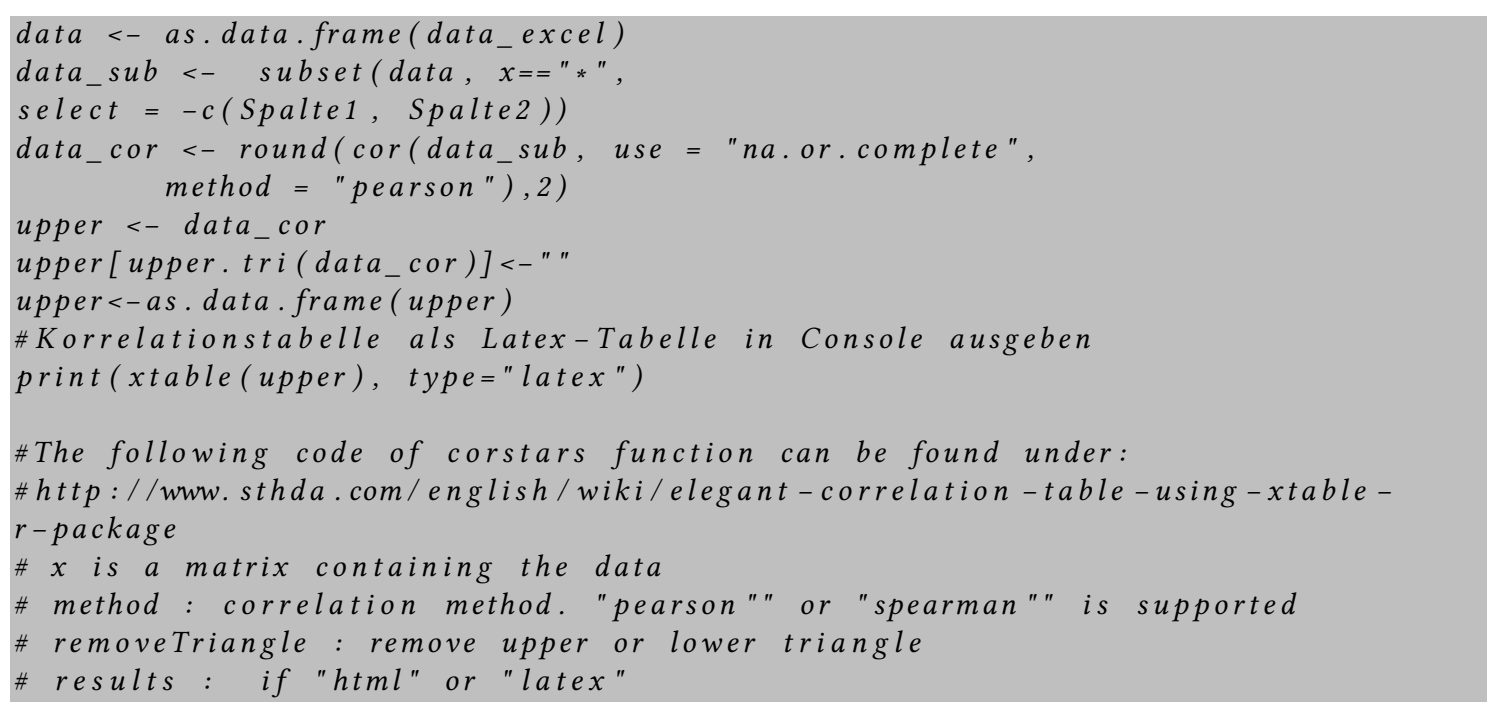




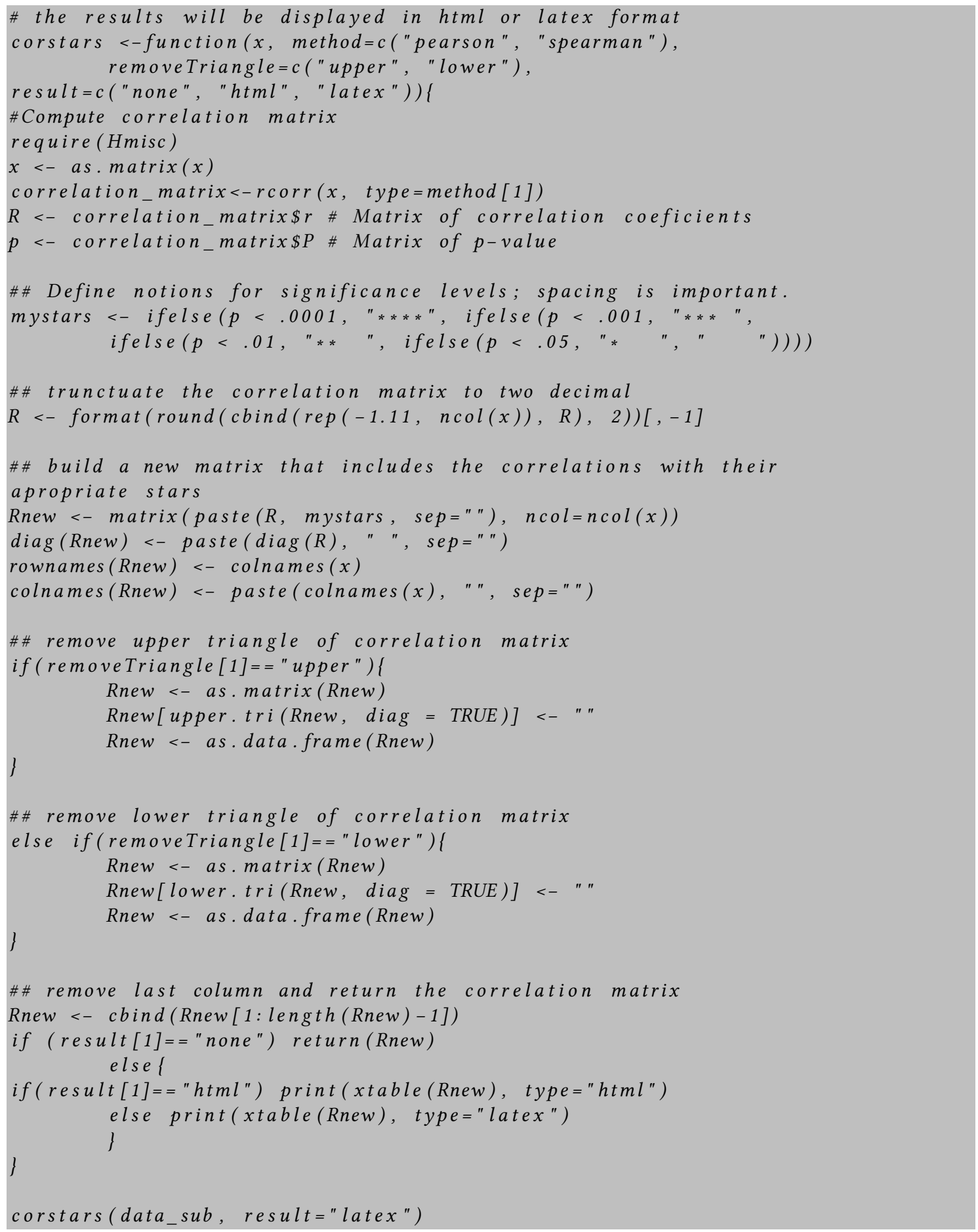




\subsection{Korrelationen mit der Wärmeleitfähigkeit}

\section{Korrelationen mit der Beschaffenheit der Faserstoffe nach der Kollektivmethode Luftstrahl-Prüfsiebung}

Tabelle 10.1: Korrelationsmatrix Fichten-Faserstoffe: Aufschlusstemperatur, SEL, Beschaffenheit nach der Kollektivmethode Luftstrahl-Prüfsiebung und Wärmeleitfähigkeit.

\begin{tabular}{rlllllll}
\hline & $\mathrm{T}$ & $\mathrm{SEL}$ & $\mathrm{Eff}$ & $\mathrm{x}_{50,3}$ & $\mathrm{x}_{97,3}$ & $\mathrm{x}_{3}$ & $\mathrm{mx}_{3}$ \\
\hline $\mathrm{T}$ & & & & & & & \\
$\mathrm{SEL}$ & $-0.63^{* *}$ & & & & & & \\
$\mathrm{EFF}$ & 0.36 & $-0.71^{* *}$ & & & & & \\
$\mathrm{x}_{50,3}$ & -0.16 & -0.26 & $0.62^{*}$ & & & & \\
$\mathrm{x}_{97,3}$ & -0.12 & -0.14 & 0.48 & $0.86^{* * * *}$ & & & \\
$\mathrm{x}_{3}$ & -0.03 & -0.17 & 0.38 & $0.71^{* *}$ & $0.97^{* * * *}$ & & \\
$\mathrm{mx}_{3}$ & -0.16 & -0.13 & 0.53 & $0.92^{* * * *}$ & $0.99^{* * * *}$ & $0.93^{* * * *}$ & \\
$\lambda_{\mathbf{1 0}}$ & $-\mathbf{0 . 7 2}$ & $\mathbf{0 . 8 1 ^ { * * * }}$ & $\mathbf{- 0 . 4 0}$ & $\mathbf{0 . 0 4}$ & $\mathbf{0 . 1 6}$ & $\mathbf{0 . 1 0}$ & $\mathbf{0 . 1 8}$
\end{tabular}

Abkürzungen: $\mathrm{T}=$ Aufschlusstemperatur, $\mathrm{SEL}=$ spezifische elektrische Leistungsaufnahme, Eff $=$ Effizienz, $x_{50,3}=$ Medianwert Prüfsieb-Maschenweite, $x_{97,3}=97 \%$-Perzentil Prüfsieb-Maschenweite, $\mathrm{x}_{3}=$ Partikelausdehnungsintervall, $\mathrm{mx}_{3}=$ mittlere Partikelausdehnung, $\lambda_{\mathbf{1 0}}=$ Wärmeleitfähigkeit. Signifikanzcode: $0,0001^{* * * *} ; 0,001^{* * *} ; 0,01^{* *} ; 0,05^{*}$

Tabelle 10.2: Korrelationsmatrix Buchen-Faserstoffe: Aufschlusstemperatur, SEL, Beschaffenheit nach der Kollektivmethode Luftstrahl-Prüfsiebung und Wärmeleitfähigkeit.

\begin{tabular}{rlllllll}
\hline & $\mathrm{T}$ & SEL & $\mathrm{Eff}$ & $\mathrm{x}_{50,3}$ & $\mathrm{x}_{97,3}$ & $\mathrm{x}_{3}$ & $\mathrm{mx}_{3}$ \\
\hline $\mathrm{T}$ & & & & & & & \\
$\mathrm{SEL}$ & $-0.63^{*}$ & & & & & & \\
$\mathrm{Eff}$ & 0.45 & $-0.72^{* *}$ & & & & & \\
$\mathrm{x}_{50,3}$ & $-0.86^{* * *}$ & 0.45 & -0.29 & & & & \\
$\mathrm{x}_{97,3}$ & $-0.66^{*}$ & $0.68^{*}$ & -0.39 & $0.65^{*}$ & & & \\
$\mathrm{x}_{3}$ & -0.56 & $0.69^{*}$ & -0.37 & 0.48 & $0.98^{* * * *}$ & & \\
$\mathrm{mx}_{3}$ & $-0.77^{* *}$ & $0.69^{*}$ & -0.39 & $0.76^{* *}$ & $0.99^{* * * *}$ & $0.93^{* * * *}$ & \\
$\lambda_{\mathbf{1 0}}$ & $-\mathbf{0 . 8 1}^{* * *}$ & $\mathbf{0 . 9 3}^{* * * *}$ & $\mathbf{- 0 . 7 0}^{* *}$ & $\mathbf{0 . 6 9}^{*}$ & $\mathbf{0 . 7 0}^{* *}$ & $\mathbf{0 . 6 8}^{*}$ & $\mathbf{0 . 7 8}^{* *}$ \\
\hline
\end{tabular}

Abkürzungen: $\mathrm{T}=$ Aufschlusstemperatur, $\mathrm{SEL}=$ spezifische elektrische Leistungsaufnahme,

Eff $=$ Effizienz, $x_{50,3}=$ Medianwert Prüfsieb-Maschenweite, $x_{97,3}=97 \%$-Perzentil Prüfsieb-Maschenweite, $\mathrm{x}_{3}=$ Partikelausdehnungsintervall, $\mathrm{mx}_{3}=$ mittlere Partikelausdehnung, $\lambda_{10}=$ Wärmeleitfähigkeit Signifikanzcode: $0,0001^{* * * *} ; 0,001^{* * *} ; 0,01^{* *} ; 0,05^{*}$

\section{Korrelationen mit der Beschaffenheit der Faserstoffe nach der Individualmethode quantitative Bildanalyse}




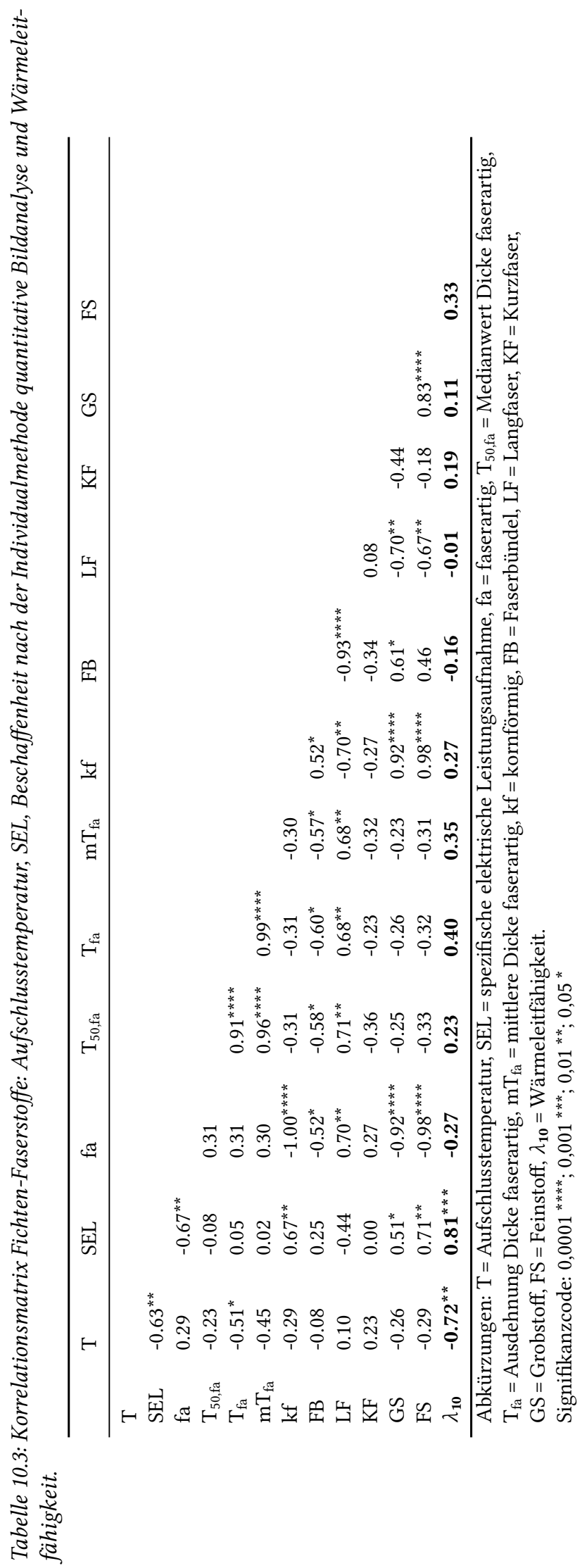




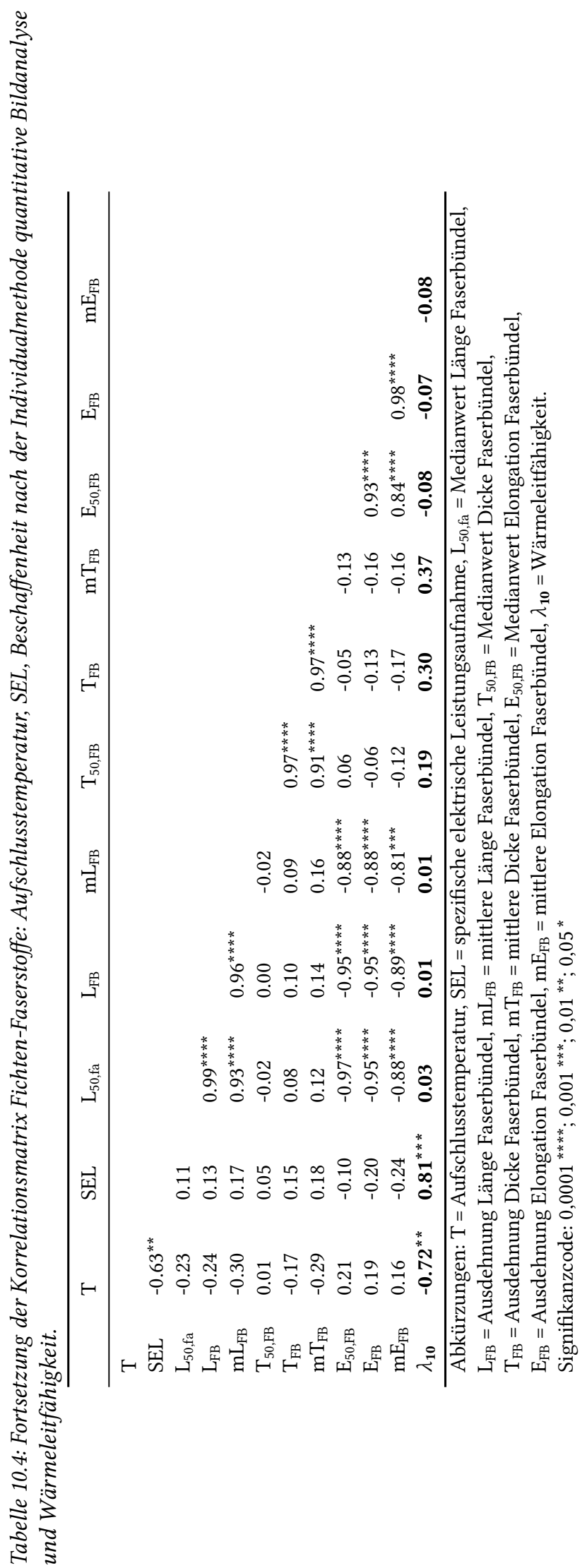




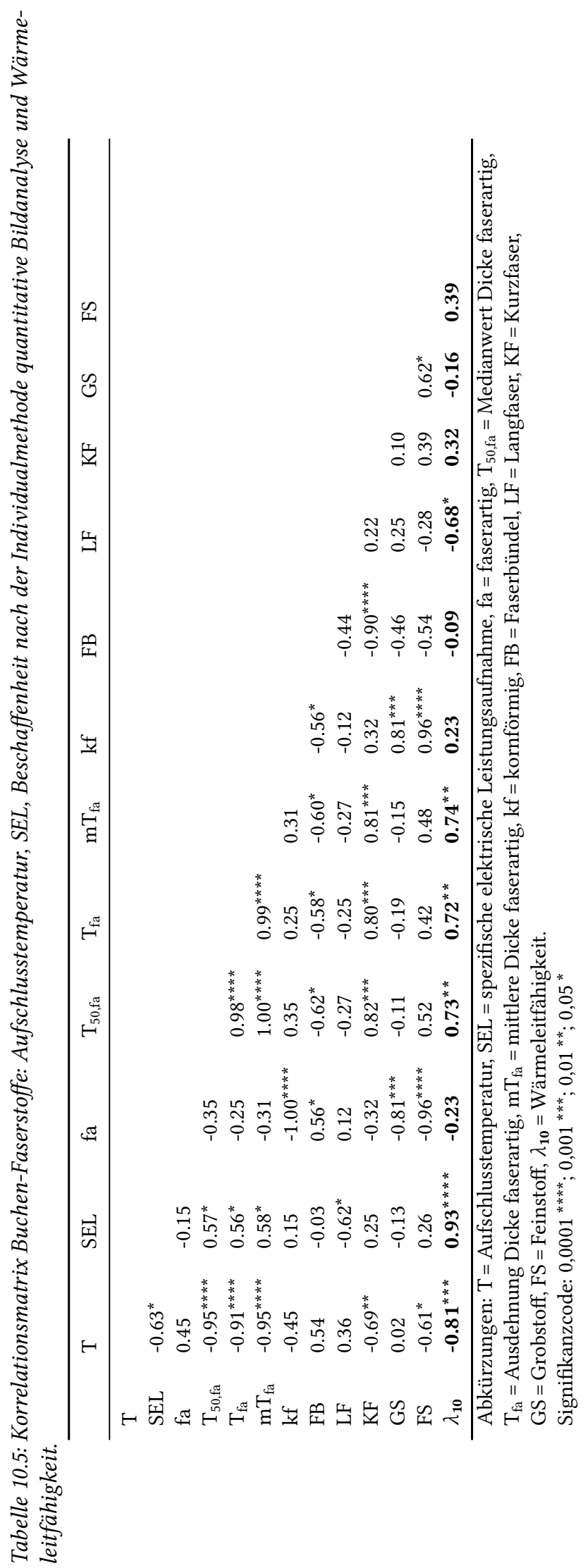




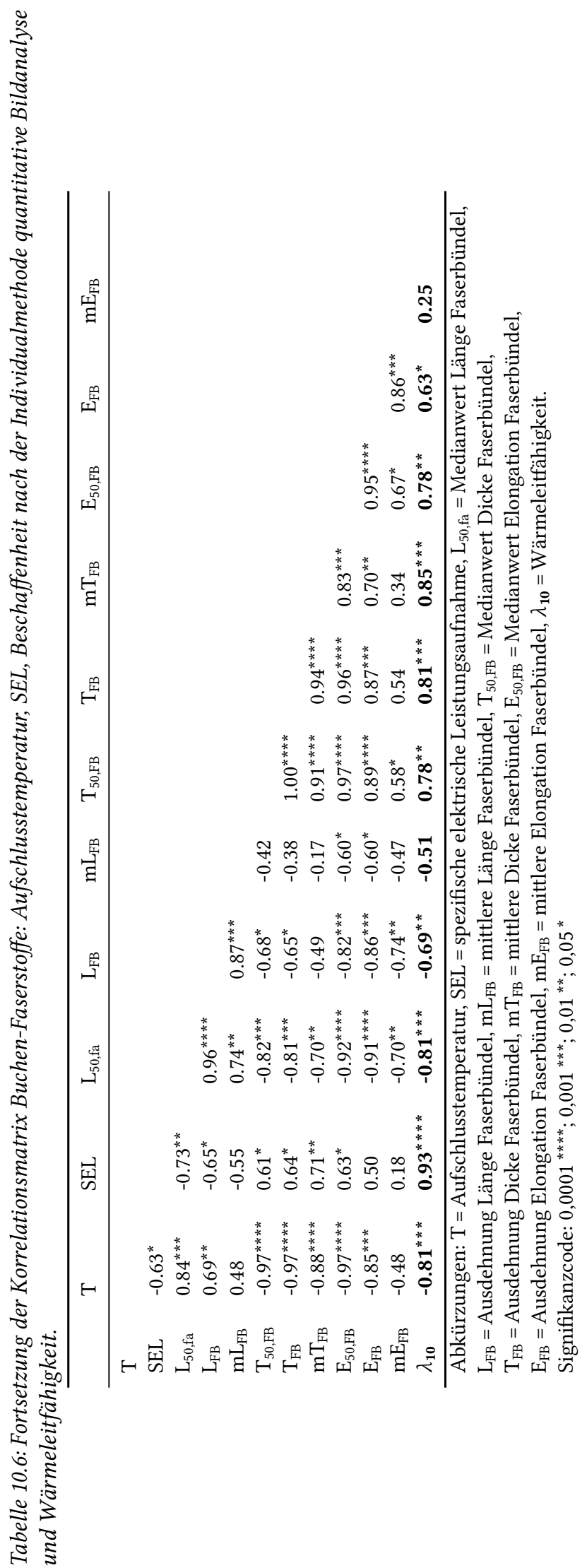




\subsection{Veröffentlichungen}

1. Barth, S., Hartmann, M., Rudolph, M., Karlinger, P., Michanickl \& A., Schemme, M. (2016). „Zusammen Maßnehmen. Verfahrensentwicklung zum Hinterspritzen von Holzfurnieren am Beispiel eines Zollstocks“. In: Kunststoffe 7/2016, S. 80-83.

2. Barth, S. \& Michanickl, A. (2017). „Fasergeometrien für Faserverbundwerkstoffe mit geringer Dichte“. Vortrag. In: 12. Holzwerkstoffkolloquium, Institut für Holztechnologie, Dresden.

3. Barth, S., Vega, J., Fuentealba, C. \& Michanickl, A. (2018). „Fiber insulation materials from Eucalyptus bark fibers - first results". Vortrag. In: 5th International Conference on Processing Technologies for Forest and Biobased Industries (PTF BPI), Technische Universität München, Freising.

4. Barth, S., Vega, F., Fuentealba, C. \& Michanickl, A. (2018). „Fiber insulation materials from Eucalyptus bark fibers - first results". In: Pro Ligno 14.4, S. 3-8.

5. Barth, S. \& Michanickl, A. (2018). „Möglichkeiten zur Optimierung der Herstellung von Holzfaserdämmstoffen “. Vortrag. In: 3. Kooperationsforum Holz als neuer Werkstoff, Bayern Innovativ, Regensburg.

6. Barth, S. \& Michanickl, A. (2019). „Importance of refiner plates for wood fiber insulation materials“. Vortrag. In: International Wood Days 2019, LAMK, Lahti, Finland.

7. Schmid, T., Barth, S. \& Michanickl, A. (2019). „Wood fiber composites for lightweight construction “. Vortrag. In: International Wood Days 2019, LAMK, Lahti, Finland.

8. Barth, S. \& Michanickl, A. (2019). „Study of refiner plates as a possibility to improve the production of wood fiber insulation materials". Vortrag. In: SWST 62nd International Convention, Society for Wood Science and Technology, Fish Camp (CA), USA.

9. Obermeier, F., Schumacher, M., Barth, S., Karlinger, P., Schemme, M. \& Michanickl, A. (2019). „Thermoplastische Hybridverbunde mit Holzfaserverstärkung“. Vortrag. In: Internationale Fachtagung Technomer, Technische Universität Chemnitz.

10. Barth, S., Schmid, T. \& Michanickl, A. (2019). „Bindemitteleinsatz und Mahlplattenentwicklung in der Herstellung von Holzfaserdämmstoffen “. Vortrag. In: 13. Holzwerkstoffkolloquium, Institut für Holztechnologie, Dresden.

11. Obermeier, F., Schumacher, M., Barth, S., Karlinger, P., Schemme, M. \& Michanickl, A. (2019). „Thermoplastische Holzfaservliese für Hybridverbunde“. Vortrag. In: 13. Holzwerkstoffkolloquium, Institut für Holztechnologie, Dresden.

12. Schumacher, M., Obermeier, F. Barth, S., Karlinger, P., Michanickl, A. \& Schemme, M. (2020). „Eignung von thermo-mechanischen Holzfasern in Vliesstoffhybridverbunden“. In: Holztechnologie 61.2, S. 40-45.

13. Barth, S. \& Michanickl, A. (2020). „Refiner plates for wood fiber based materials“. Vortrag. In: International Wood Days 2020, LAMK, Lahti, Finland. 
14. Schmid, T., Barth, S. \& Michanickl, A. (2020): „Wood fiber reinforced composites \& nonwovens. New perspectives on the usage of wood fibers". Vortrag. In: International Wood Days 2020, LAMK, Lahti, Finland.

15. Obermeier, F., Schumacher, M., Barth, S., Karlinger, P., Schemme, M. \& Michanickl, A. (2020). "Thermoplastische Hybridverbunde mit Holzfaserverstärkung: Leichtbau mit Naturfasern“. In: Plastverarbeiter 2/2020, S. 34-36.

16. Obermeier, F., Schumacher, M., Barth, S., Karlinger, P., Michanickl, A., Schemme, M. \& Altstädt, V. (2021). „Verstärkung von Polypropylen mit Holzfasern durch Direkt-Compoundierung“. In: Zeitschrift Kunststofftechnik 17.2, S. 88-111.

17. Barth, S., Schmid, T. \& Michanickl, A. (2021). „A method to determine the wetting of wood fibers with thermoplastic". In: Wood and Fibre Science 53.2, S. 157-159. 